\title{
Group-level cortical functional connectivity patterns using fNIRS: Assessing the effect of bilingualism in young infants
}

This document includes the following information:

- fNIRS device specifications, and information describing the data preprocessing and analysis steps, parameters and functions employed in this study (supplementary table 1).

- Group level data quality assessment and replication analysis (supplementary figure 1).

- ICA model order selection (supplementary figure 2).

- Supplementary results: temporal group ICA analysis (supplementary figures 3 and 4 and supplementary table 2).

- Supplementary results: example of functional connectivity matrices at the individual and group level (supplementary figure 5).

- Supplementary results: connICA analysis (supplementary figure 6 and supplementary table 3).

- References.

- Data quality assessment figures. 


\begin{tabular}{|c|c|c|}
\hline \multicolumn{3}{|c|}{ SYSTEM SPECIFICATIONS } \\
\hline Company and model & \multicolumn{2}{|l|}{ NIRx NIRScout, CW-NIRS device } \\
\hline Wavelengths & \multicolumn{2}{|l|}{$[760,850]$} \\
\hline Sampling frequency & \multicolumn{2}{|l|}{$8.93 \mathrm{~Hz}$} \\
\hline Number of sources & \multicolumn{2}{|l|}{16 (14 analysed - occipital excluded) } \\
\hline Number of detectors & \multicolumn{2}{|l|}{24 (19 analysed - occipital excluded) } \\
\hline Number of channels & \multicolumn{2}{|l|}{52 (46 analysed - occipital excluded) } \\
\hline Channel localization & \multicolumn{2}{|l|}{ Head-based fiducial locations (10-20 system) } \\
\hline Source-Detector distances & \multicolumn{2}{|l|}{ [20 - 45 mm, see main text Fig. 1] } \\
\hline \multicolumn{2}{|r|}{ PARAMETERS } & FUNCTION/TOOLBOX \\
\hline \multicolumn{3}{|l|}{ Data preprocessing } \\
\hline 1) Intensity to optical density & & Homer $^{l}$ : hmrIntensity2OD \\
\hline 2) Motion detection and censoring & Visually & Matlab: Plot \\
\hline 3) Motion correction & $\begin{array}{l}\text { [Wavelet: } \mathrm{d} 4 \text {; threshold: } 0.02 \text {; boundary: reflection; } \\
\text { chsearch: moderate; nscale: extreme] }\end{array}$ & BrainWavelet Toolbox ${ }^{2}$ \\
\hline 4) Optical density to $\mathrm{HbO}$ and $\mathrm{HbR}$ & DPF: $[5.3,4.2]$ & Homer2: hmrOD2conc \\
\hline 5) Filter and global signal regression & HPF: $4^{\text {th }}$ order Legendre polynomials; LPF: $0.09 \mathrm{~Hz}$ & In house script: GitHub \\
\hline \multicolumn{3}{|l|}{ Data analysis } \\
\hline $\begin{array}{l}\text { Temporal group ICA } \\
\text { connICA }\end{array}$ & $\begin{array}{l}15 \text { independent components } \\
11 \text { independent components }\end{array}$ & $\begin{array}{l}\text { ICASSO Toolbox }{ }^{3} \\
\text { ConnICA Toolbox }\end{array}$ \\
\hline \multicolumn{3}{|l|}{ Statistical analysis } \\
\hline $\begin{array}{l}\text { One-way analysis of variance - } \\
\text { ANOVA }\end{array}$ & Language background [Bilingual, Spanish, Basque] & Matlab: anova1 \\
\hline Bayesian statistics - ANOVA & Language background [Bilingual, Spanish, Basque] & $J A S P$ \\
\hline
\end{tabular}

Supplementary Table 1. Information describing the fNIRS device specifications, and the data preprocessing and analysis steps, parameters and functions employed in this study. CW: Continuous wave; DPF: Differential pathlength factor; HPF: High pass filter; LPF: Low pass filter; ICA: Independent component analysis.

In house scripts will be made publicly available at: https://github.com/borjablanco/RS_4months

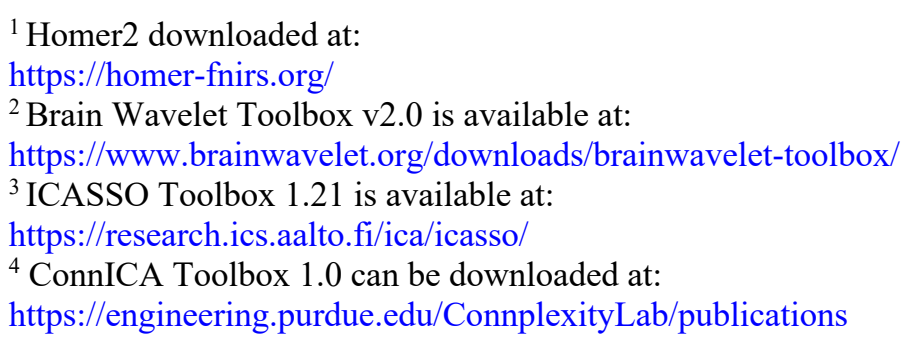



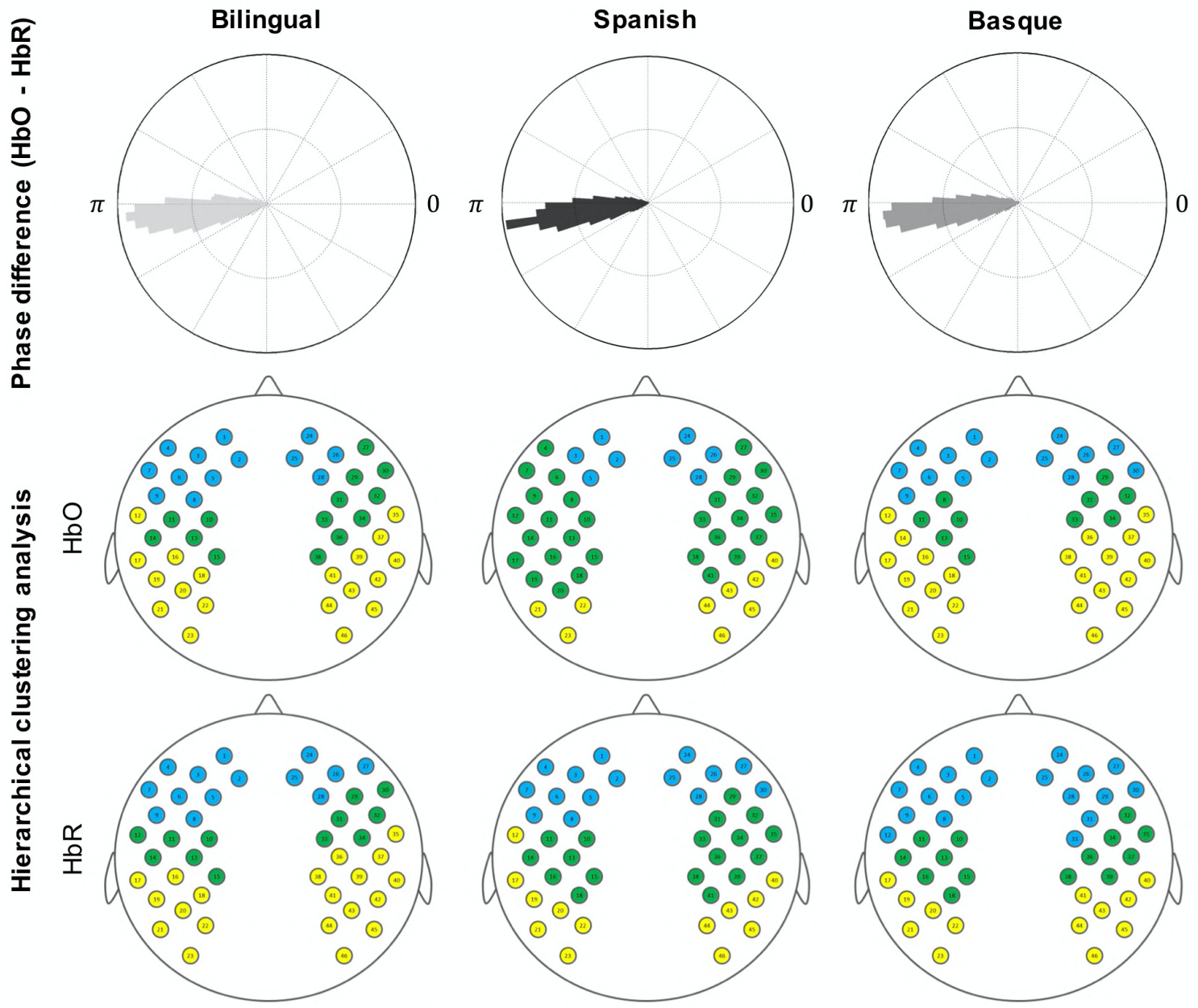

Cluster 1

Cluster 2

Cluster 3

Supplementary Figure 1. Group-level data quality assessment plots replicating two previous infant resting-state functional connectivity studies using fNIRS. First row shows the channelwise average phase difference (hPod value, Watanabe et al., 2017) between $\mathrm{HbO}$ and $\mathrm{HbR}$ in each experimental group. The three groups show a similar pattern characterized by an antiphase state between $\mathrm{HbO}$ and $\mathrm{HbR}$, replicating previous outcomes. Second and third rows in this figure show the results of a hierarchical clustering approach (Homae et al., 2010) in which channels' time series are clustered based on similarity. A similar cluster configuration can be observed across groups in $\mathrm{HbO}$ and $\mathrm{HbR}$. Cluster 1 is formed by channels located in the most anterior part of both hemispheres. Cluster 2 comprises channels located in middle brain regions. Channels located in the most posterior part of the setup are grouped together in cluster 3 . 


\section{Model order selection for Independent Component Analysis}

A recurrent issue in studies using independent component analysis (ICA) to examine resting-state functional connectivity is how to determine the number of IC to be estimated. Here we propose a data-driven approach to determine the number of estimated ICA components by computing two metrics that exploit specific defining properties of ICA estimation and fNIRS data: 1) the robustness of the components across multiple realization of the ICA (Himberg et al., 2004) and 2) the assumed similarity between the $\mathrm{HbO}$ and $\mathrm{HbR}$ derived IC.

First, a valid interpretation of the results requires selecting only those independent components that are considered robust; being robustness defined by the identifiability of the component across multiple runs of the ICA algorithm with different random initializations. A principal component analysis (PCA) is commonly performed prior to ICA to reduce the dimensionality of the data and/or remove noise components. The number of ICA components to be estimated usually corresponds with the number of PCA components that are retained. We evaluated the robustness of the estimated IC across multiple PCA thresholds by using ICASSO (Himberg et al., 2004; Damaraju et al., 2014). Concretely, 100 realizations of ICA were computed for different PCA thresholds (i.e., 60, 65, 70, 75, 80, 85, 90, 95 and 99) representing the percentage of data variance explained. For the two ICA methods employed in the current work (temporal group ICA and connICA), ICASSO quality index (Iq) values were obtained for each component and for each PCA threshold (Supplementary Figure 2 - volcano plots). This index quantifies the robustness of the estimated components across ICA realizations, with values ranging between 0 (low robustness) and 1 (high robustness). In the temporal group ICA approach, higher $I q$ values (i.e. more robustness) were obtained at the lower PCA thresholds (60\% and $65 \%$ explained variance). For higher thresholds, the first 15-20 IC also showed high Iq values. However, these values start decaying as the number of IC increased and were particularly low for PCA thresholds above $80 \%$. A similar trend can be observed for connICA. The highest $I q$ values were obtained at the lower PCA thresholds ( $60 \%-70 \%$ explained variance), whereas the $I q$ values for higher PCA thresholds were only high up to the first 15 IC and then progressively decreased.

Second, the expected statistical association between $\mathrm{HbO}$ and $\mathrm{HbR}$ chromophores can be used as a metric for evaluating the validity of the IC extracted from the ICA algorithm. A high correlation between the extracted $\mathrm{HbO}$ and $\mathrm{HbR}$ IC or maps implying consistency across $\mathrm{Hb}$ chromophores can be interpreted as a measure of physiological reliability (Villringer and Chance, 1997; Wolf et al., 2002; Obrig and Villringer, 2003). In the temporal group ICA approach, IC are estimated from $\mathrm{HbO}$ and $\mathrm{HbR}$ time series, which are expected to be negatively correlated. A highly similar spatial configuration in the extracted spatial maps of both chromophores (i.e., channel weights) is expected, but with opposite sign (i.e., negative correlation). In our temporal group ICA analysis $\mathrm{HbO}$ and $\mathrm{HbR}$ spatial maps displayed a strong negative correlation at the lower PCA thresholds - up to $75 \%$ of explained variance (Supplementary Figure 2 - volcano plots). For higher thresholds, correlation between $\mathrm{HbO}$ and $\mathrm{HbR}$ components remained negative, but values decreased considerably. For the connICA approach, a strong positive correlation between the $\mathrm{HbO}$ and $\mathrm{HbR}$ independent functional components is expected, as they are estimated from the $\mathrm{HbO}$ and $\mathrm{HbR}$ functional connectivity matrices, which show alike topology across chromophores. The analysis for the connICA method yielded similar results as the previous method. At the lower PCA thresholds $(60 \%$ and $65 \%$ explained variance) the correlation between $\mathrm{HbO}$ and $\mathrm{HbR}$ components was notably high $(r \geq$ 0.7). At higher PCA thresholds, correlation between components remained positive, but with lower correlation values between $\mathrm{HbO}$ and $\mathrm{HbR}$ components.

Based on these two metrics (i.e., robustness of IC, and correlation between the extracted IC from $\mathrm{HbO}$ and $\mathrm{HbR}$ data) an appropriate choice for PCA model order, and in turn for the subsequent ICA method, corresponds to approximately $60 \%$ of the explained variance in both approaches (i.e., tGICA and connICA). To reinforce this choice, the variance explained by each IC was computed by calculating the accumulative data variance explained by the components. This analysis showed that for the two approaches with the selected $60 \%$ threshold, we are able to explain the largest amount of data variance, while also obtaining the highest values in our 
robustness and consistency metrics. At higher PCA thresholds, explaining the same amount of variance would require including components that are not robust, or which do not show the expected association between $\mathrm{HbO}$ and $\mathrm{HbR}$. Based on this information, in the temporal group ICA approach we selected 15 principal components corresponding to $60 \%$ explained variance. As a reference, a method for automatic dimensionality selection based on Laplace approximation (Minka et al., 2001) yielded a result of 27 components (i.e., $73.5 \%$ explained variance). For the connICA method we considered 11 principal components, which corresponds to $60 \%$ explained variance. For this method the Laplacian approximation produced a value of 17 components (i.e., $66.34 \%$ explained variance).
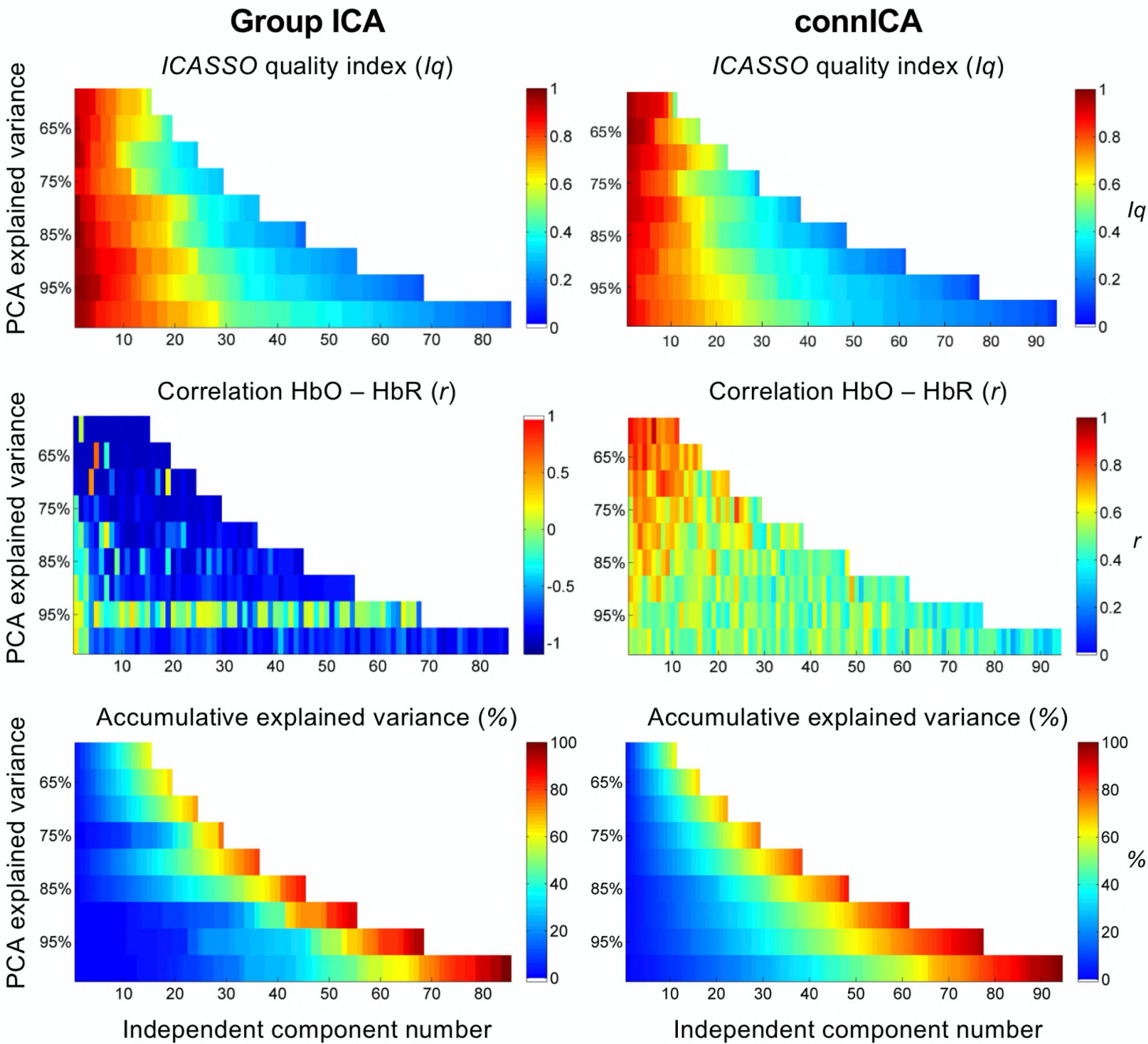

Accumulative explained variance (\%)

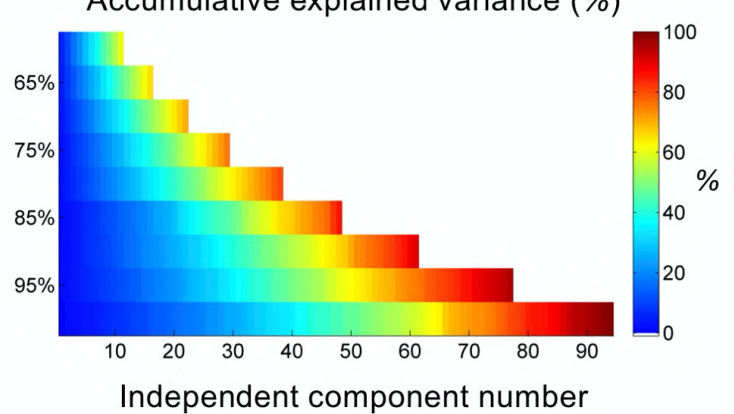

Supplementary Figure 2. Model order selection criteria for temporal group ICA (first column) and connICA (second column) methods at different PCA thresholds indicating the percentage of explained variance (y axis). First row depicts the cluster quality index (Iq) for each component as estimated using ICASSO, which represents component stability across multiple ICA realizations. Second row shows the correlation between the estimated $\mathrm{HbO}$ and $\mathrm{HbR}$ components. Third row shows the components' accumulative explained variance, being the maximum amount of explained variance determined by the specific PCA threshold. 


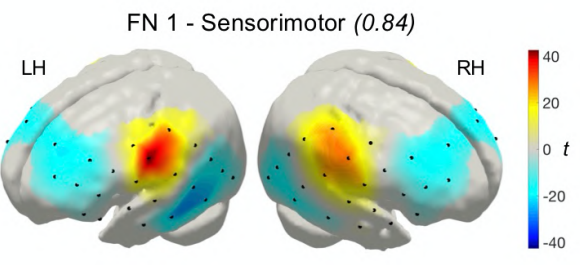

FN 2 - Sensorimotor (0.76)

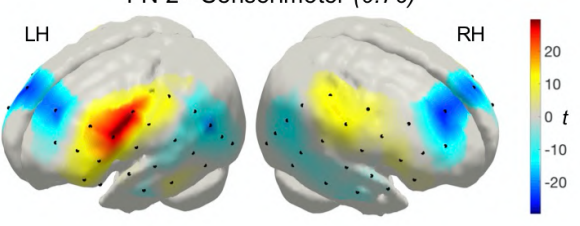

FN 3 - Sensorimotor (0.72)

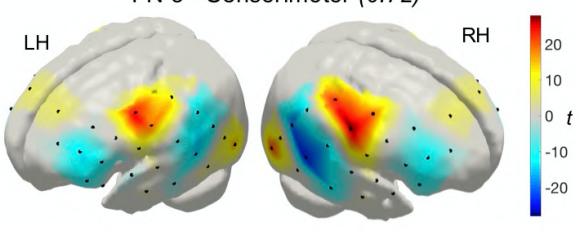

FN 4 - Auditory (0.49)

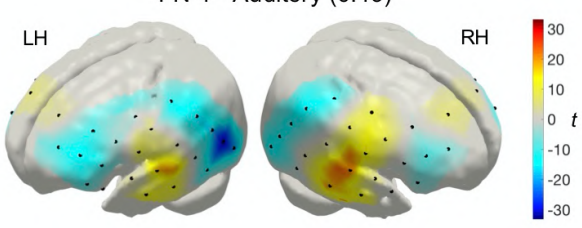

FN 5 - Language (0.89)

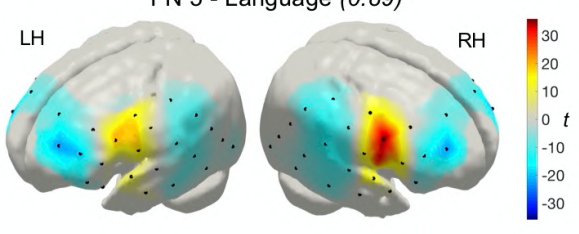

FN 6 - Frontal (0.91)

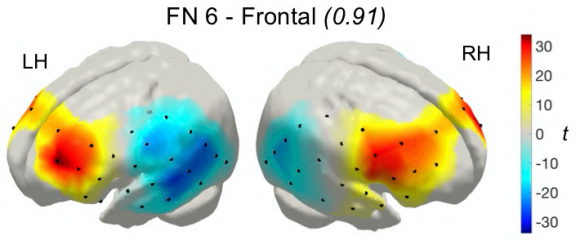

FN 7 - Default-Mode (0.8)

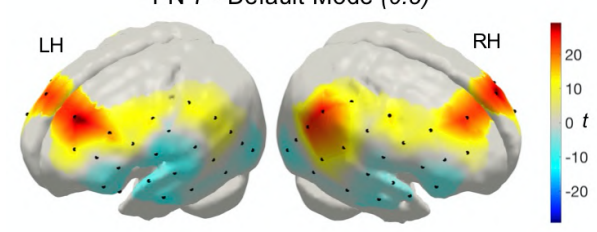

$\mathrm{HbO}$

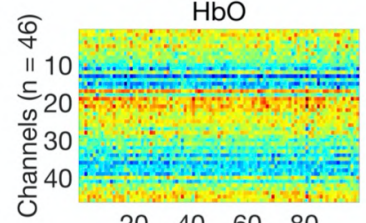

$\mathrm{HbO}$

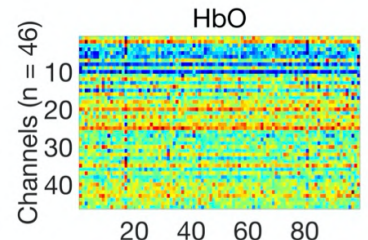

$\mathrm{HbO}$

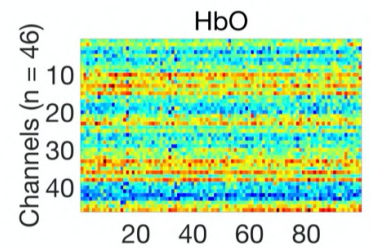

$\mathrm{HbO}$

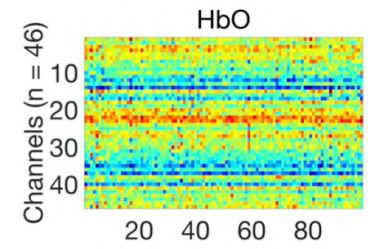

$\mathrm{HbO}$

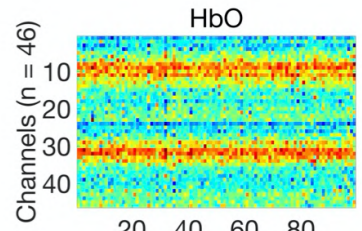

$20 \quad 40 \quad 60 \quad 80$
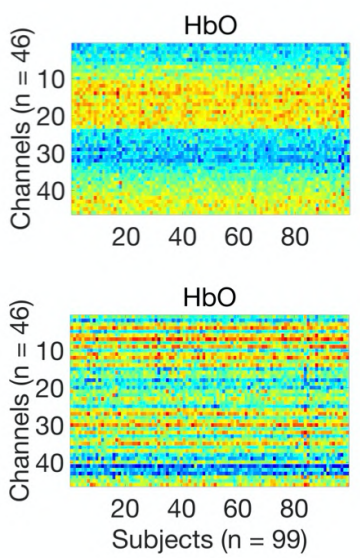

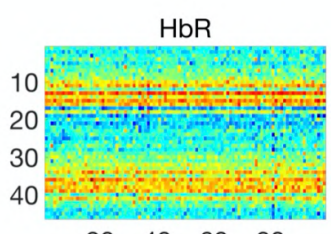

$20 \quad 40 \quad 60 \quad 80$

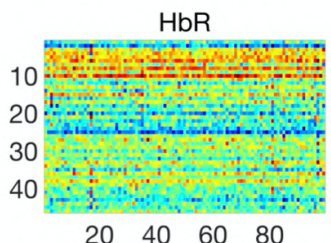

$20 \quad 40 \quad 60 \quad 80$

$\mathrm{HbR}$

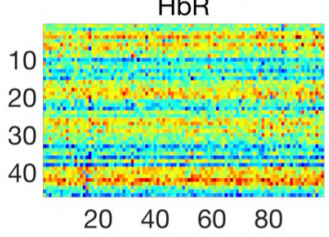

$\mathrm{HbR}$

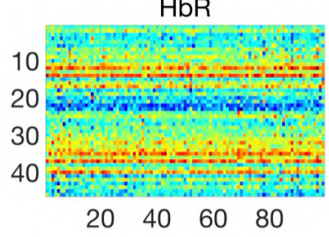

$\mathrm{HbR}$

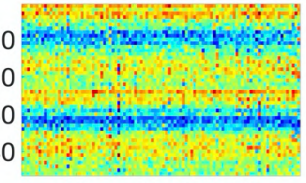

$20 \quad 40 \quad 60 \quad 80$

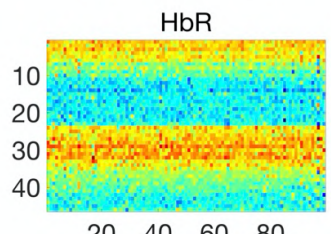

$20 \quad 40 \quad 60 \quad 80$

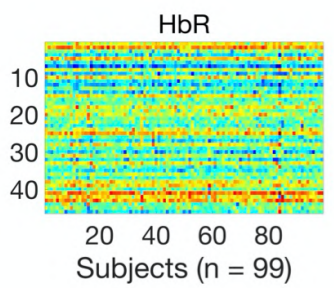

Figure continues in next page 


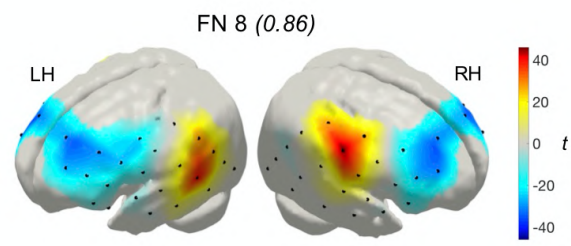

FN $9(0.82)$

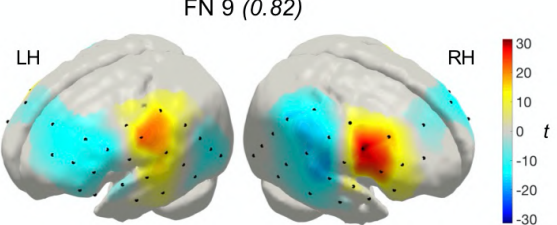

FN $10(0.77)$

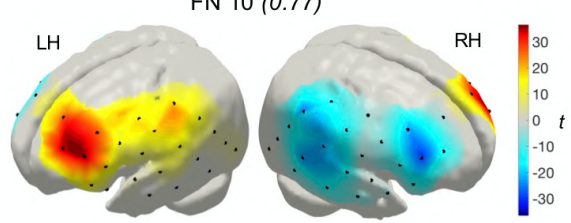

FN $11(0.73)$
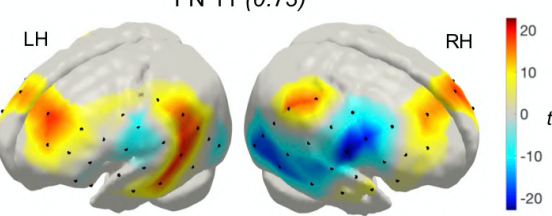

FN $12(0.68)$
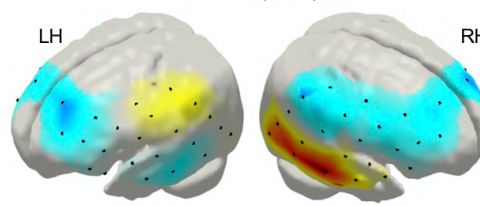

$\mathrm{RH}$
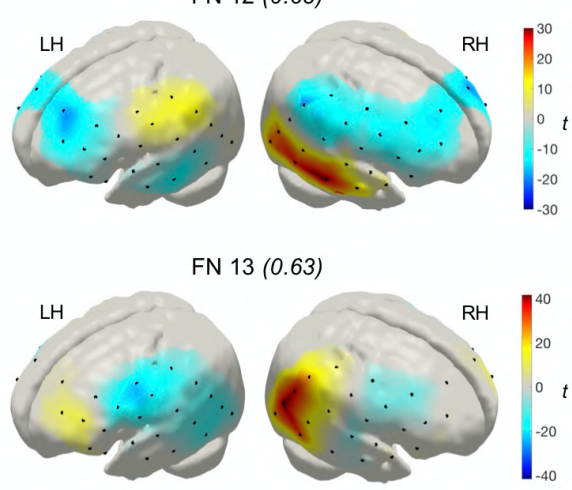

FN $14(0.63)$
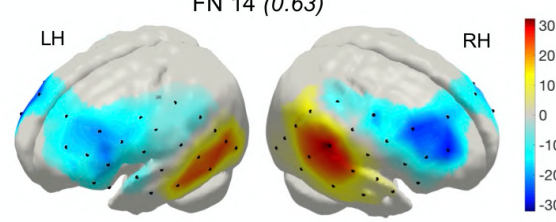

FN $15(0.91)$
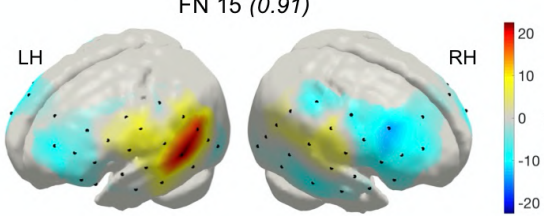
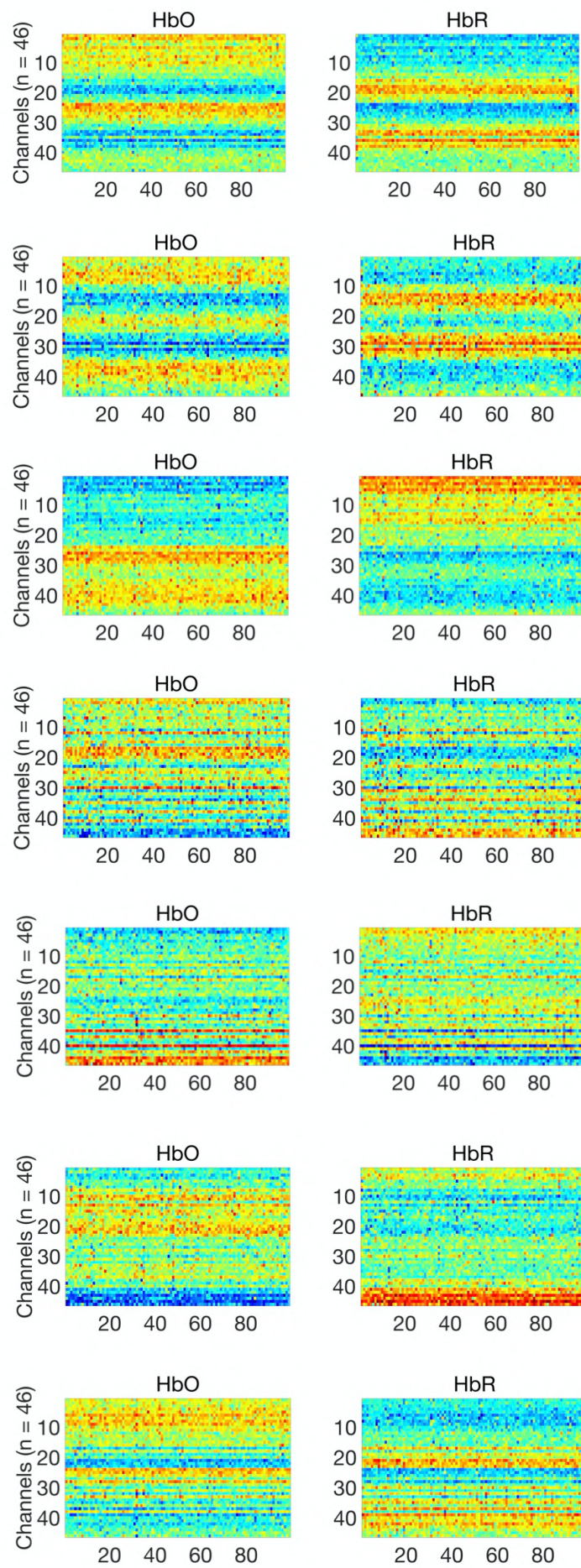

$\mathrm{HbR}$
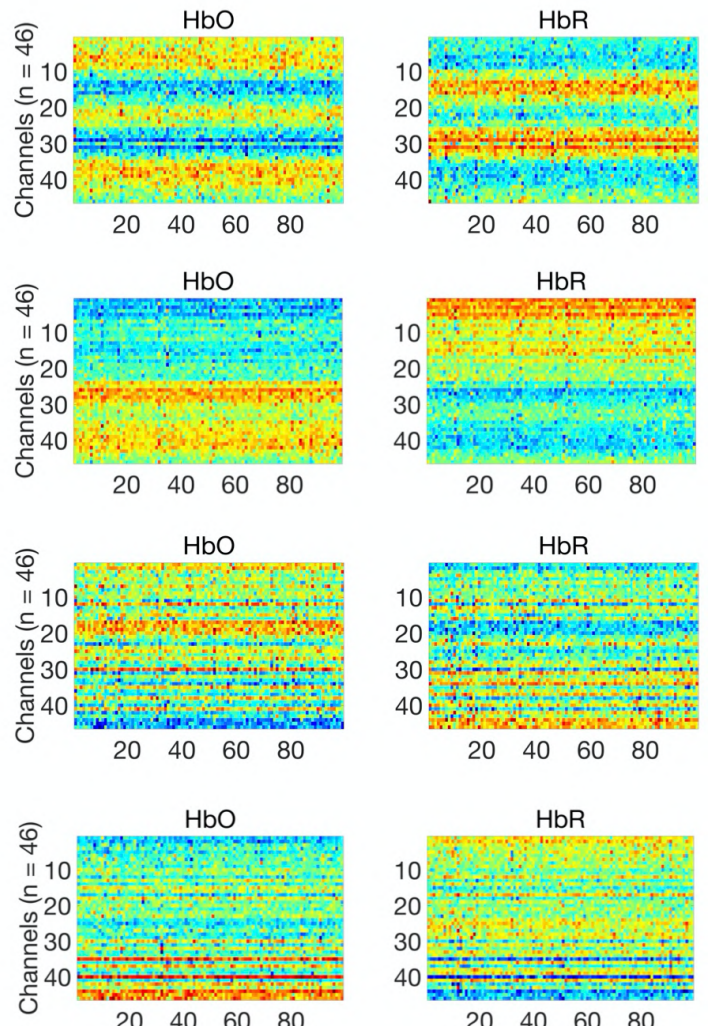

$20 \quad 40 \quad 60 \quad 80$
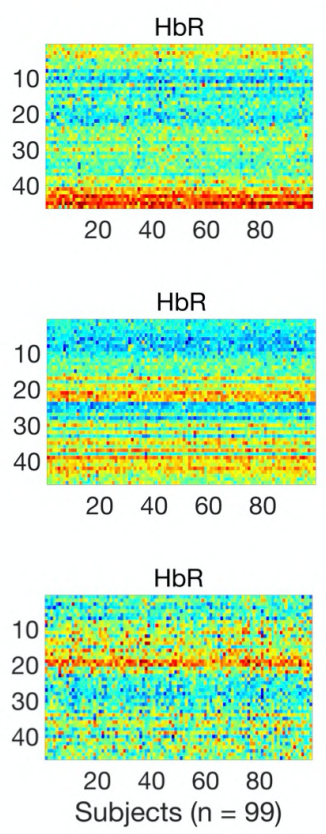

Supplementary Figure 3. Functional networks extracted with the temporal group ICA approach ( $\mathrm{n}=$ 15 , Iq values appear in parentheses). Due to the high similarity between $\mathrm{HbO}$ and $\mathrm{HbR}$ components, the associated spatial maps are displayed for HbR only. Functional networks were reconstructed to the subject space using dual regression. Subject-level maps for $\mathrm{HbO}$ and $\mathrm{HbR}$ are represented in matrix form in the middle and rightmost columns. 


\begin{tabular}{|c|c|c|c|c|}
\hline Funtional Network (FN) & $I q$ & $r$ & $\begin{array}{c}\text { ssd }(\%) \\
\text { total }=100\end{array}$ & $\begin{array}{c}\text { ssd (\%) } \\
\text { total }=60\end{array}$ \\
\hline FN 1 Sensorimotor & 0.84 & -0.99 & 8.8 & 4.1 \\
\hline FN 2 Sensorimotor & 0.76 & -0.98 & 4.3 & 3.9 \\
\hline FN 3 Sensorimotor & 0.72 & -0.99 & 5.5 & 3.9 \\
\hline FN 4 Auditory & 0.49 & -0.98 & 5.6 & 3.9 \\
\hline FN 5 Language & 0.89 & -0.99 & 7.4 & 4.0 \\
\hline FN 6 Frontal & 0.91 & -0.99 & 10.5 & 4.2 \\
\hline FN 7 Default-Mode & 0.80 & -0.97 & 6.2 & 4.0 \\
\hline FN 8 & 0.86 & -0.99 & 8.9 & 4.1 \\
\hline FN 9 & 0.82 & -0.99 & 7.2 & 4.0 \\
\hline FN 10 & 0.77 & -0.98 & 7.3 & 4.0 \\
\hline FN 11 & 0.73 & -0.98 & 4.5 & 3.9 \\
\hline FN 12 & 0.68 & -0.98 & 5.7 & 3.9 \\
\hline FN 13 & 0.63 & -0.98 & 5.1 & 3.9 \\
\hline FN 14 & 0.63 & -0.99 & 9.0 & 4.1 \\
\hline FN 15 & 0.91 & 0.02 & 3.8 & 3.8 \\
\hline
\end{tabular}

Supplementary Table 2. Temporal group ICA model order evaluation metrics for the PCA threshold selected in this study (i.e., $60 \%$ - 15 ICs). Sum of squared differences (ssd) are computed with respect to the data after PCA (total $=100 \%$ ) and with respect to the original data without PCA (total $=60 \%$ ). $\mathrm{Iq}=I C A S S O$ cluster quality index; $\mathrm{r}=$ correlation coefficient between $\mathrm{HbO}$ and $\mathrm{HbR}$ spatial maps. 

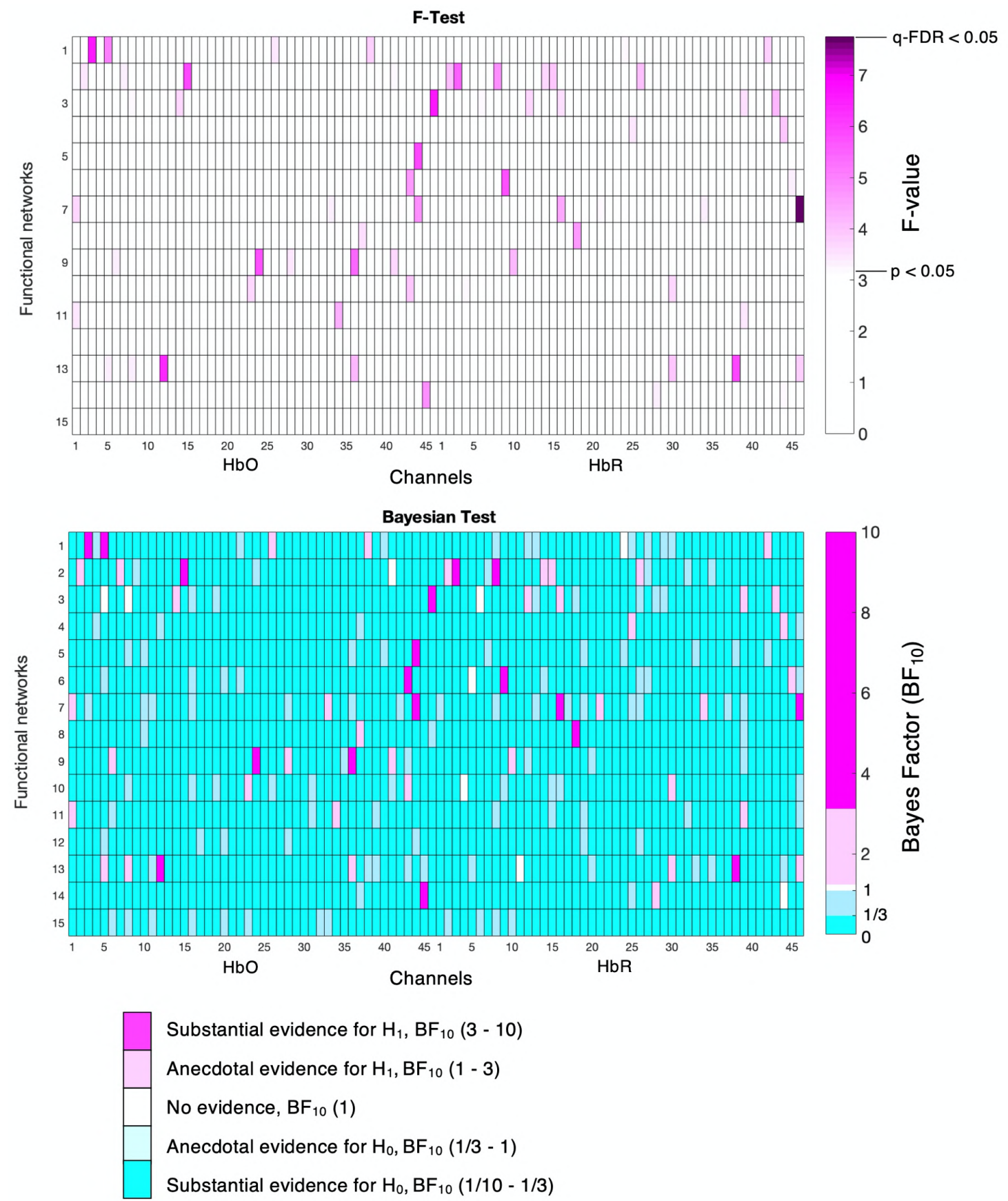

Supplementary Figure 4. Results of channelwise between group statistical comparisons (i.e., frequentist F-test) on the prominence of the extracted functional networks showed weak effects on isolated channels which were not consistent across $\mathrm{HbO}$ and $\mathrm{HbR}$ and which not survived multiple comparisons correction (FDR corrected among 46 channels, $\mathrm{q}<0.05$ ). Group comparisons based on Bayesian statistics (Wetzles and Wagenmakers, 2012; Keysers et al., 2020) showed an overall increased likelihood for the null model $\mathrm{H}_{0}$. A partial correspondence between frequentist and Bayesian tests can be observed in those channels showing potential evidence for $\mathrm{H}_{1}$, but these effects are only present on isolated channels, and are not consistent across $\mathrm{HbO}$ and $\mathrm{HbR} . \mathrm{BF}_{10}=$ Bayes Factor. 


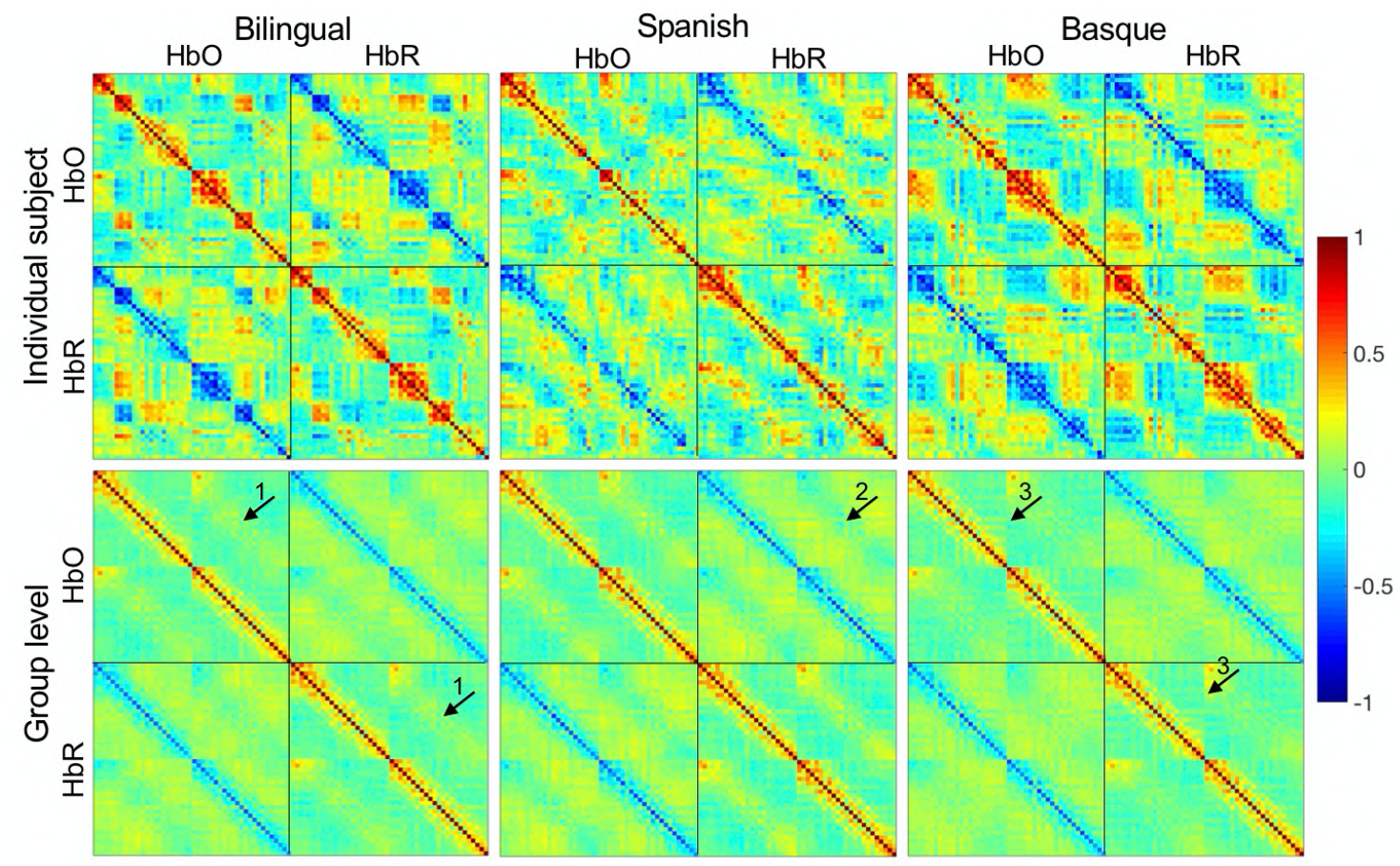

Supplementary Figure 5. Functional connectivity matrices of representative individual subjects (first row) and at the group level (second row) for each experimental group. Functional connectivity matrix for $\mathrm{HbO}$ and $\mathrm{HbR}$ is shown in the top-left part and bottom-right part, respectively. Functional connectivity matrix representing the correlation between $\mathrm{HbO}$ and $\mathrm{HbR}$ is shown in the top-right part. Plots are symmetric with respect to the main diagonal. Channels are ordered based on their location, from anterior to posterior and from left to right. This allows visualizing an increased correlation between homotopic channels in $\mathrm{HbO}$ and $\mathrm{HbR}$ (see arrow 1), an increased negative correlation between homotopic channels between $\mathrm{HbO}$ and $\mathrm{HbR}$ (see arrow 2) and a clear delimitation of left and right hemispheres (see arrow 3). 

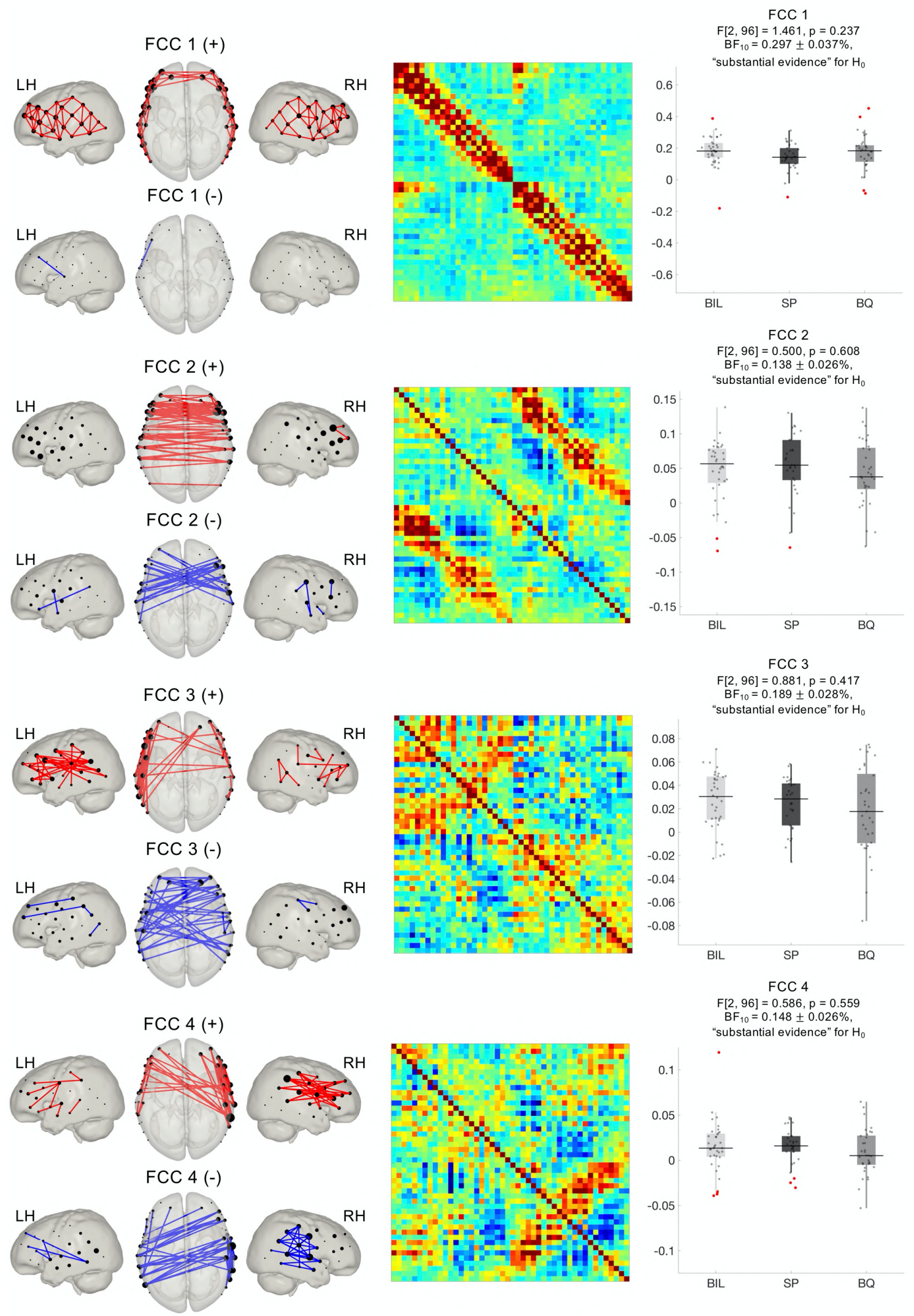

Figure continues in next page 

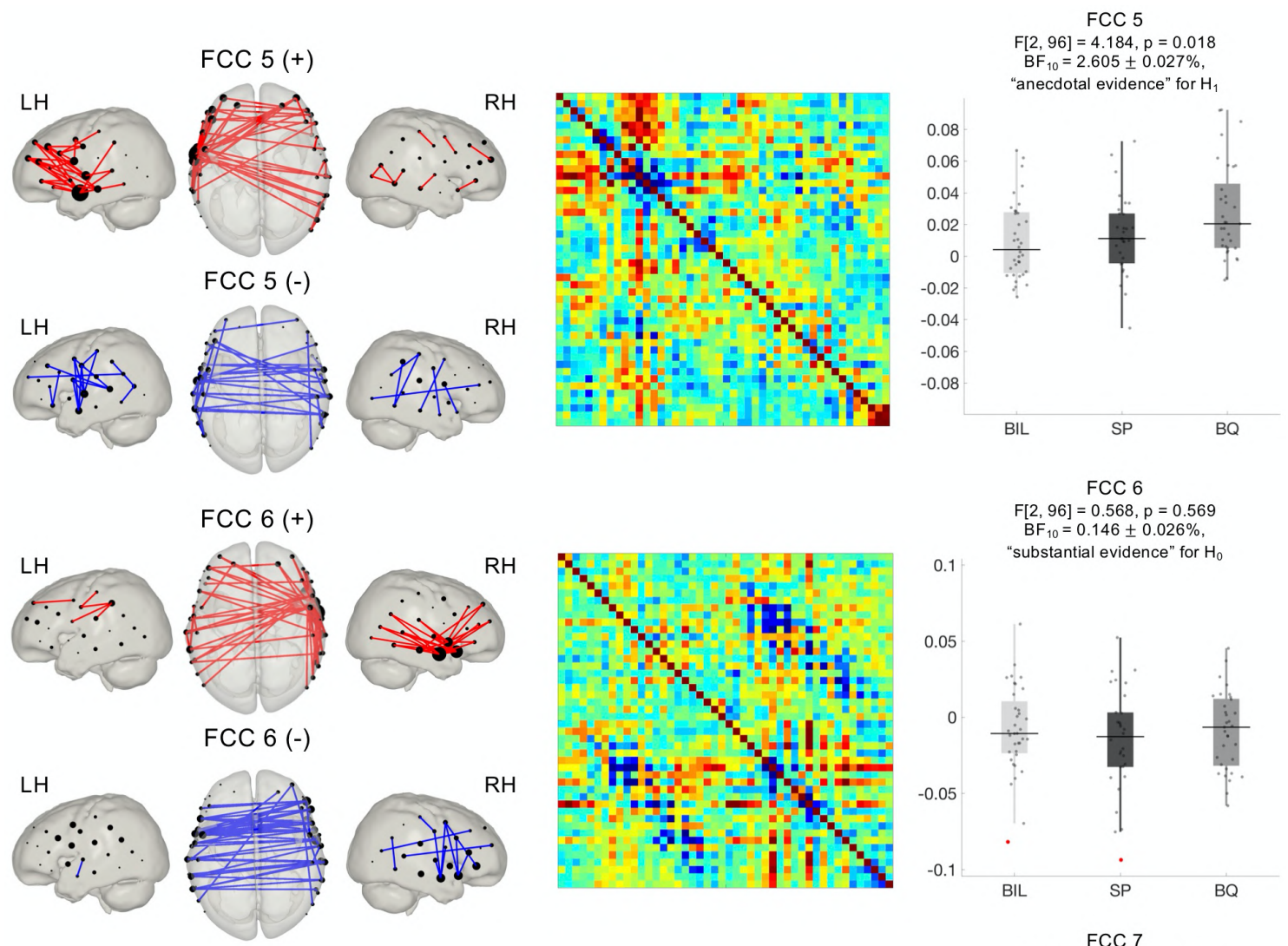

FCC 7 (+)
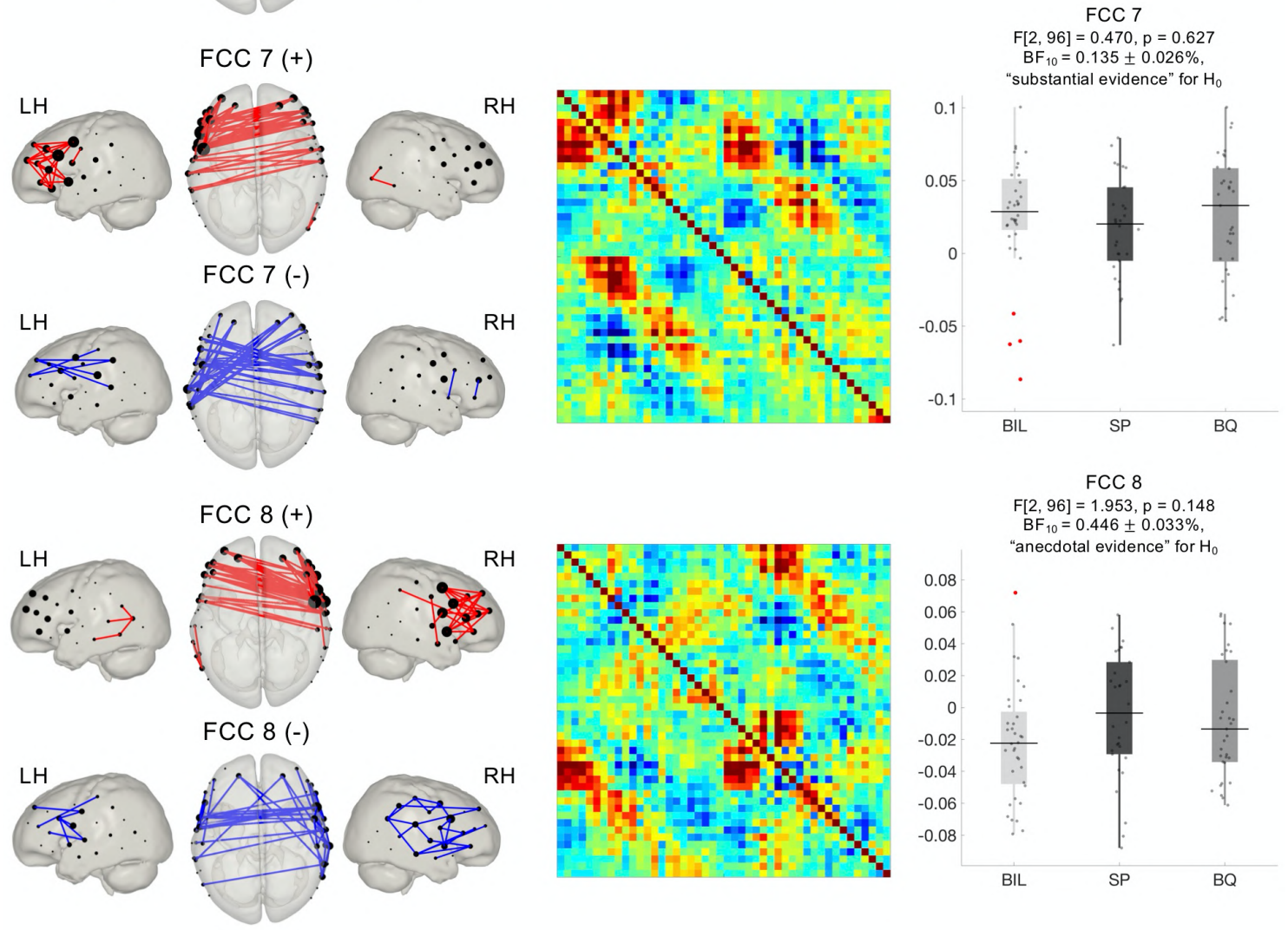

Figure continues in next page 

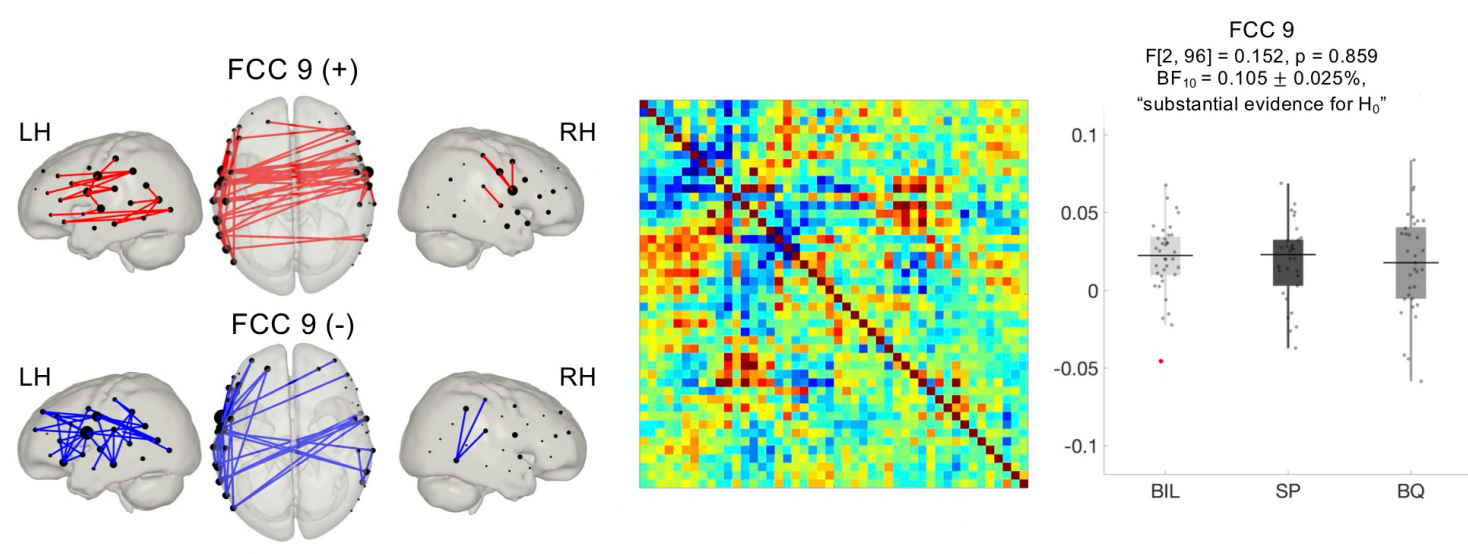

FCC $10(+)$
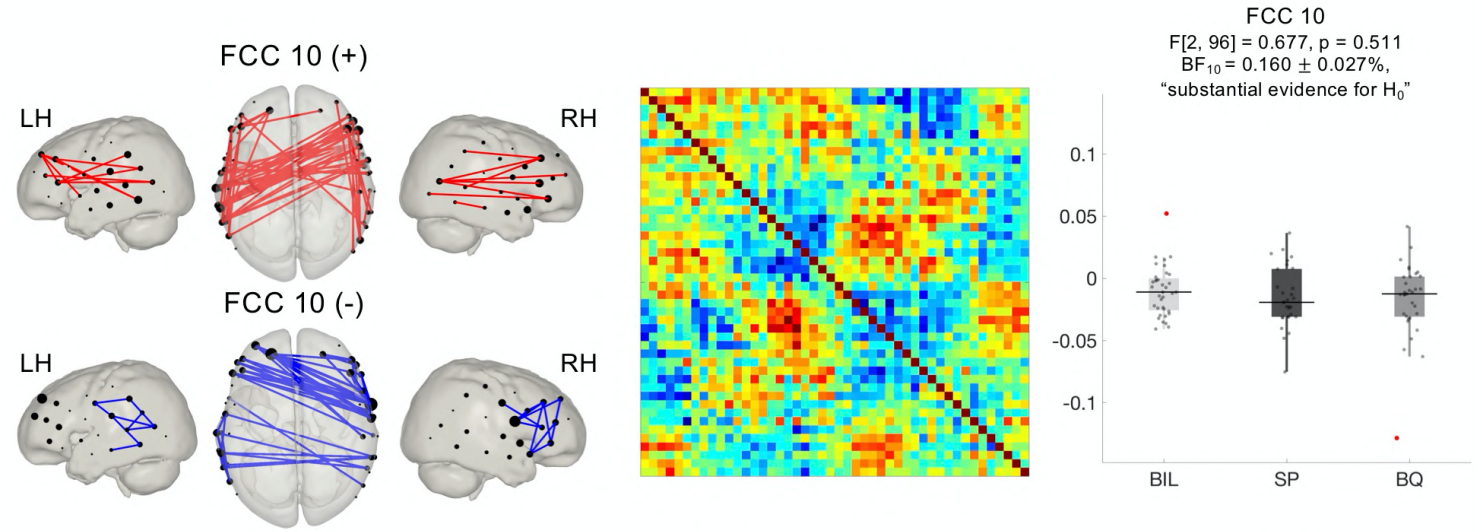

FCC $11(+)$
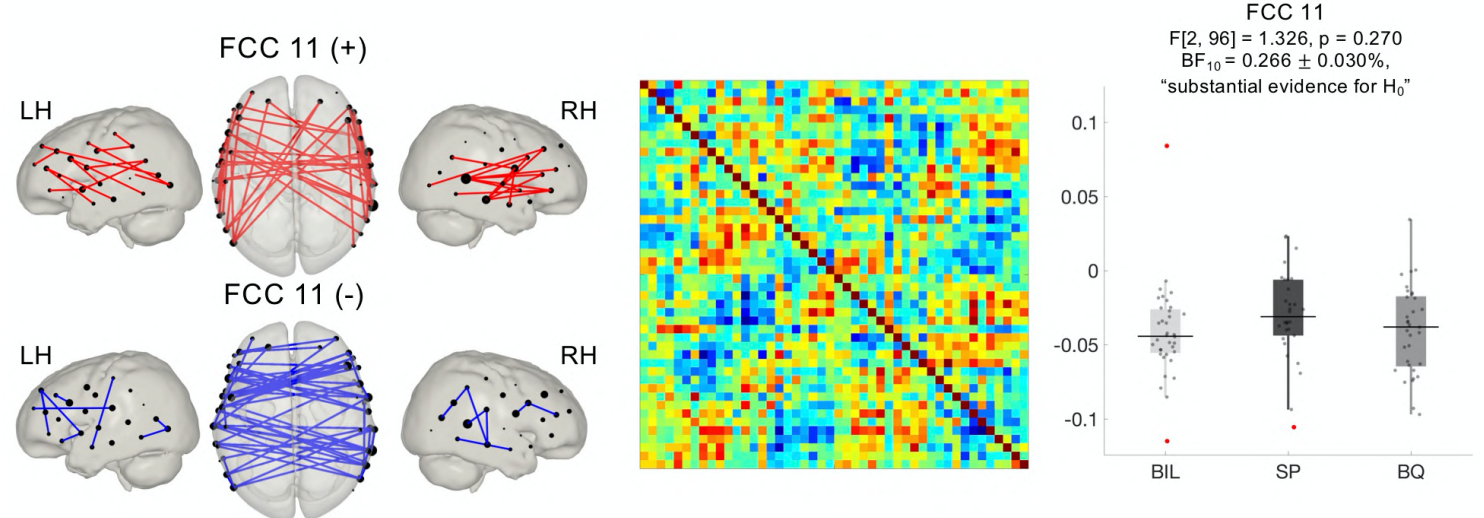

Supplementary Figure 6. Functional connectome components (FCC, positive + and negative -) obtained with the connICA method $(n=11)$. Leftmost column shows the positive and negative parts of the components which are represented as nodes and edges (top 10\% connections) in a brain template. Due to the high similarity between $\mathrm{HbO}$ and $\mathrm{HbR}$ components, brain maps are shown for HbR only. The middle column shows the components as reconstructed in their original form (i.e., adjacency matrices), with $\mathrm{HbO}$ and $\mathrm{HbR}$ displayed in the upper and lower triangular sections of the matrix respectively. These matrices represent connectivity values but are not restricted to the Pearson's correlation [-1 1] range (Amico et al., 2017). Statistical comparisons based on frequentist F-test on the weights for each component (right column) revealed no differences between groups (FDR corrected among 11 components, $\mathrm{q}<0.05)$. The analysis based on Bayesian statistics showed substantial evidence for $\mathrm{H}_{0}$ in 9 out of 11 FCC. FCC 5 showed anecdotal evidence for $\mathrm{H}_{1}$ and FCC 8 demonstrated anecdotal evidence for $\mathrm{H}_{0}$. 


\begin{tabular}{ccccc}
\hline $\begin{array}{c}\text { Funtional Connectome } \\
\text { Component (FCC) }\end{array}$ & $\boldsymbol{I q}$ & $\boldsymbol{r}$ & $\begin{array}{c}\text { ssd } \\
(\mathbf{1 0 0} \%)\end{array}$ & $\begin{array}{c}\text { ssd } \\
(\mathbf{6 0} \%)\end{array}$ \\
\hline FCC 1 & 0.96 & 0.95 & 24 & 5.5 \\
FCC 2 & 0.91 & 0.88 & 7.2 & 5.5 \\
FCC 3 & 0.50 & 0.70 & 7.4 & 5.4 \\
FCC 4 & 0.94 & 0.72 & 7.8 & 5.4 \\
FCC 5 & 0.91 & 0.80 & 7.3 & 5.5 \\
FCC 6 & 0.91 & 0.83 & 7.3 & 5.4 \\
FCC 7 & 0.90 & 0.90 & 8.8 & 5.6 \\
FCC 8 & 0.84 & 0.84 & 7.3 & 5.4 \\
FCC 9 & 0.88 & 0.72 & 7.6 & 5.5 \\
FCC 10 & 0.90 & 0.74 & 8.0 & 5.4 \\
FCC 11 & 0.71 & 0.67 & 7.6 & 5.4 \\
\hline
\end{tabular}

Supplementary Table 3. Model order evaluation metrics for the PCA threshold selected in the connICA approach (i.e., 60\% - 11 independent components). Sum of squared differences (ssd) are computed with respect to the data after PCA (total $=100 \%$ ) and with respect to the original data without PCA (total $=60 \%$ ). Iq $=$ ICASSO cluster quality index; $\mathrm{r}=$ correlation coefficient between $\mathrm{HbO}$ and $\mathrm{HbR}$ components. 


\section{References}

Amico E, Marinazzo D, Di Perri C, Heine L, Annen J, Martial C, Dzemidzic M, Kirsch M, Bonhomme V, Laureys S, Goñi J. 2017. Mapping the functional connectome traits of levels of consciousness. Neuroimage, 148, 201-211. https://doi.org/10.1016/j.neuroimage.2017.01.020.

Damaraju E, Caprihan A, Lowe JR, Allen EA, Calhoun VD, Phillips JP. 2014. Functional connectivity in the developing brain: a longitudinal study from 4 to 9 months of age. Neuroimage, 84, 169-80. https://doi.org/10.1016/j.neuroimage.2013.08.038.

Himberg J, Hyvärinen A, Esposito F. 2004. Validating the independent components of neuroimaging time series via clustering and visualization. Neuroimage, 22(3), 1214-1222. https://doi.org/10.1016/j.neuroimage.2004.03.027.

Homae F, Watanabe H, Otobe T, Nakano T, Go T, Konishi Y, Taga G. 2010. Development of global cortical networks in early infancy. J. Neurosci. 30(14), 4877-4882. https://doi.org/10.1523/JNEUROSCI.5618-09.2010.

Keysers C, Gazzola V, Wagenmakers E. 2020. Using Bayes factor hypothesis testing in neuroscience to establish evidence of absence. Nat Neurosci 23, 788-799. https://doi.org/10.1038/s41593-020-0660-4.

Minka TP. 2001. Automatic choice of dimensionality for PCA. Adv. Neural Inf. Process Syst. 598-604.

Morey RD, Rouder JN, Jamil T, Urbanek S, Forner K, Ly A, 2018. BayesFactor.

Obrig H, Villringer A. 2003. Beyond the visible - Imaging the human brain with light. J. Cereb. Blood Flow Metab. 23(1), 1-18. https://doi.org/10.1097/01.WCB.0000043472.45775.29.

Villringer A, Chance B. 1997. Non-invasive optical spectroscopy and imaging of human brain function. Trends Neurosci. 20(10), 435-442. https://doi.org/10.1016/S0166-2236(97)01132-6.

Watanabe H, Shitara Y, Aoki Y, Inoue T, Tsuchida S, Takahashi N, Taga G. 2017. Hemoglobin phase of oxygenation and deoxygenation in early brain development measured using fNIRS. Proc. Natl. Acad. Sci. USA, 114(9), E1737-E1744. https://doi.org/10.1073/pnas.1616866114.

Wetzels R, Wagenmakers EJ. 2012. A default Bayesian hypothesis test for correlations and partial correlations. Psychon. Bull. Rev. 19(6), 1057-1064. https://doi.org/10.3758/s13423-012-0295-x.

Wolf M, Wolf U, Toronov V, Michalos A, Paunescu LA, Choi JH, Gratton E. 2002. Different time evolution of oxyhemoglobin and deoxyhemoglobin concentration changes in the visual and motor cortices during functional stimulation: a near-infrared spectroscopy study. Neuroimage, 16(3), 704-712. https://doi.org/10.1006/nimg.2002.1128. 


\section{Data quality assessment figures}

For each participant ( $\mathrm{n}=99$ ), two figures comprising various plots of the different steps of our data quality assessment routine are displayed in this supplementary material. The last five figures on Pages 215-219, represent the time series for the five datasets that were discarded due to insufficient data qualty. A brief explanation of the figures is provided below.

\section{Figure 1}

This figure presents the time series of the complete recording (560 seconds) for the two wavelengths ( 760 and $850 \mathrm{~nm}$ ) used by our fNIRS system.

Top row: Raw intensity data.

Middle row: Optical density data after conversion from raw intensity data.

Bottom row: Optical density data after artifact correction using the wavelet-based despike method (Patel et al., 2014).

\section{Figure 2}

This figure shows various data quality assessment plots at different preprocessing steps. Rows 1 and 2 display the $\mathrm{HbO}$ and $\mathrm{HbR}$ time series. The third row shows the power spectral density of $\mathrm{HbO}$ and $\mathrm{HbR}$ concentration data at each preprocessing step. In the bottom row, and for each column (i.e., preprocessing step), leftmost plot shows the functional connectivity matrix. In this plot, the functional connectivity matrix for $\mathrm{HbO}$ and $\mathrm{HbR}$ is shown in the top-left part and bottom- right part, respectively. The functional connectivity matrix representing the correlation between $\mathrm{HbO}$ and $\mathrm{HbR}$ is shown in the top-right part (expected negative). Note that these matrices are symmetric with respect to the main diagonal. For preprocessed data, after filtering and global signal regression, the standard functional connectivity matrix and the functional connectivity matrix computed using robust regression (Santosa et al., 2017) are presented. The histogram plots in polar coordinates presented on the right part show the phase difference between $\mathrm{HbO}$ and $\mathrm{HbR}$ time-series (hPod value, Watanabe et al., 2017) at each preprocessing step.

Left column: raw $\mathrm{HbO}$ and $\mathrm{HbR}$ data.

Middle column: $\mathrm{HbO}$ and $\mathrm{HbR}$ time series after filtering.

Right column: $\mathrm{HbO}$ and $\mathrm{HbR}$ preprocessed data, after filtering and global signal regression. 

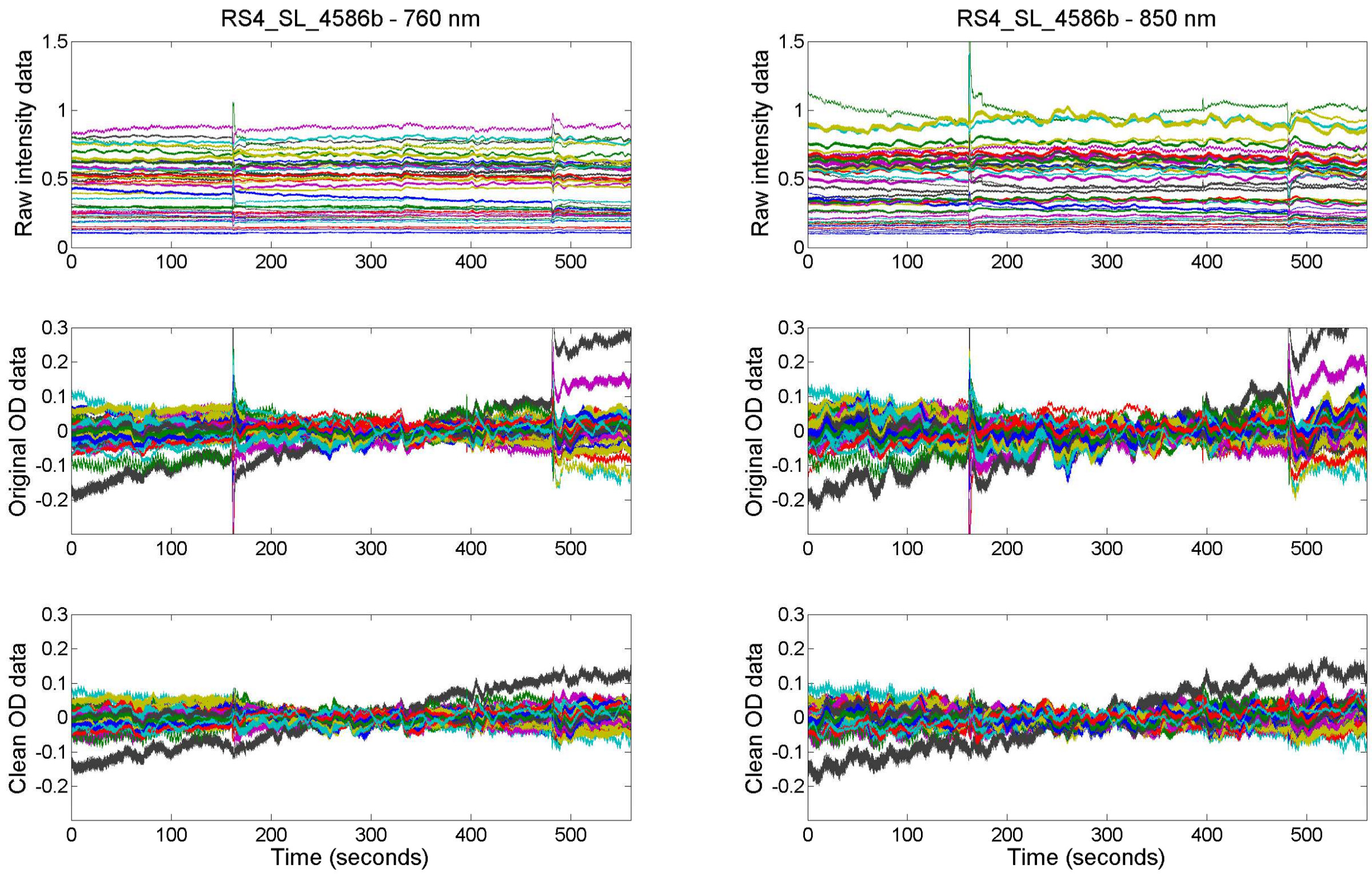

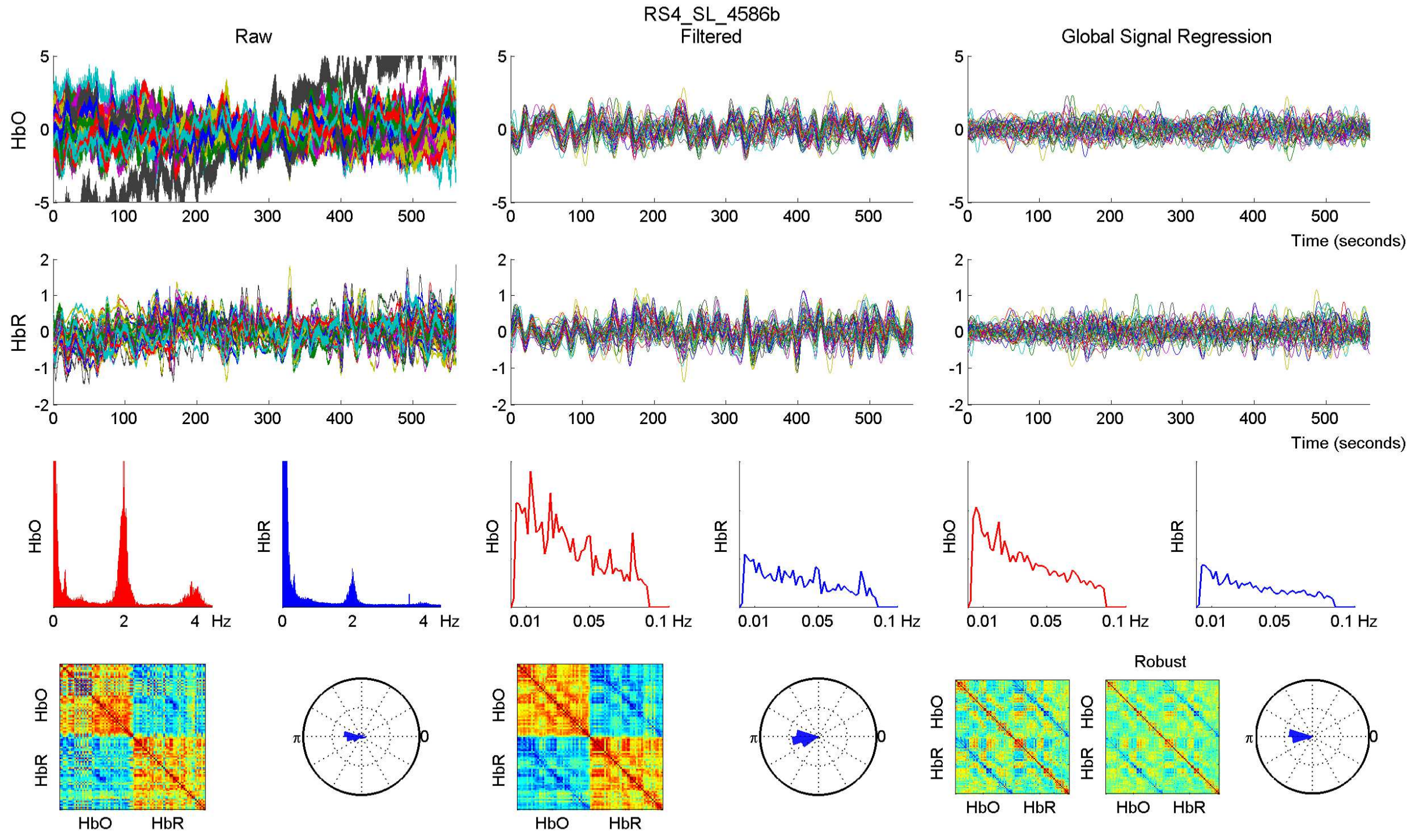

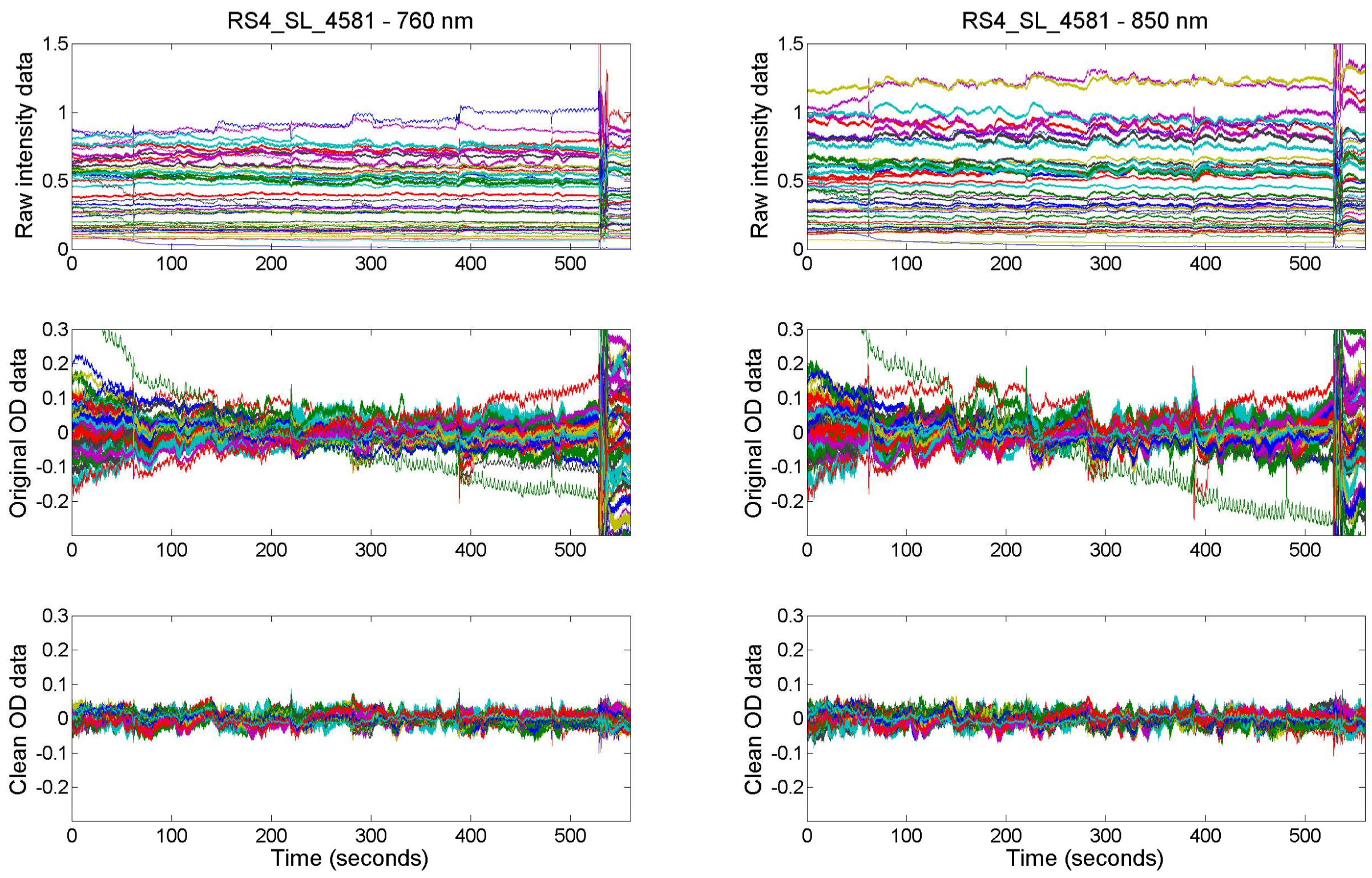
Raw
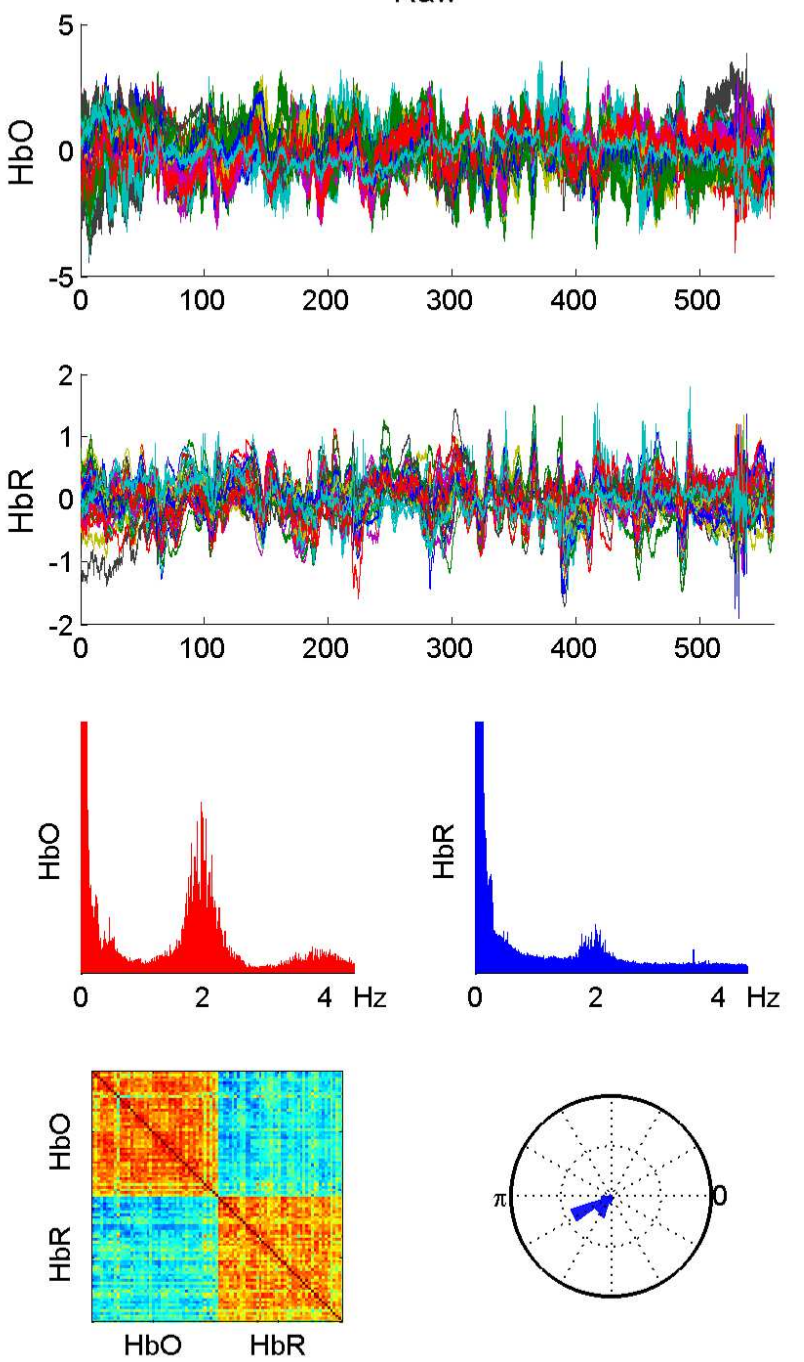

RS4 SL 4581

Filtered
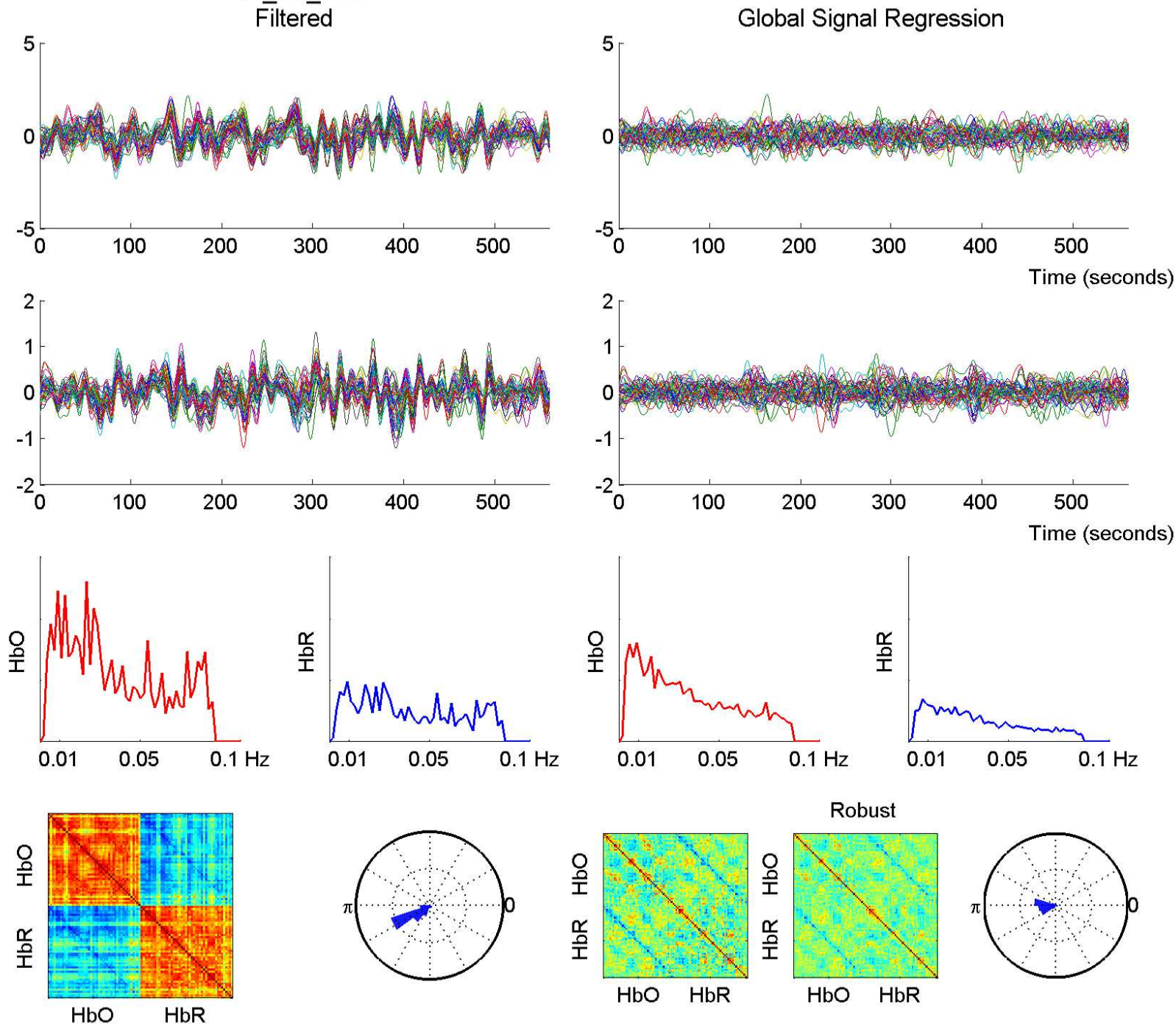

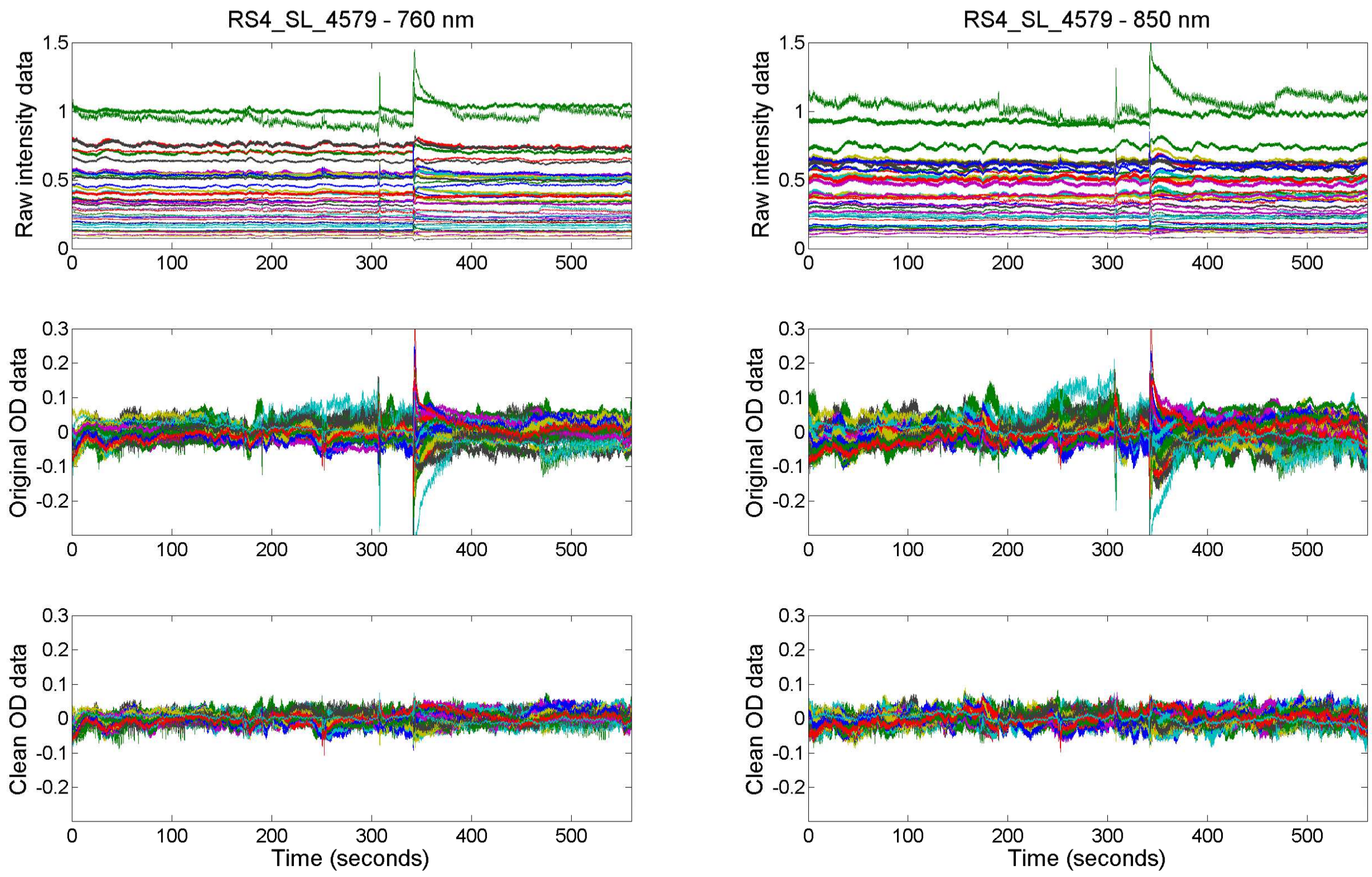
Raw

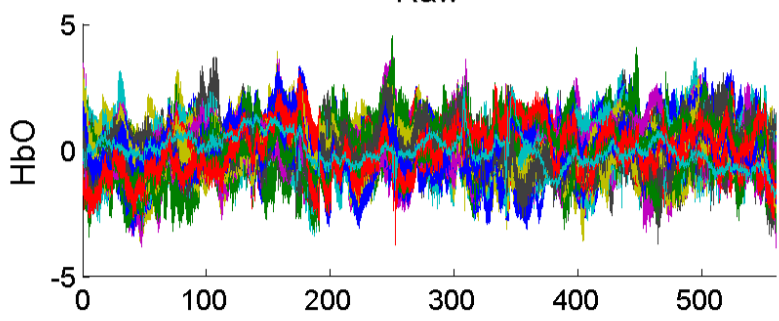

2
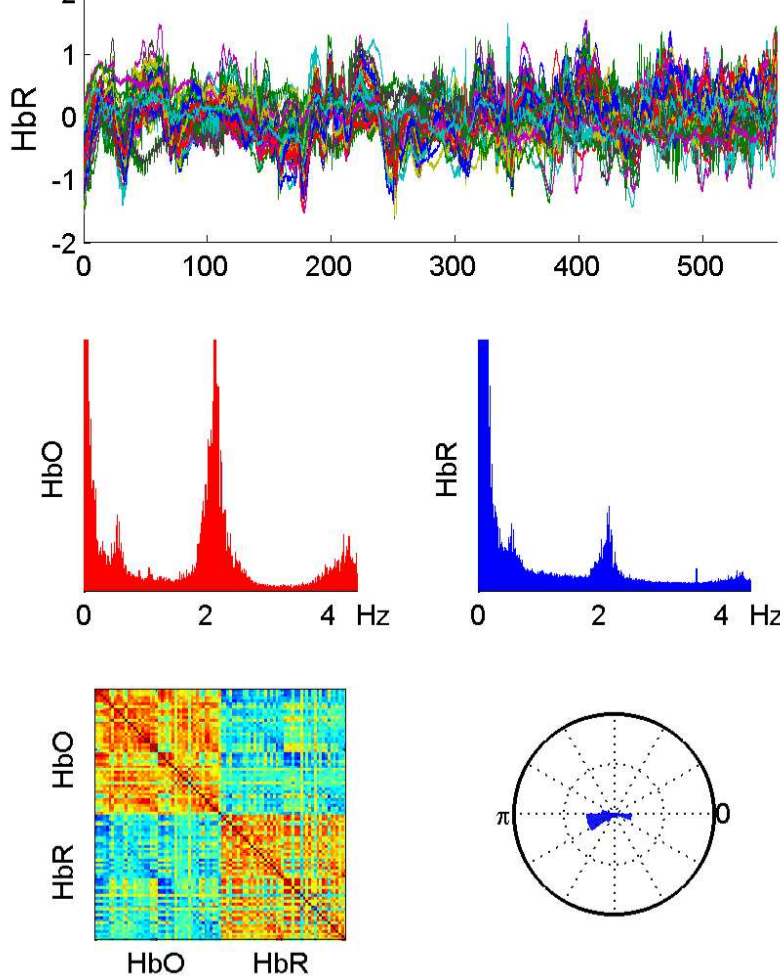

RS4 SL 4579

Filtered
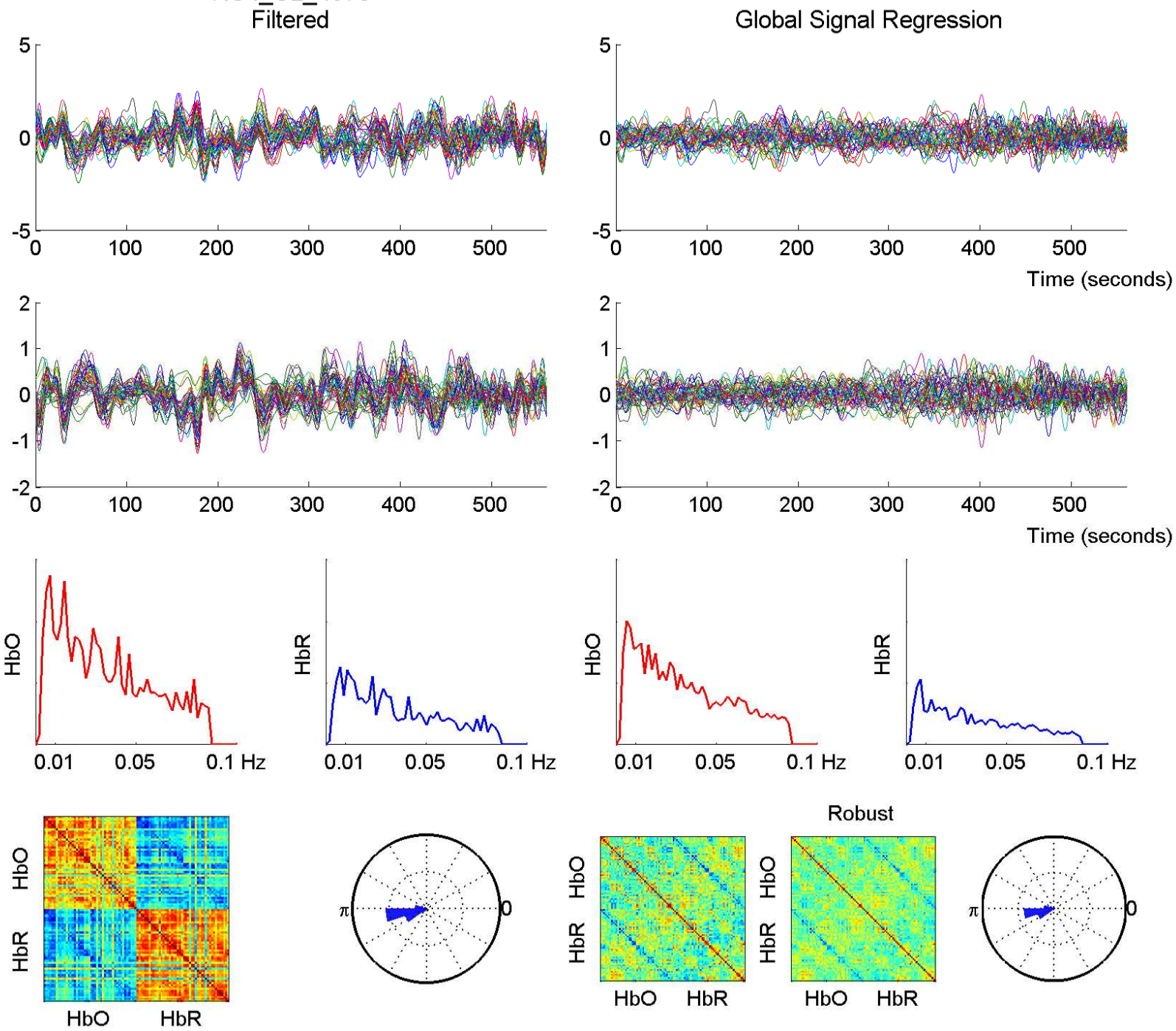
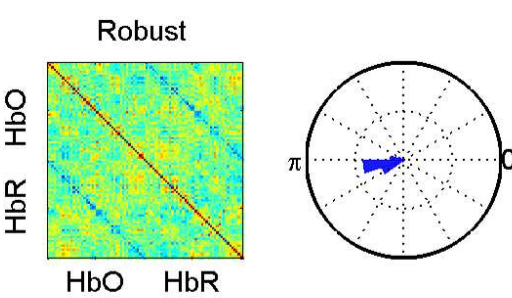

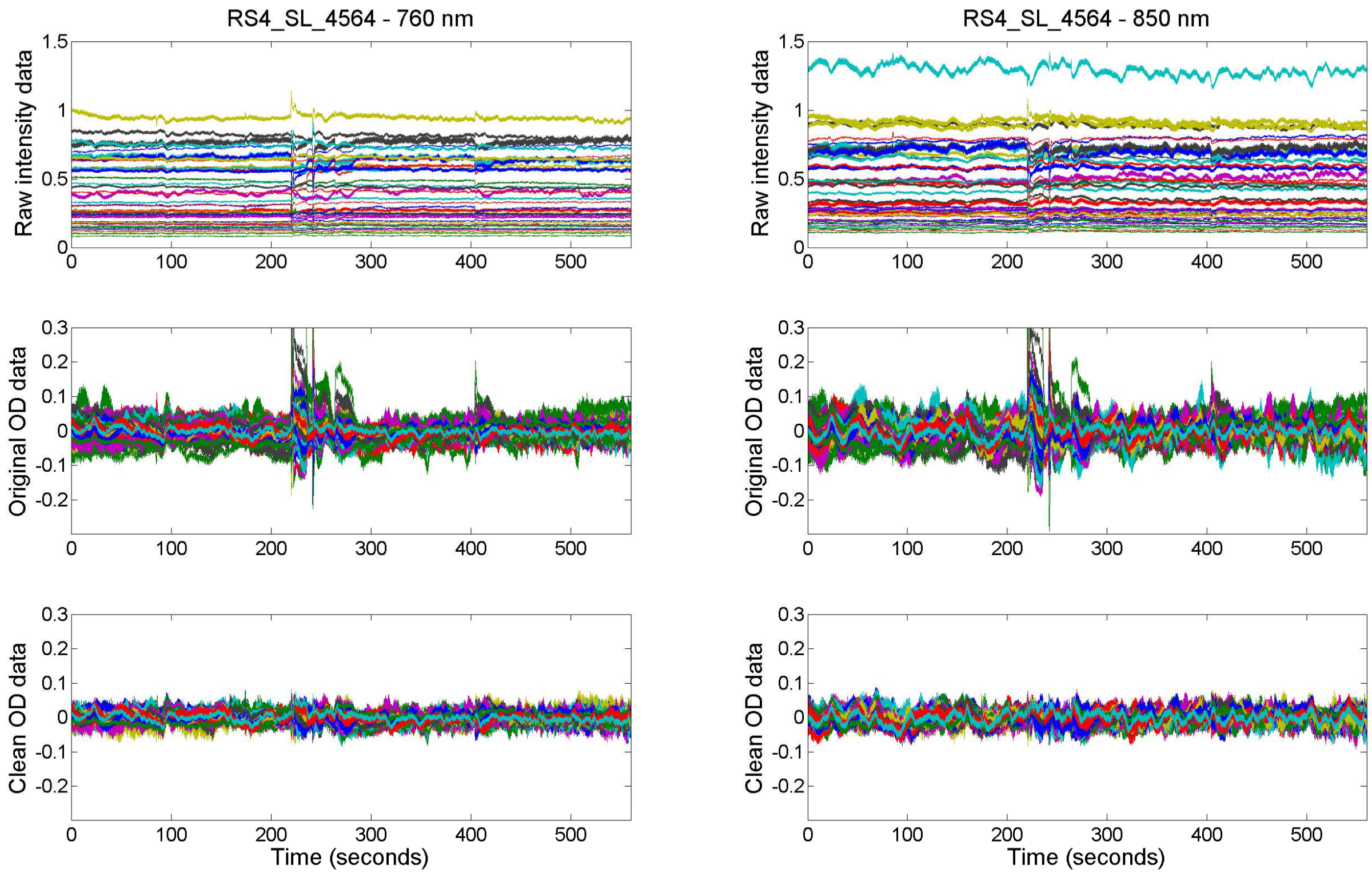
Raw

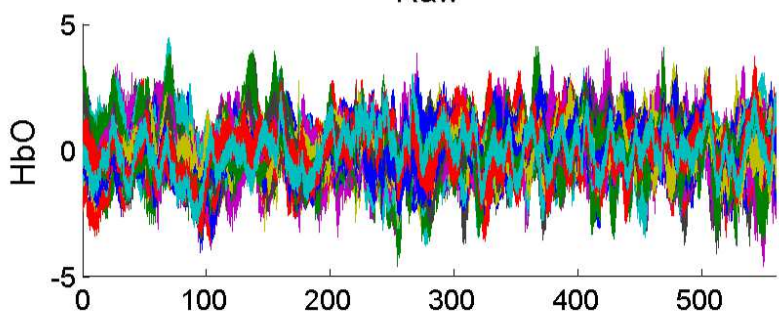

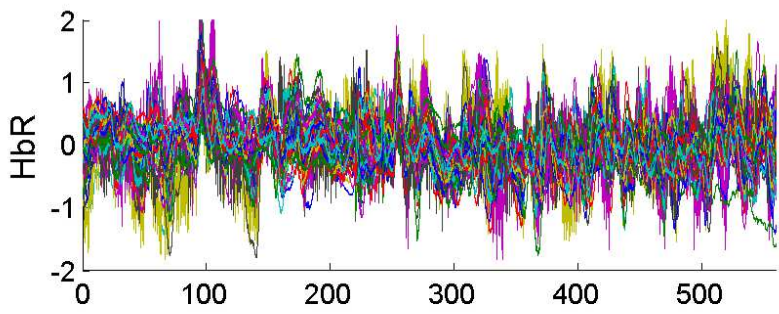
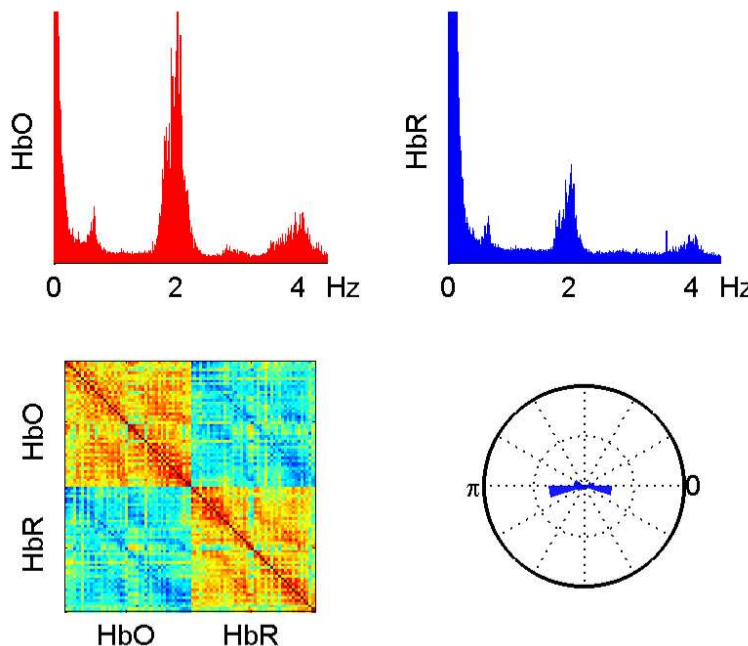

RS4_SL_4564

Filtered

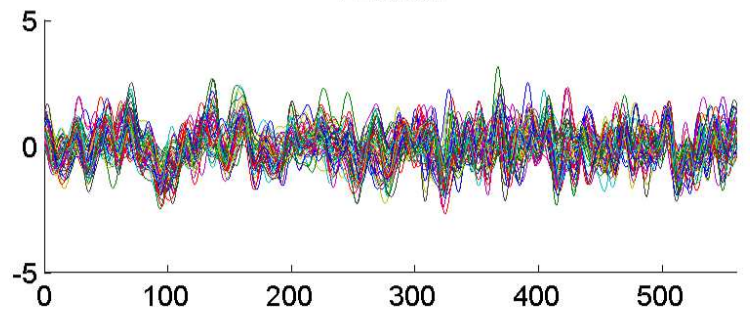

2
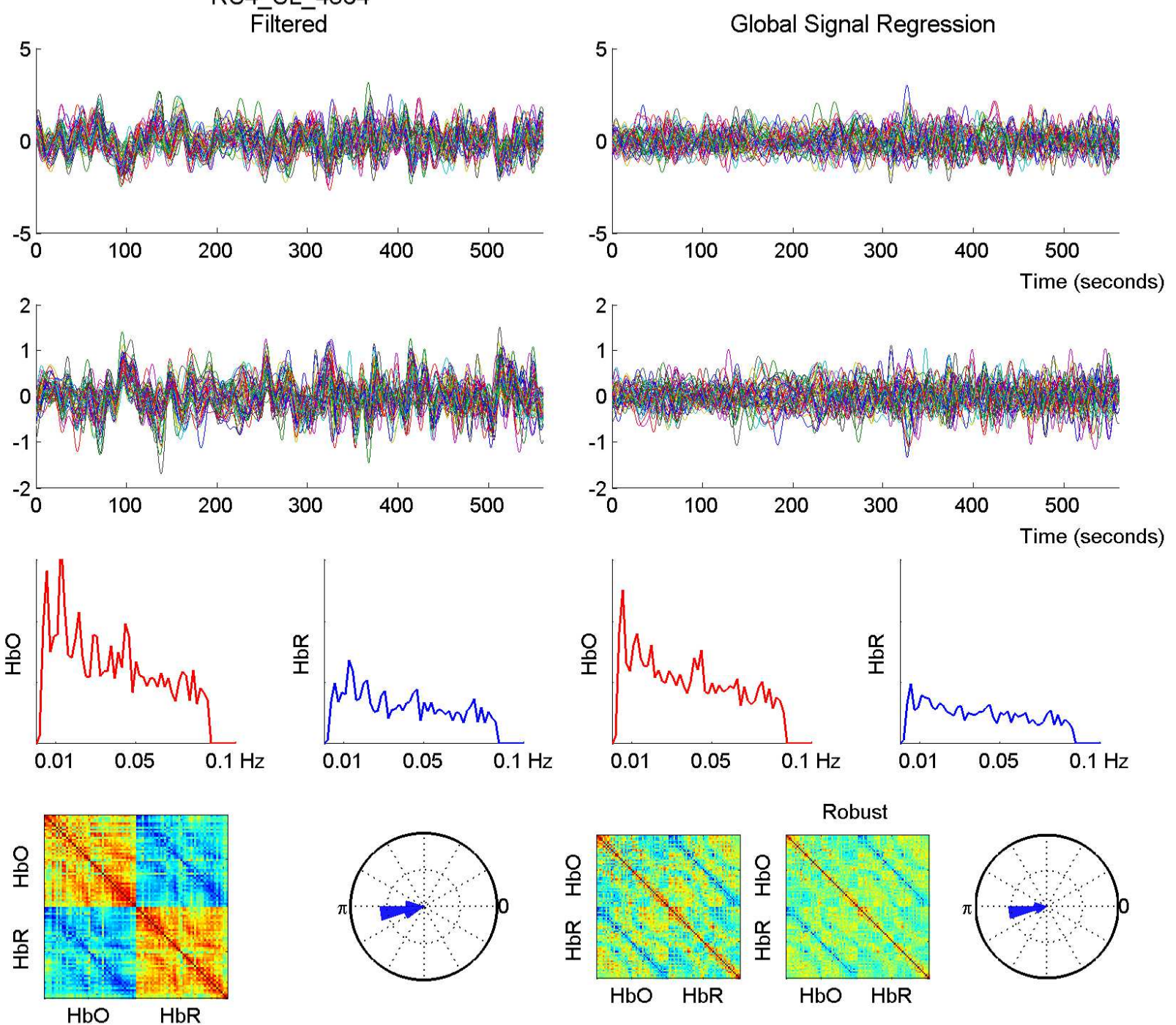
RS4 SL $4563-760 \mathrm{~nm}$
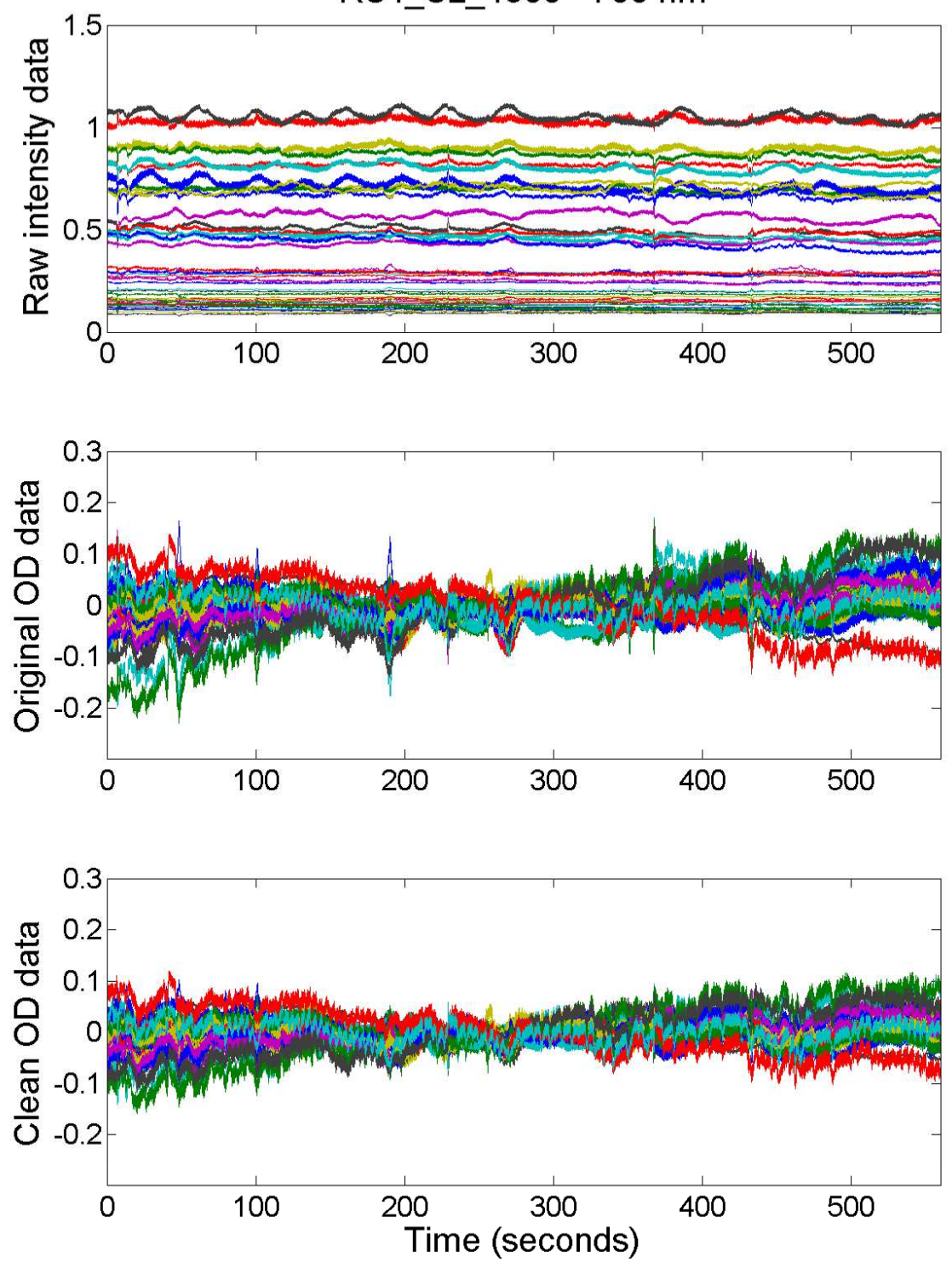

RS4 SL $4563-850 \mathrm{~nm}$
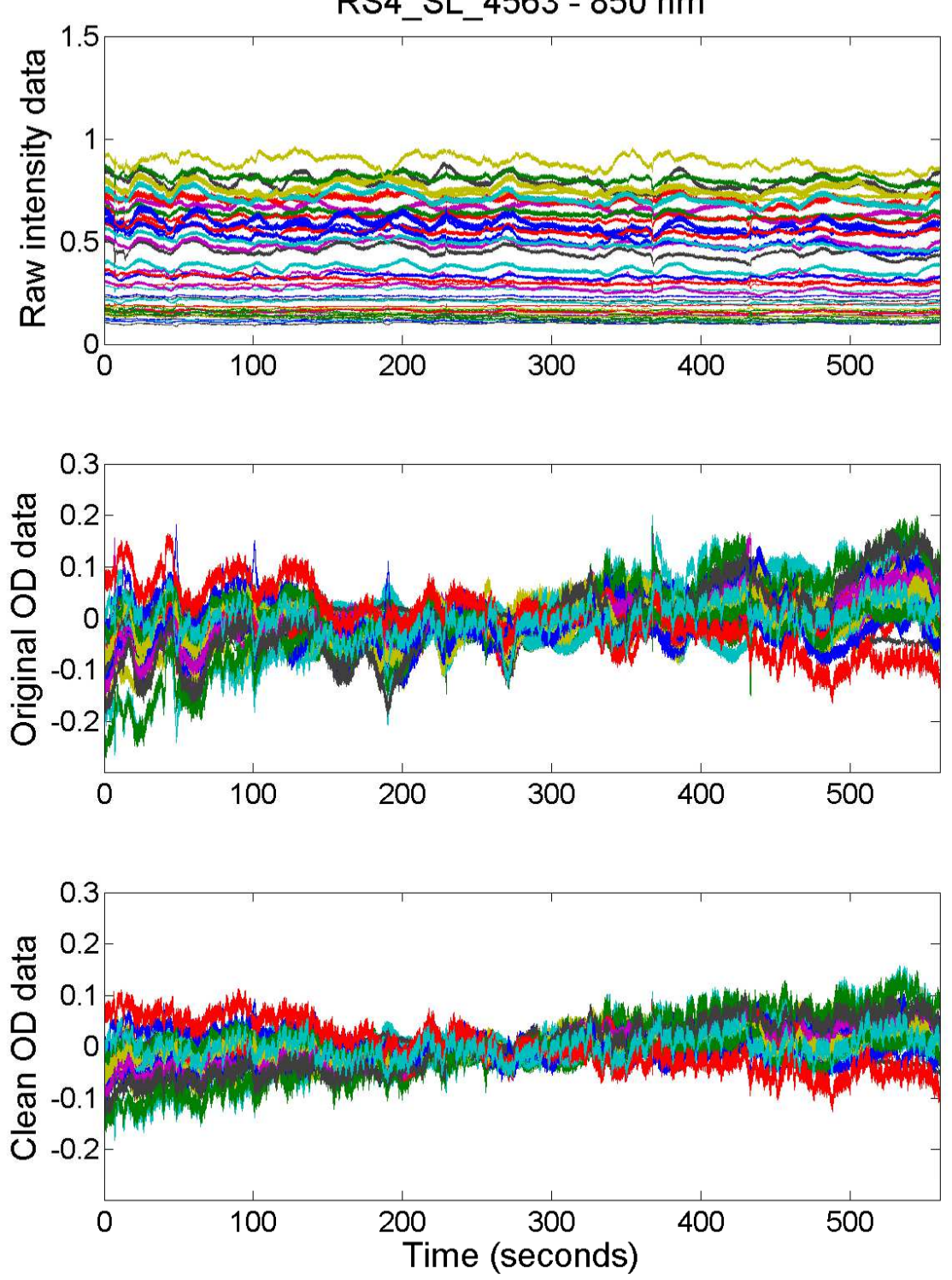
RS4_SL_4563
Raw

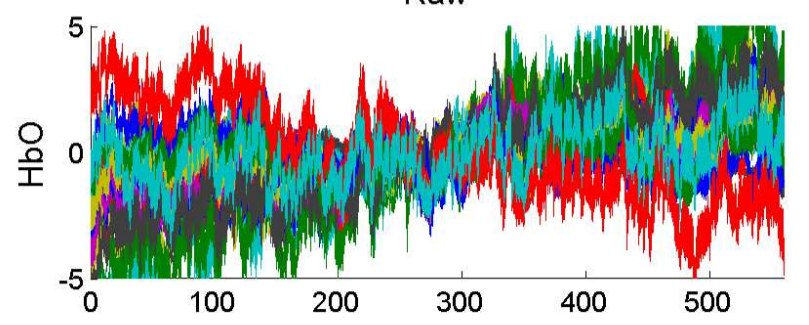

2
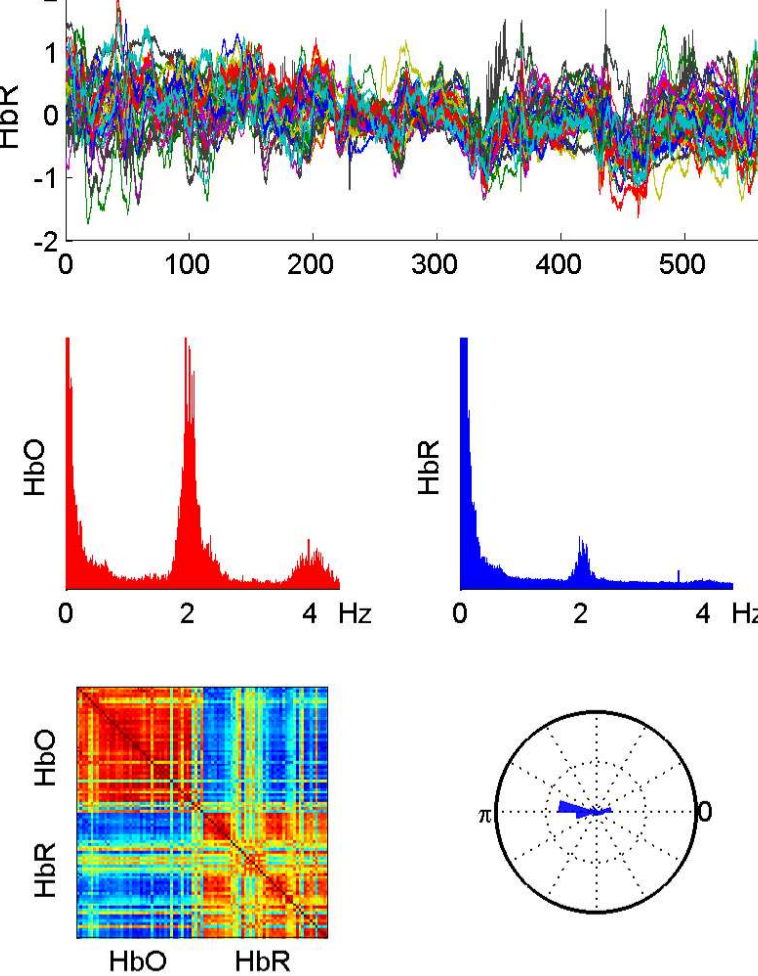
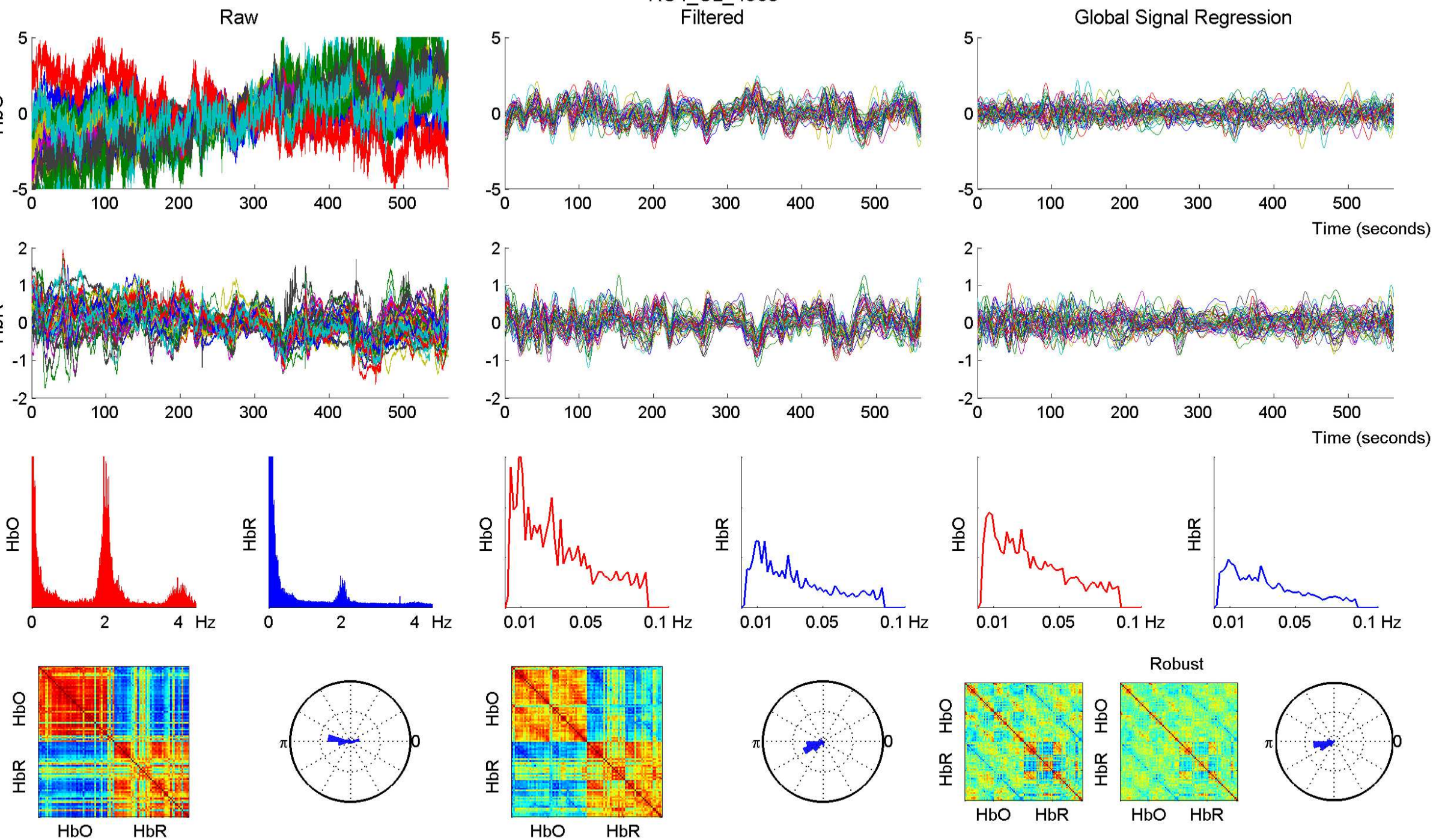

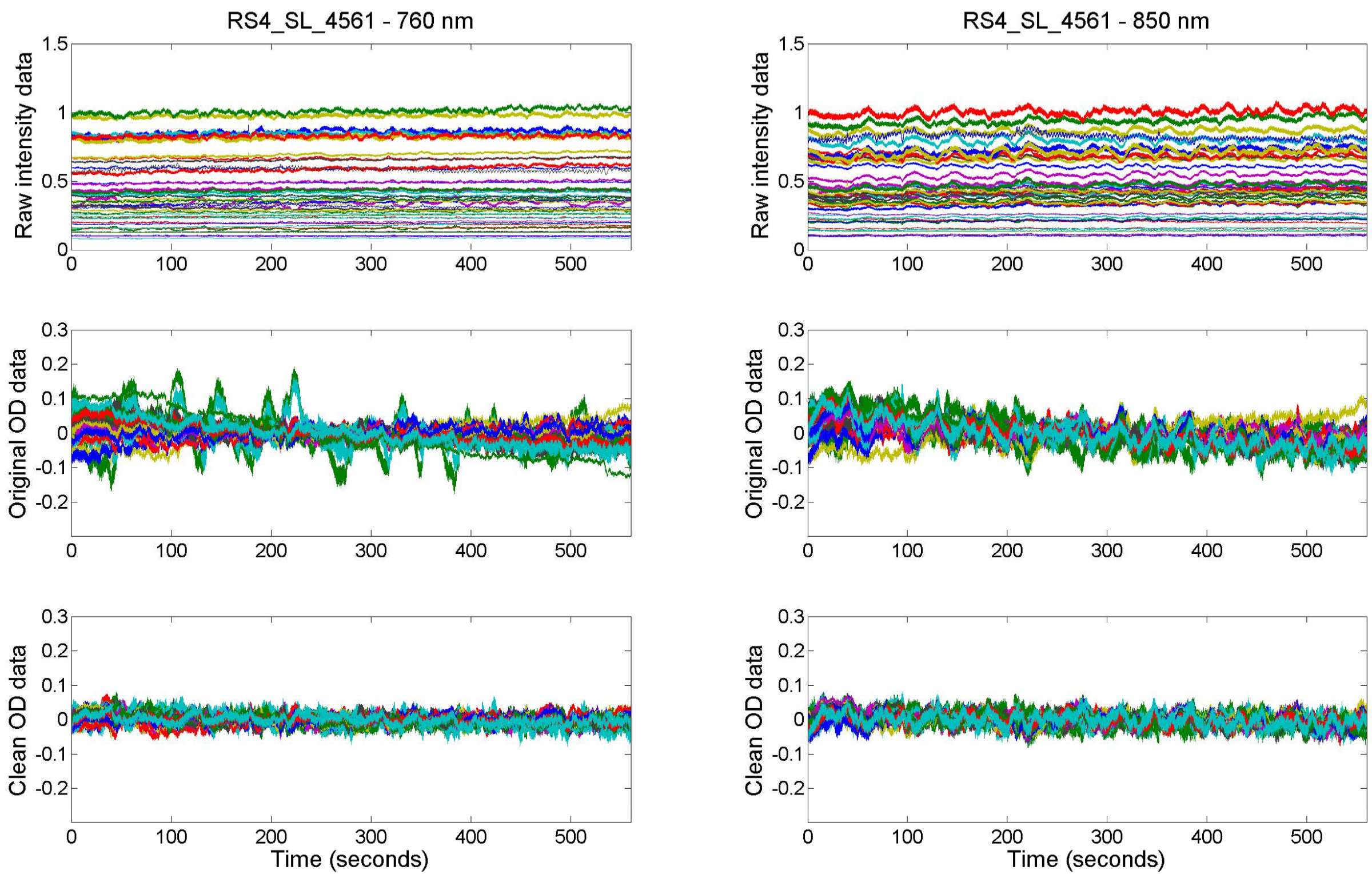
Raw
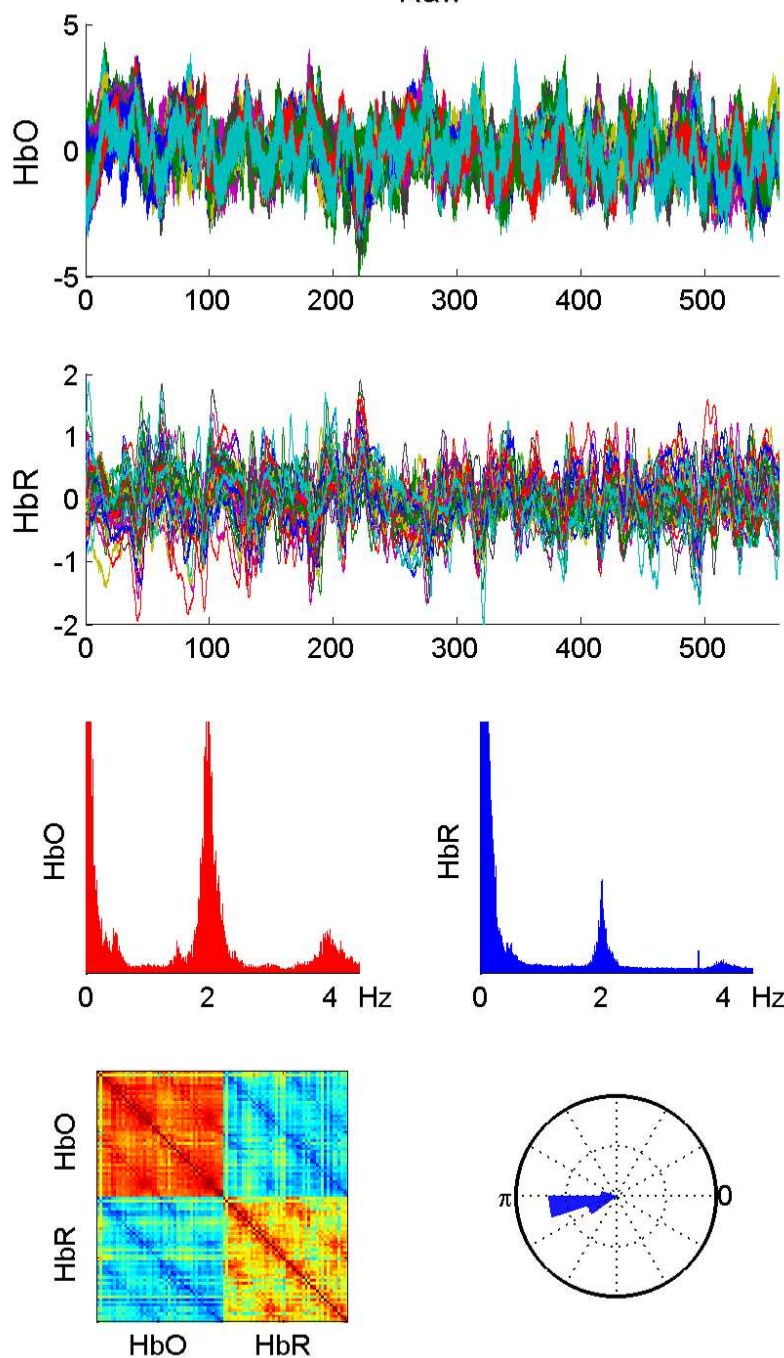

RS4_SL_4561

Filtered
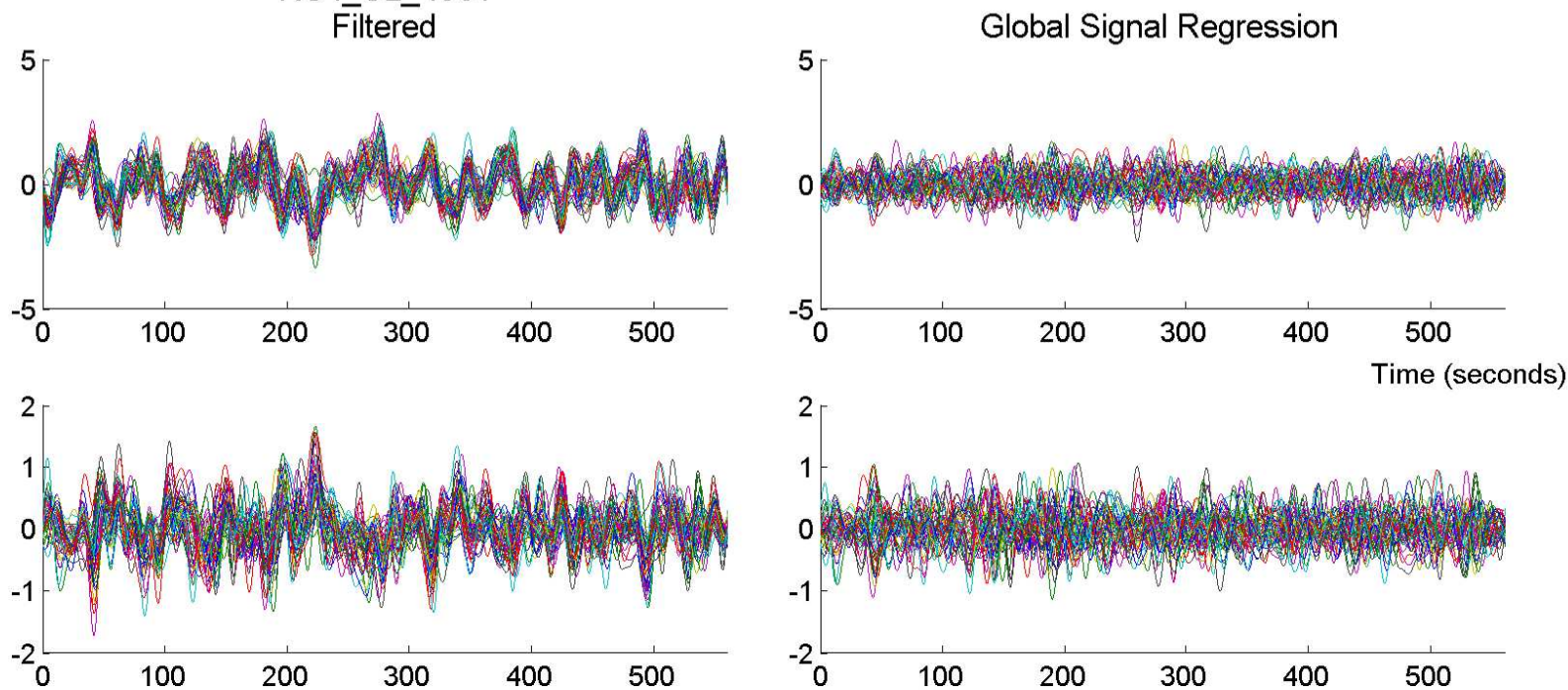

2
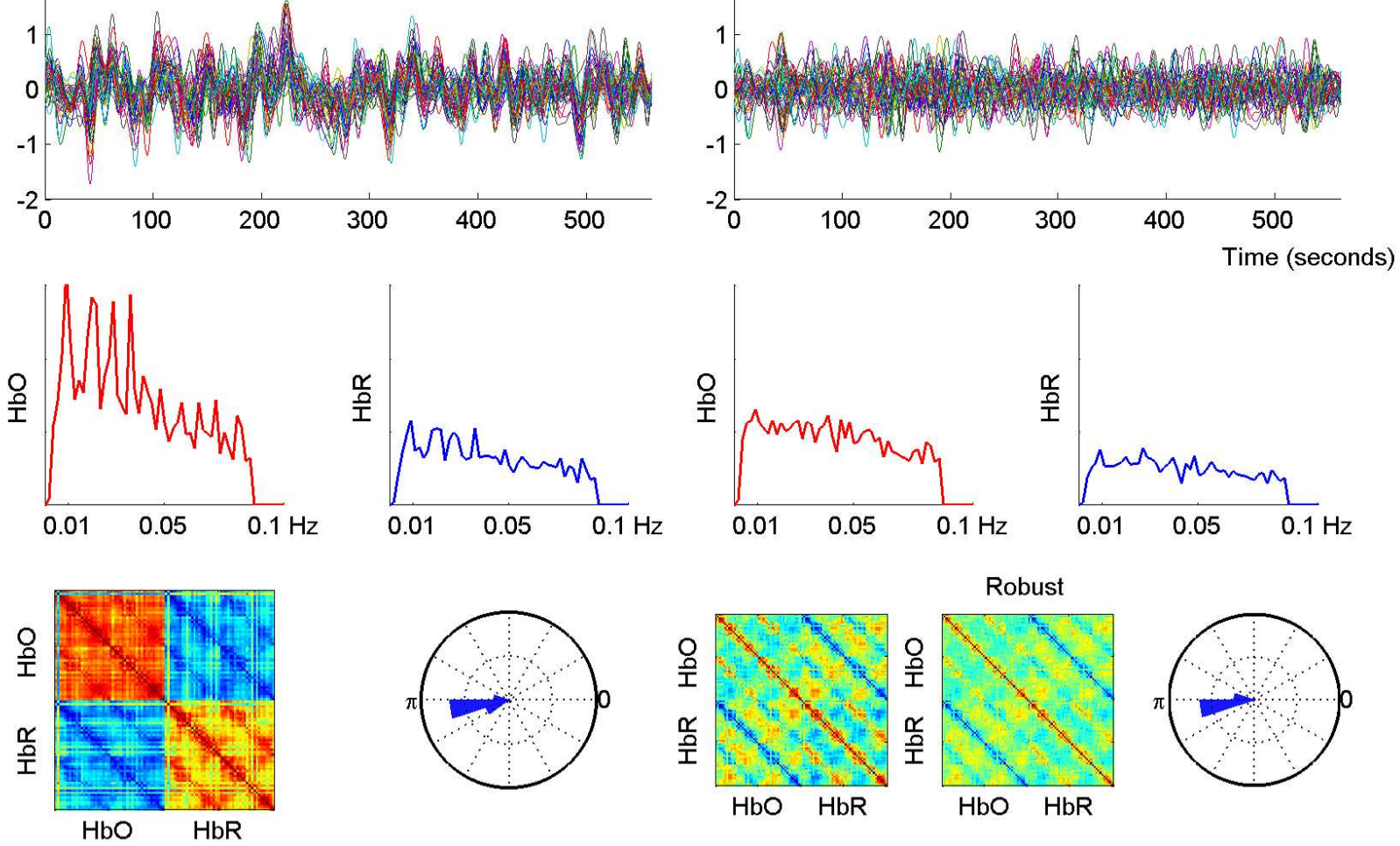

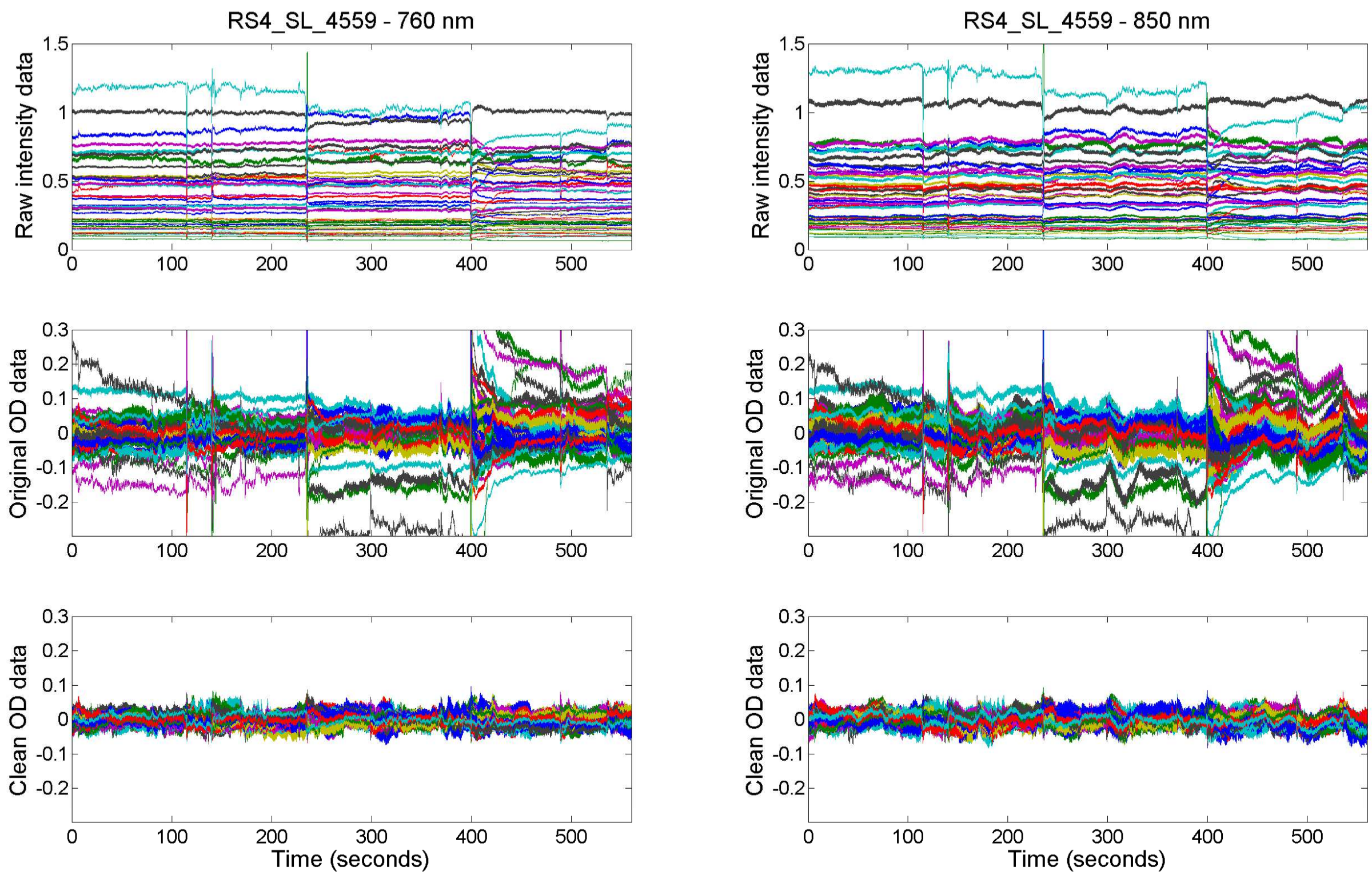
RS4_SL_4559

Raw
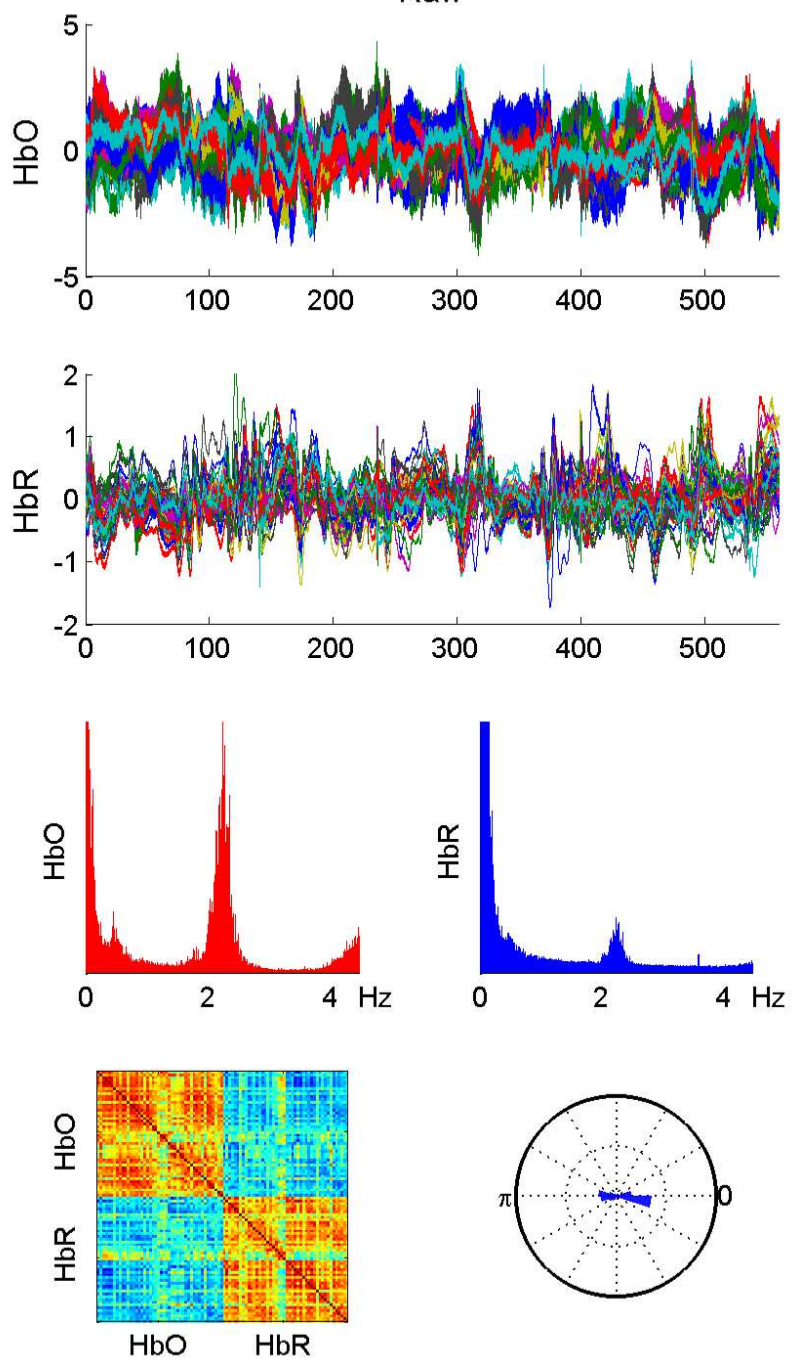
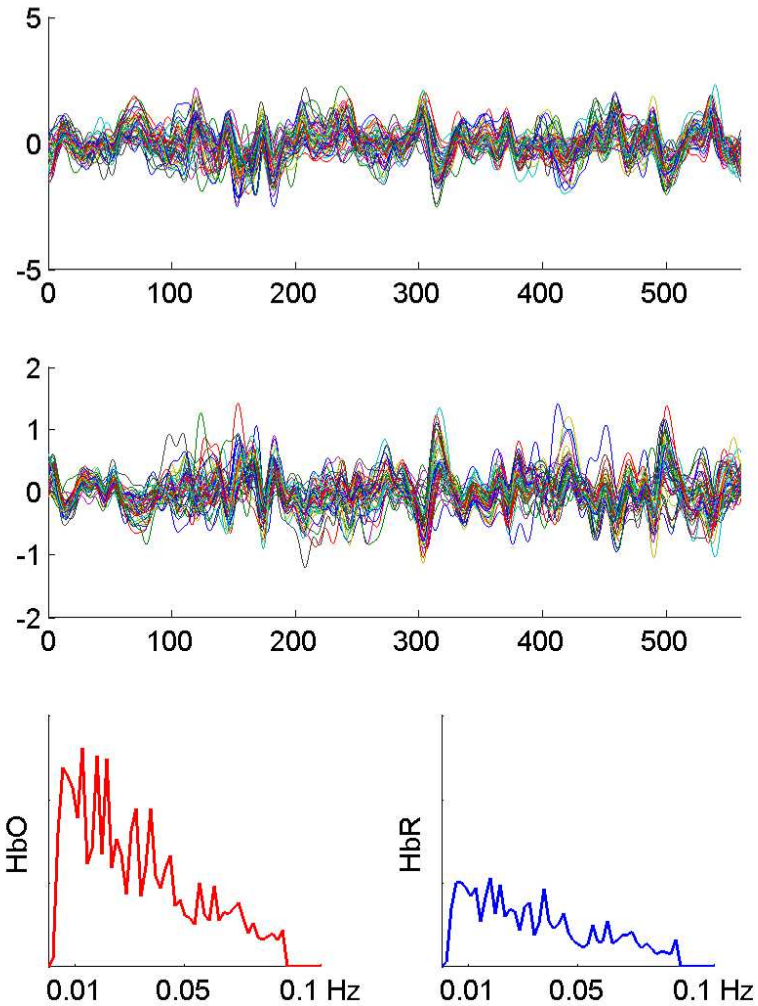

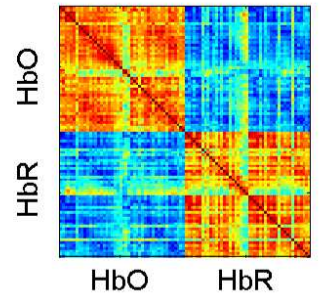

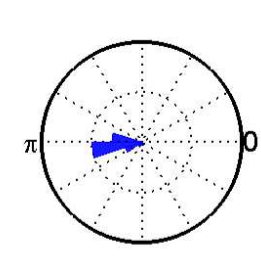

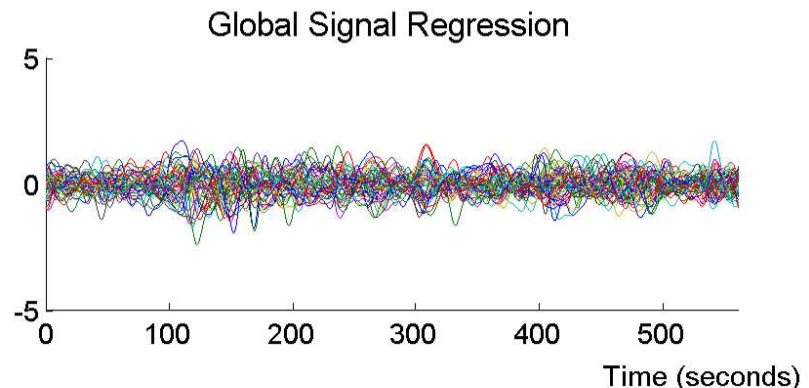

2
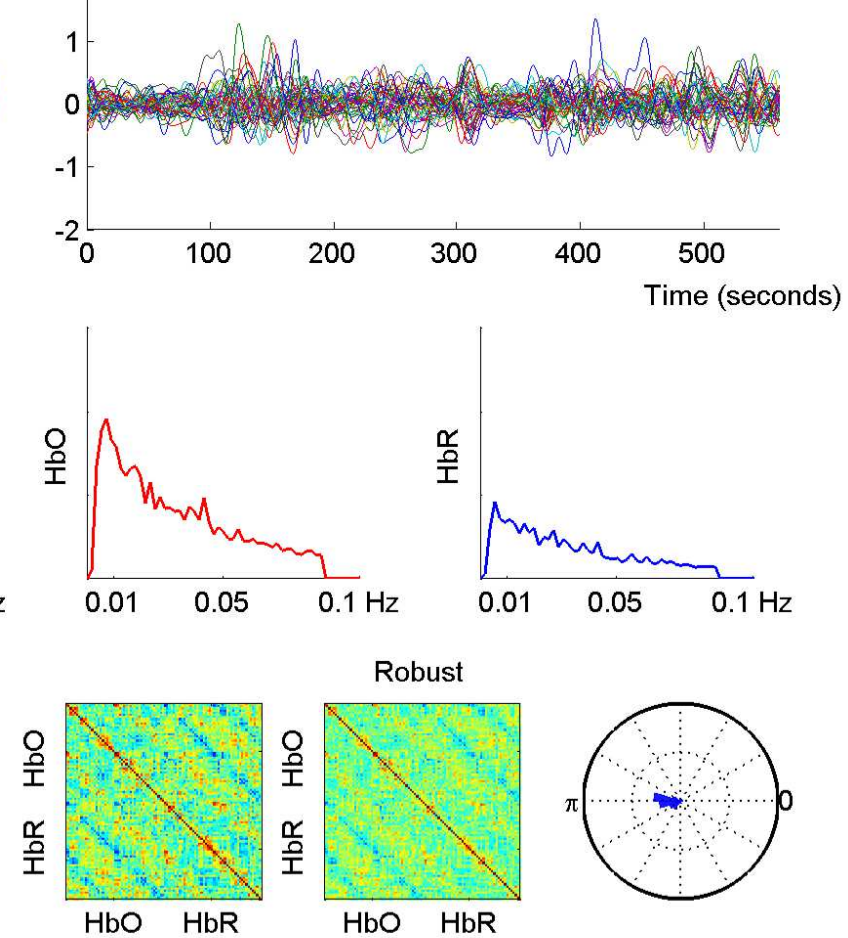

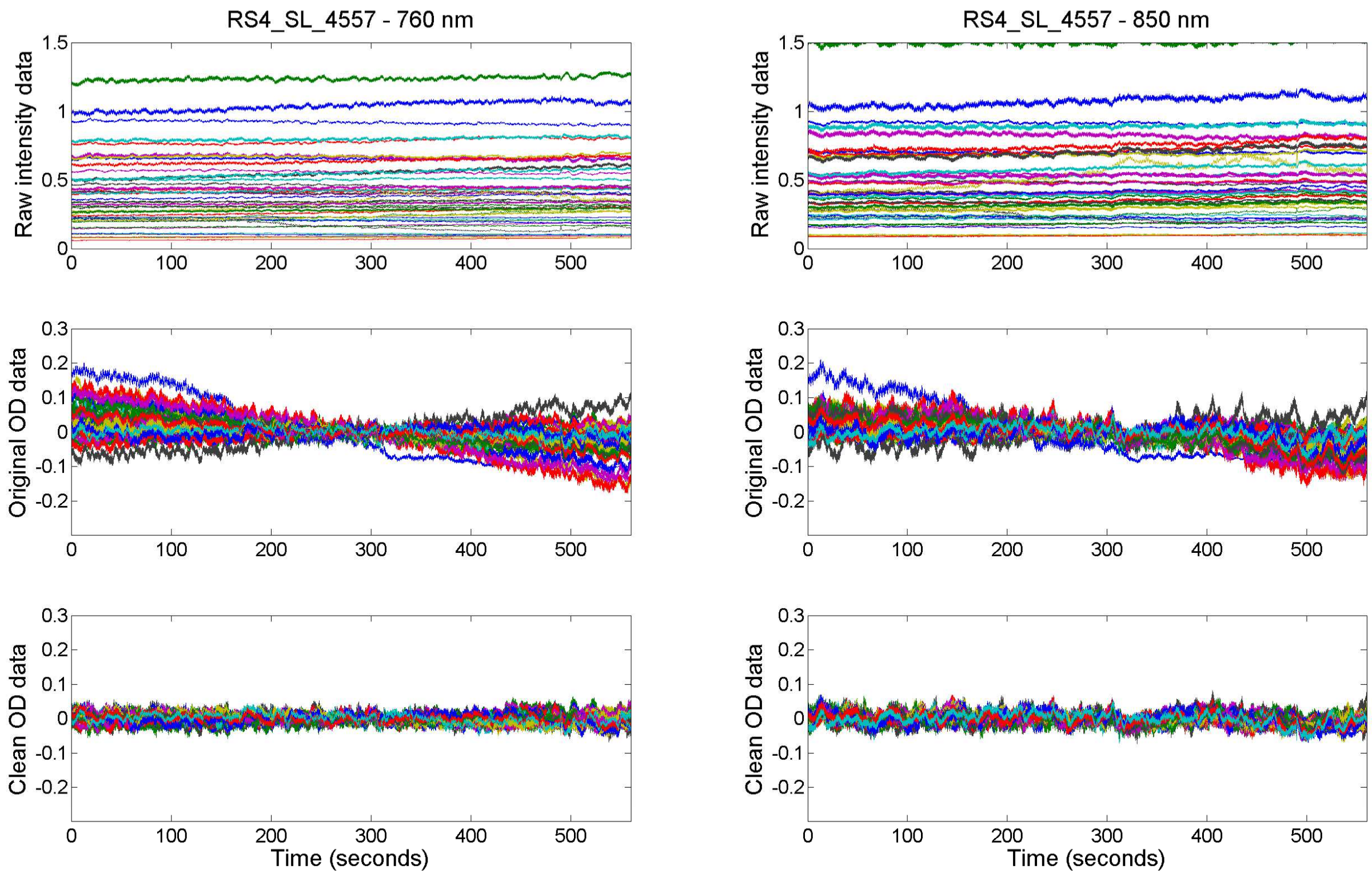
Raw
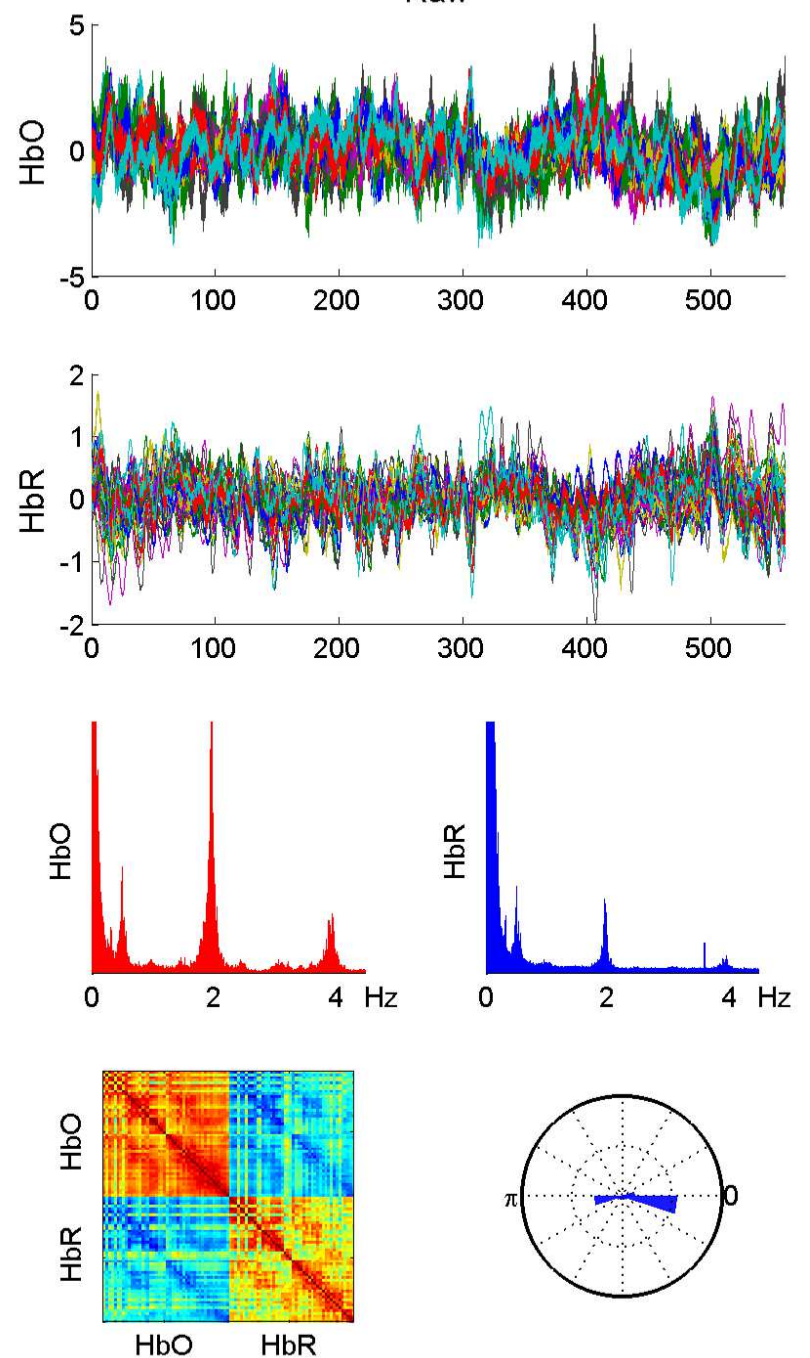

RS4_SL_4557

Filtered
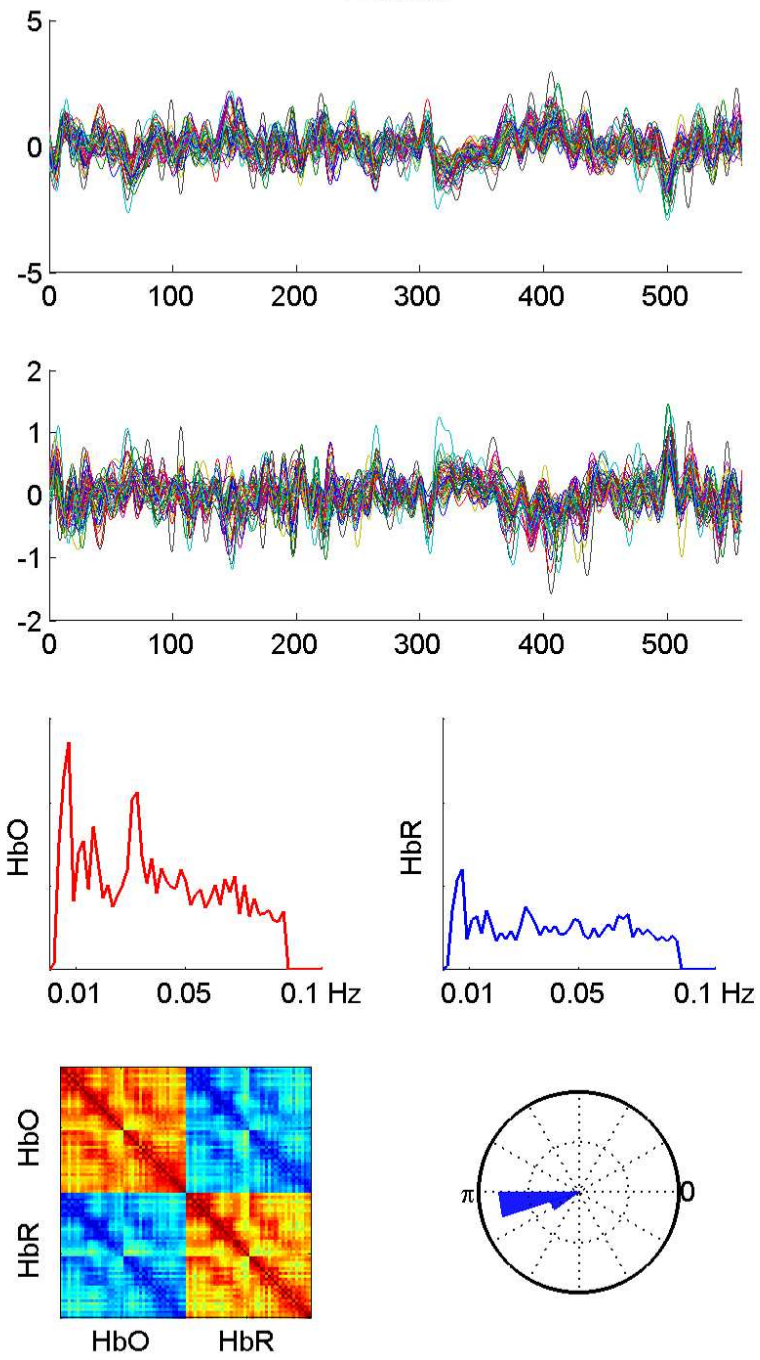

Global Signal Regression

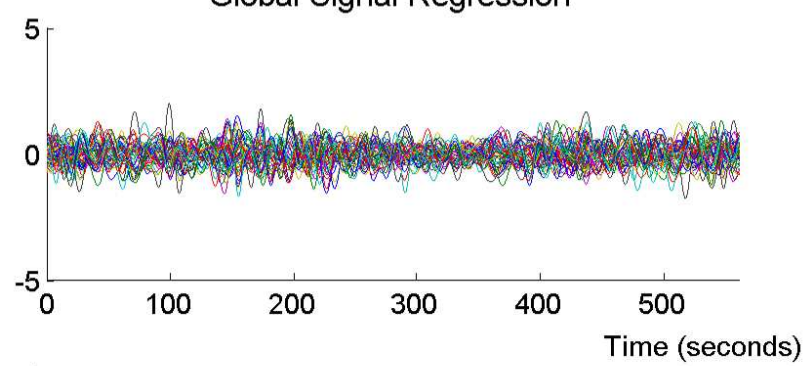

2
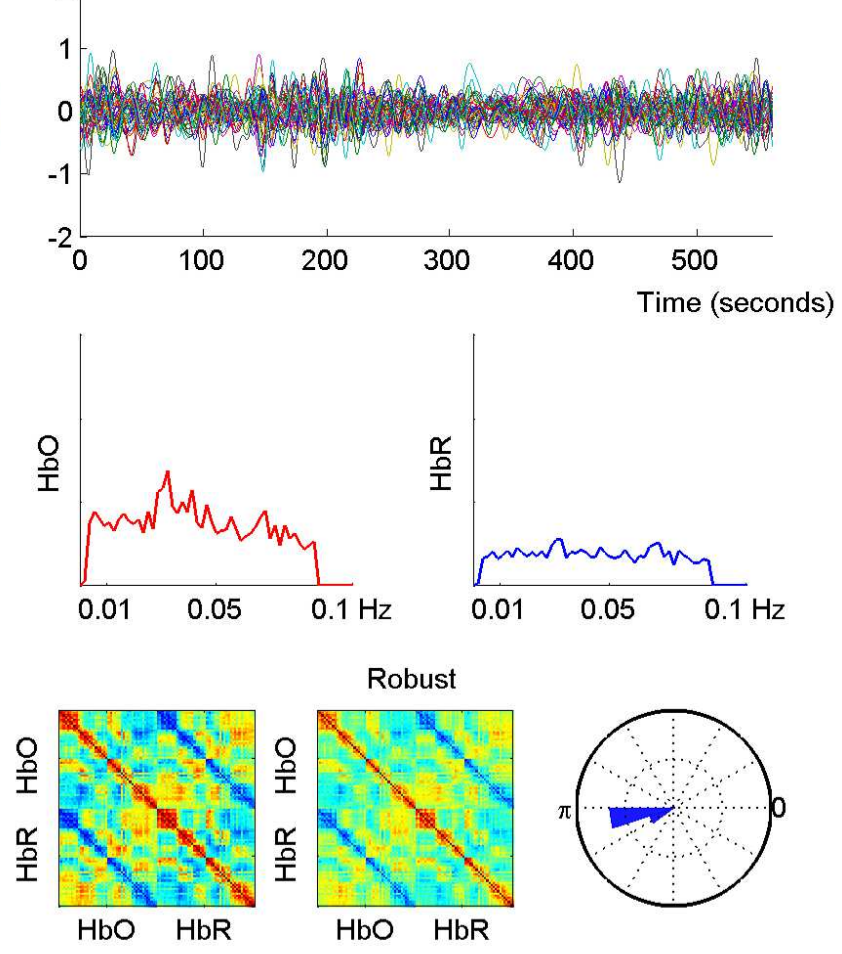

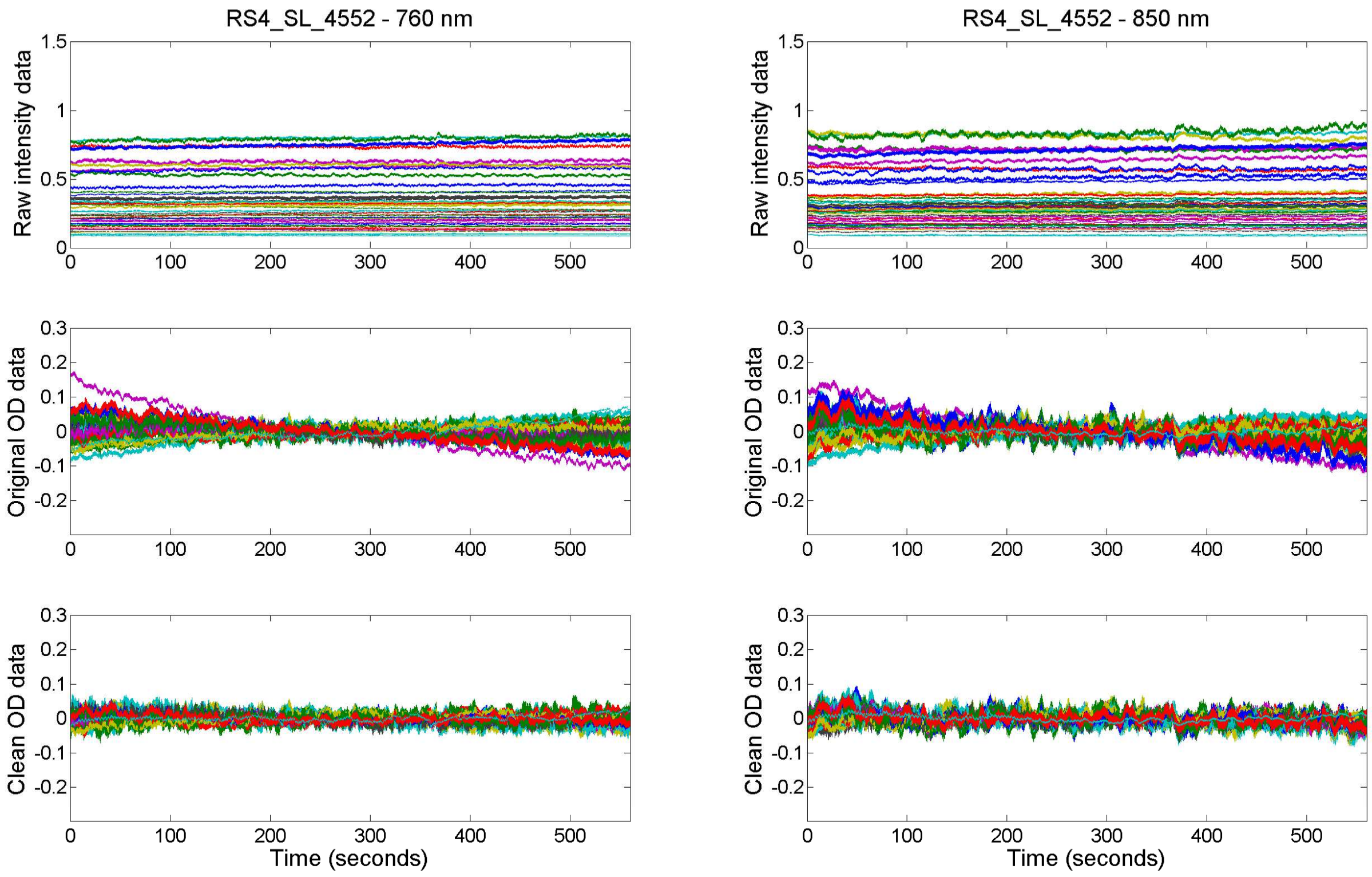
Raw
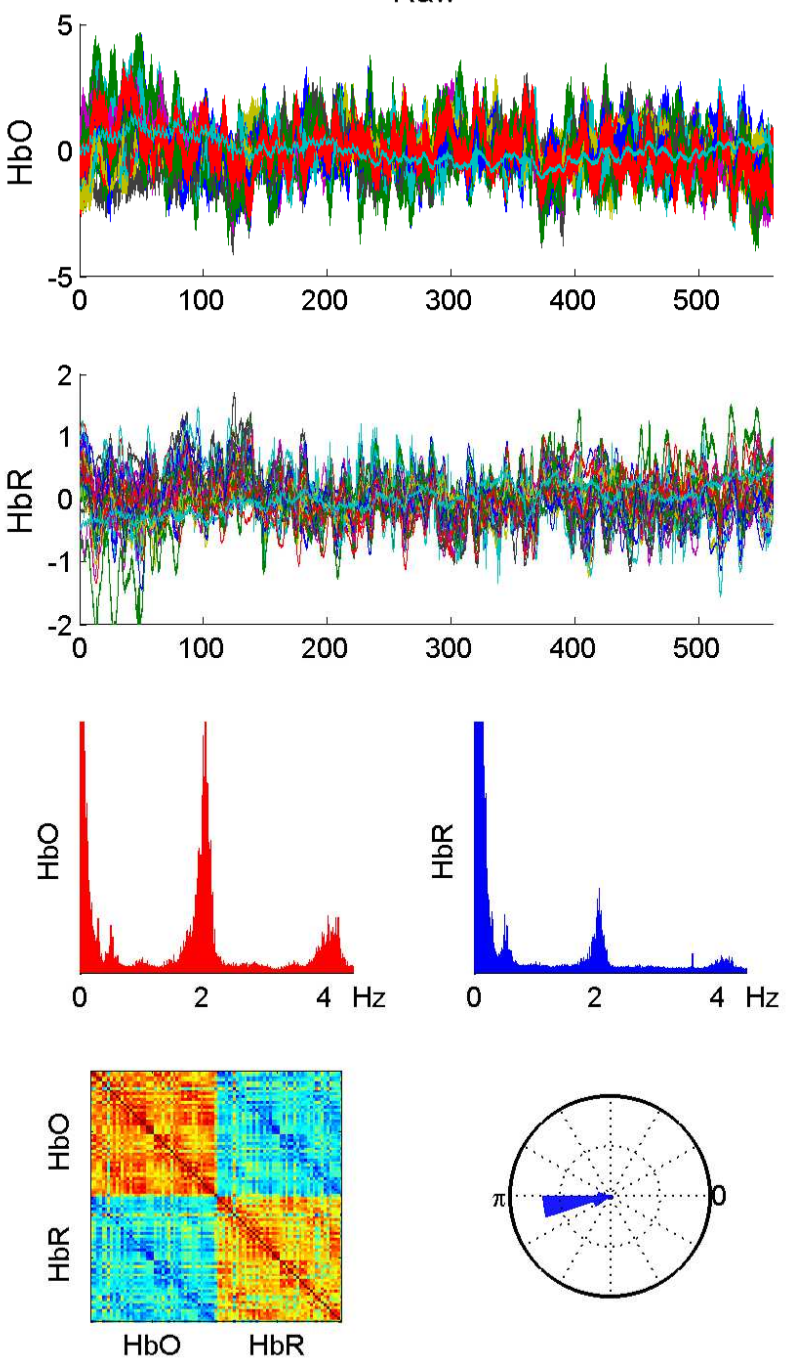

RS4_SL_4552

Filtered
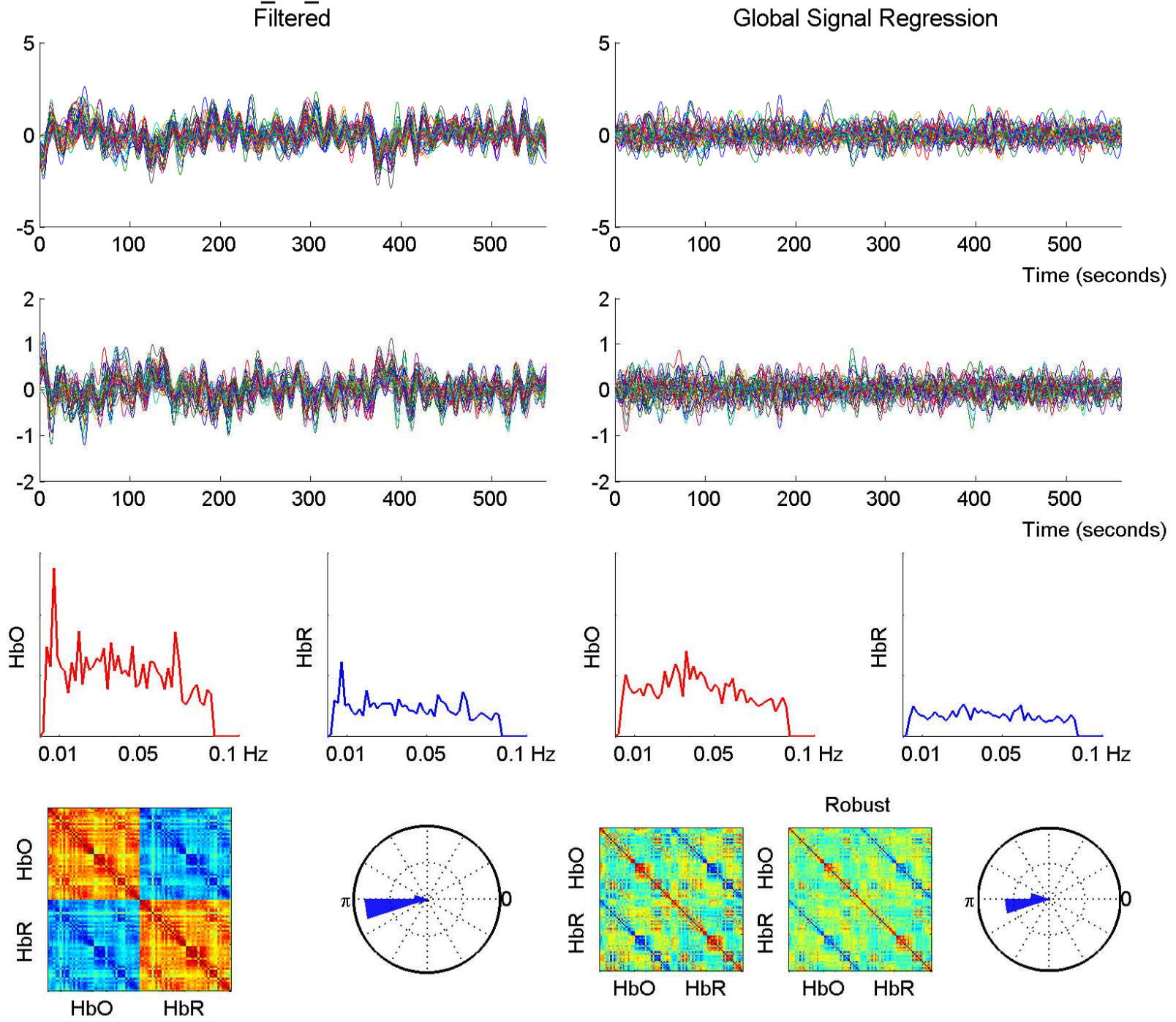

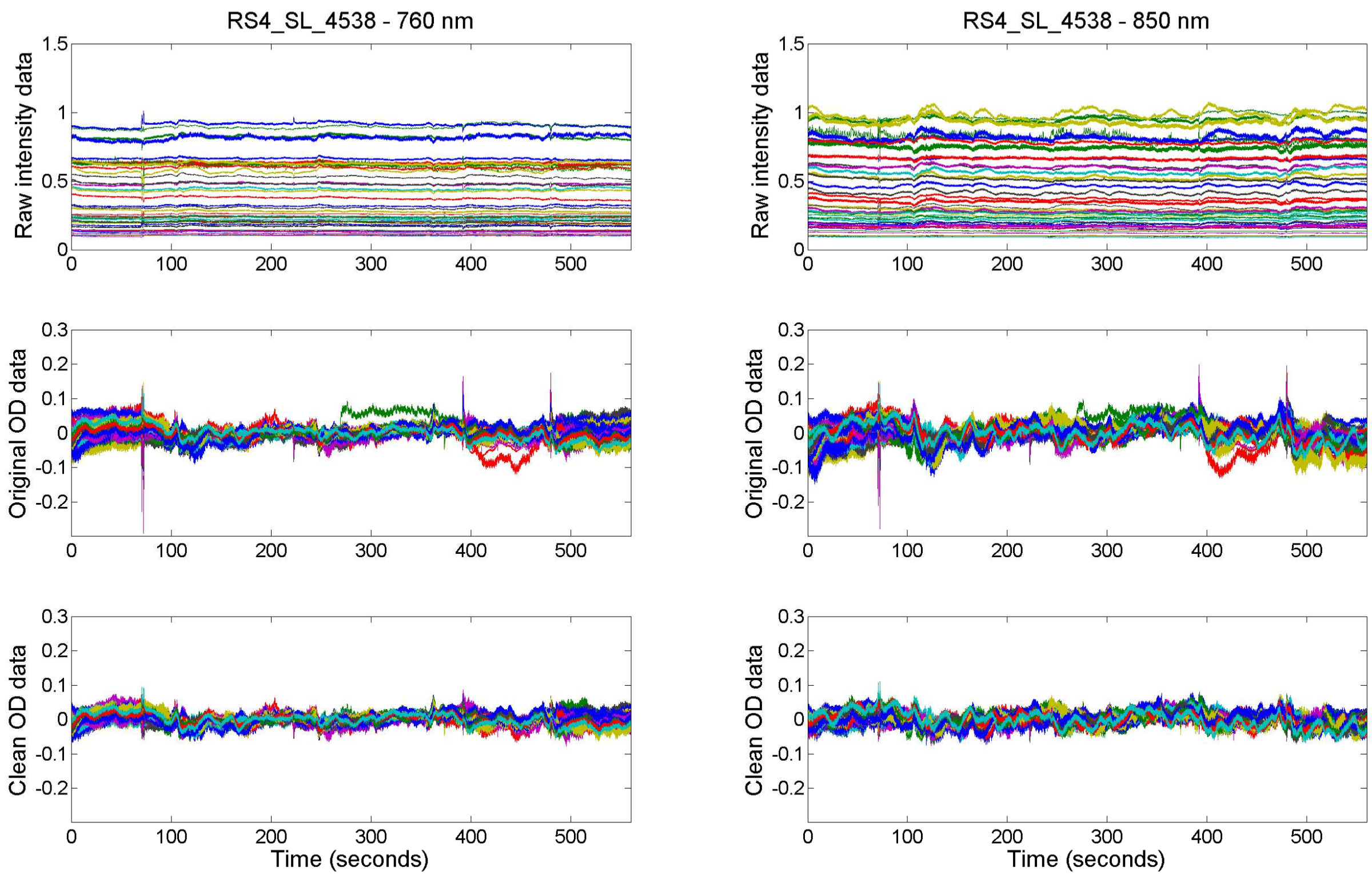
Raw
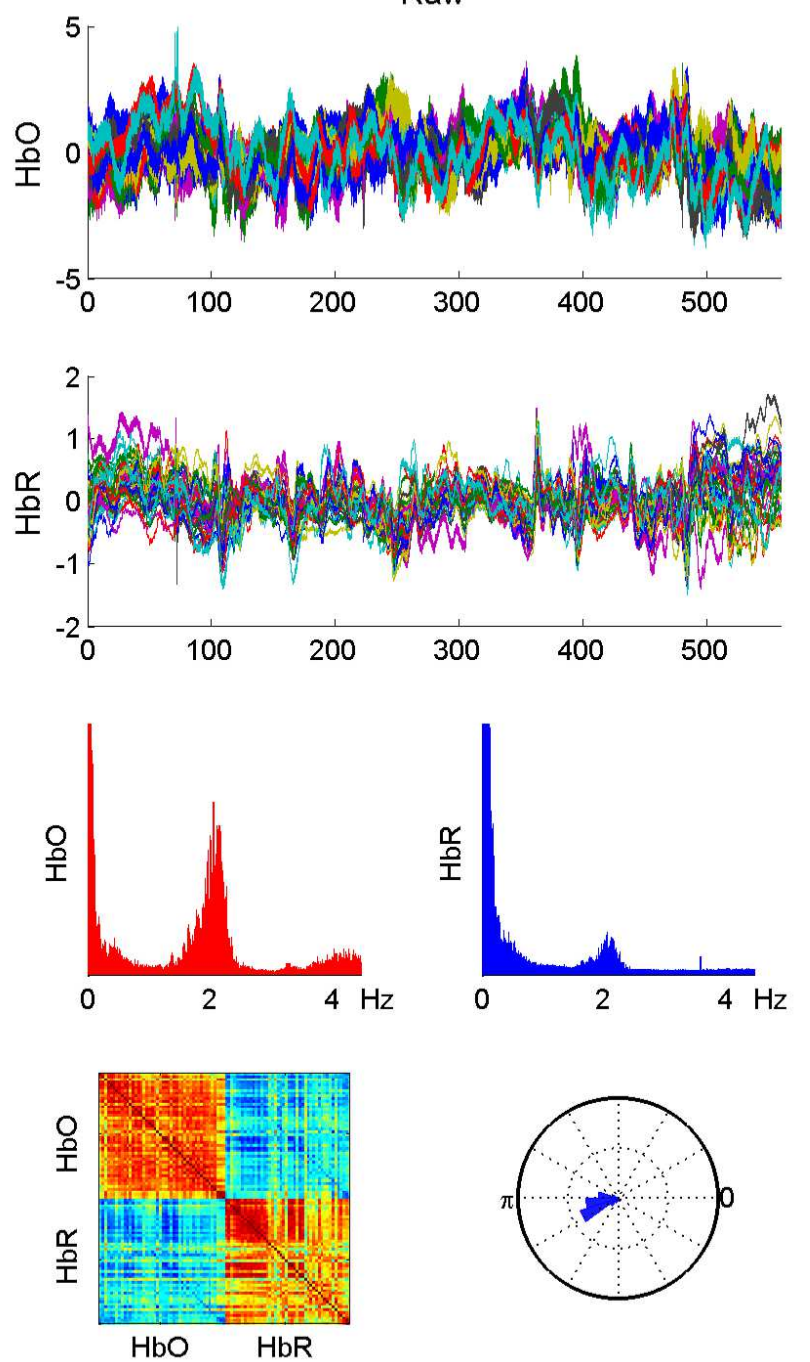

RS4 SL 4538
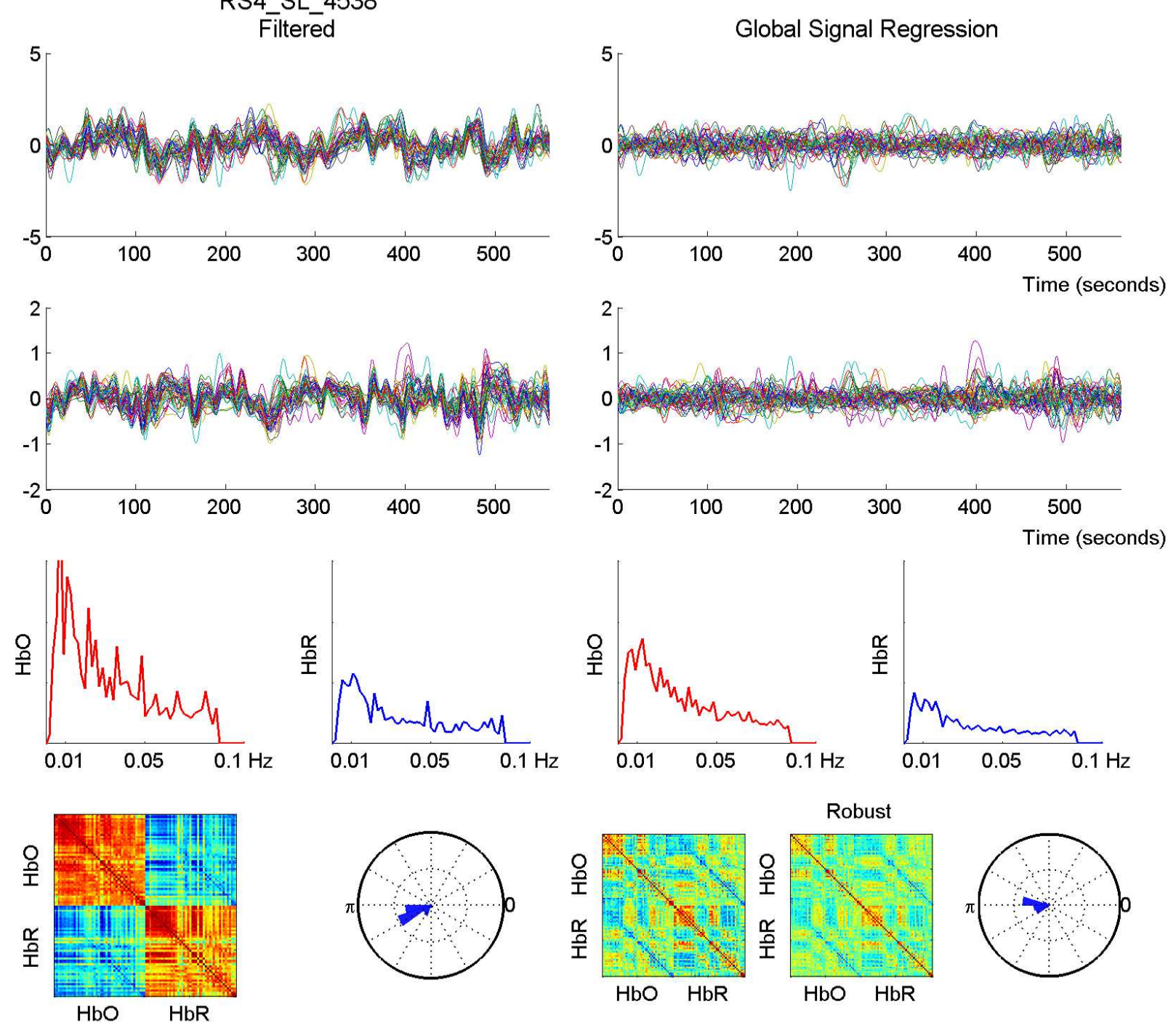

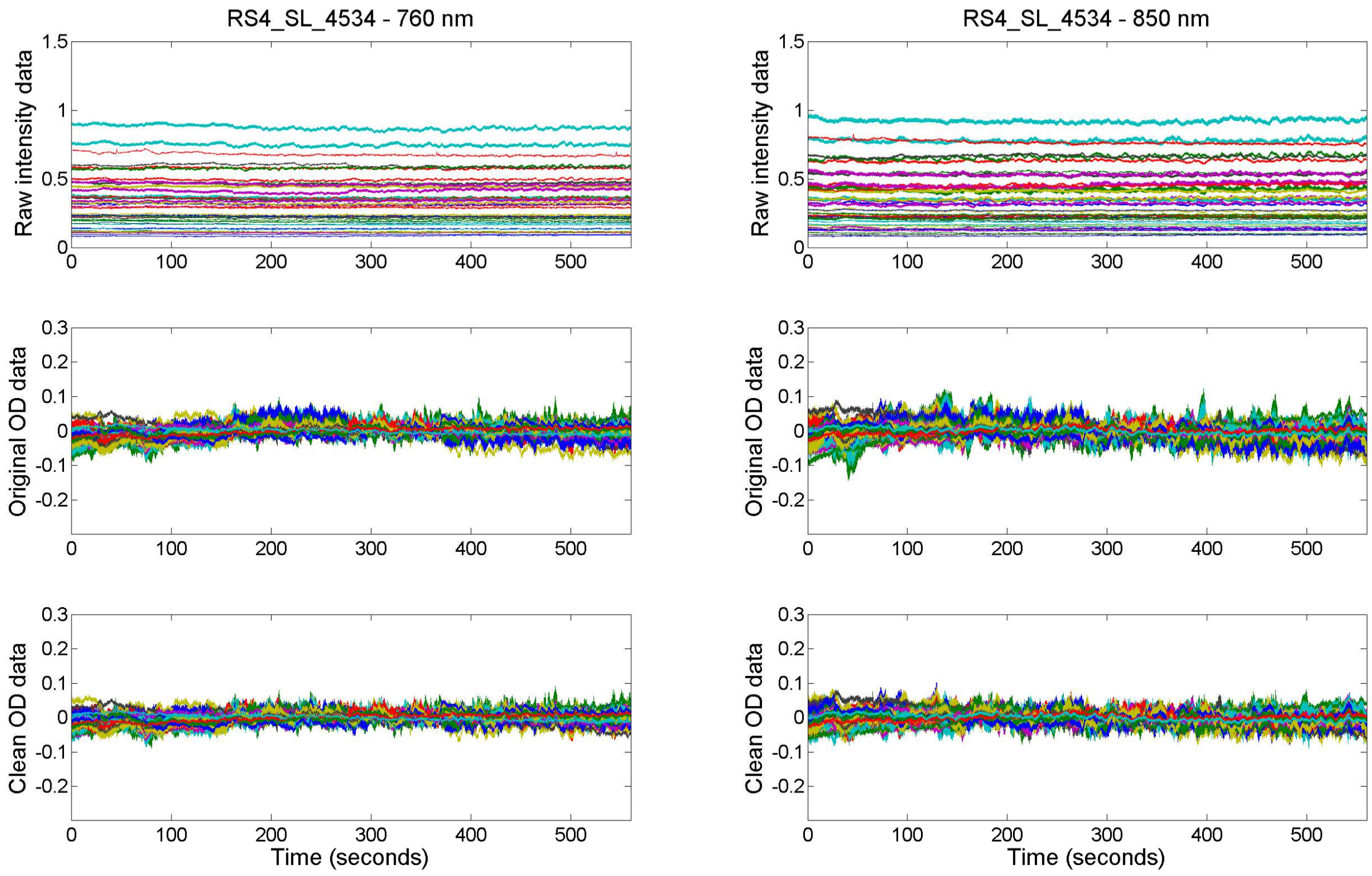
Raw

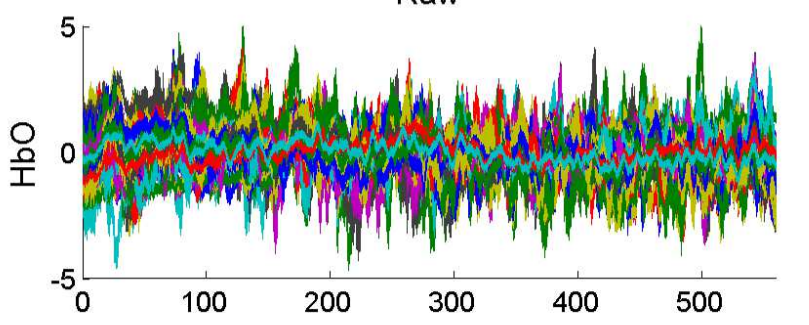

2
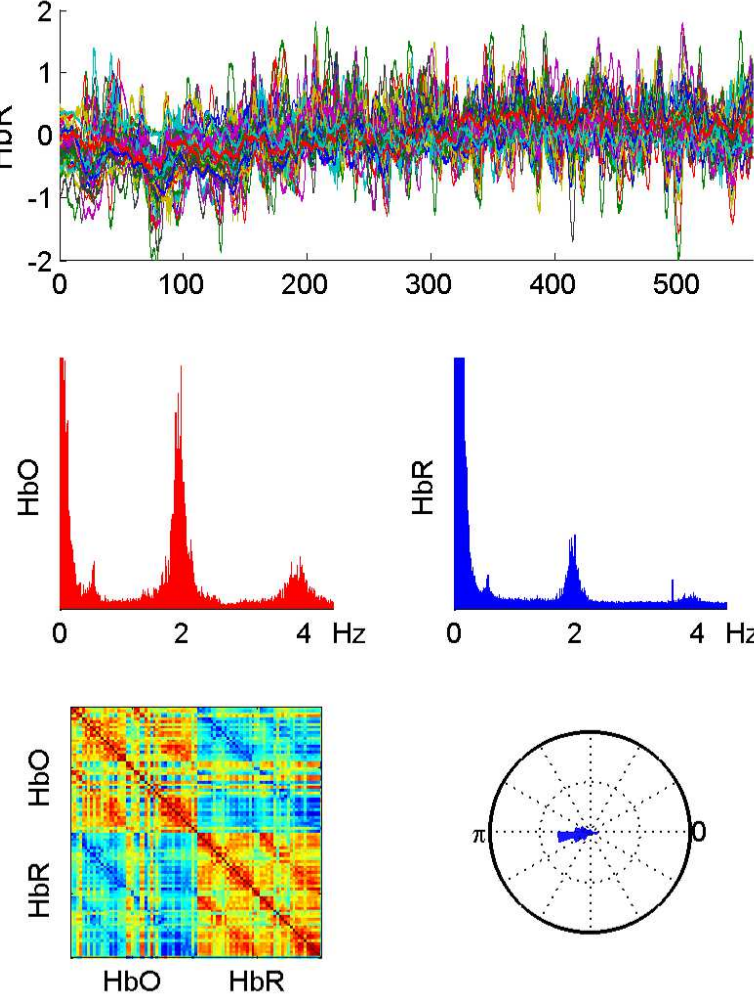

RS4_SL_4534

Filtered

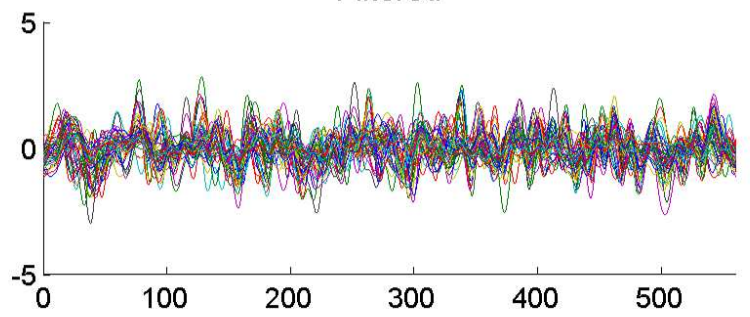

2
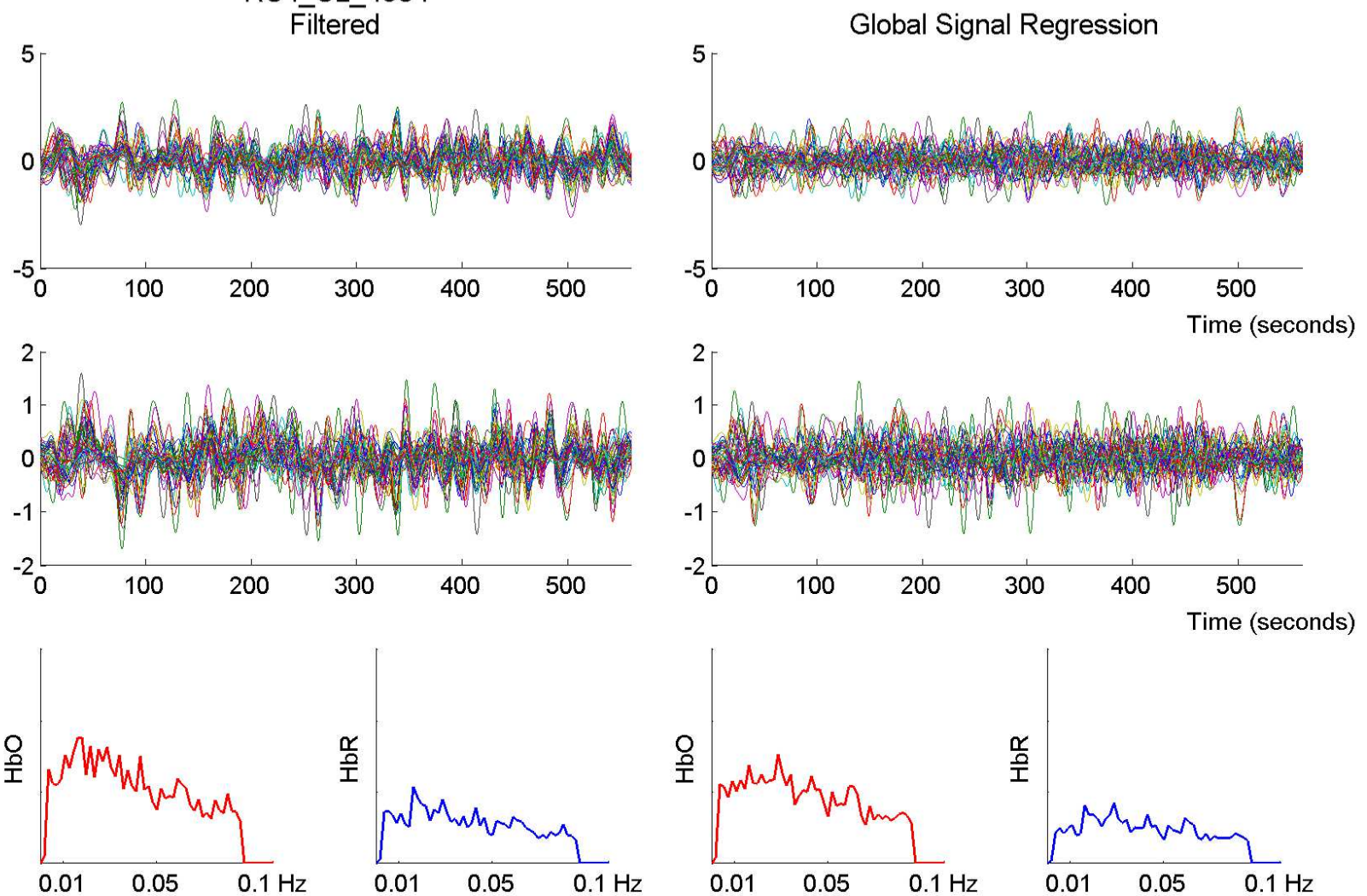

2
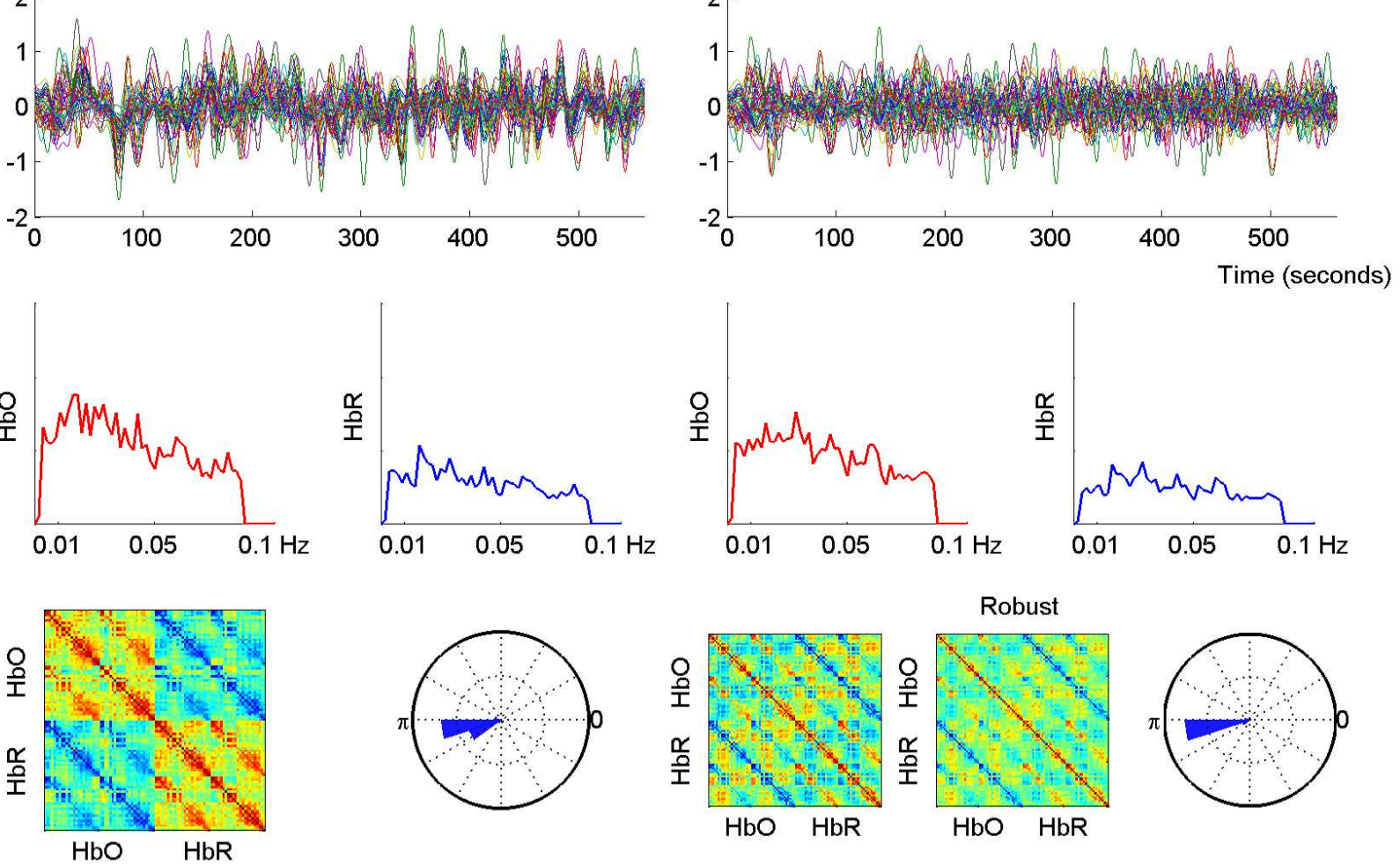

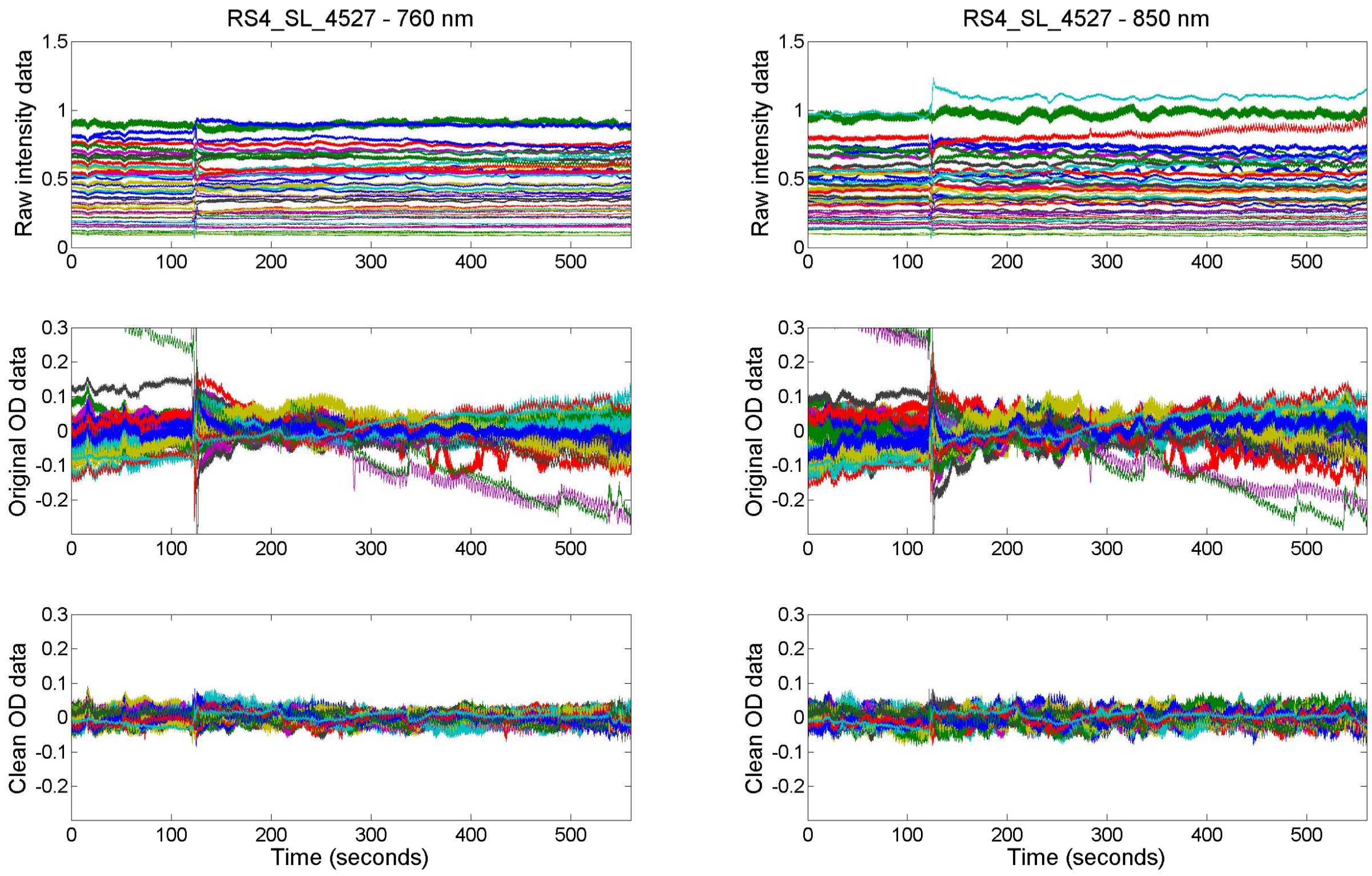
Raw
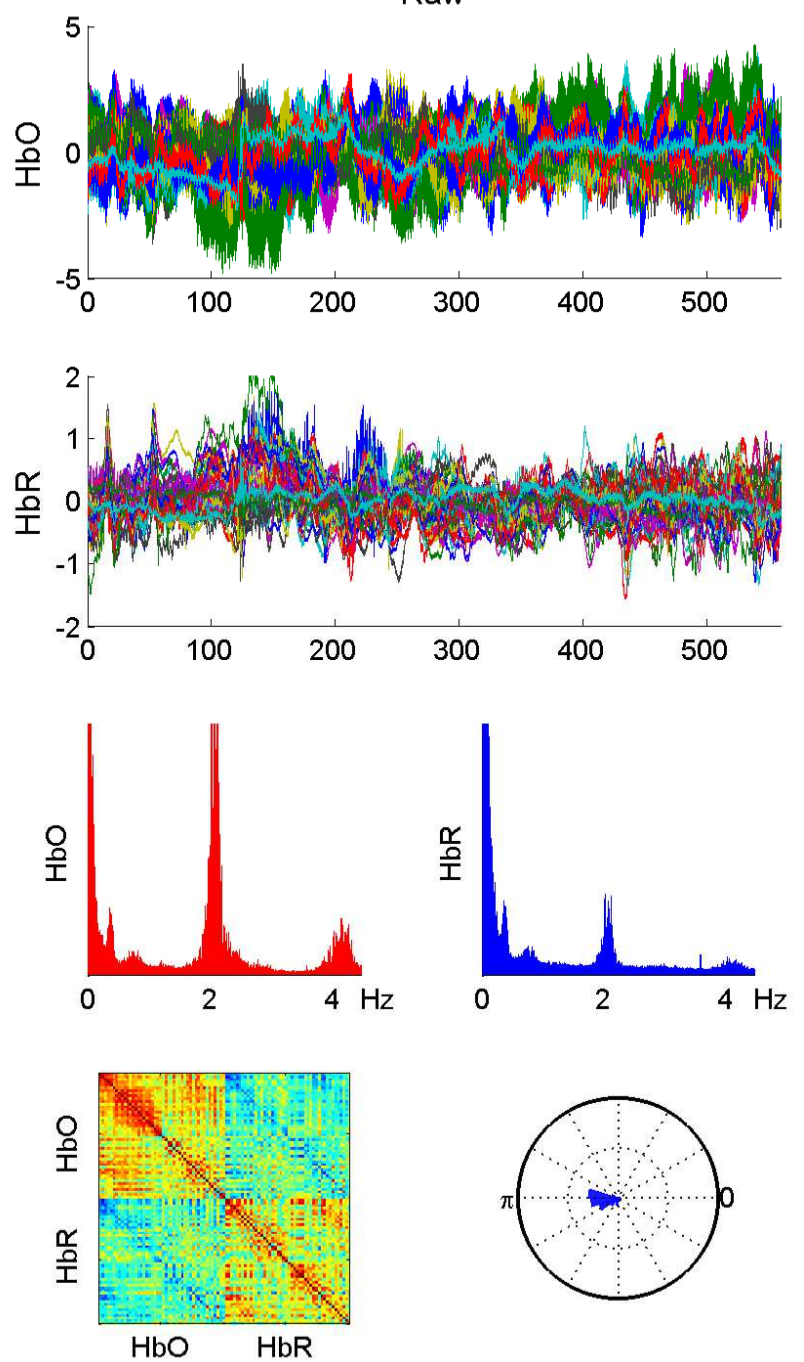

RS4 SL 4527

Filtered
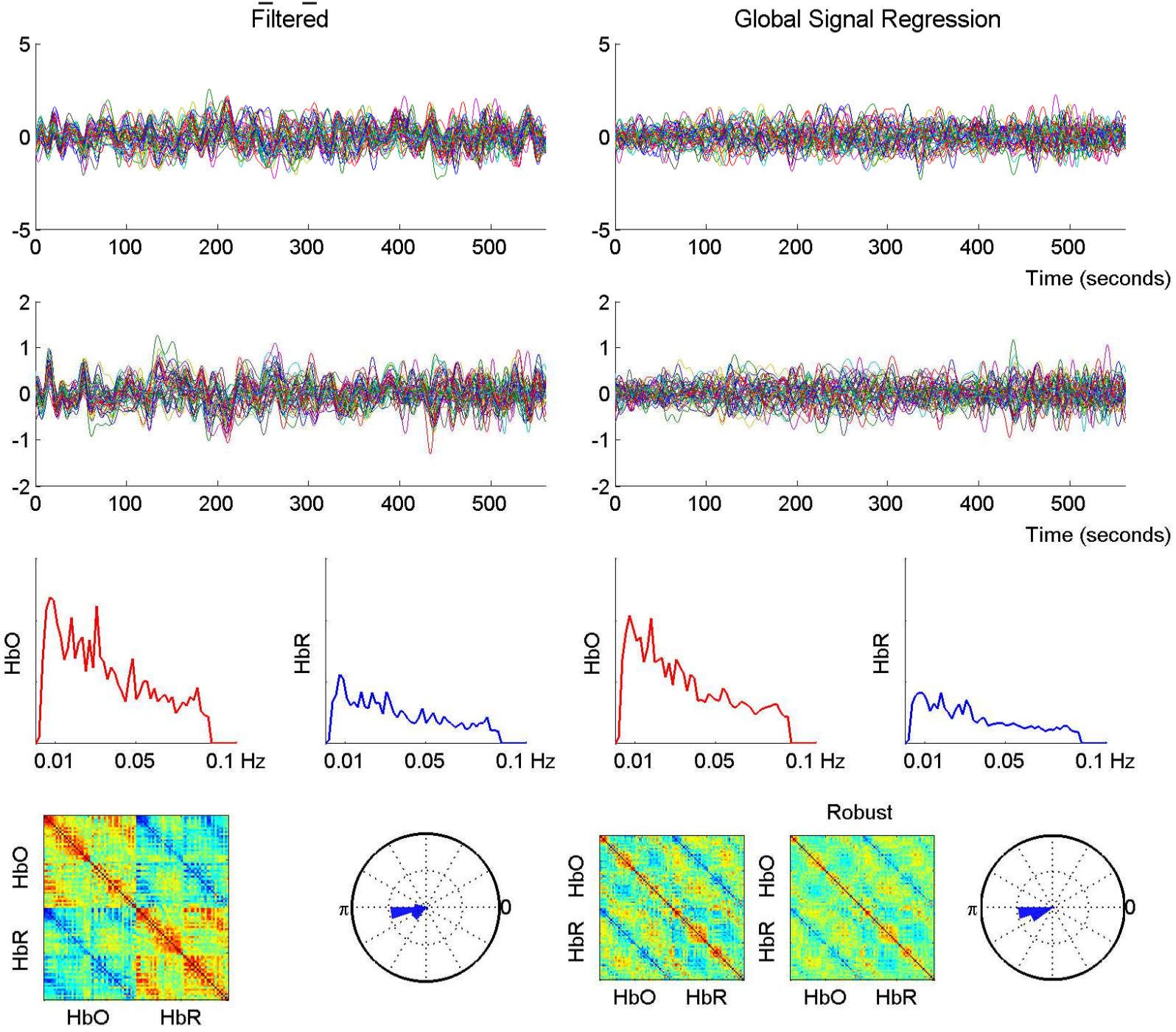
RS4 SL 4526 - 760 nm
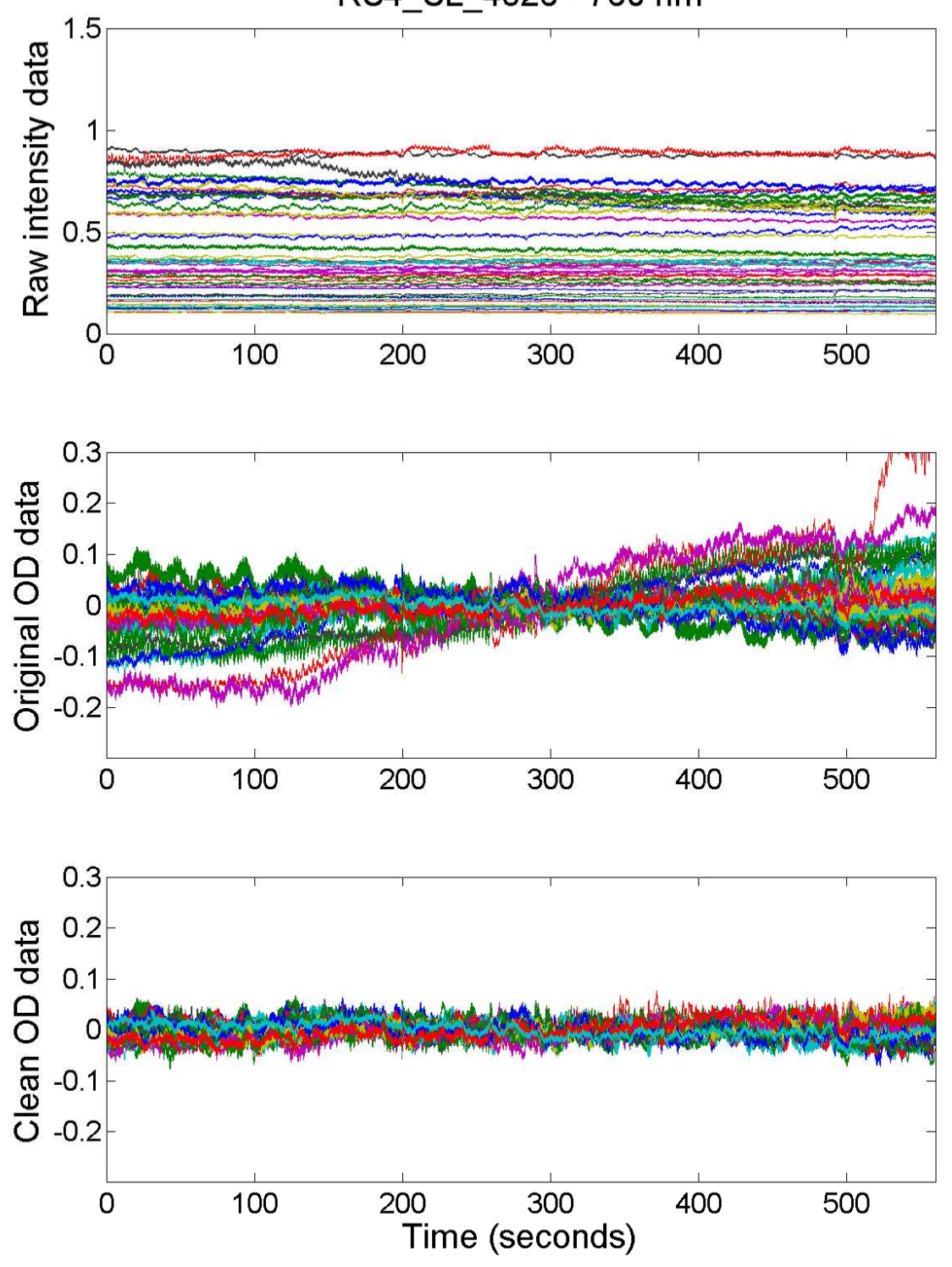

RS4 SL $4526-850 \mathrm{~nm}$
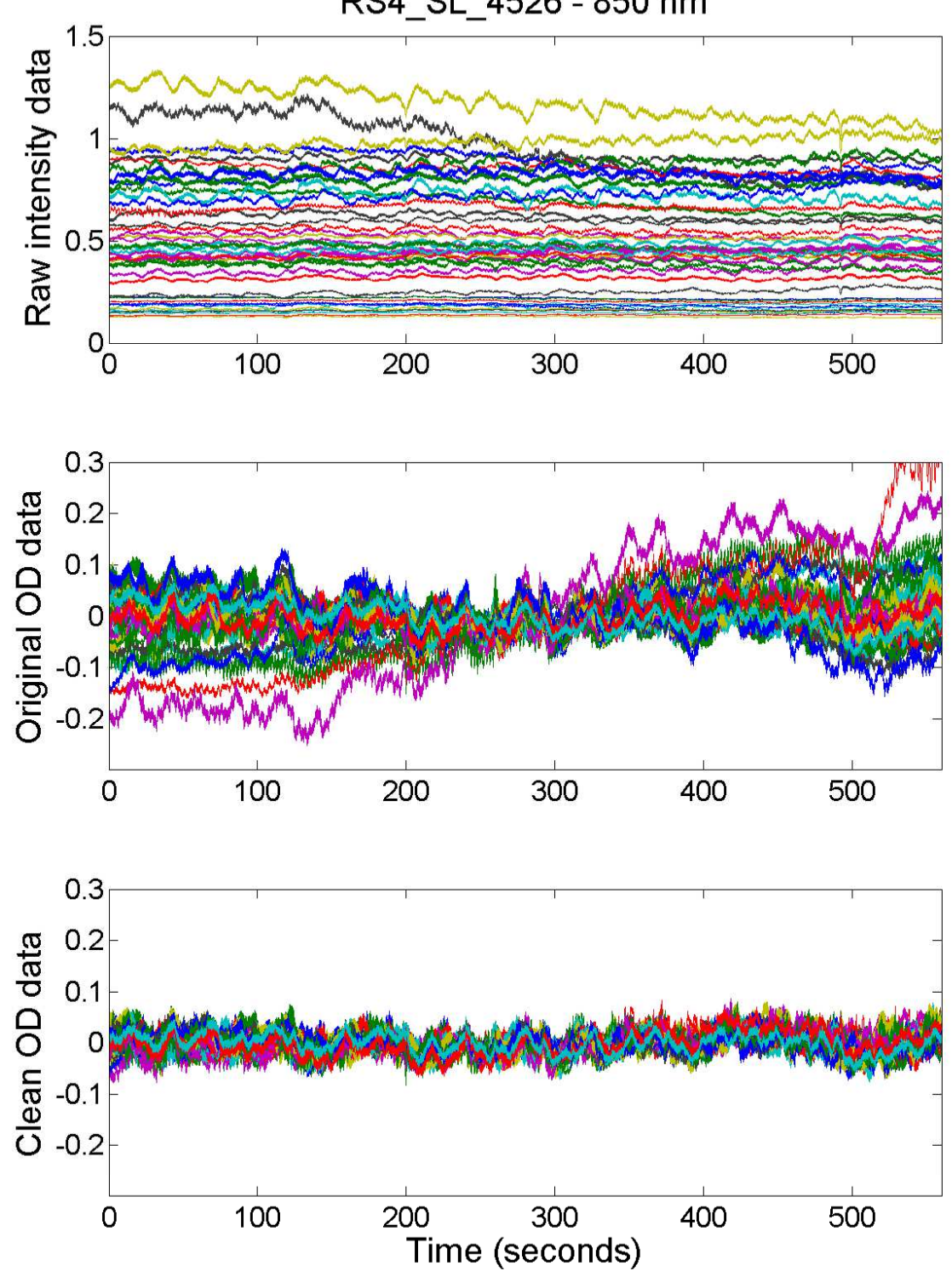
Raw
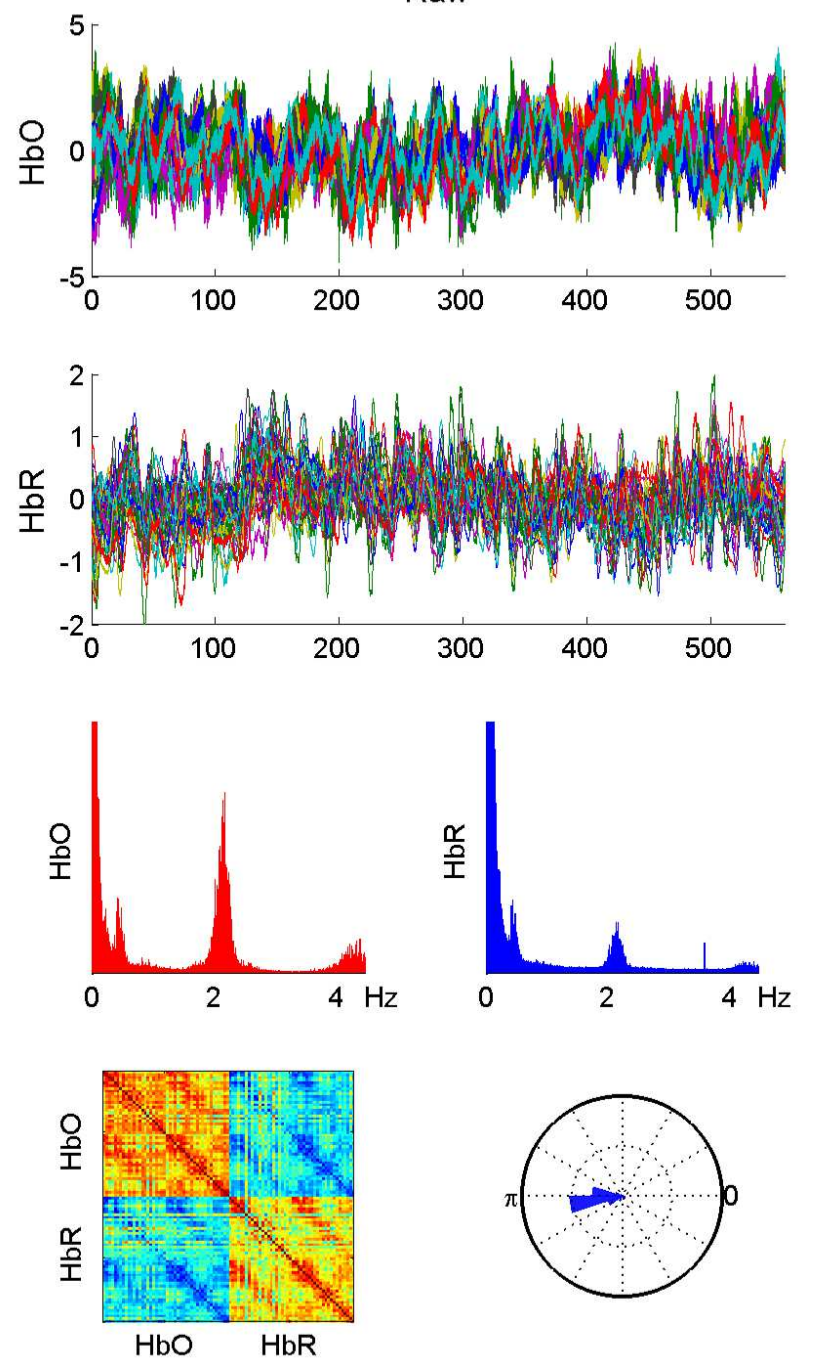

RS4_SL_4526

Filtered

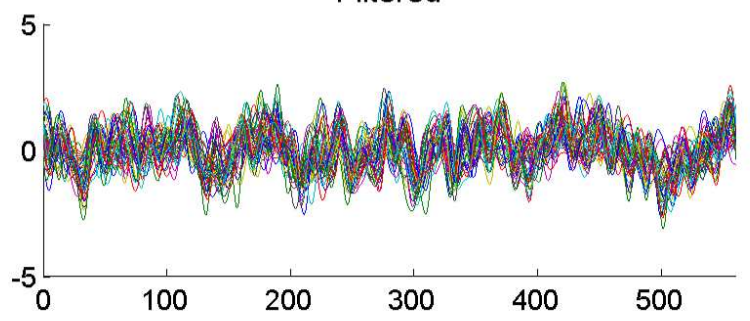

2
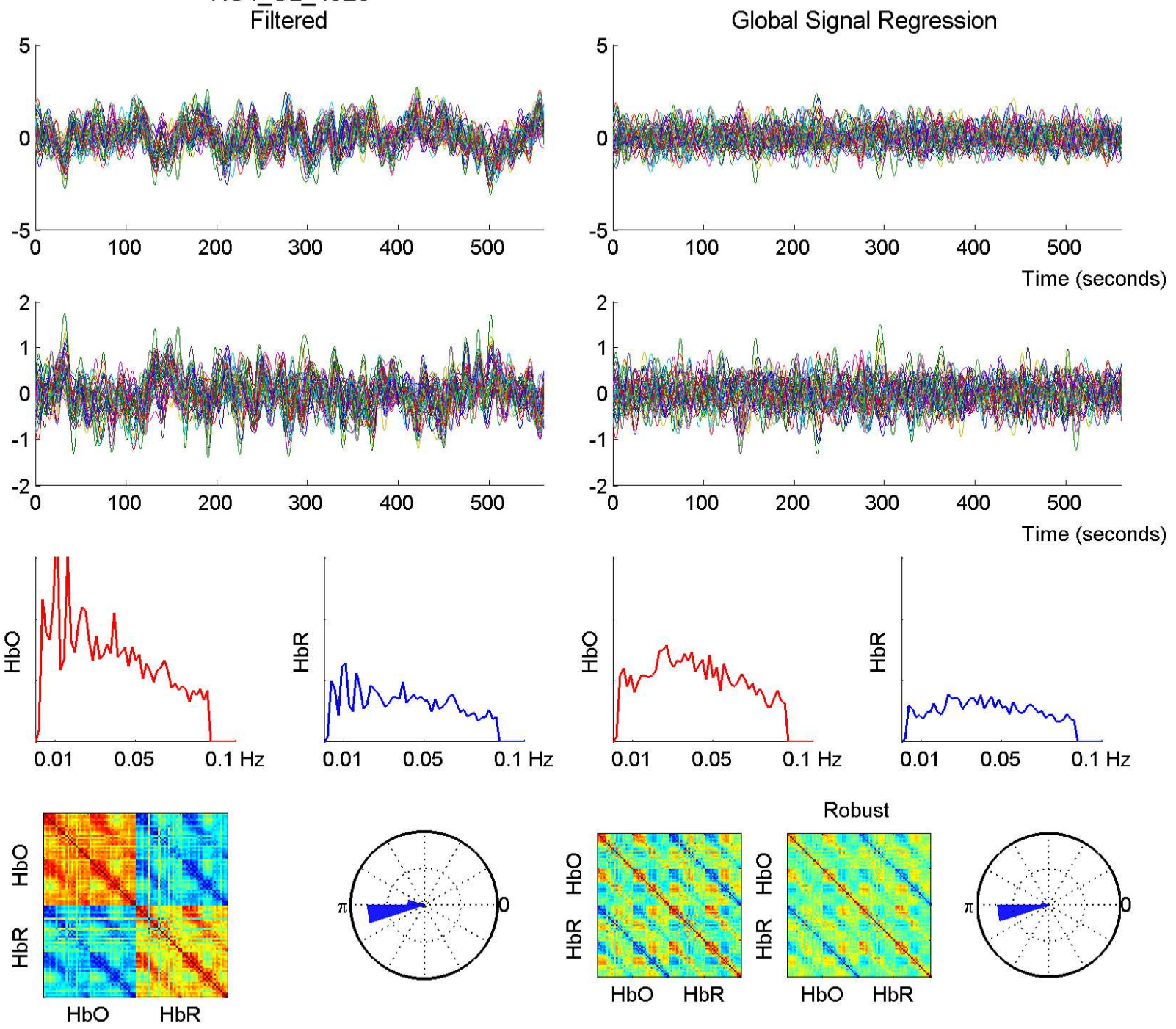

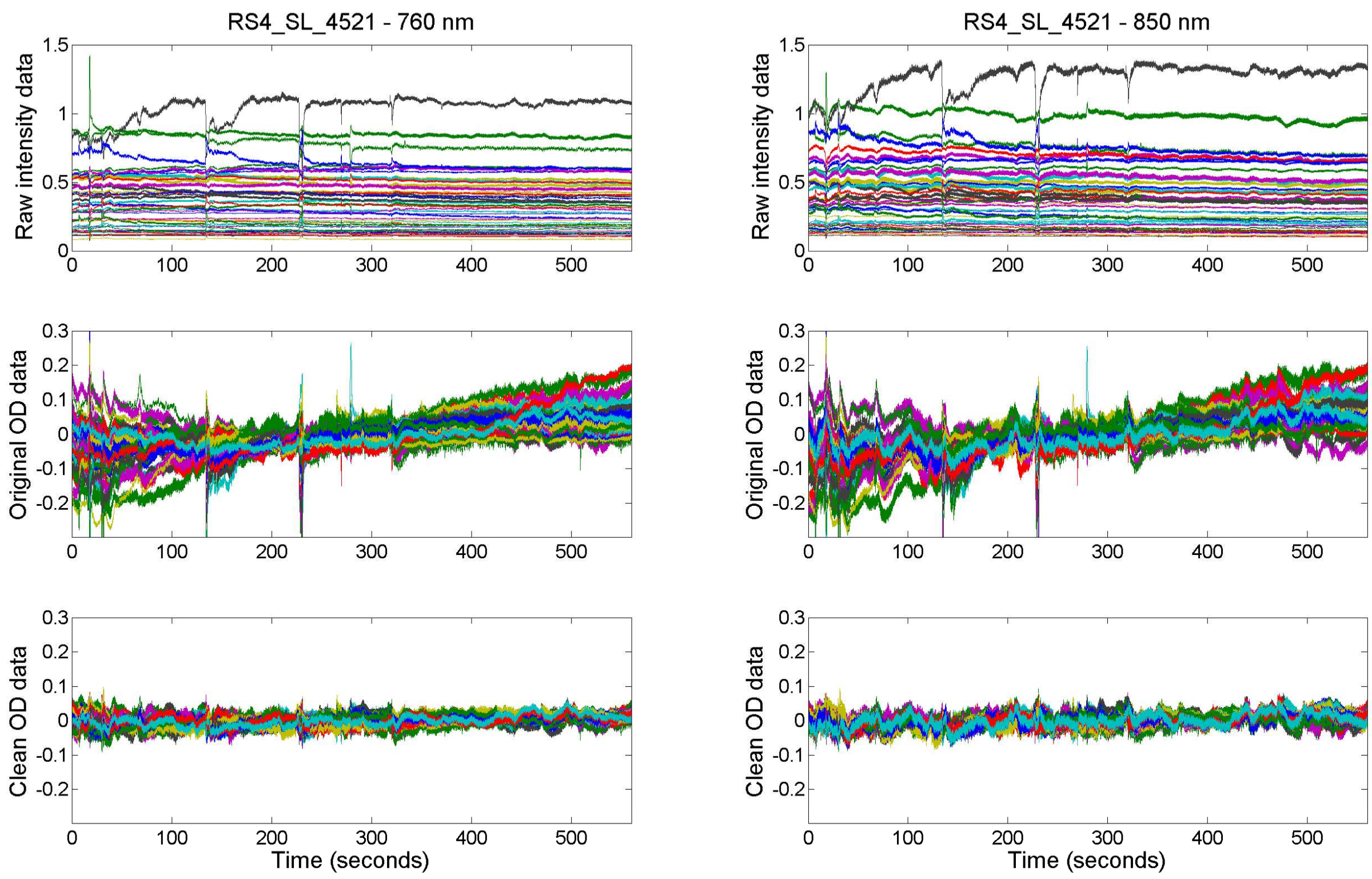
Raw
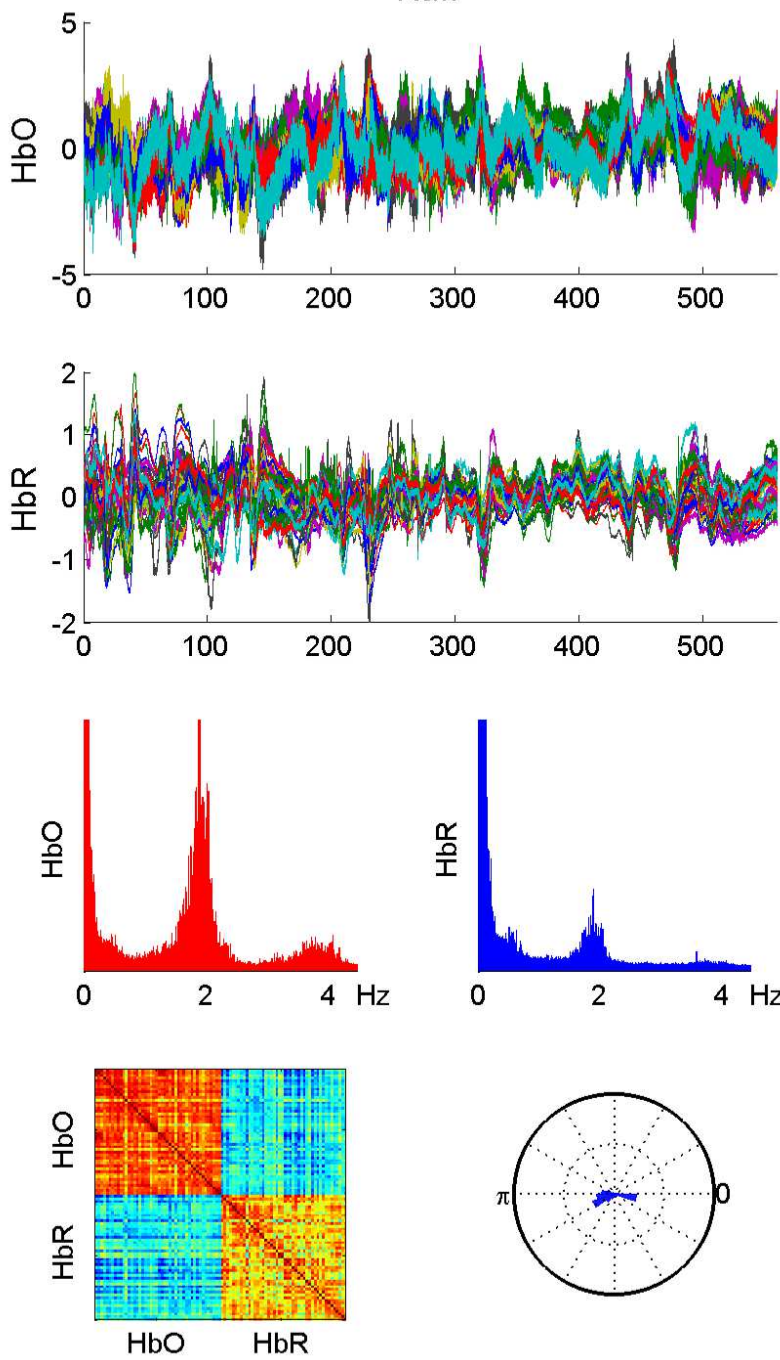

RS4 SL 4521

Filtered
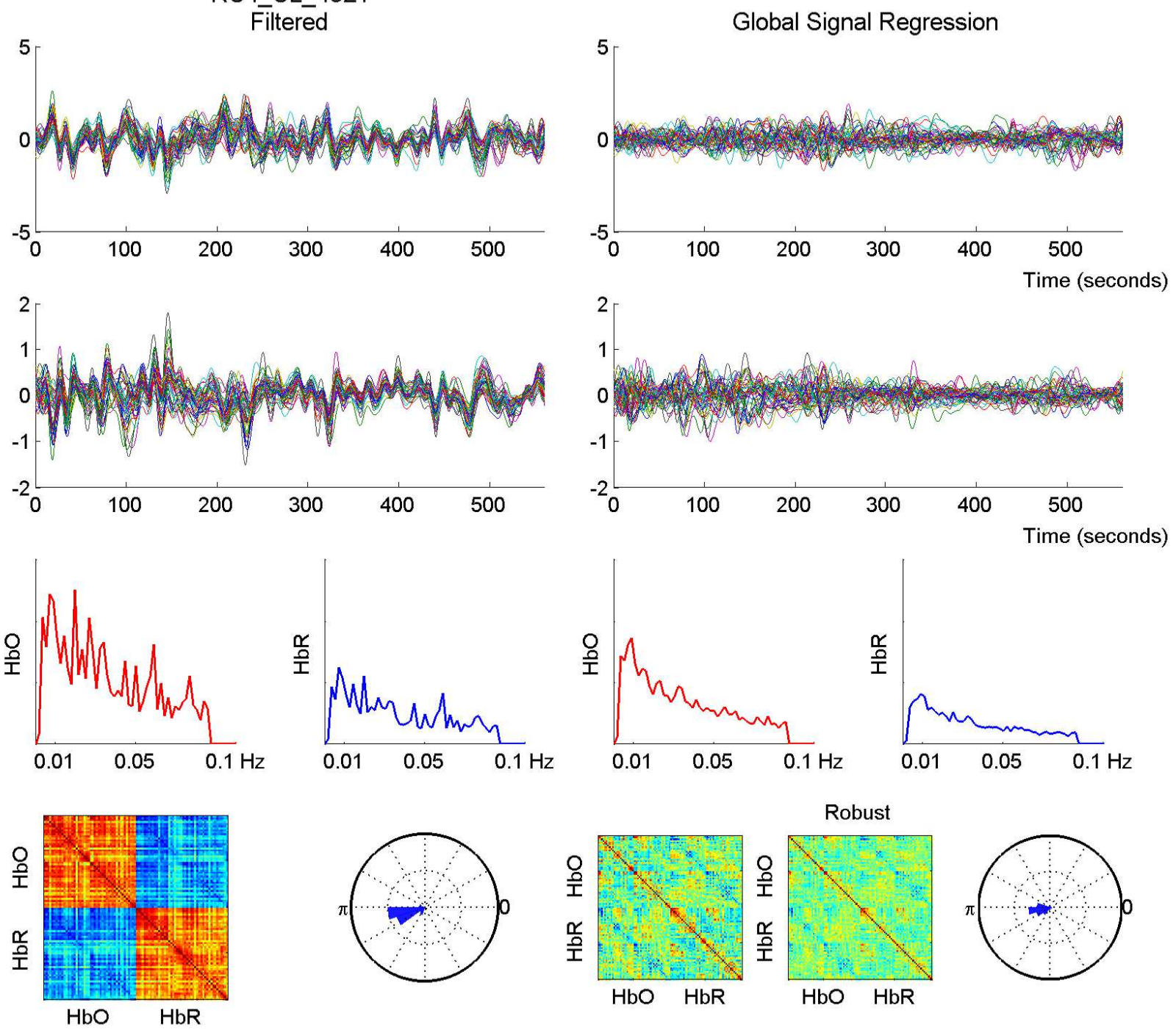
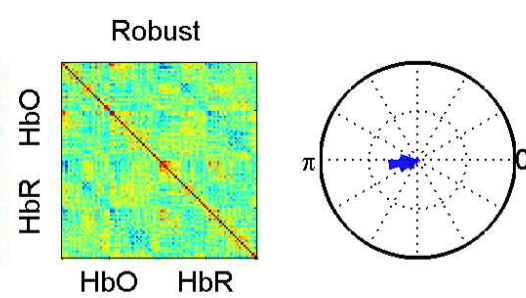
RS4 SL 4518 - 760 nm
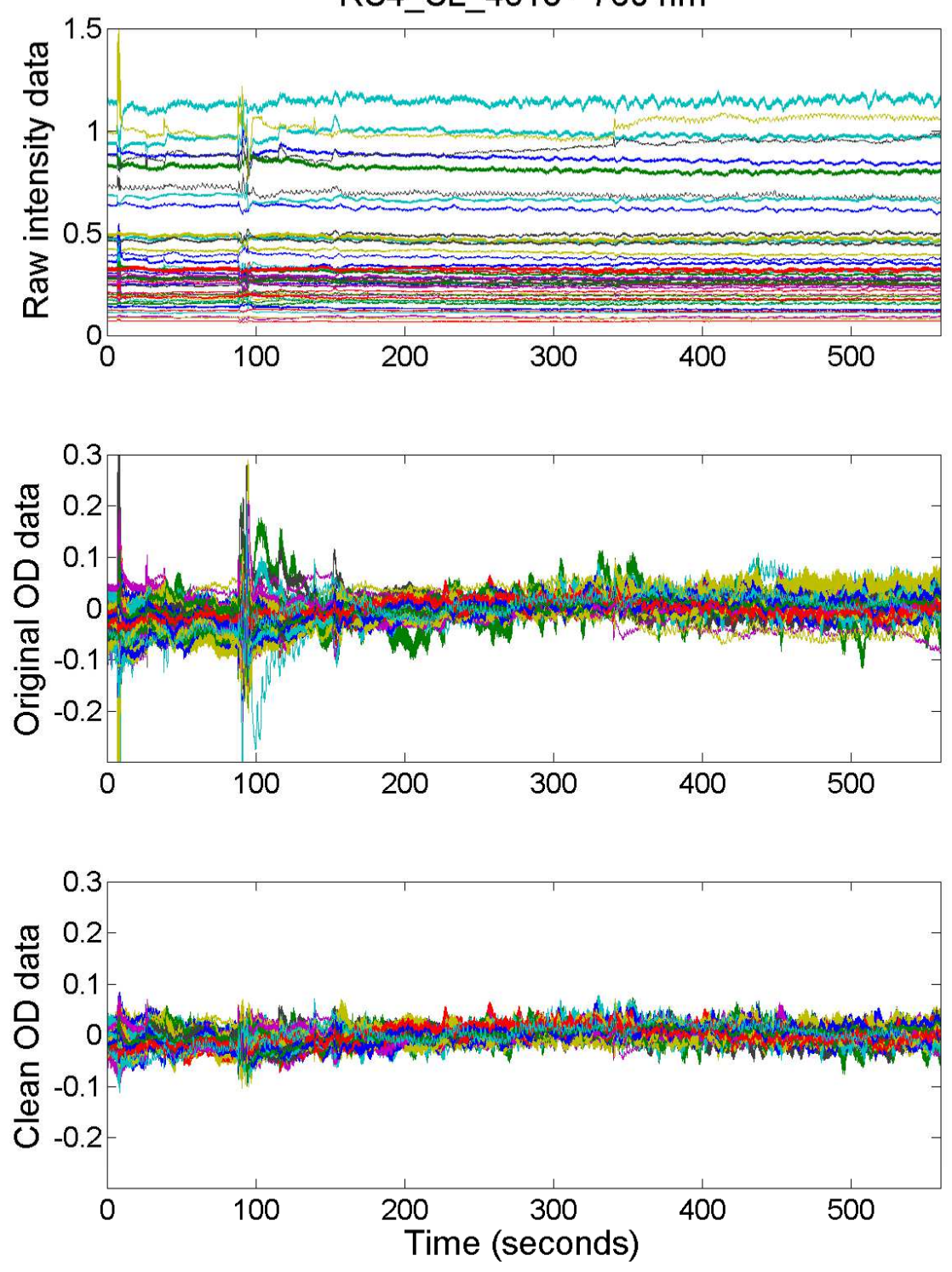

RS4 SL $4518-850 \mathrm{~nm}$
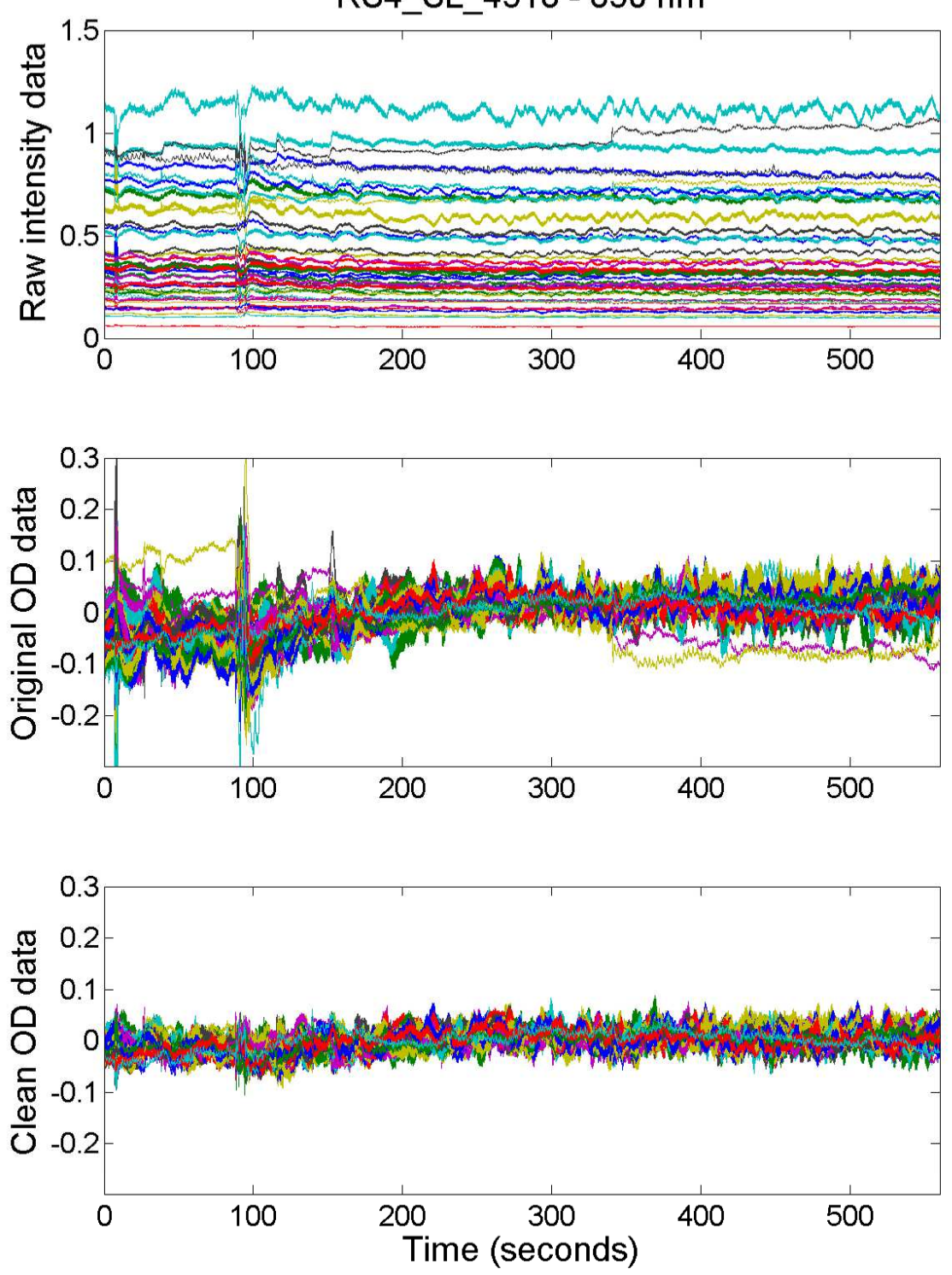
Raw
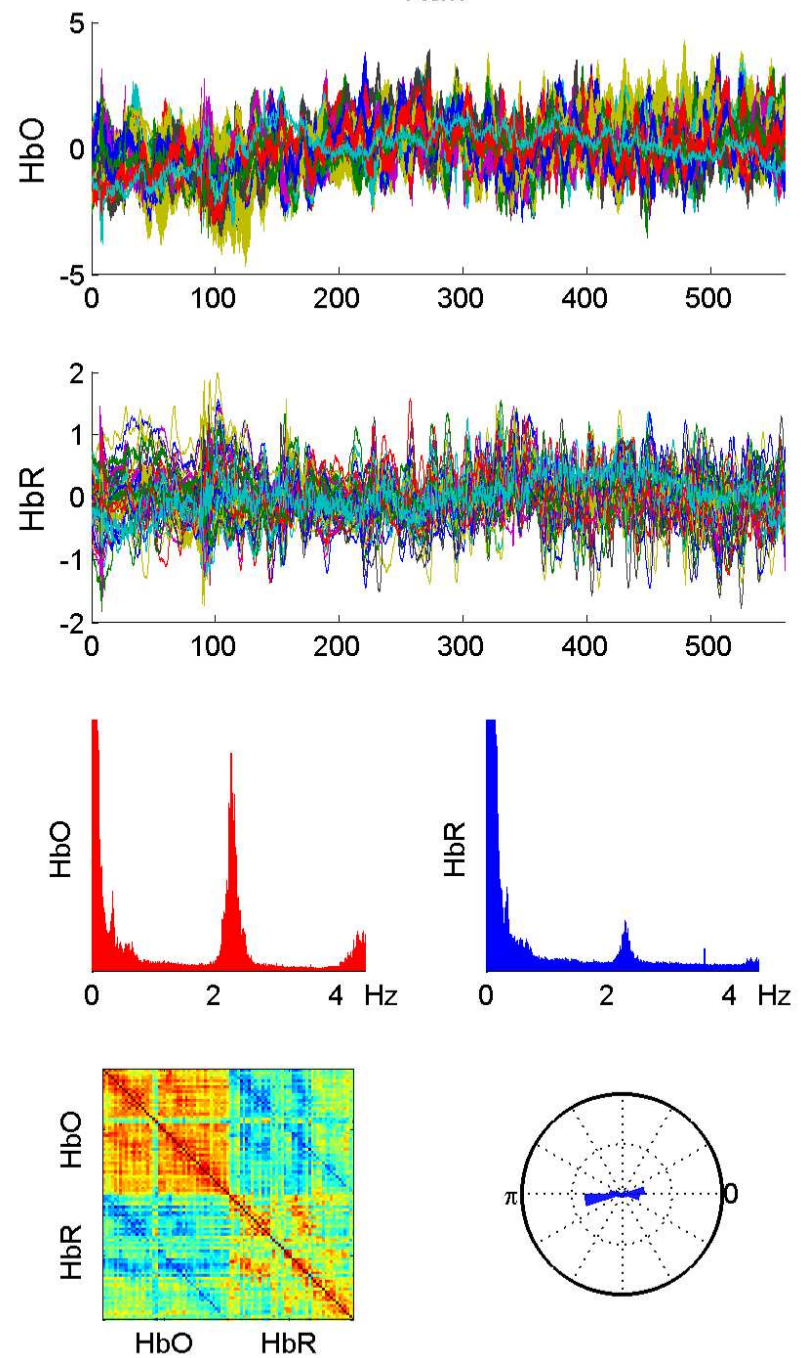

RS4 SL 4518

Filtered
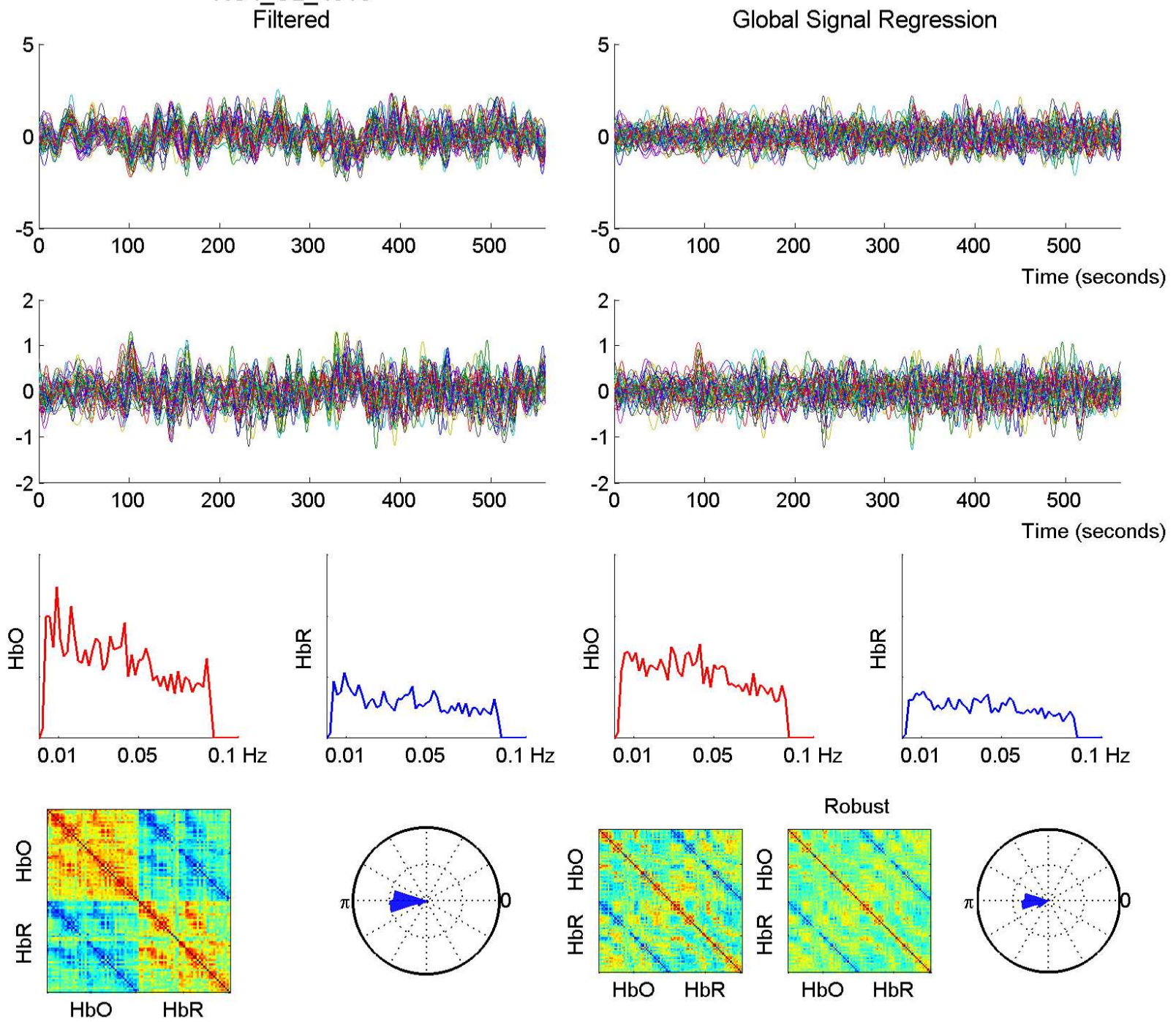
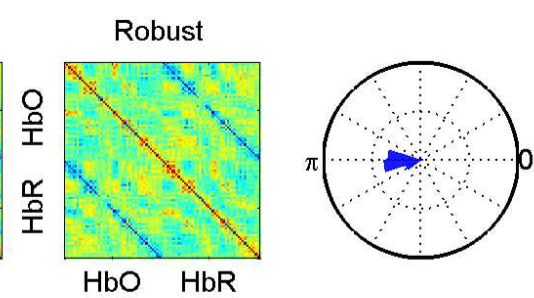
RS4 SL 4515 - 760 nm
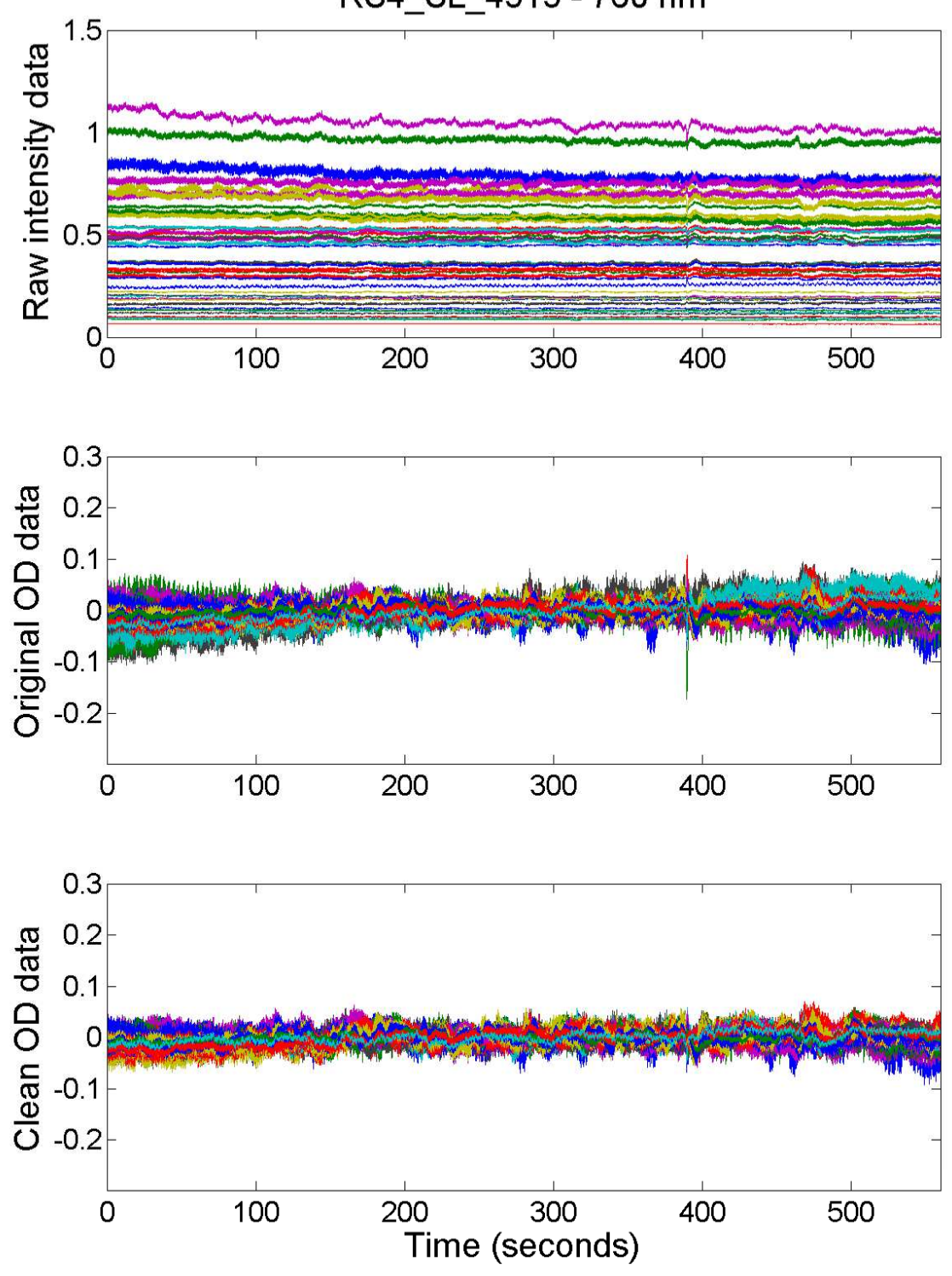

RS4 SL $4515-850 \mathrm{~nm}$
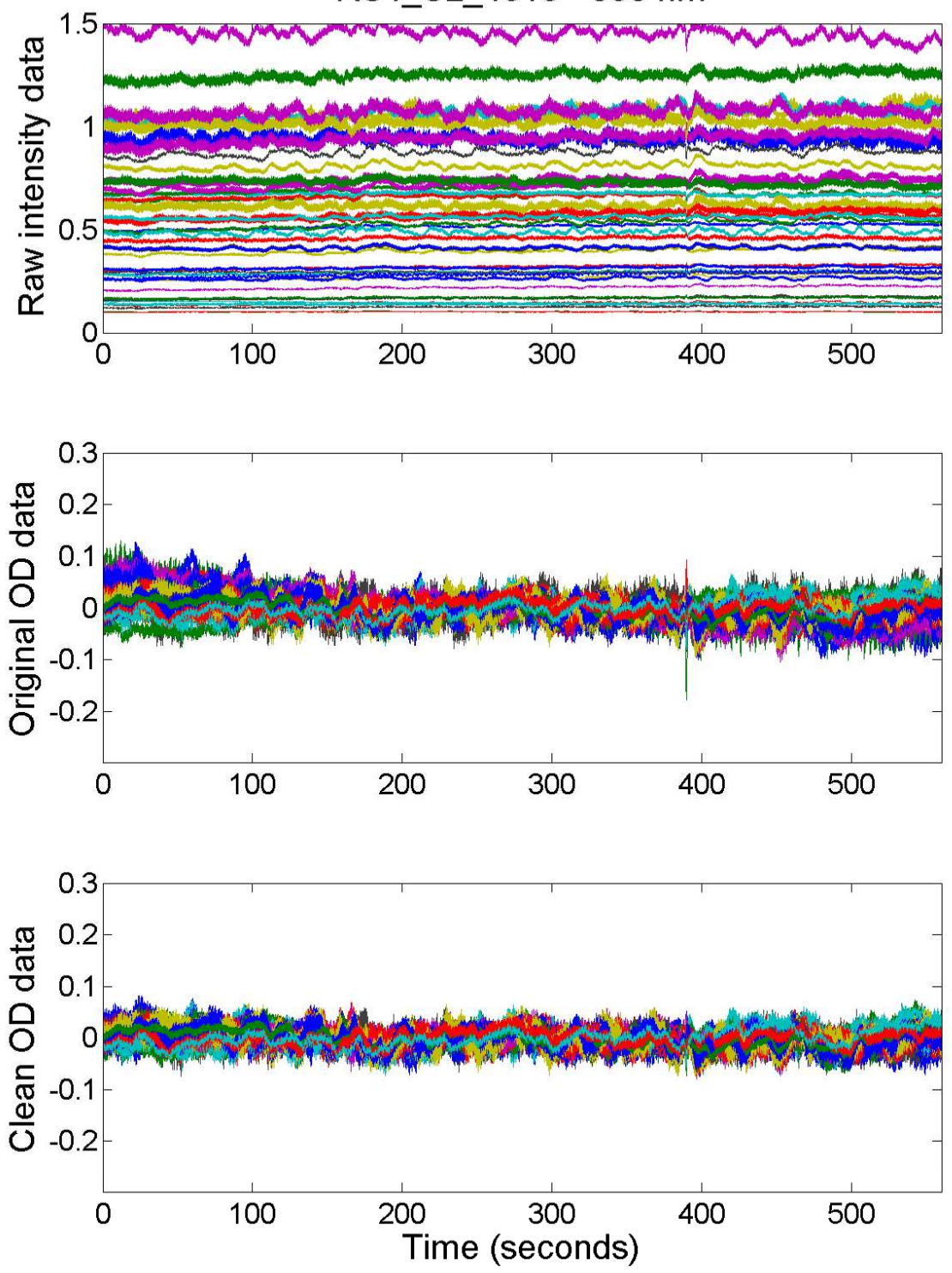
Raw
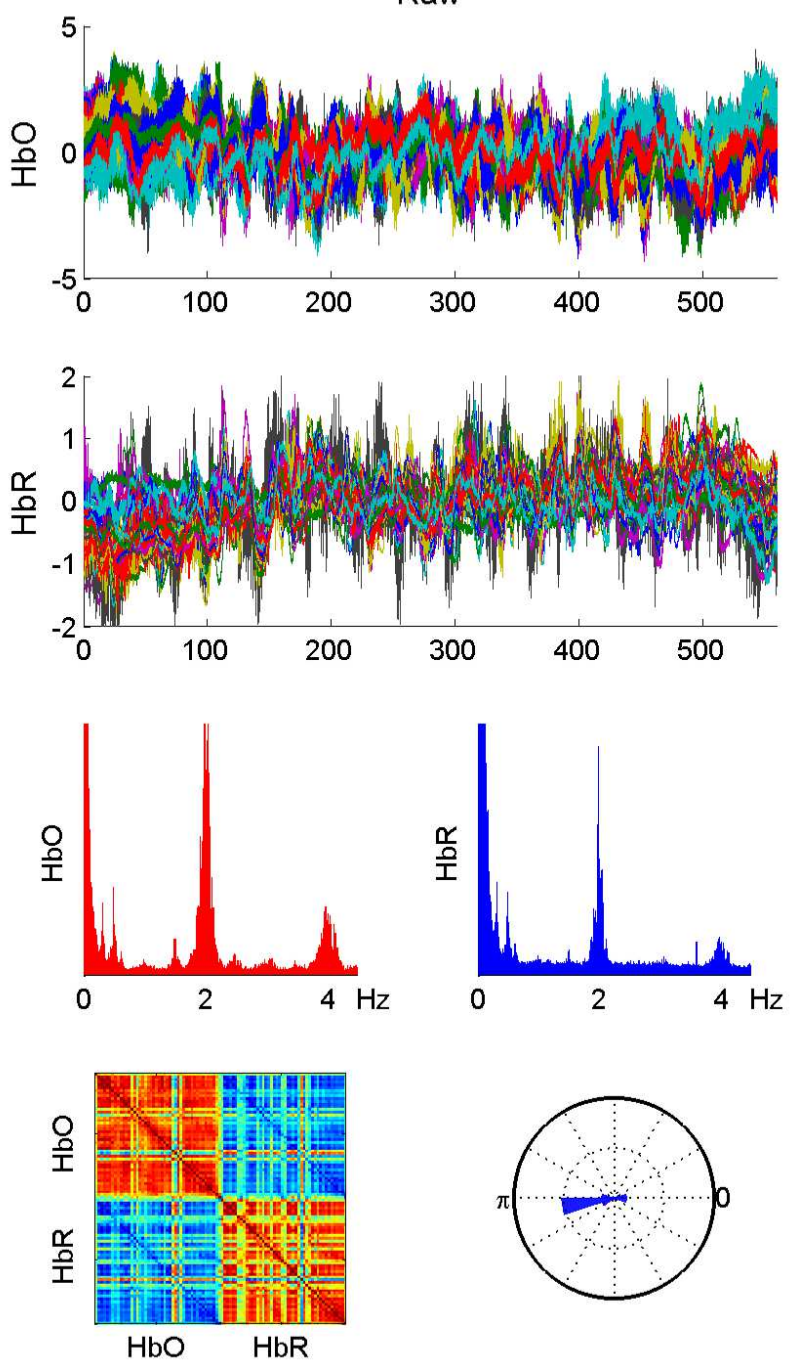

RS4 SL 4515

Filtered
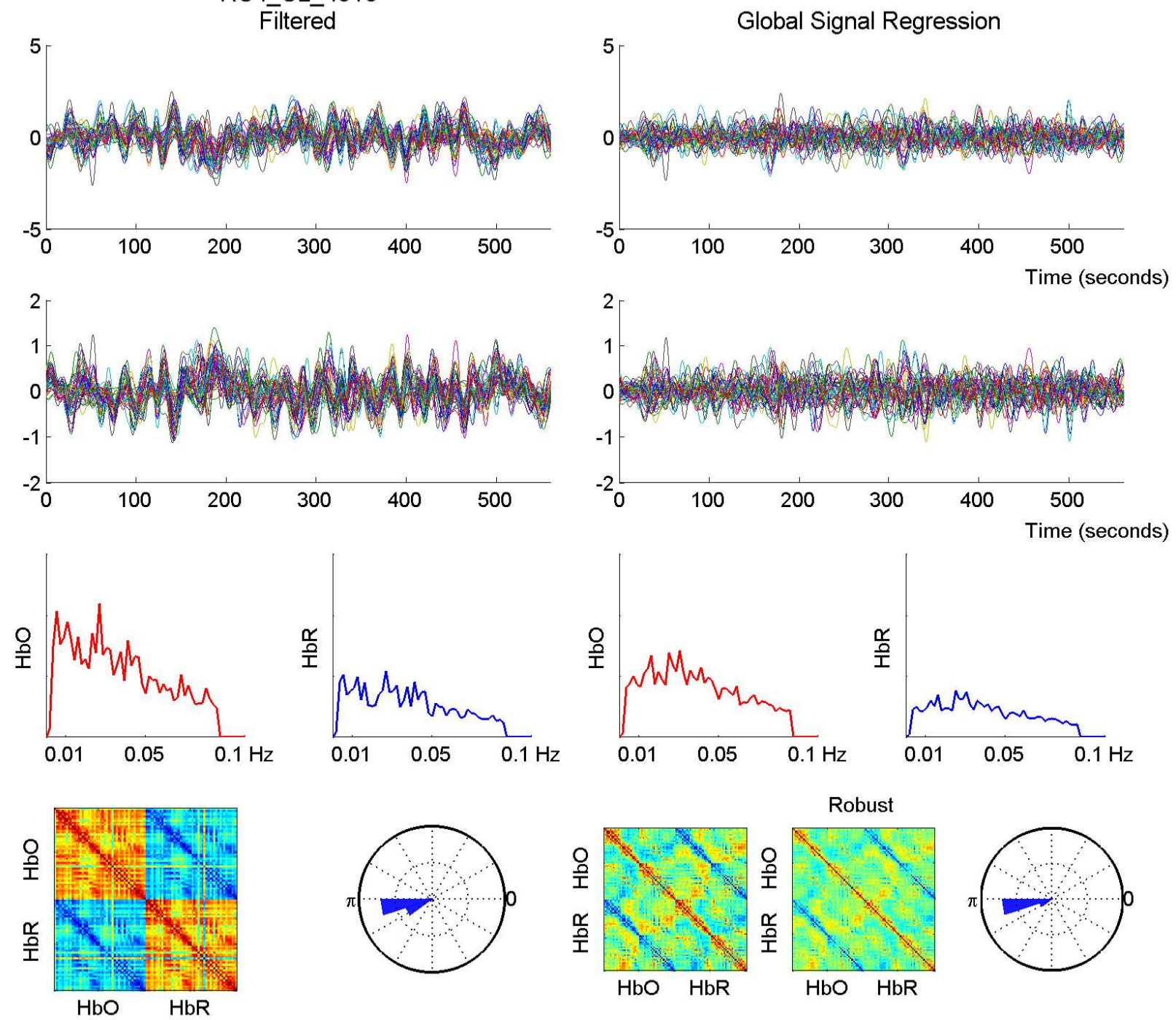

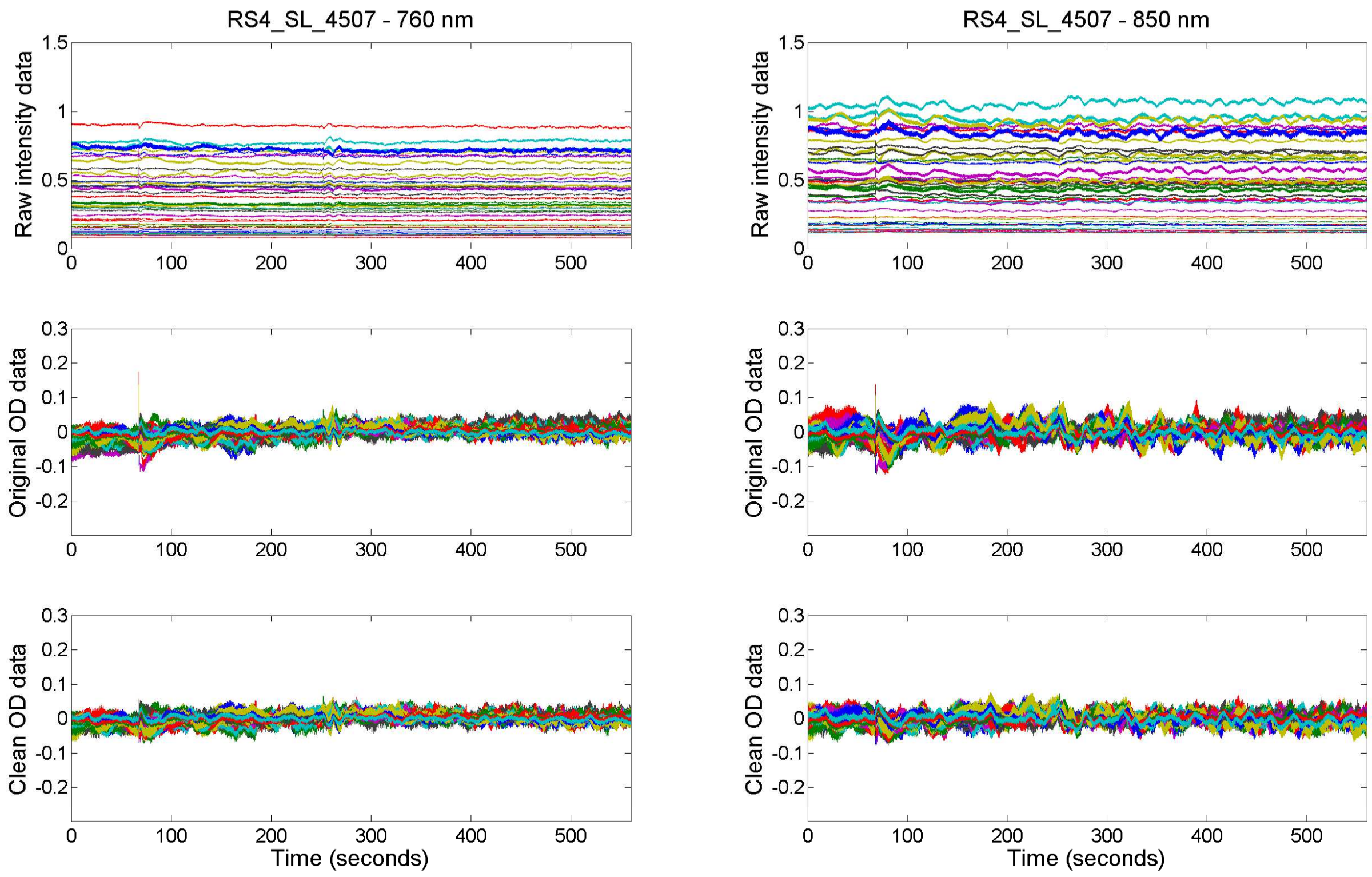
Raw
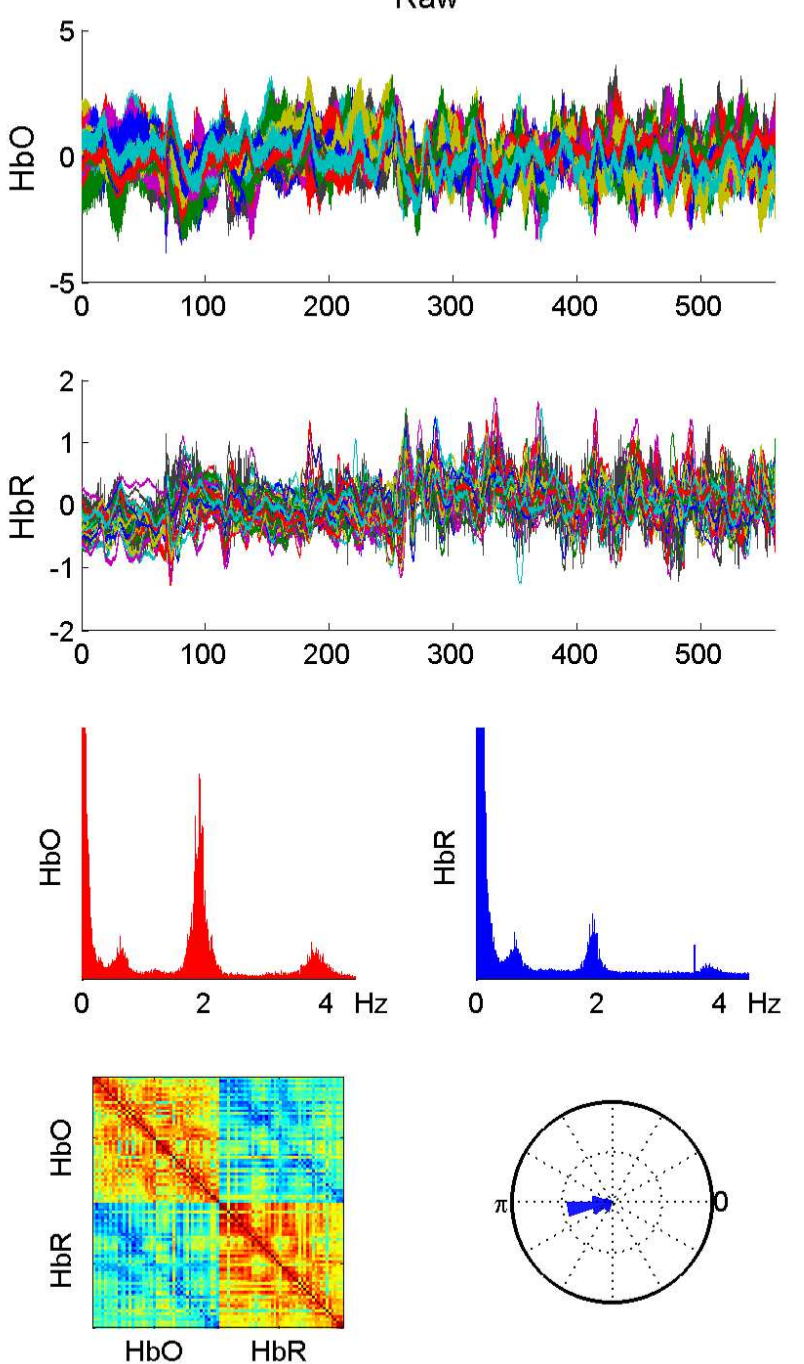

RS4_SL_4507

Filtered
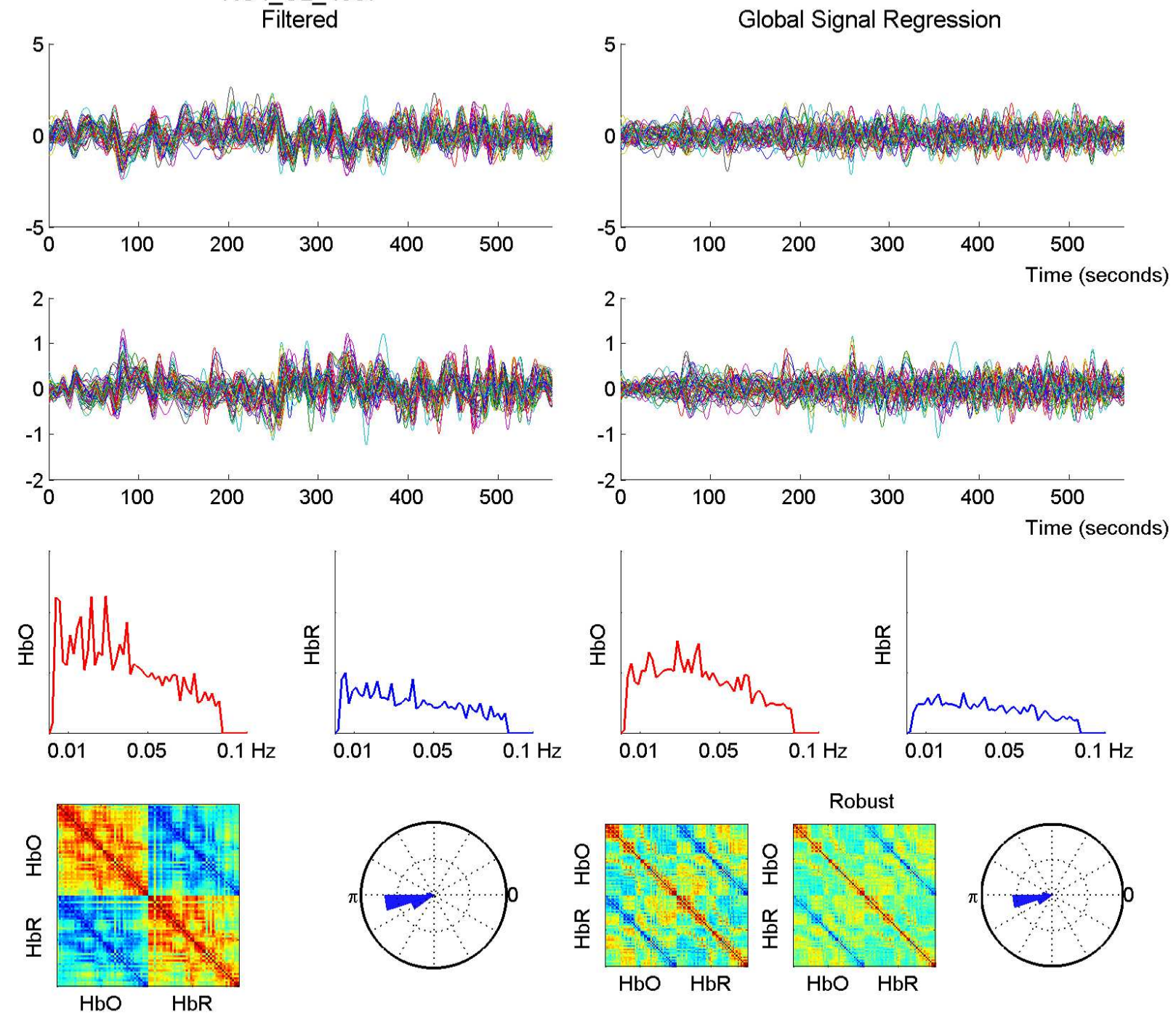
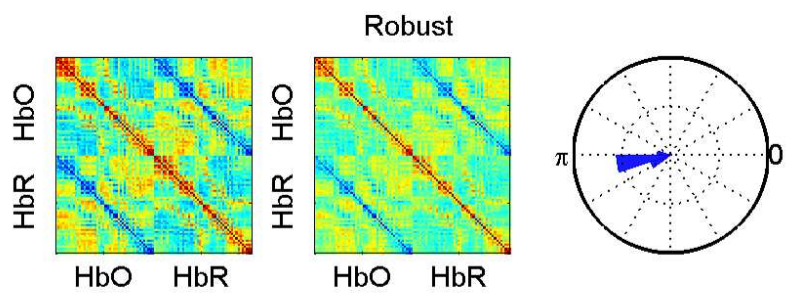
RS4 SL 4505 - 760 nm
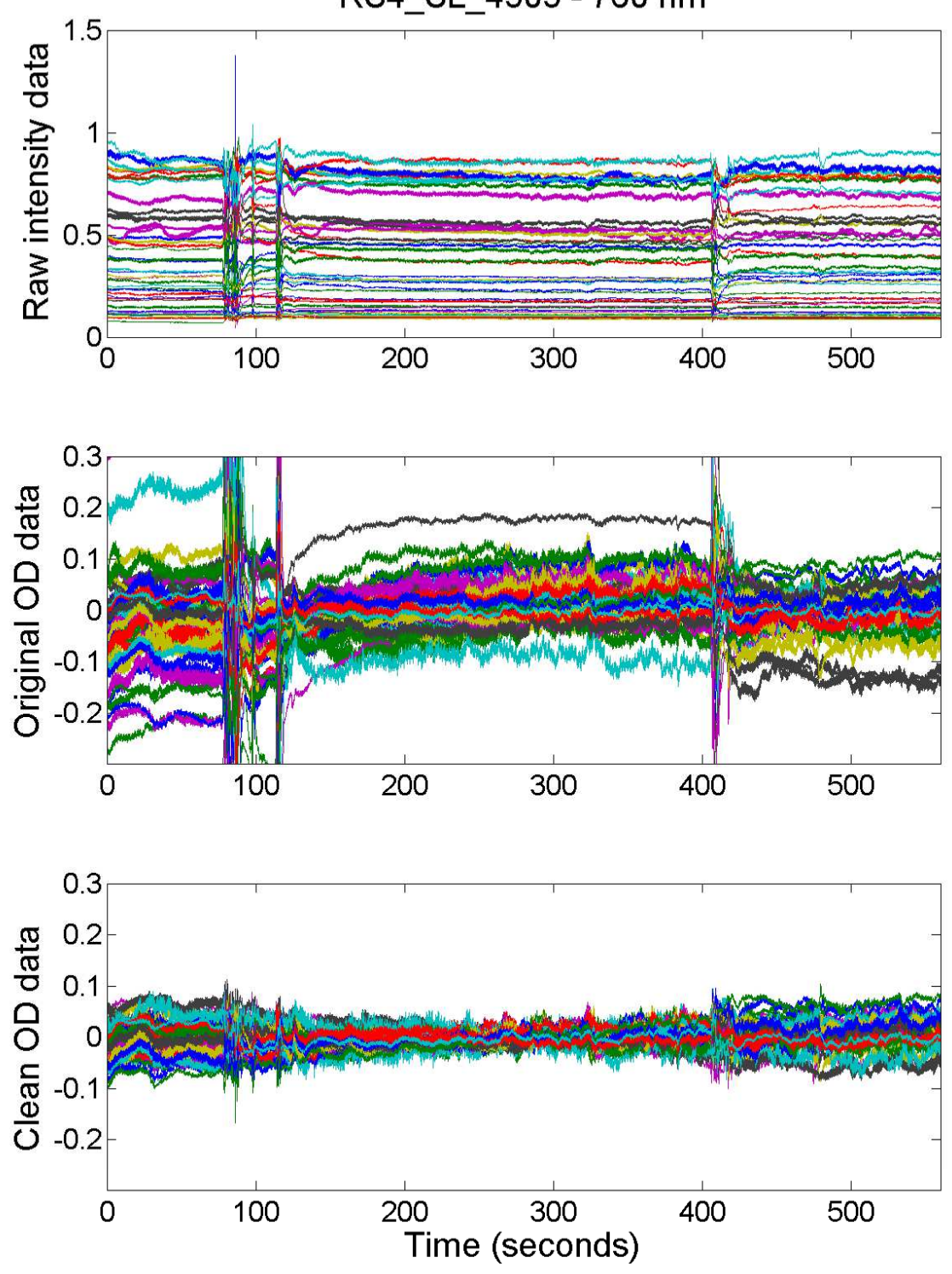

RS4 SL $4505-850 \mathrm{~nm}$
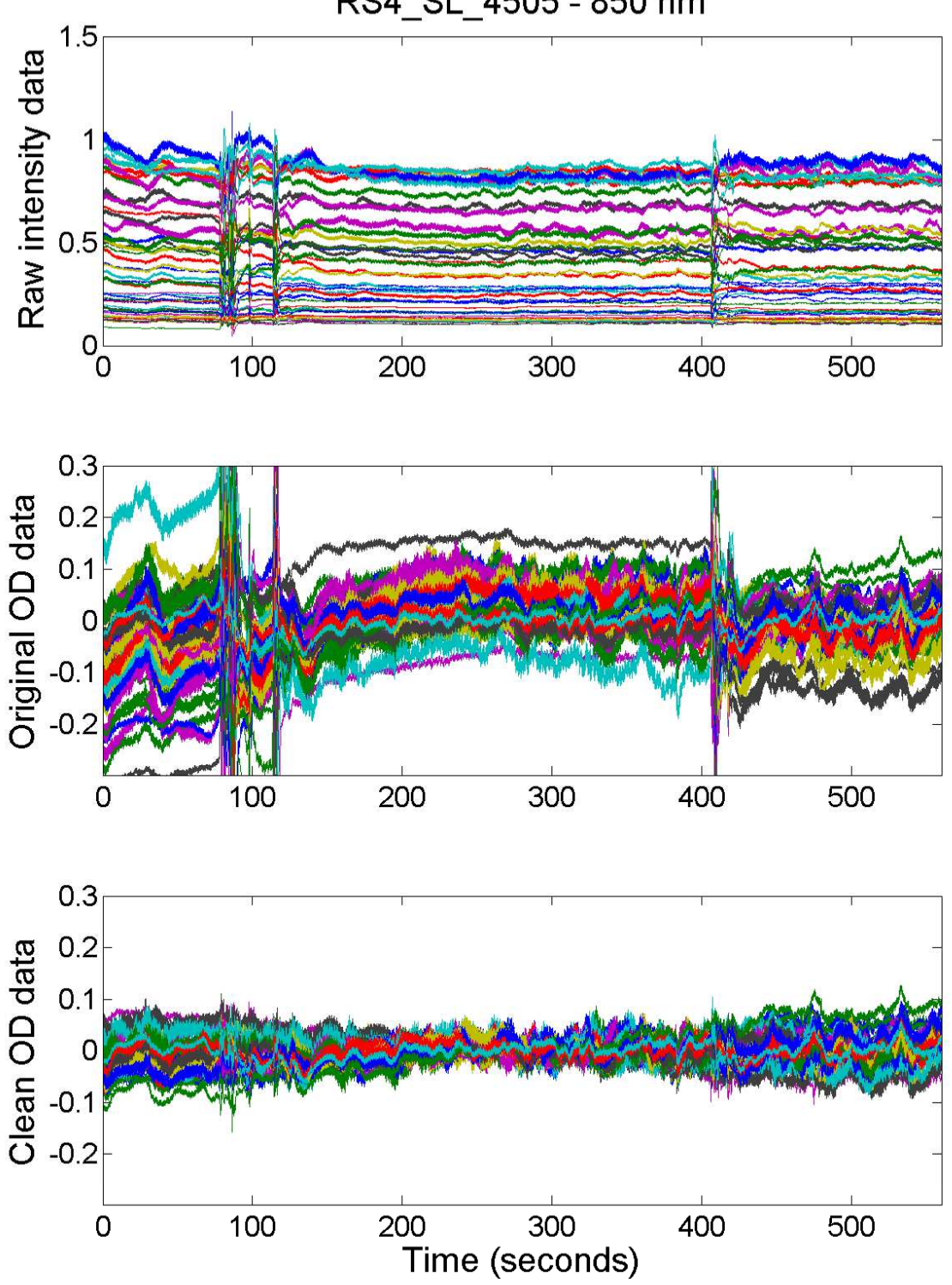
Raw
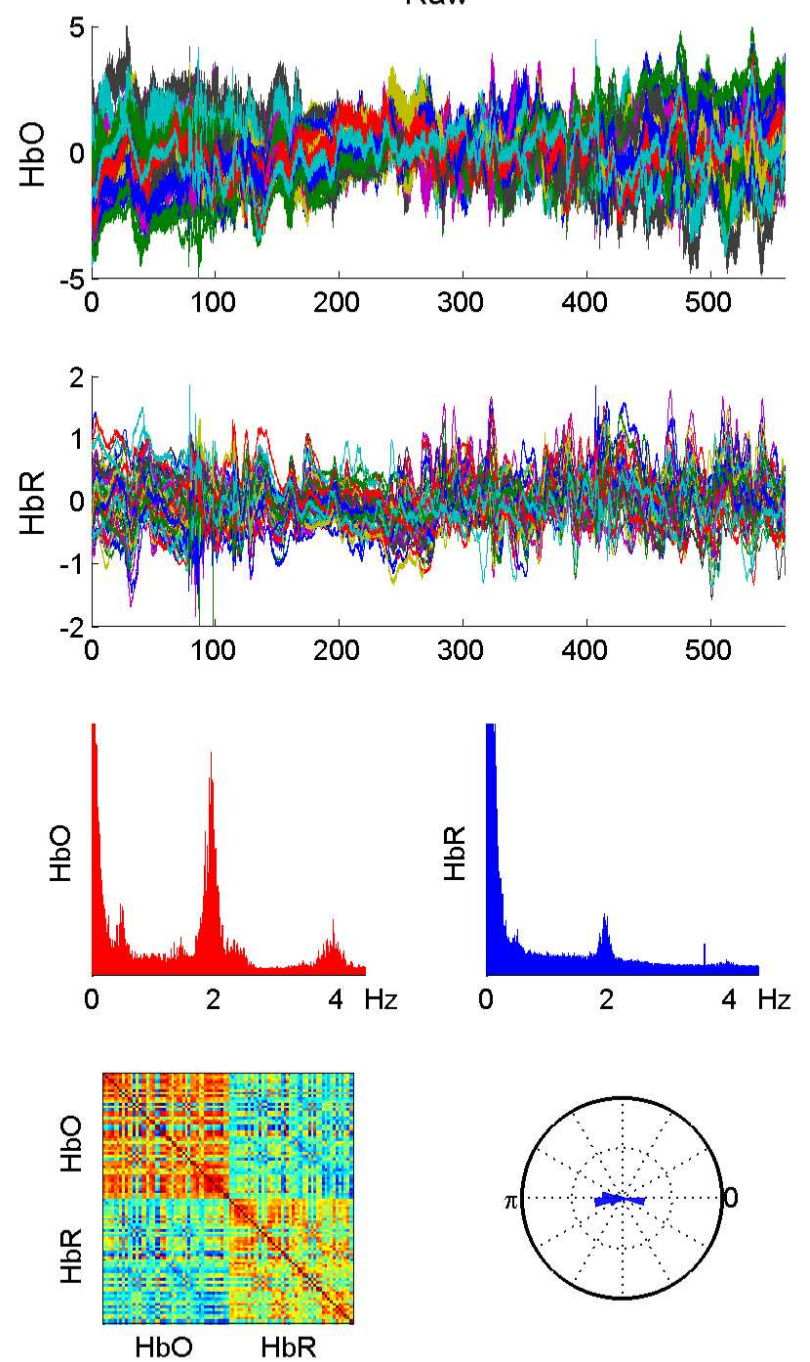

RS4 SL 4505

Filtered
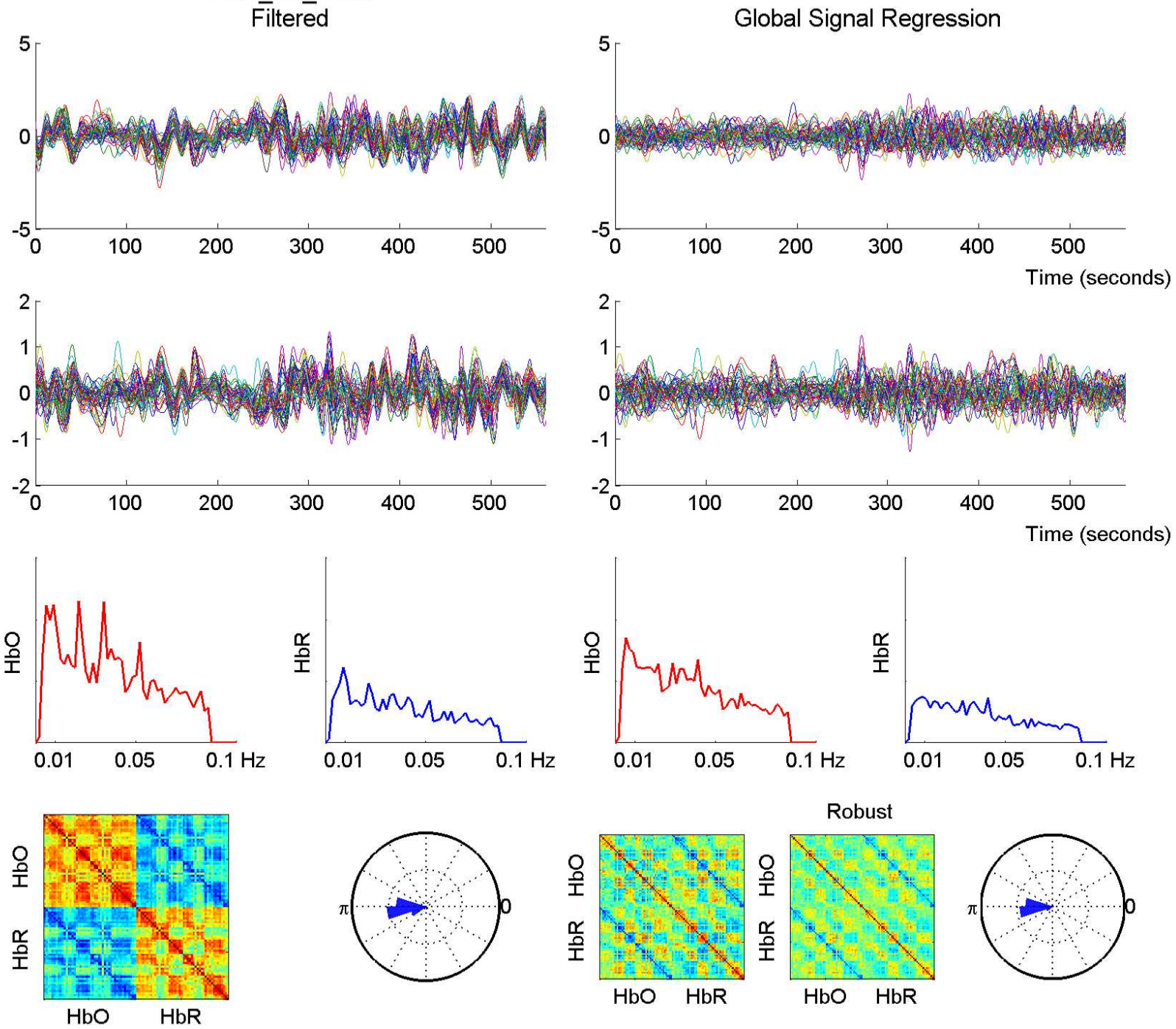
RS4 SL 4498 - 760 nm
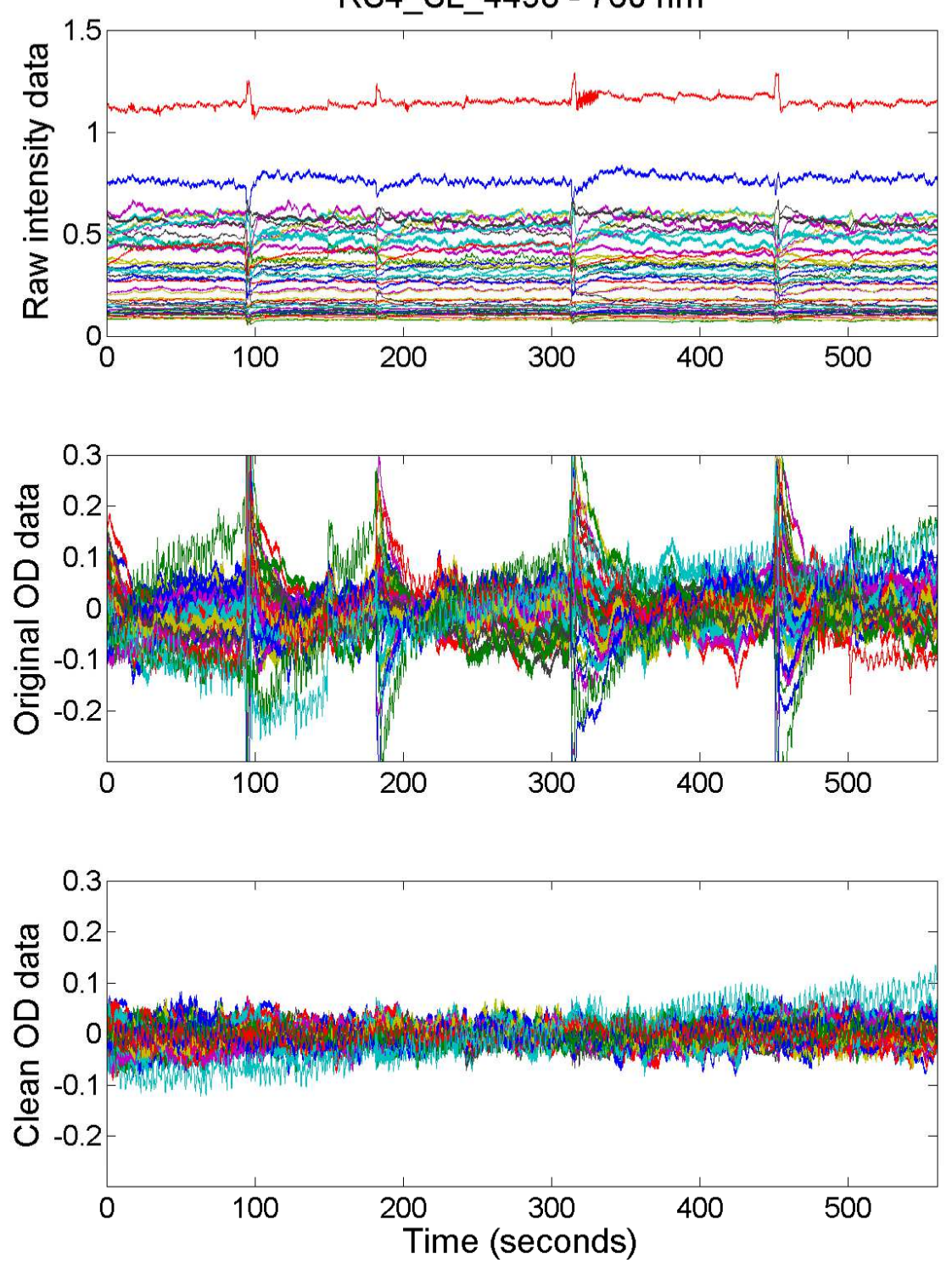

RS4 SL $4498-850$ nm
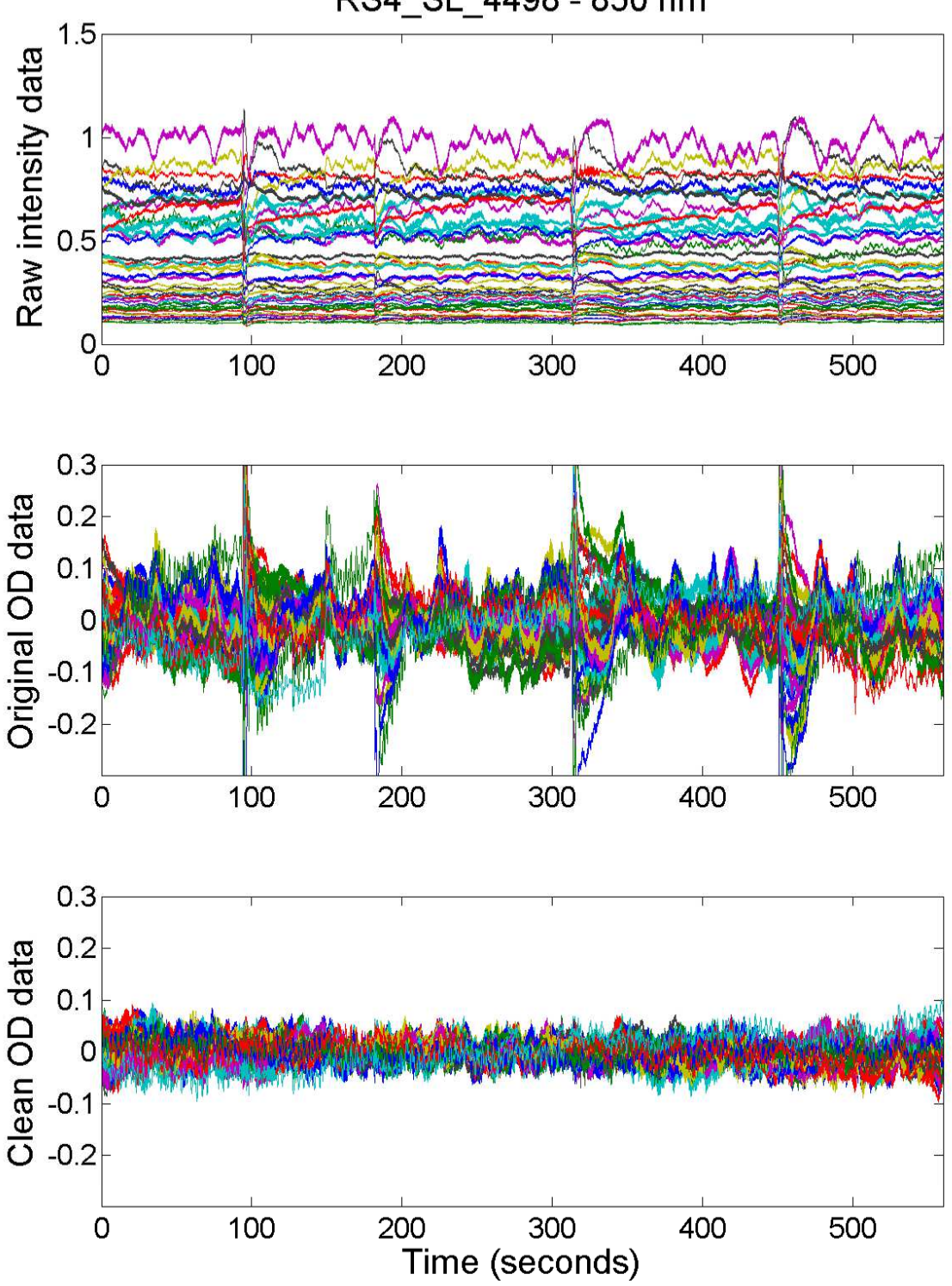
Raw

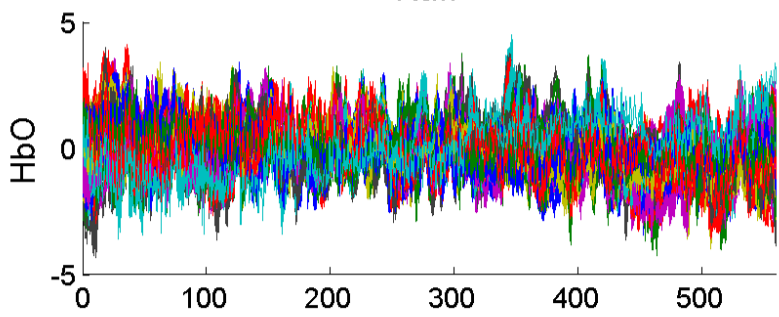

2

옹
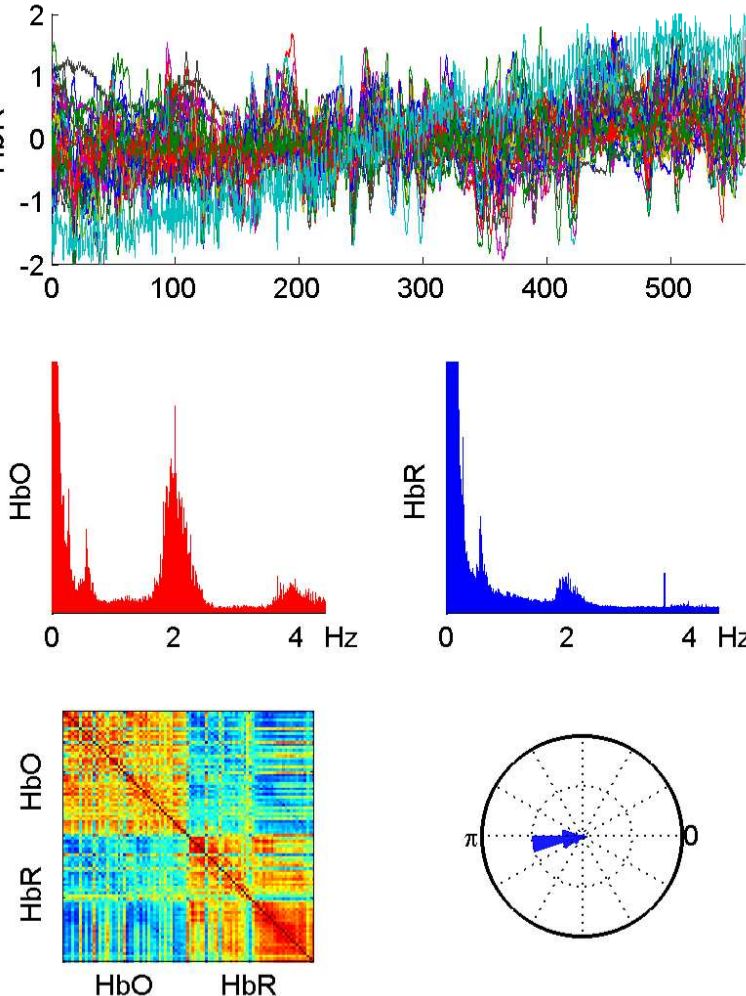

RS4_SL_4498

Filtered
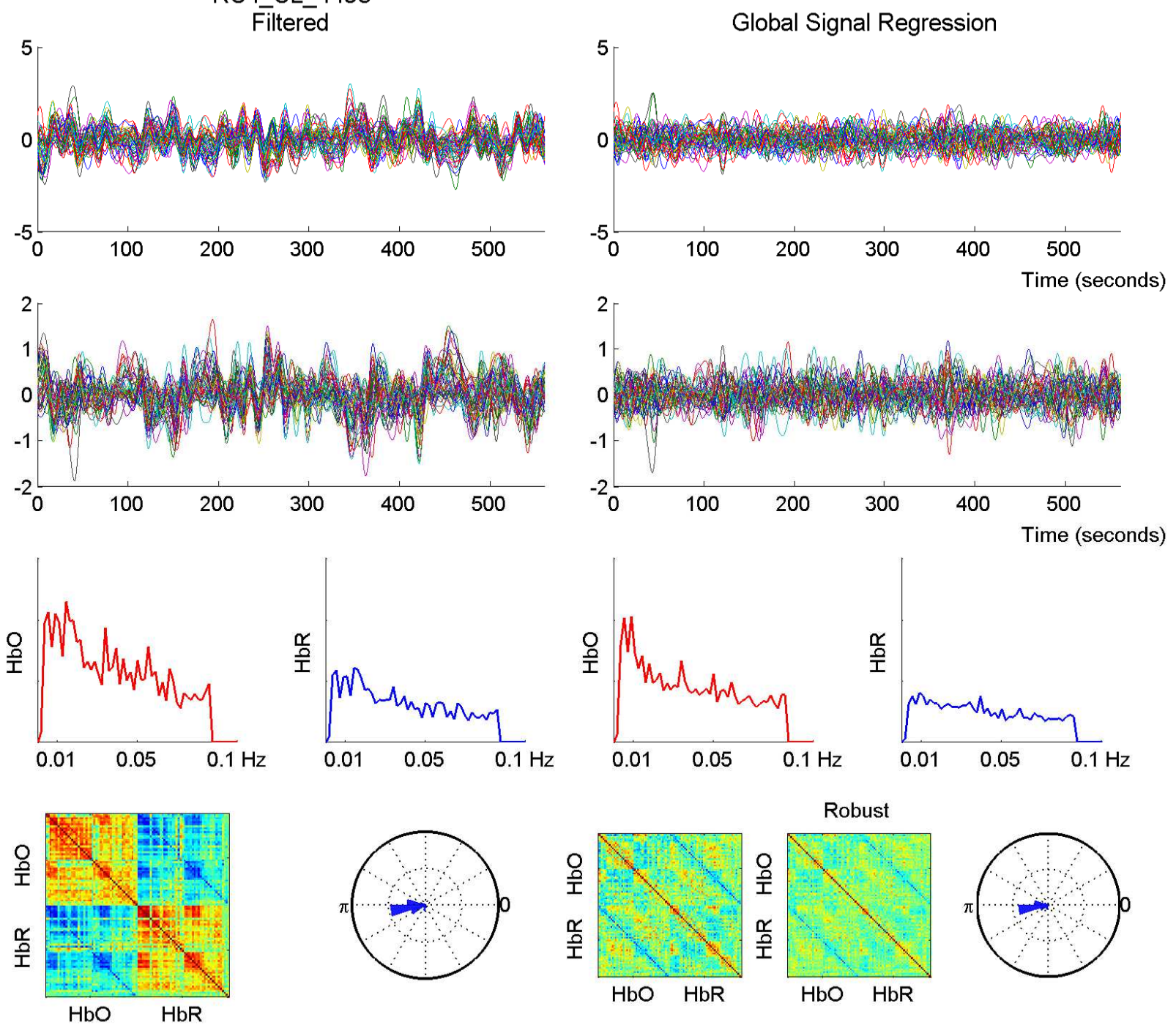
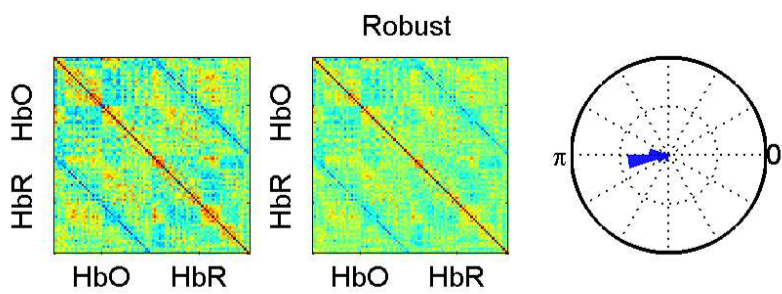

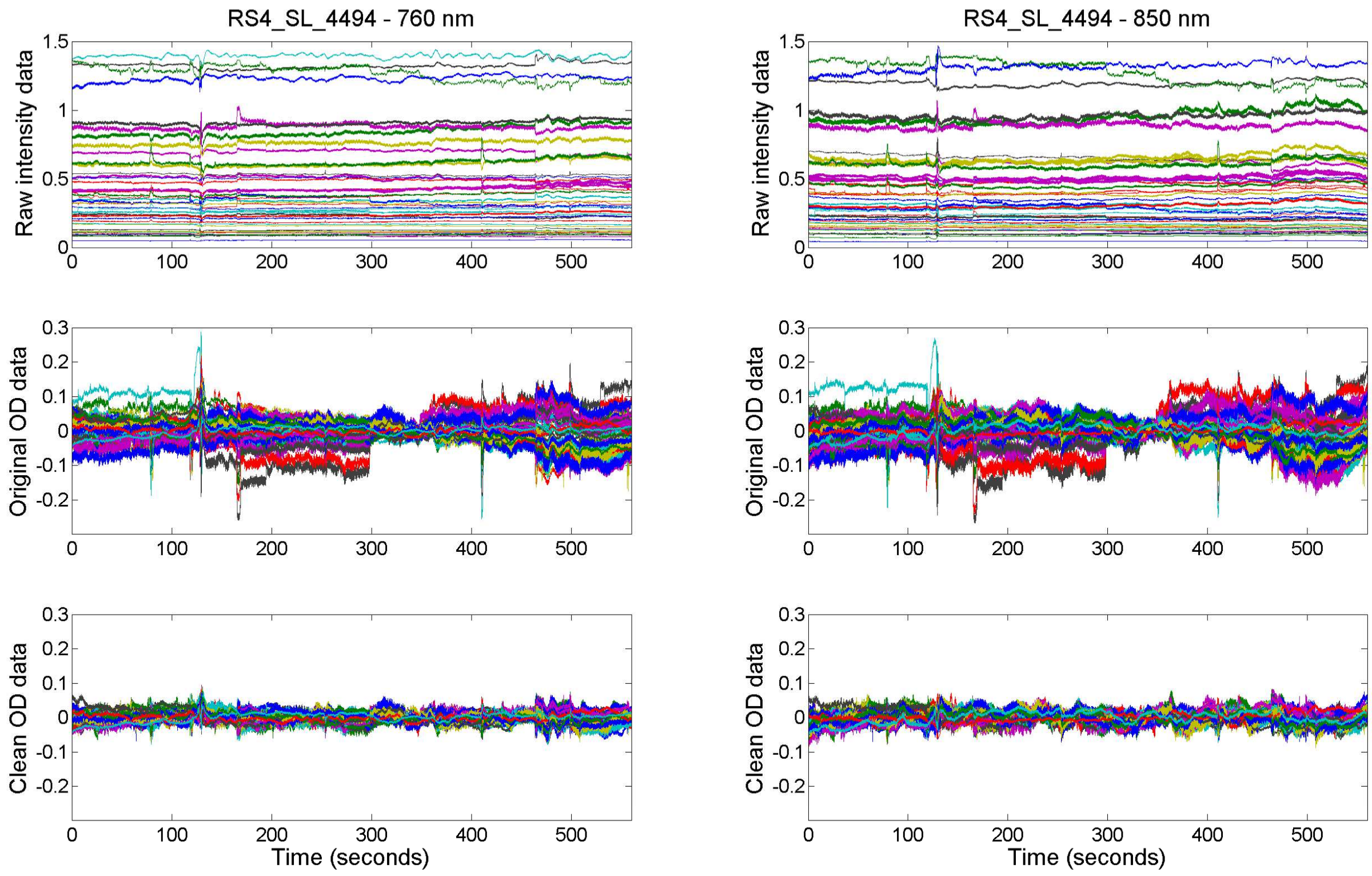
RS4_SL_4494

Raw
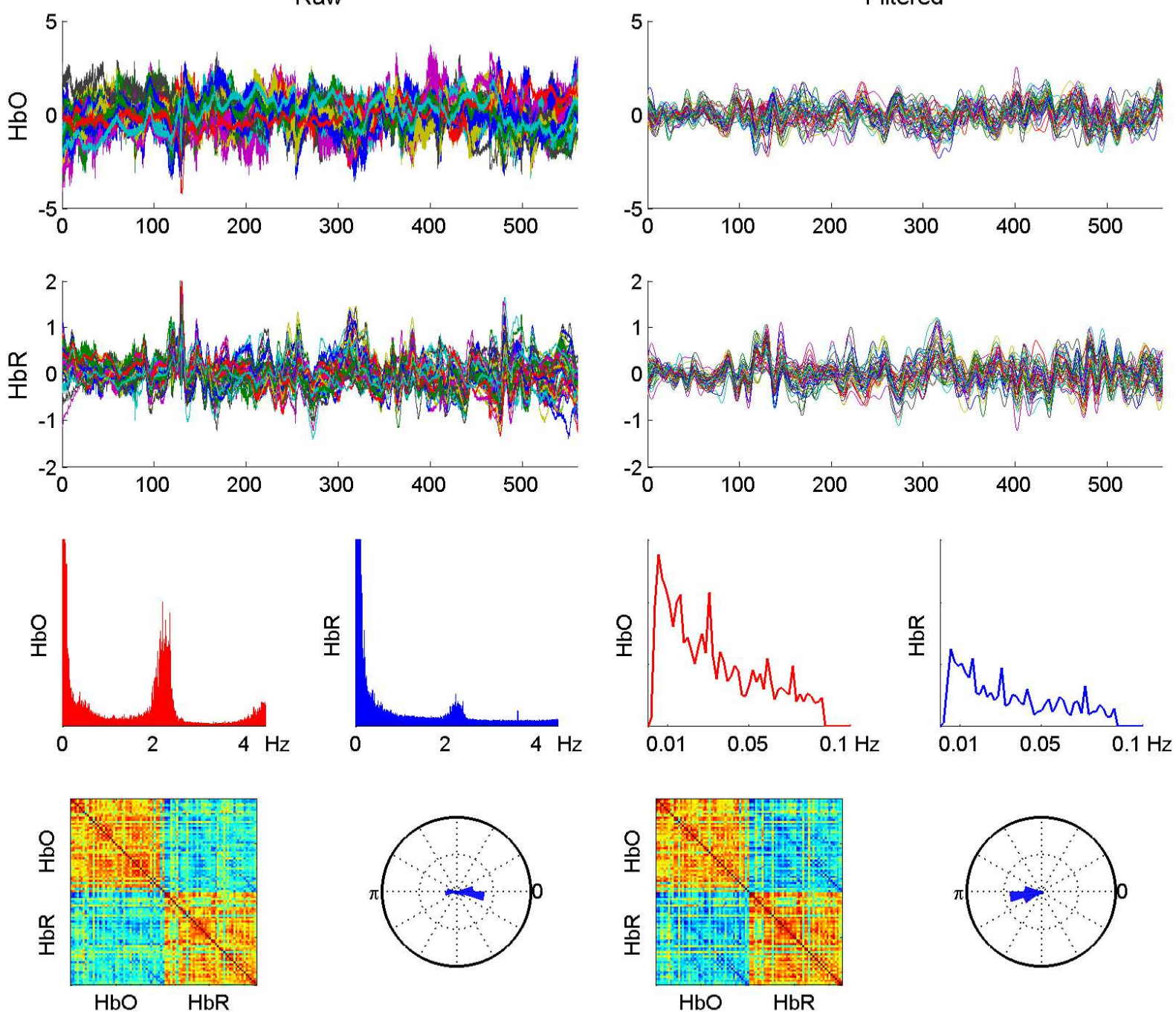
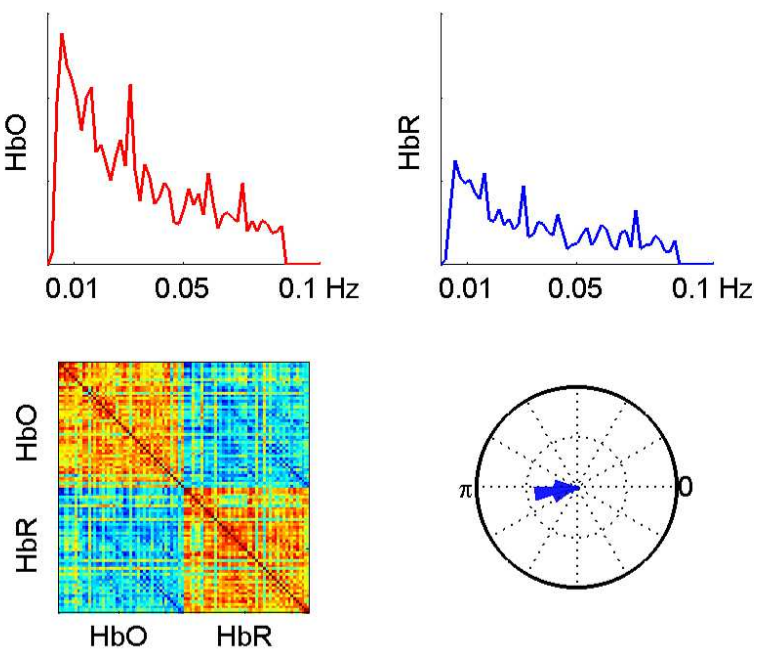
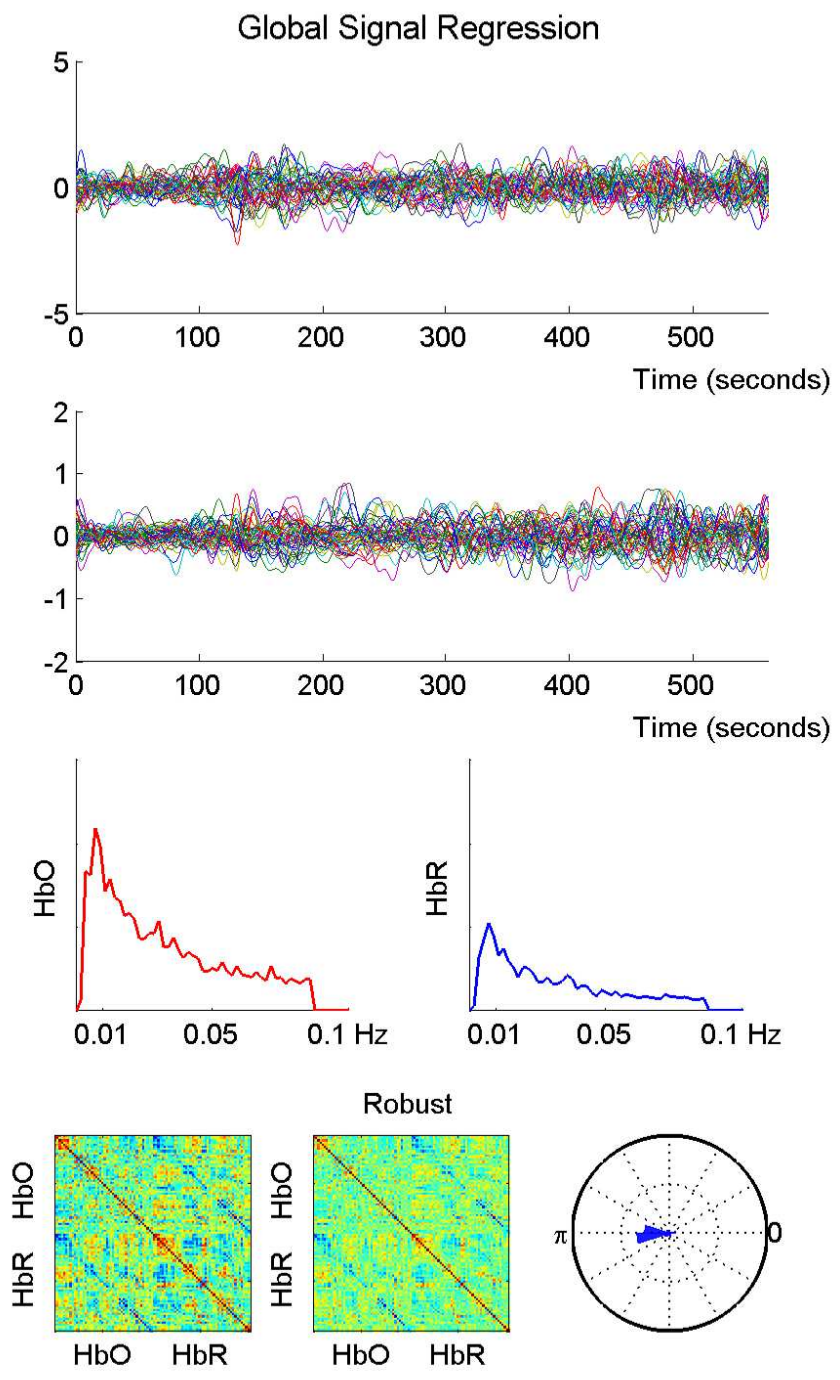
RS4 SL 4492 - 760 nm
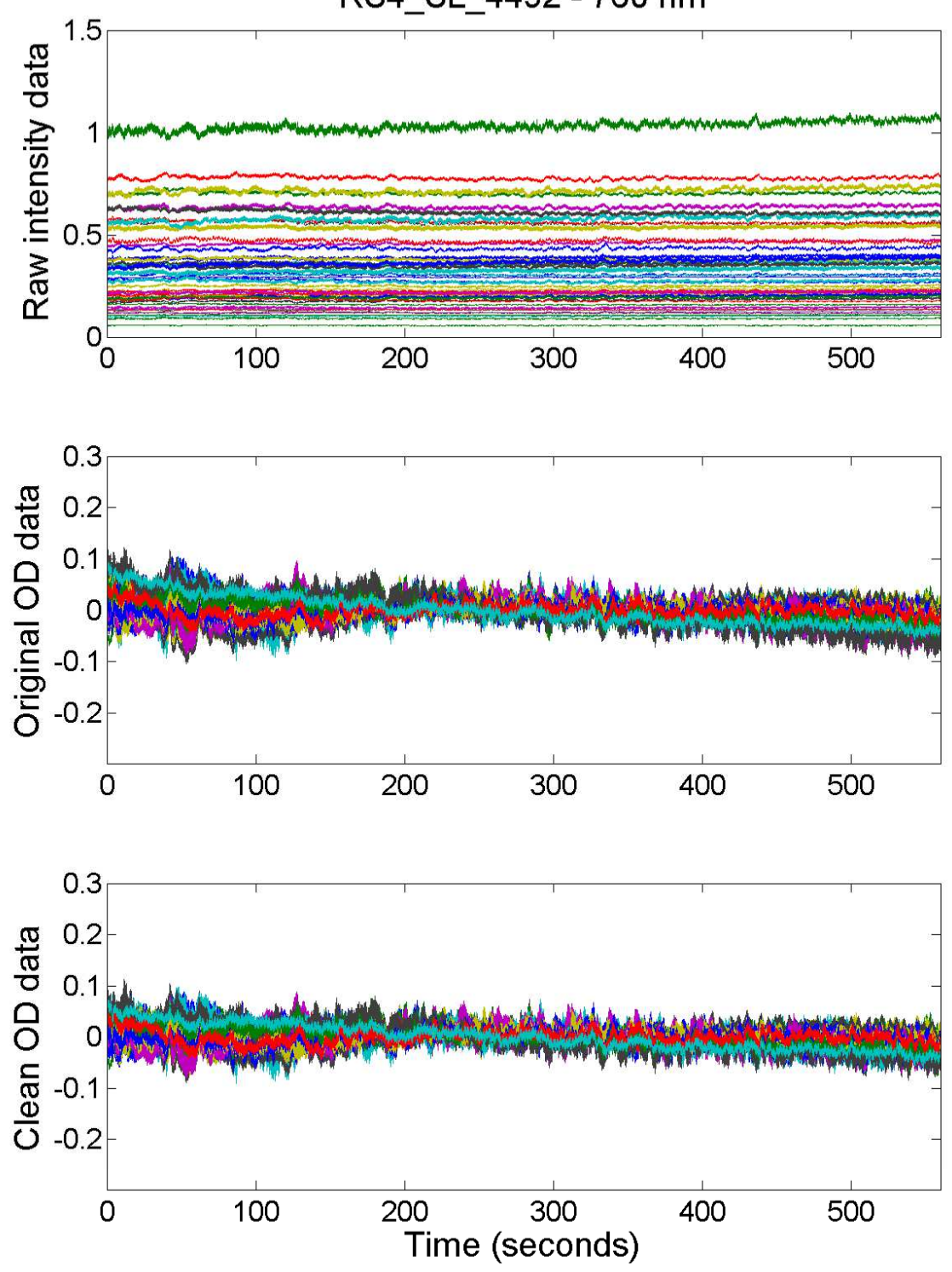

RS4 SL $4492-850 \mathrm{~nm}$
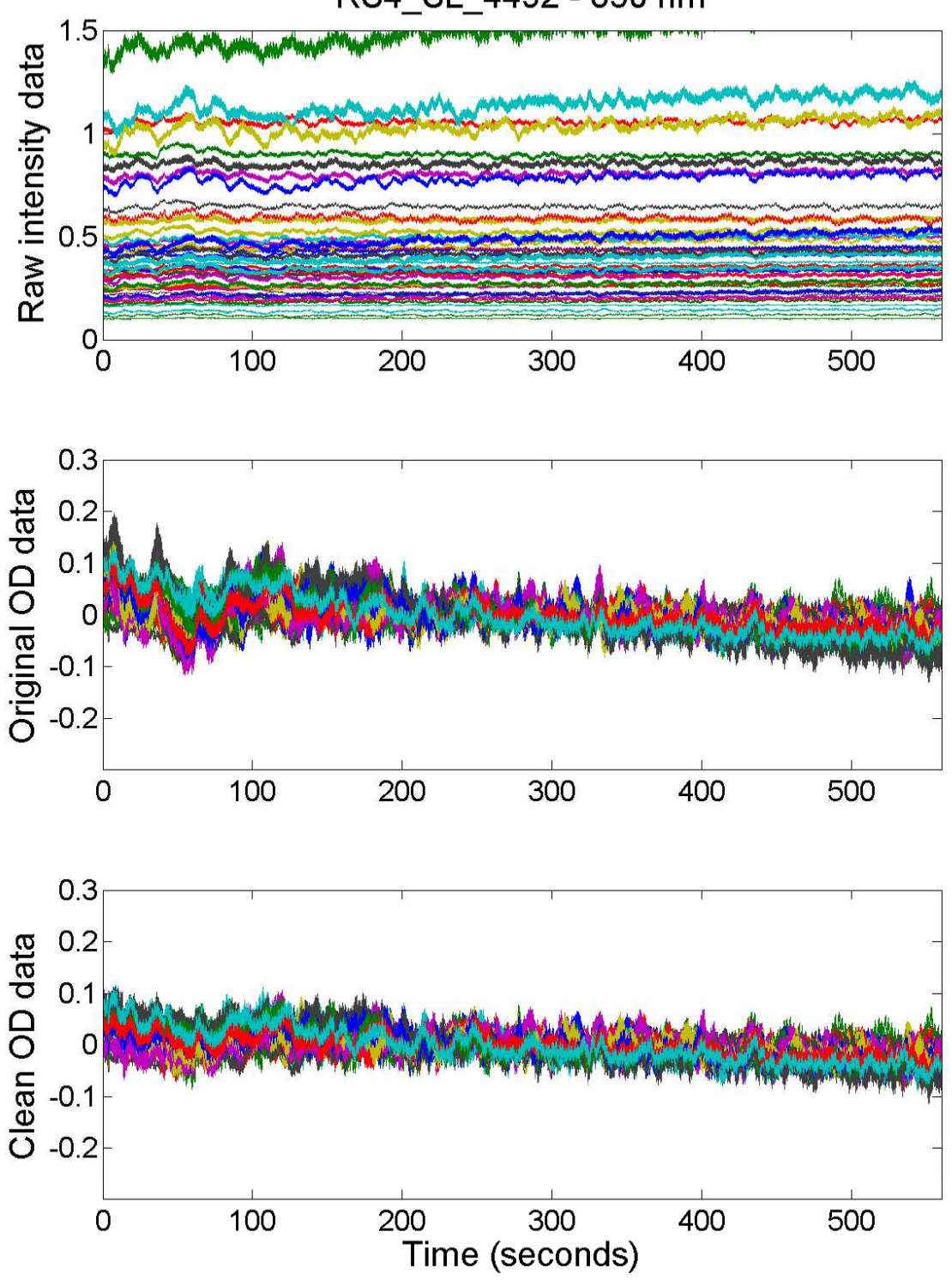
RS4 SL 4492

Raw

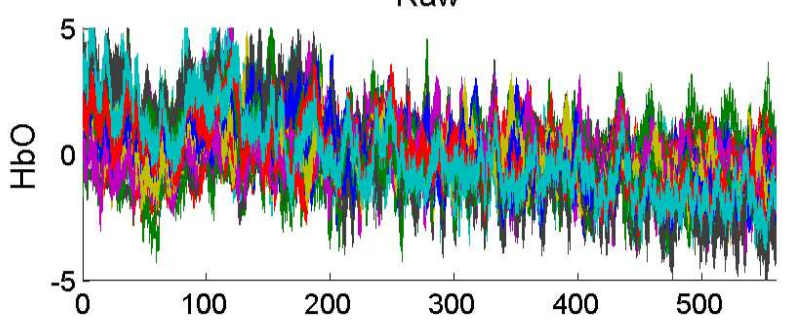

$$
5
$$
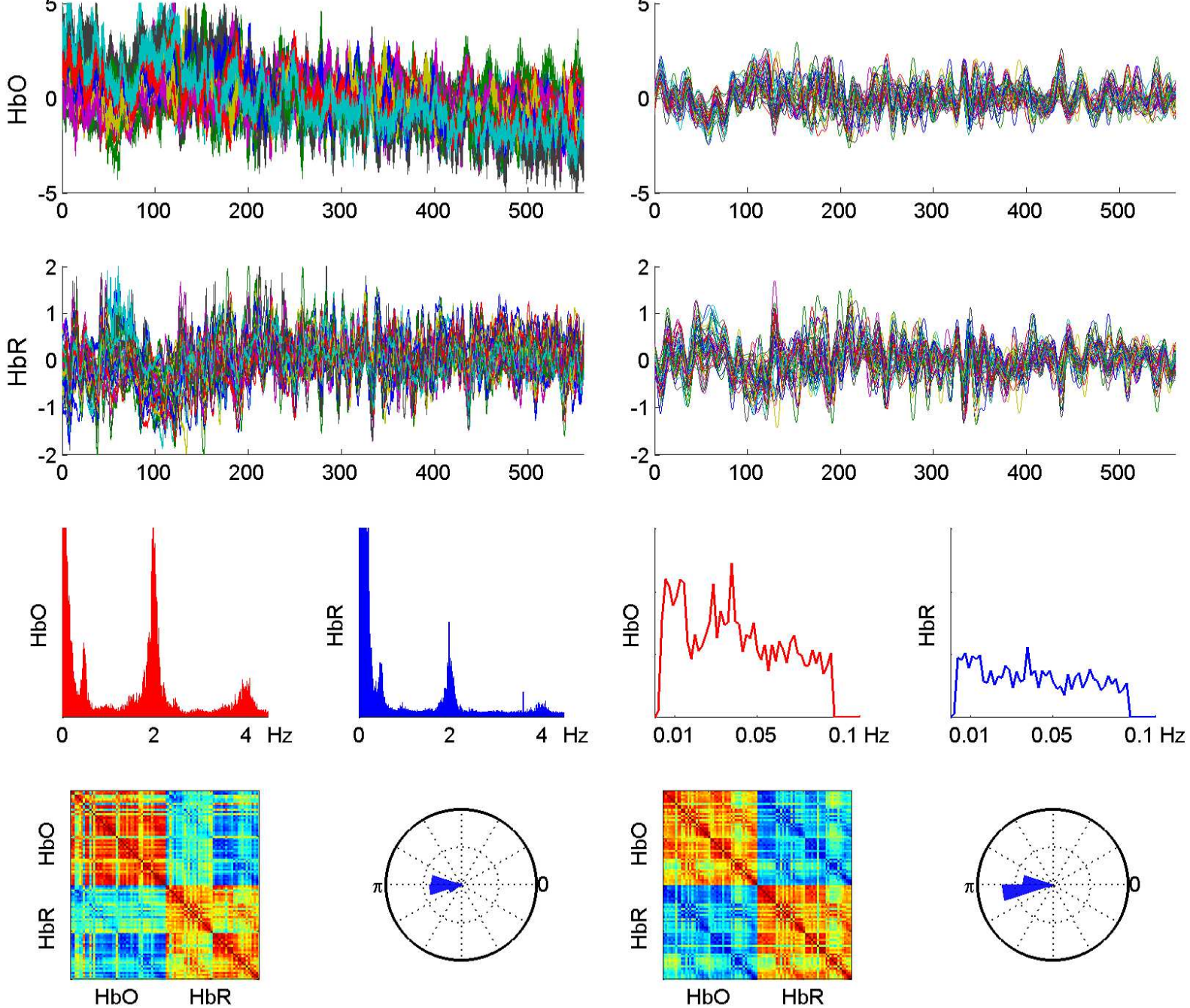
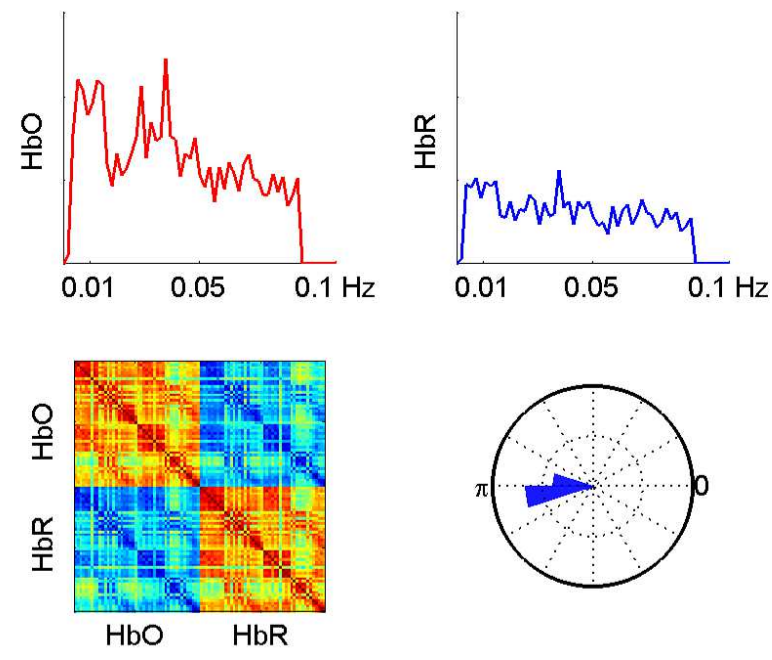

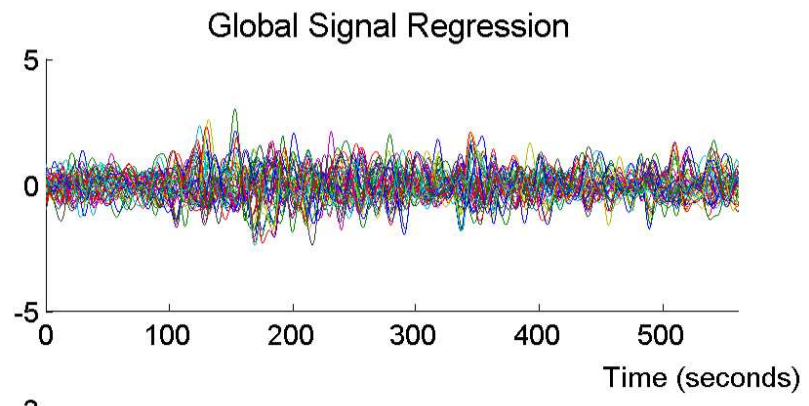

2
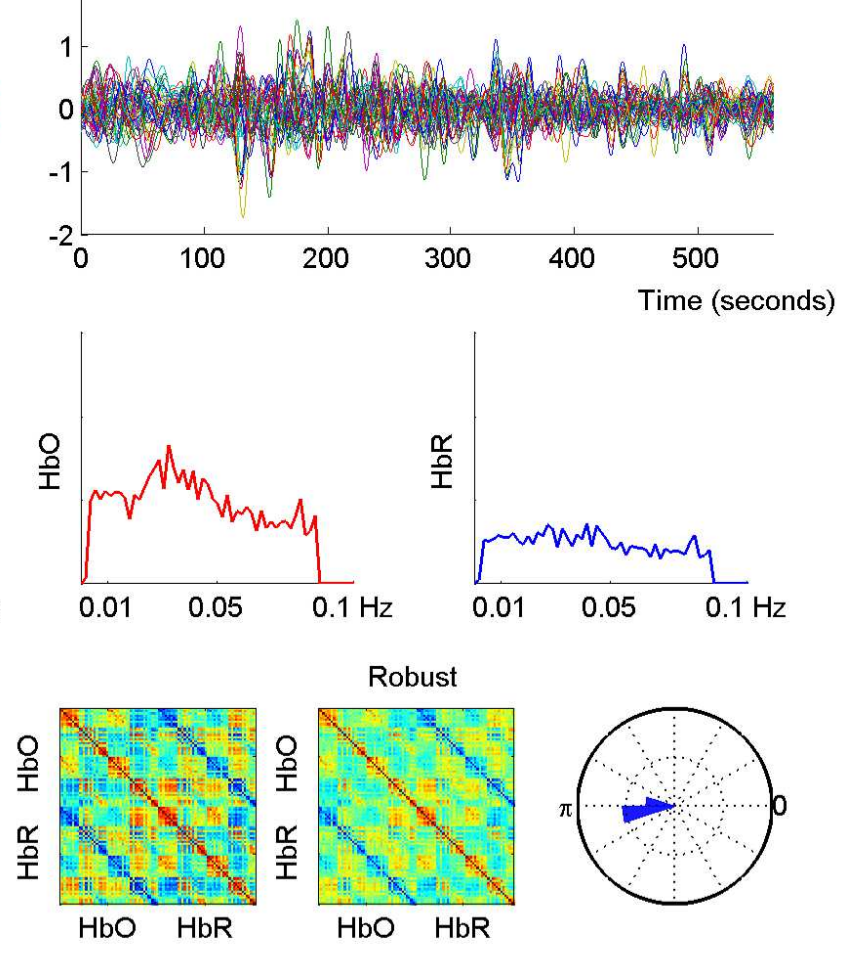
RS4 SL 4487 - $760 \mathrm{~nm}$
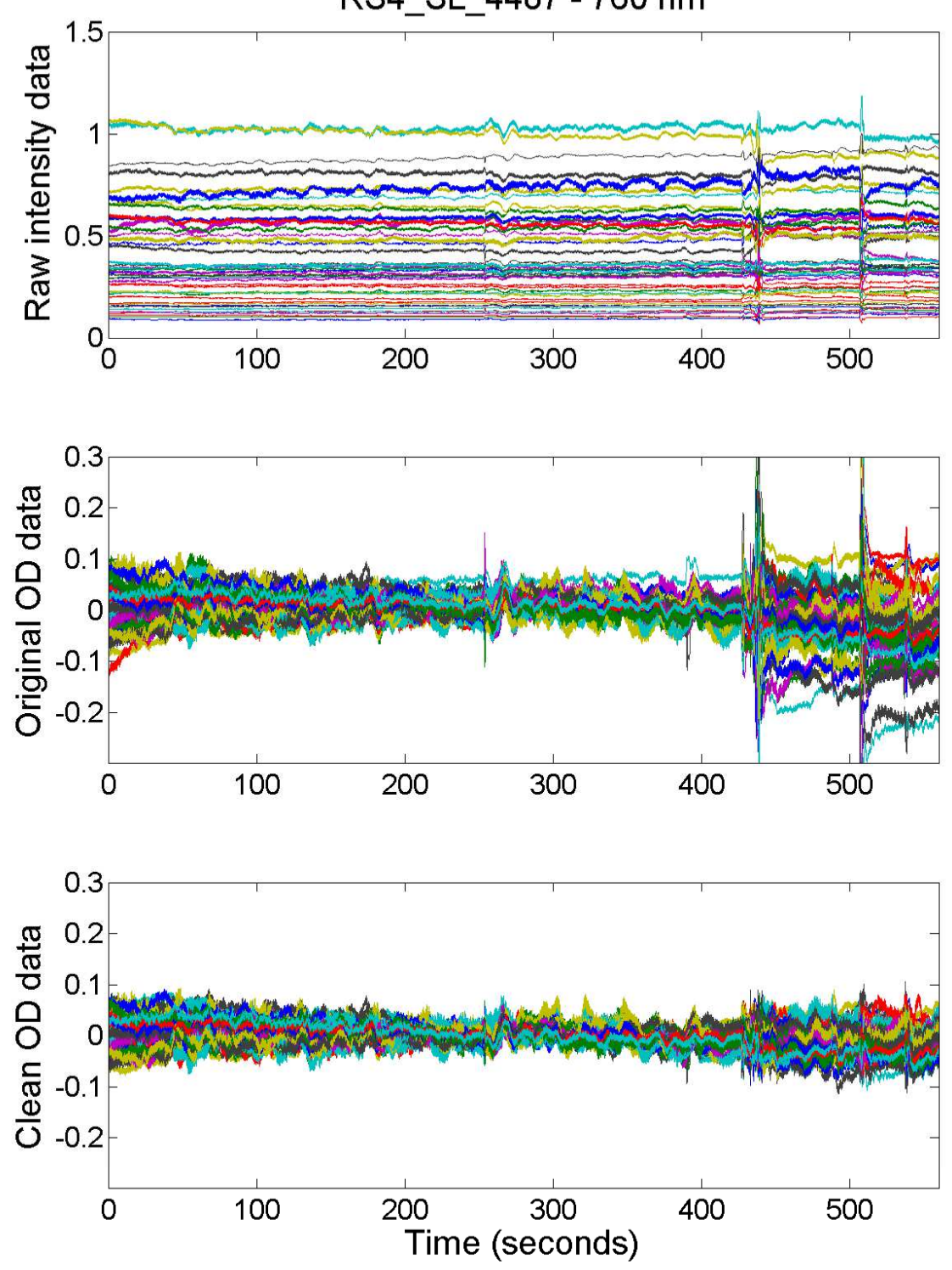

RS4 SL $4487-850 \mathrm{~nm}$
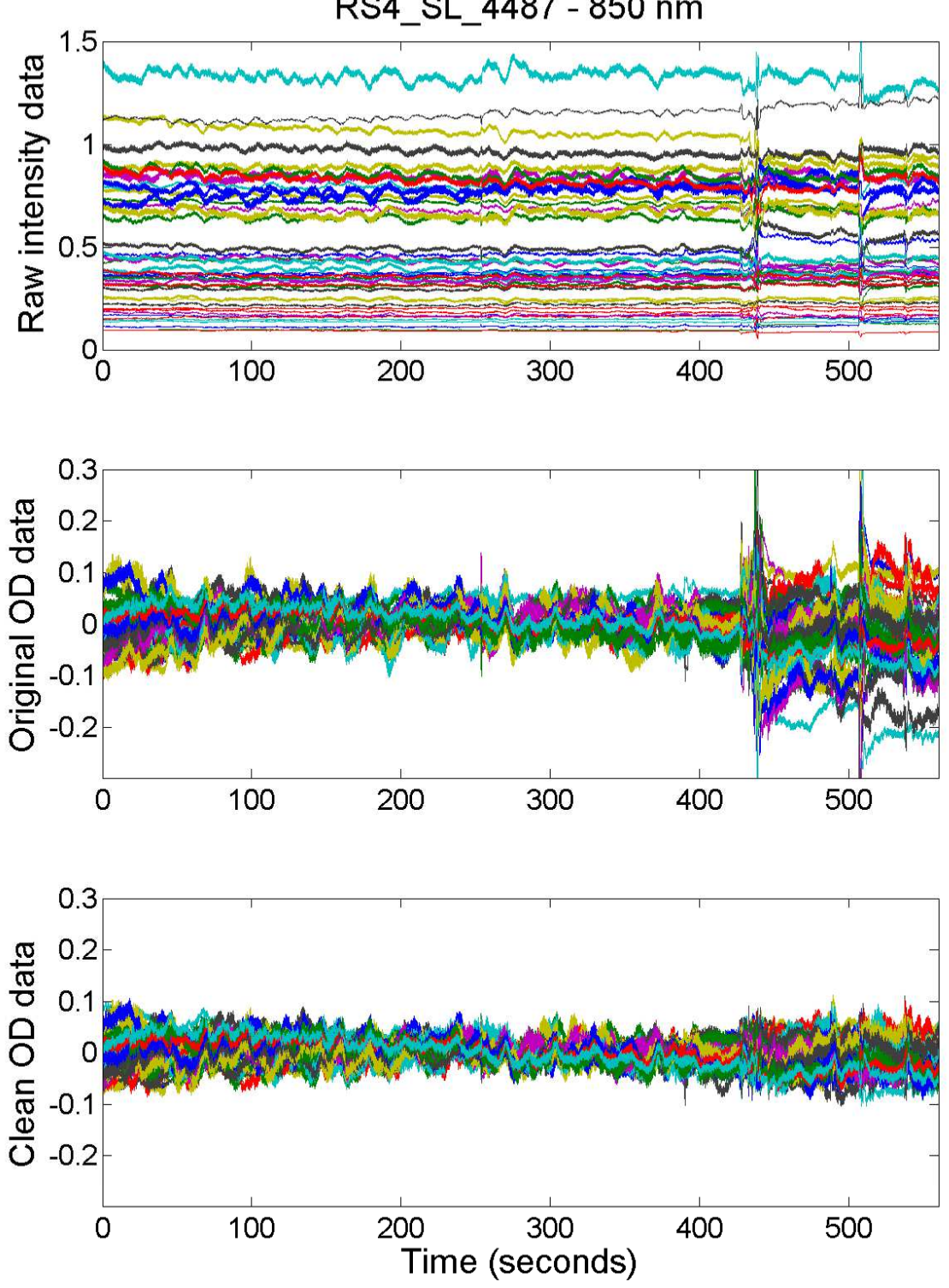
Raw
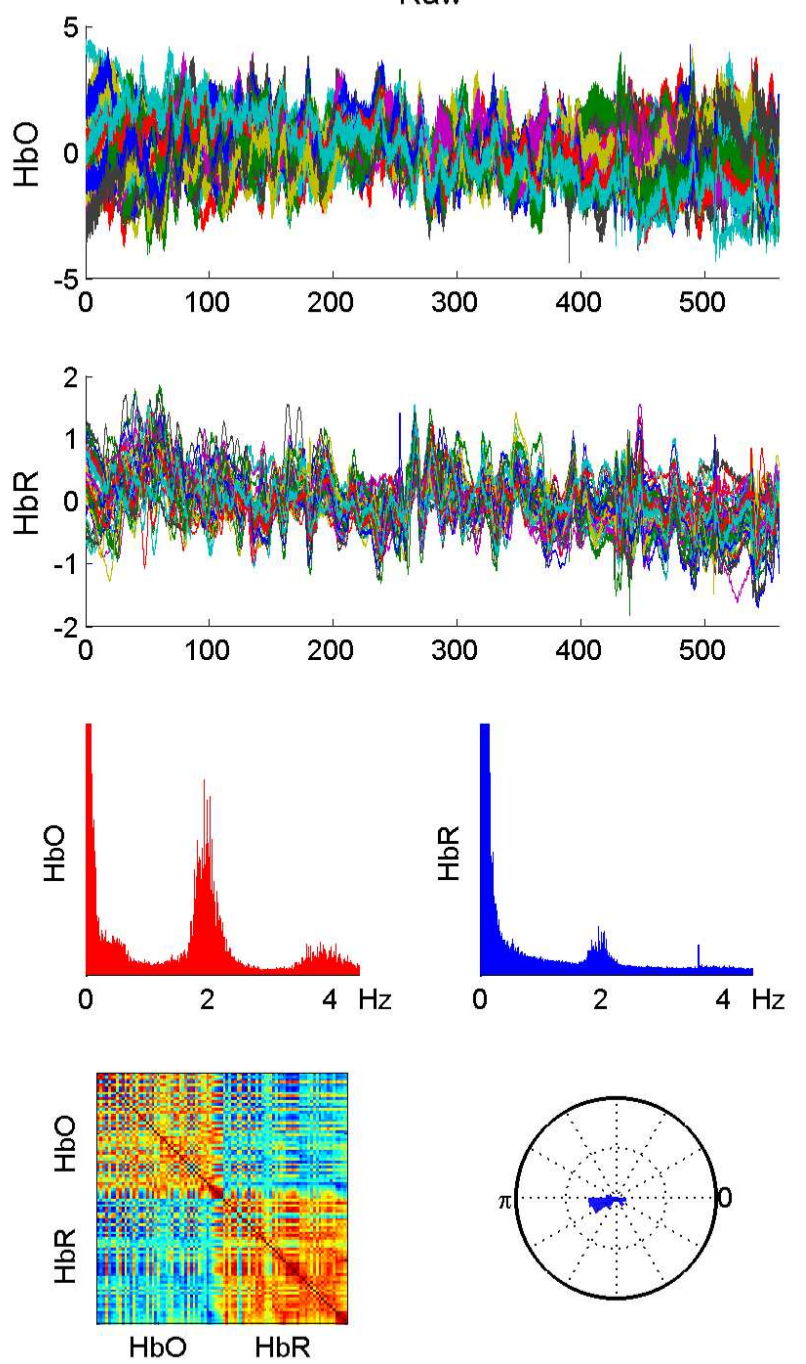

RS4 SL 4487

Filtered
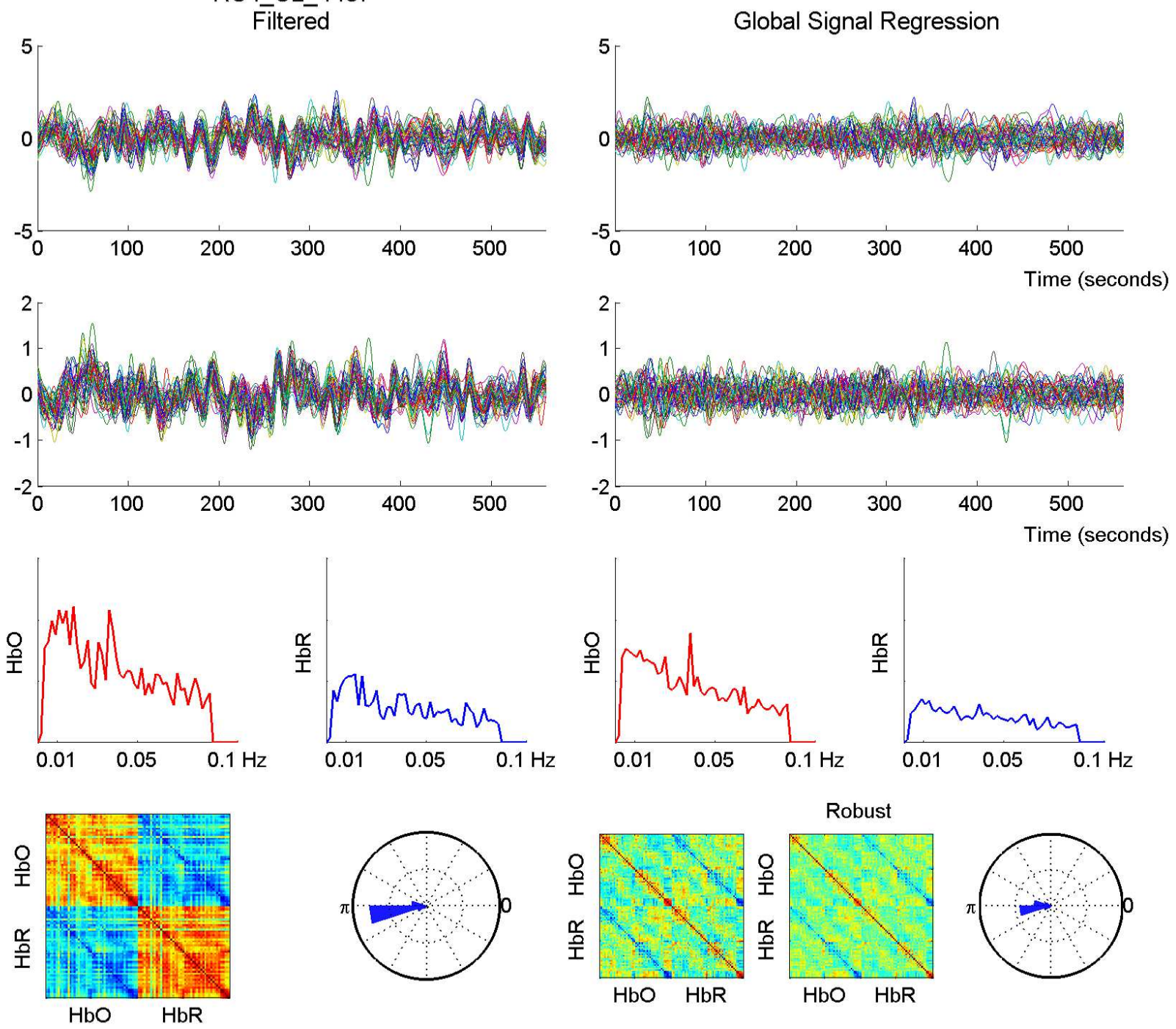
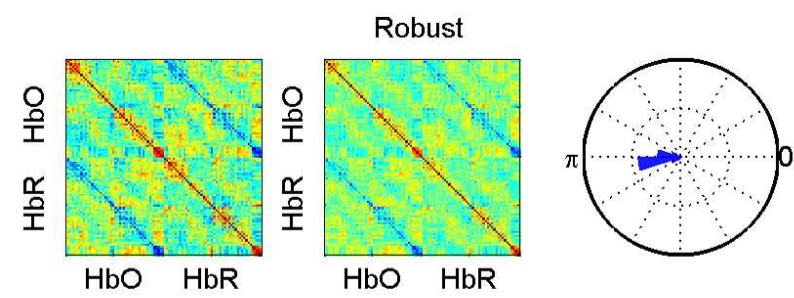

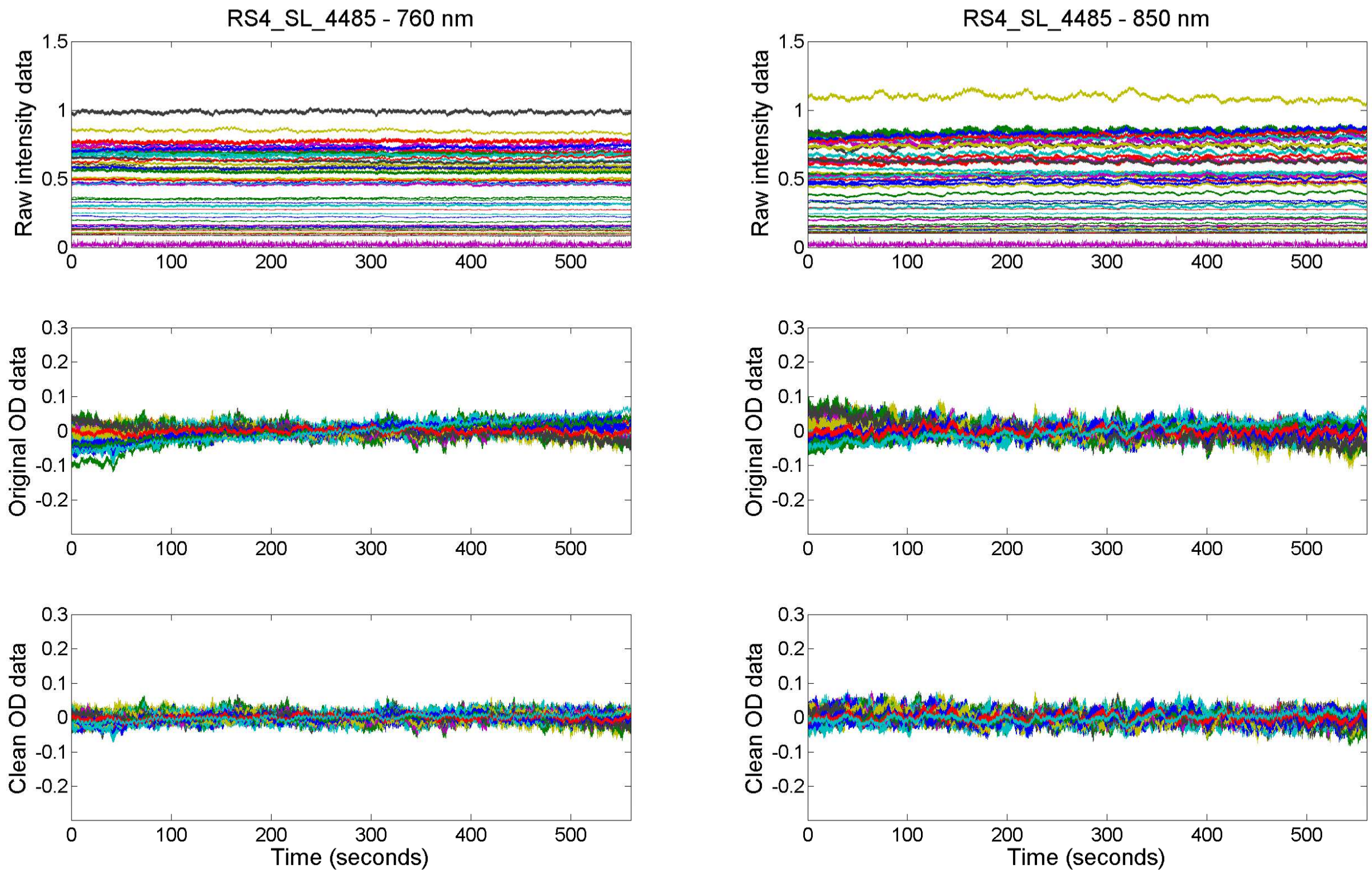
Raw

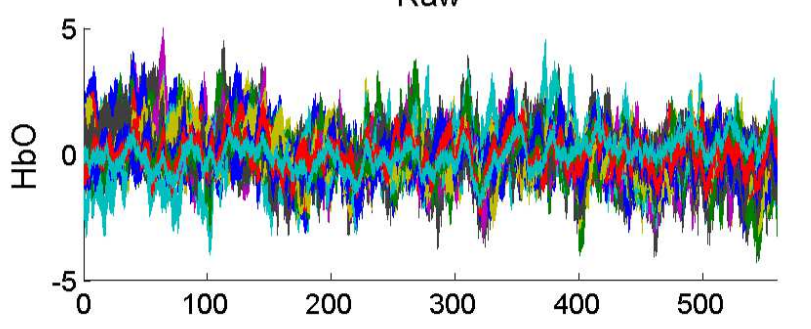

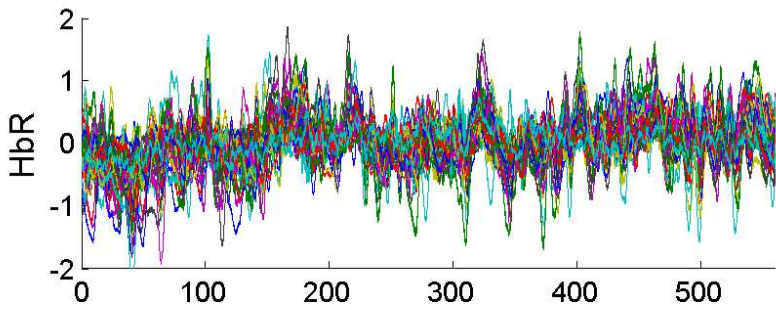
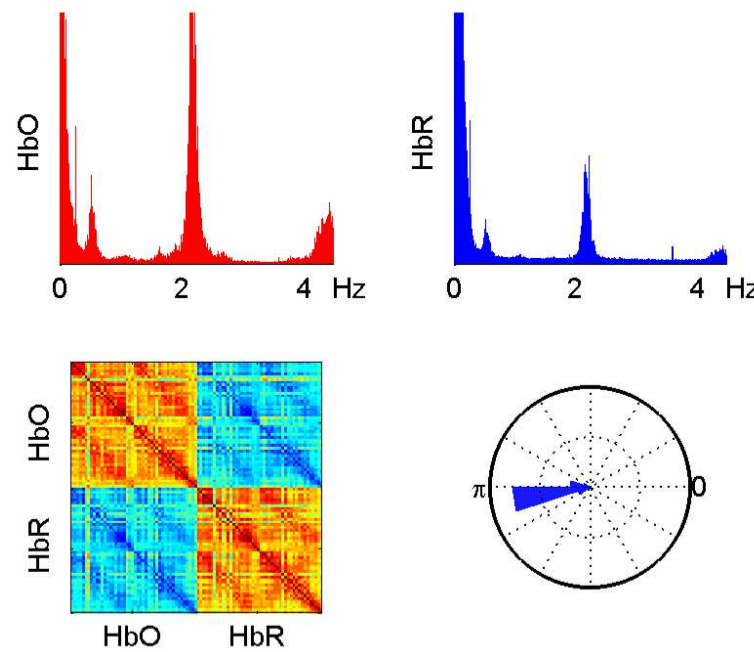

RS4 SL 4485

Filtered
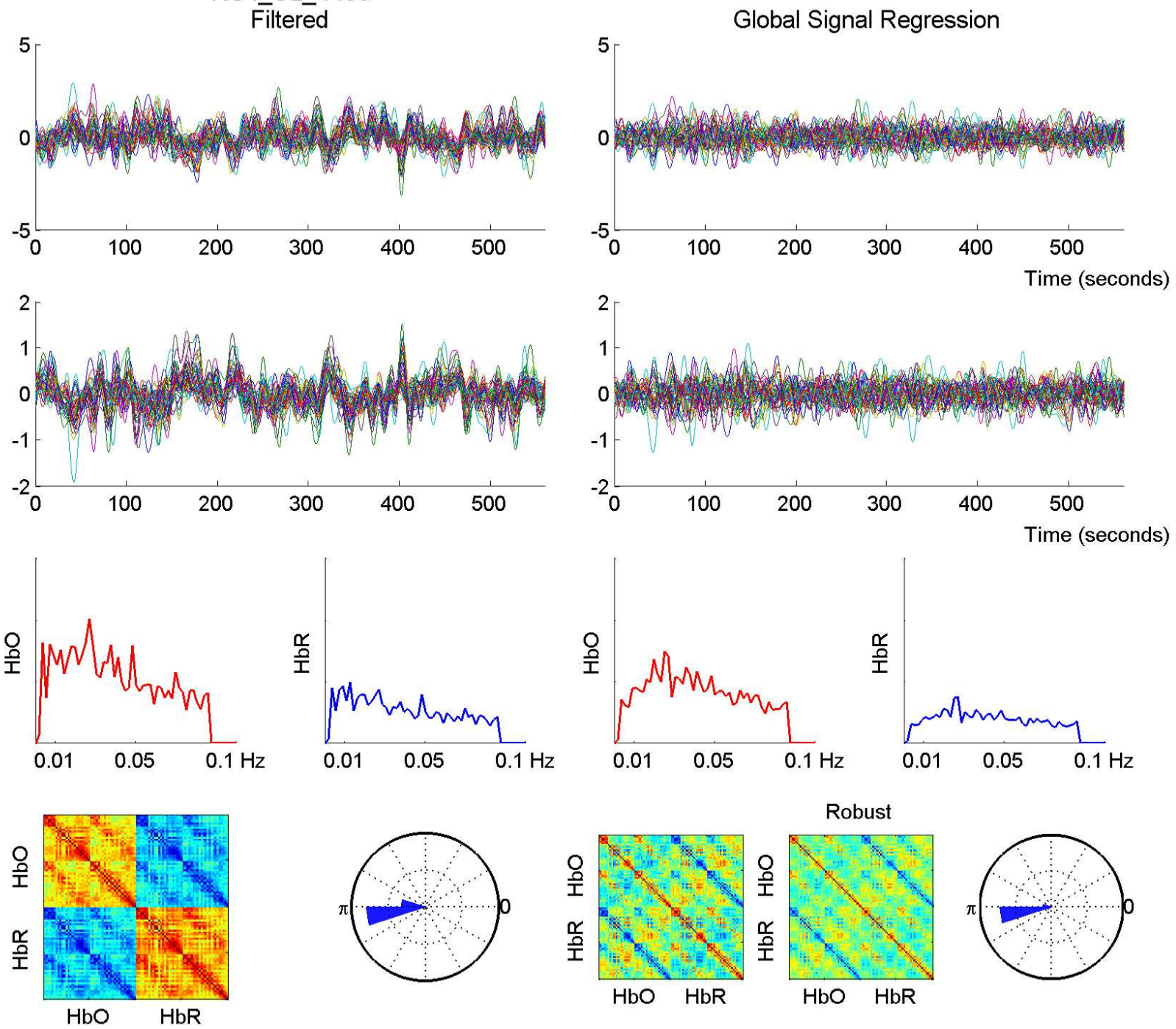
RS4 SL $4483-760 \mathrm{~nm}$
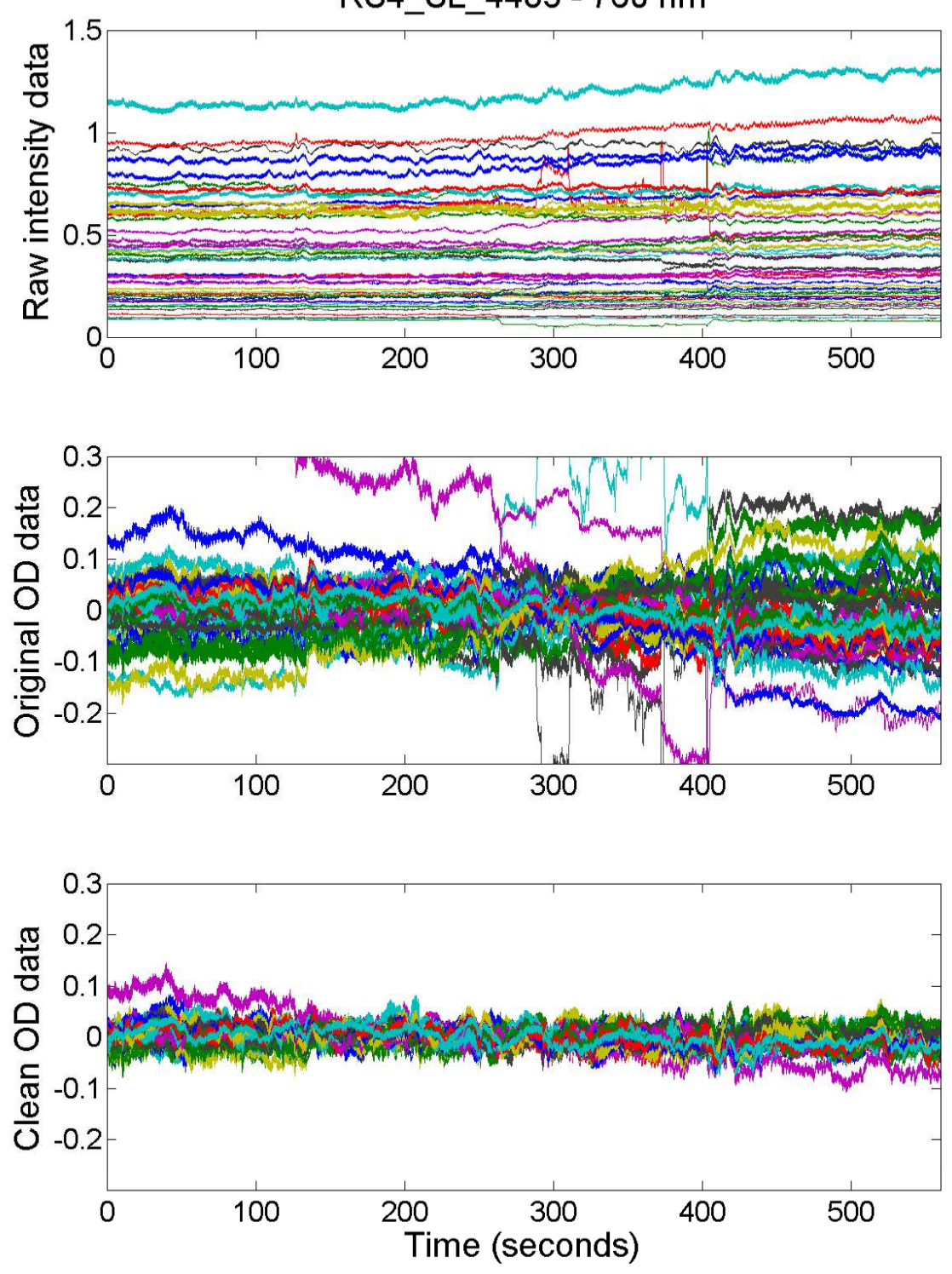

RS4 SL $4483-850 \mathrm{~nm}$
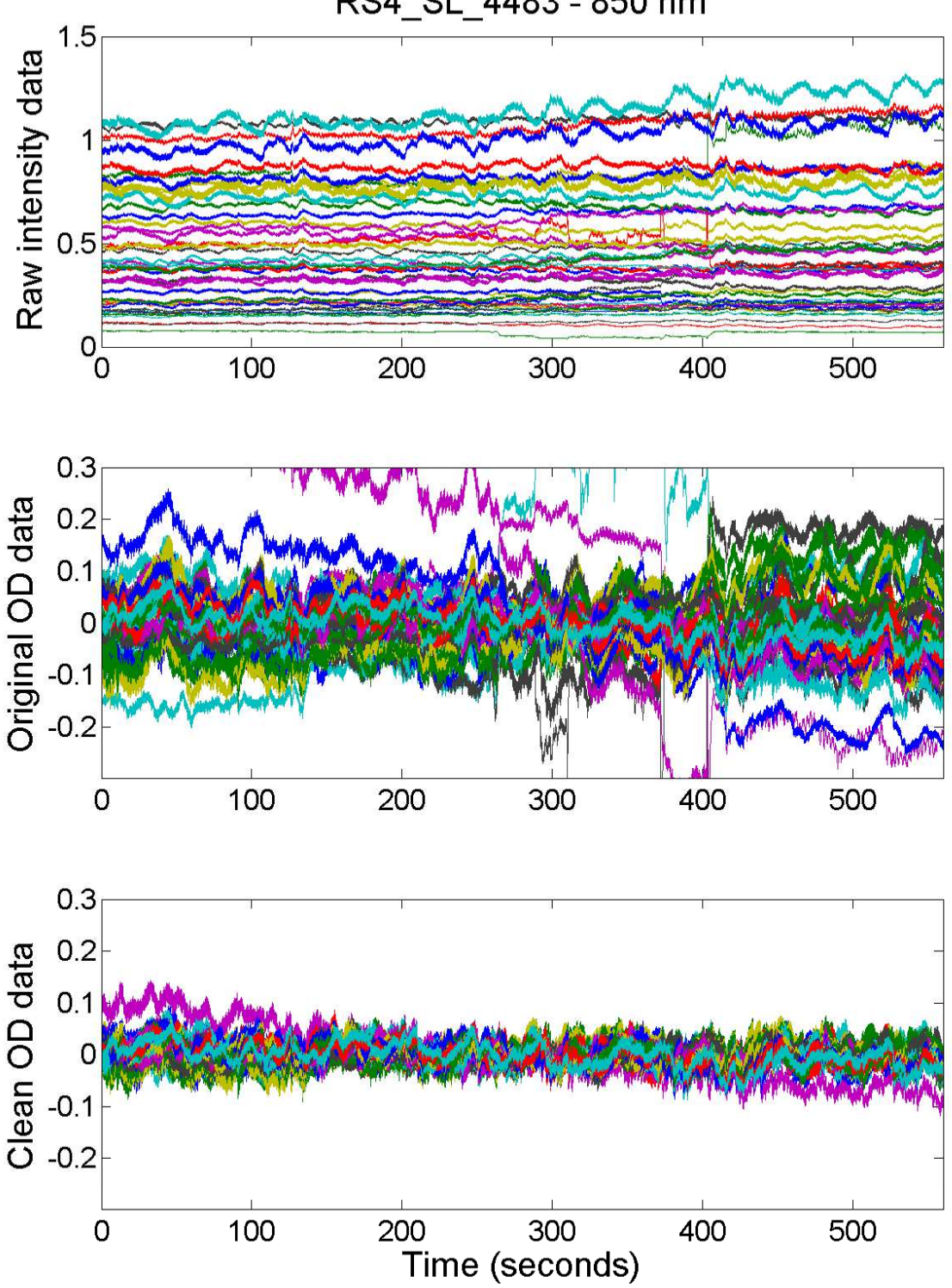
RS4_SL_4483

Raw
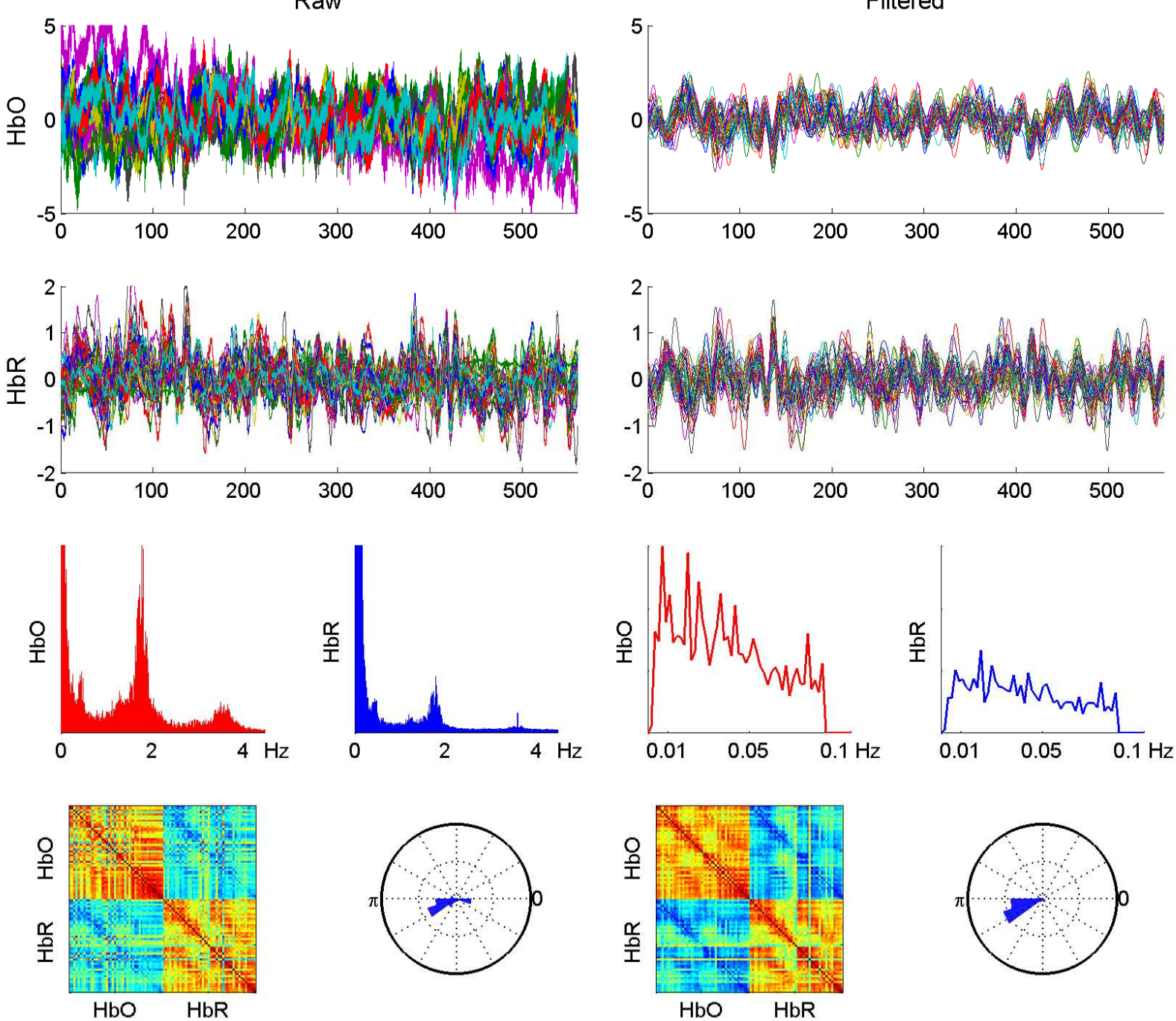
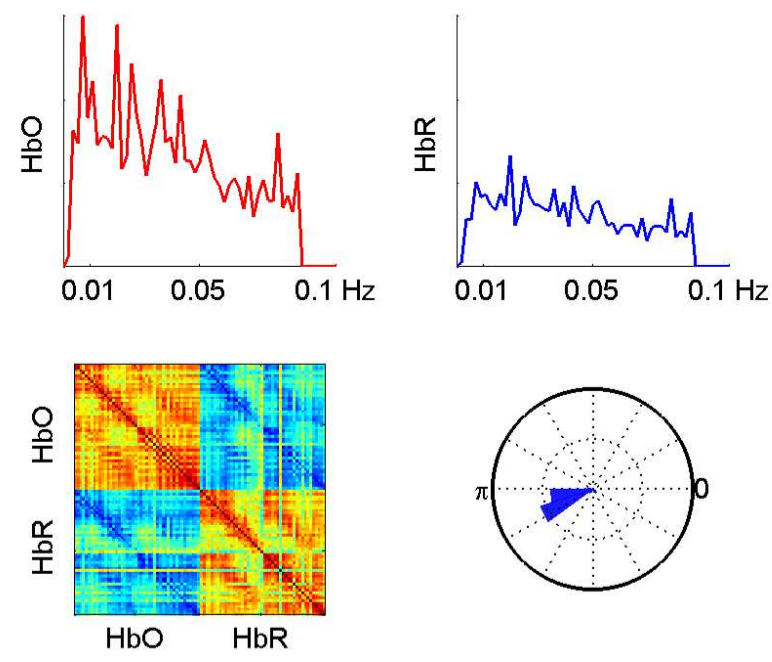

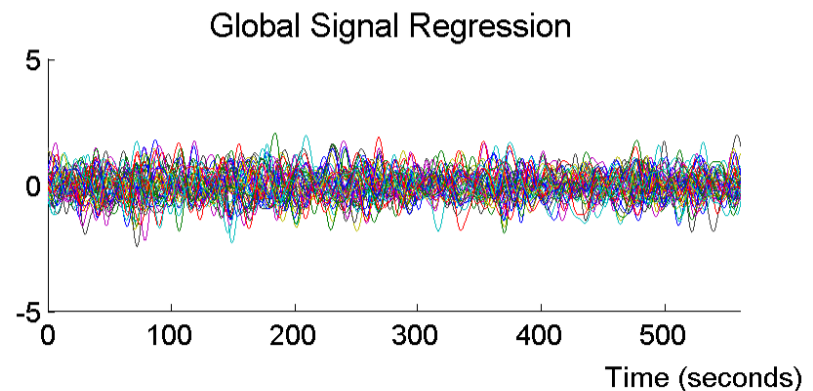

2
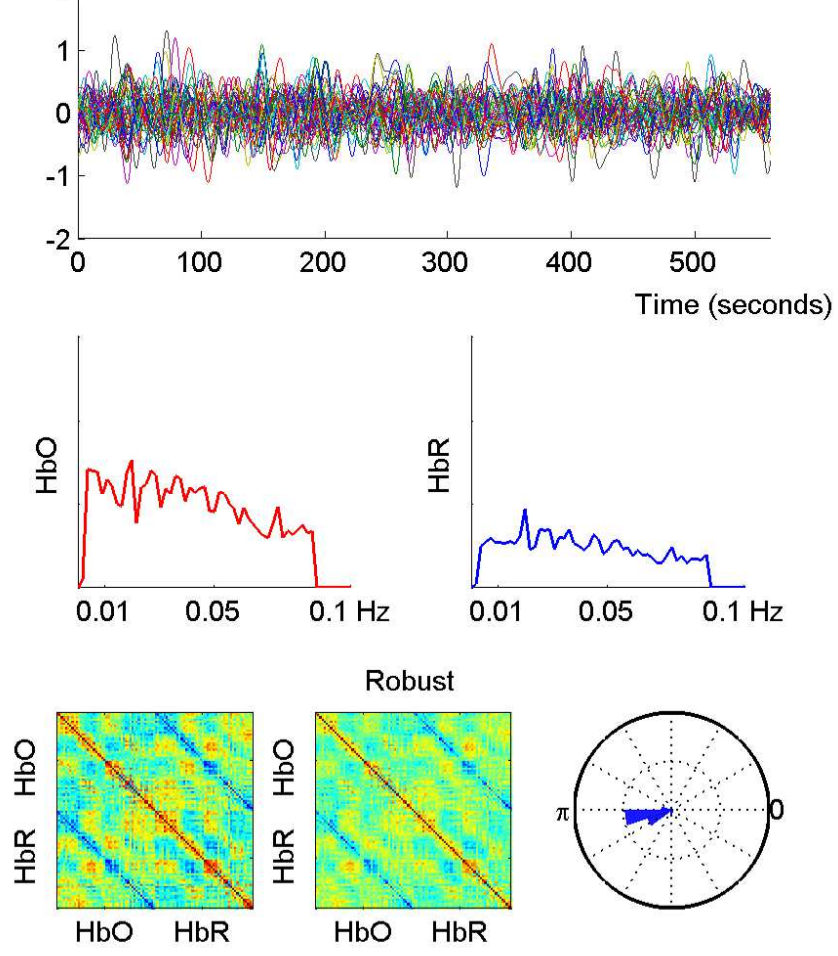

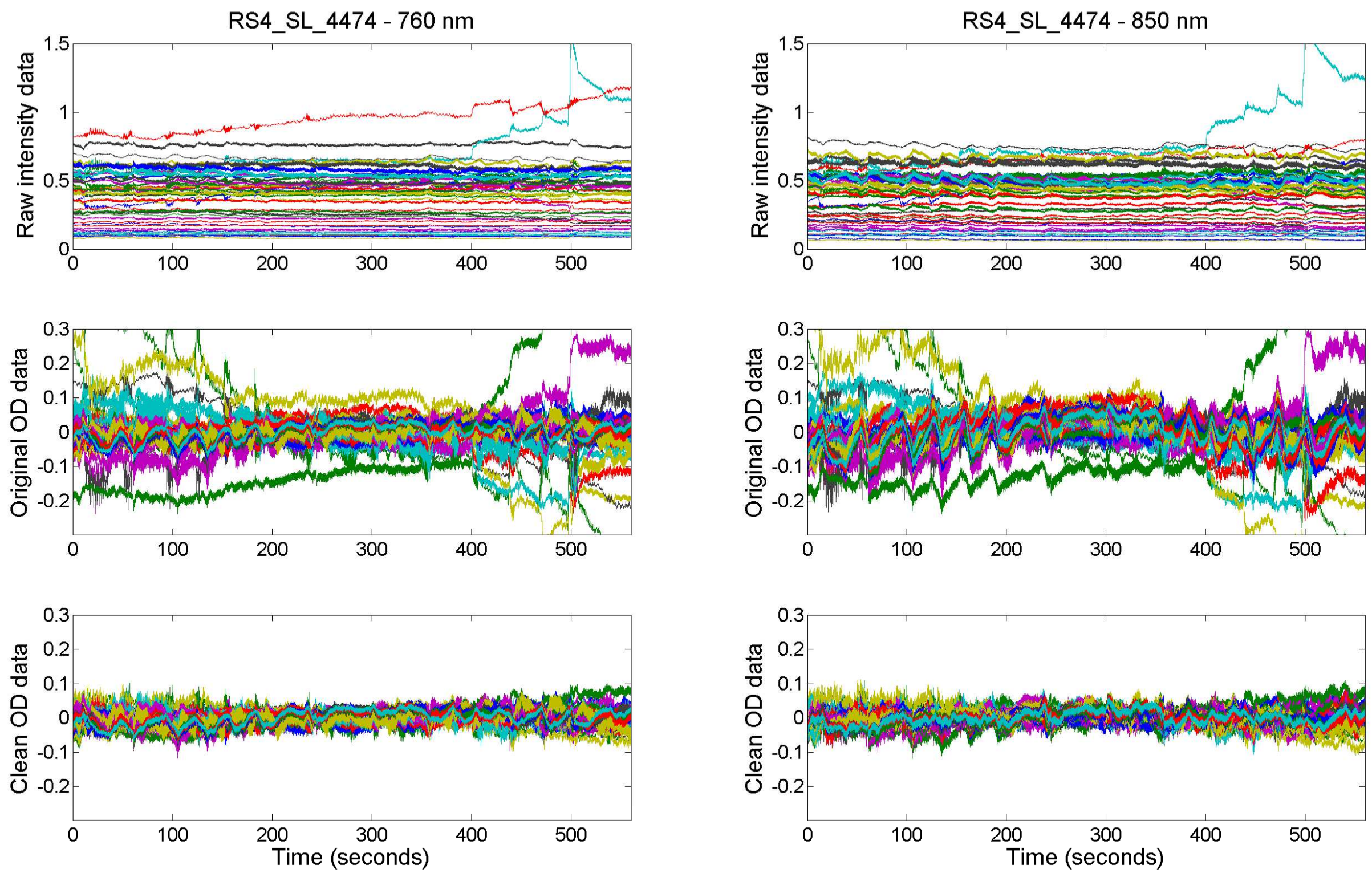
Raw
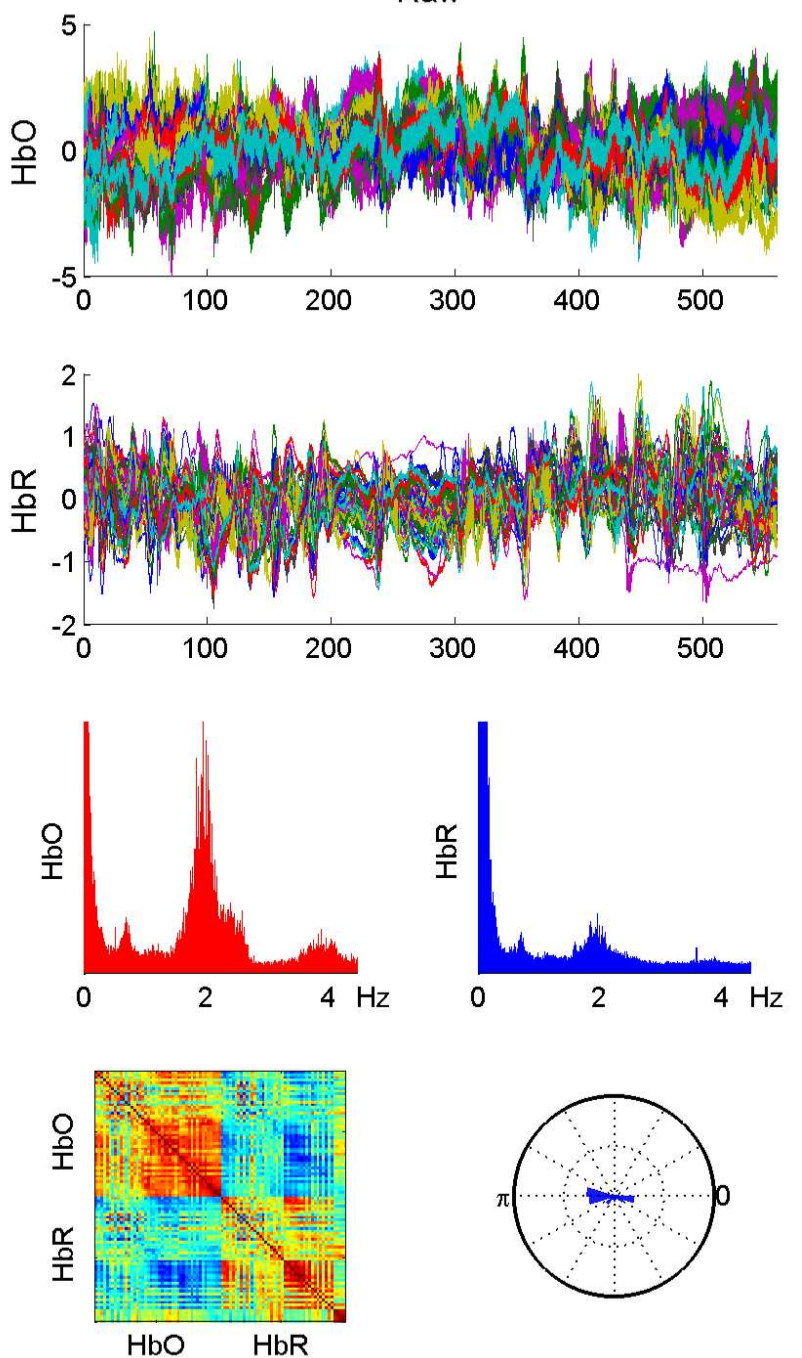

RS4 SL 4474

Filtered
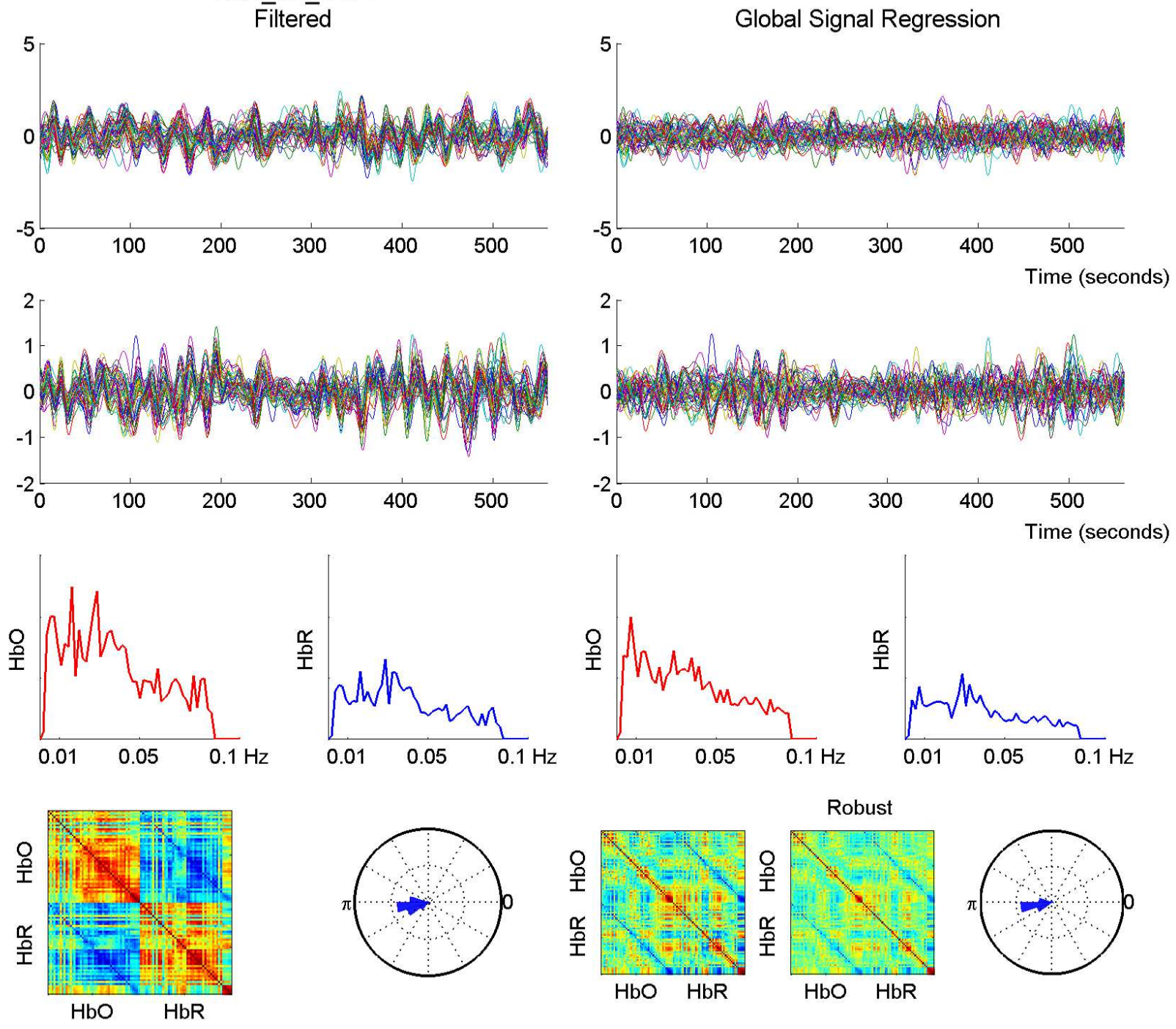
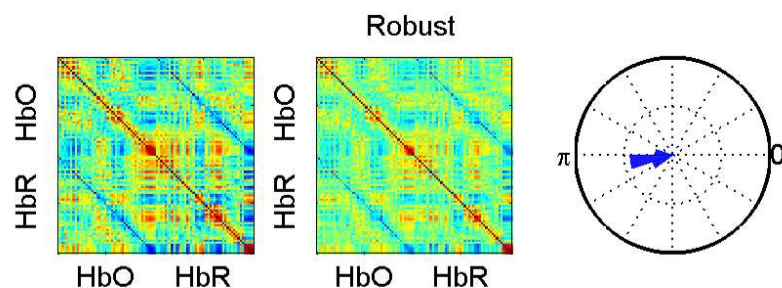
RS4 SL 4472 - 760 nm
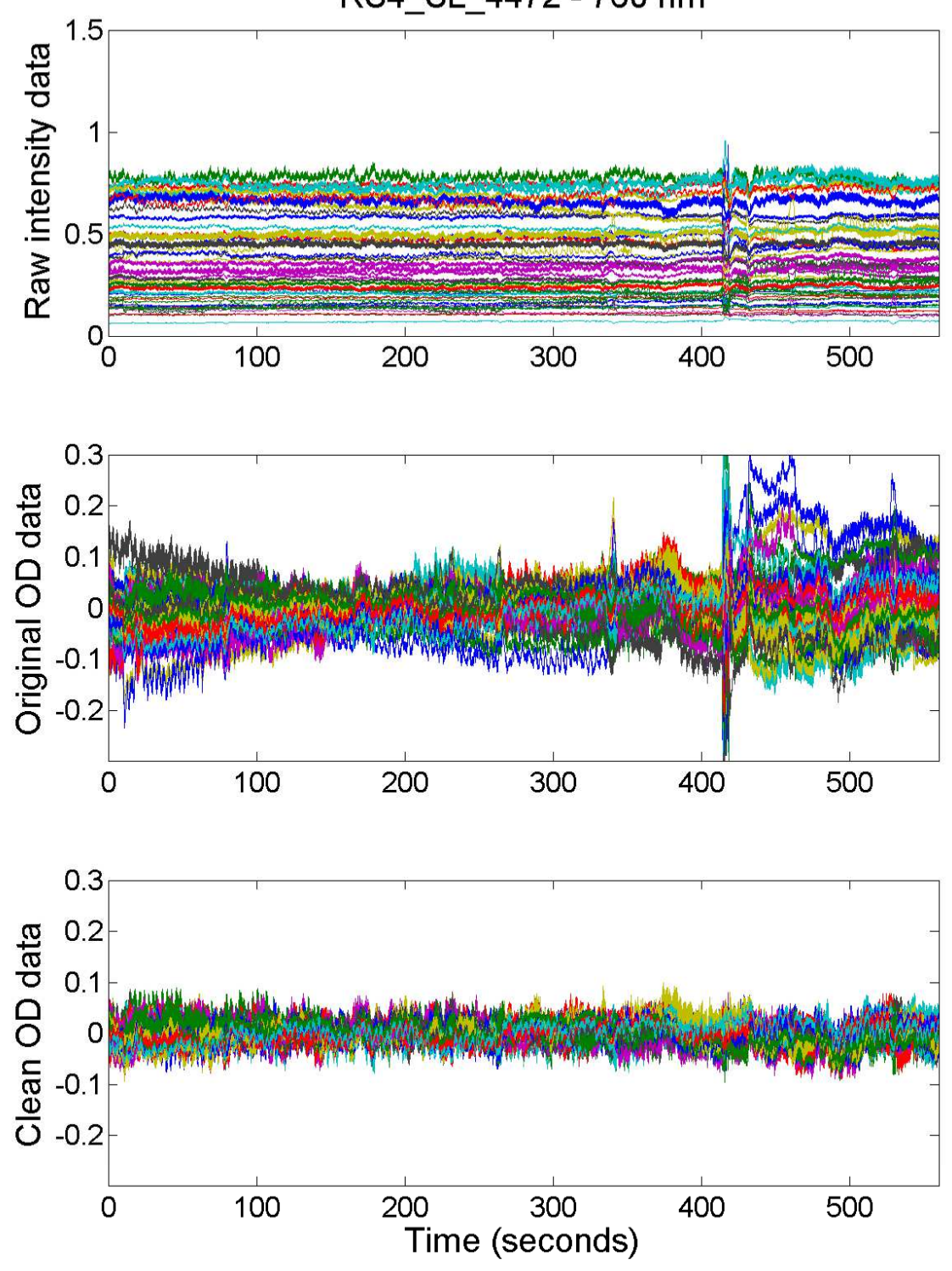

RS4 SL $4472-850 \mathrm{~nm}$
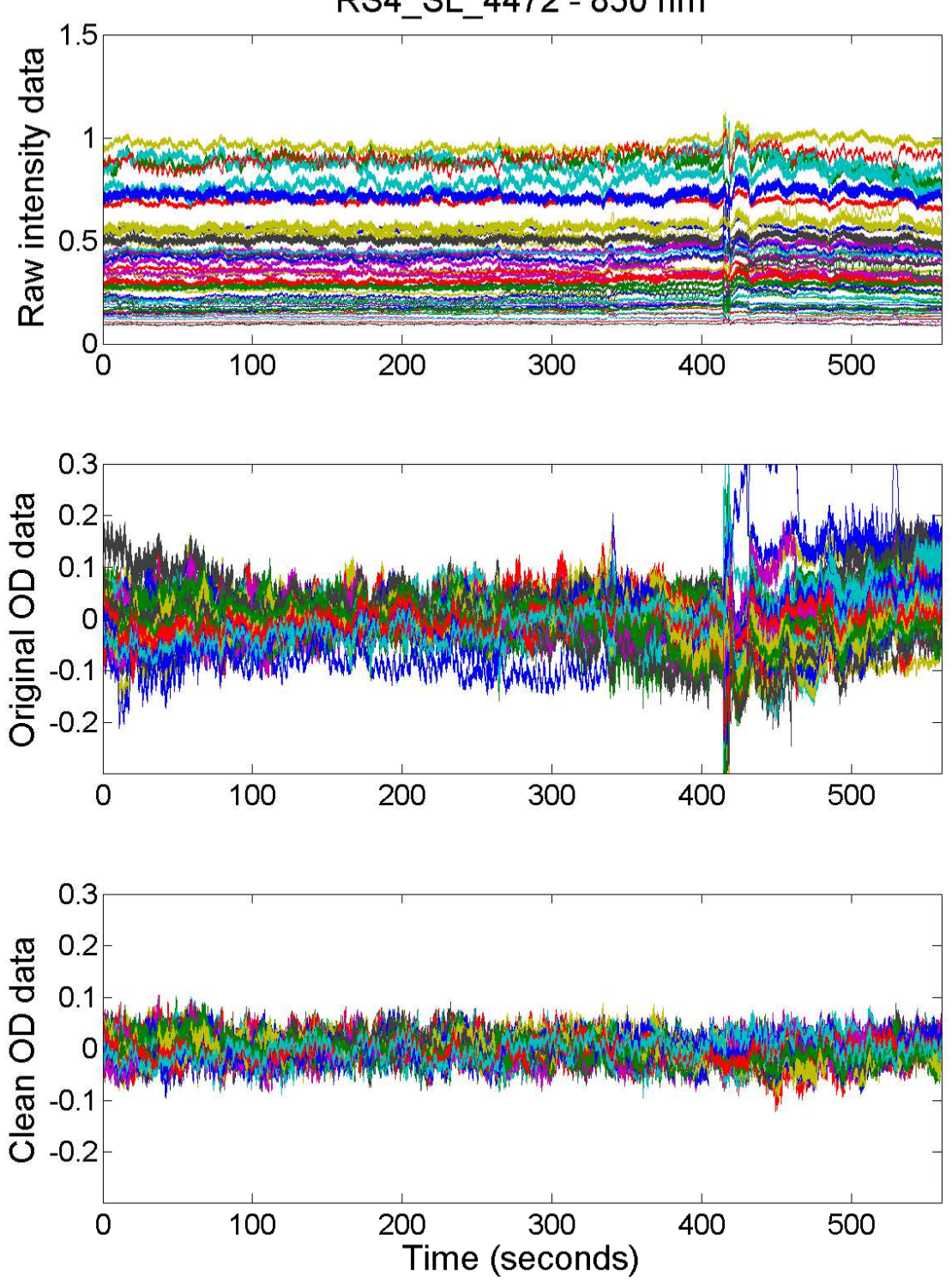
Raw

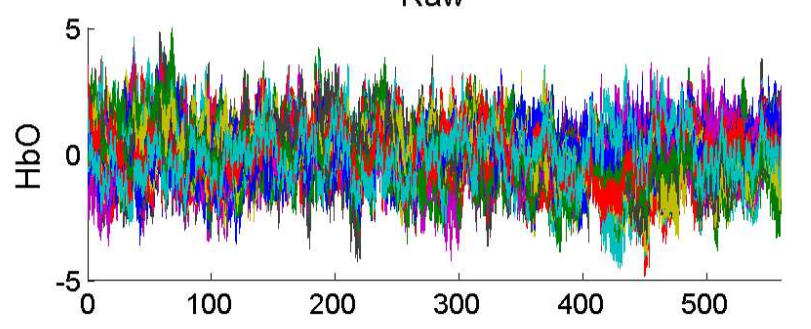

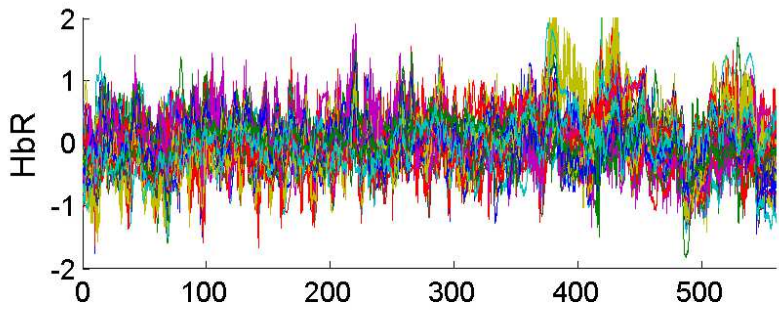
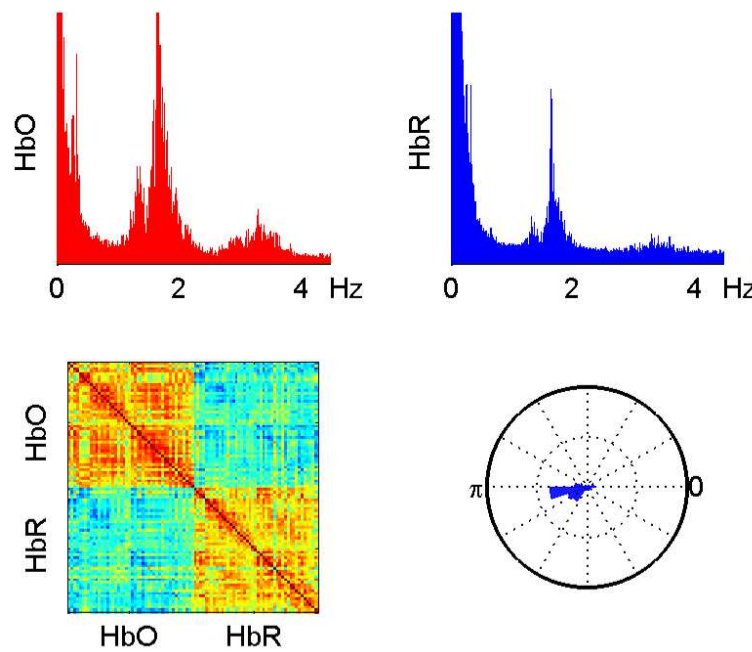

RS4_SL_4472

Filtered
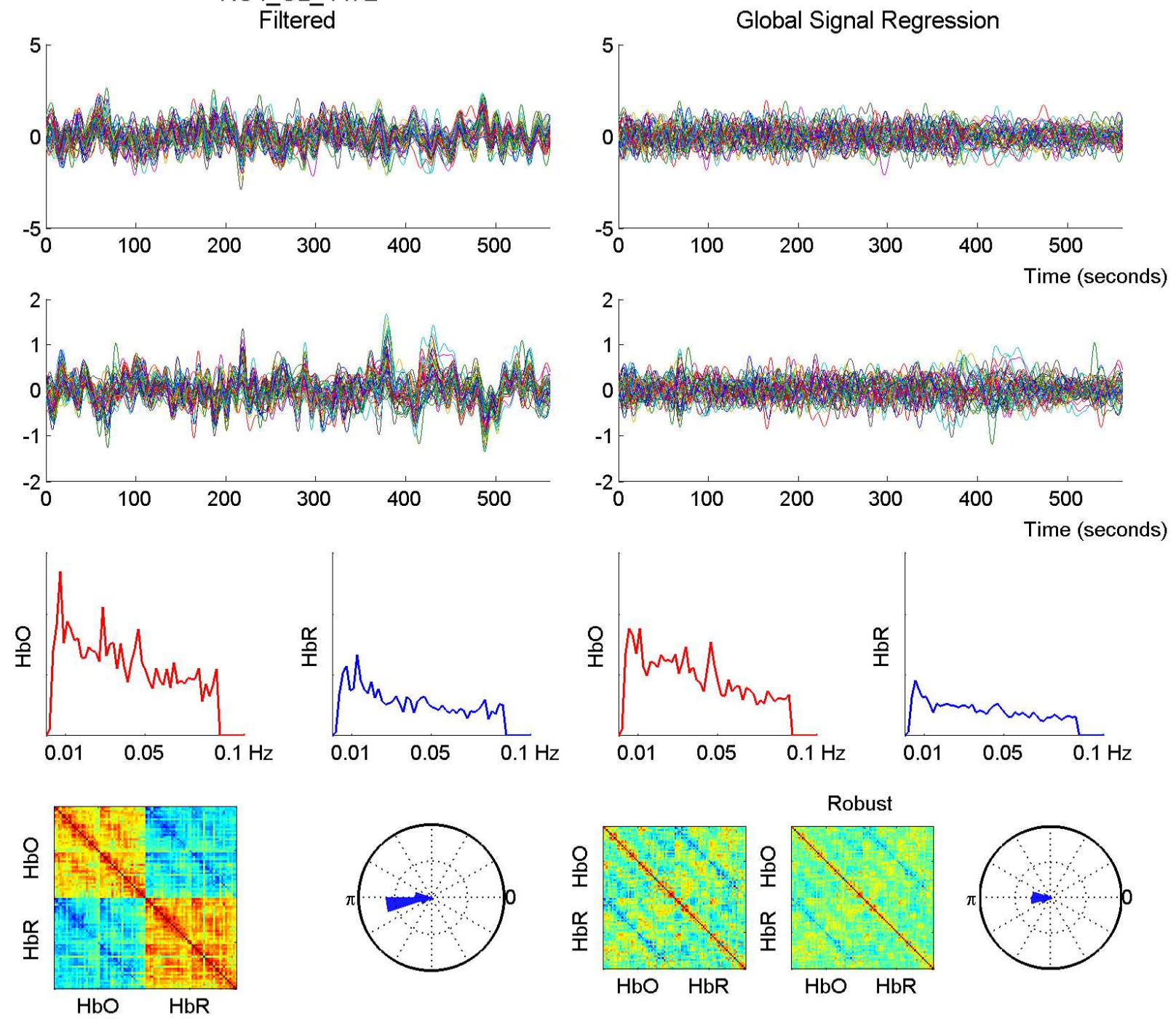
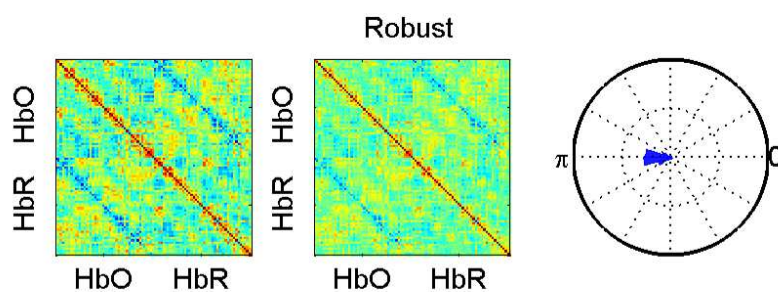
RS4 SL 4468 - 760 nm
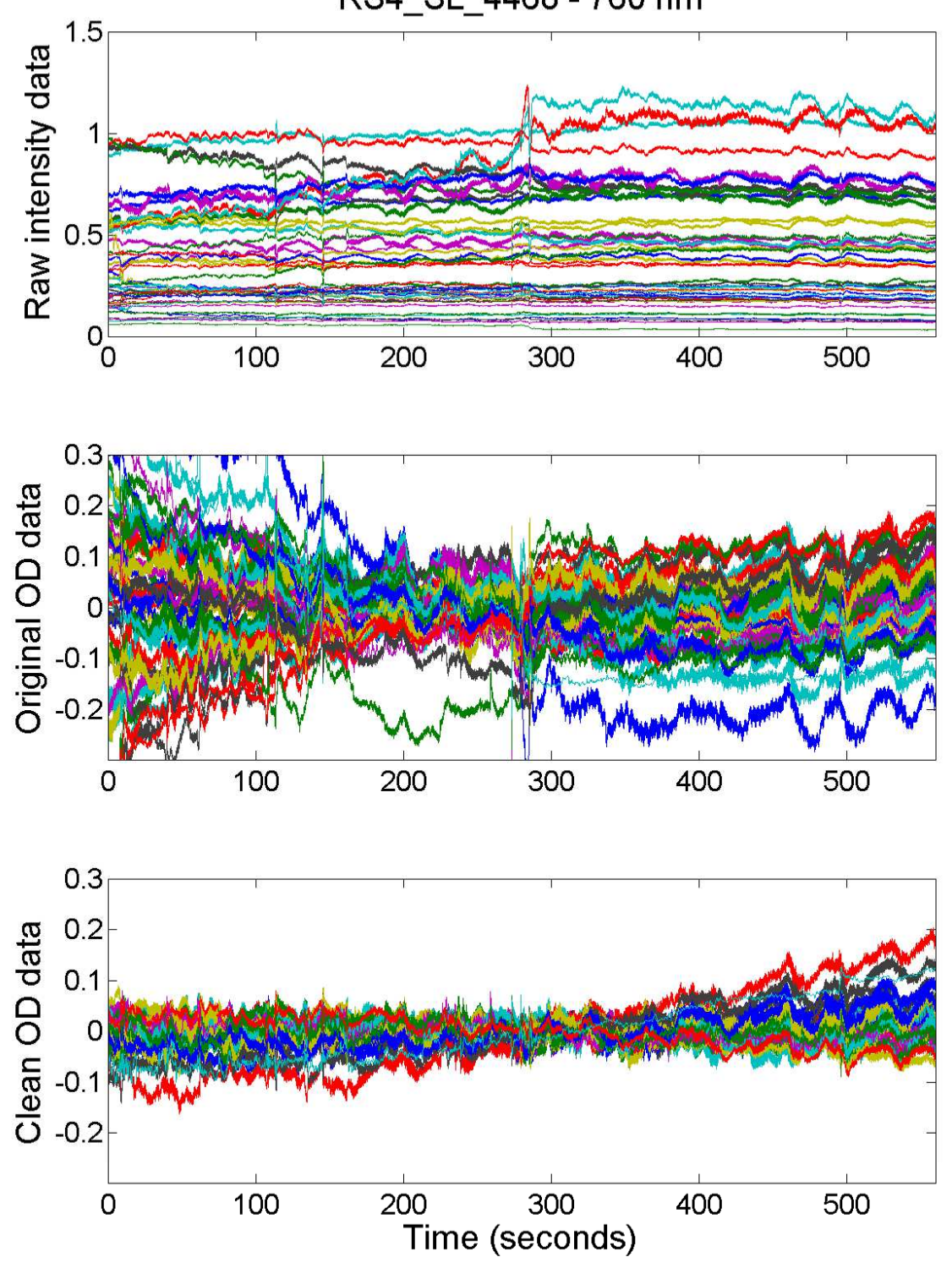
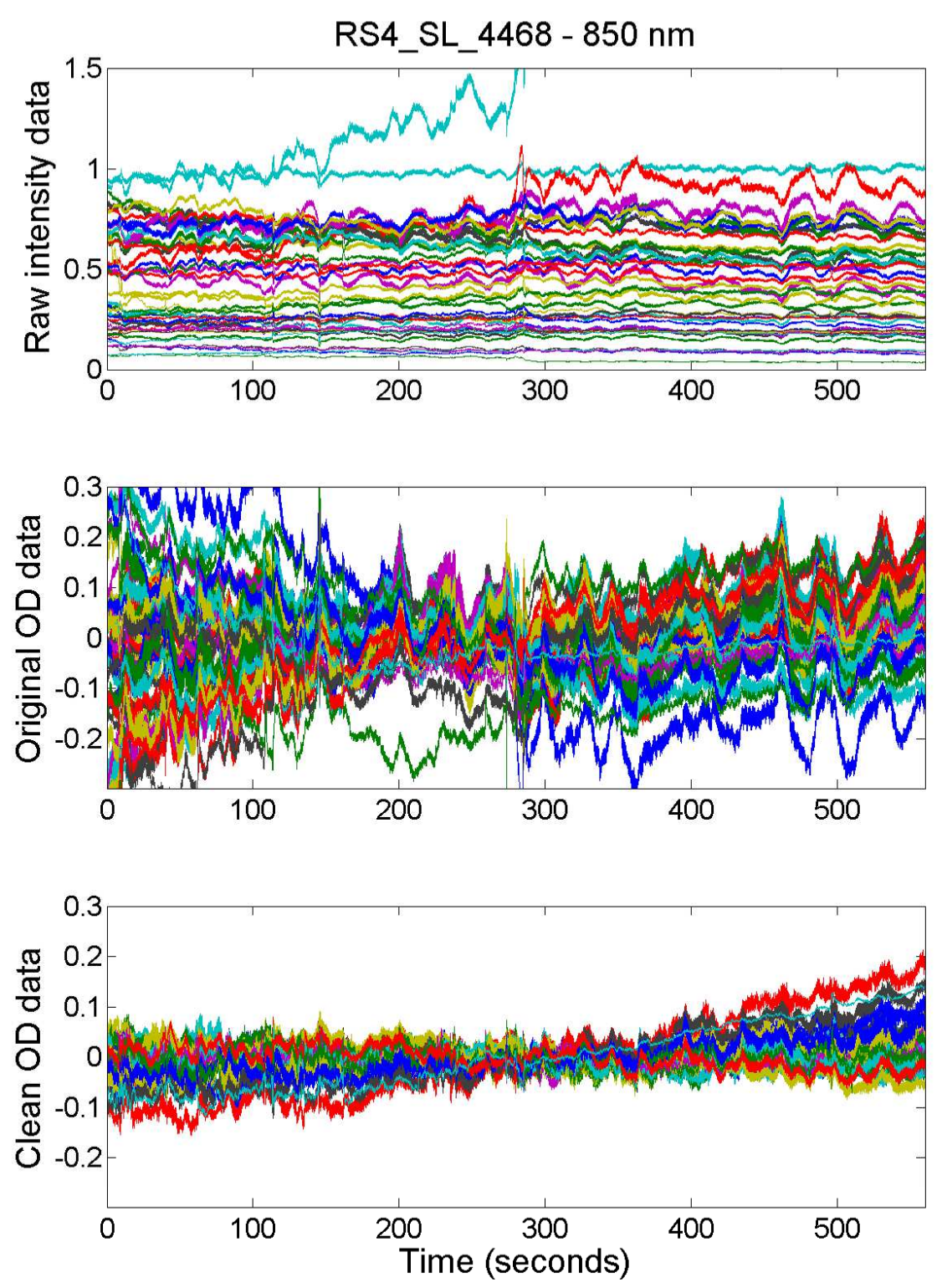
Raw
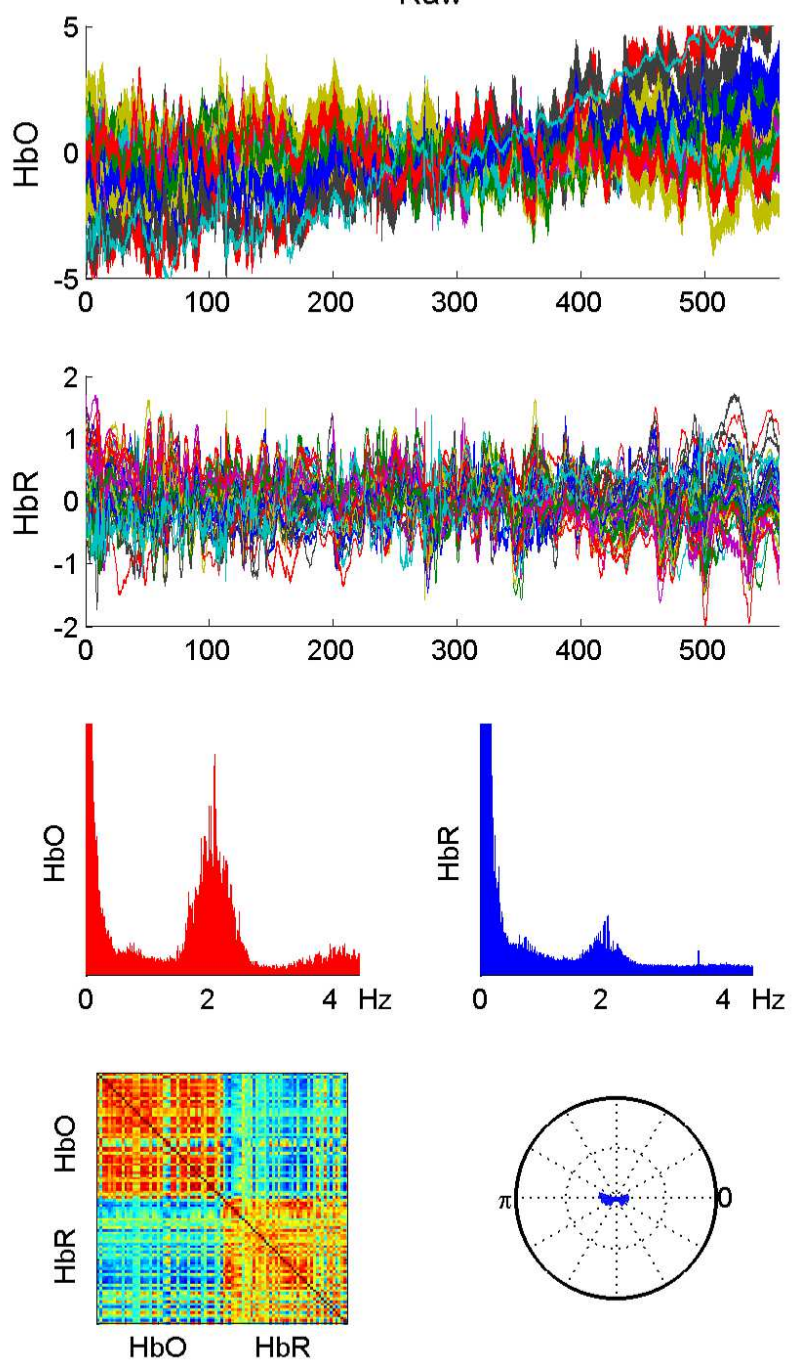

RS4 SL 4468

Filtered
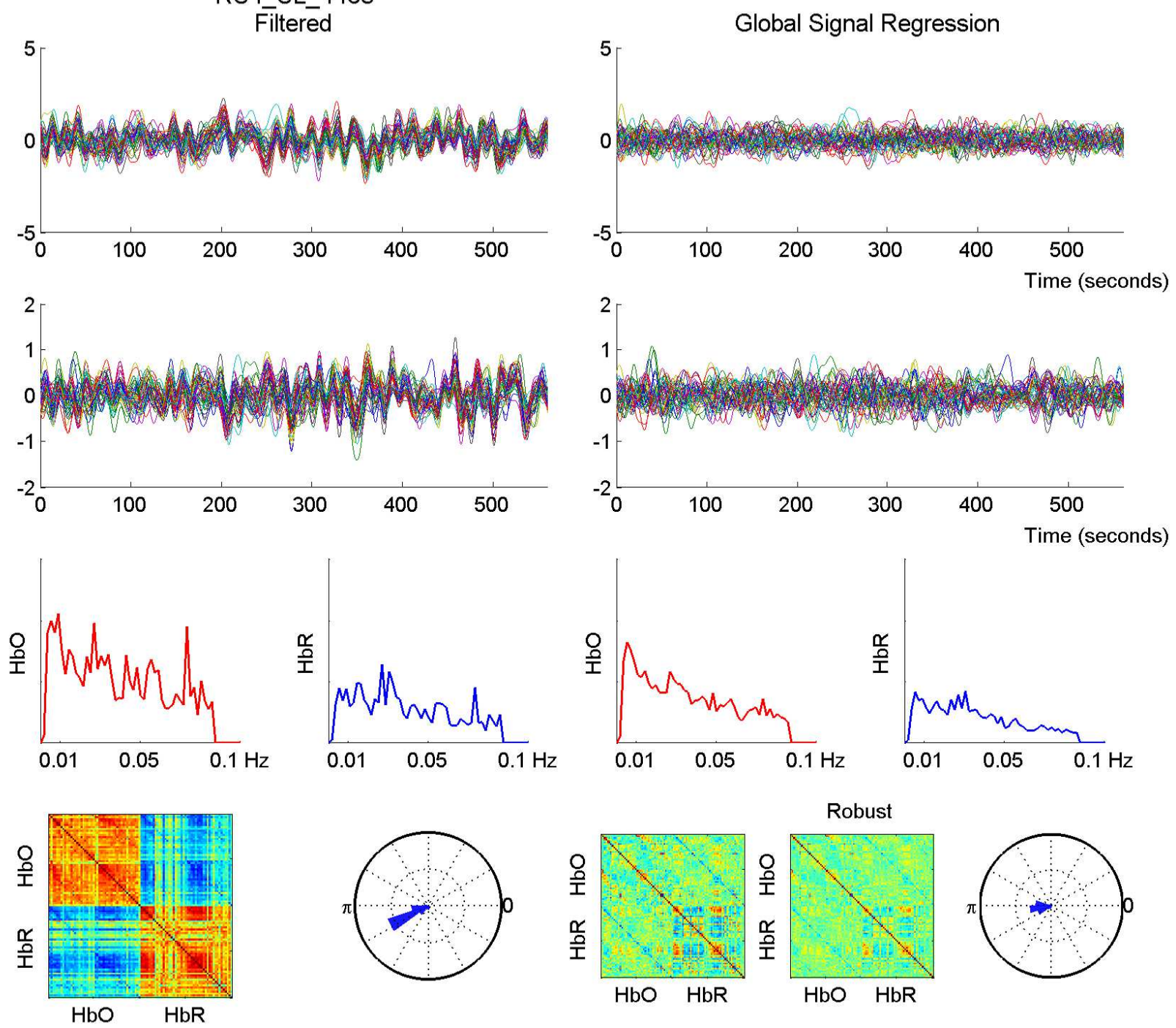

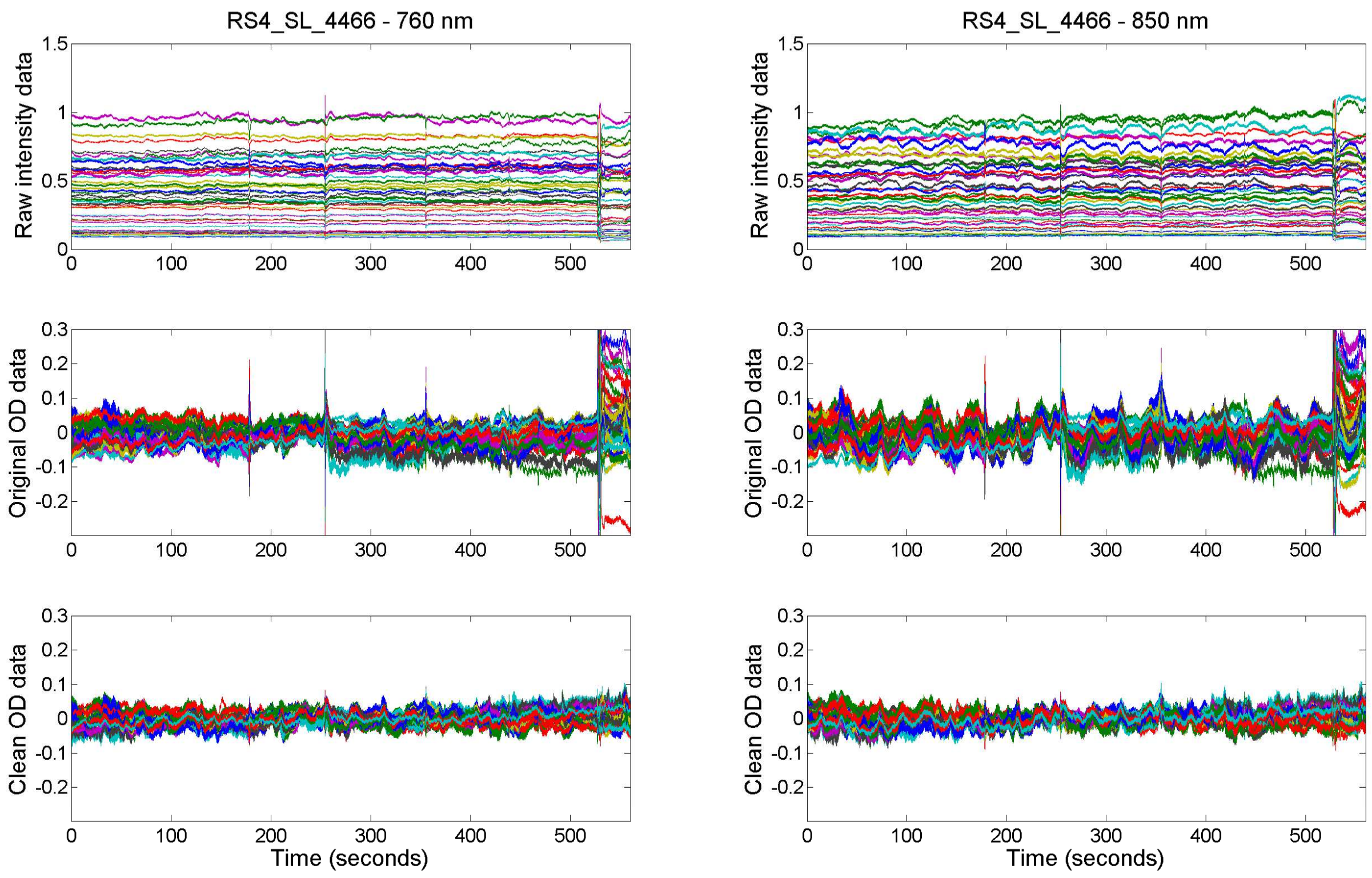
Raw

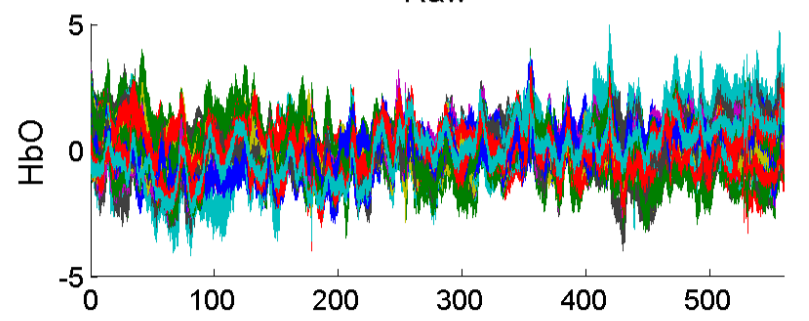

2

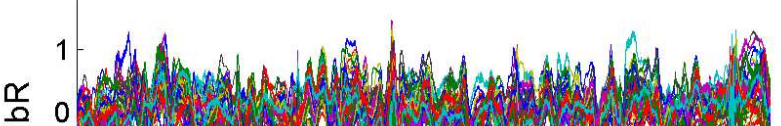

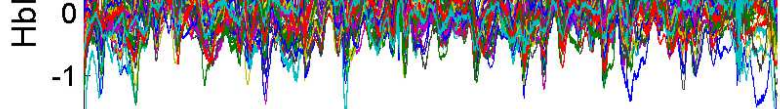
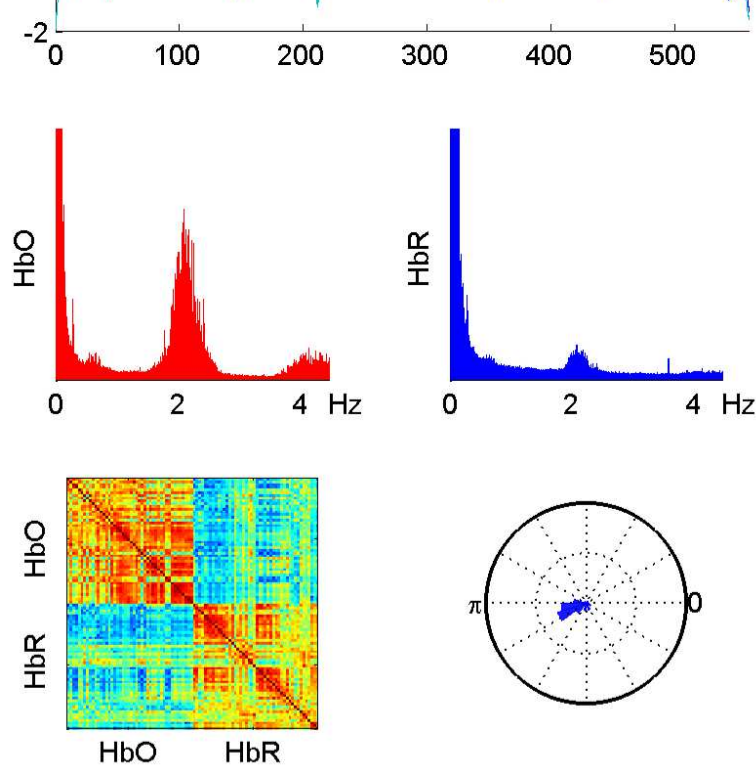

RS4 SL 4466

Filtered
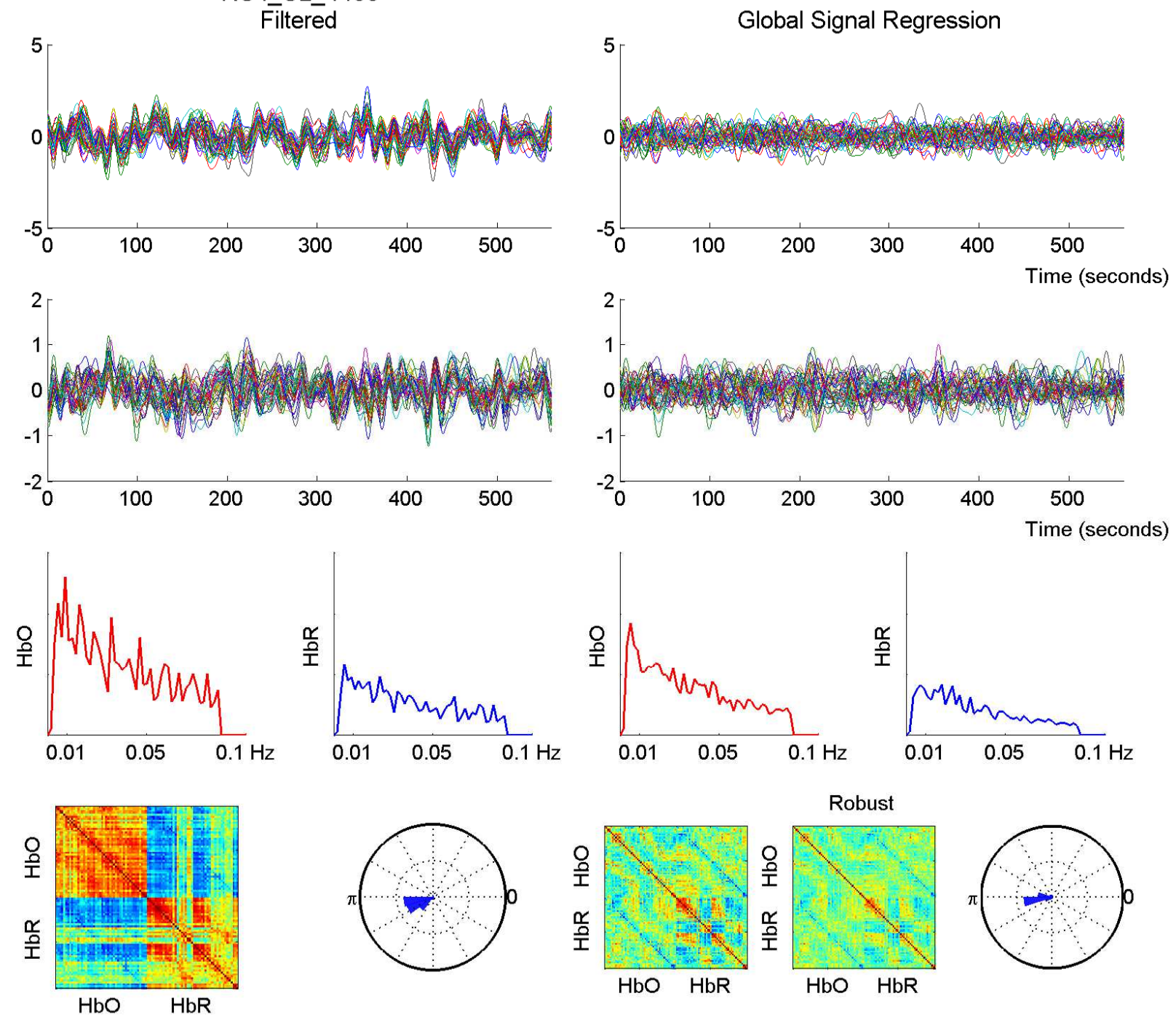
RS4 SL $4465-760$ nm
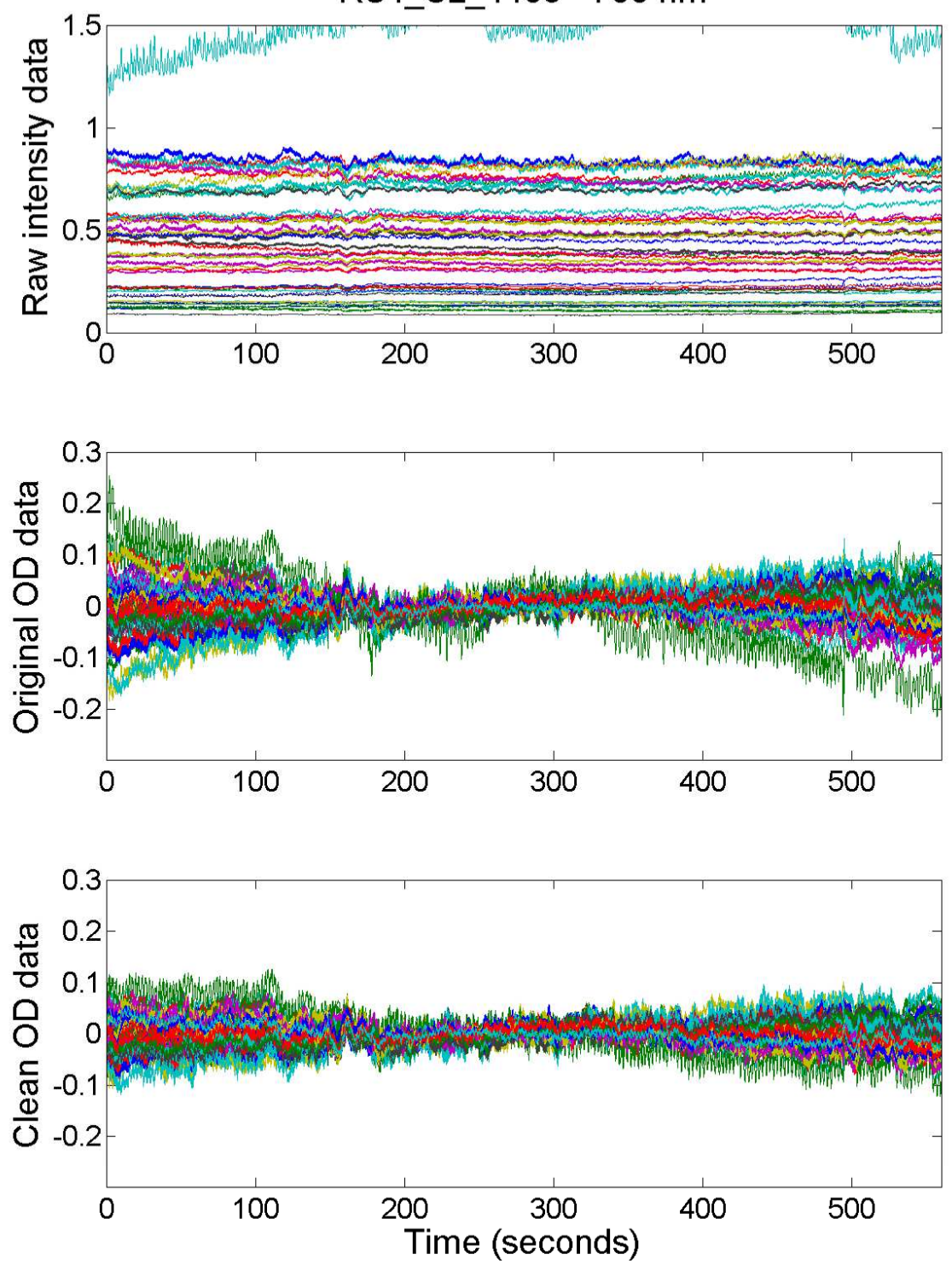

RS4 SL $4465-850 \mathrm{~nm}$
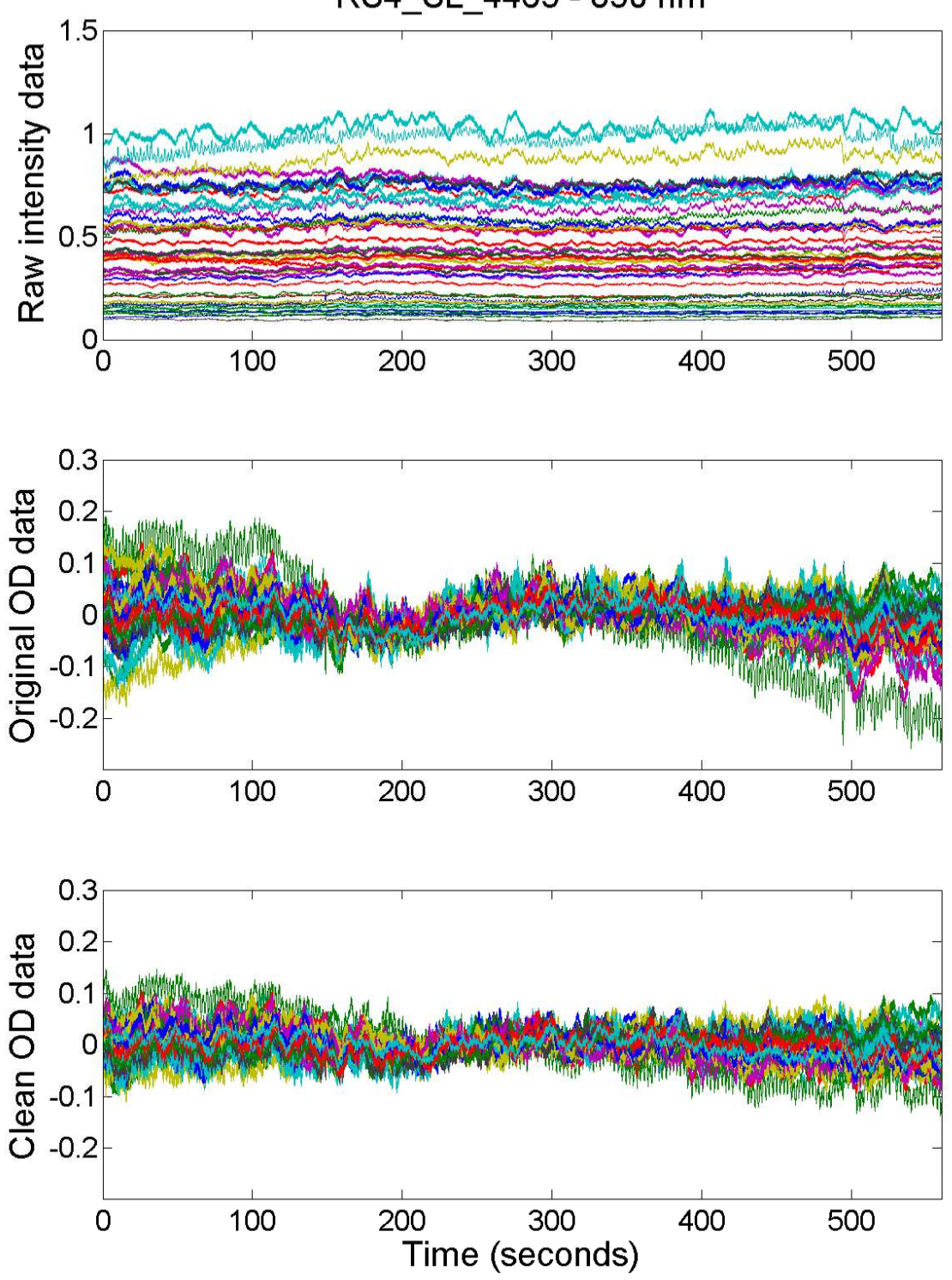
Raw

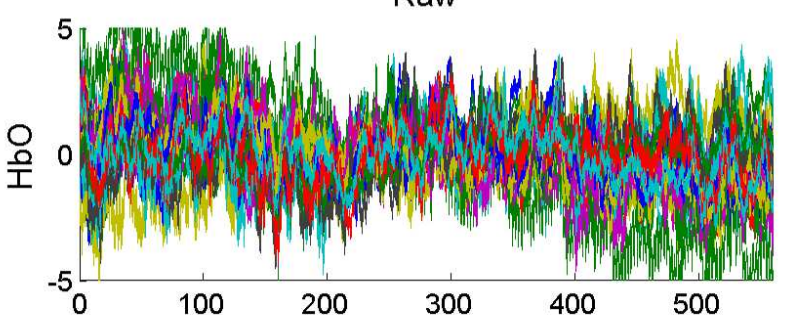

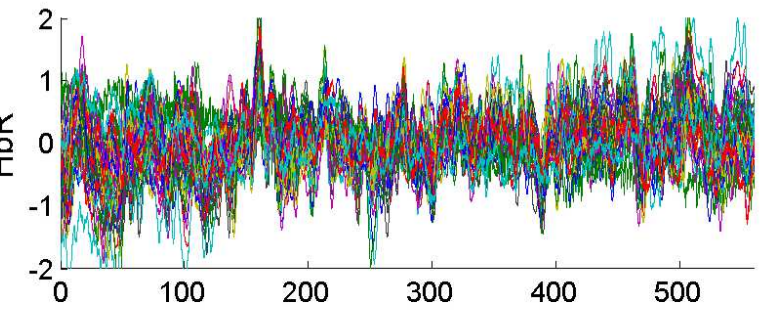
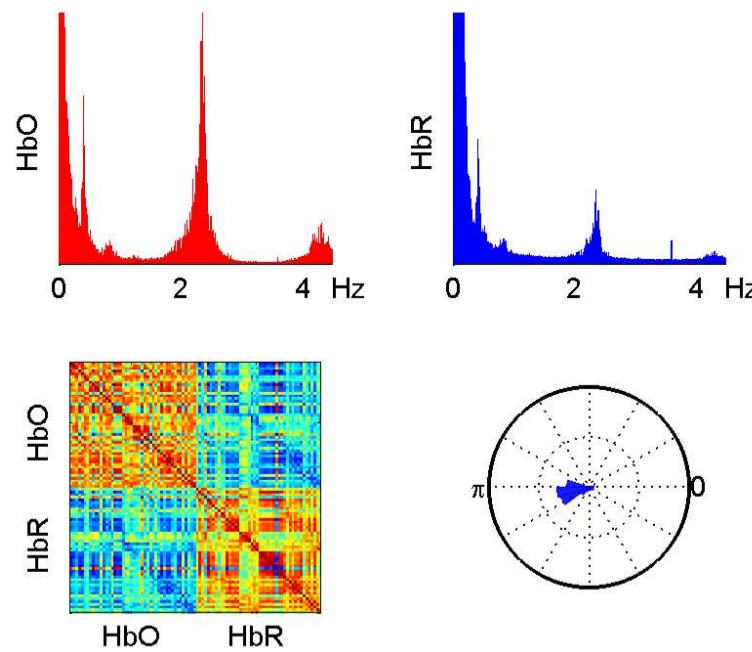

RS4 SL 4465

Filtered
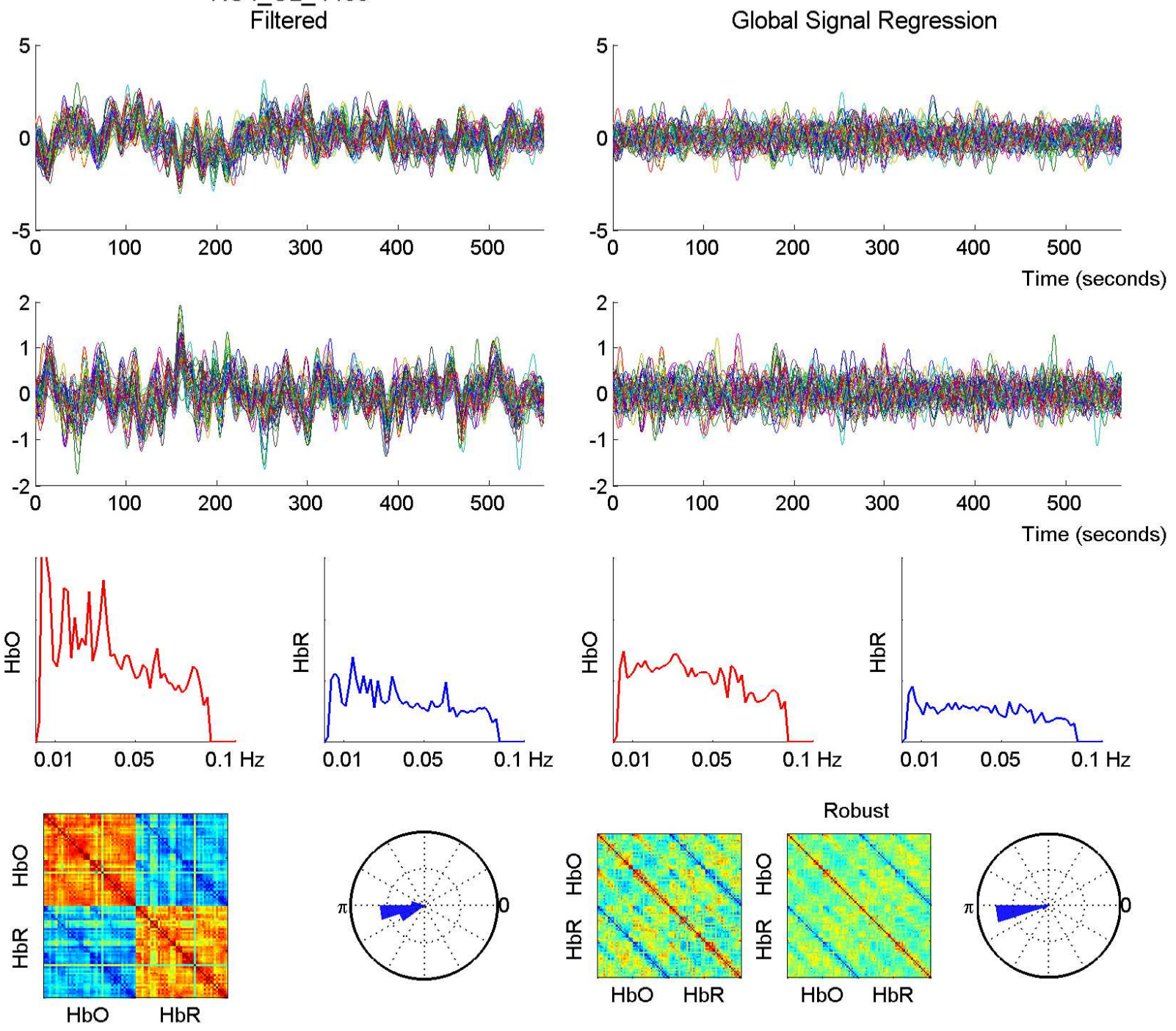
RS4 SL 4464 - 760 nm
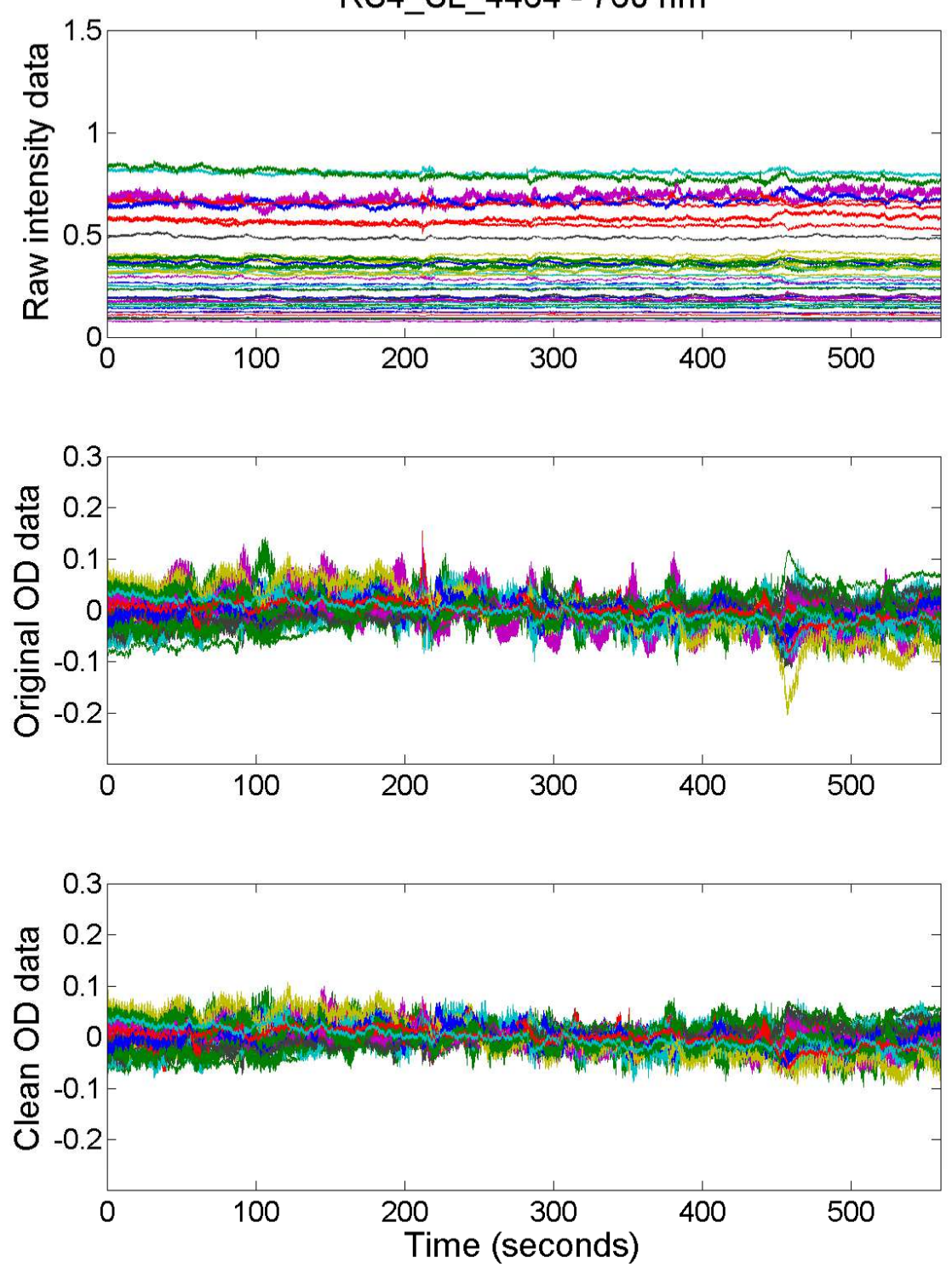

RS4 SL $4464-850 \mathrm{~nm}$
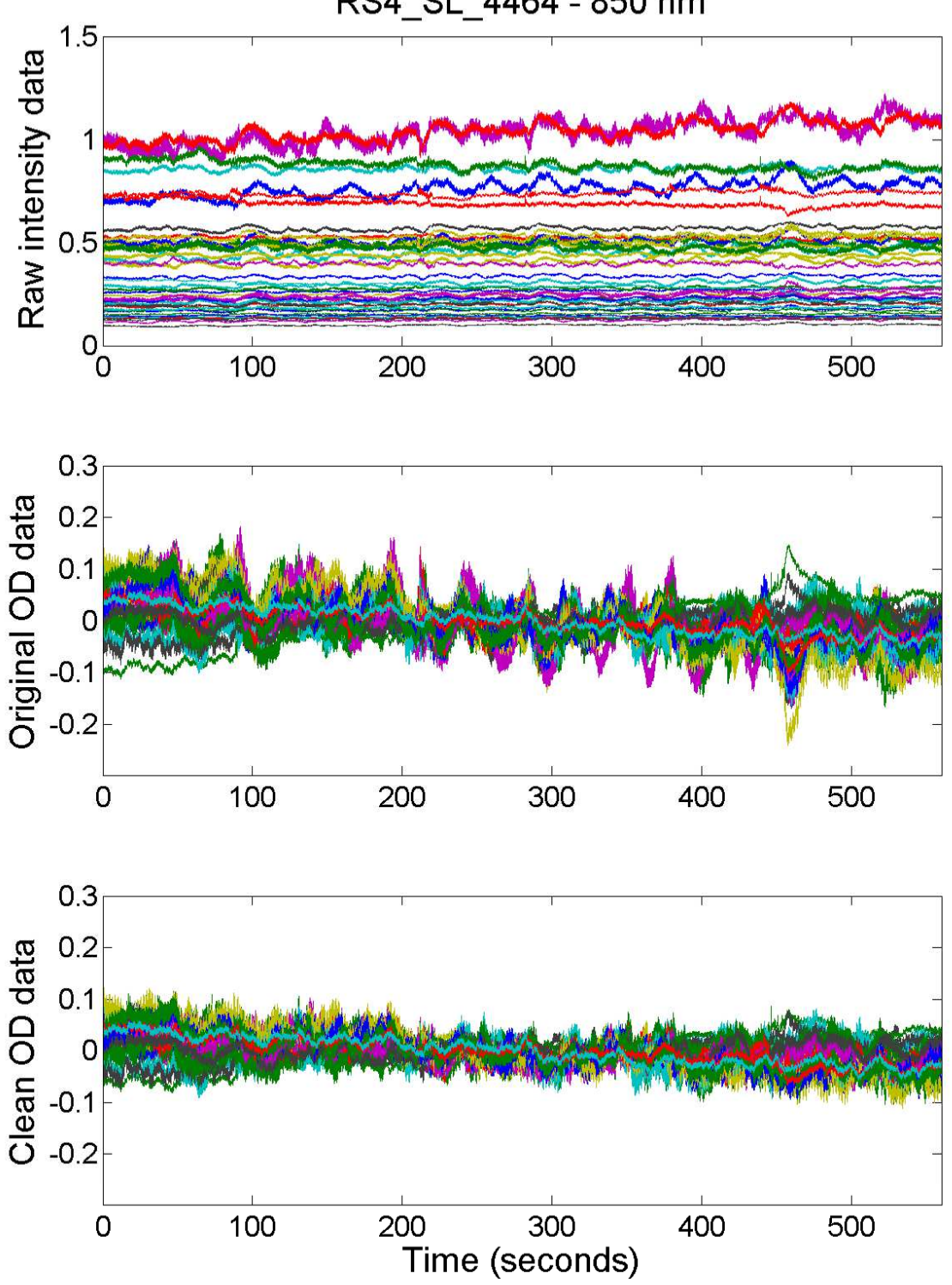
Raw
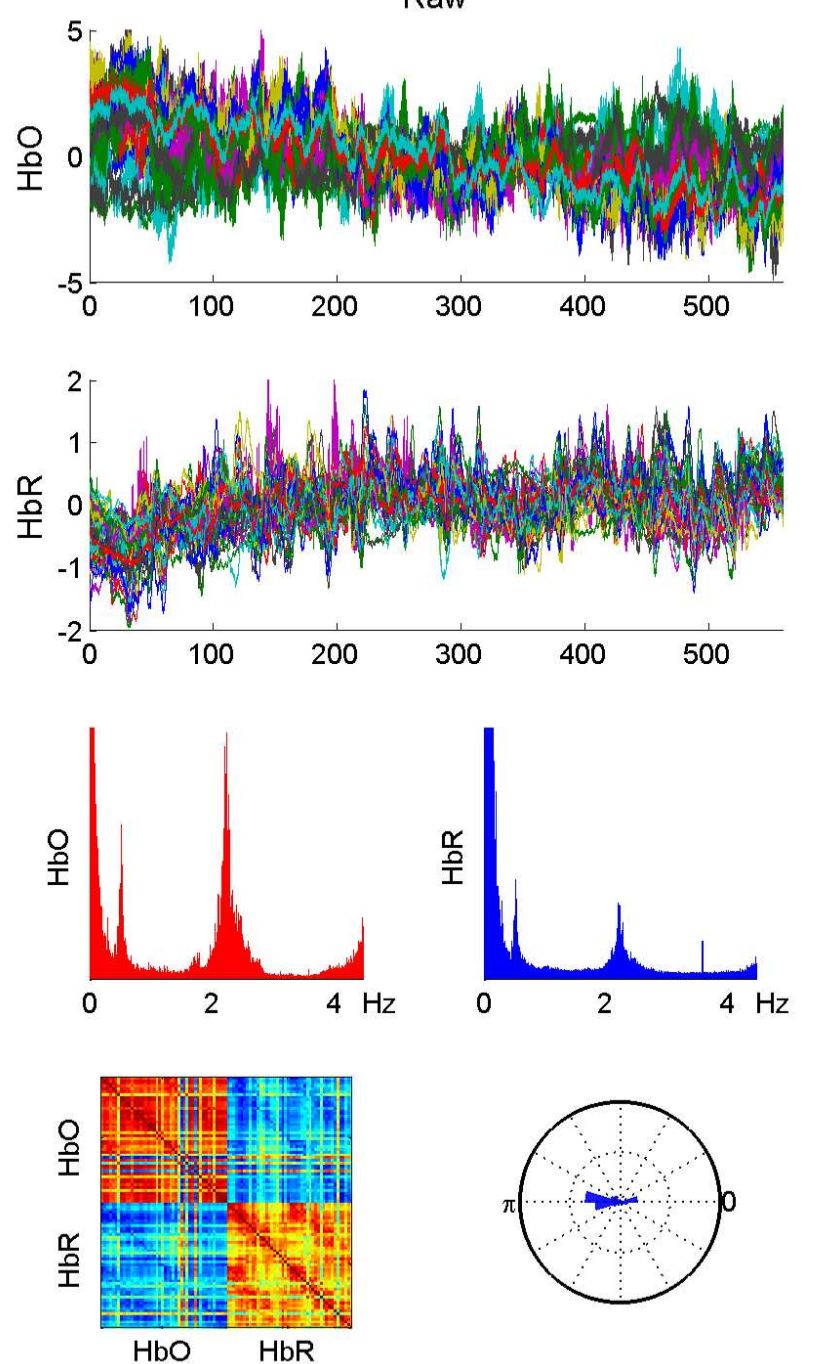

RS4_SL_4464

Filtered
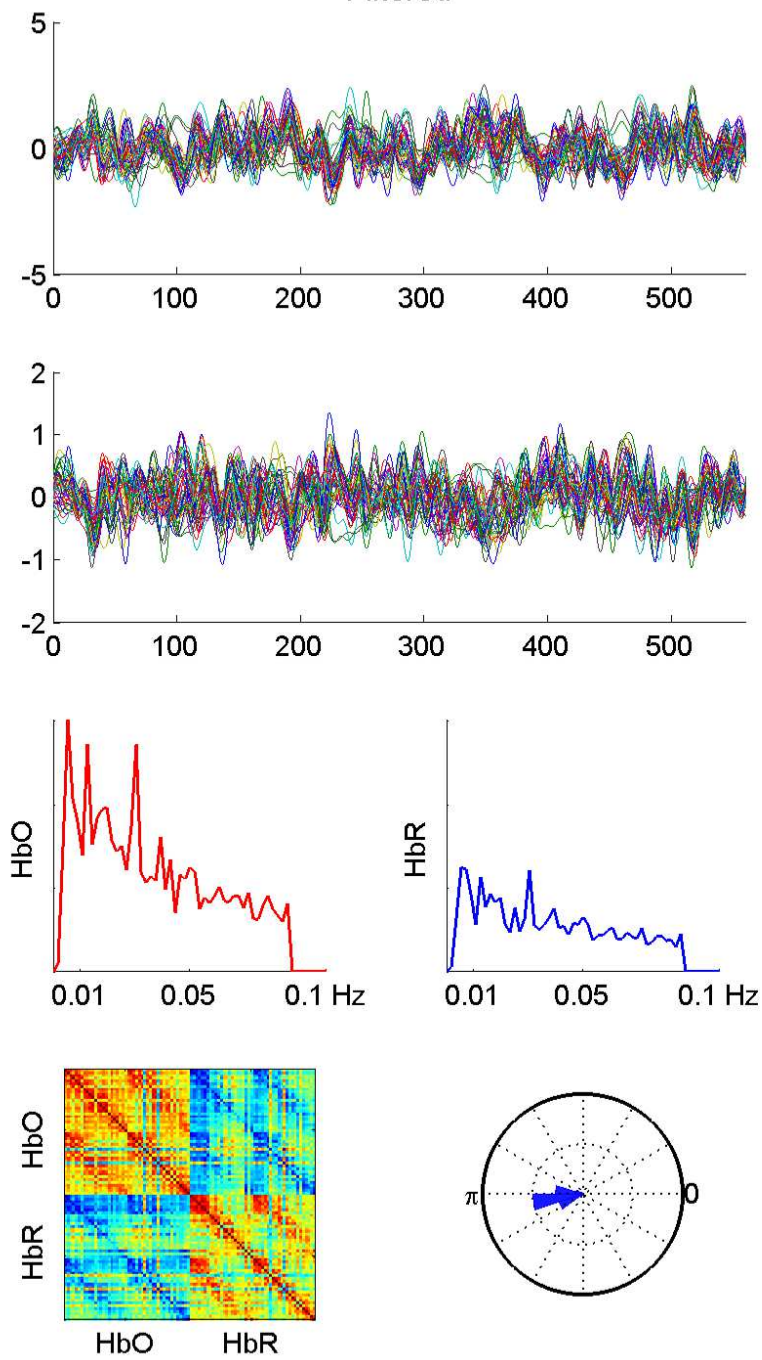

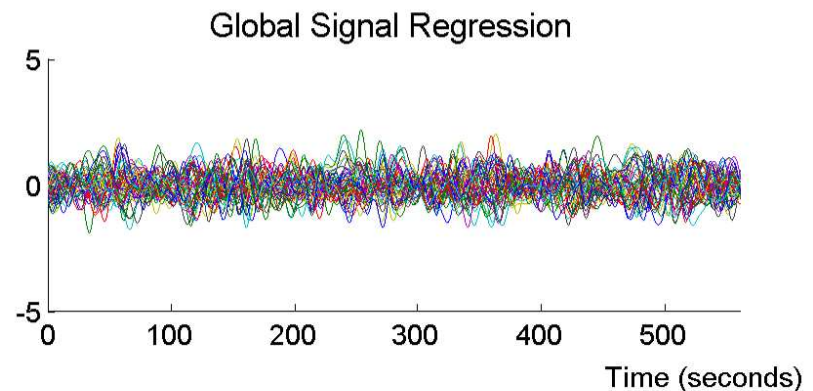

2
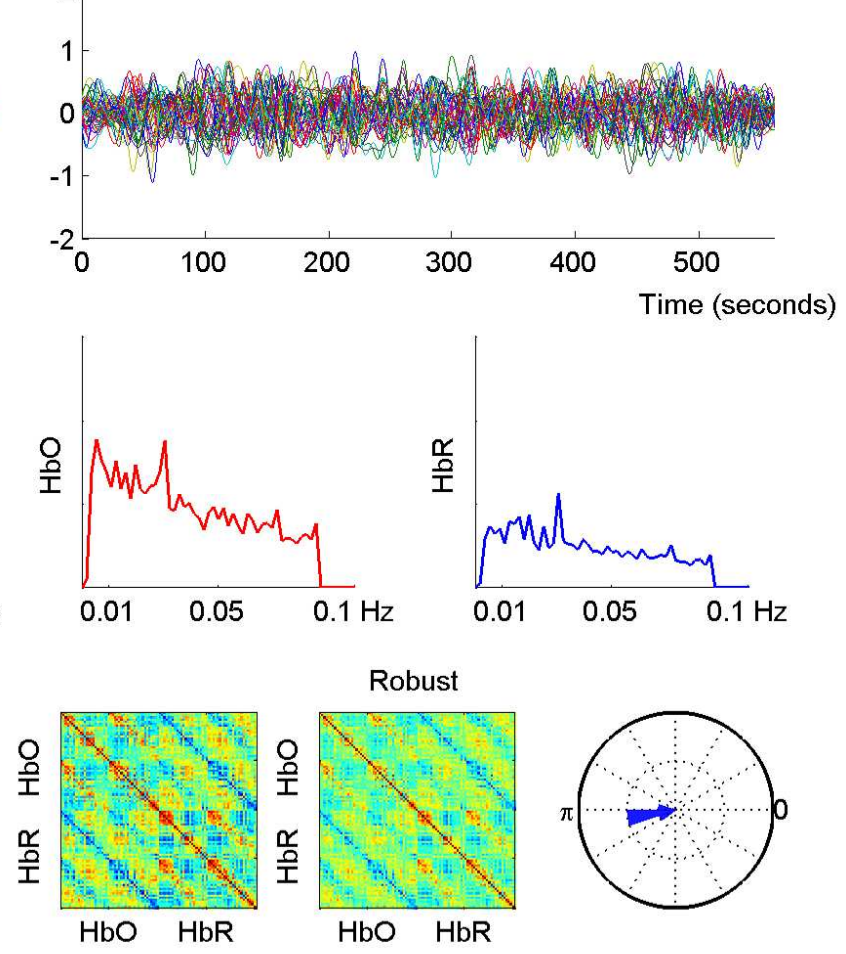

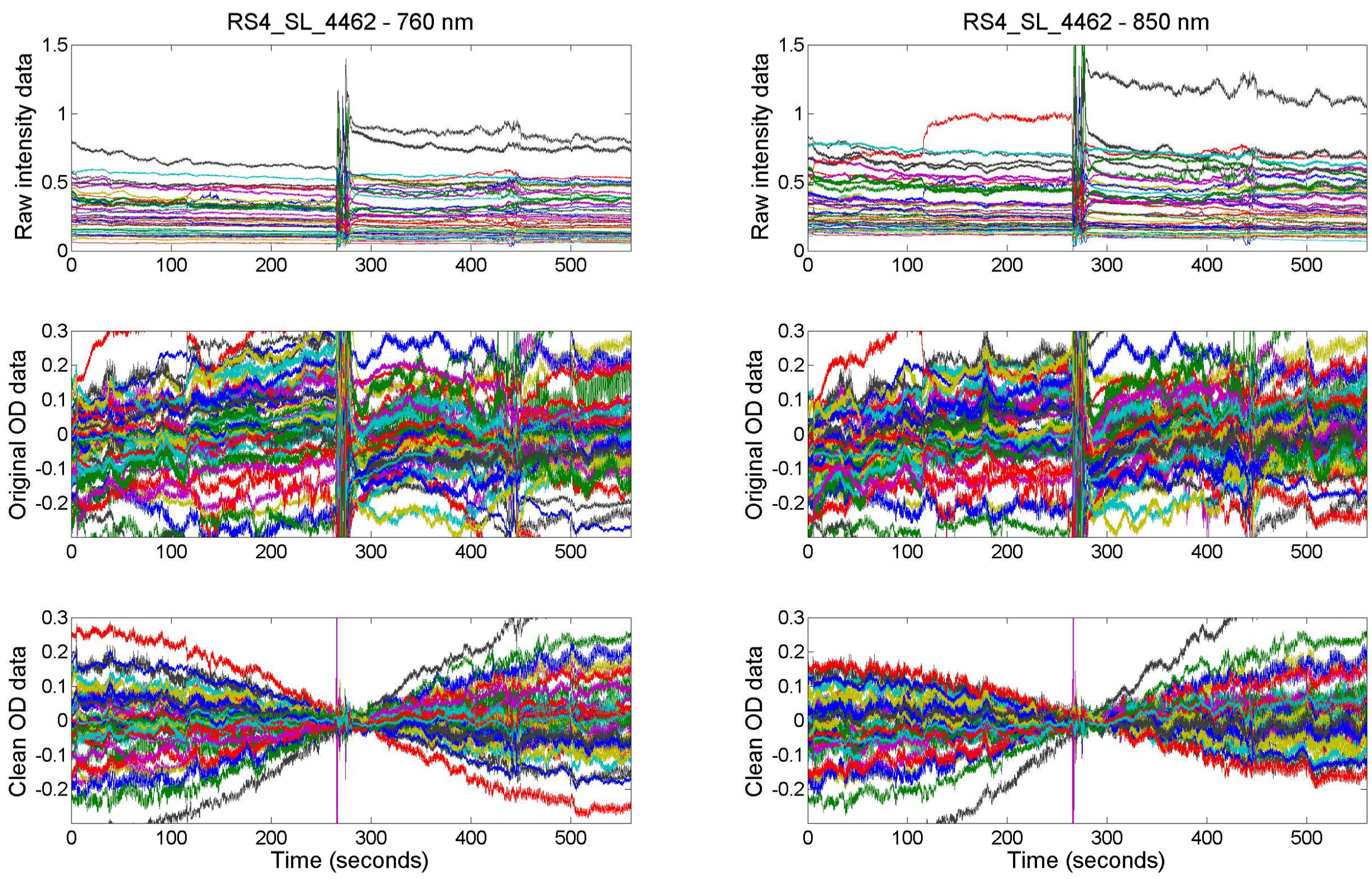

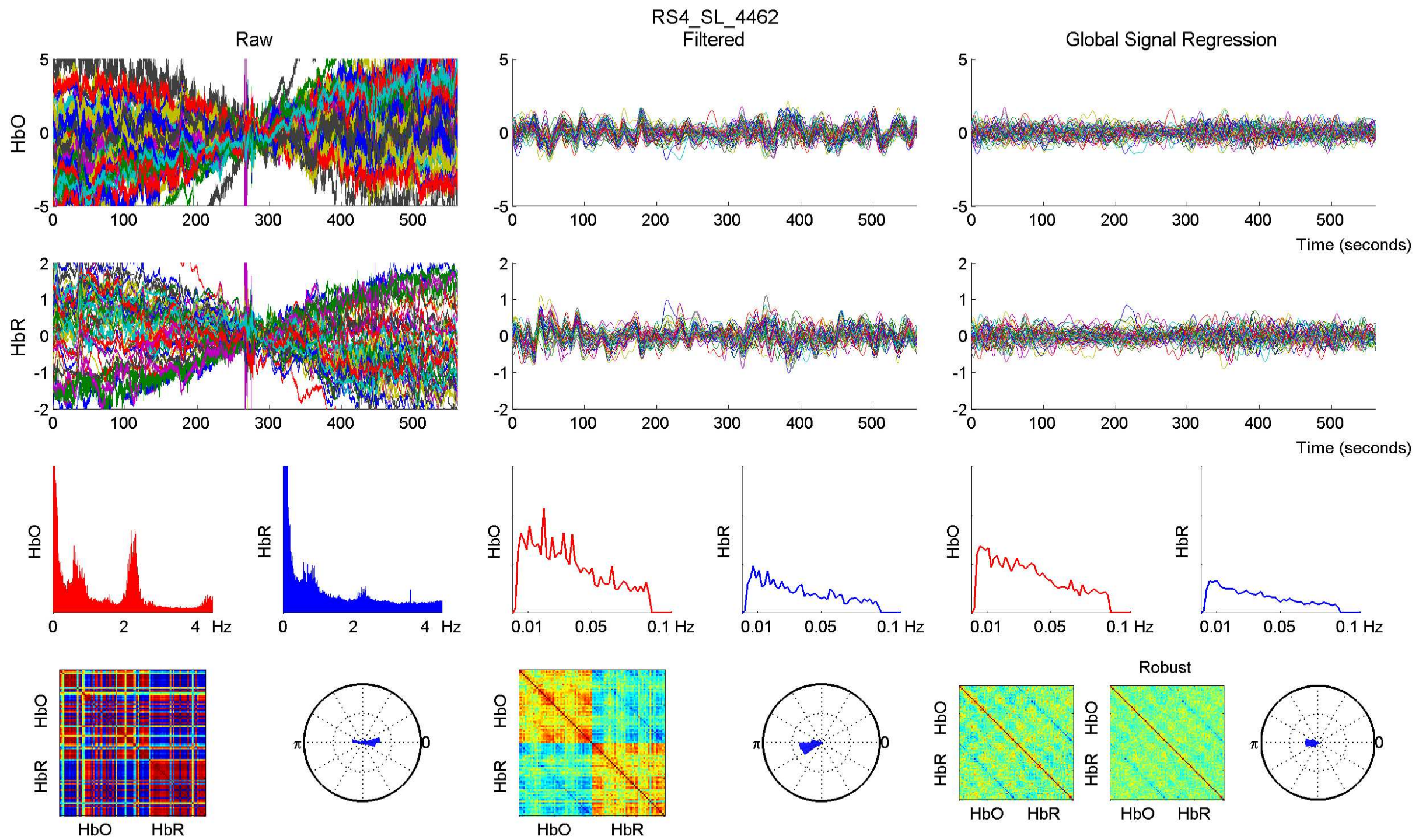
RS4 SL 4459 - 760 nm
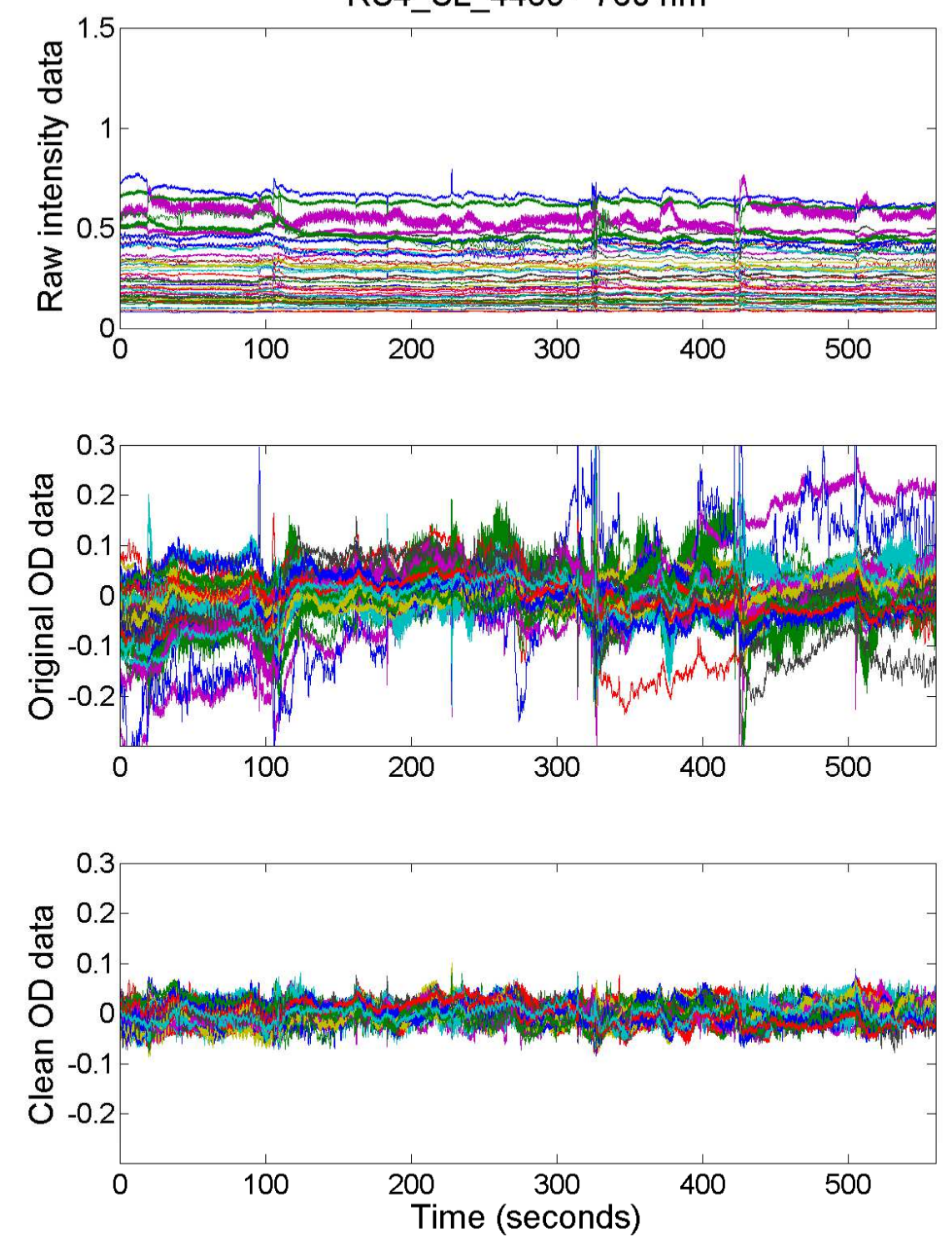

RS4 SL $4459-850 \mathrm{~nm}$
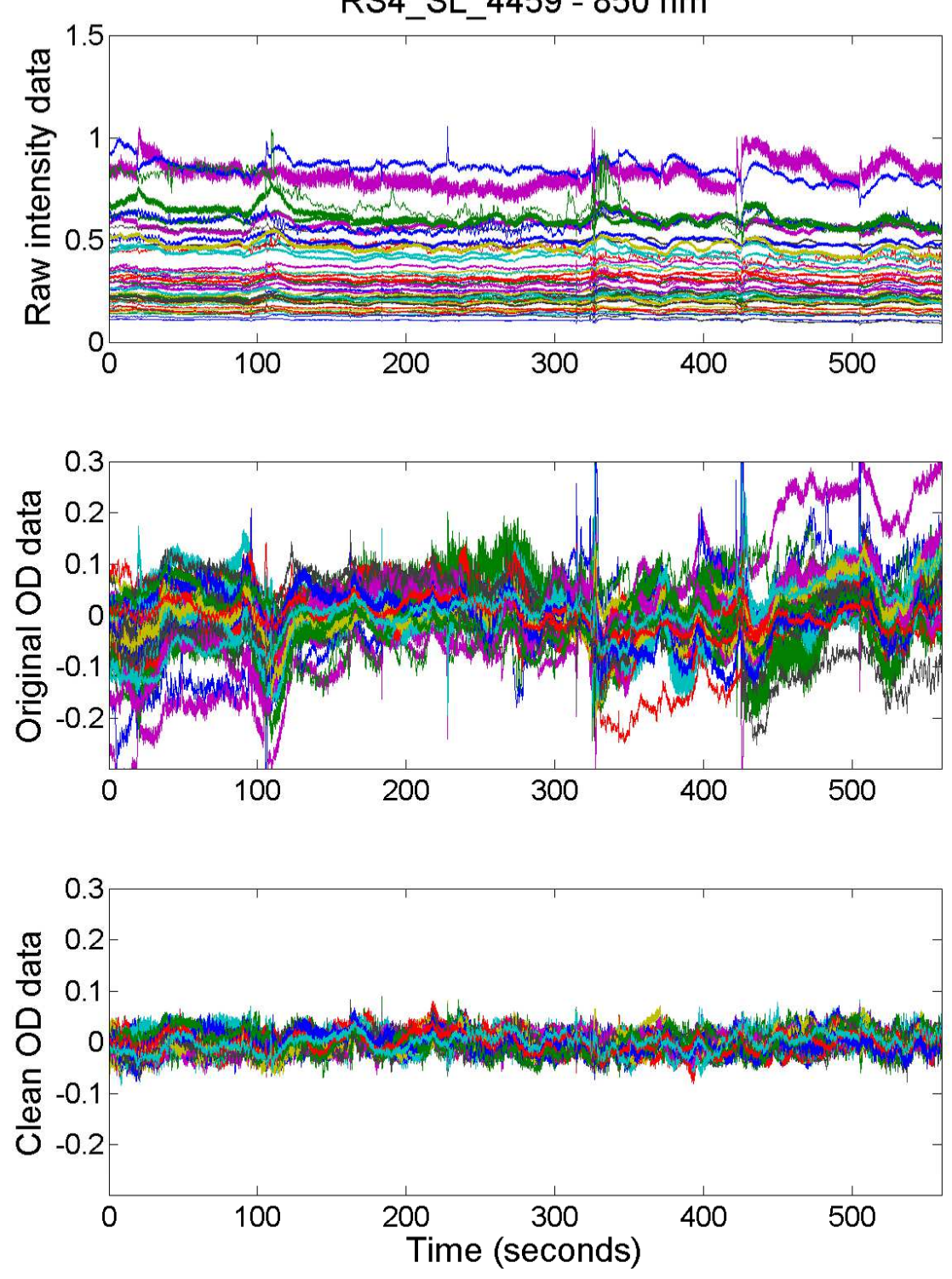
Raw
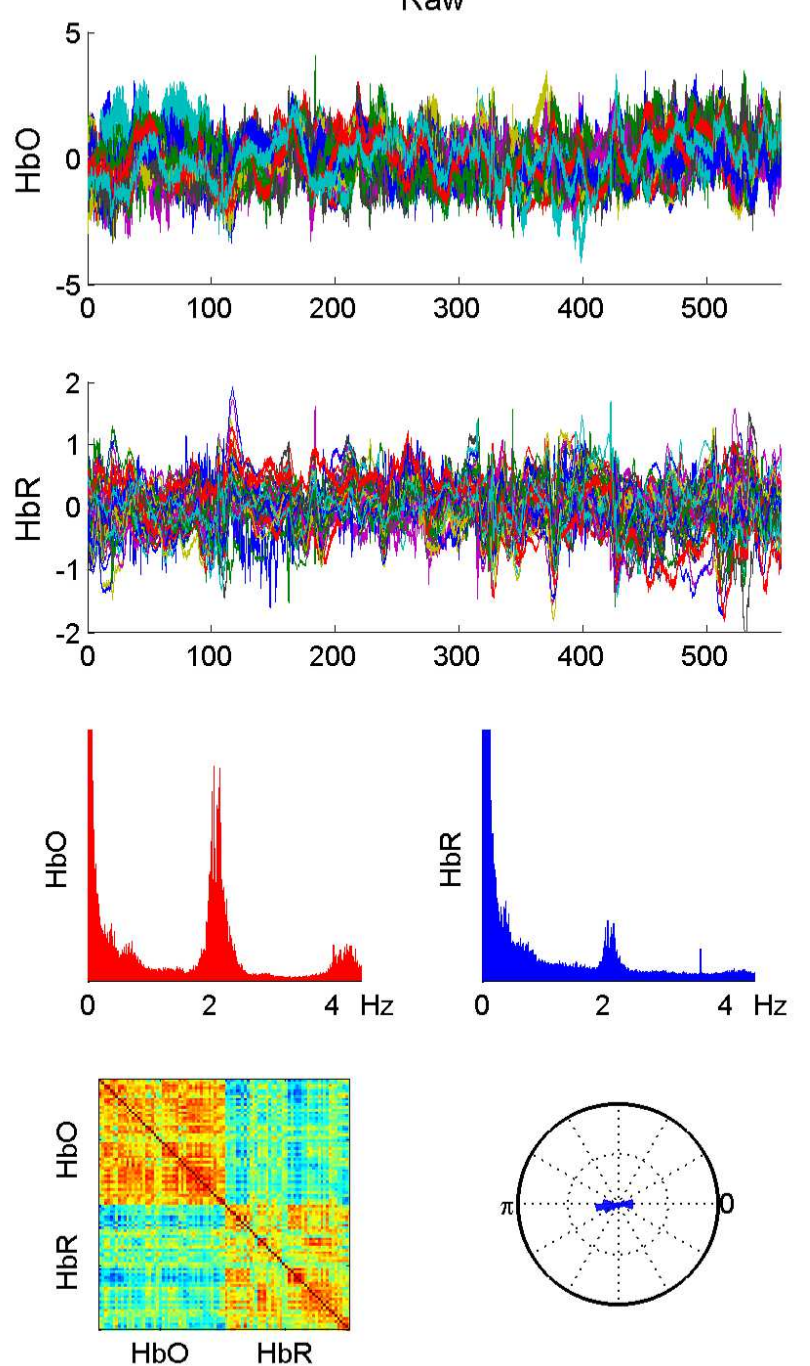

RS4_SL_4459

Filtered
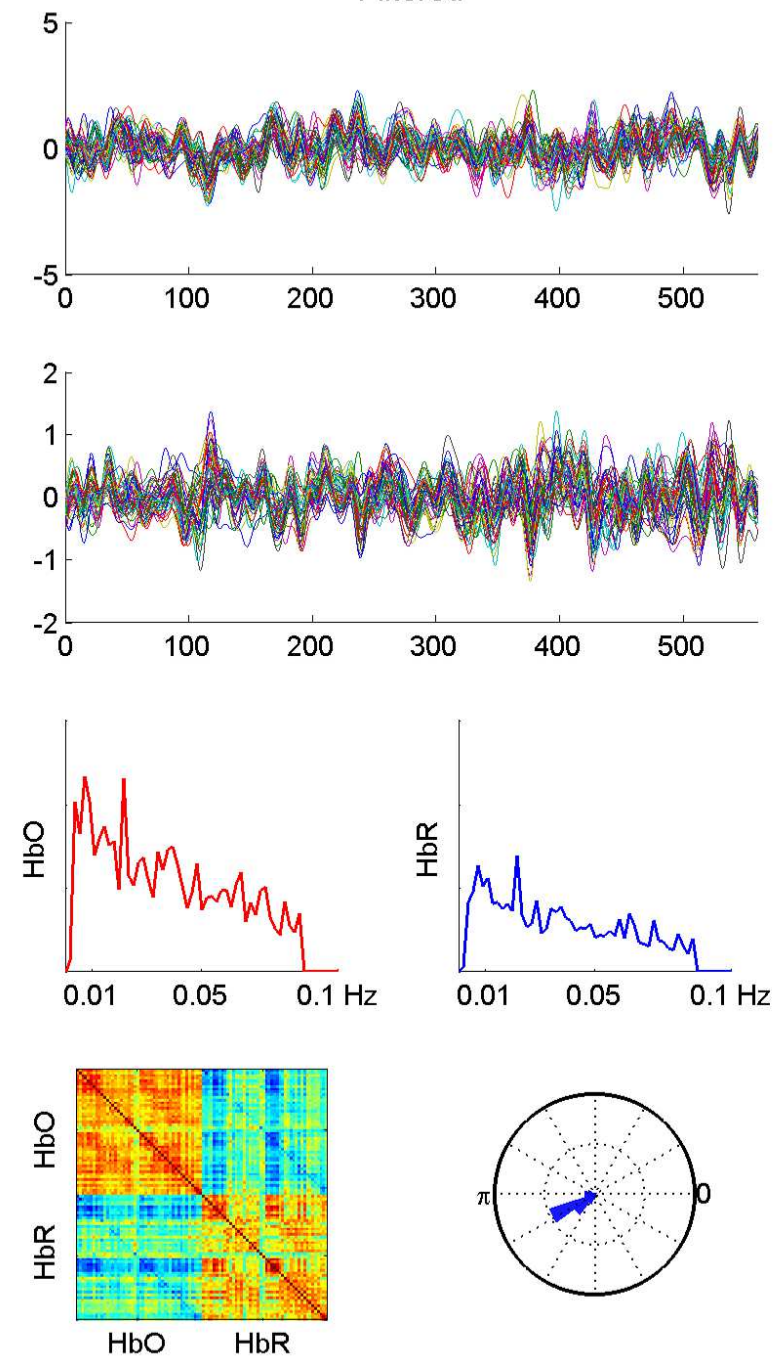

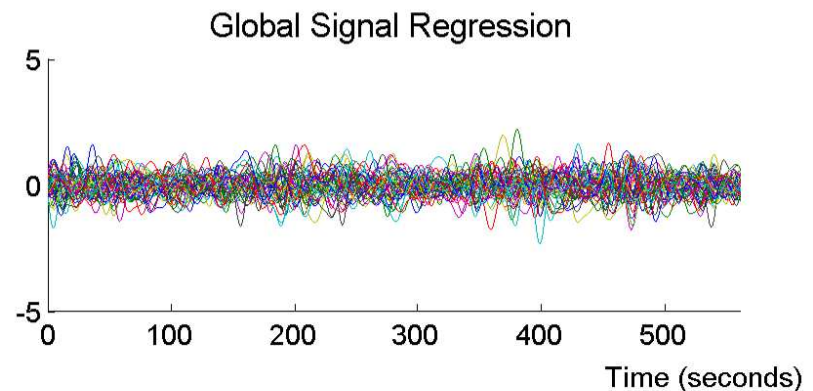

2
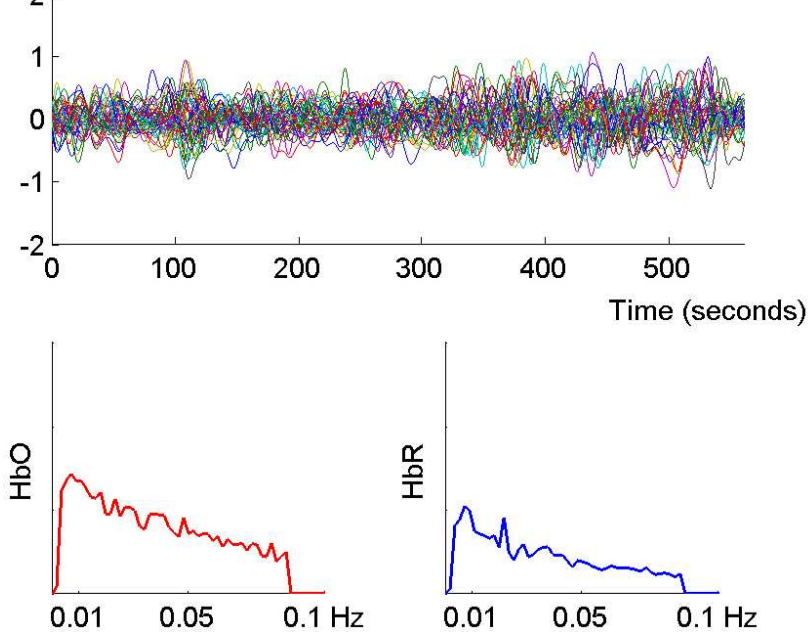
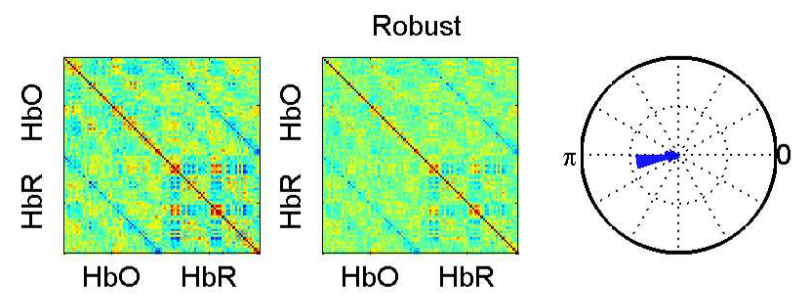
RS4 SL 4457 - 760 nm
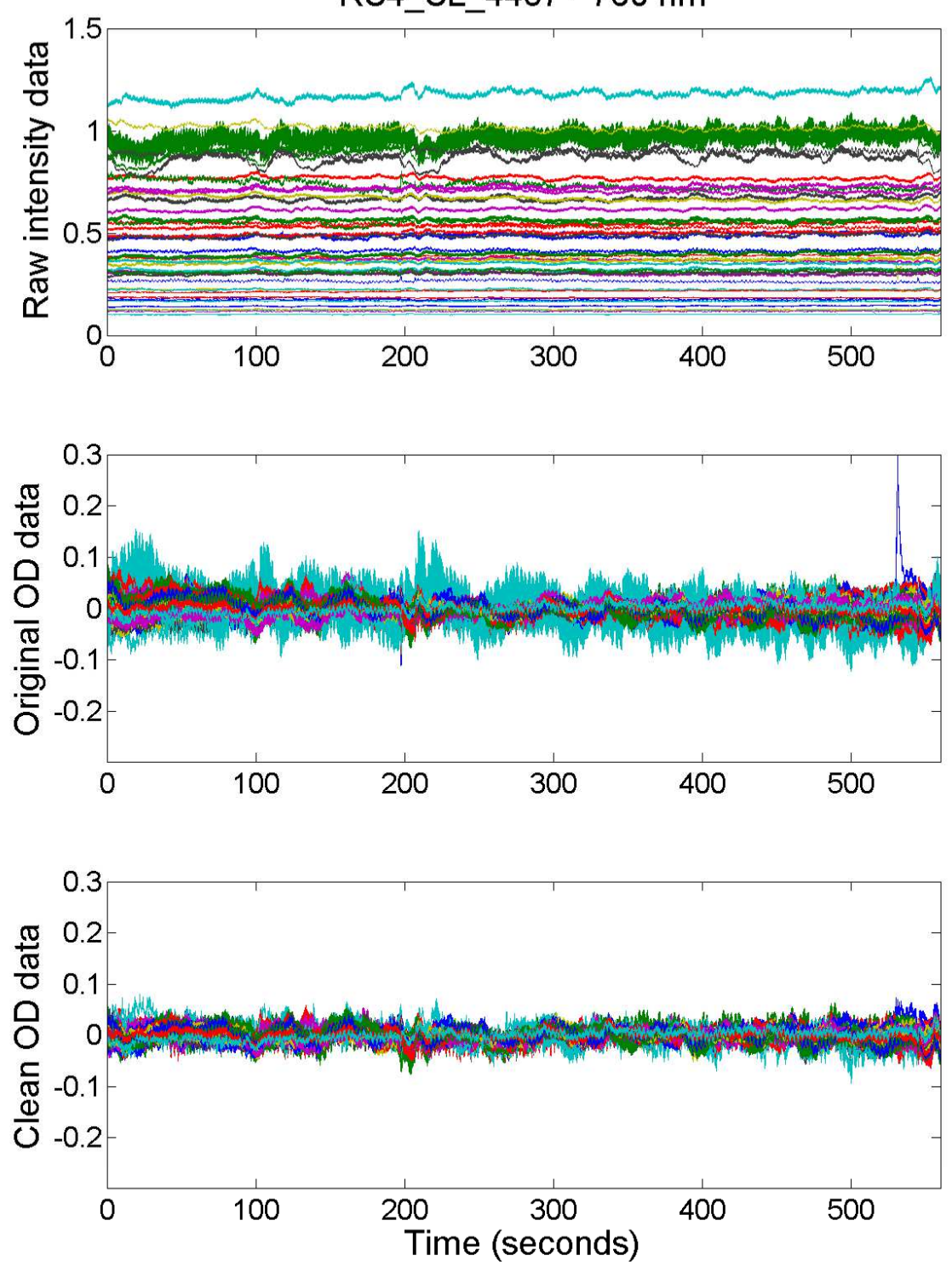

RS4 SL $4457-850 \mathrm{~nm}$
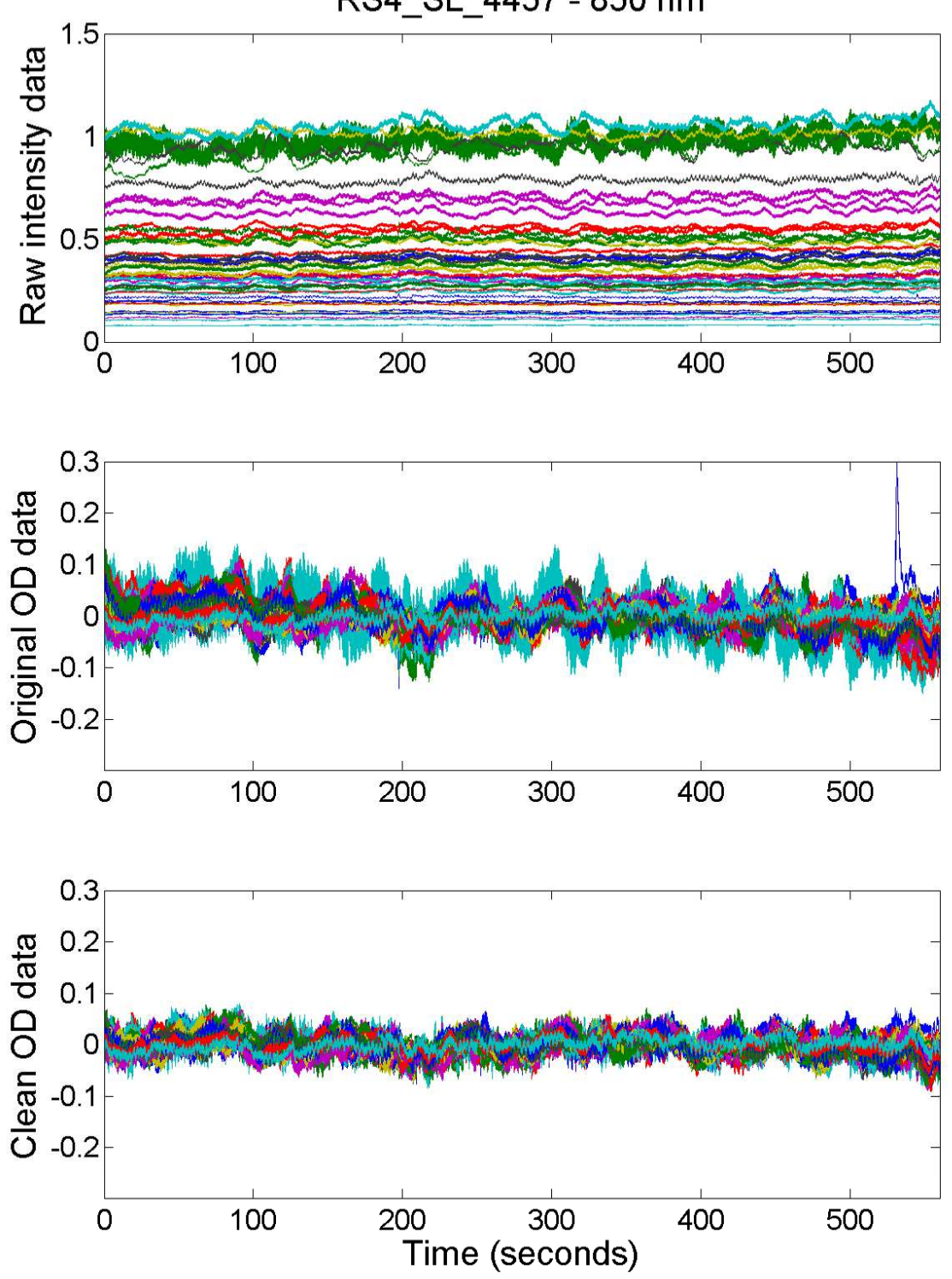
Raw

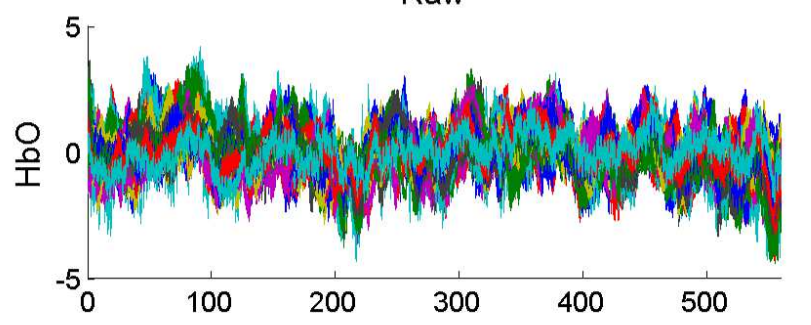

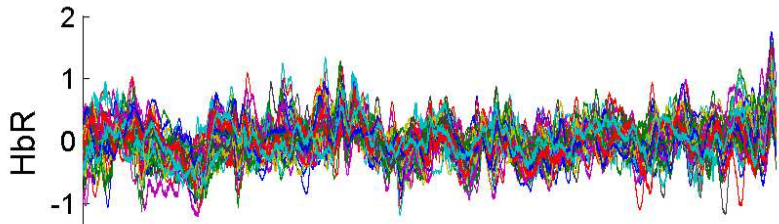
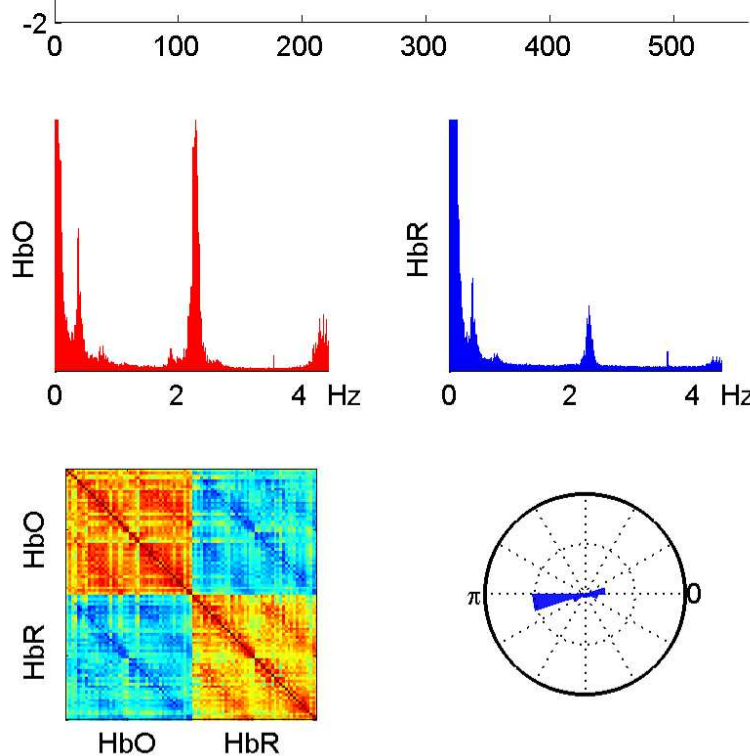

RS4 SL 4457

Filtered
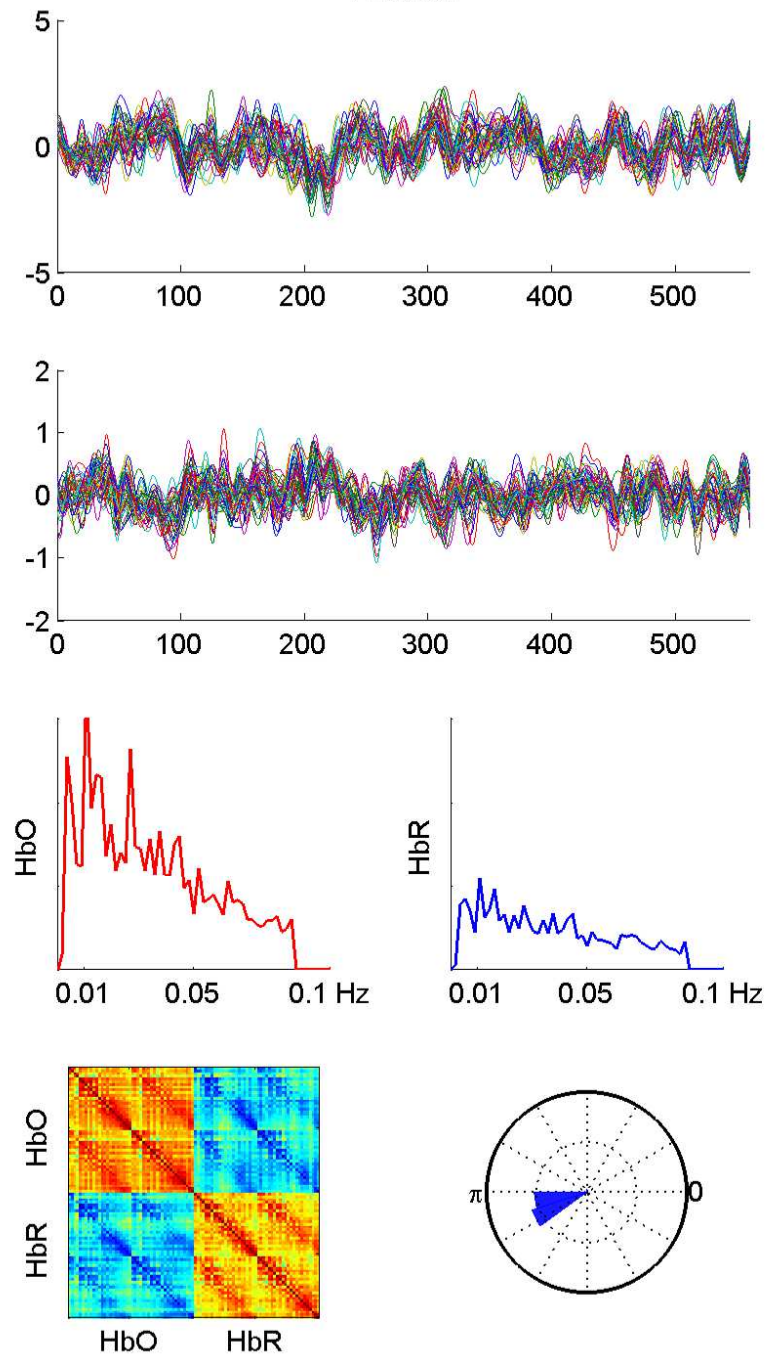

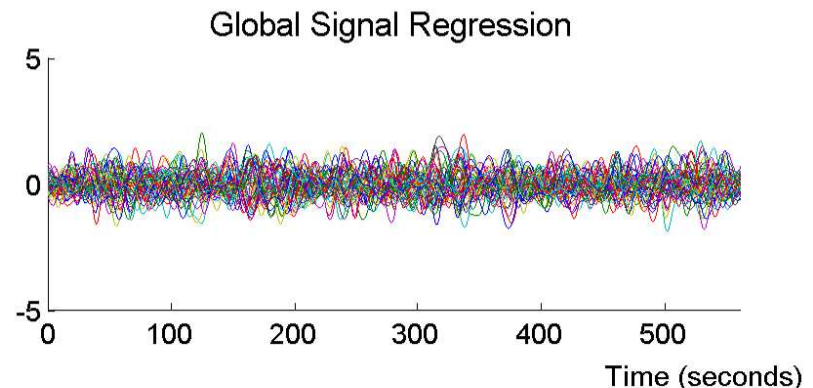

2
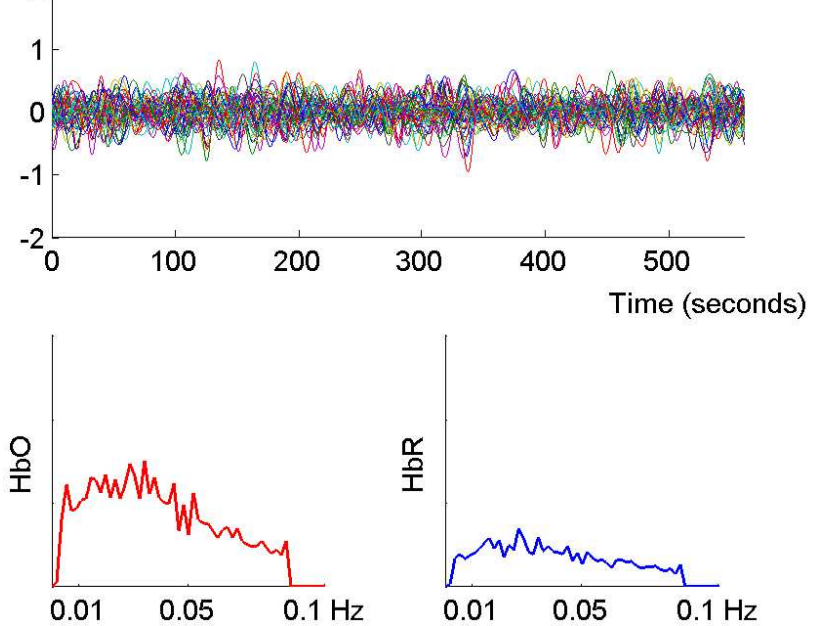

$\frac{\alpha}{1}$

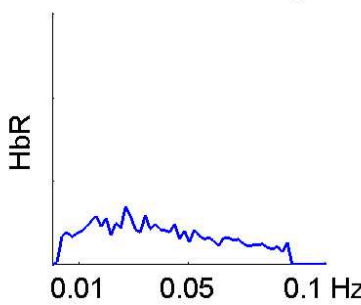

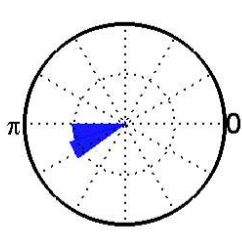

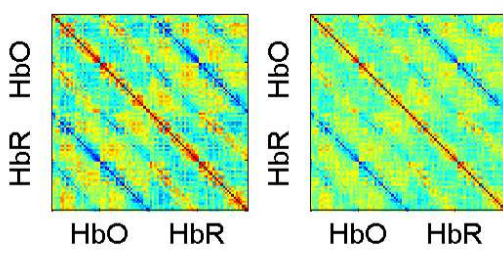

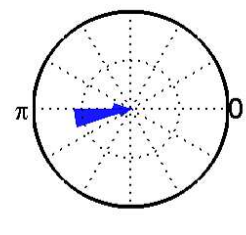



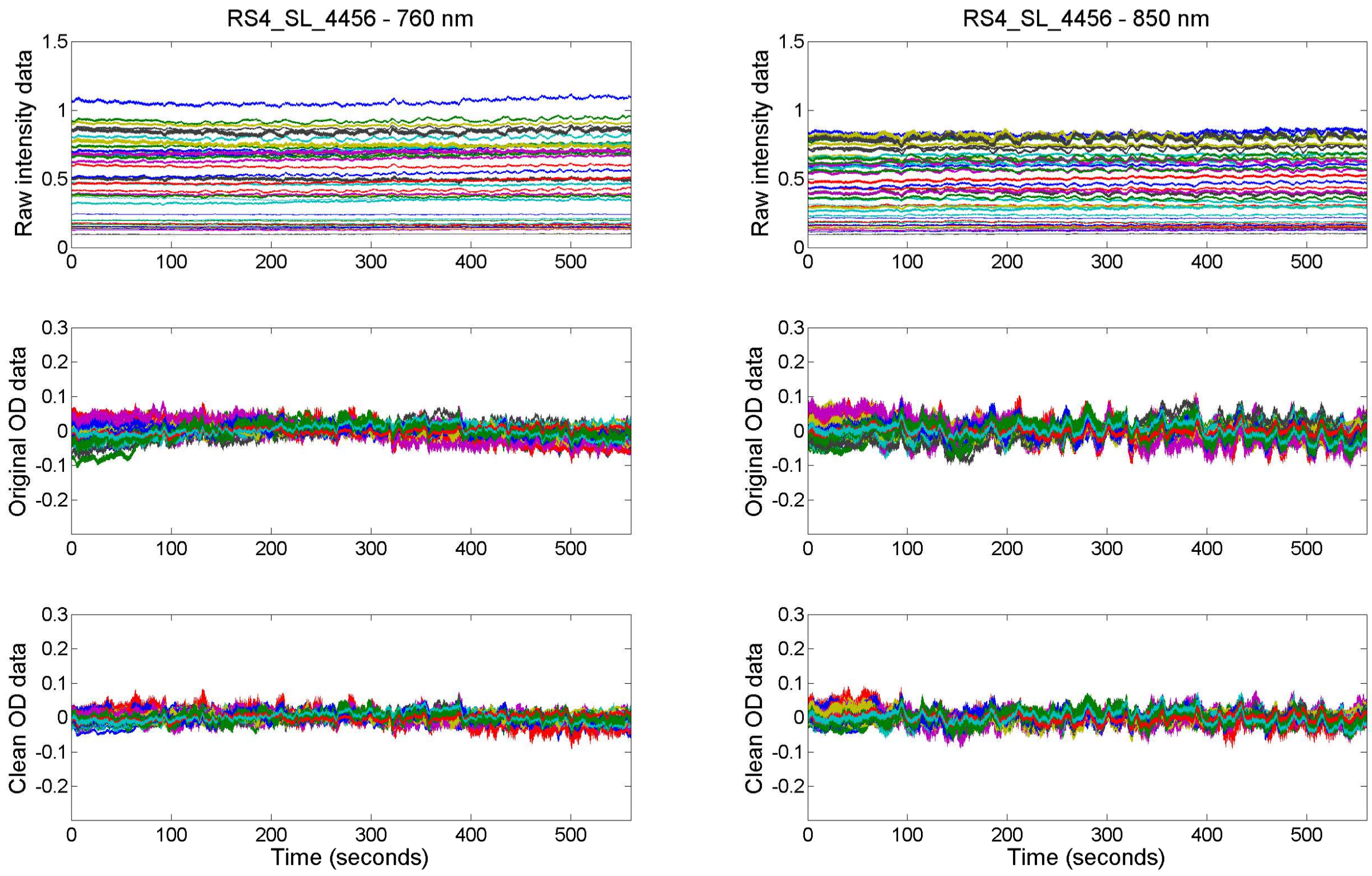
Raw

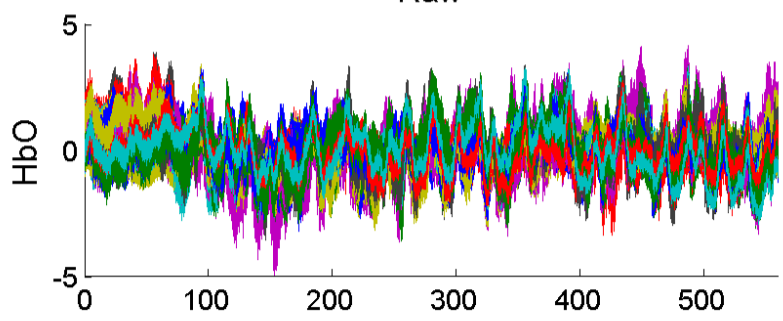
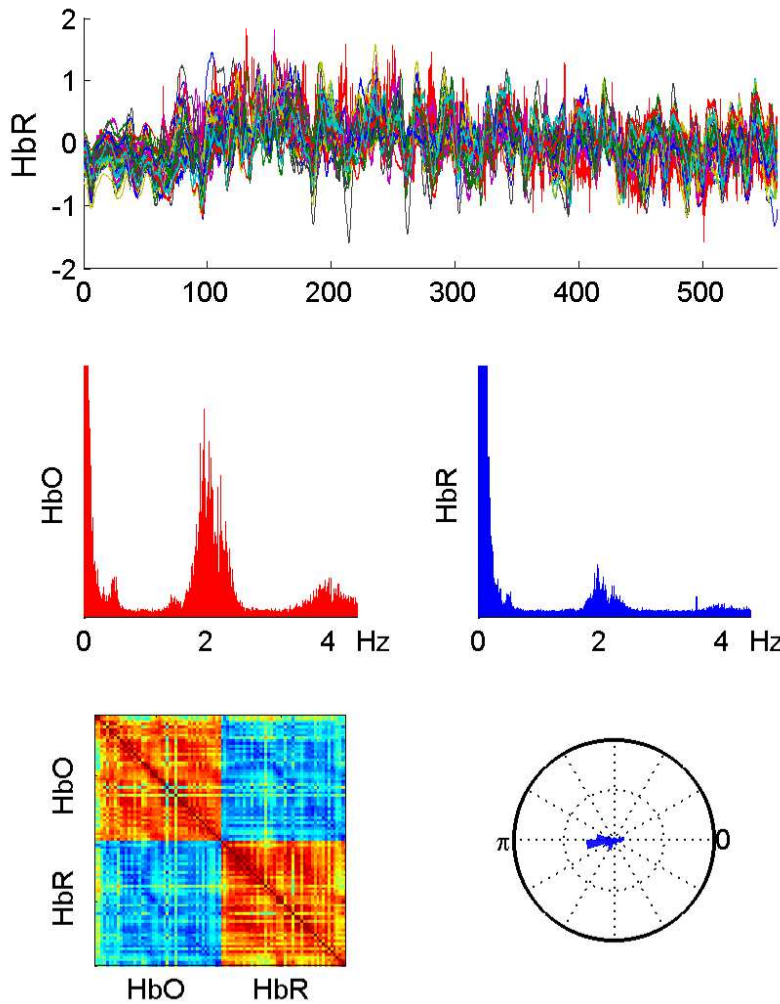

RS4_SL_4456

Filtered
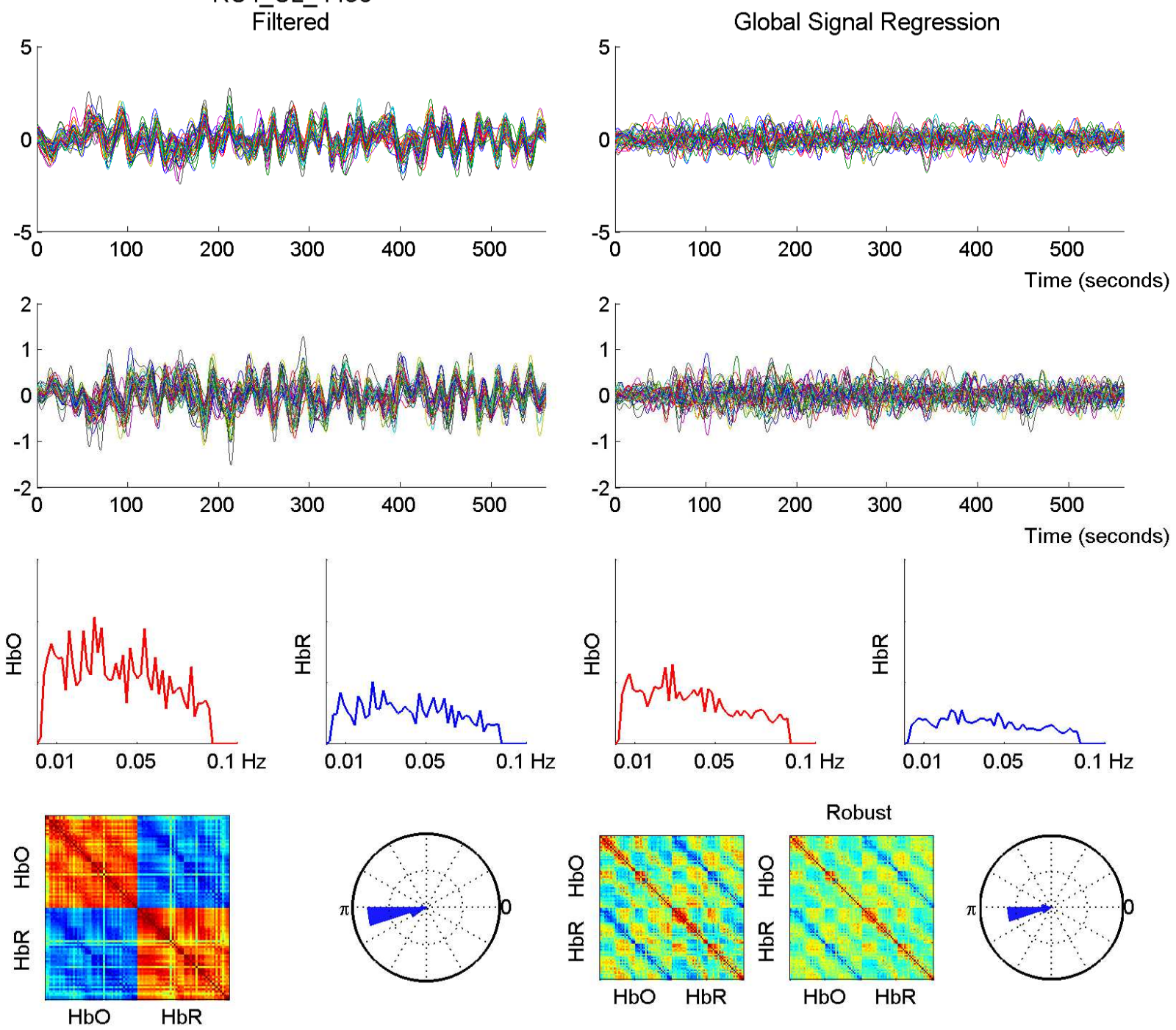

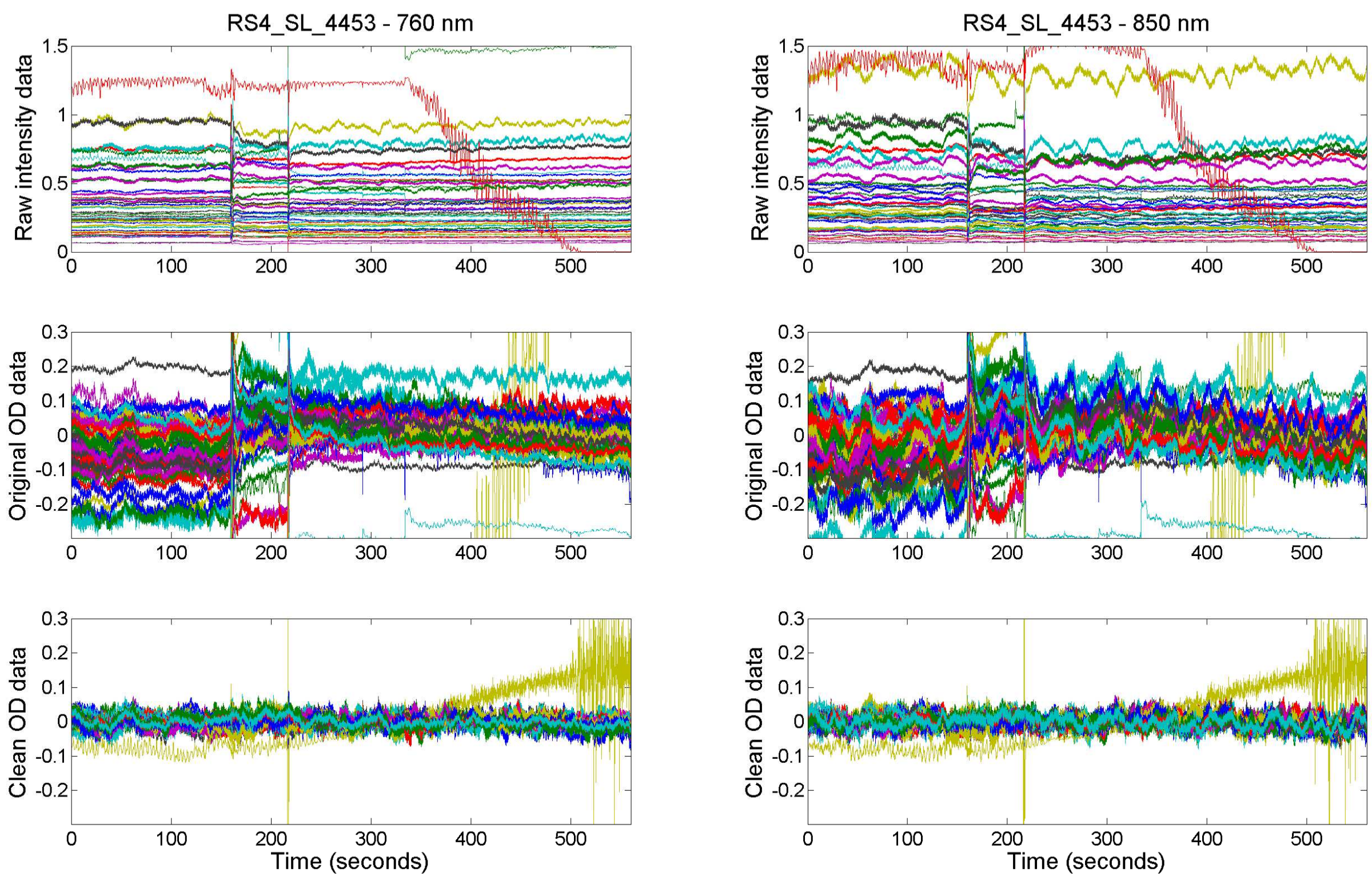
Raw
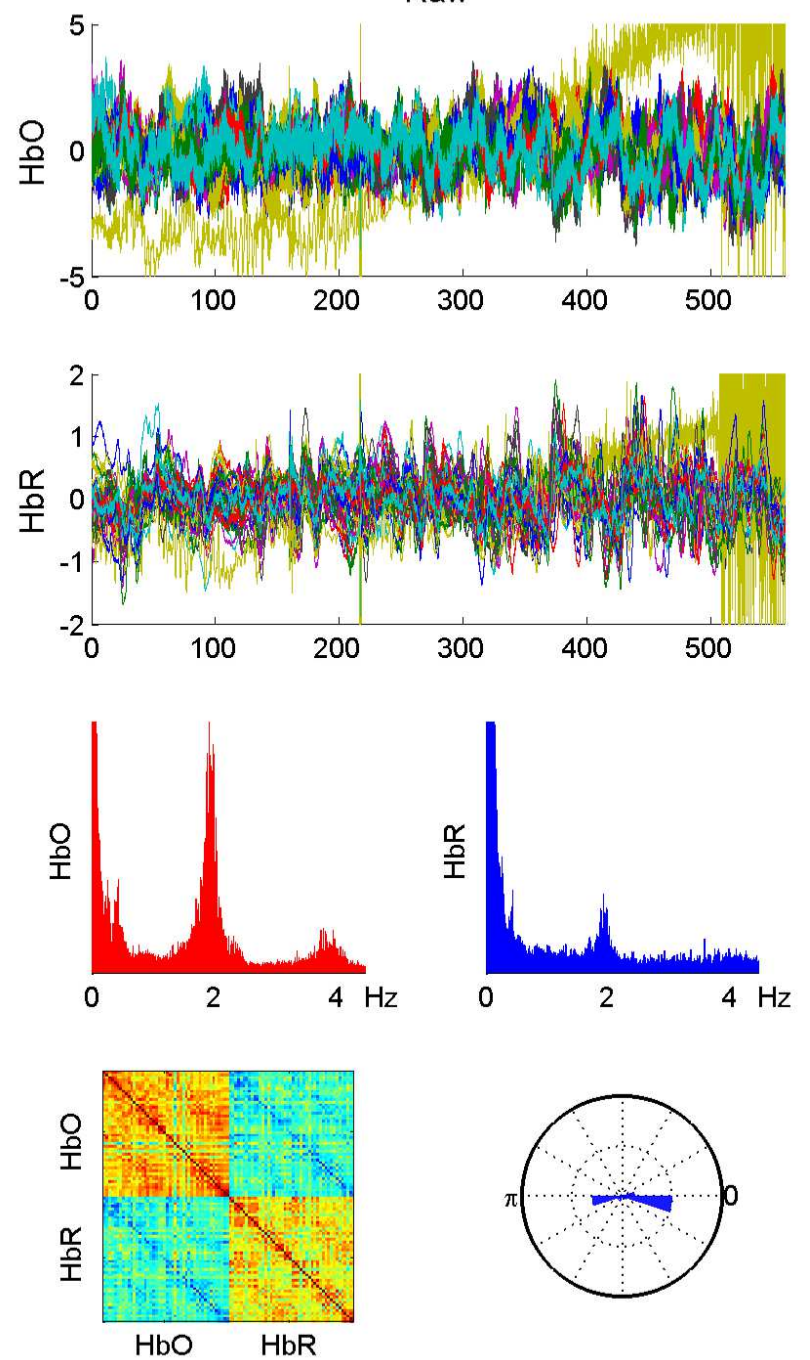

RS4_SL_4453

Filtered
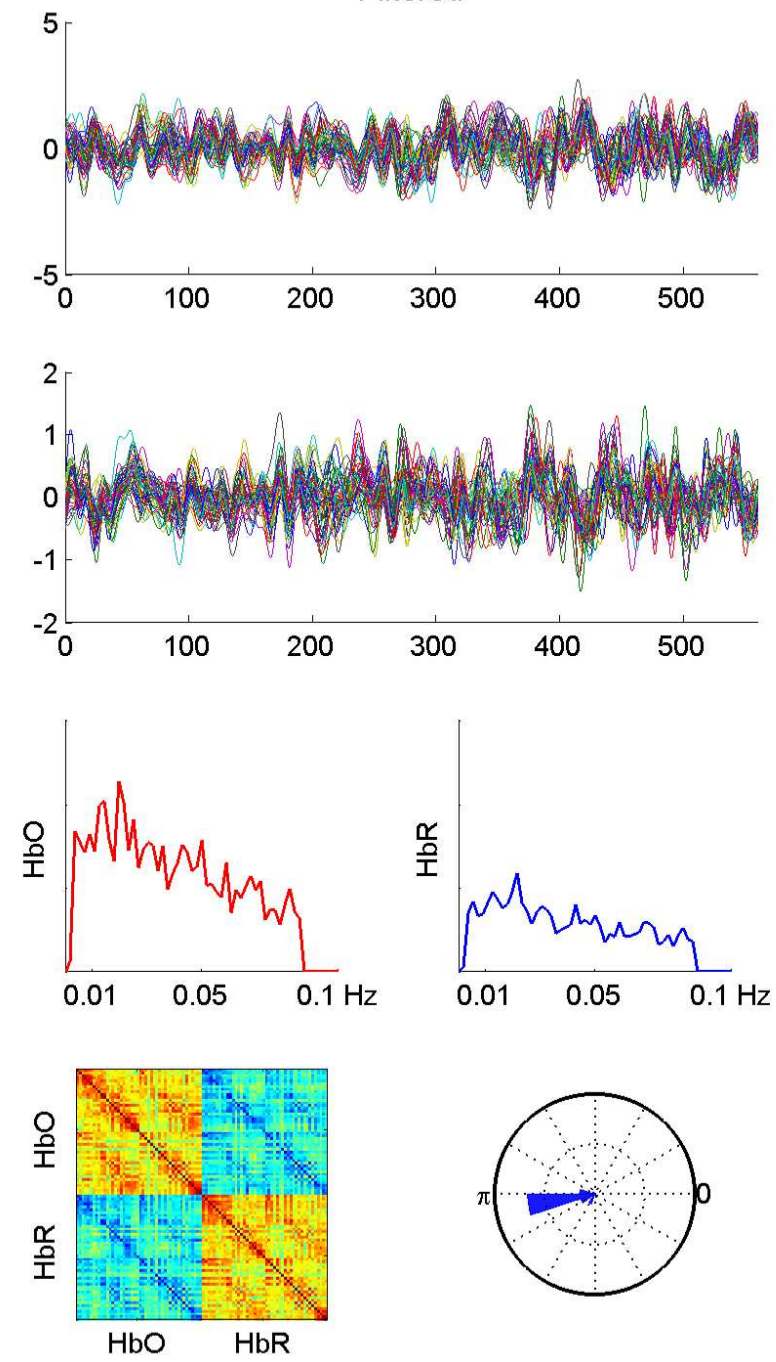

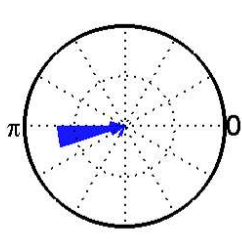

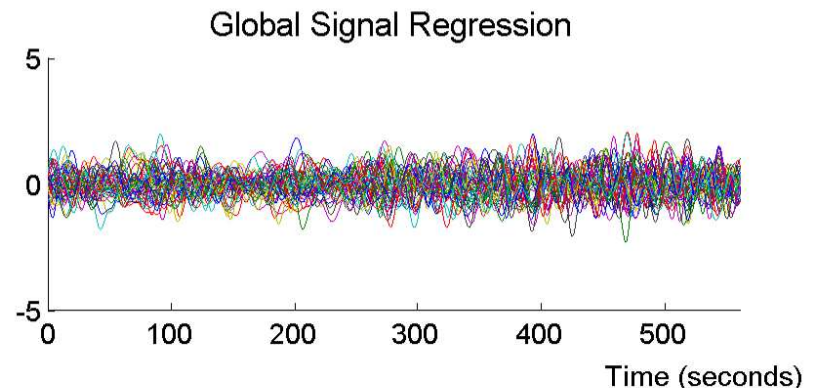

2
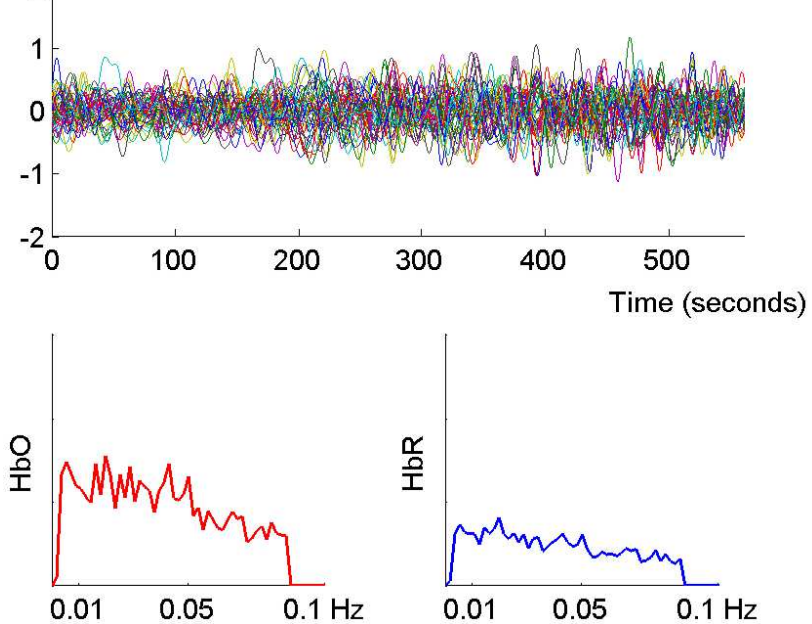
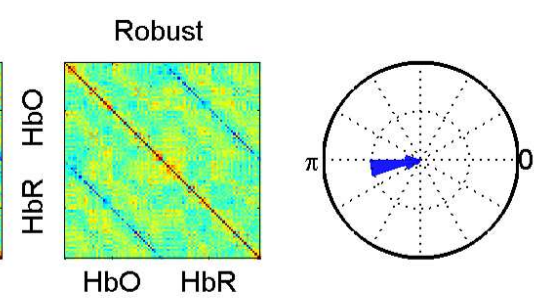

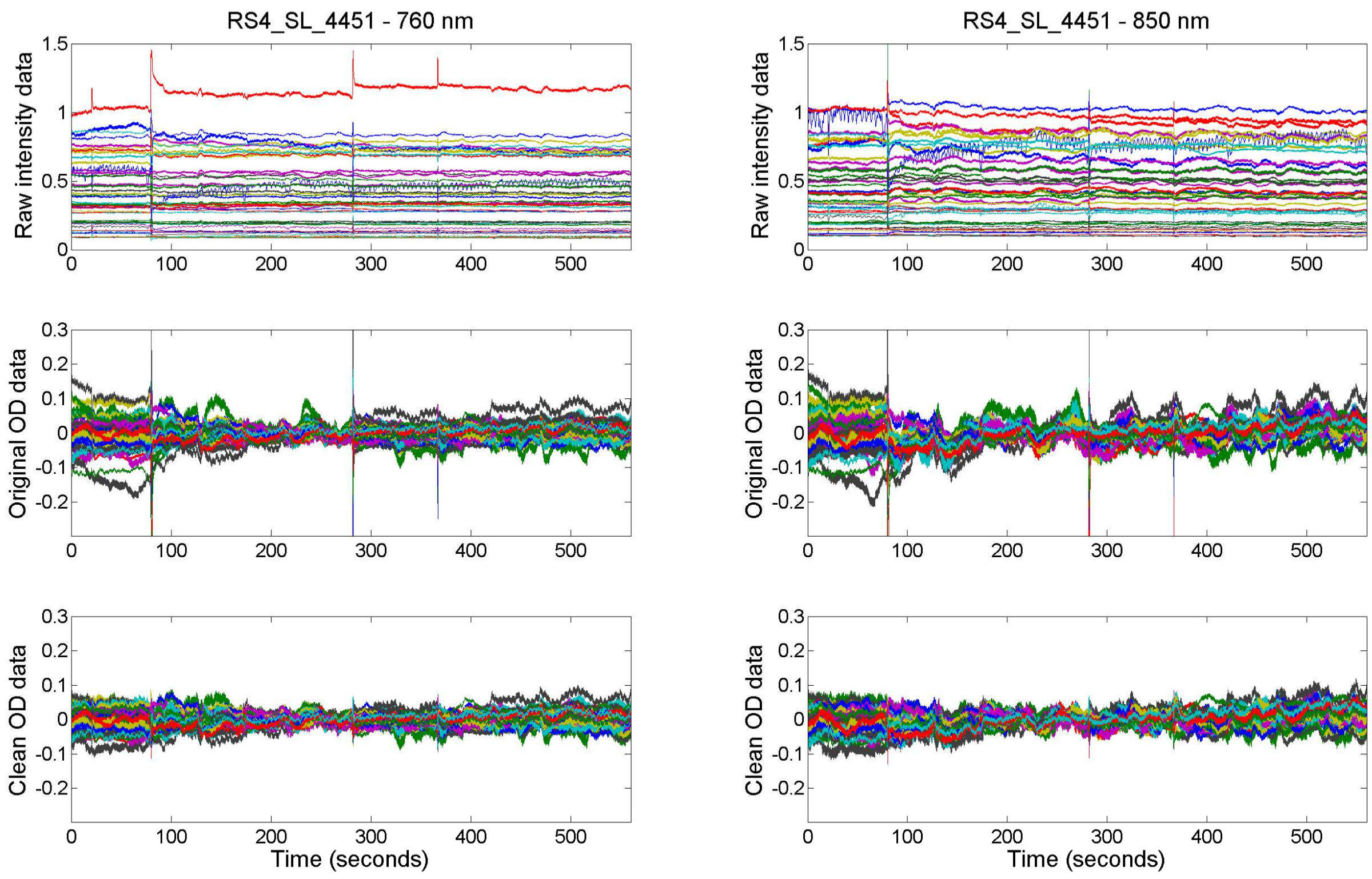
RS4 SL 4451

Raw
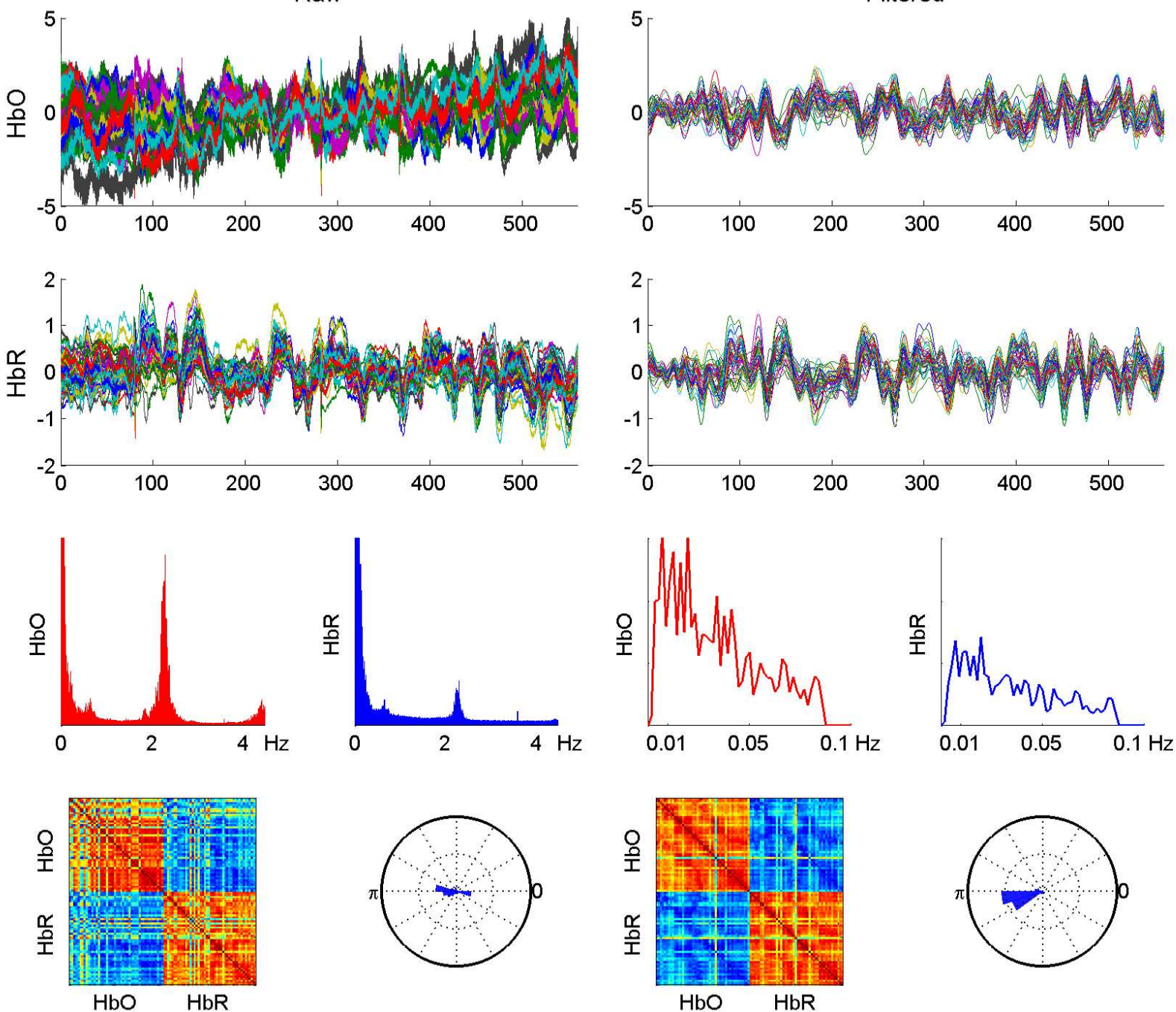
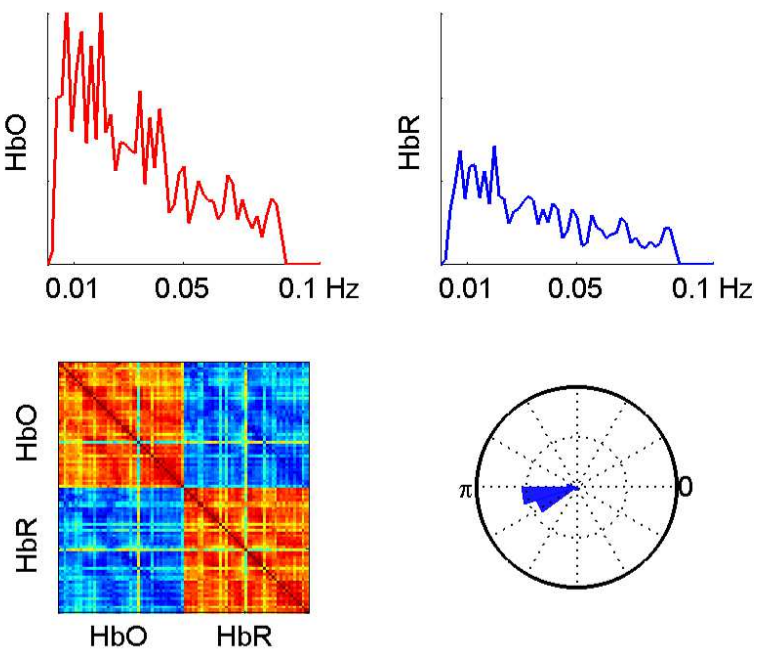

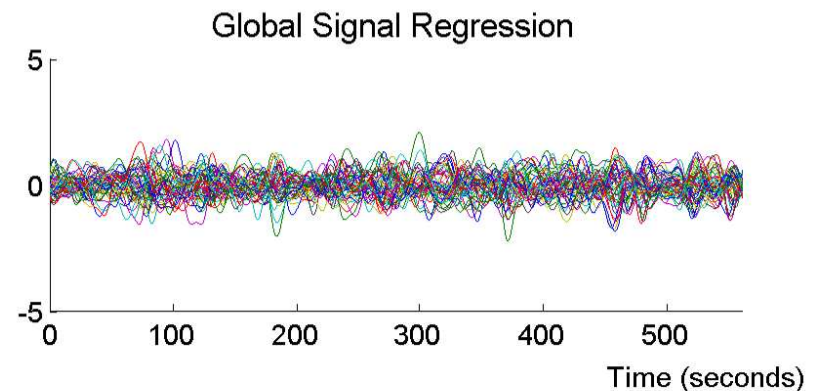

2
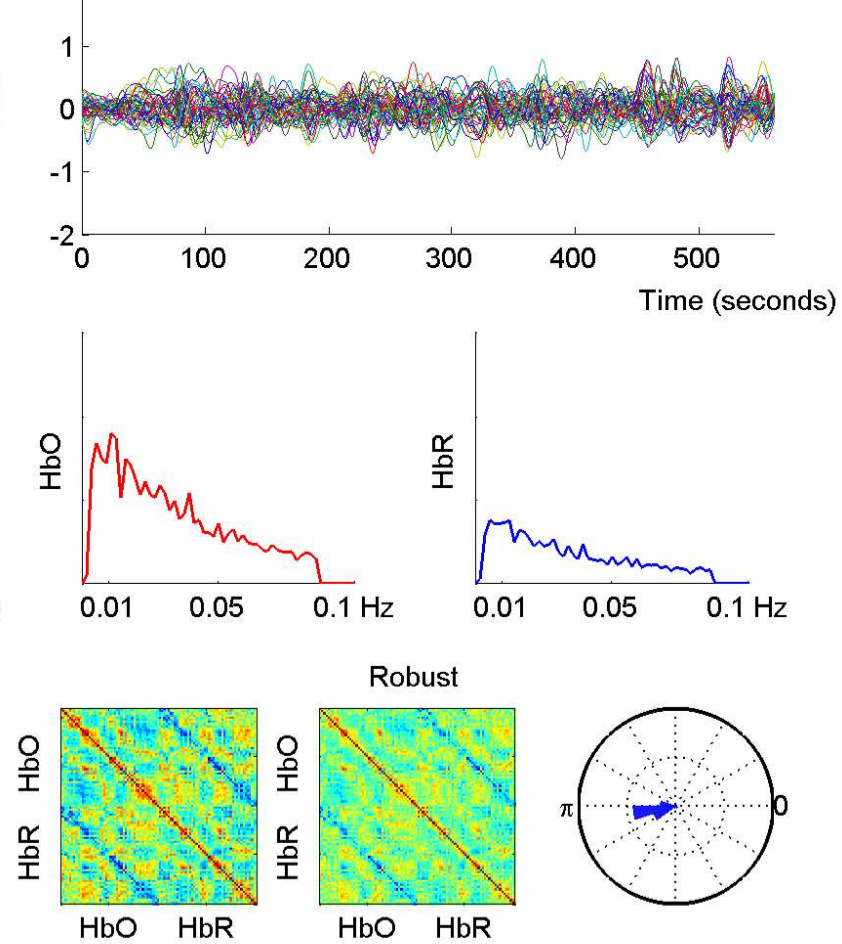

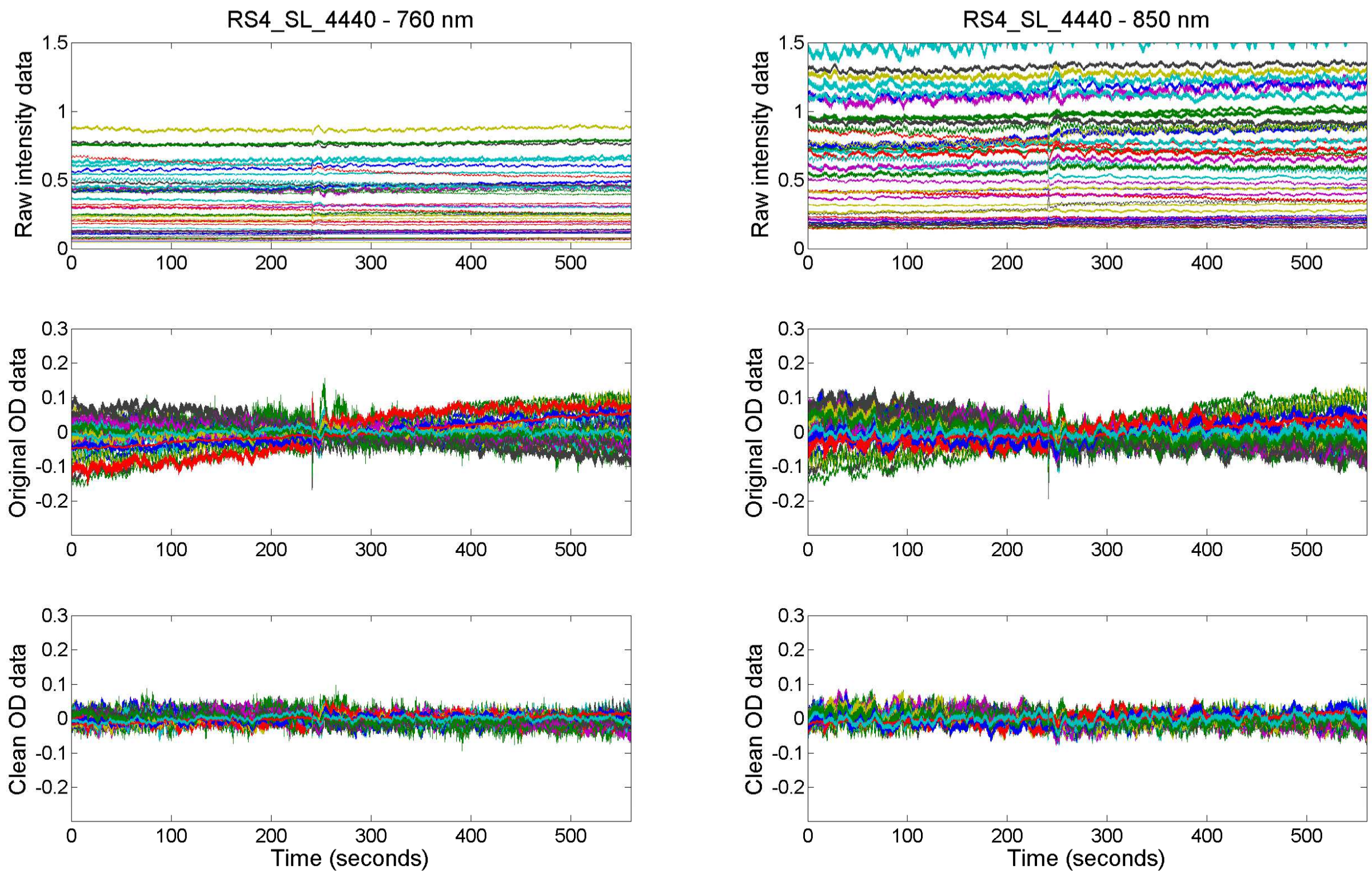
Raw
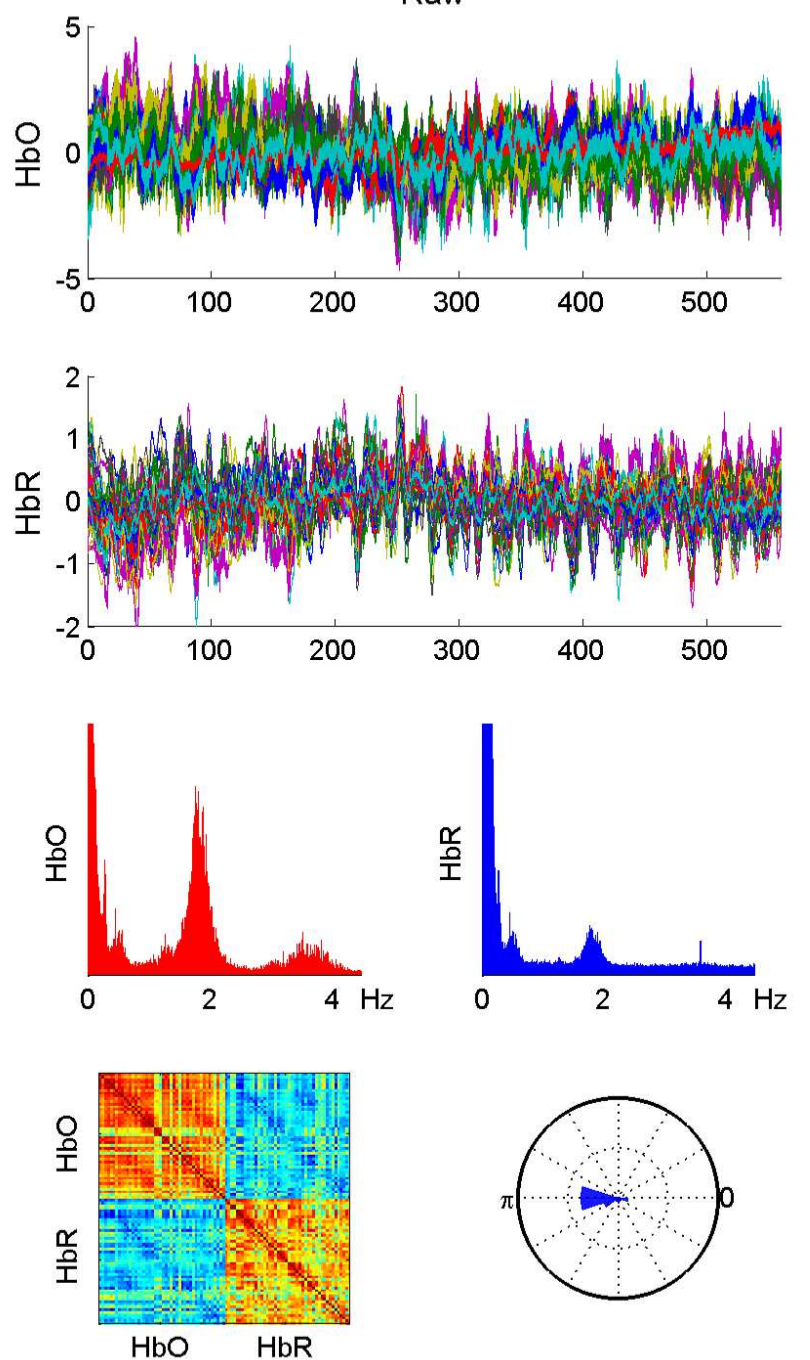

RS4 SL 4440

Filtered
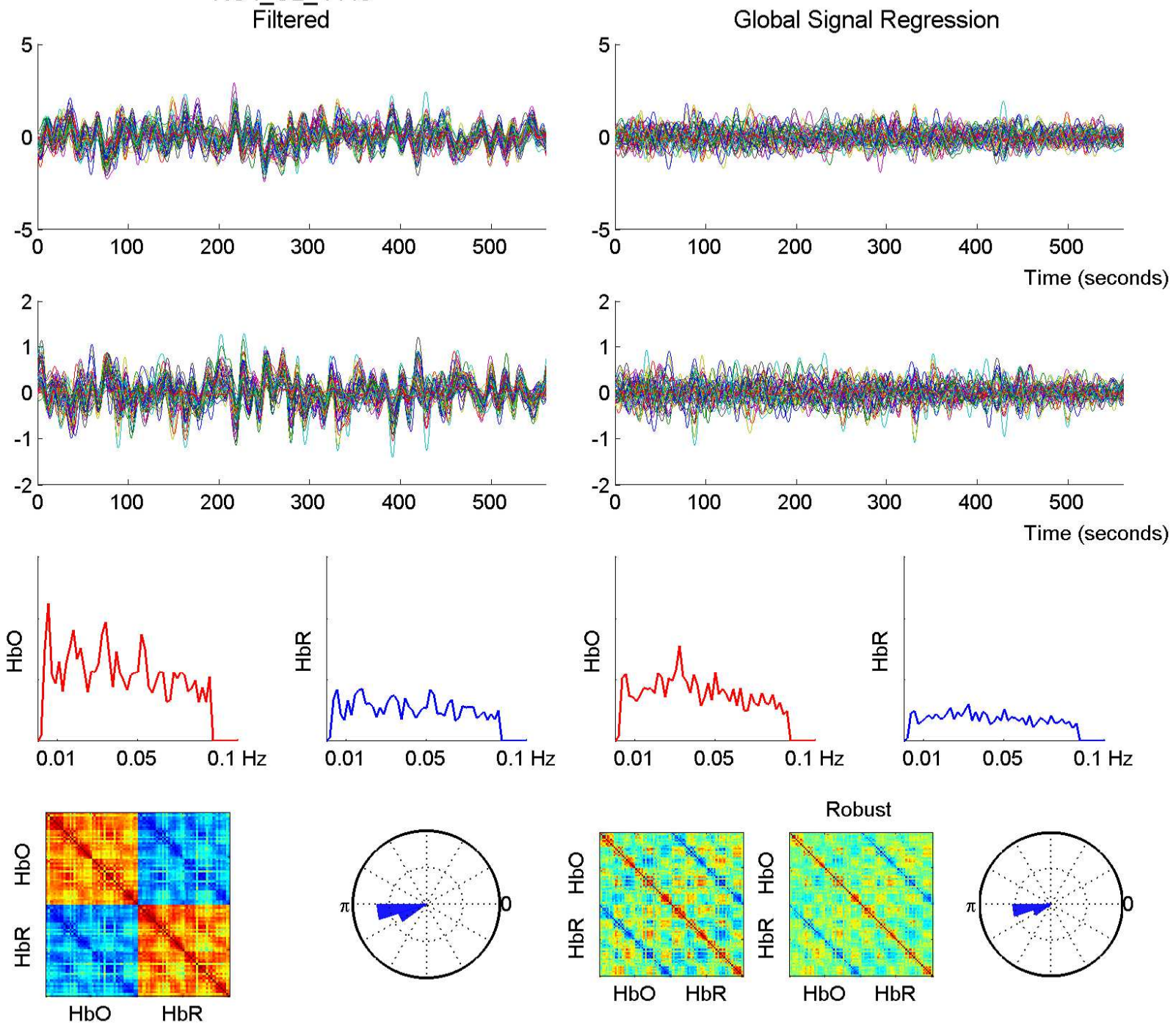
RS4 SL 4437 - 760 nm
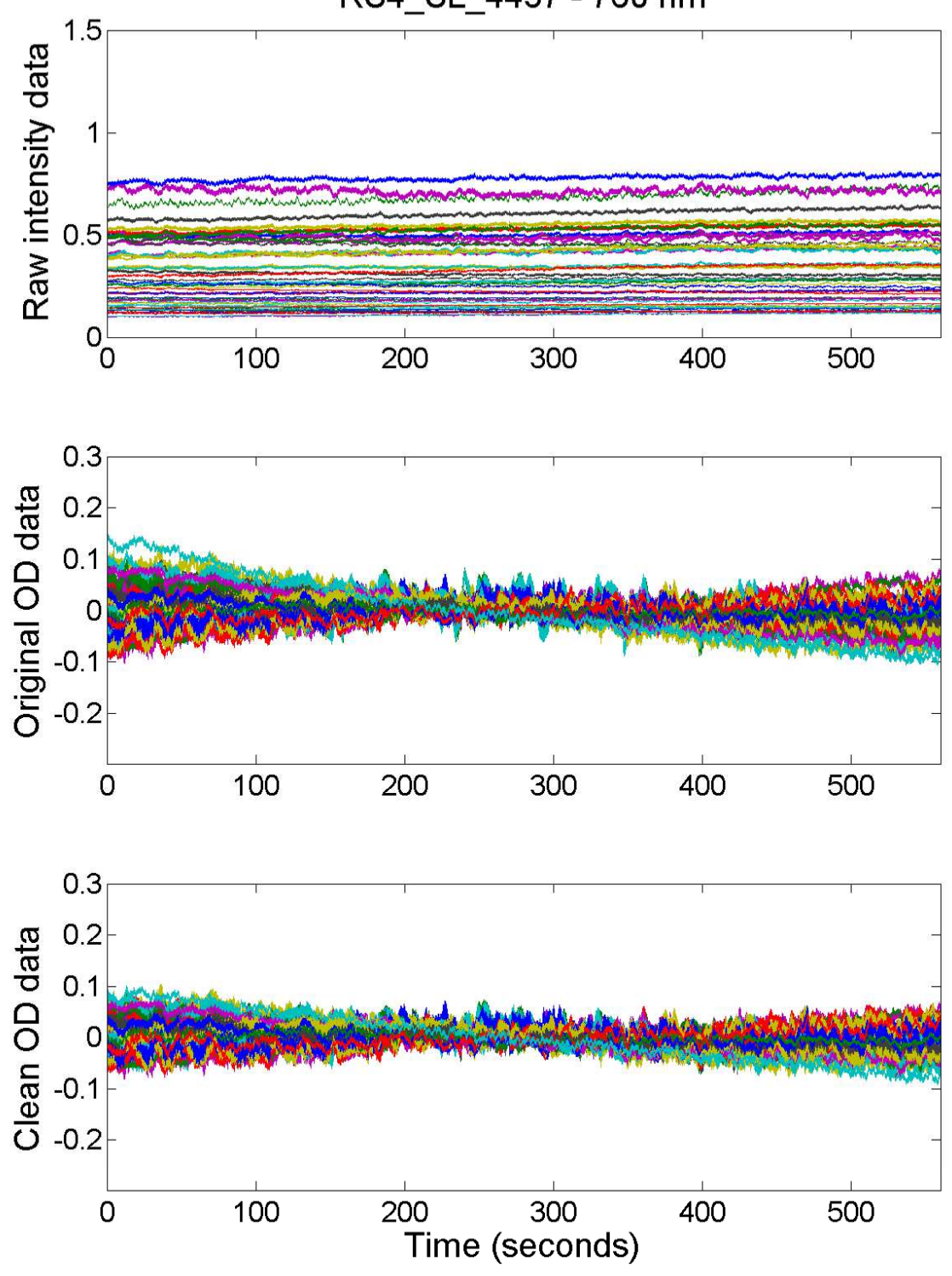

RS4 SL $4437-850 \mathrm{~nm}$
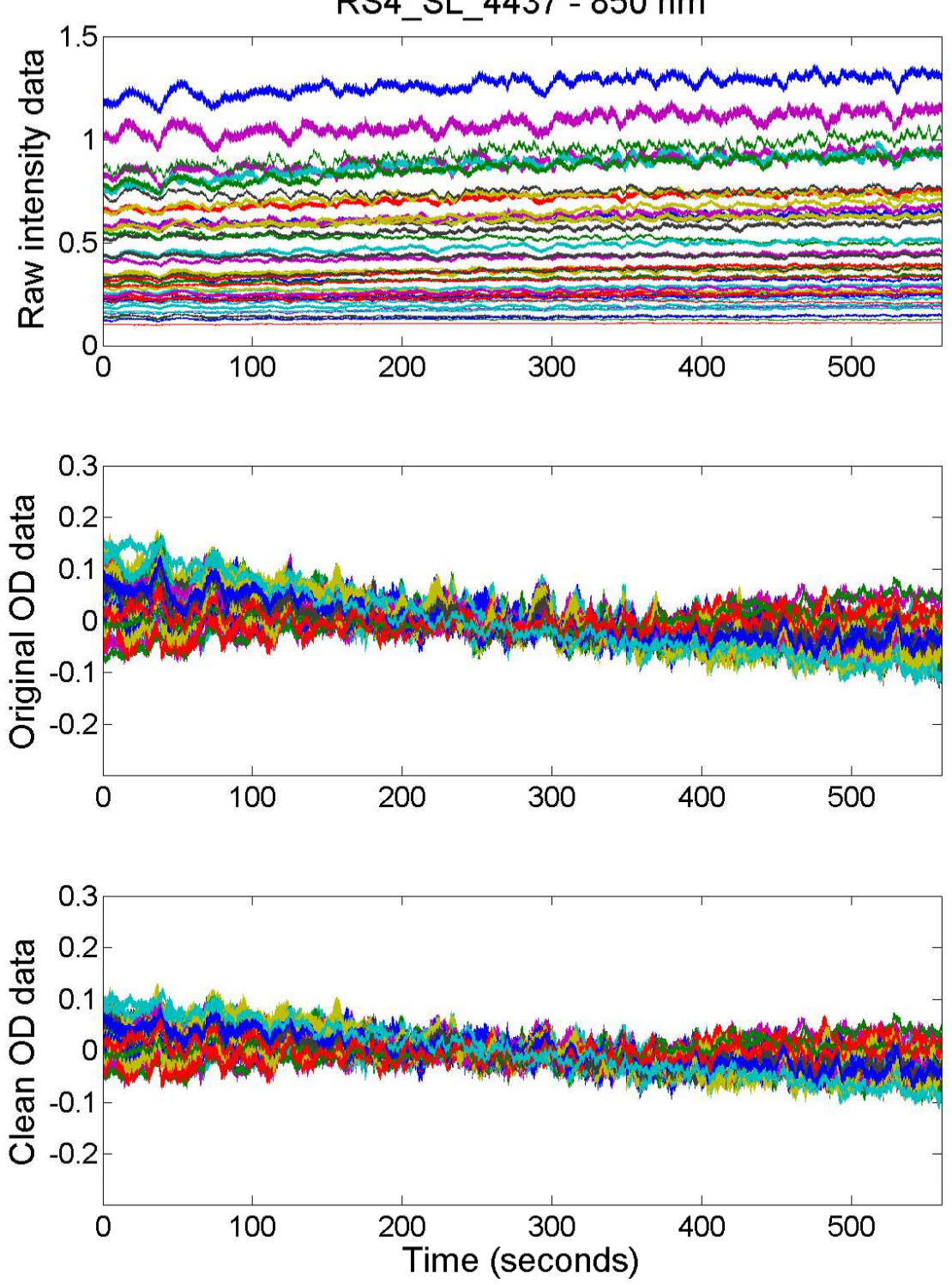
Raw

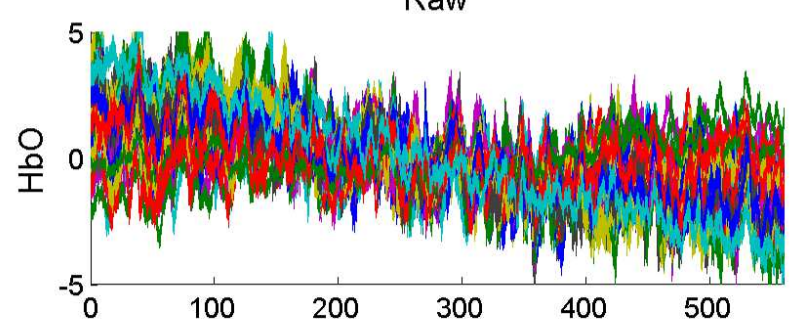

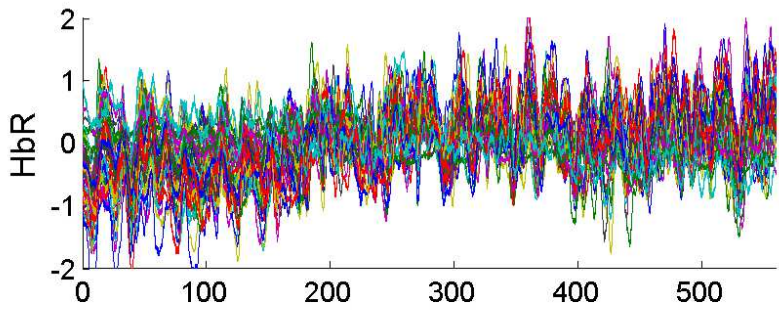
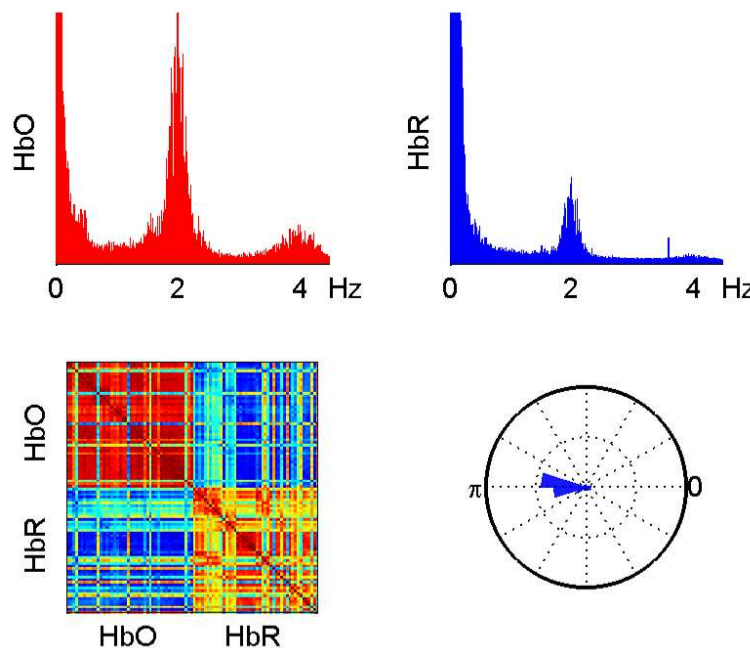

RS4_SL_4437

Filtered
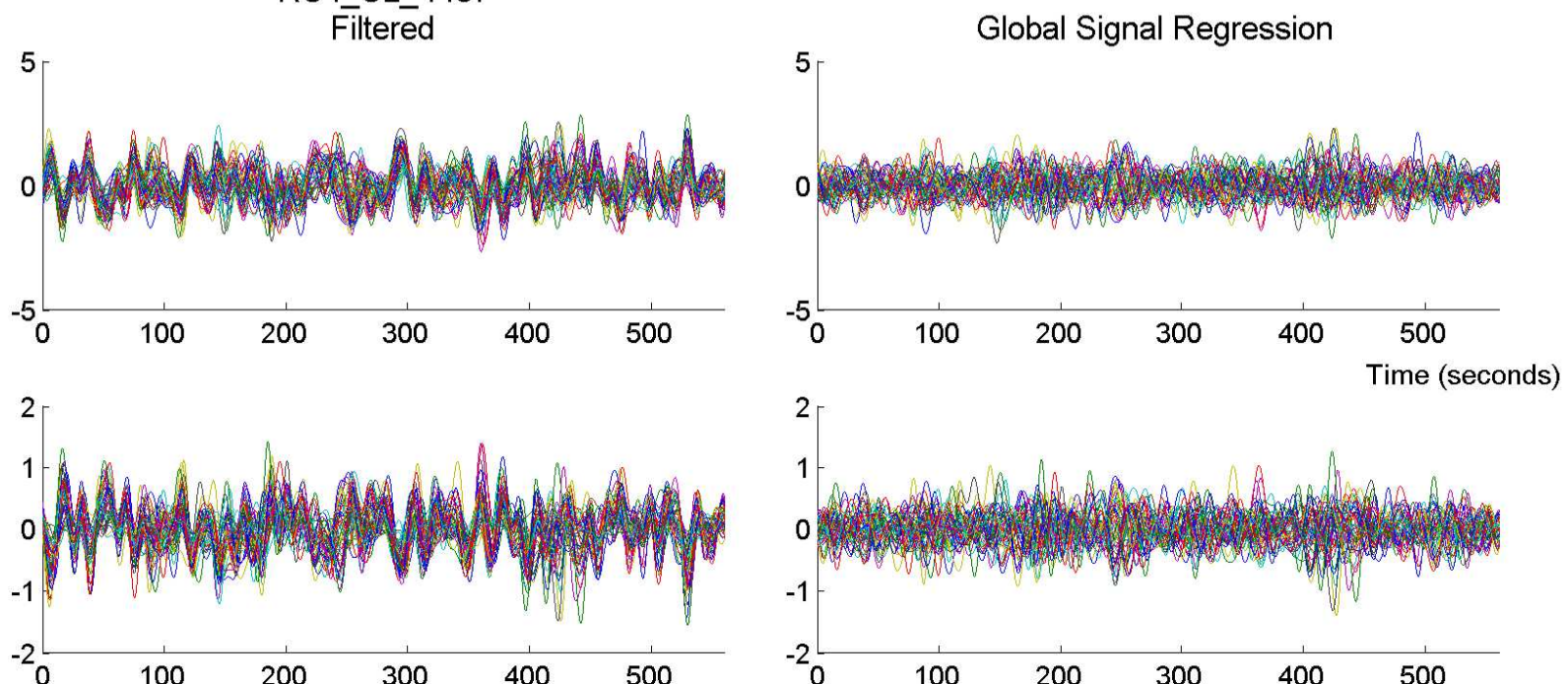

2
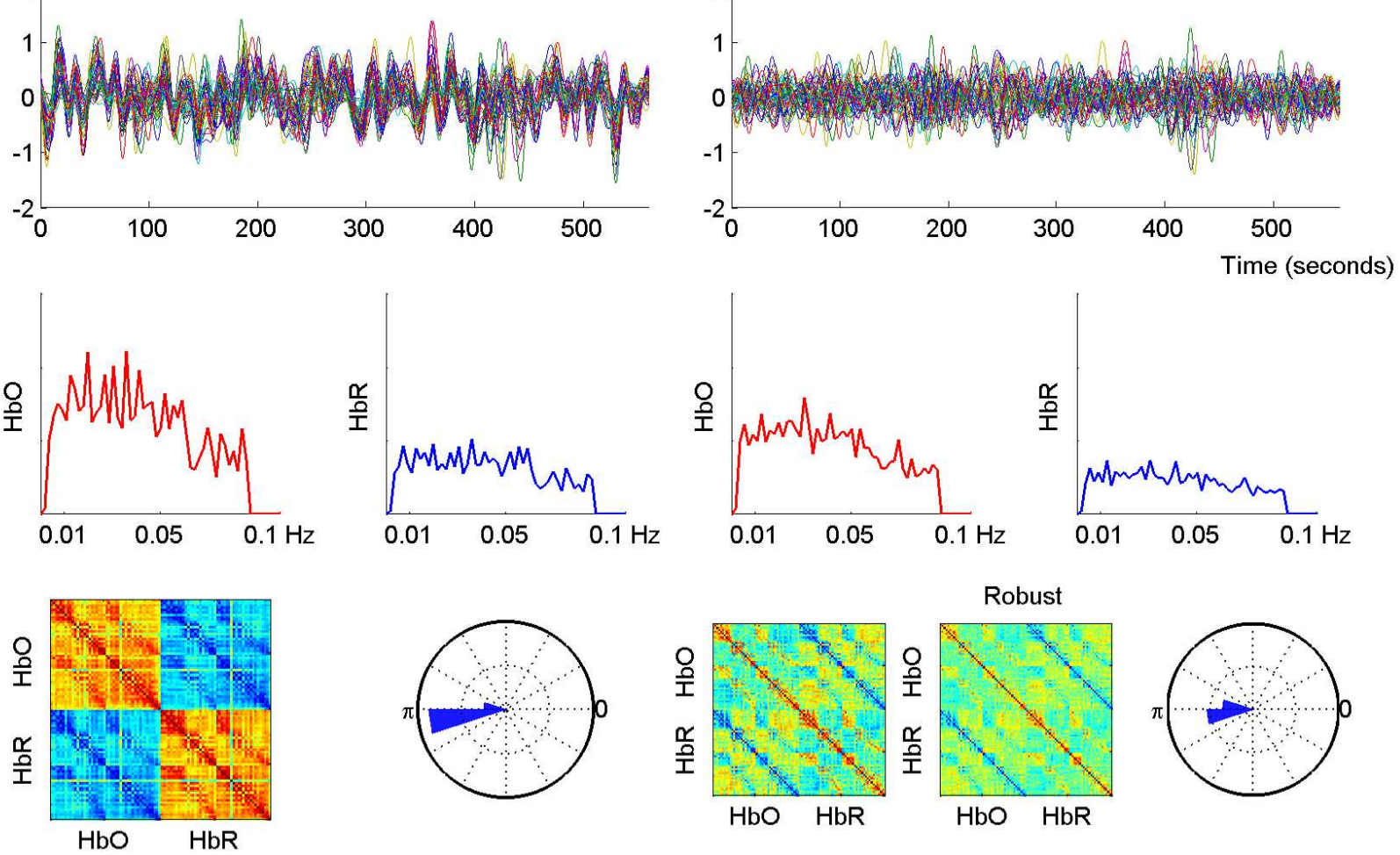
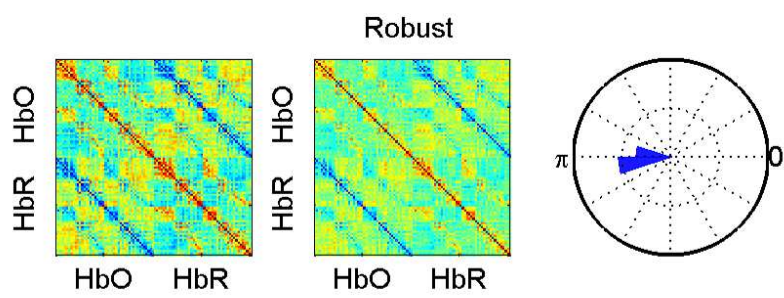

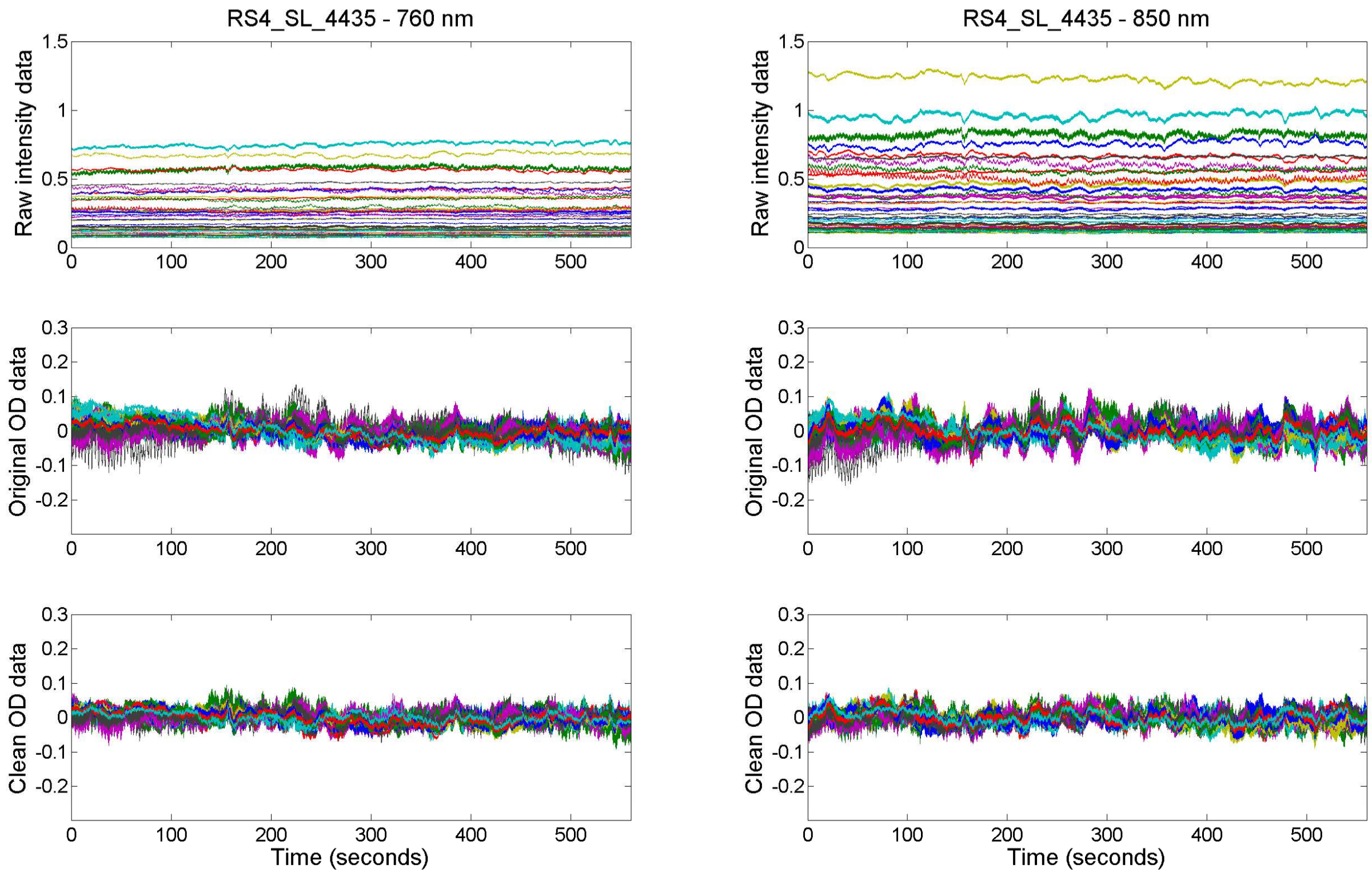
Raw
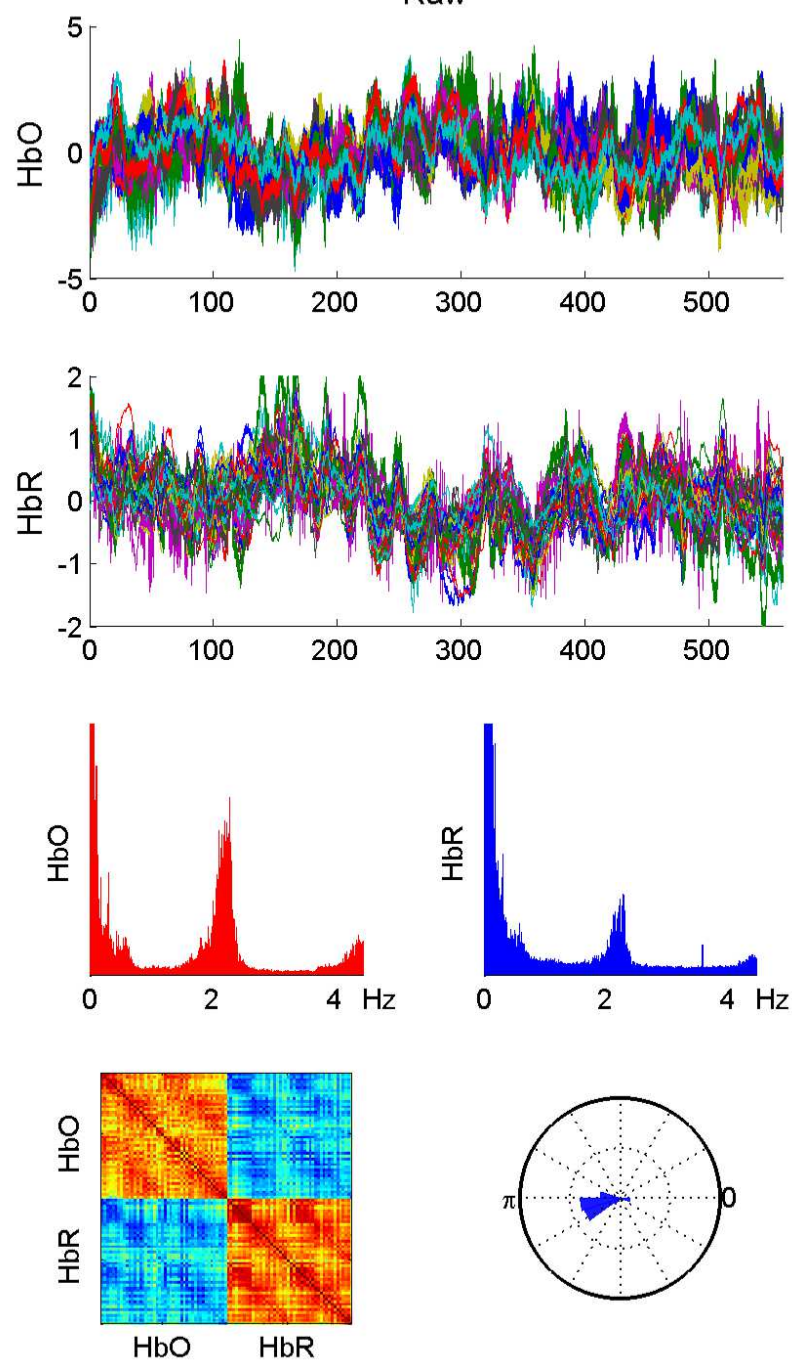

RS4 SL 4435

Filtered
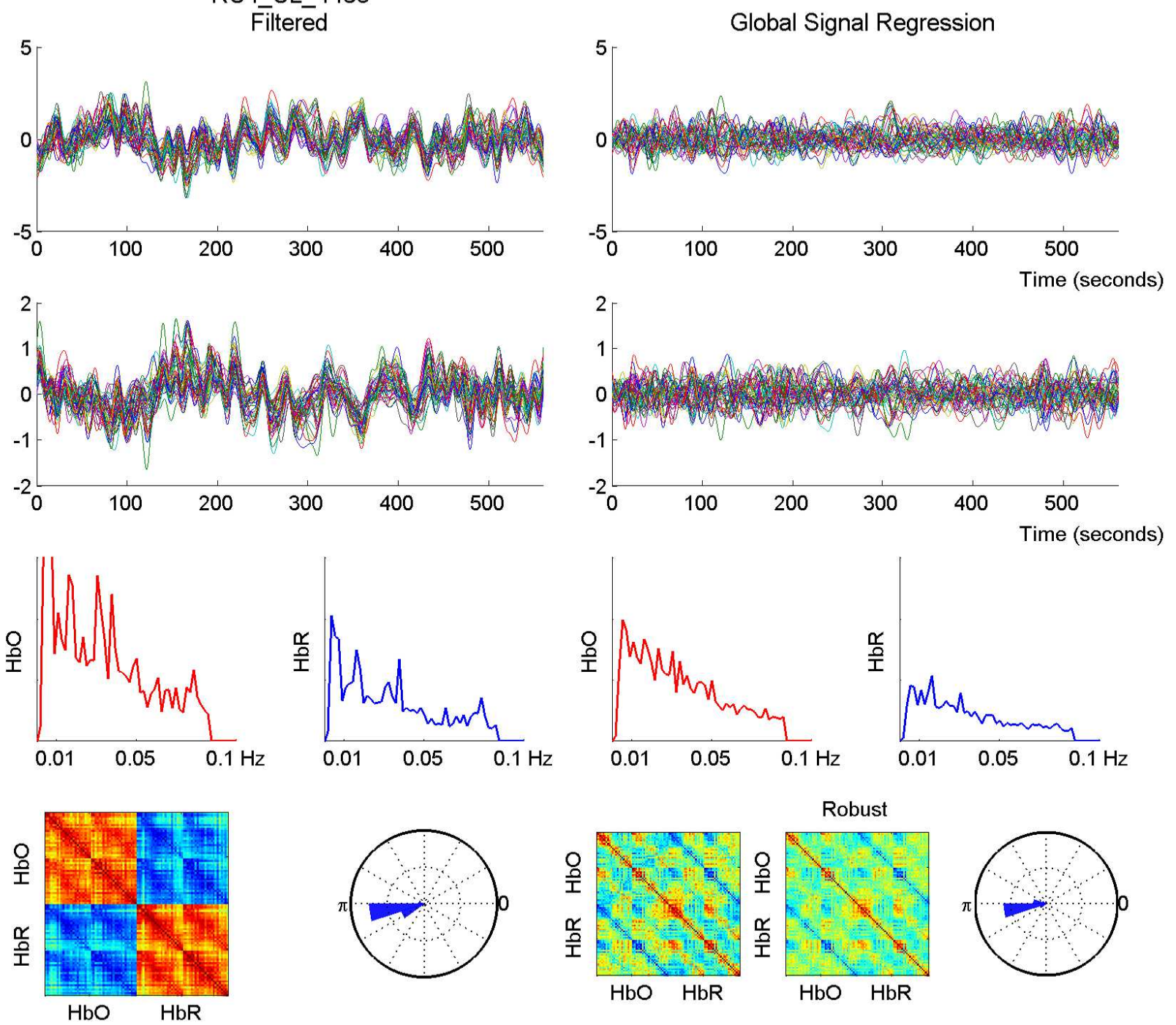

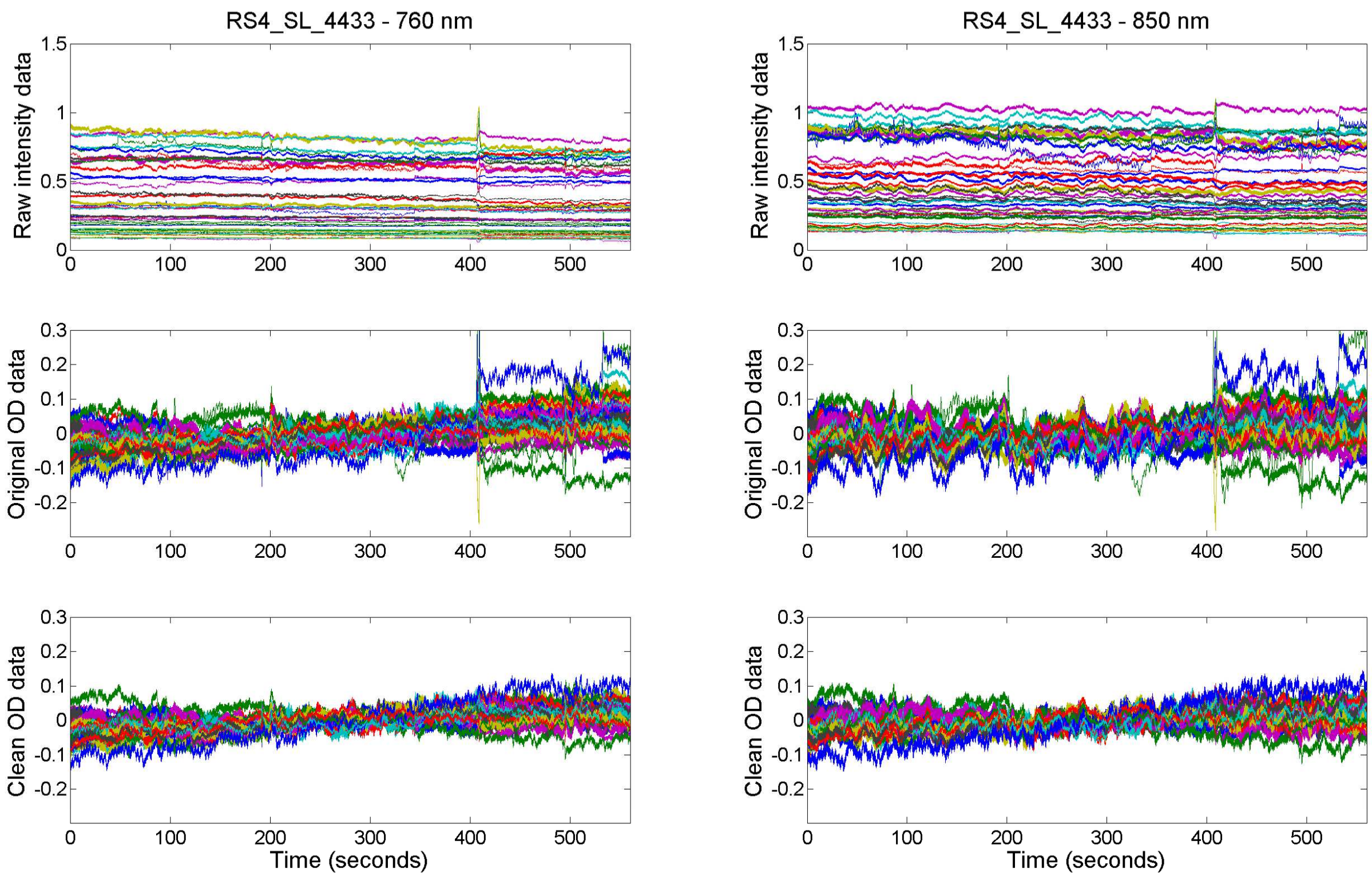
RS4_SL_4433

Raw

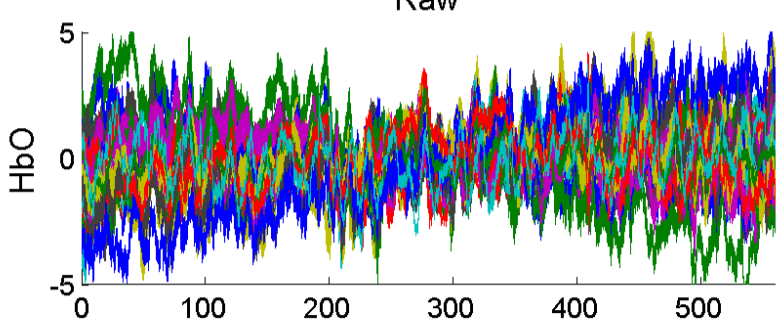

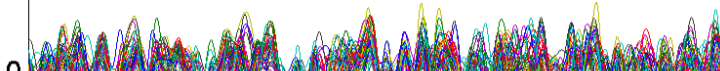

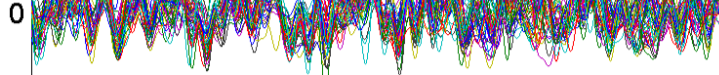
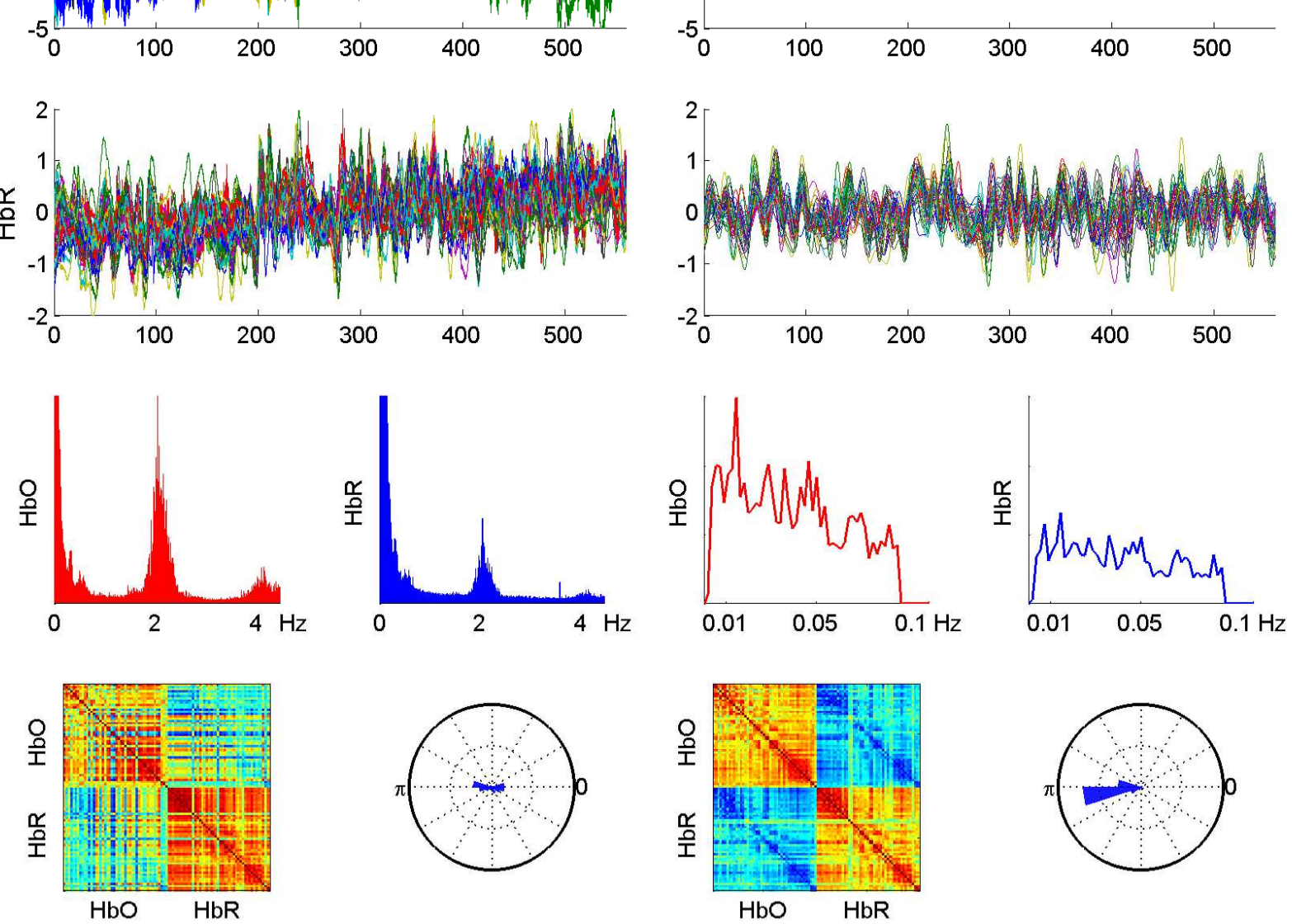
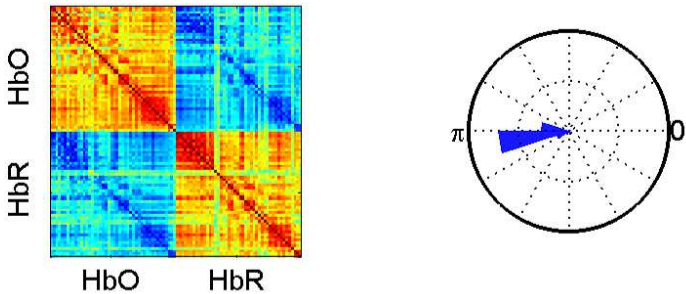

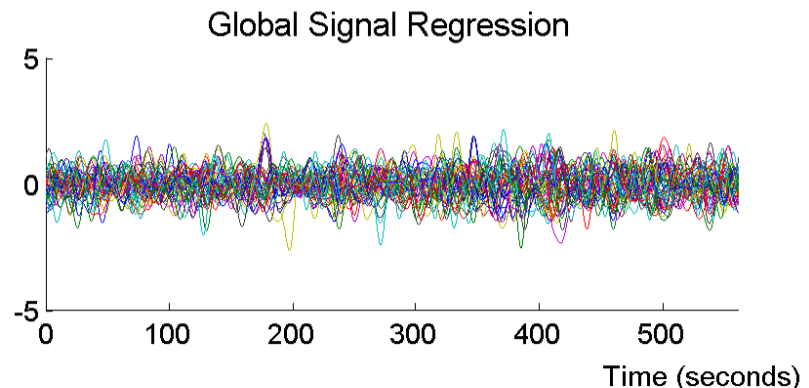

2
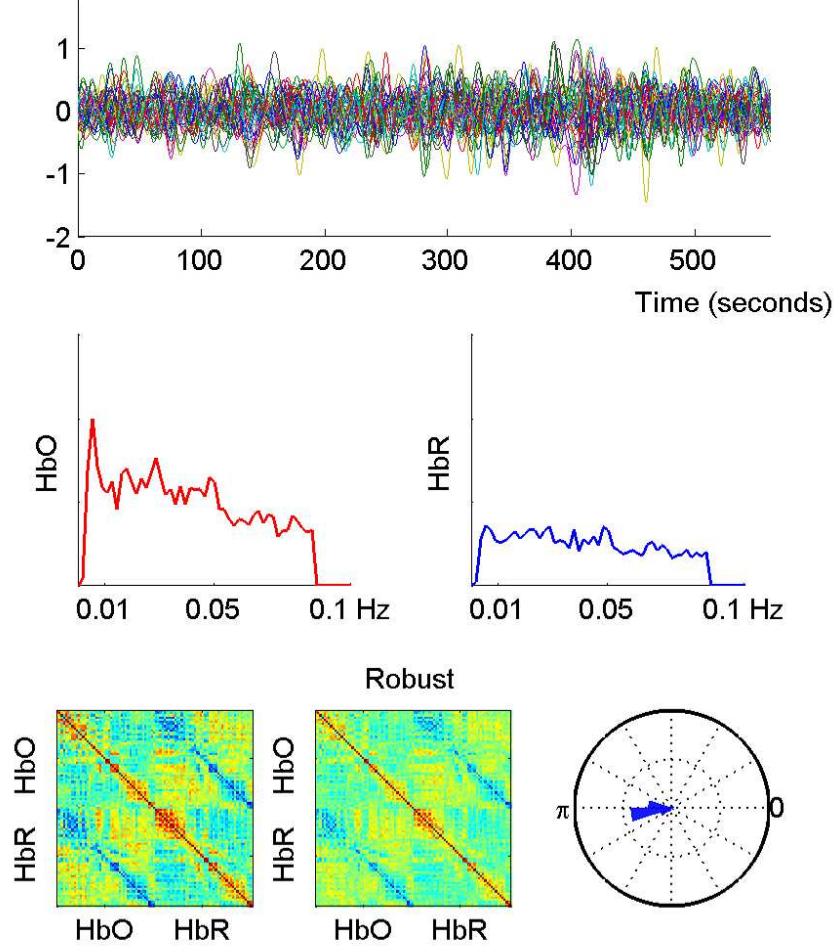

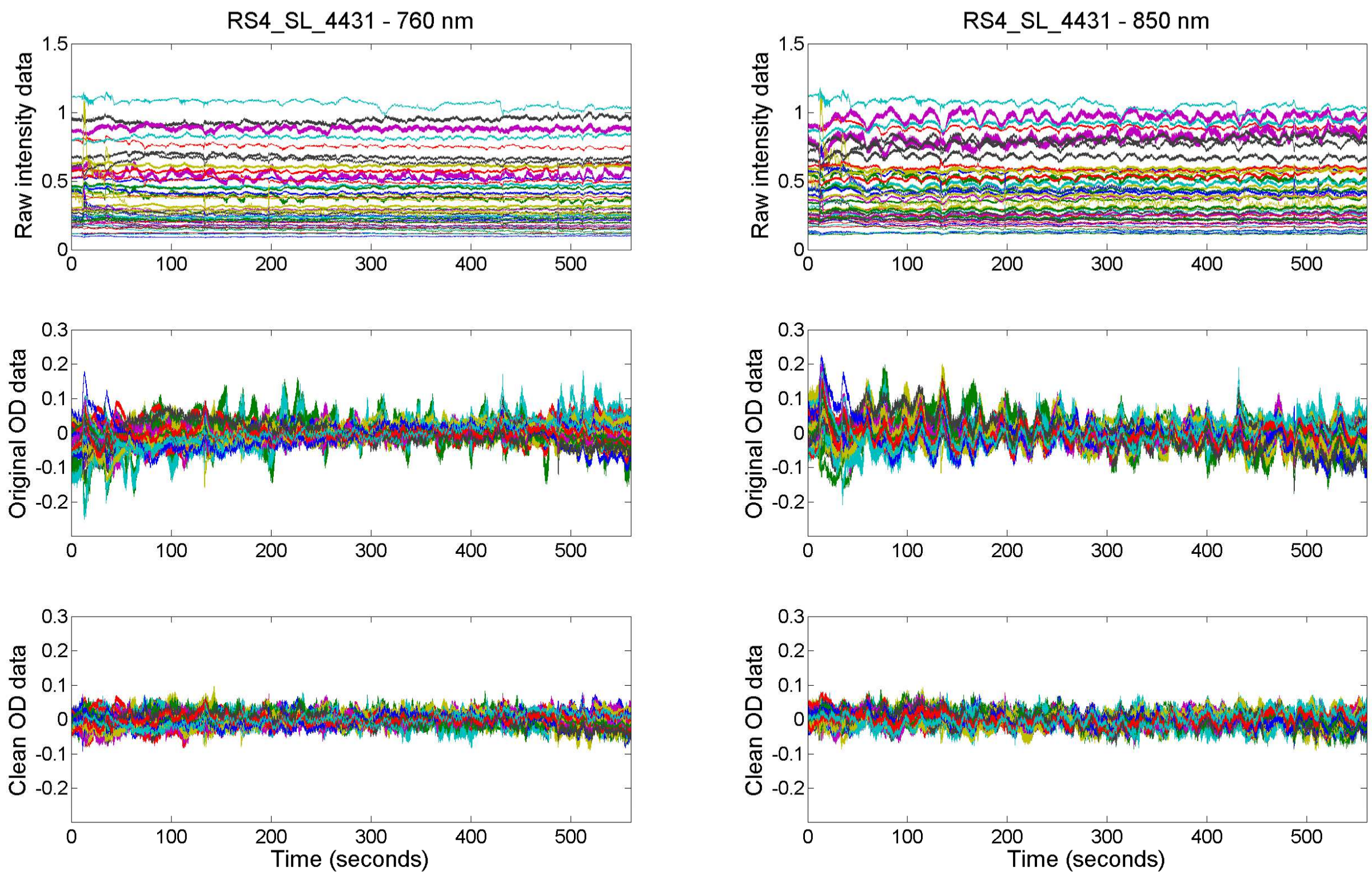
Raw

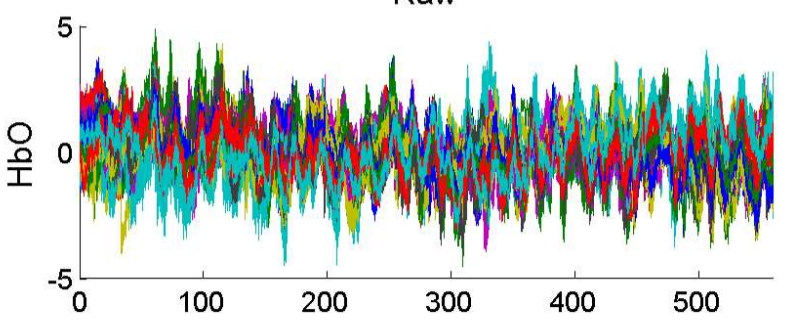

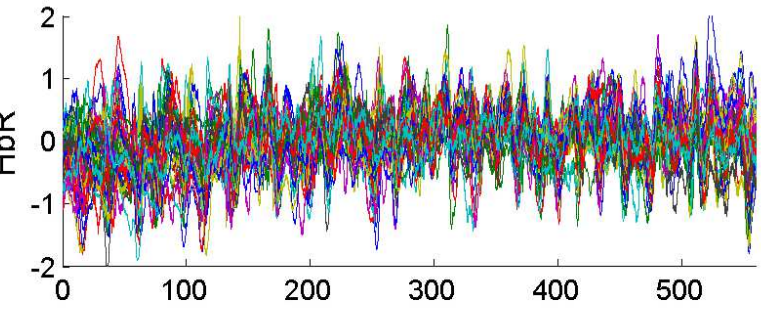
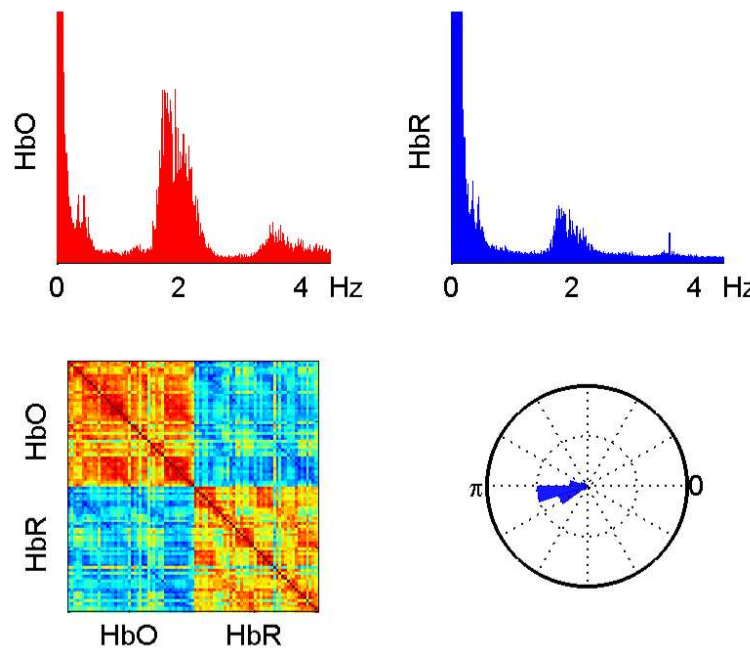

RS4_SL_4431

Filtered

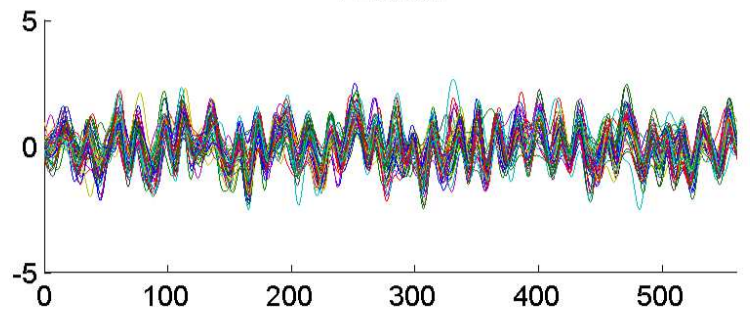

2

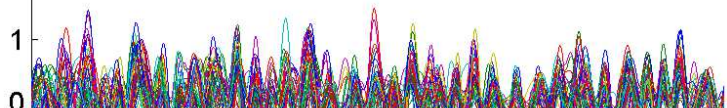

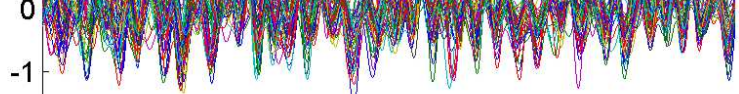
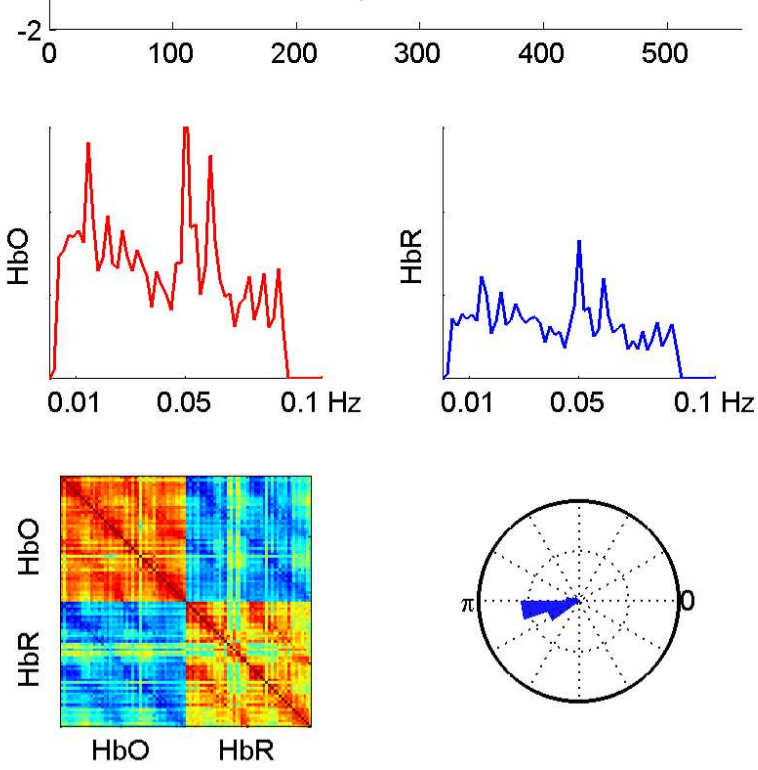

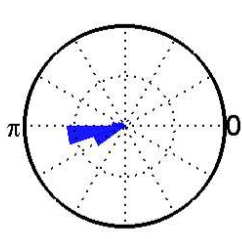

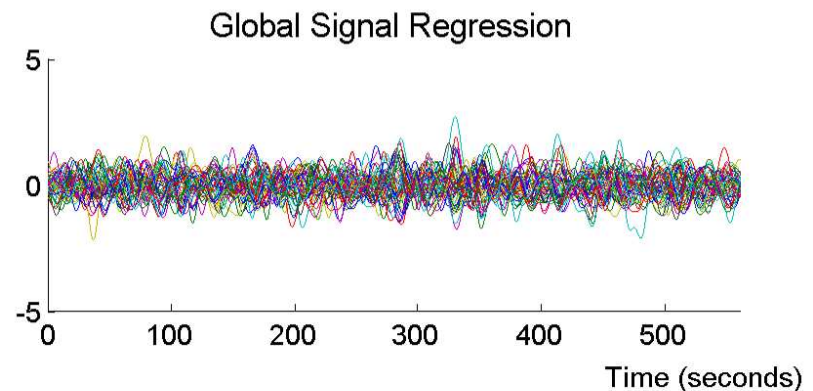

2
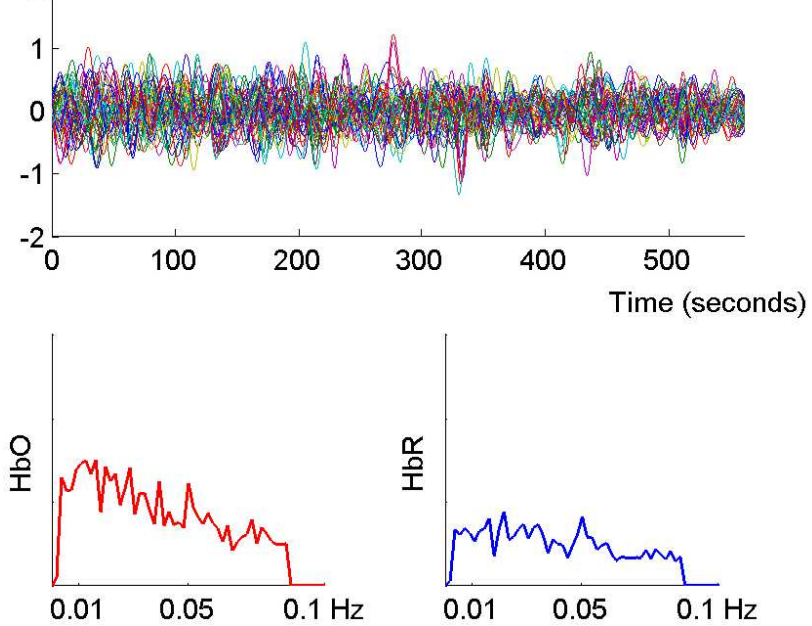

Robust

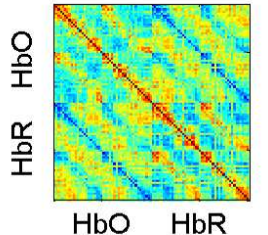

옹

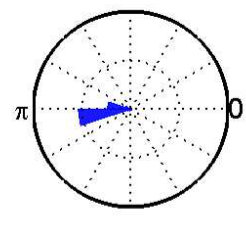



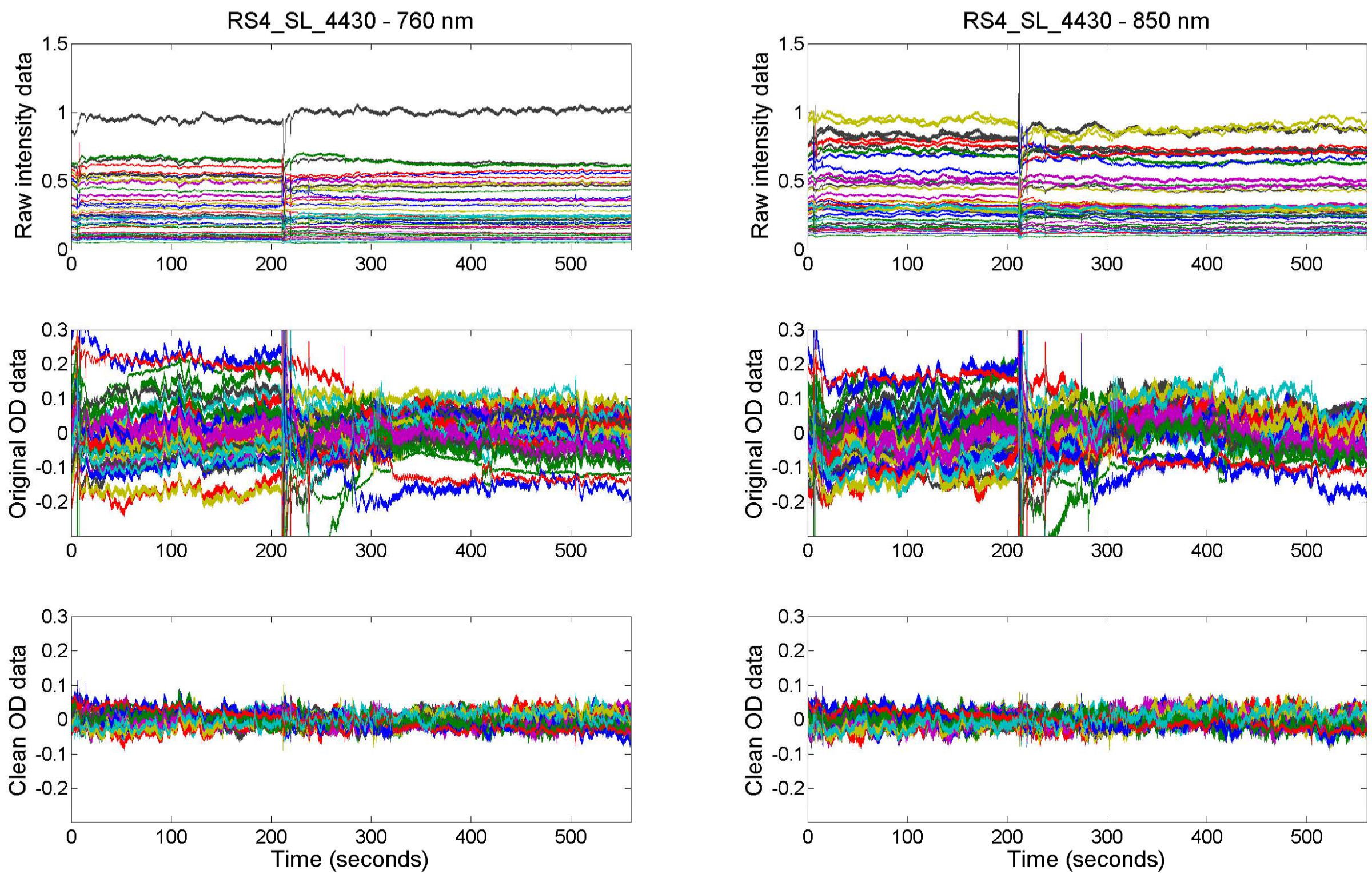
Raw

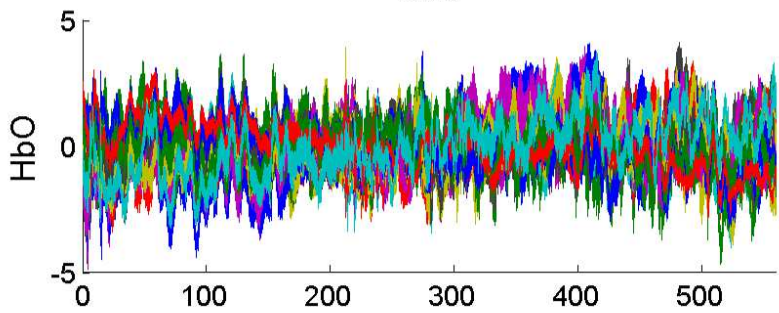

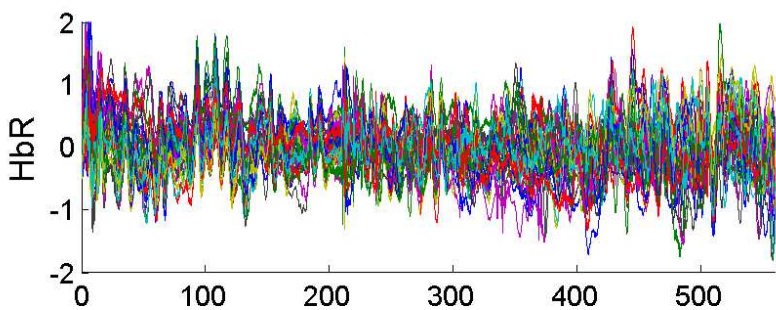
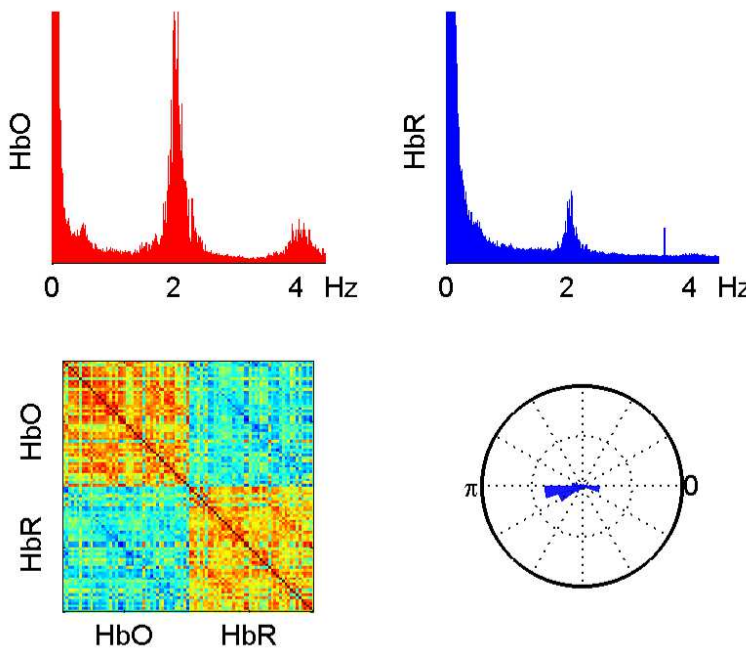

RS4_SL_4430

Filtered
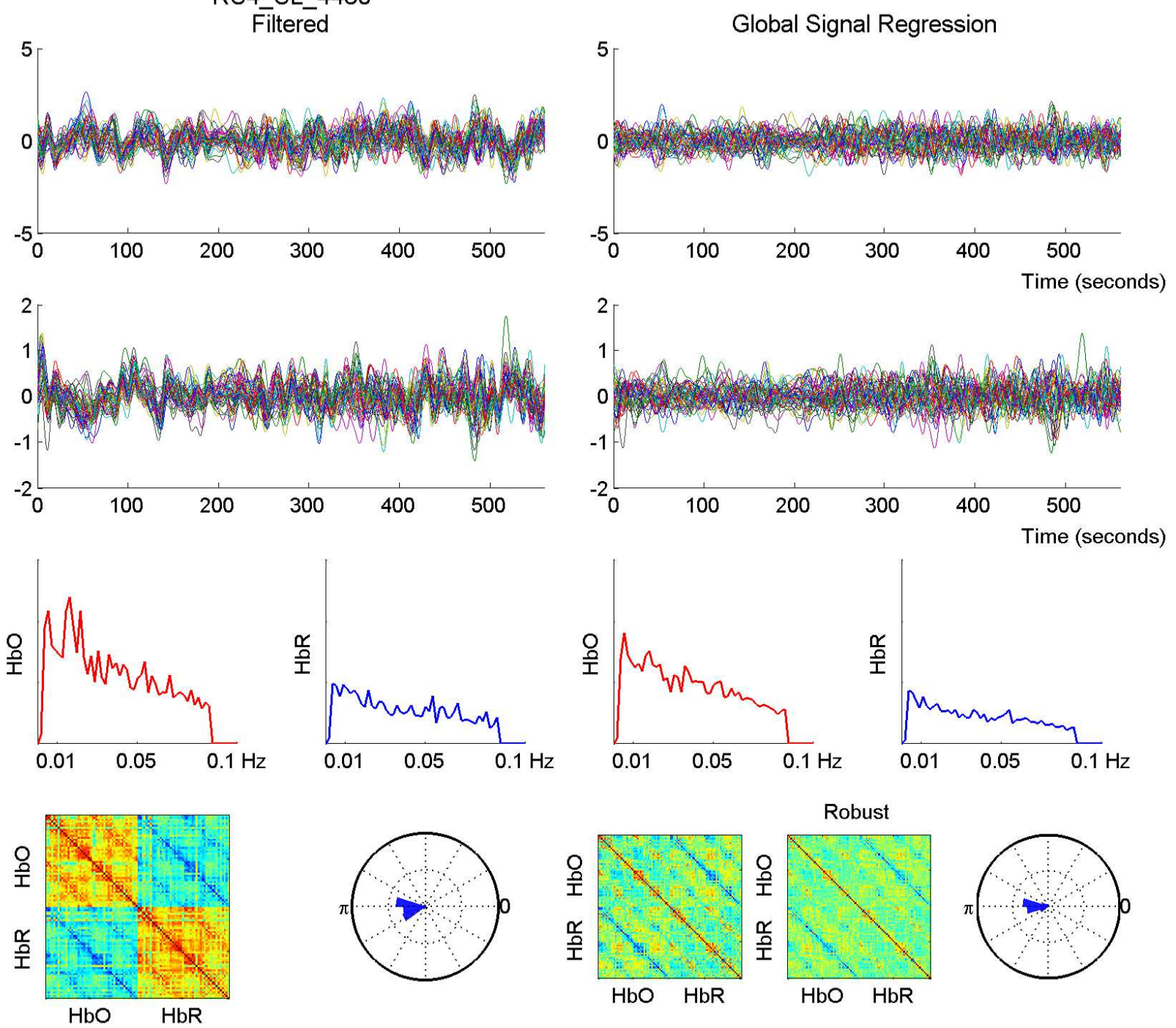
RS4 SL 4422 - 760 nm
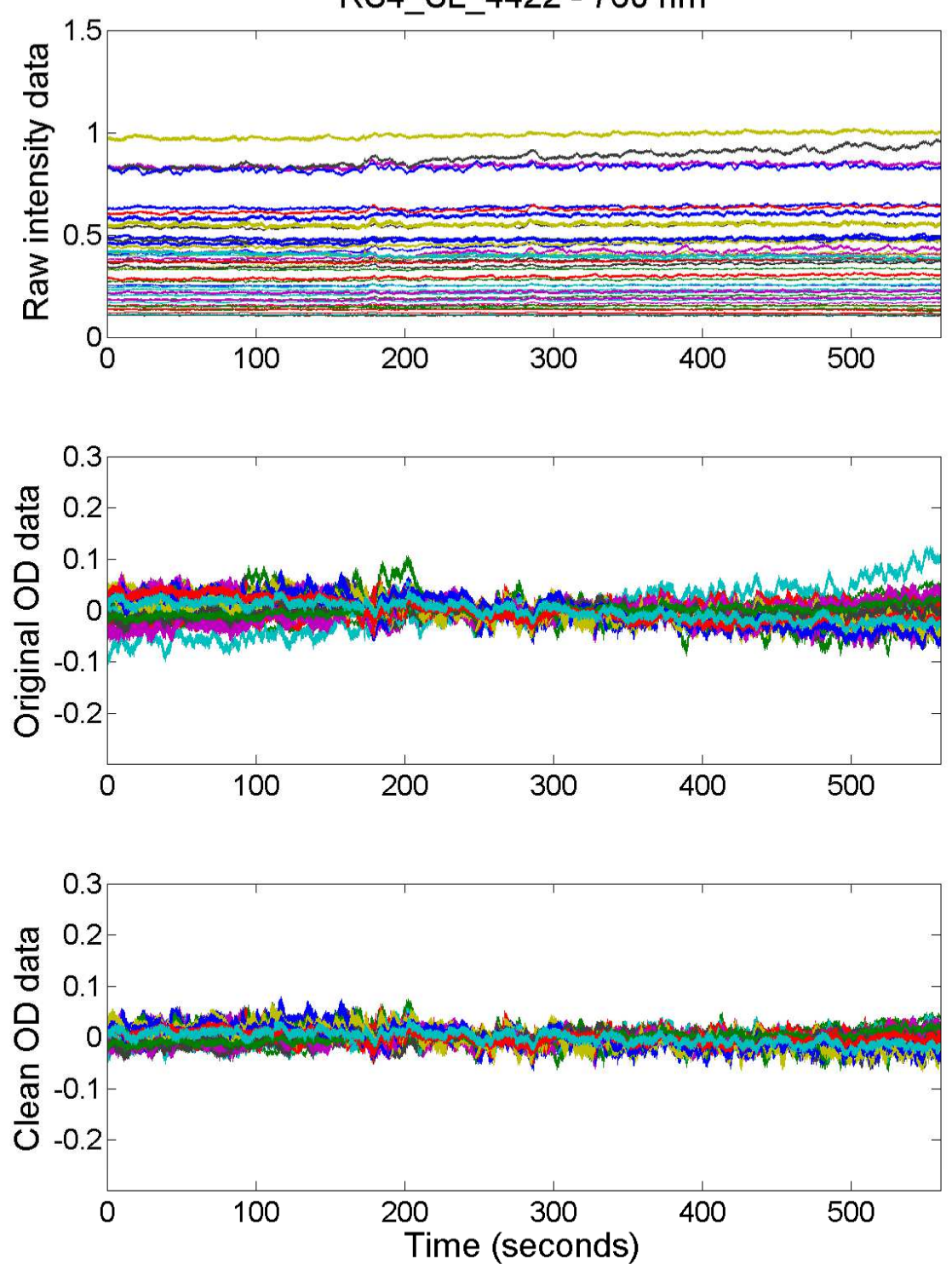

RS4 SL $4422-850 \mathrm{~nm}$
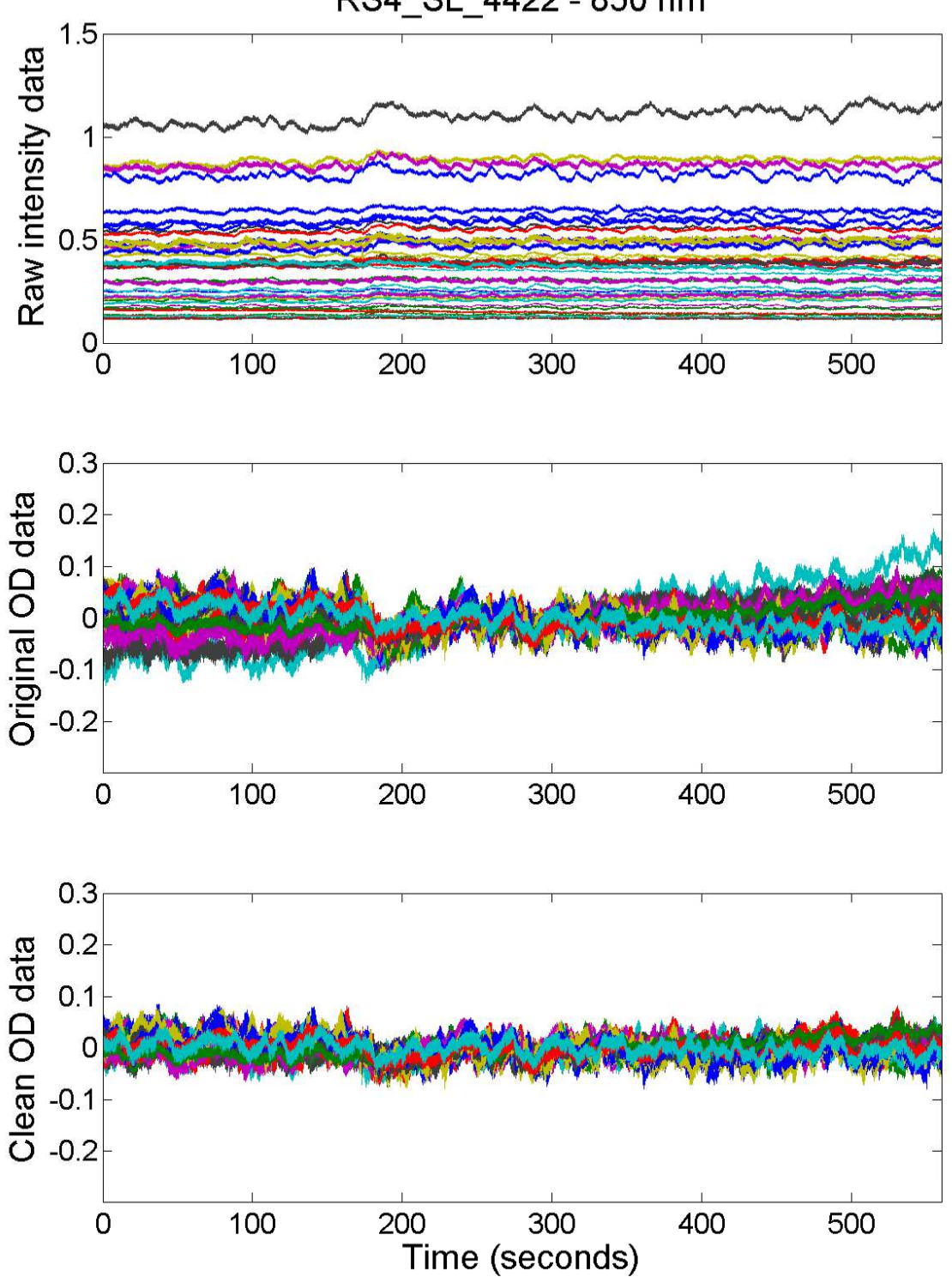
Raw

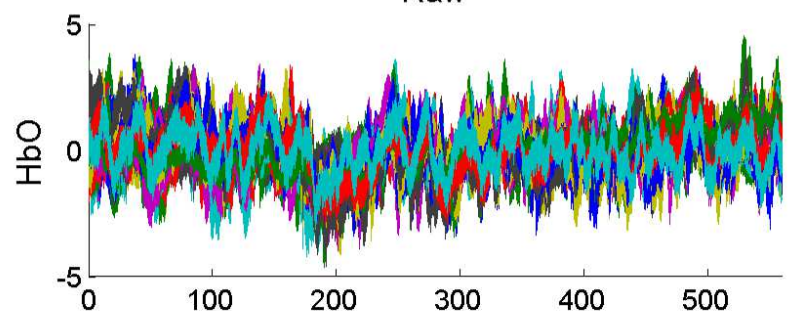

2

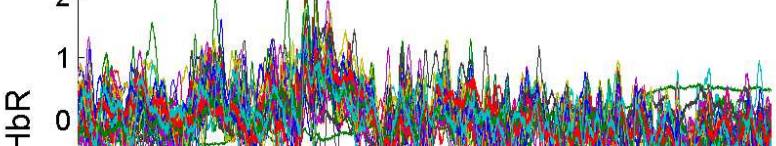

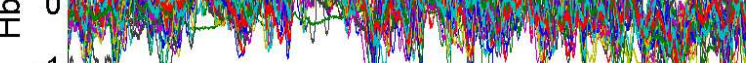
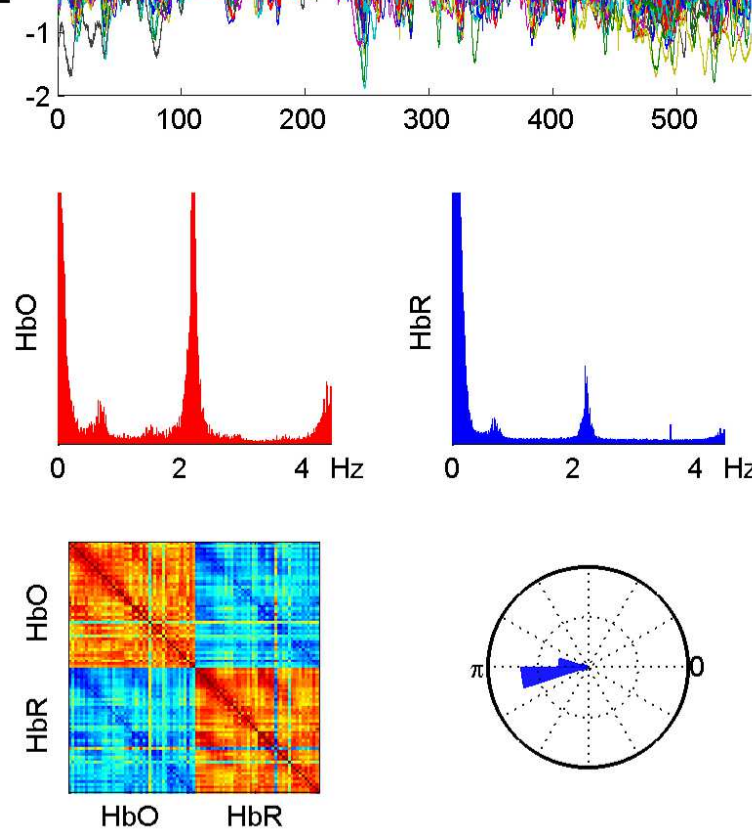

RS4 SL 4422

Filtered

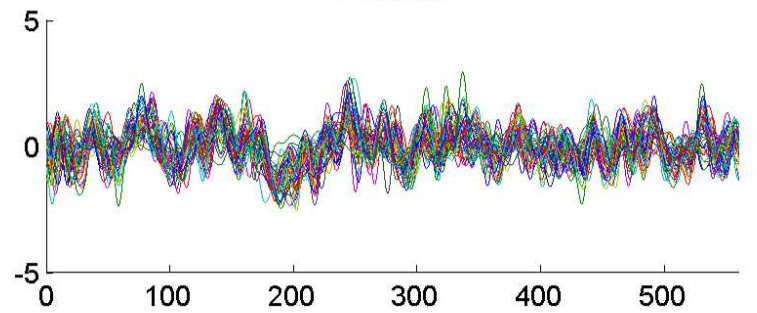

2
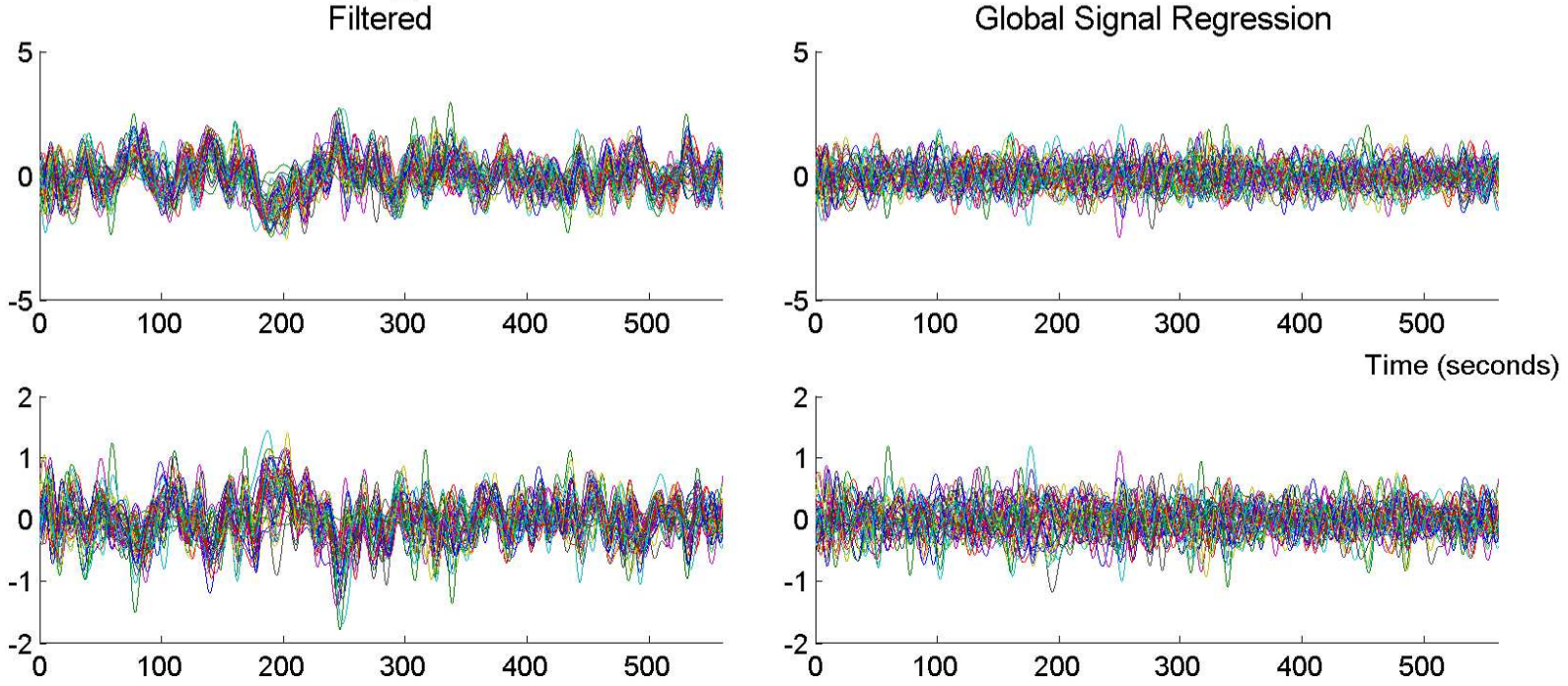

2
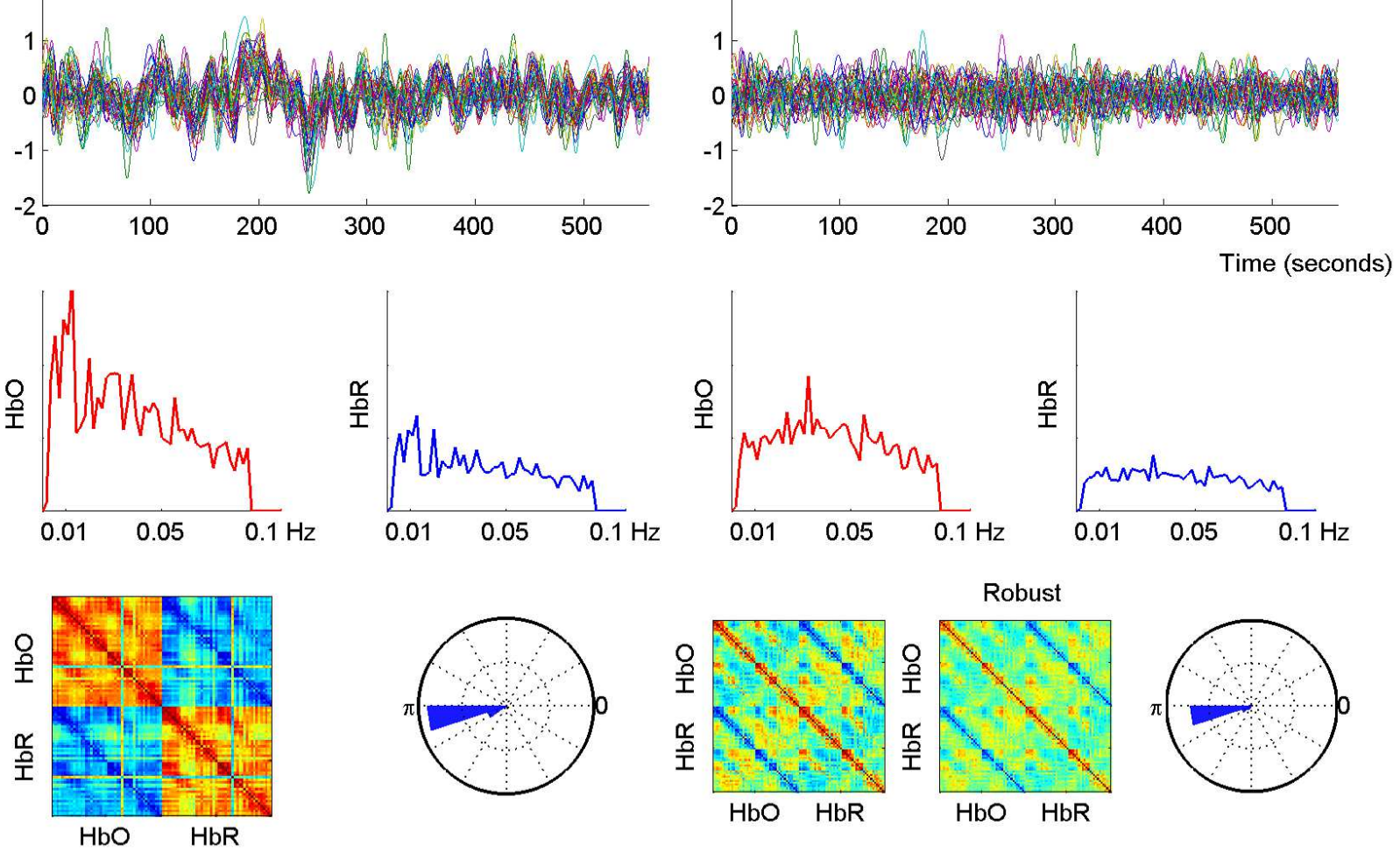
RS4 SL 4416 - 760 nm
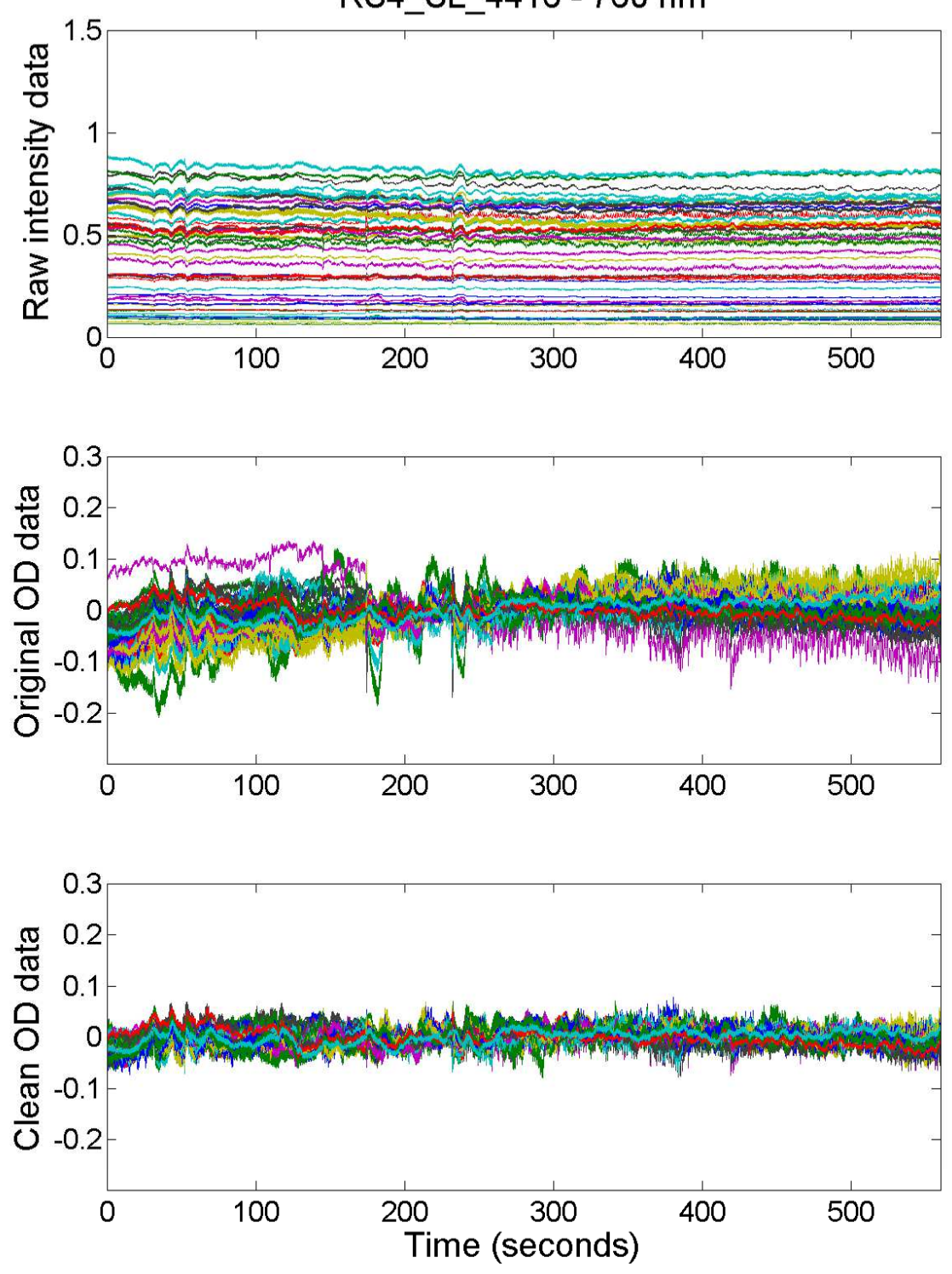

RS4 SL $4416-850 \mathrm{~nm}$
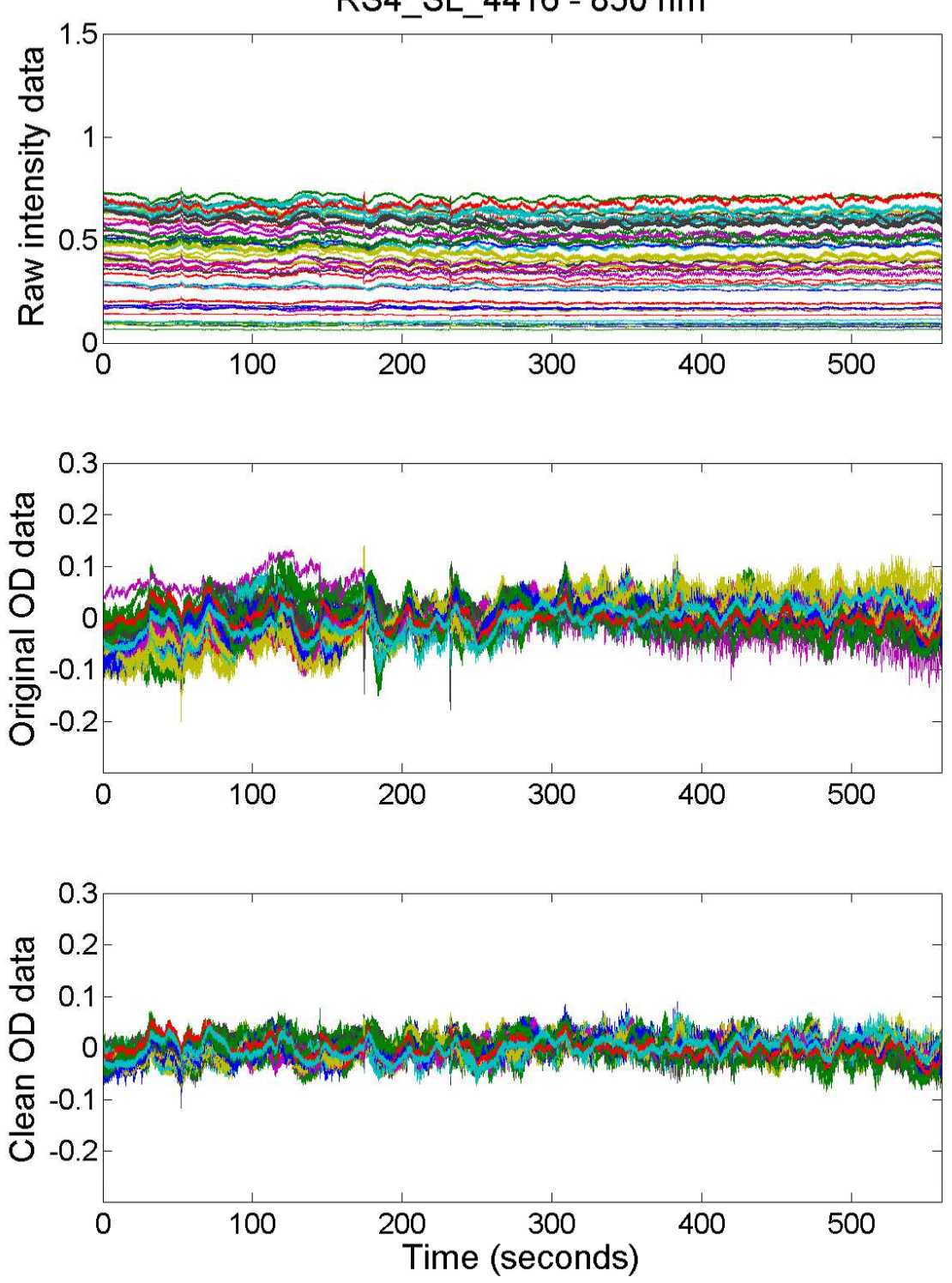
RS4_SL_4416

Filtered
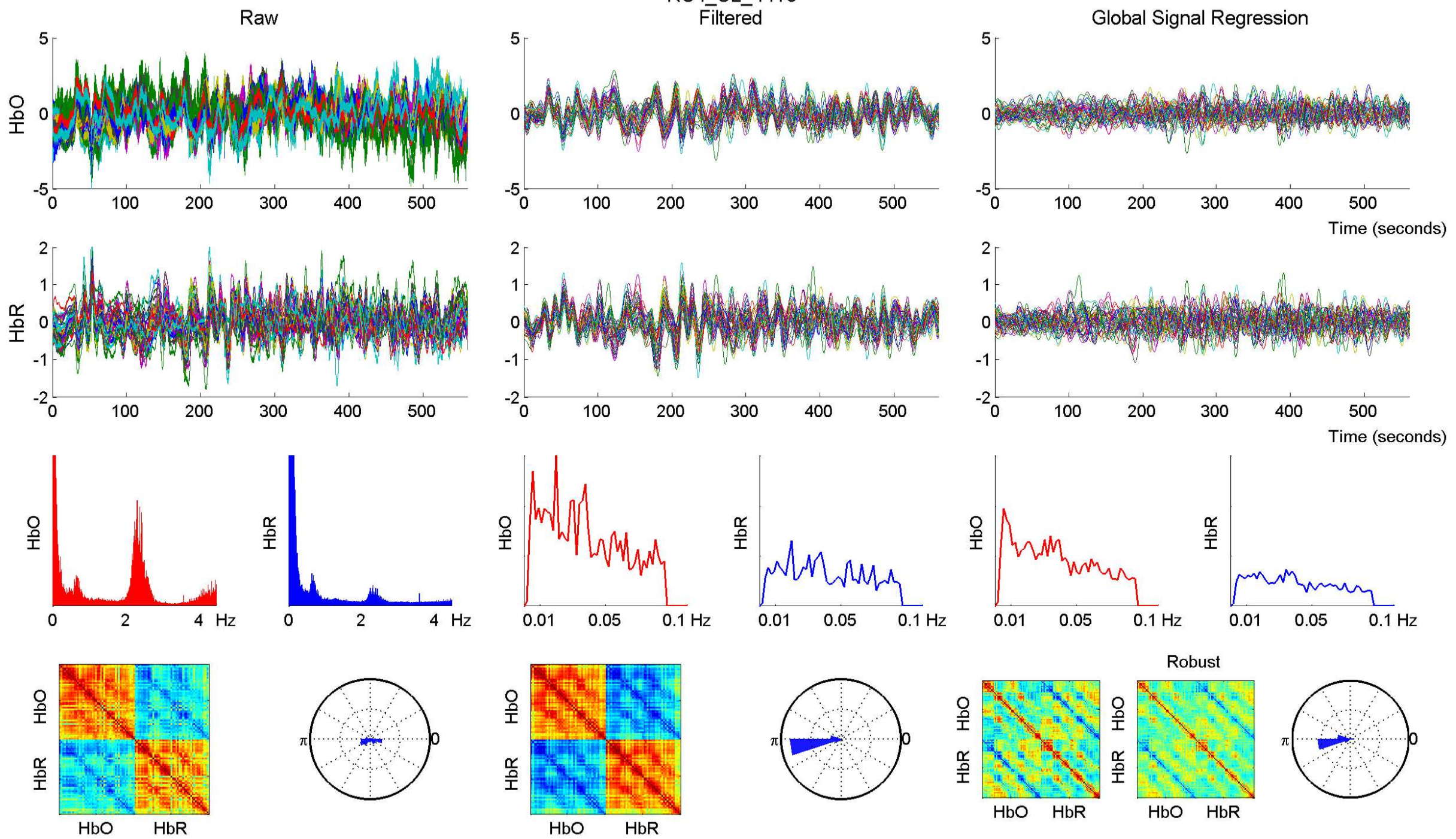
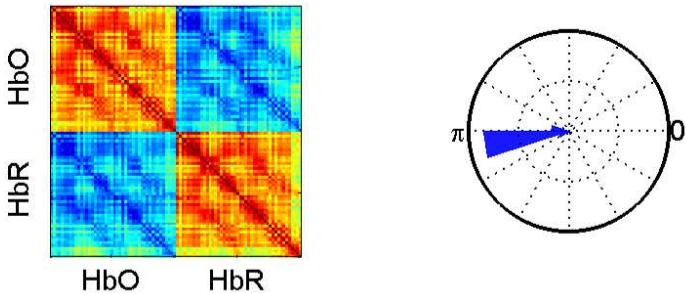
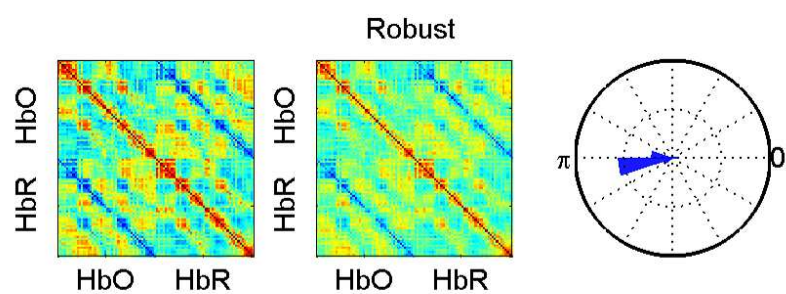
RS4 SL 4415 - 760 nm
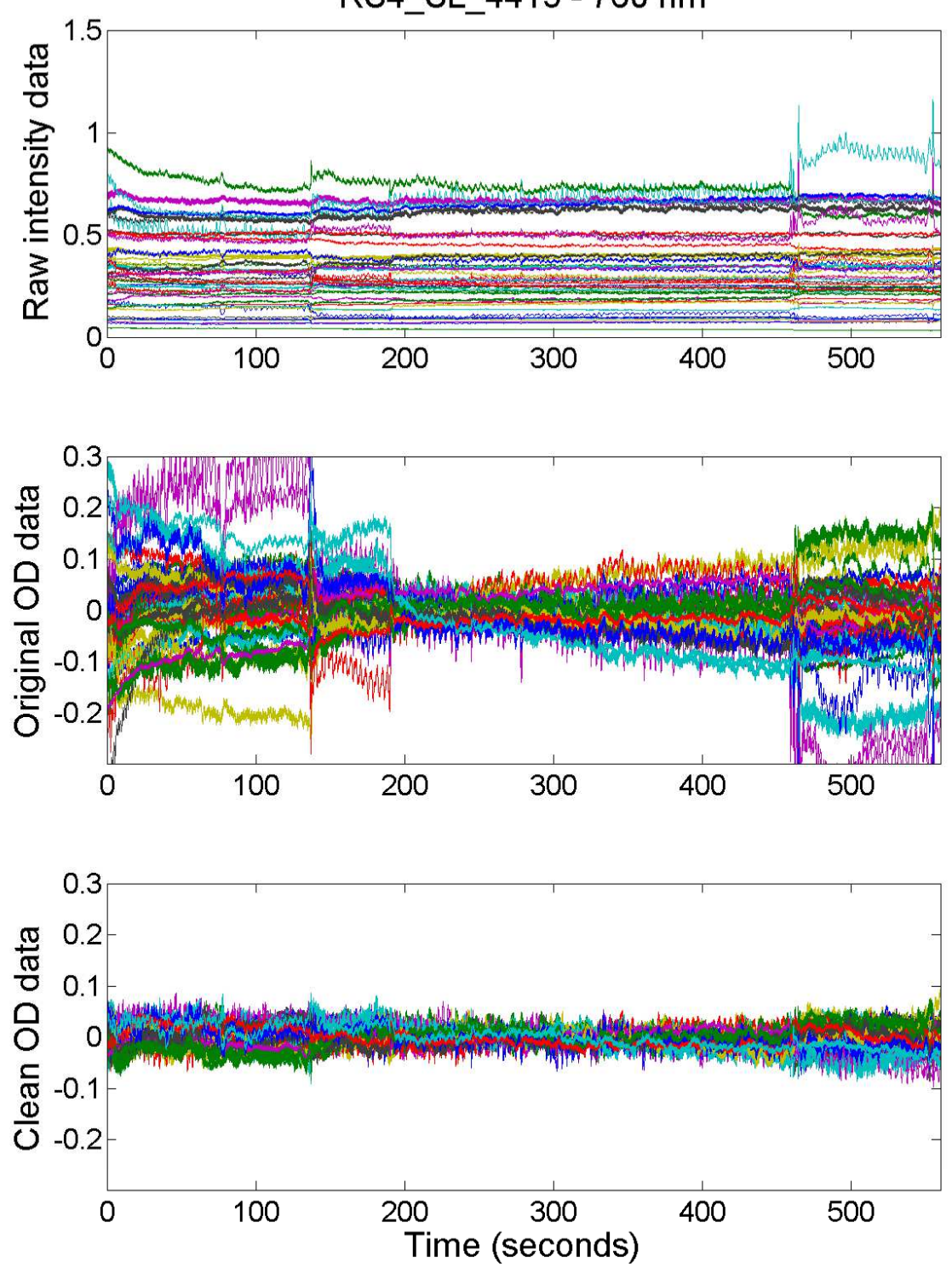

RS4 SL $4415-850 \mathrm{~nm}$
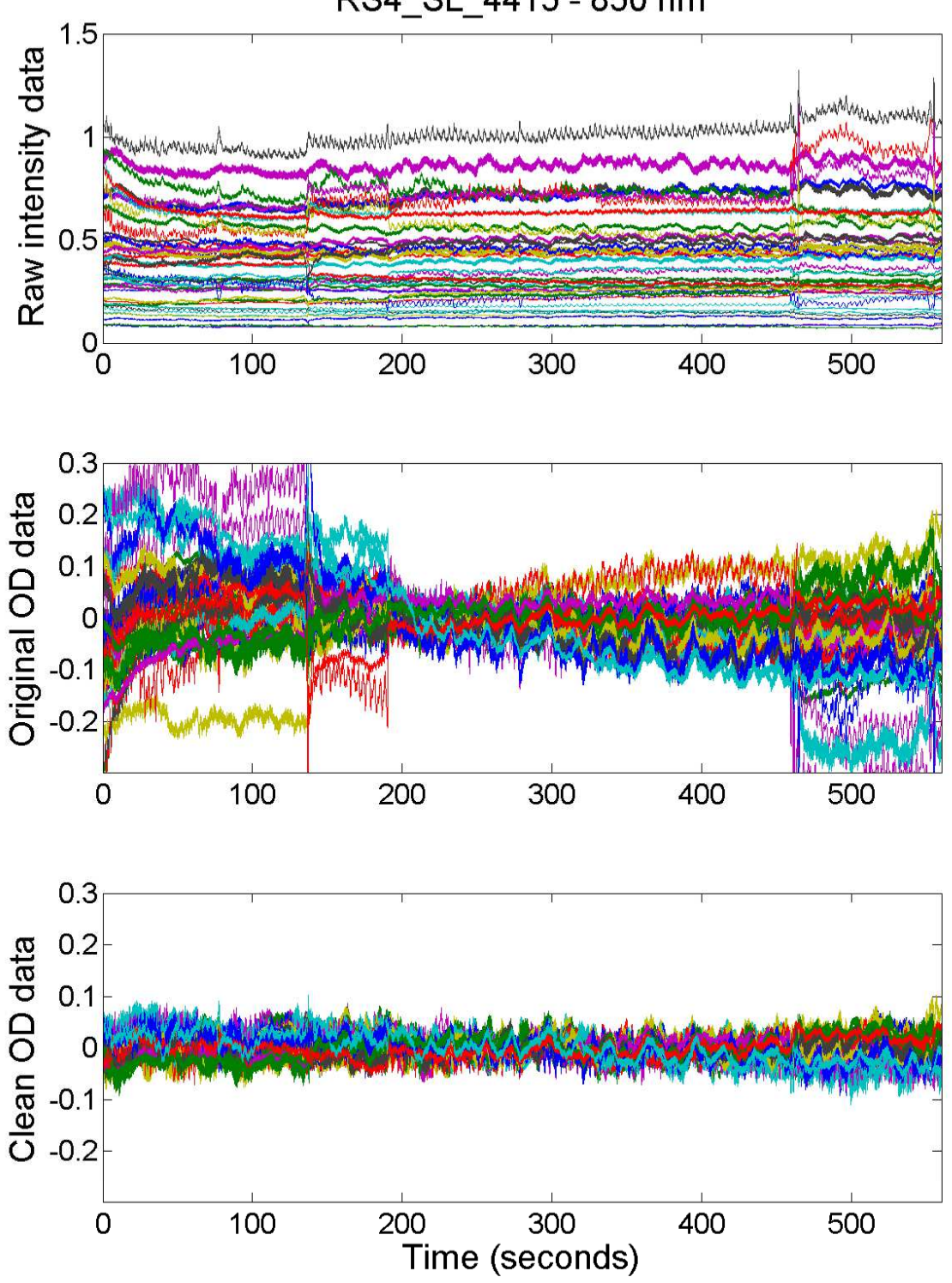
Raw

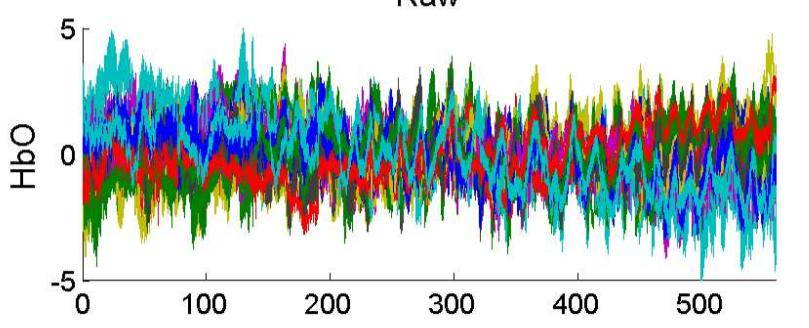

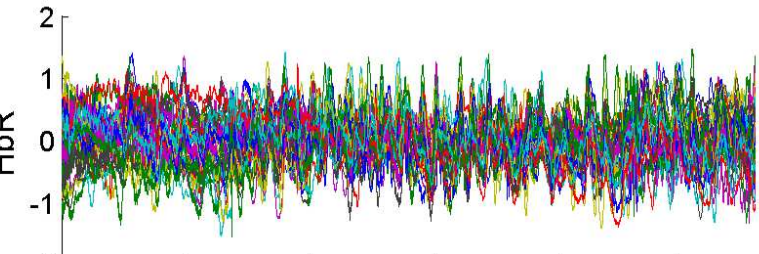
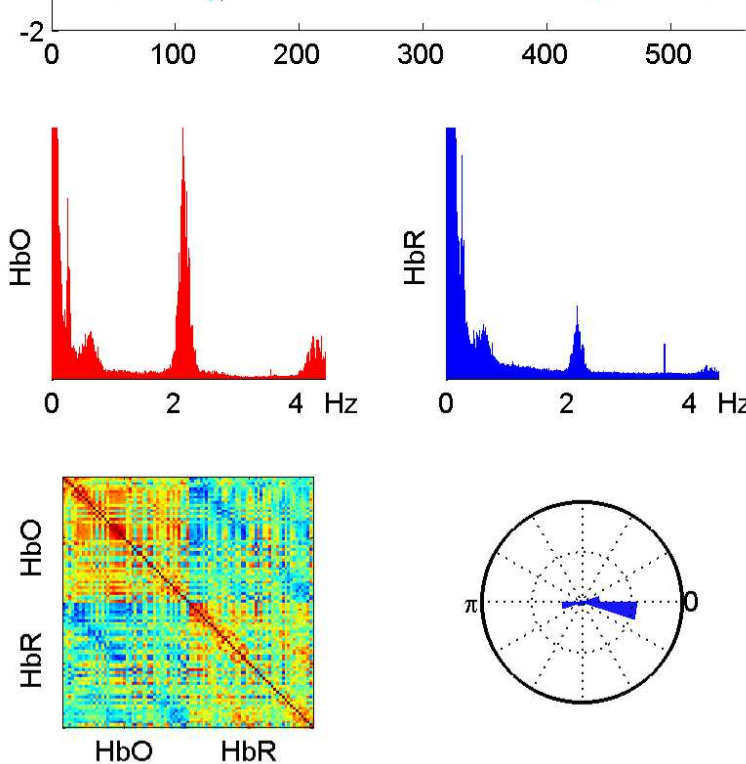

RS4_SL_4415

Filtered
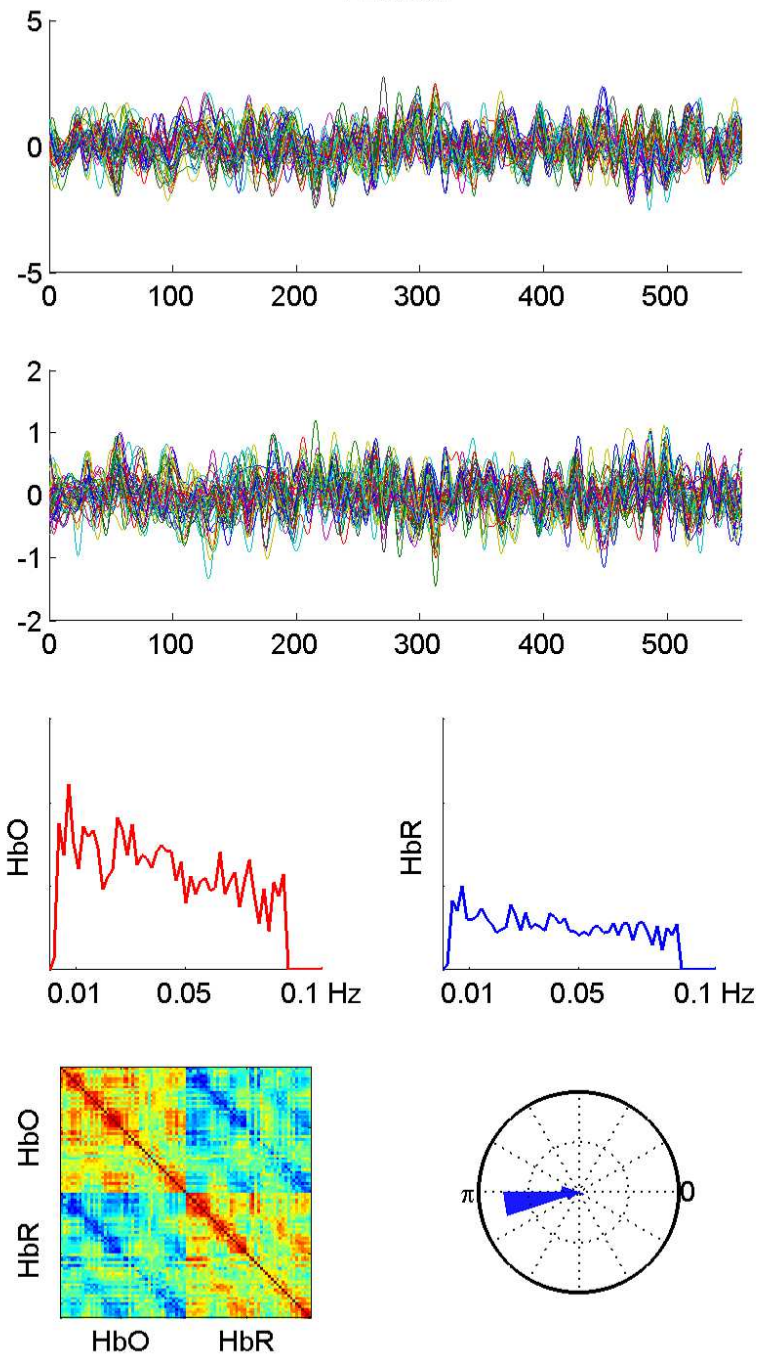

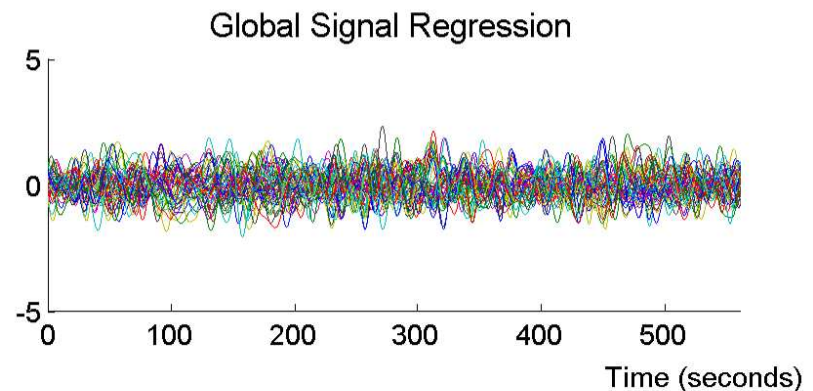

2
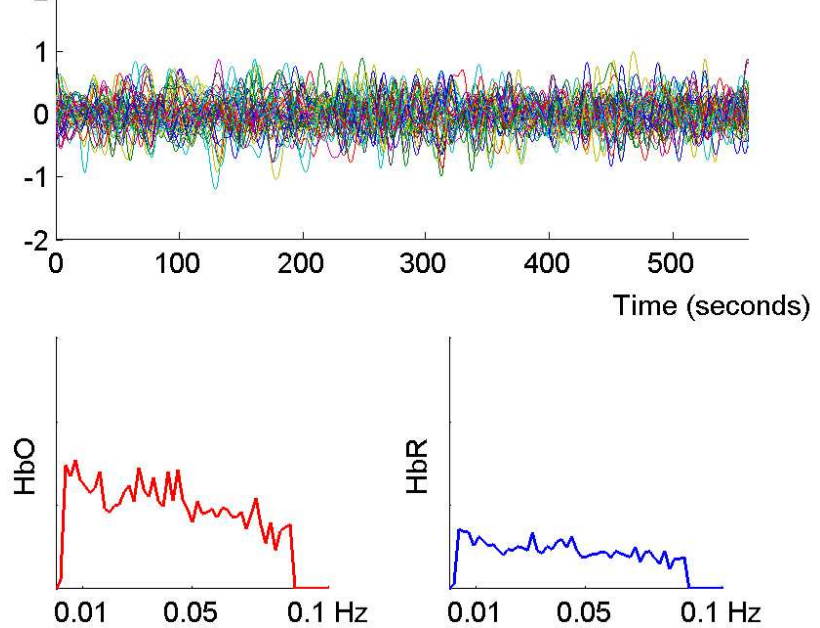
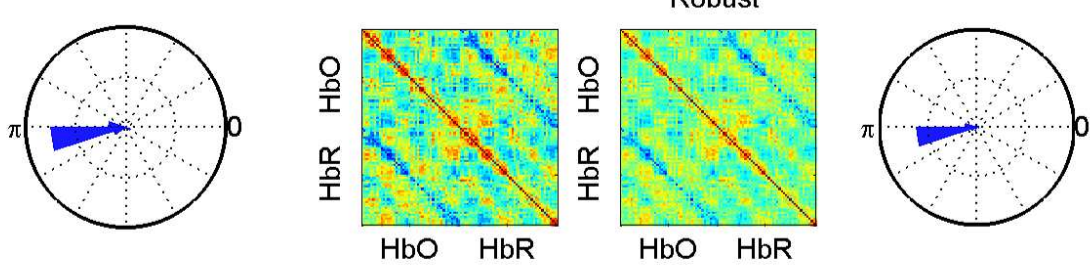

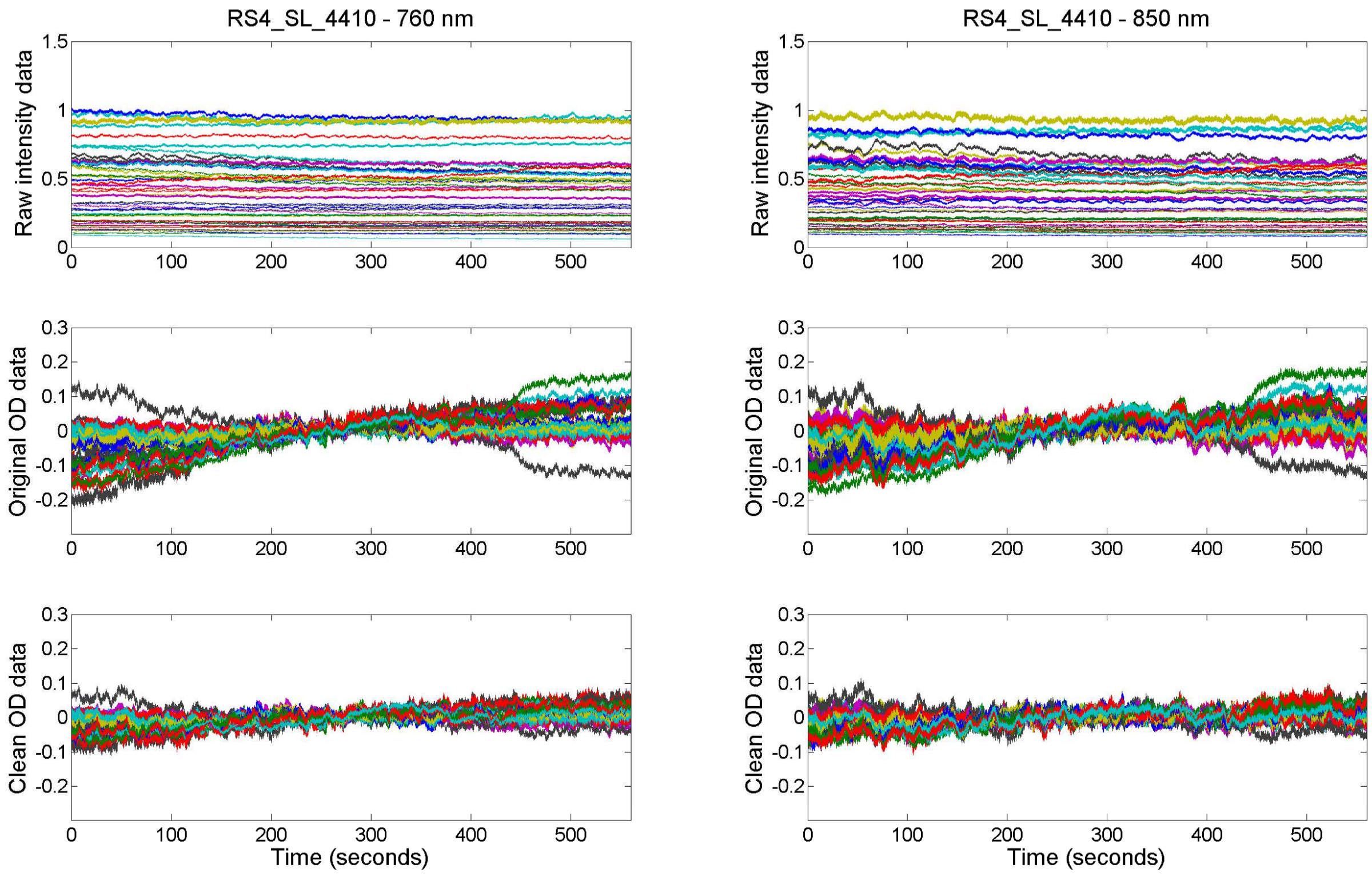
Raw
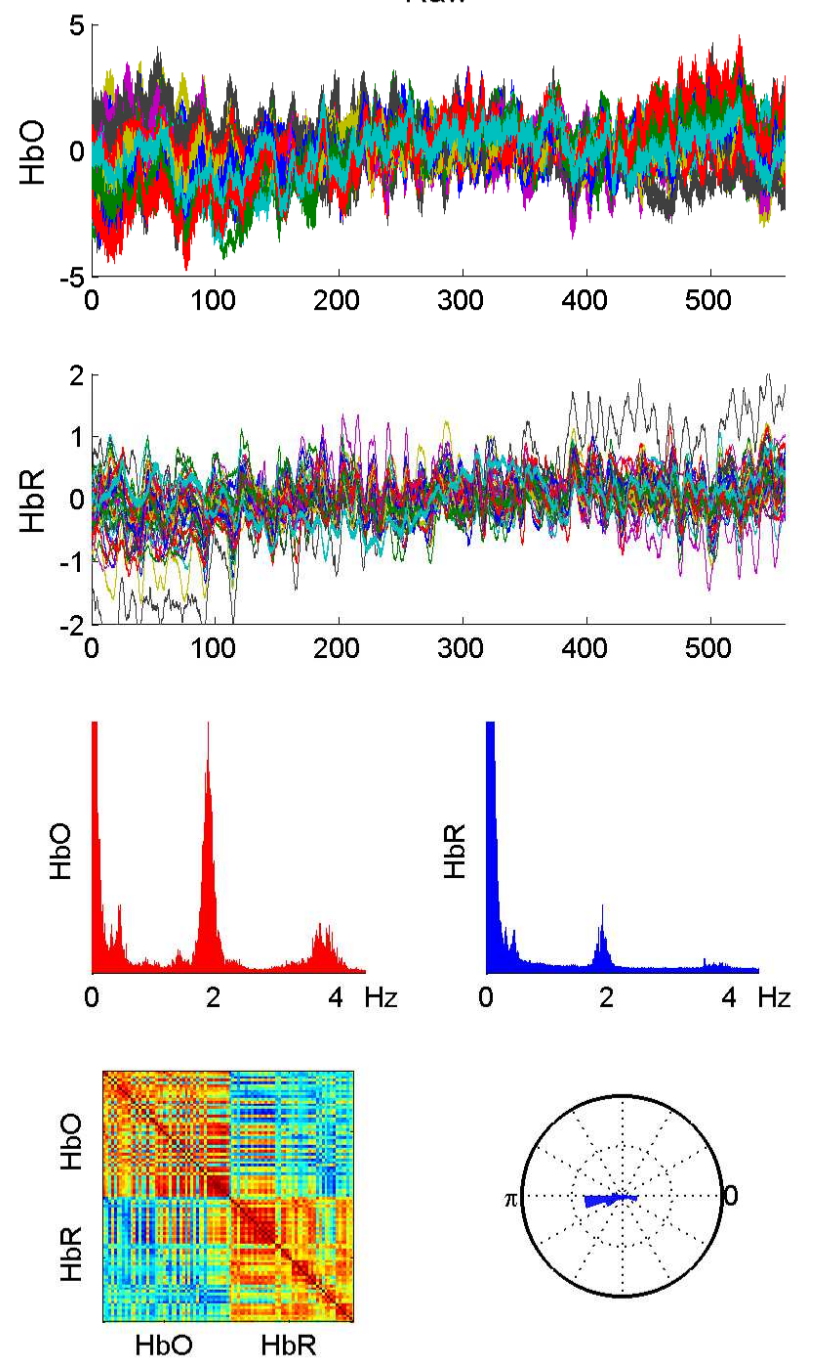

RS4 SL 4410

Filtered
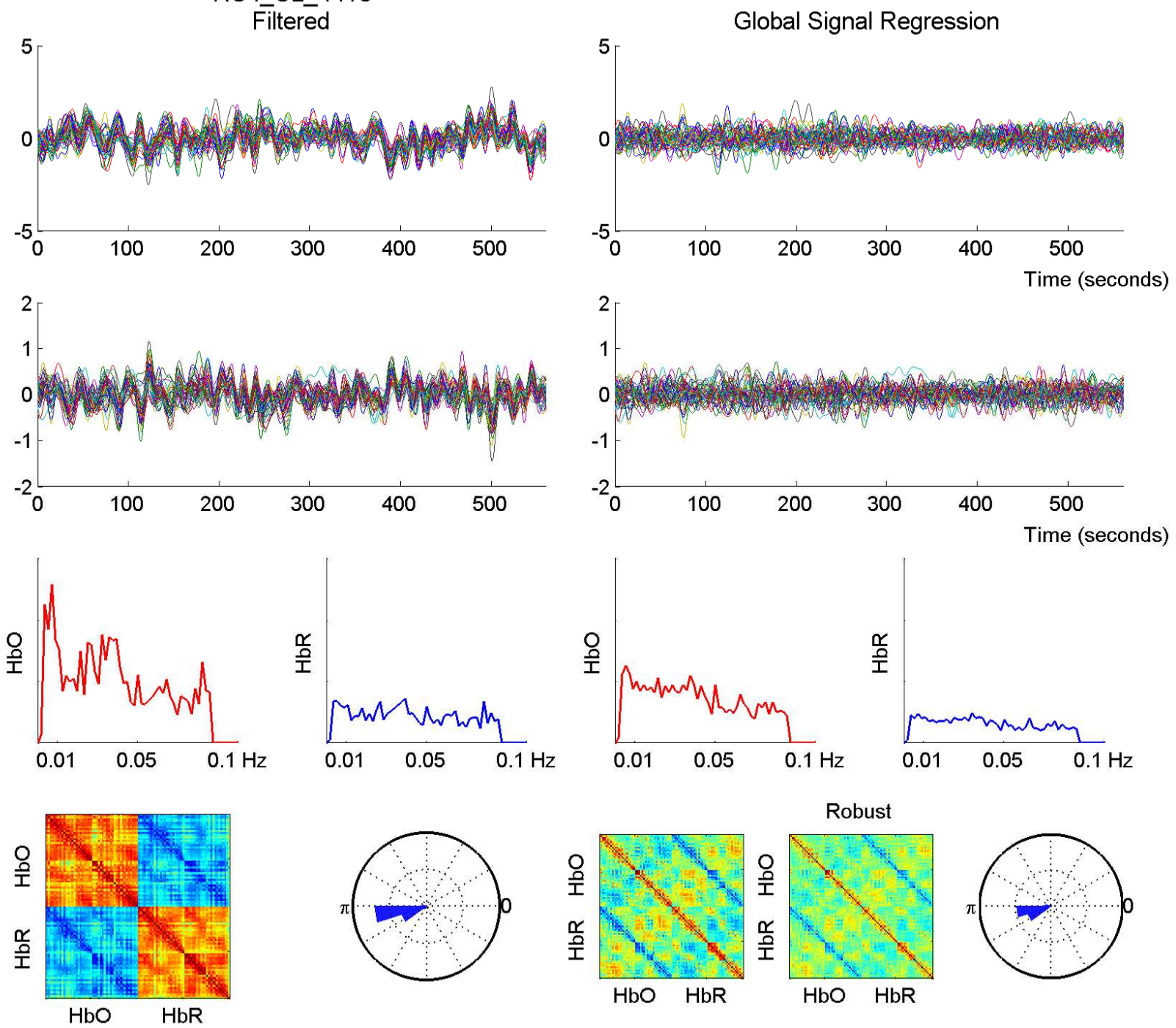

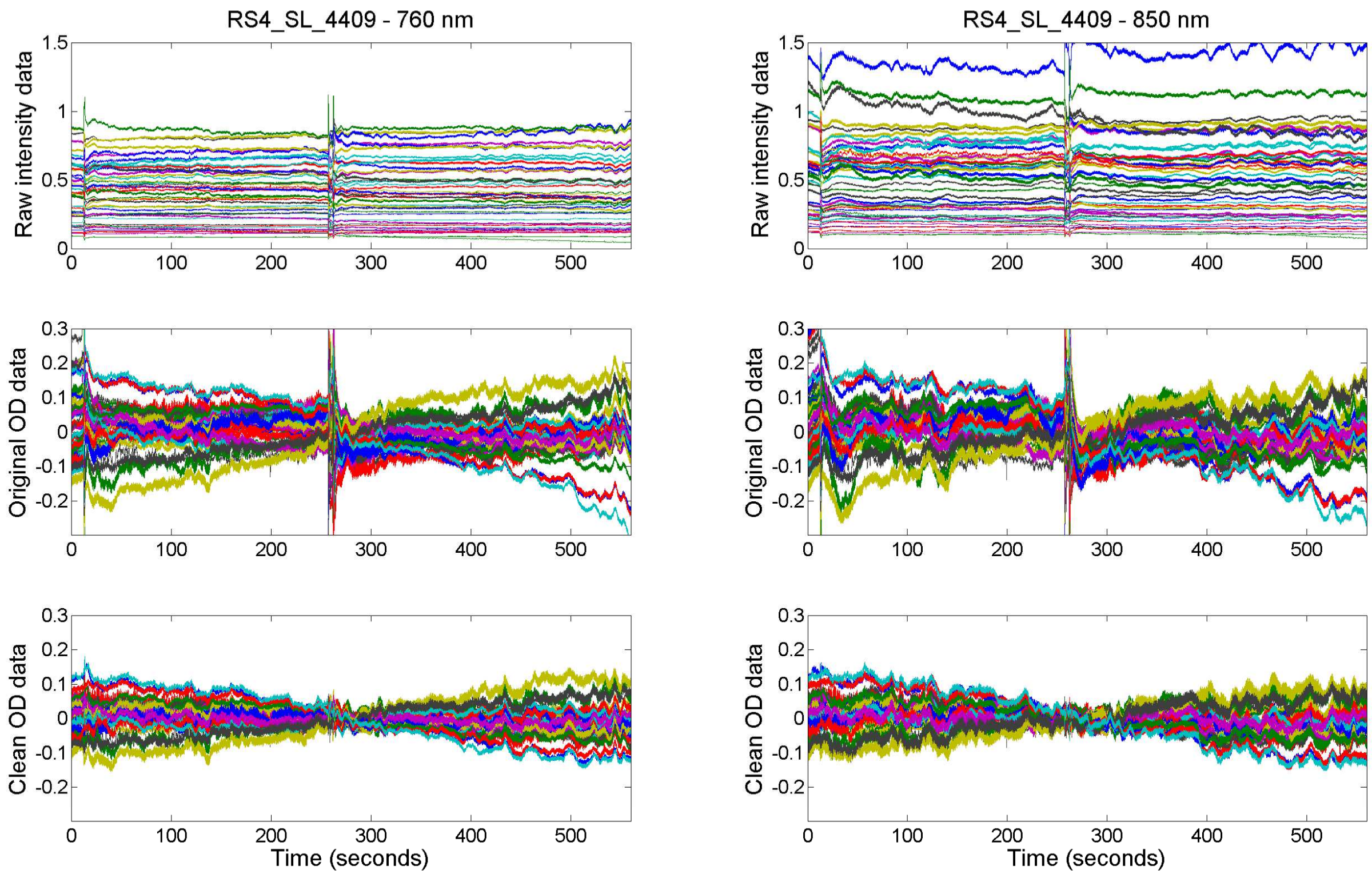
RS4_SL_4409

Raw
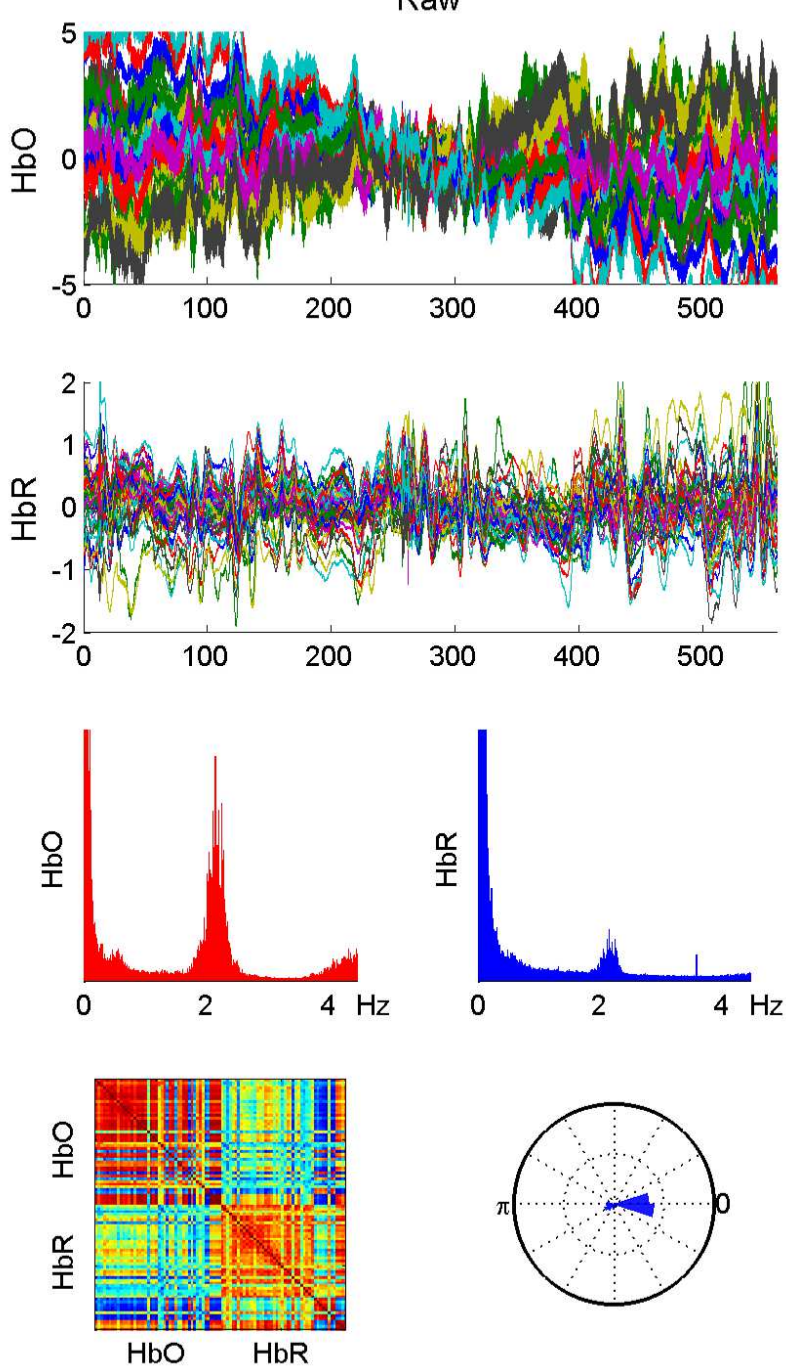

Filtered
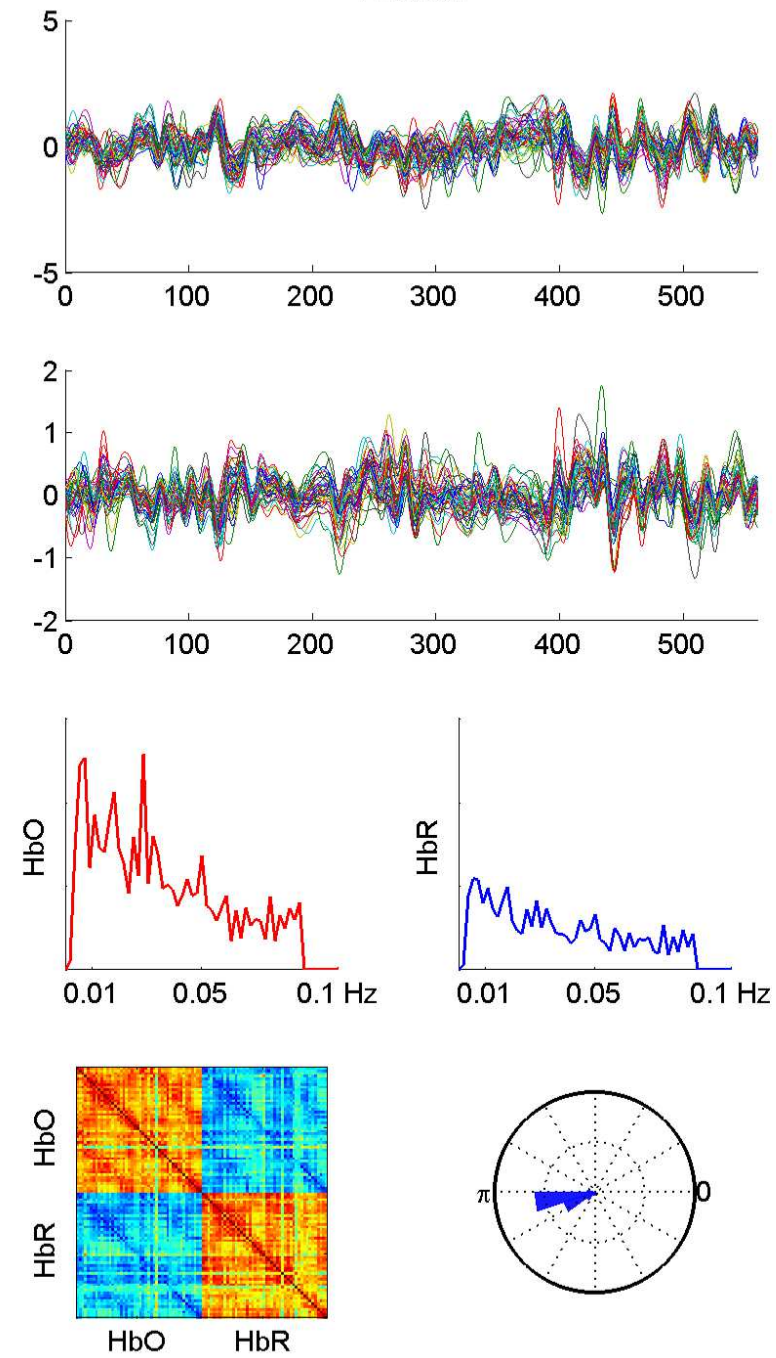
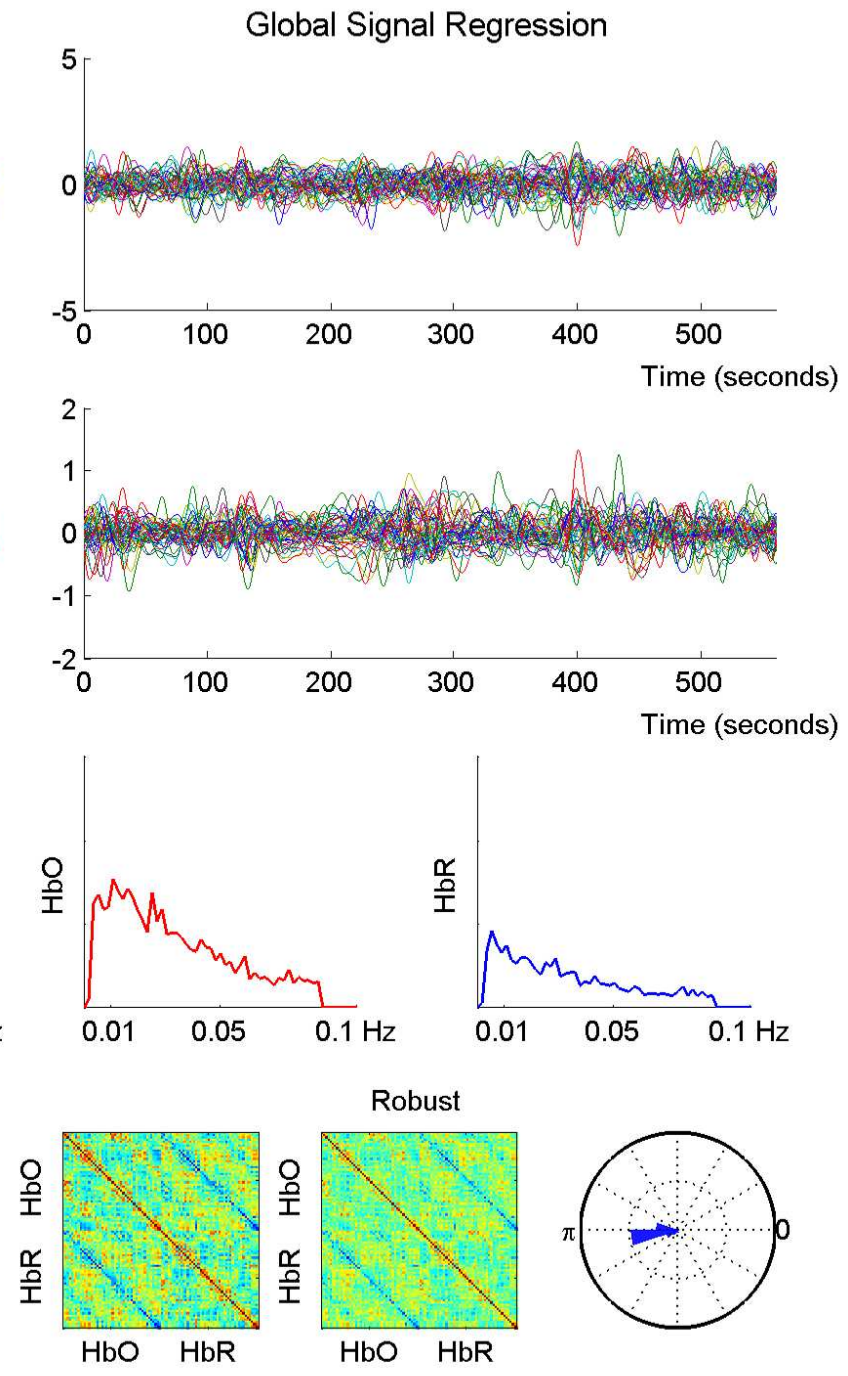

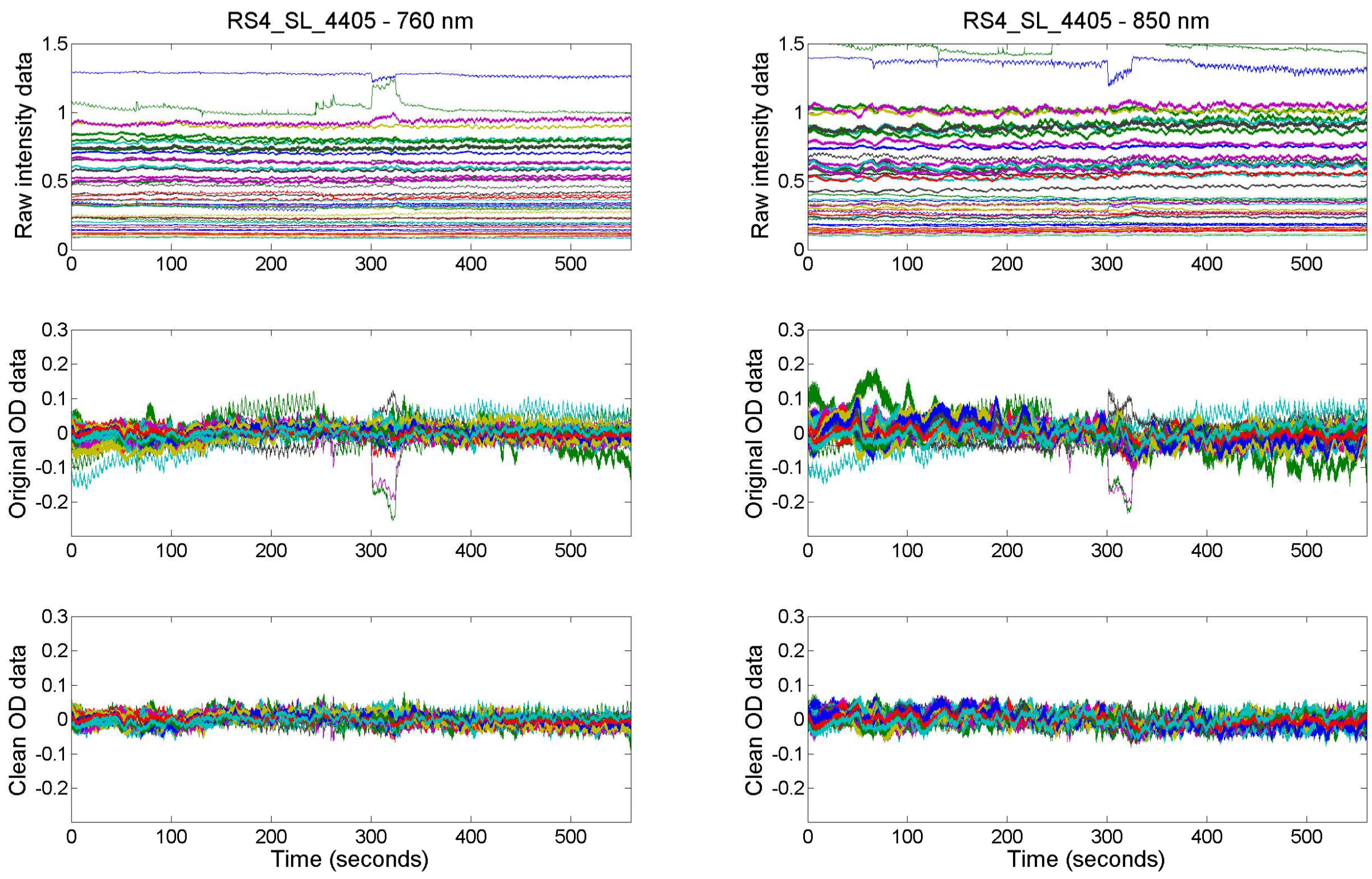
RS4_SL_4405

Raw

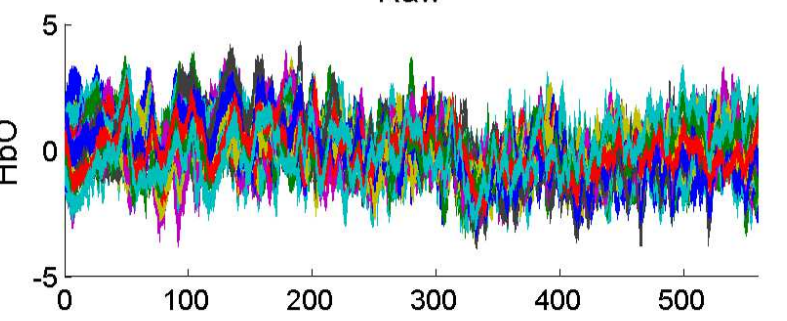

${ }_{-2}^{2}{ }_{0}^{1}$
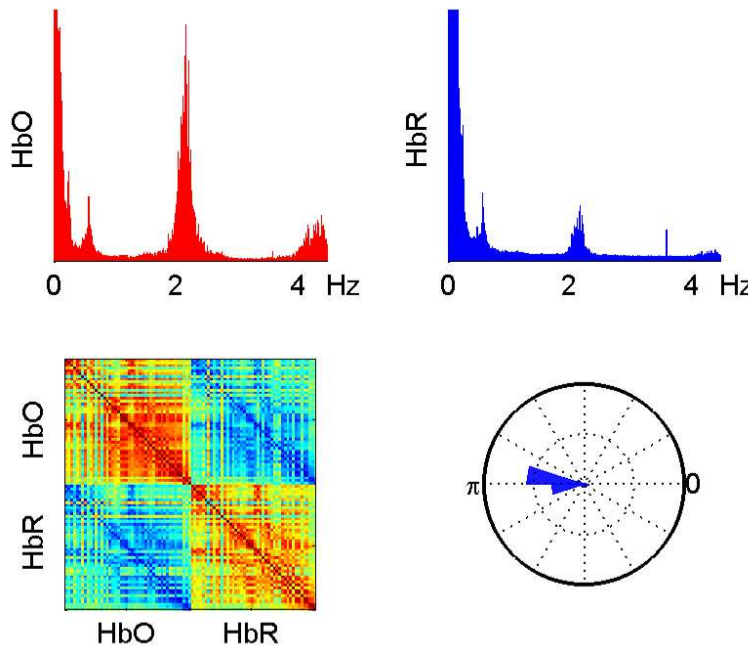

5

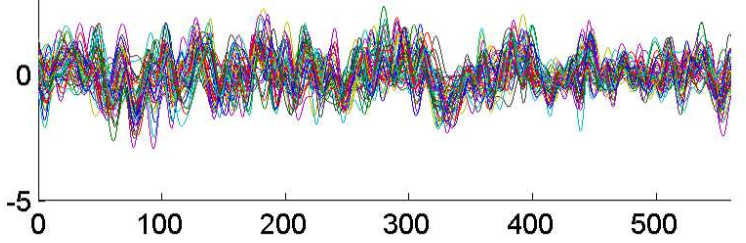

Filtered
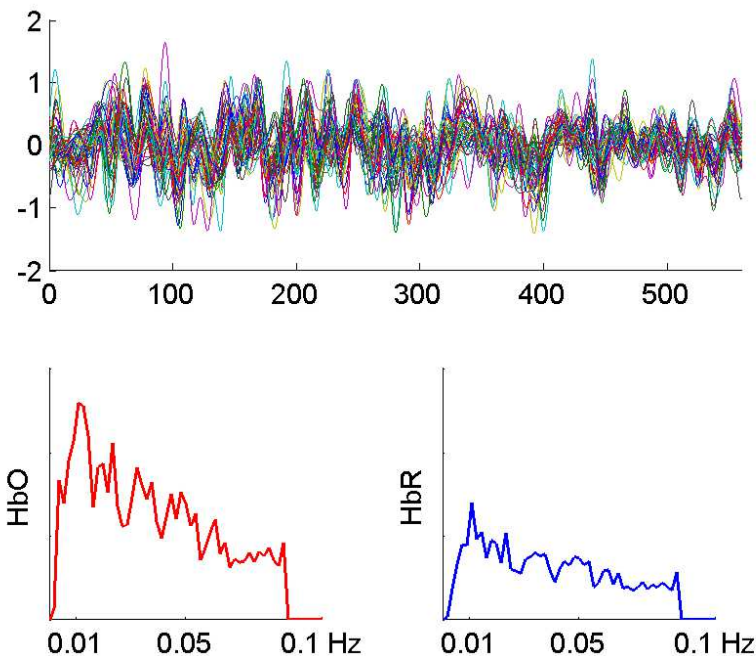

웅
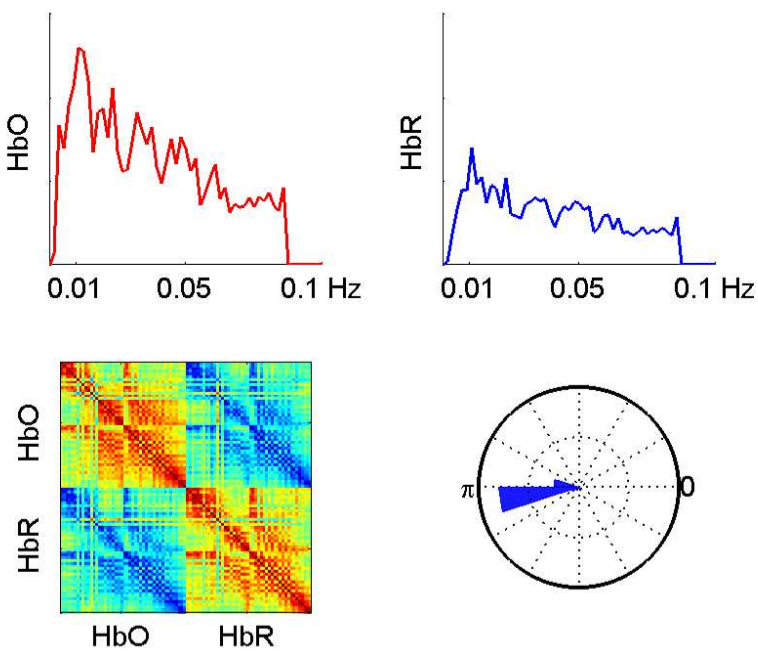
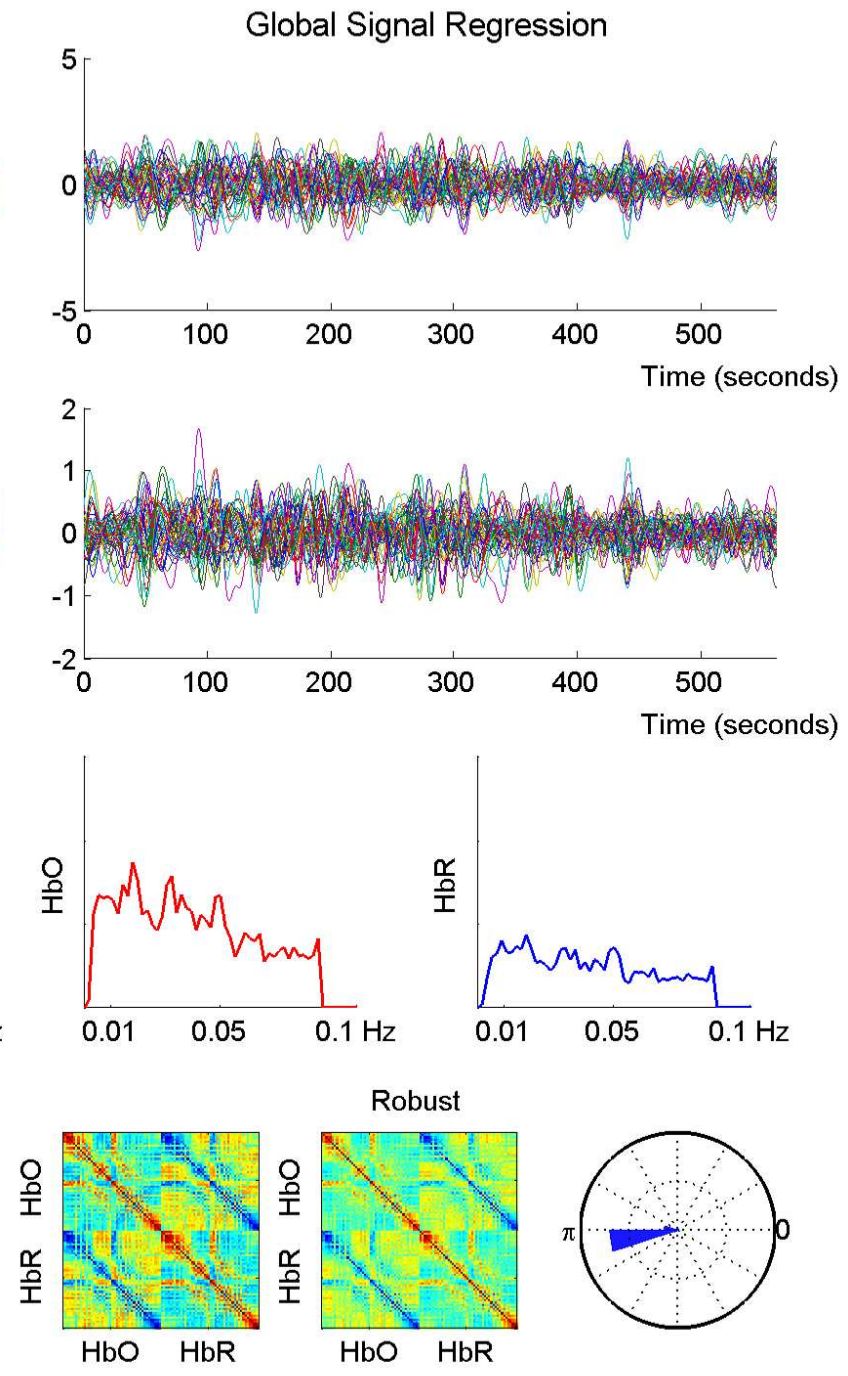

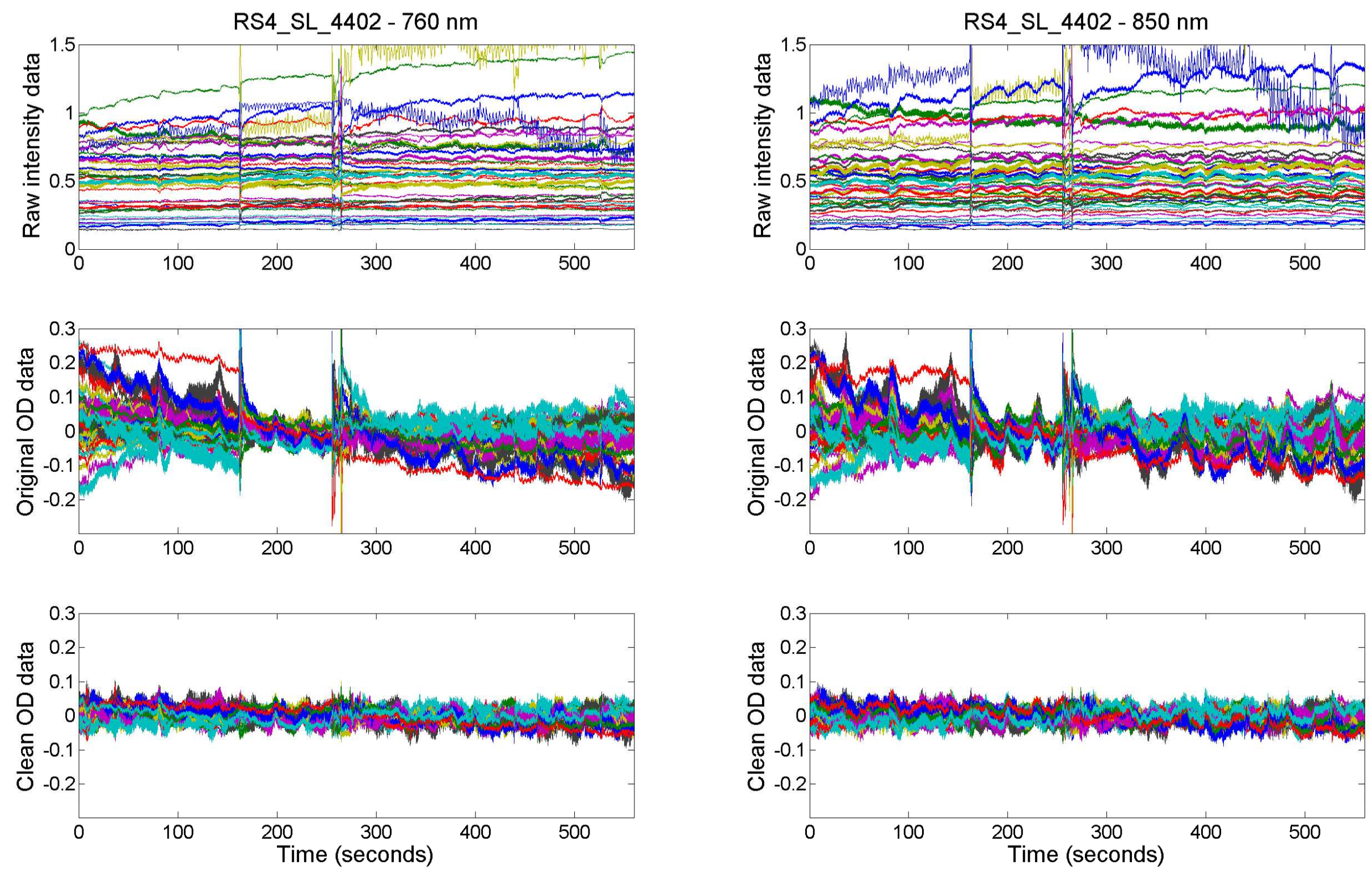
Raw

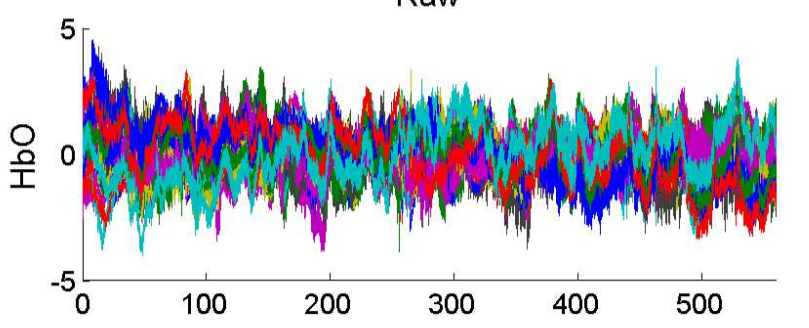

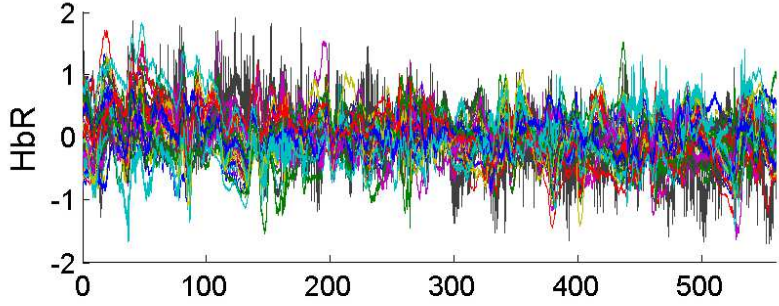
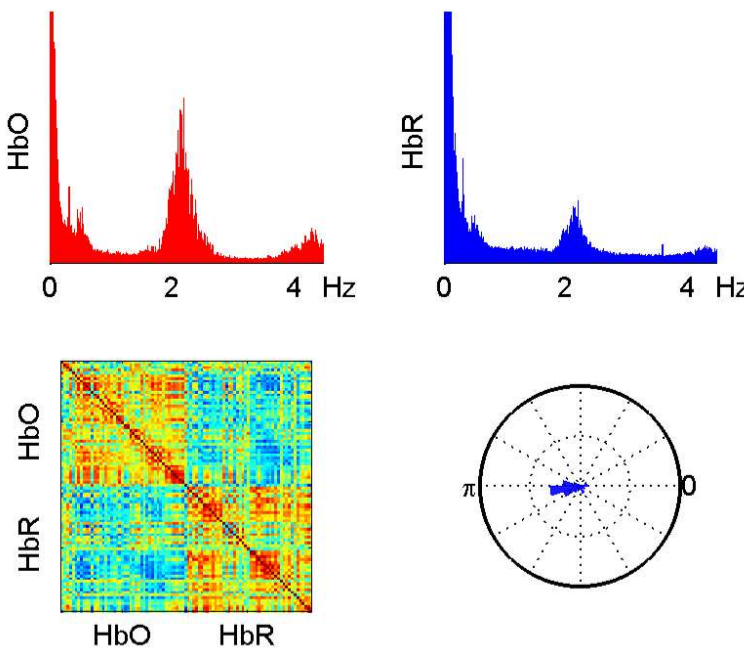

RS4_SL_4402

Filtered
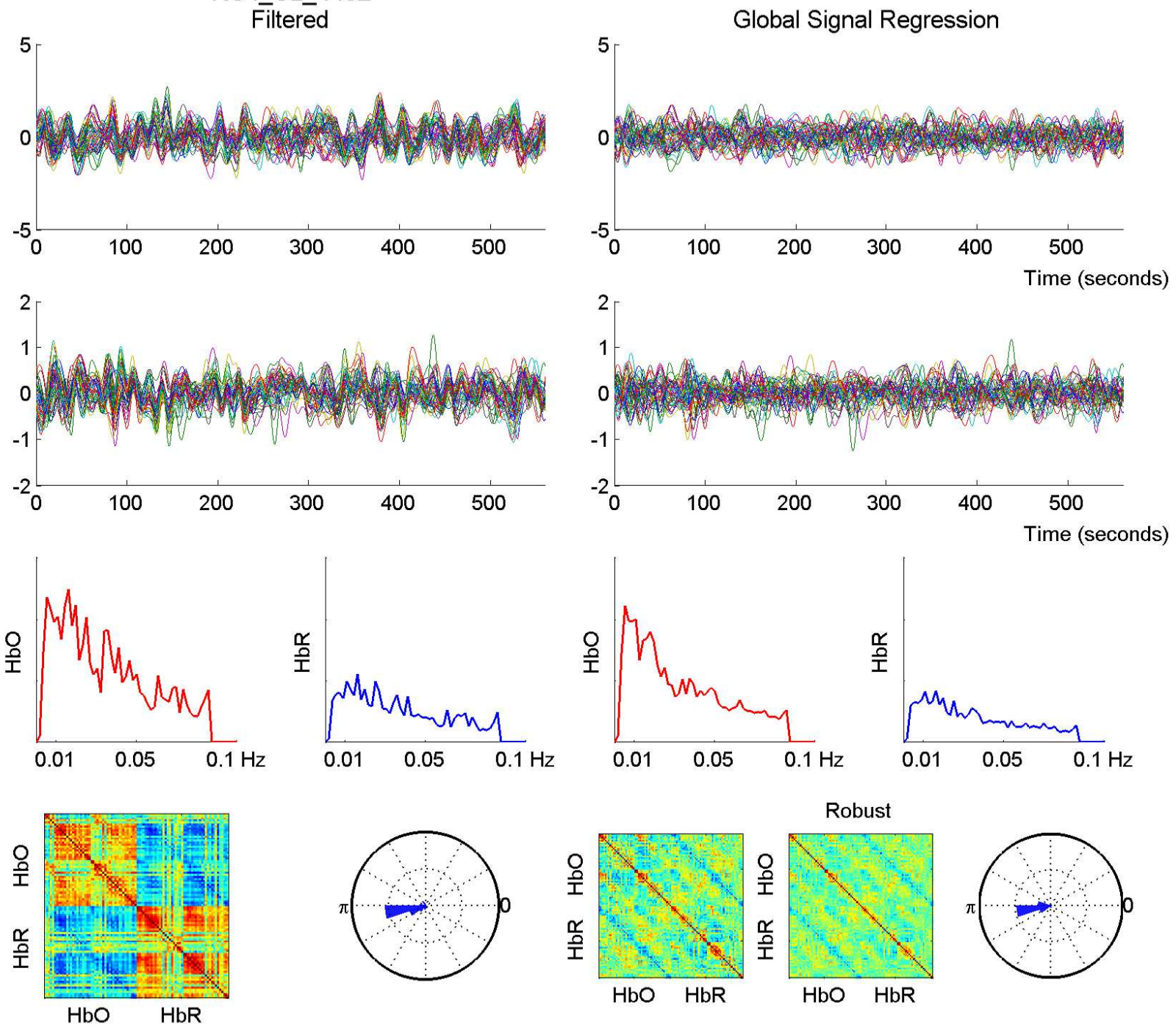

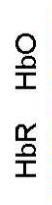

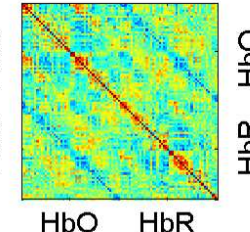

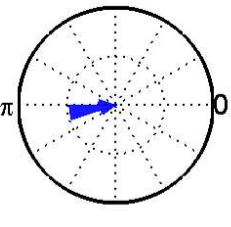

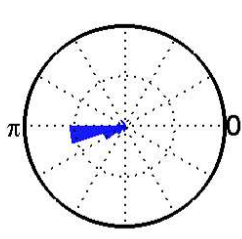

Robust

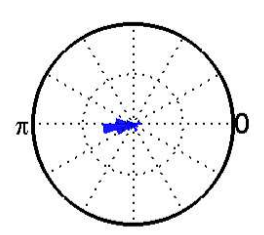


RS4 SL 4397 - 760 nm
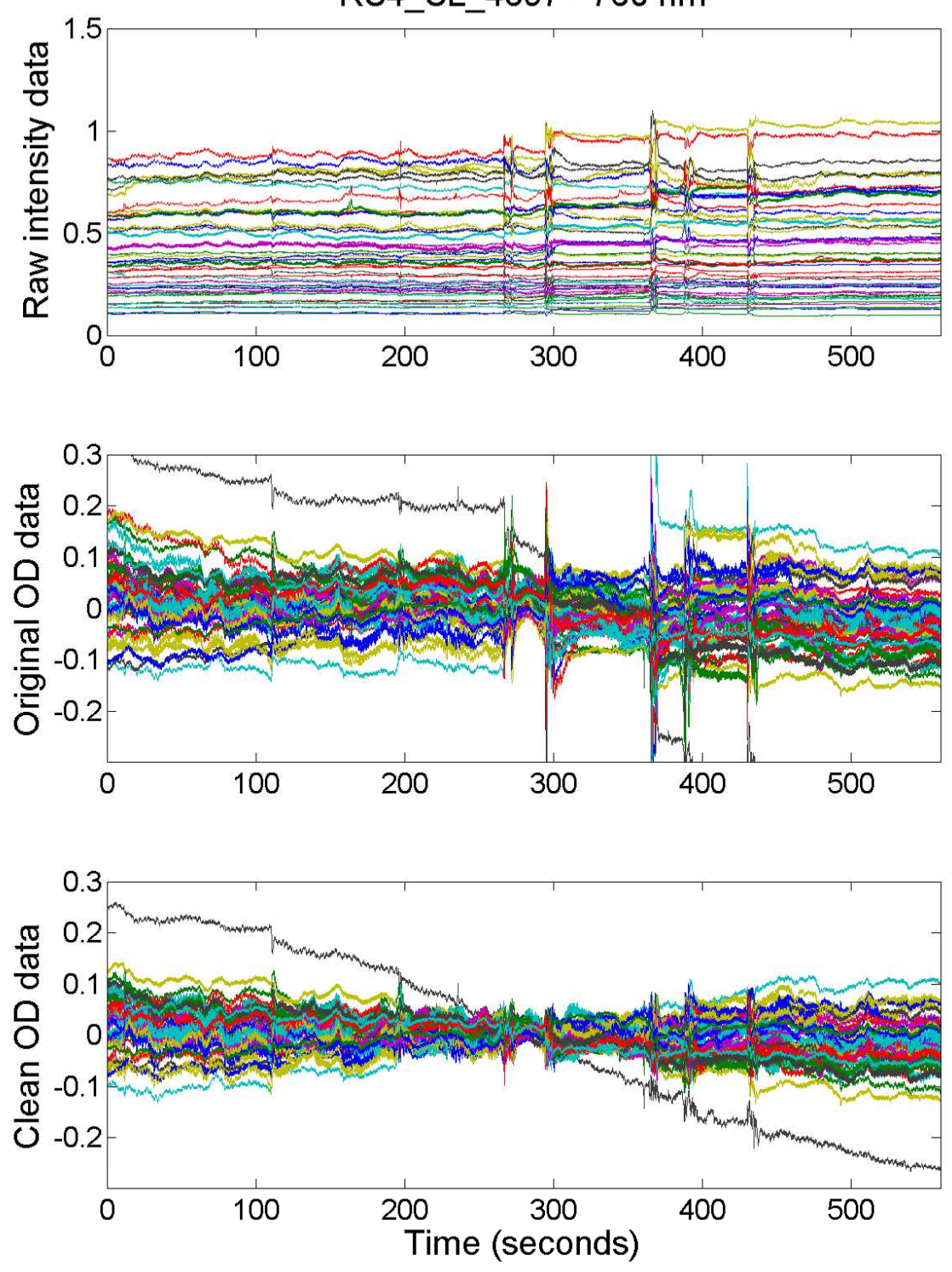

RS4 SL $4397-850 \mathrm{~nm}$
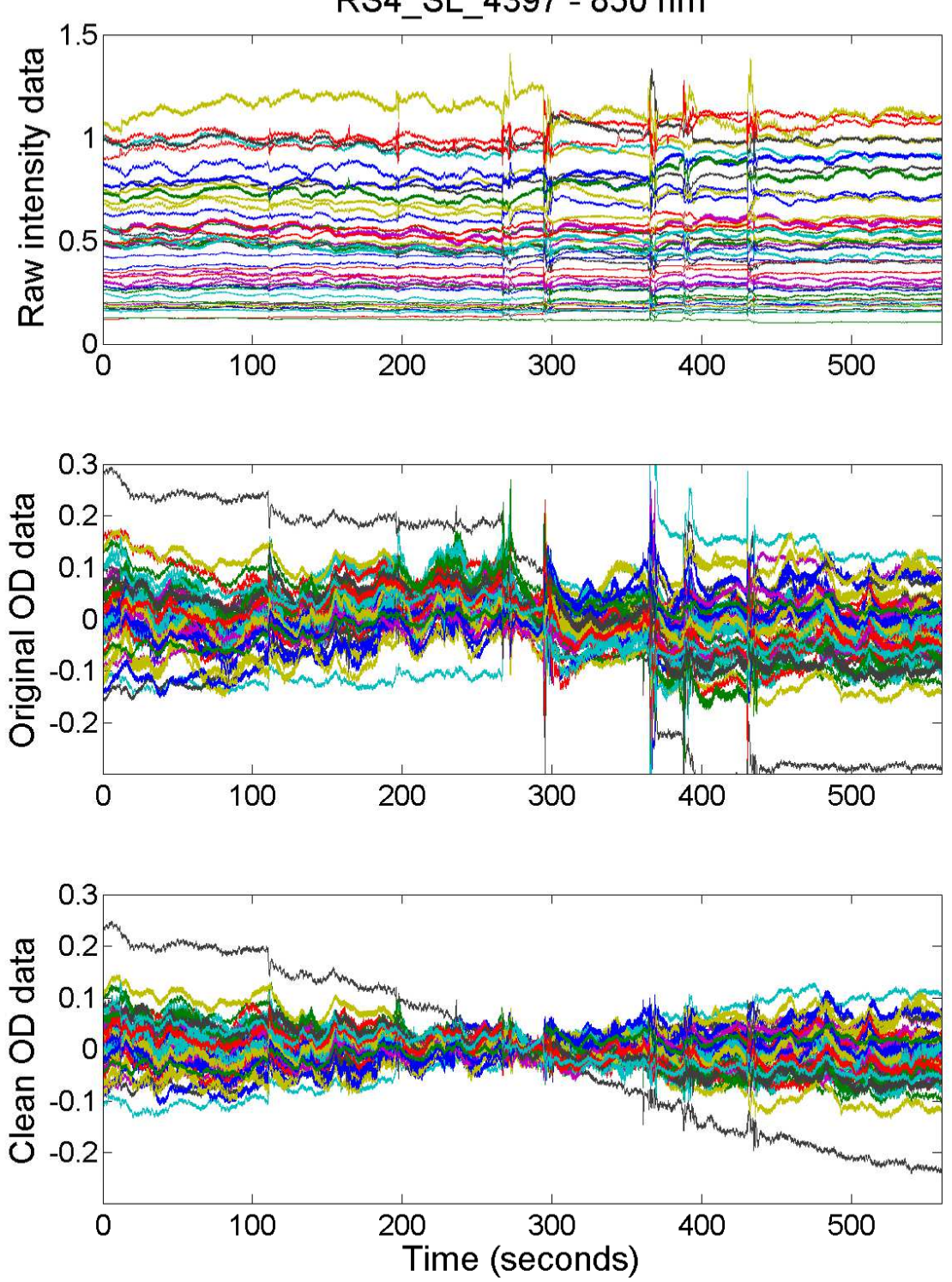
RS4_SL_4397

Raw
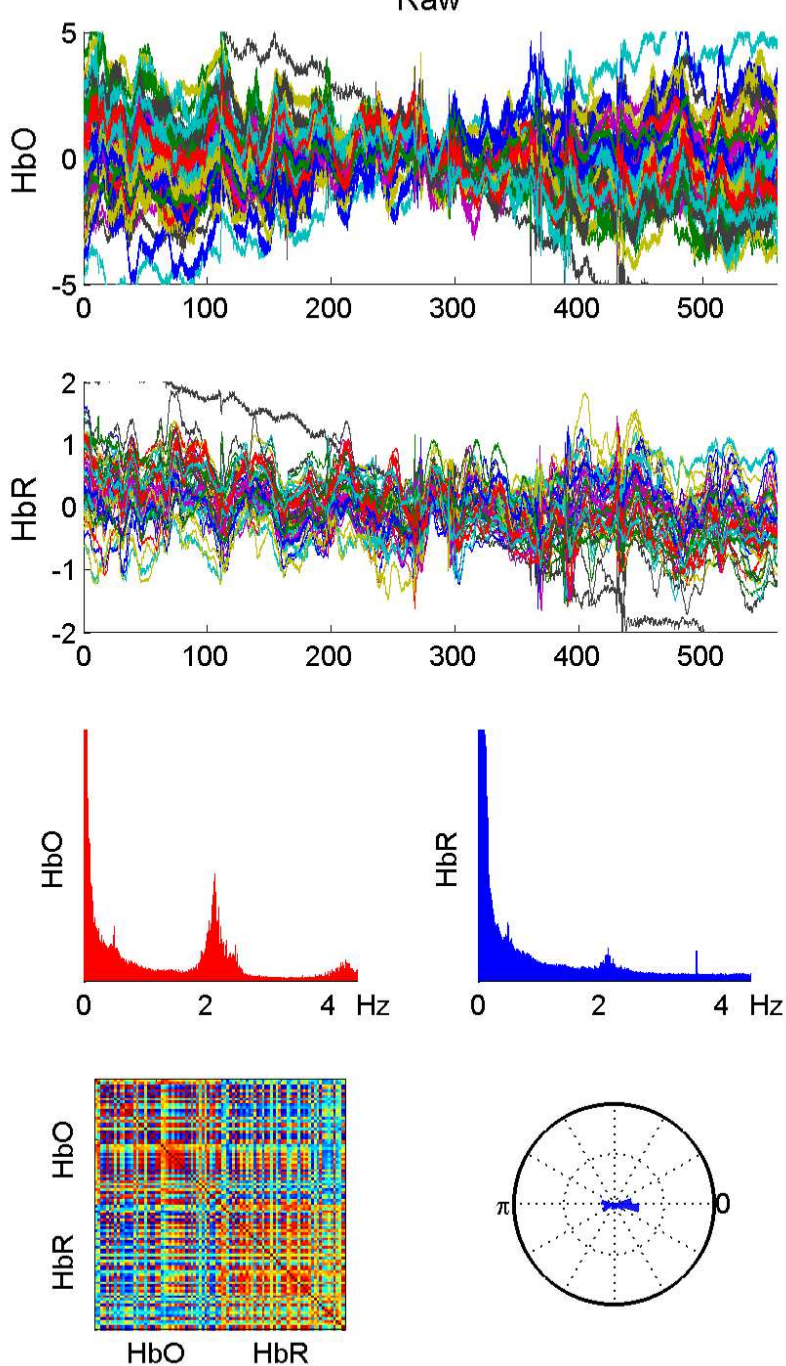

Filtered
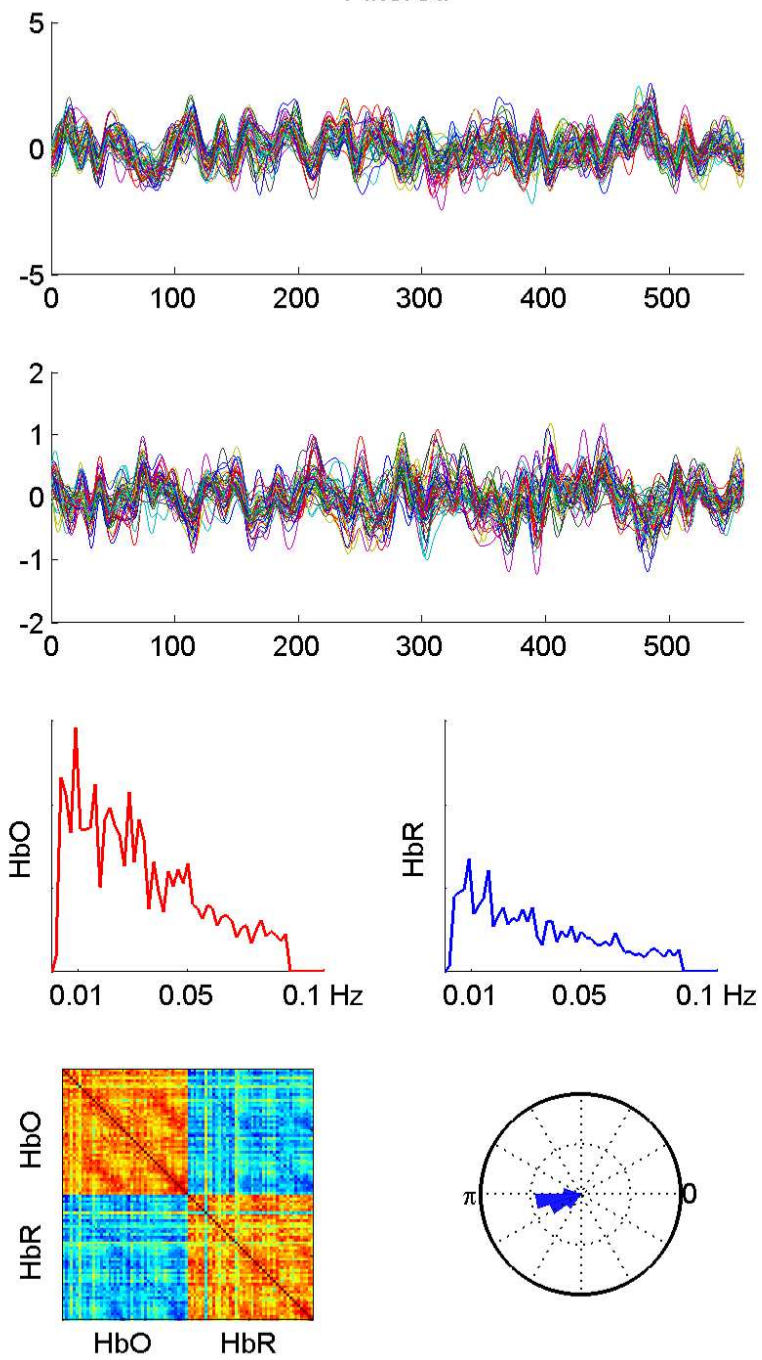
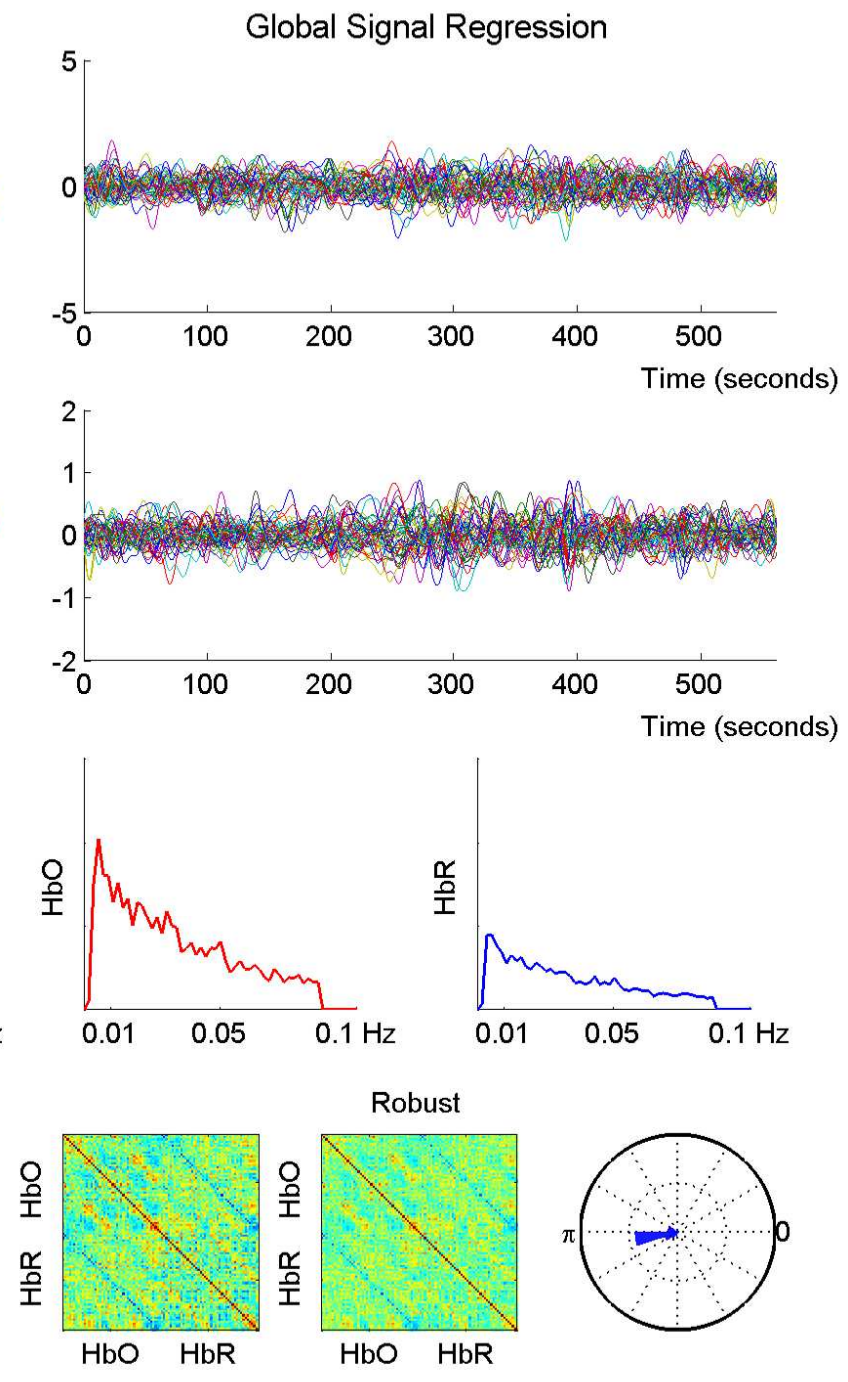
RS4 SL 4395 - 760 nm
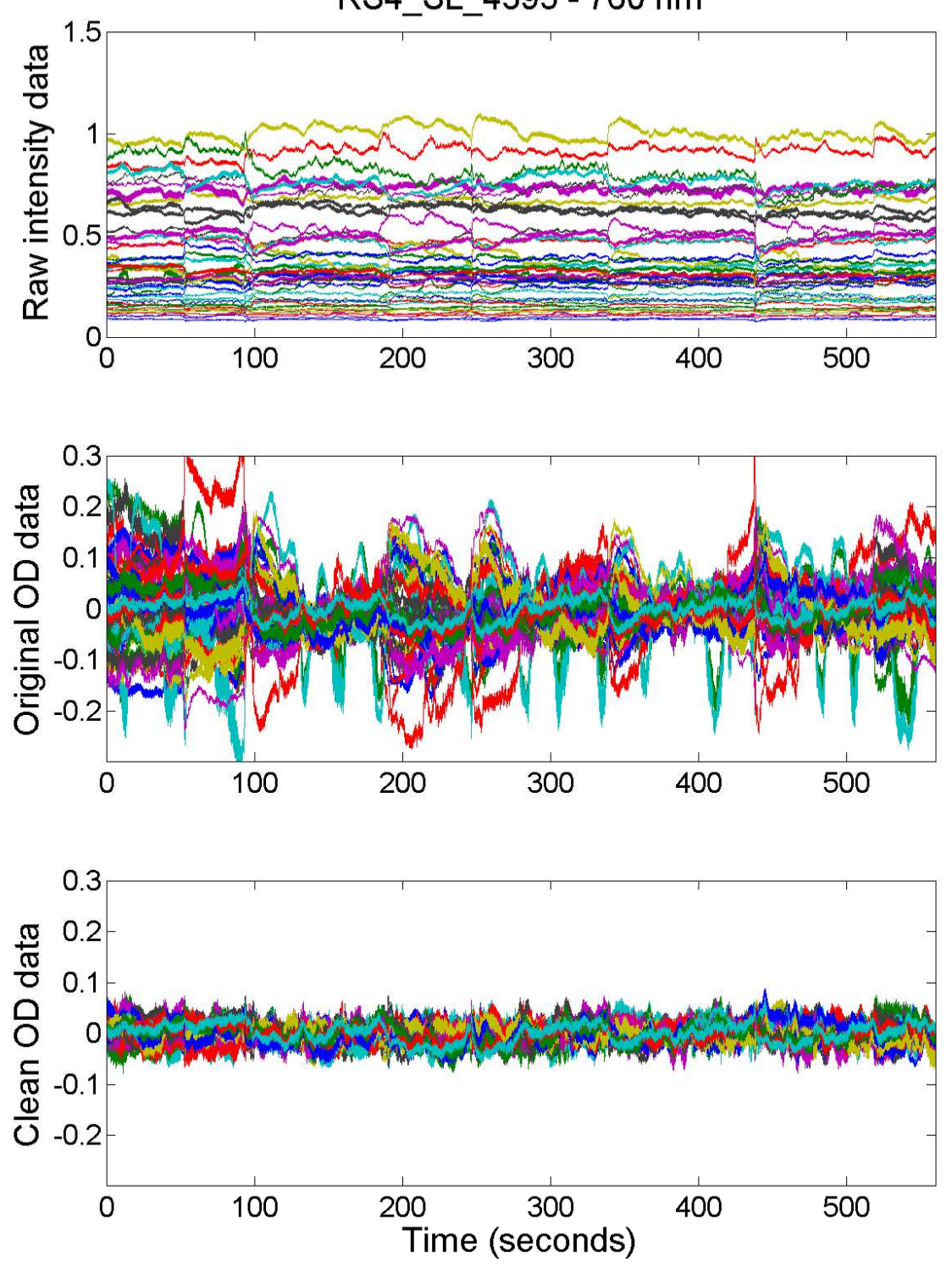

RS4 SL $4395-850 \mathrm{~nm}$
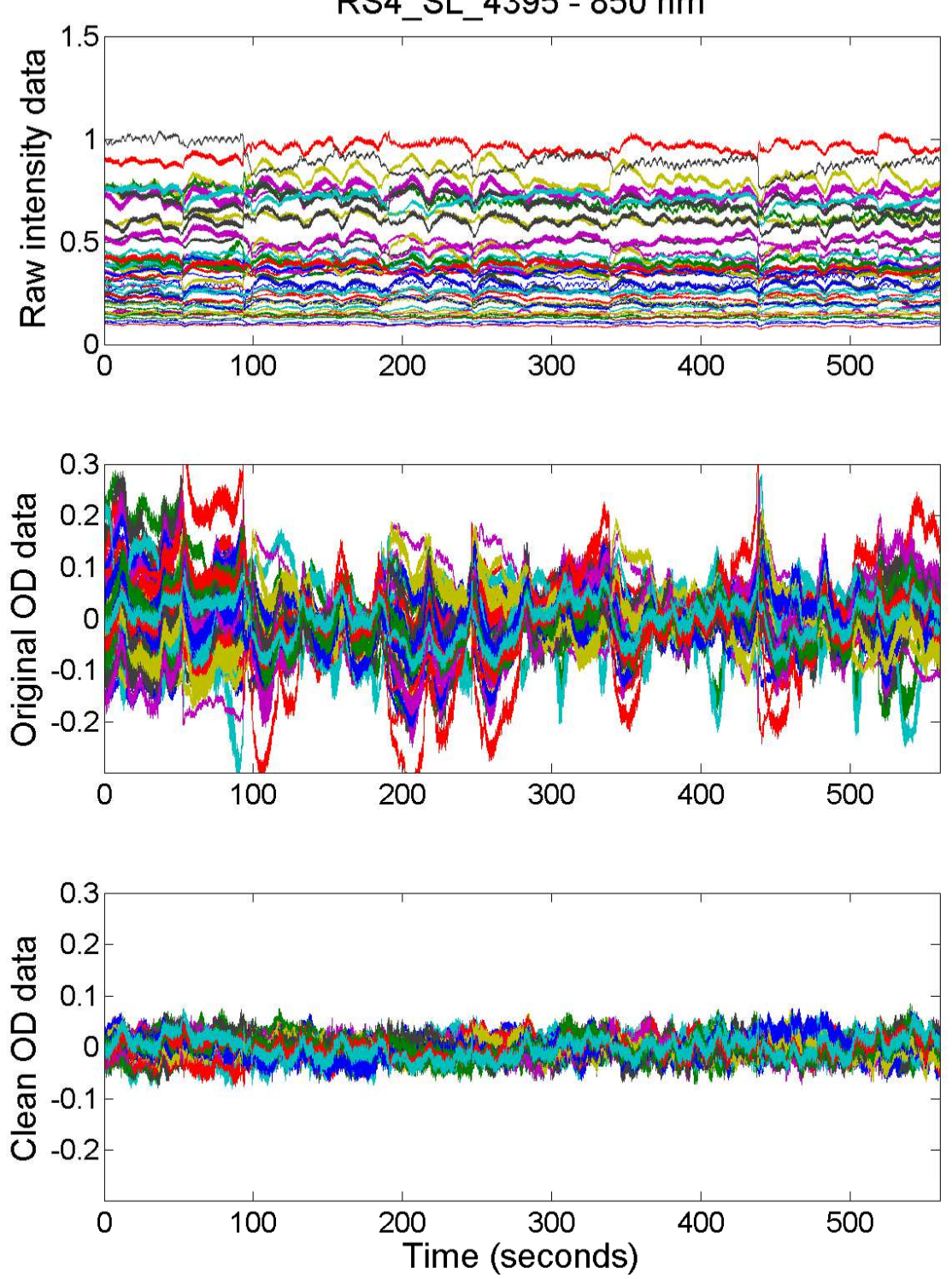
Raw
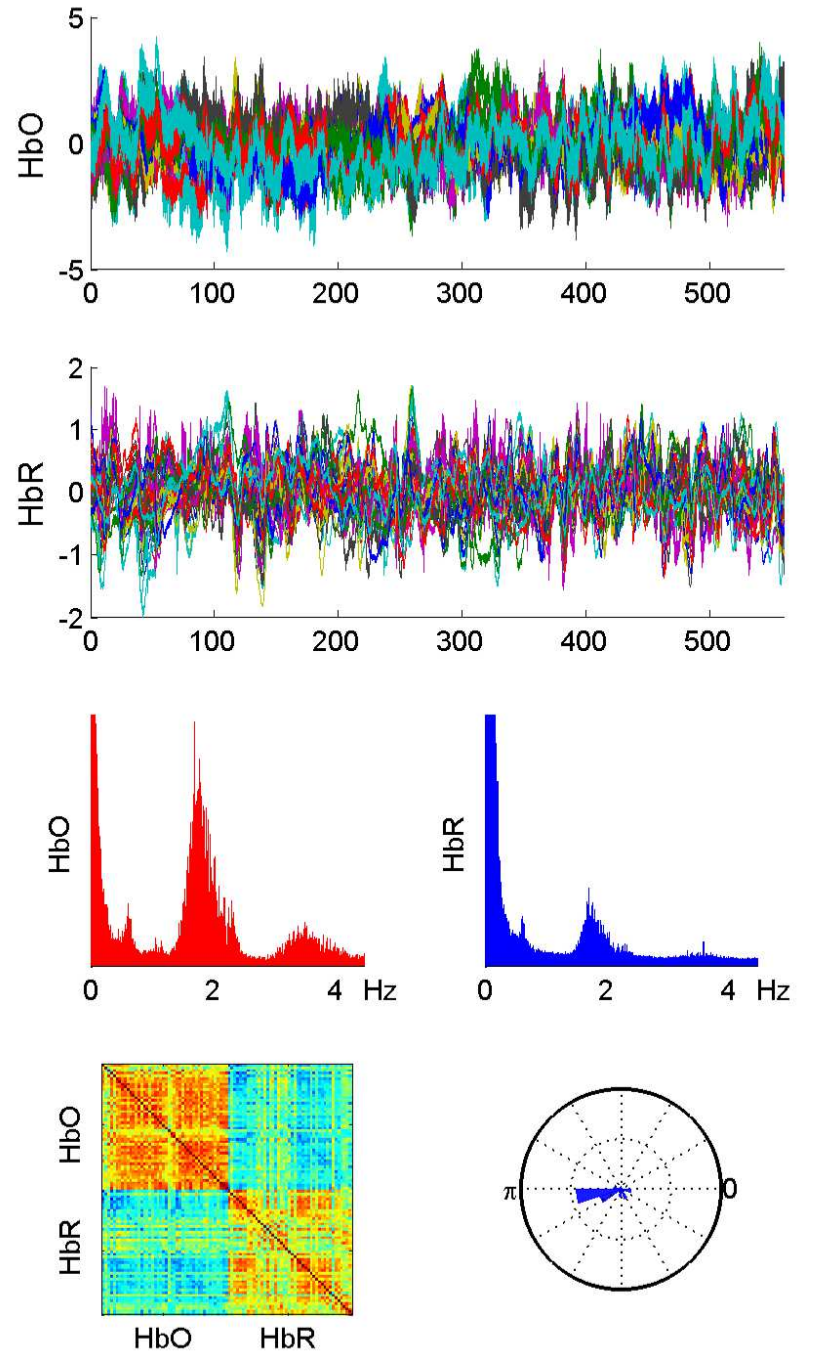

RS4_SL_4395

Filtered

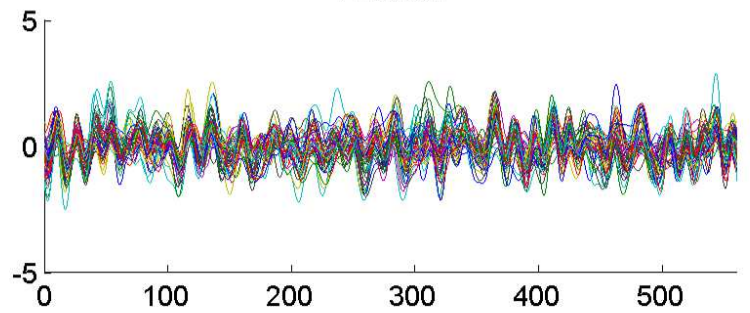

2
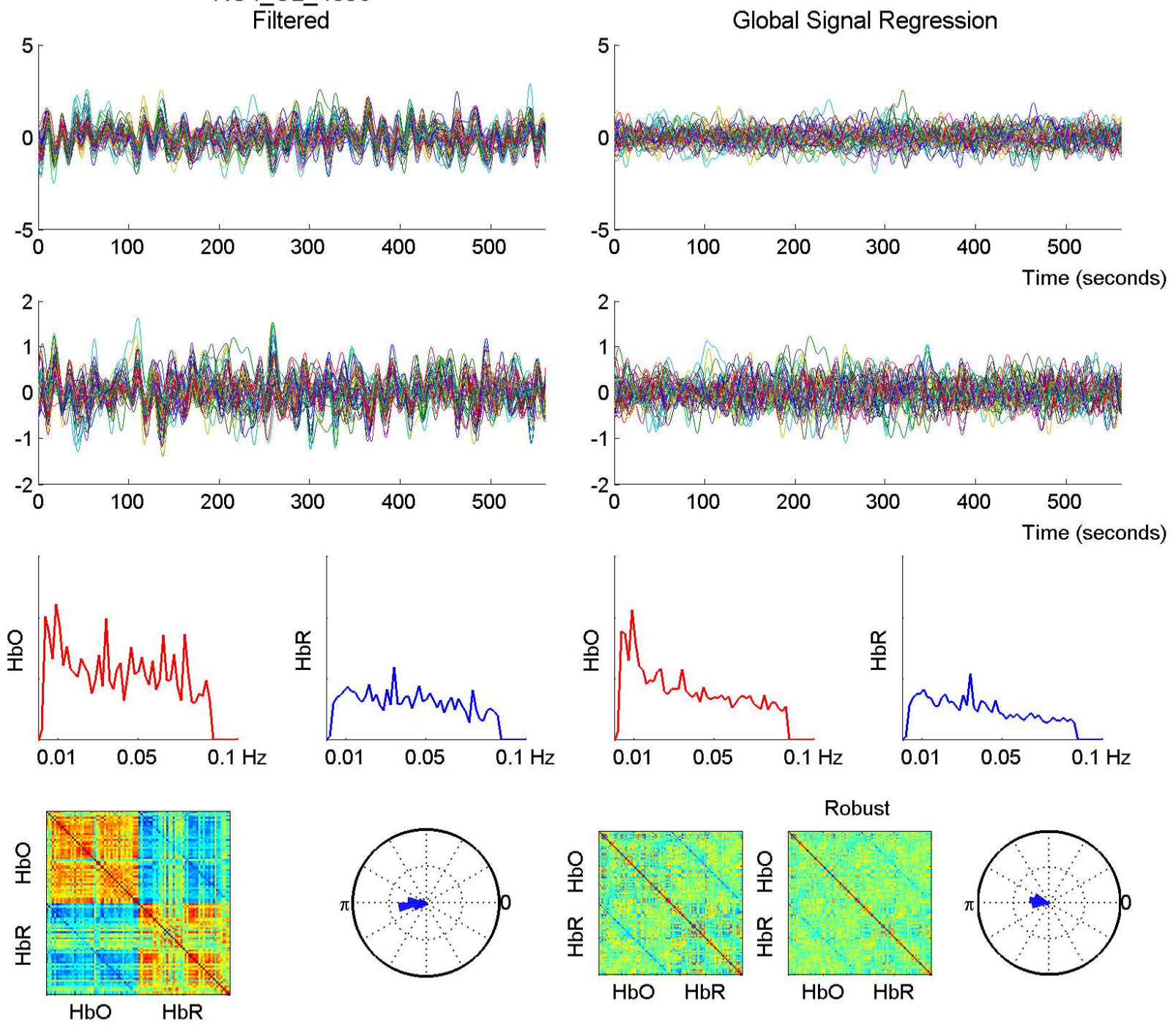
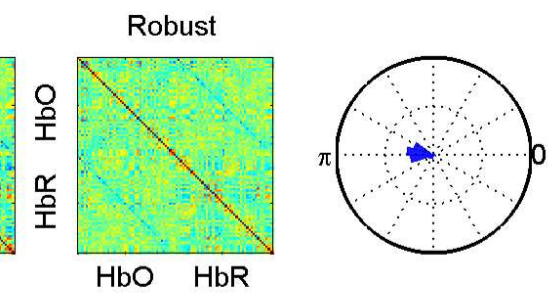

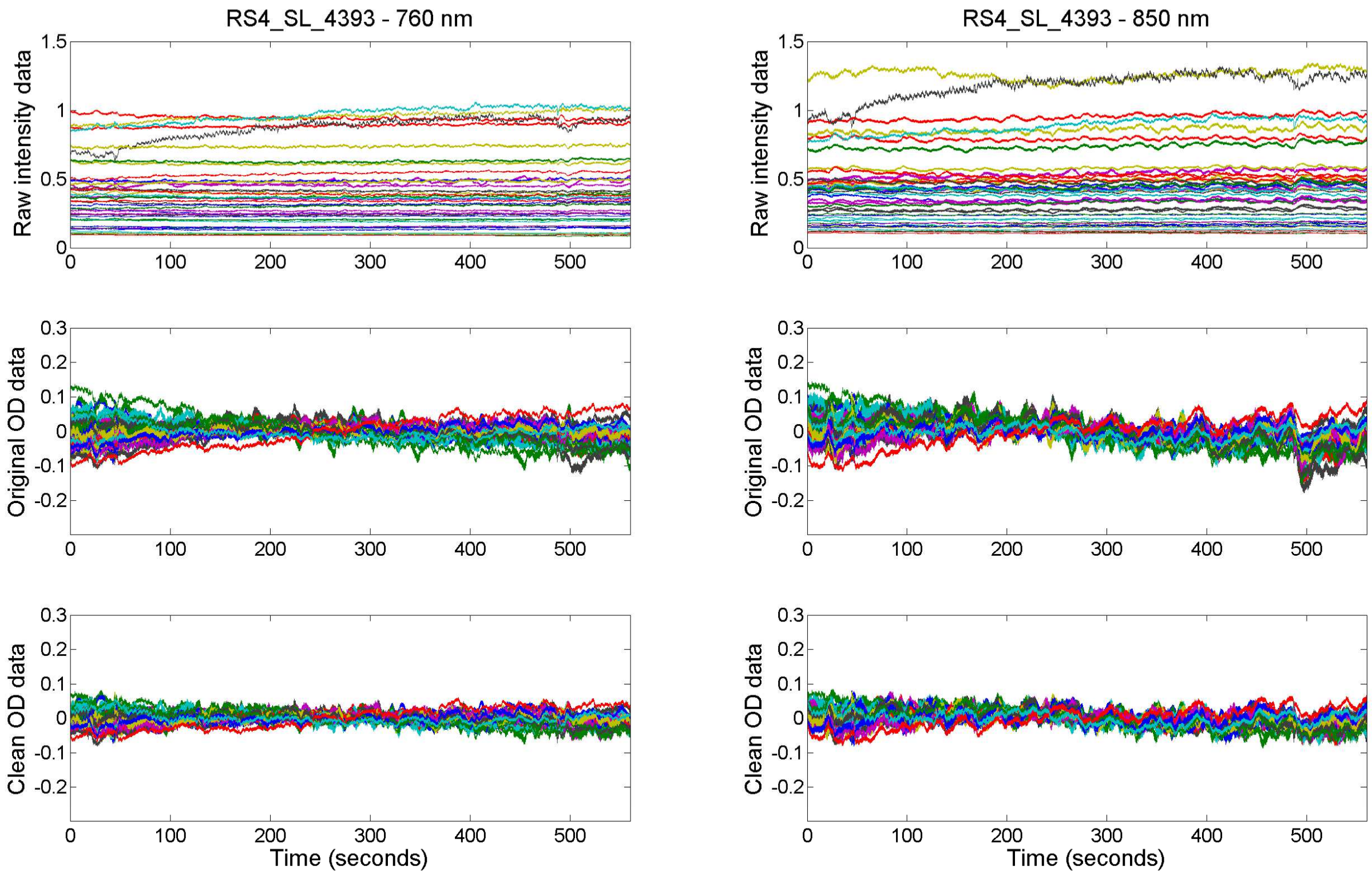
Raw
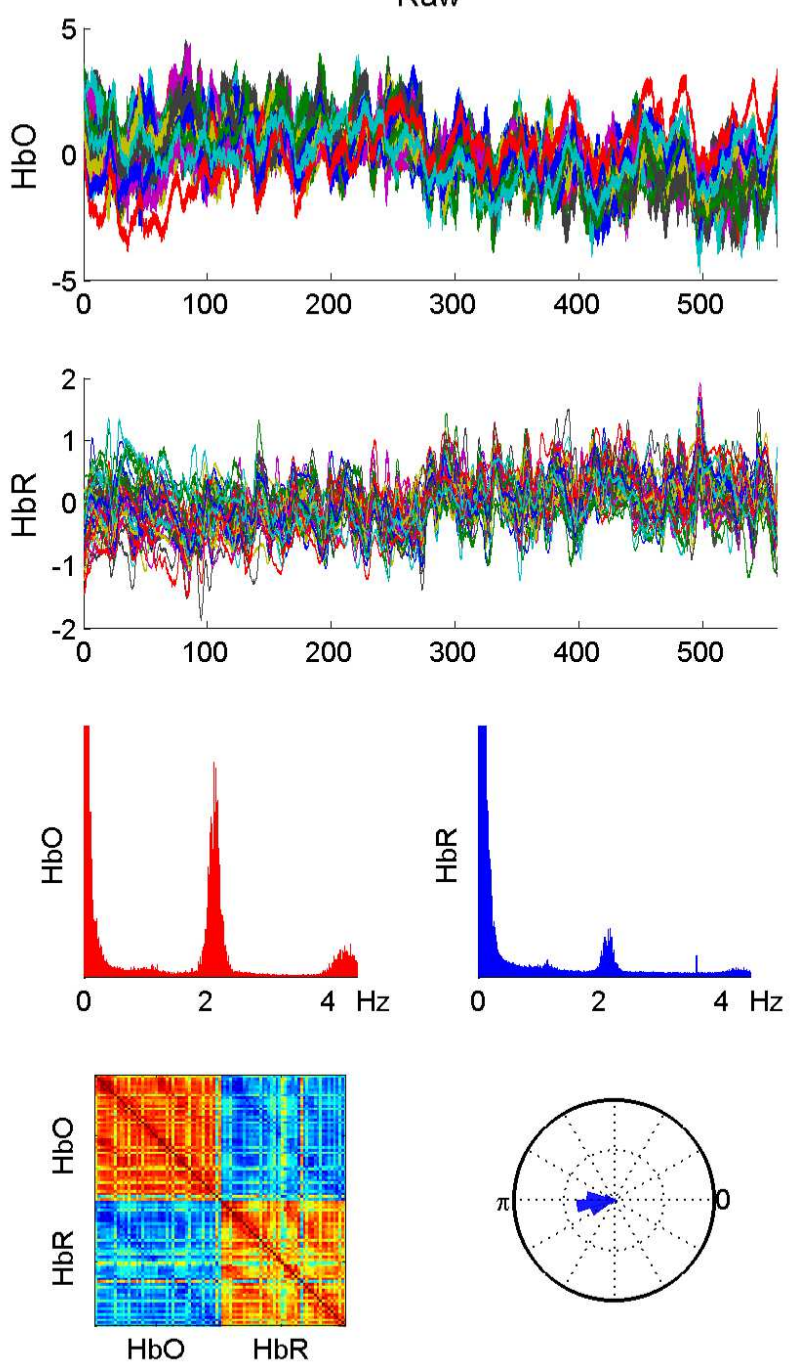

RS4 SL 4393

Filtered

5
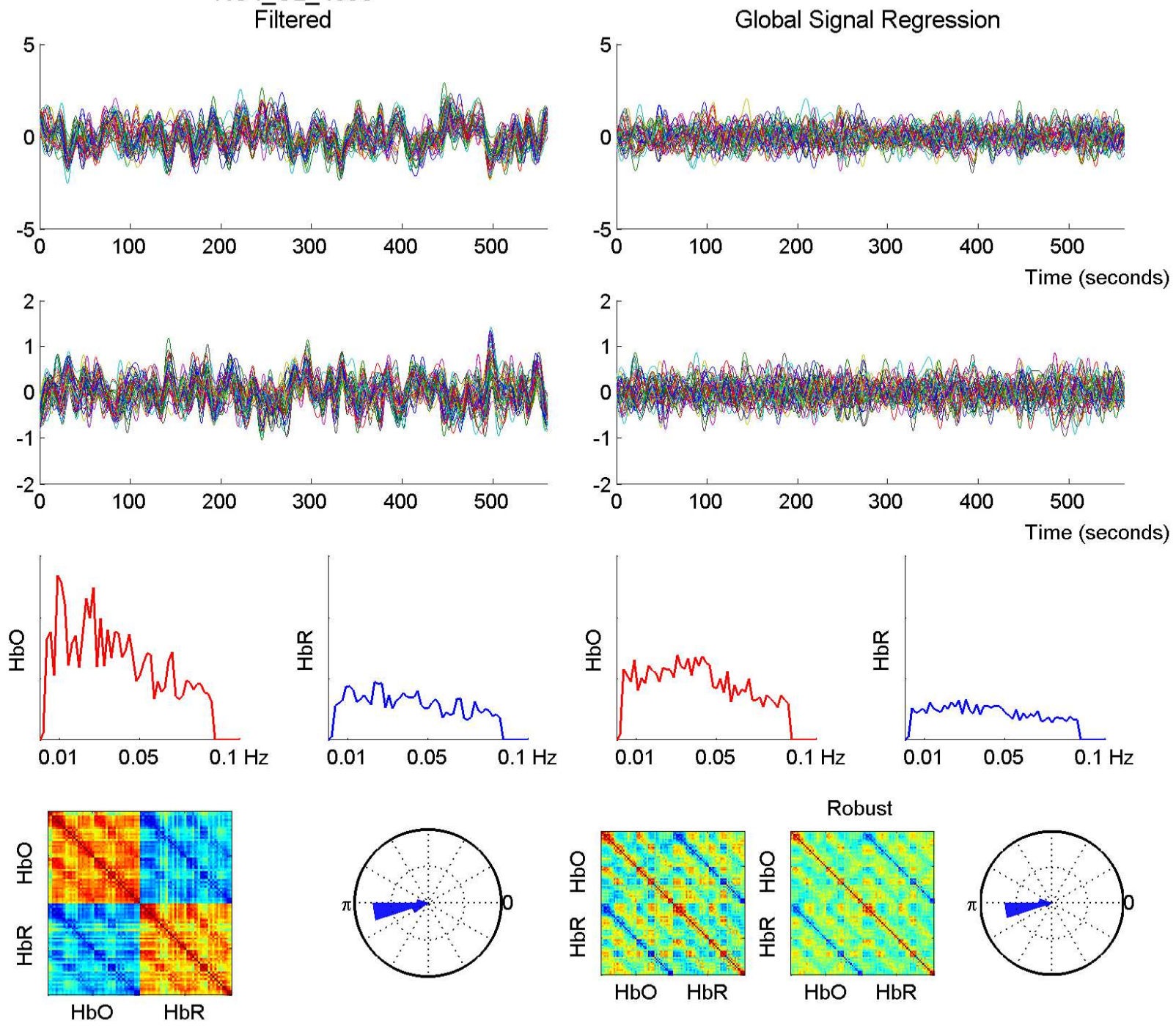
RS4 SL 4385 - 760 nm
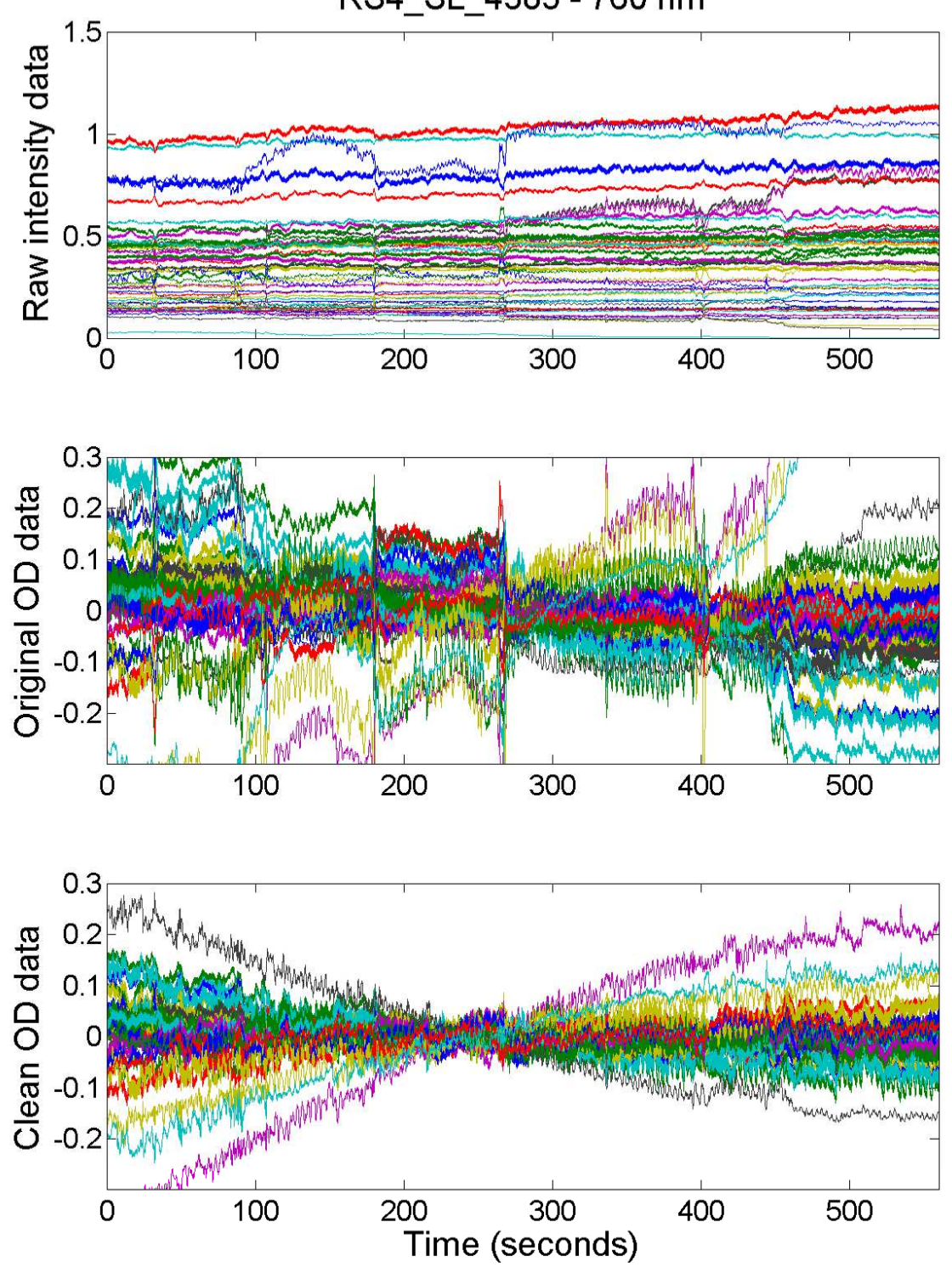

RS4 SL 4385 - 850 nm
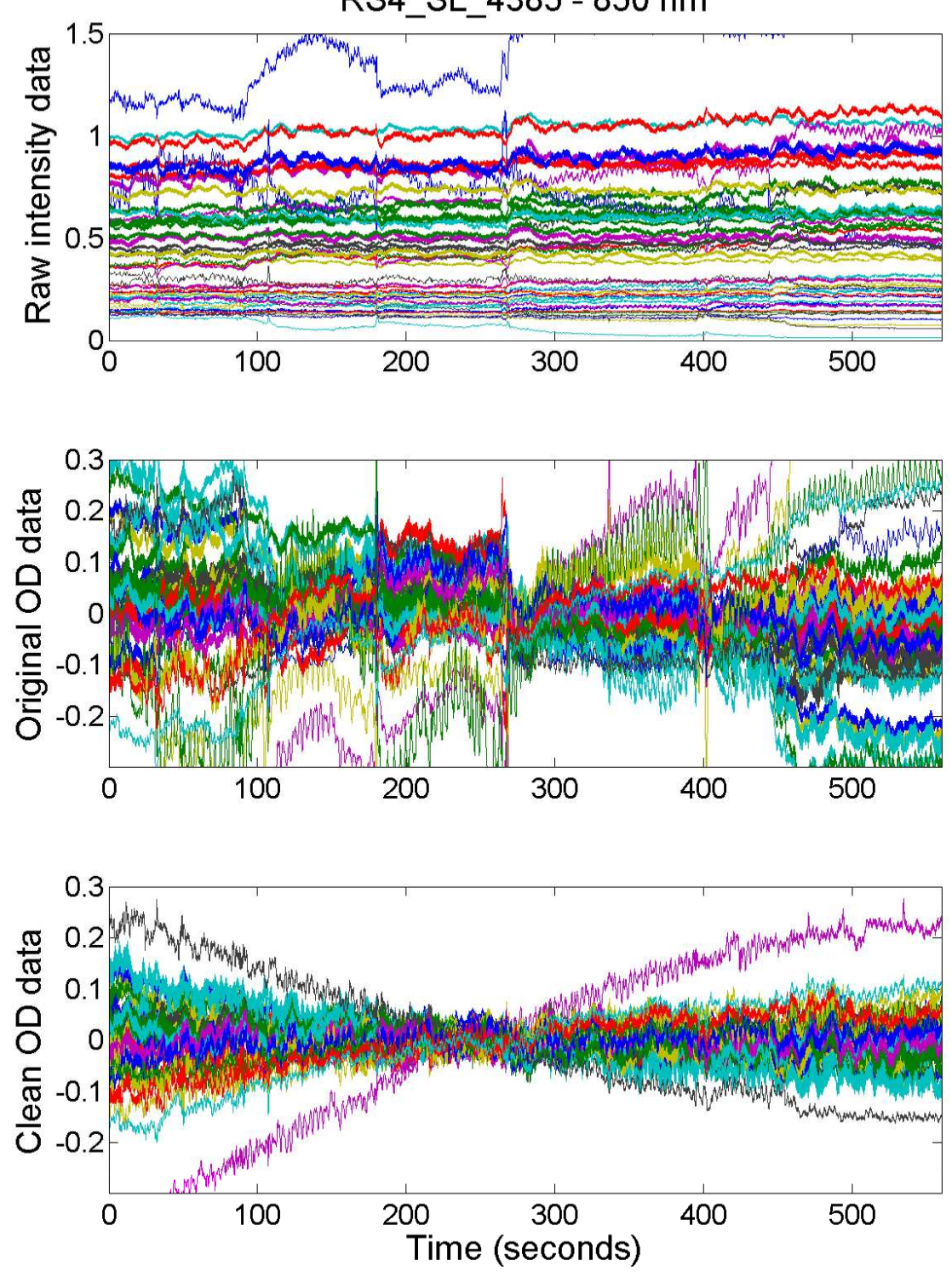
RS4_SL_4385

Raw
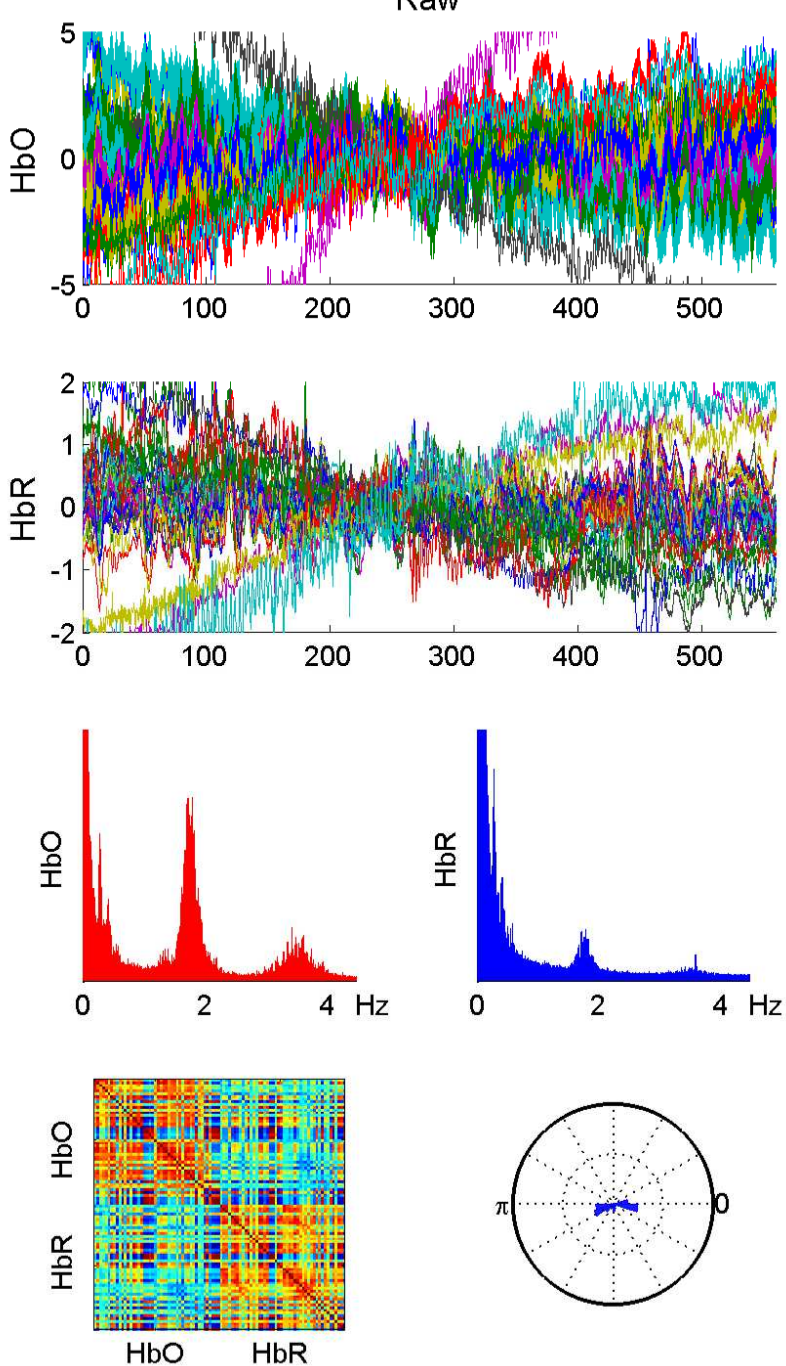
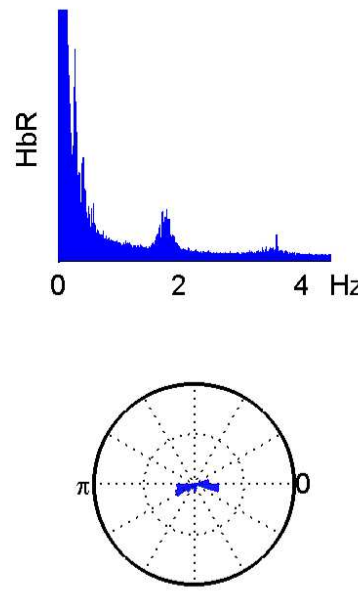

Filtered
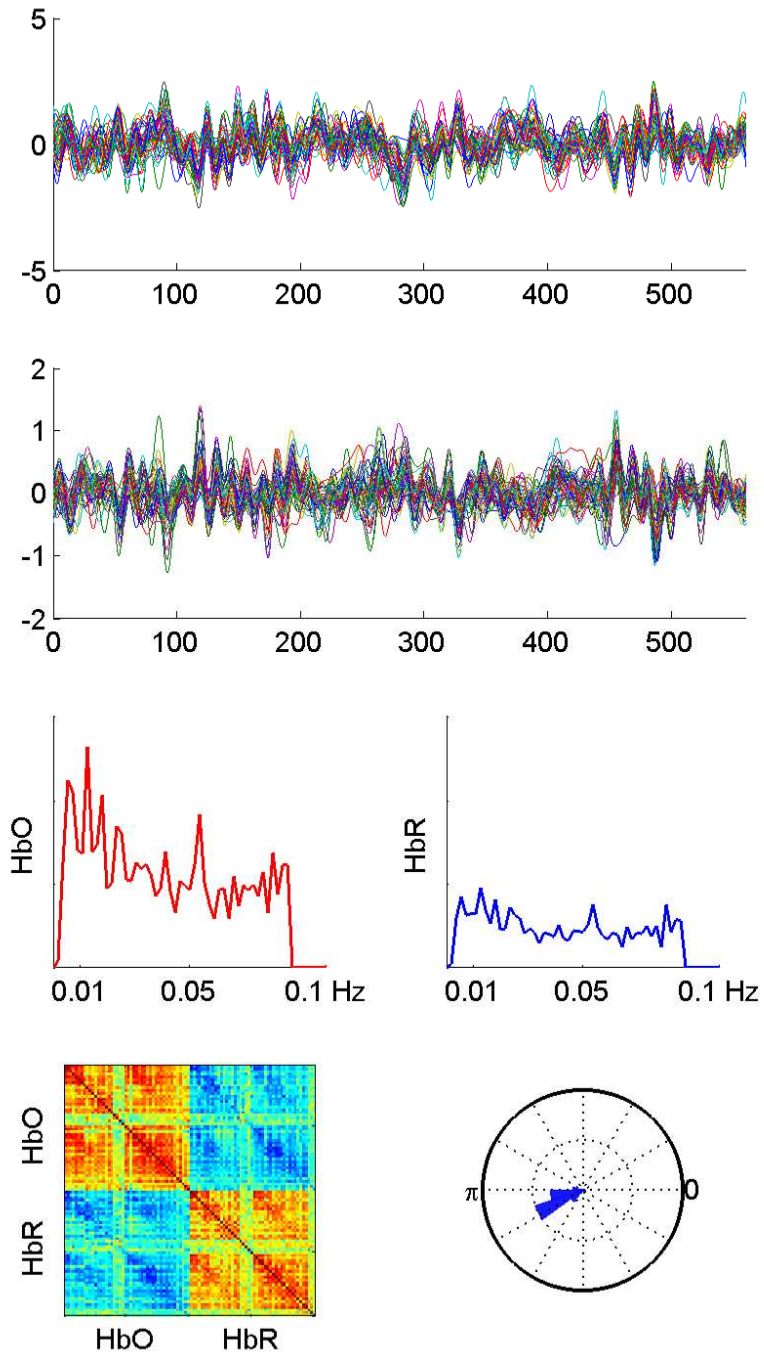
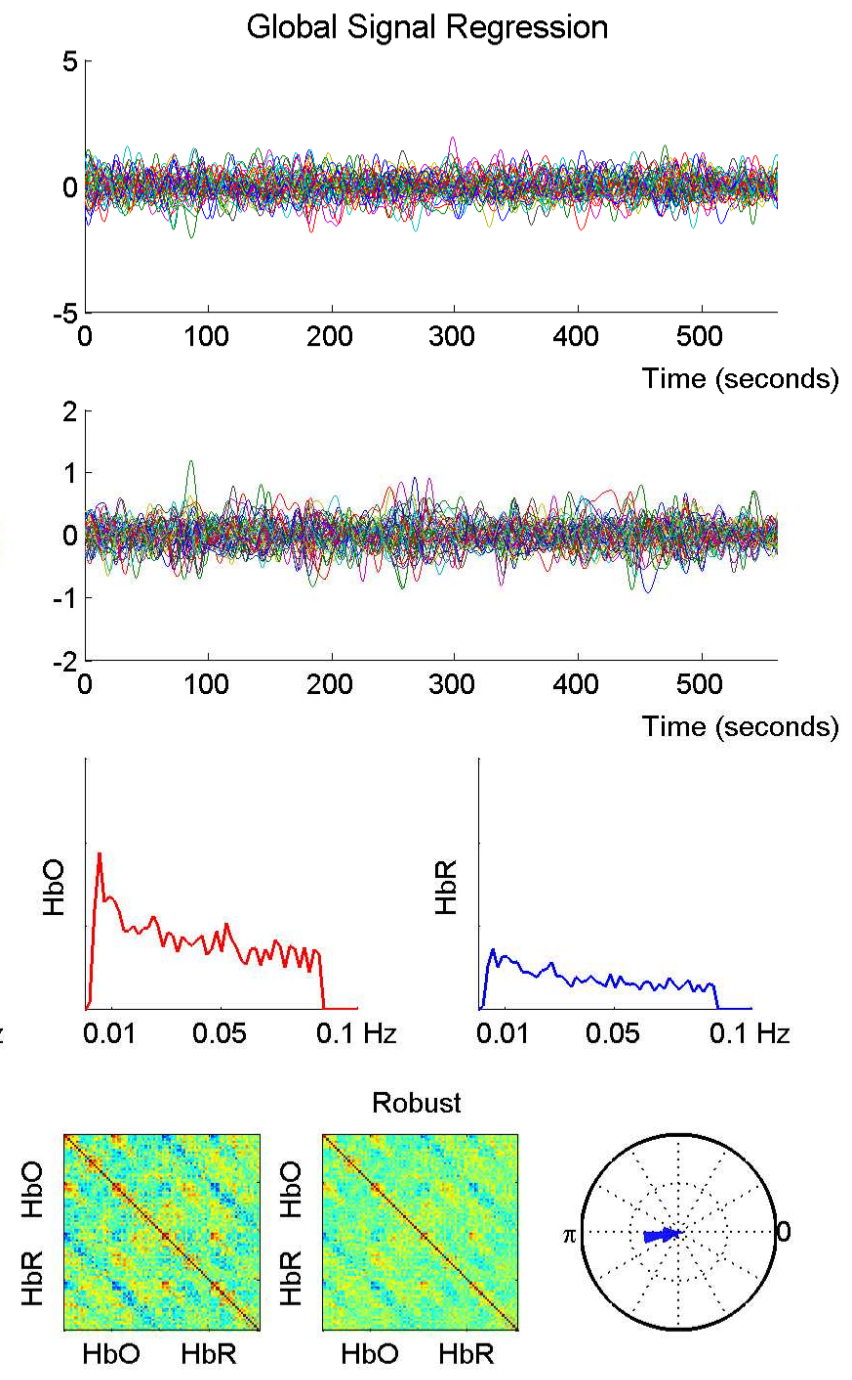

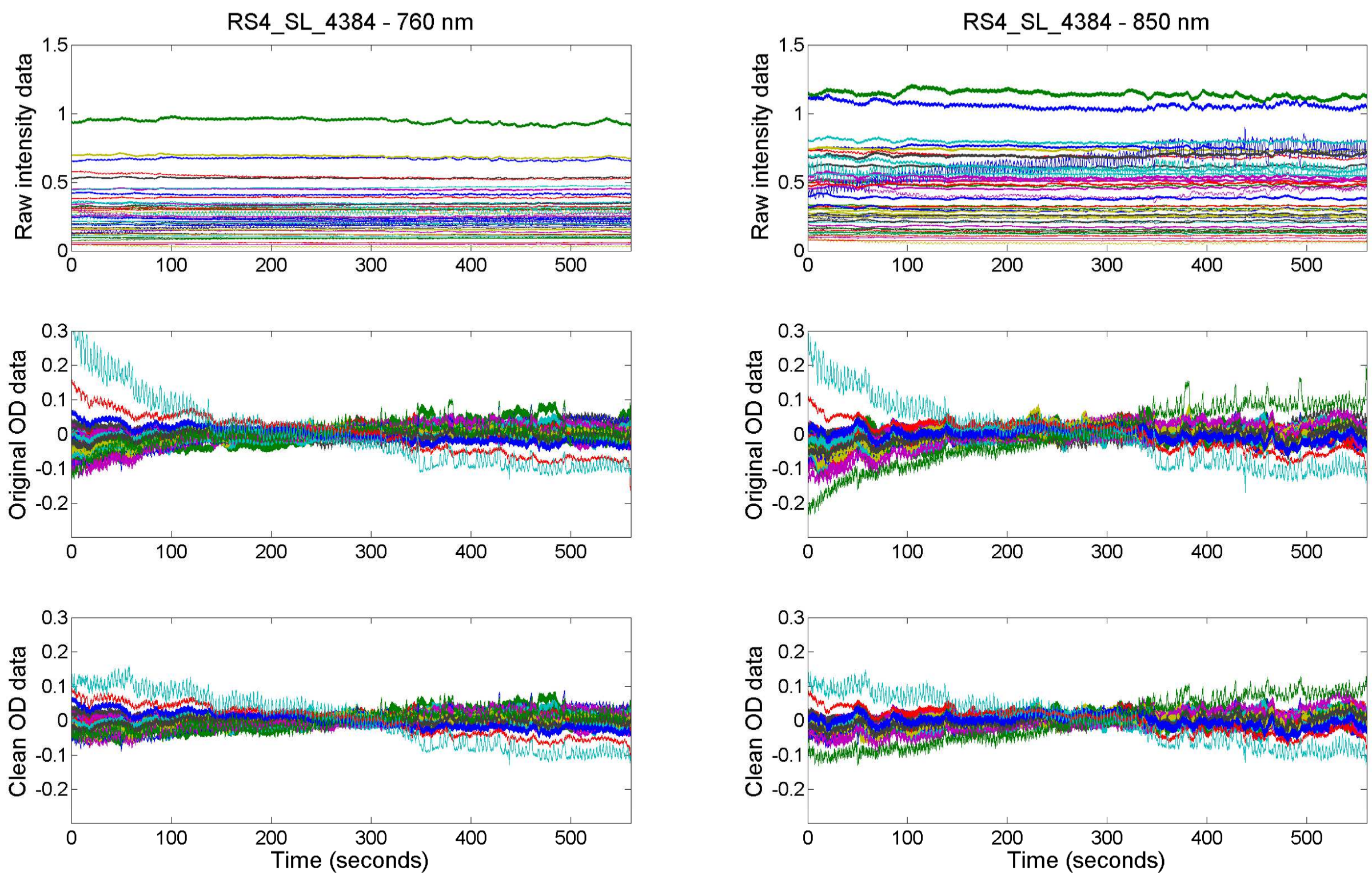

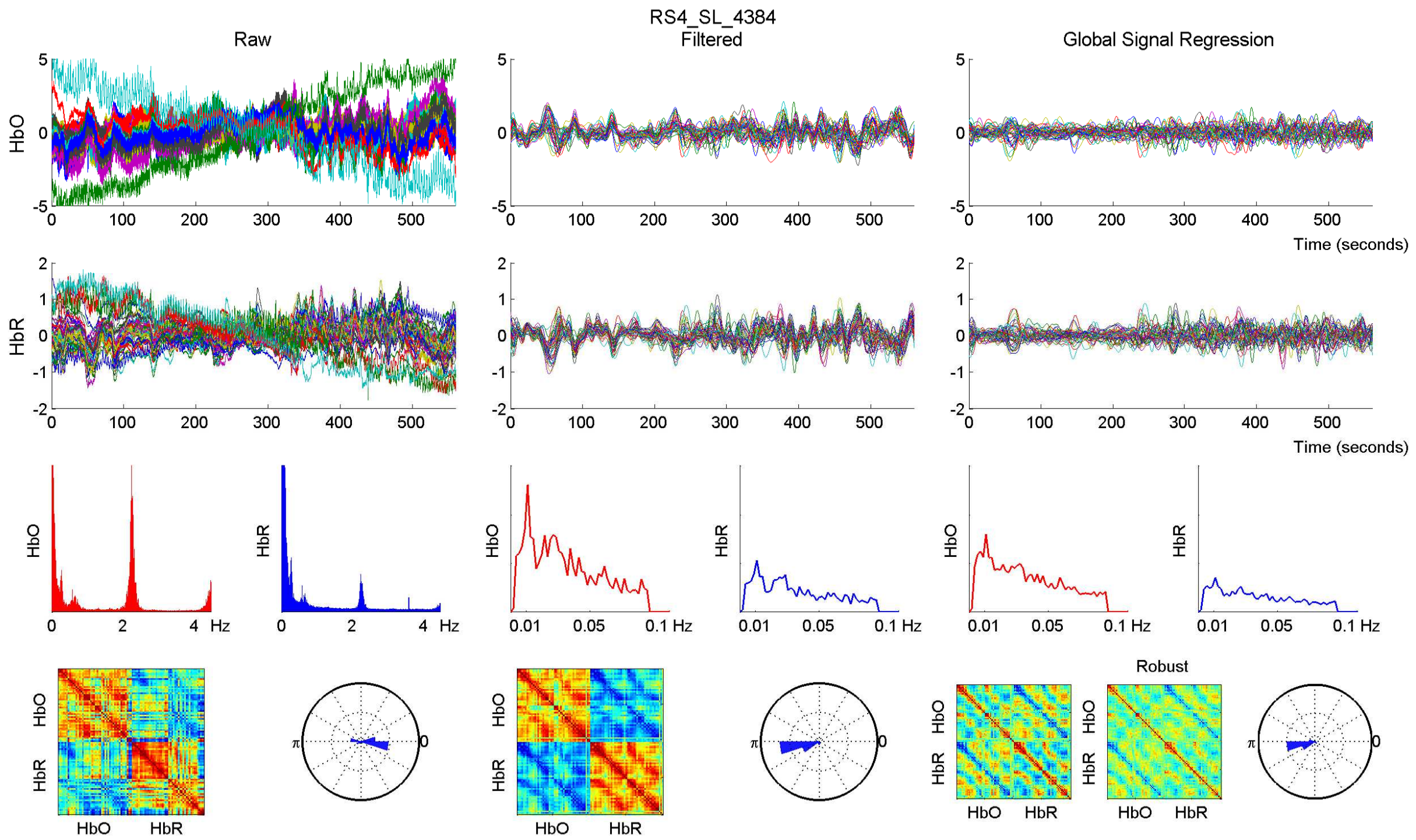

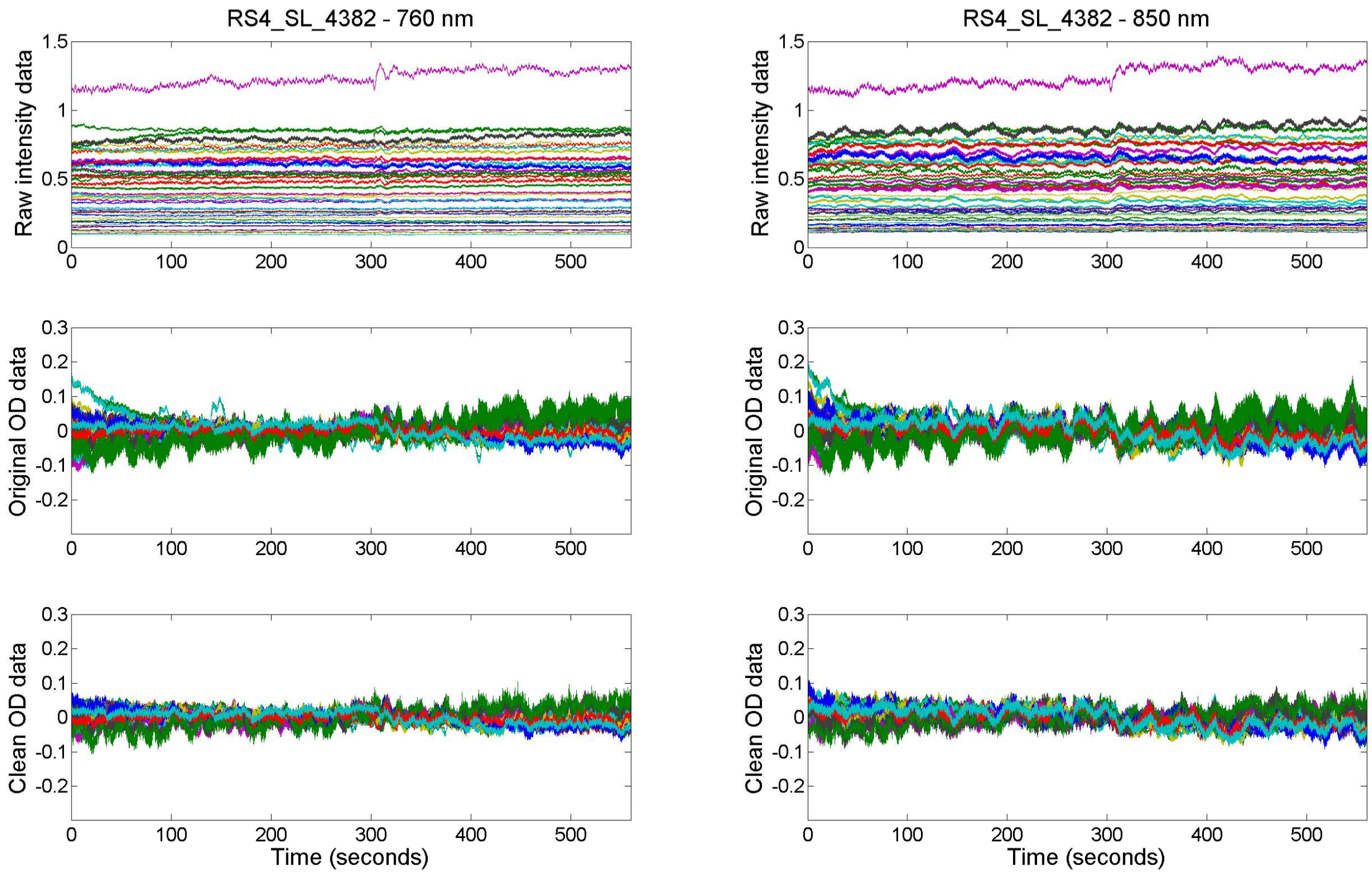
Raw

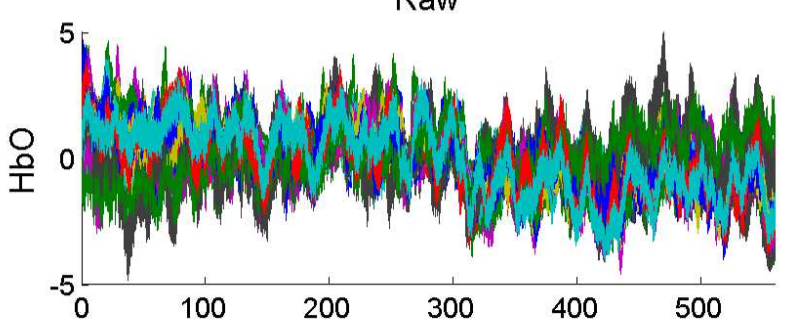

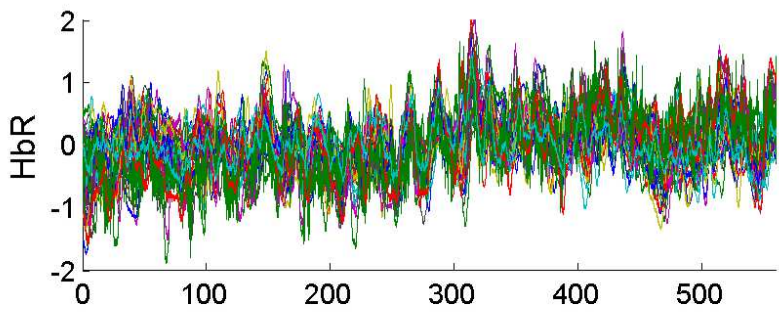
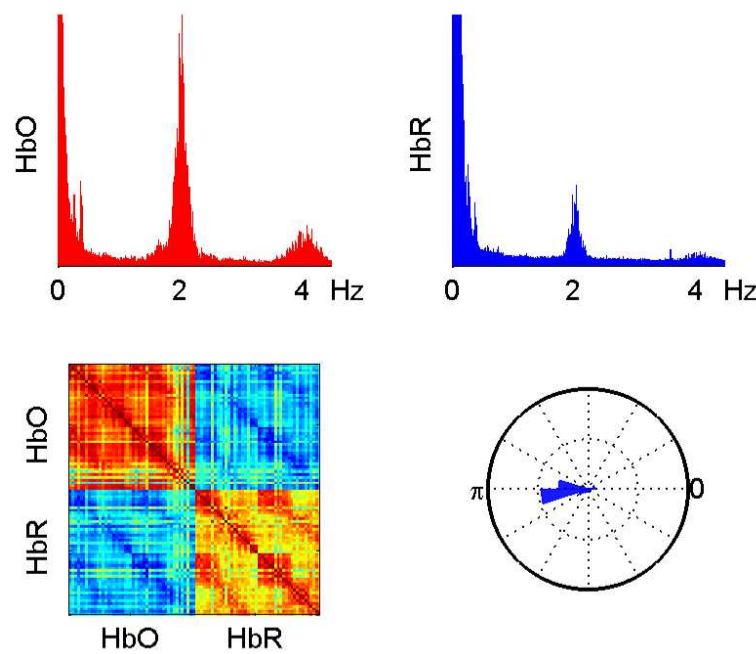

RS4_SL_4382

Filtered
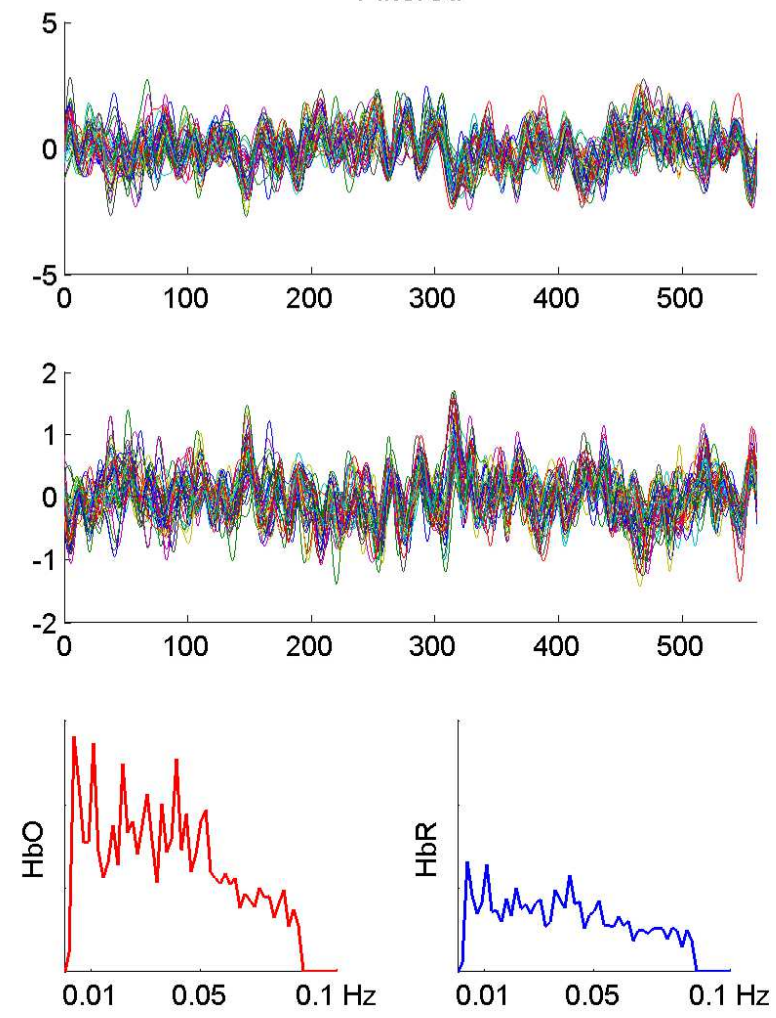

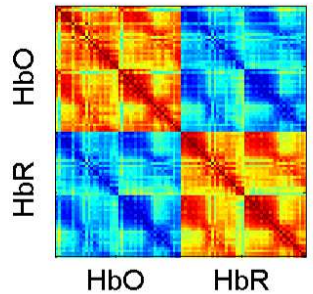

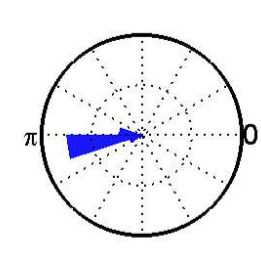

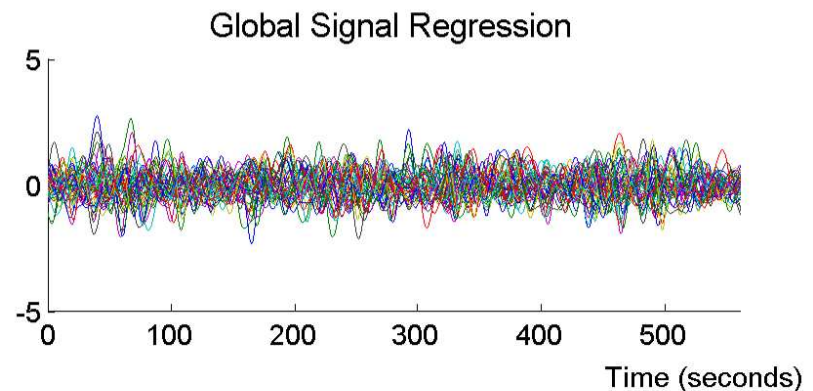

2

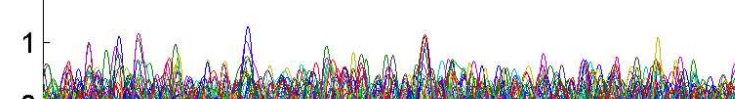
0 -1. $-1$
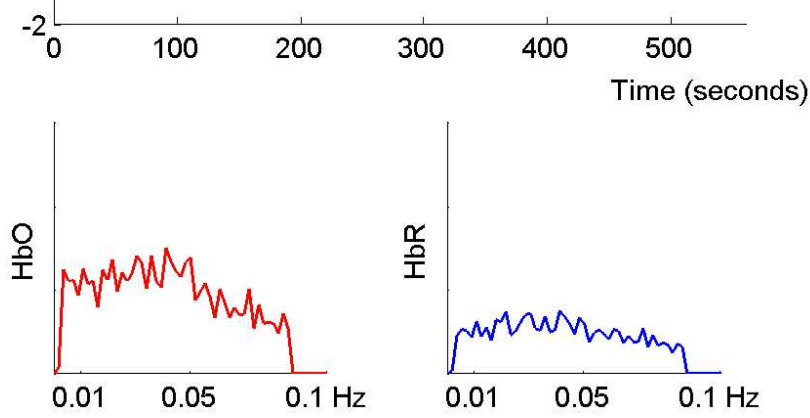
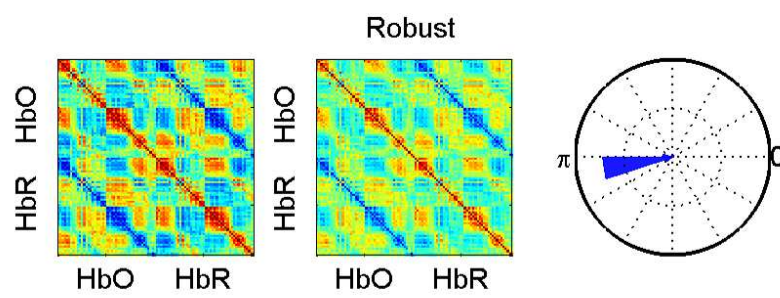

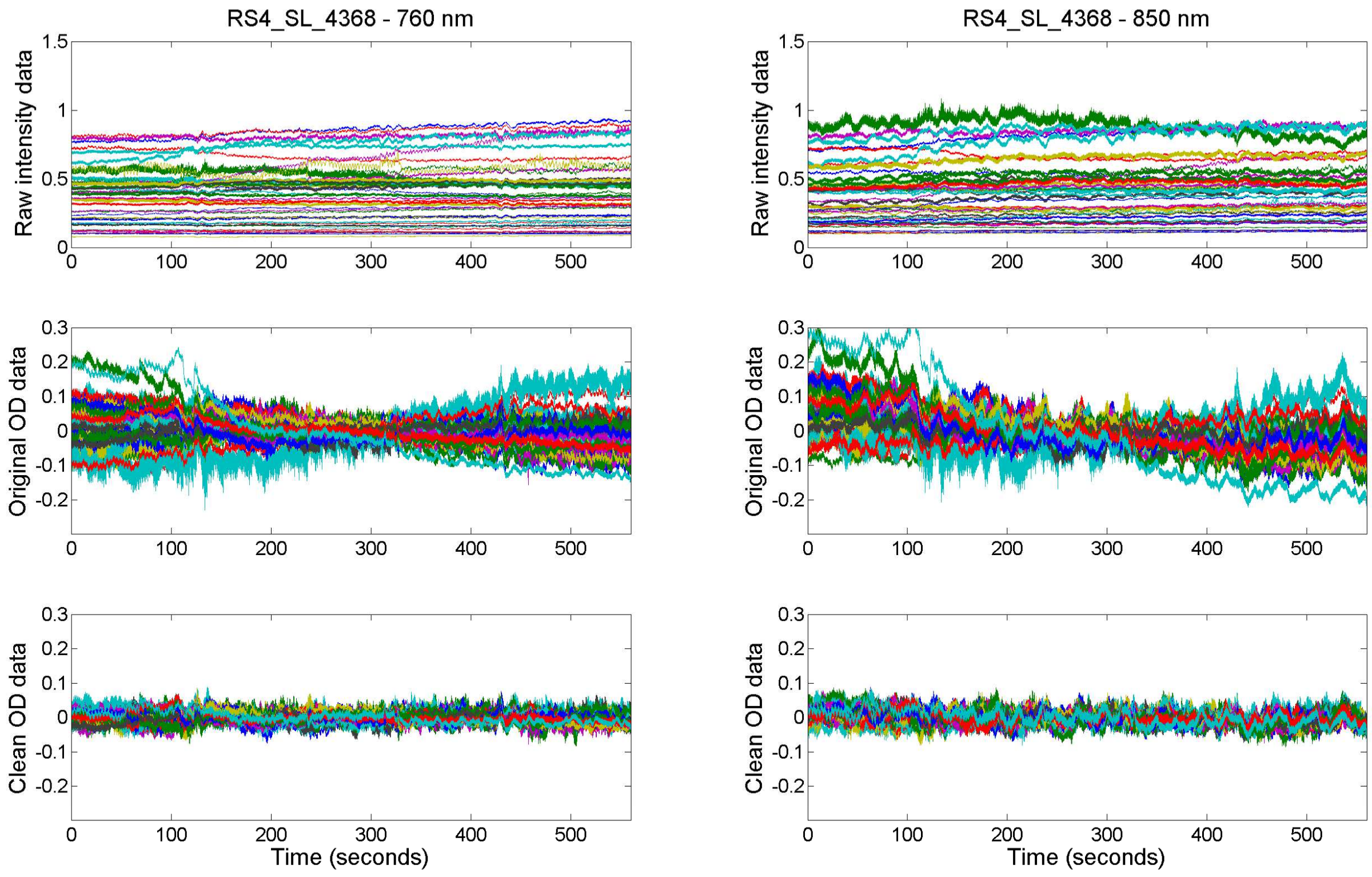
RS4_SL_4368

Raw

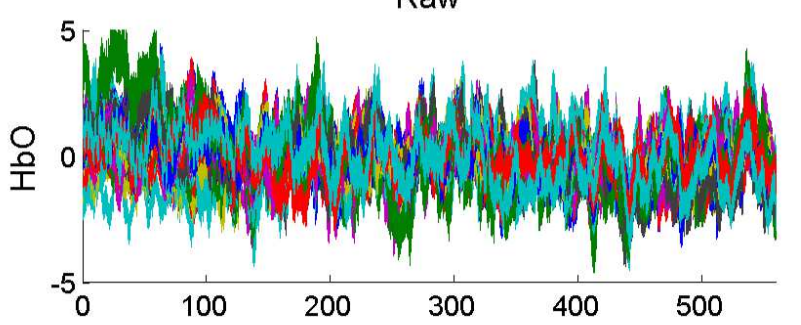

5

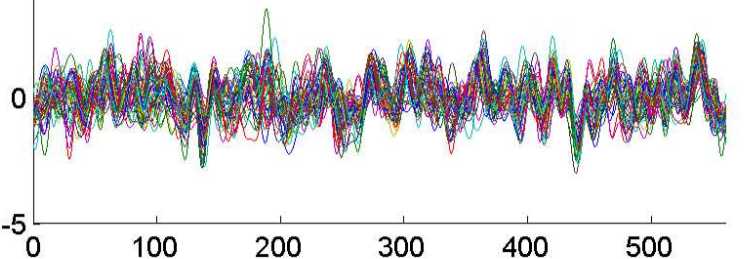

2

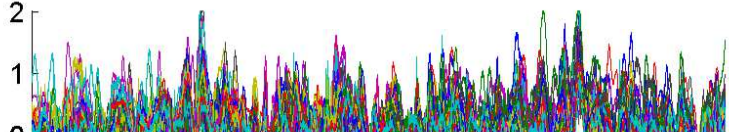

옹
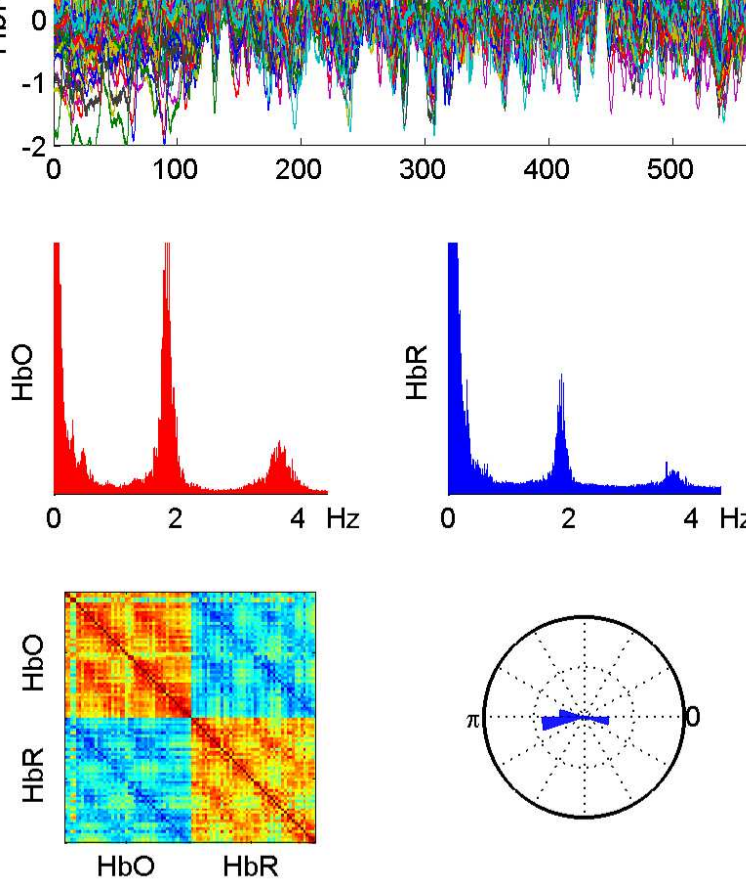
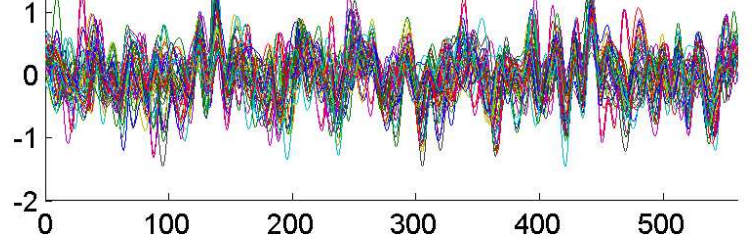
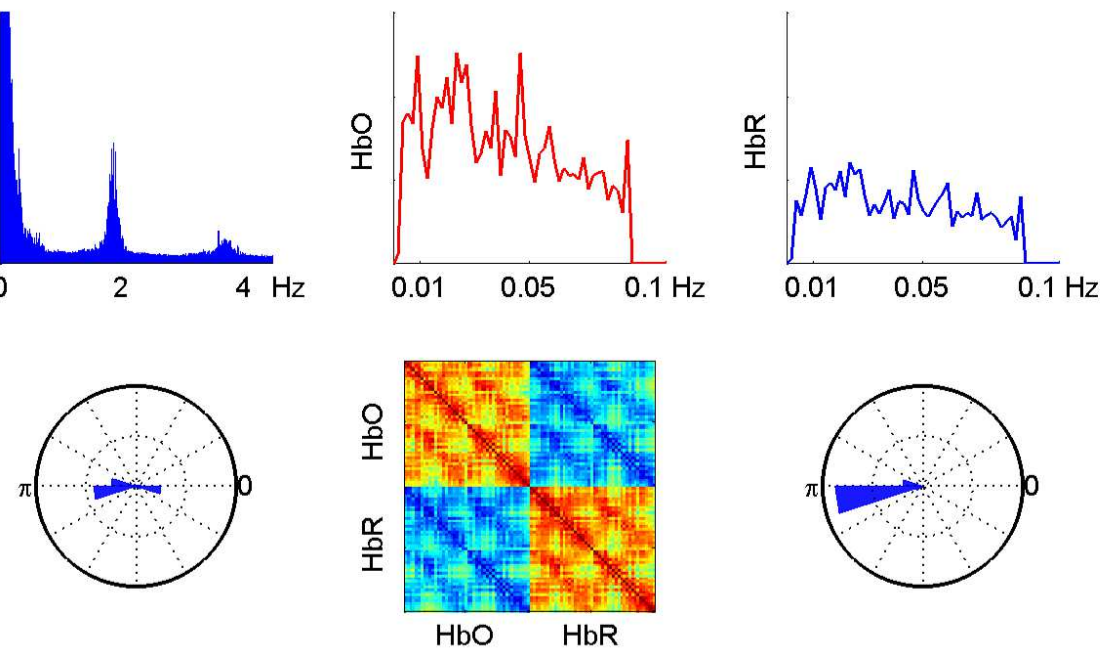

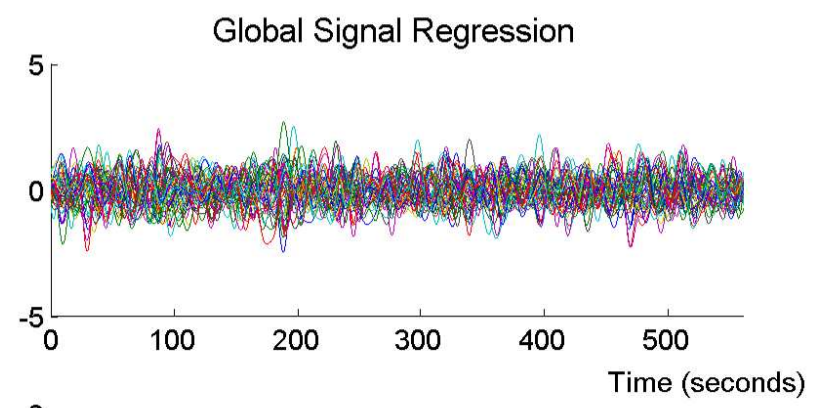

2
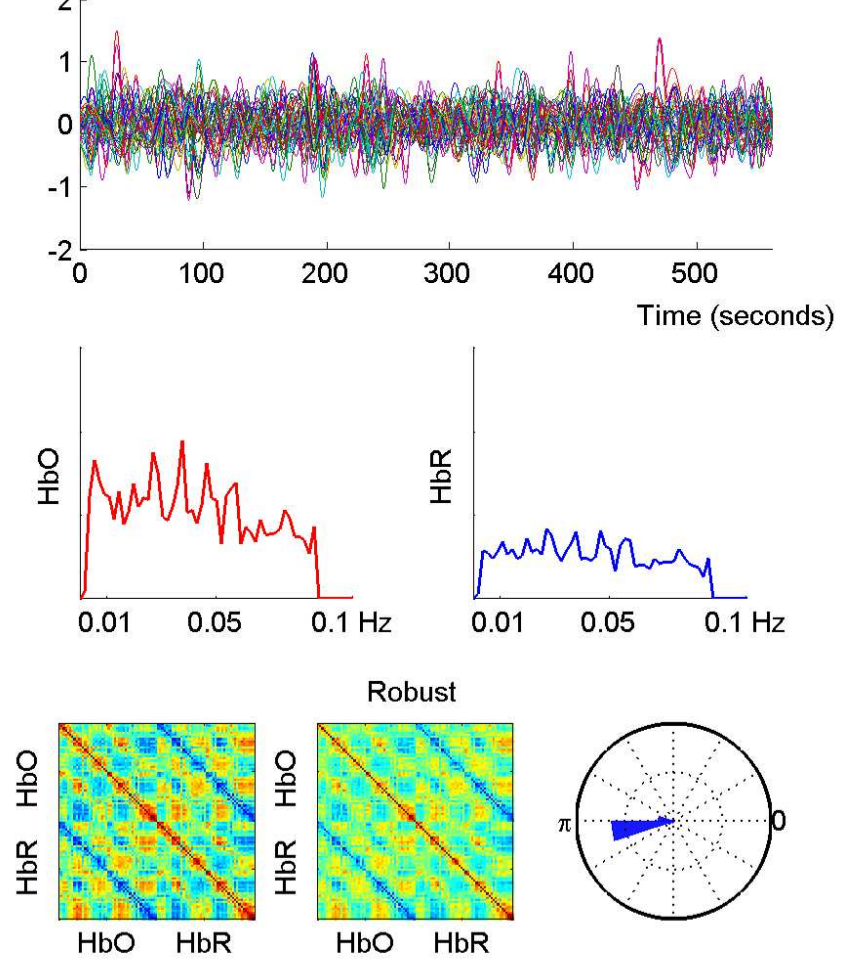

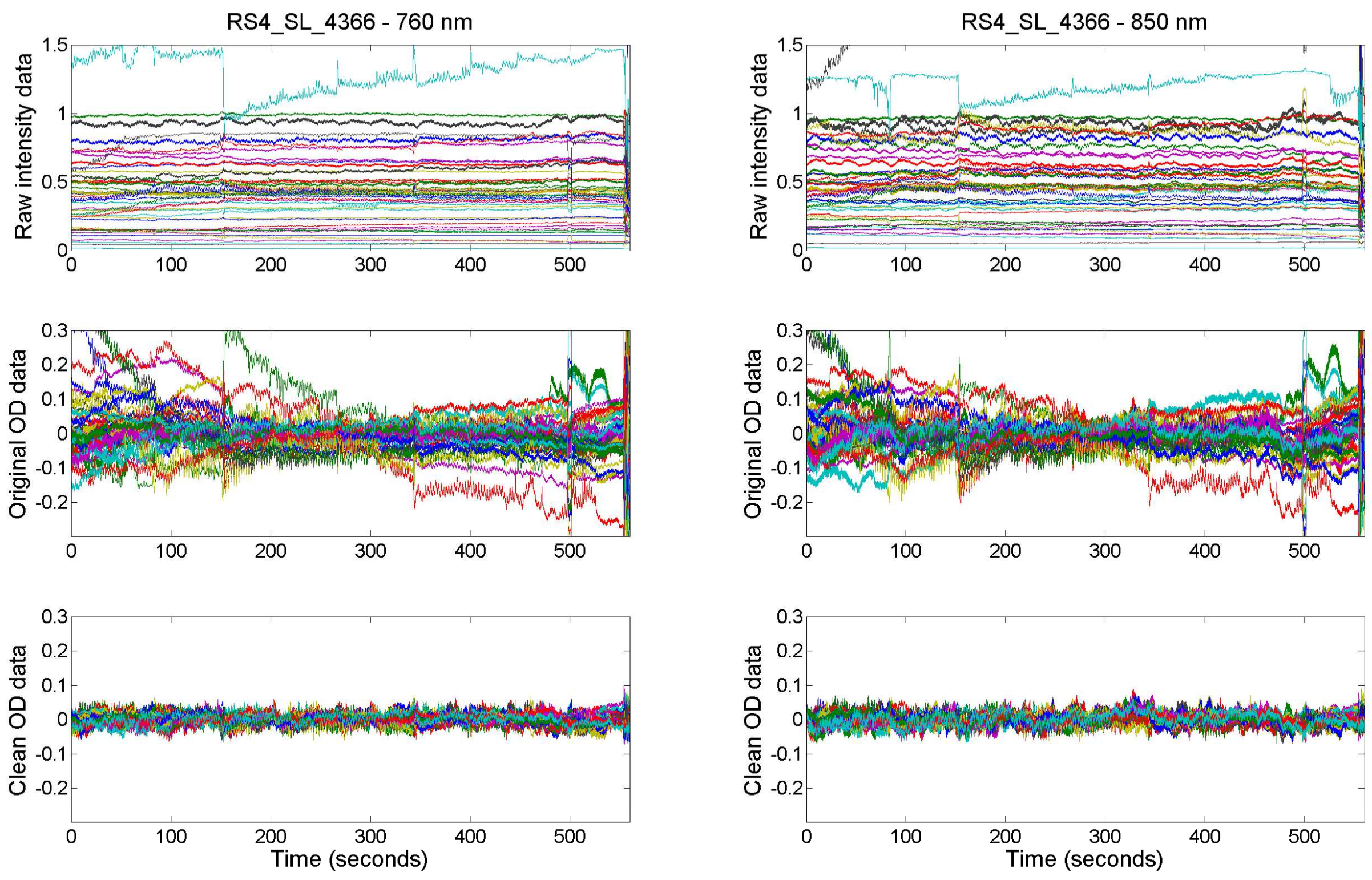
Raw

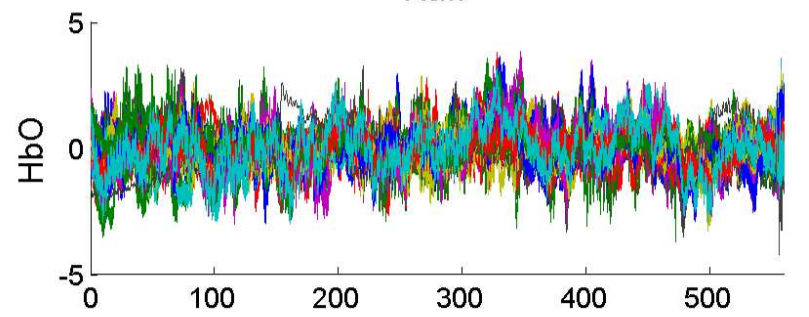

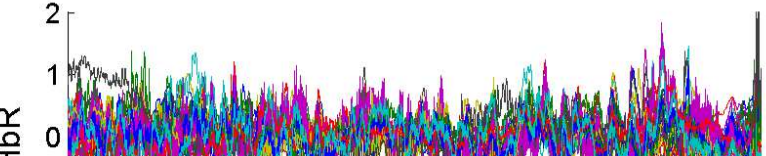

工
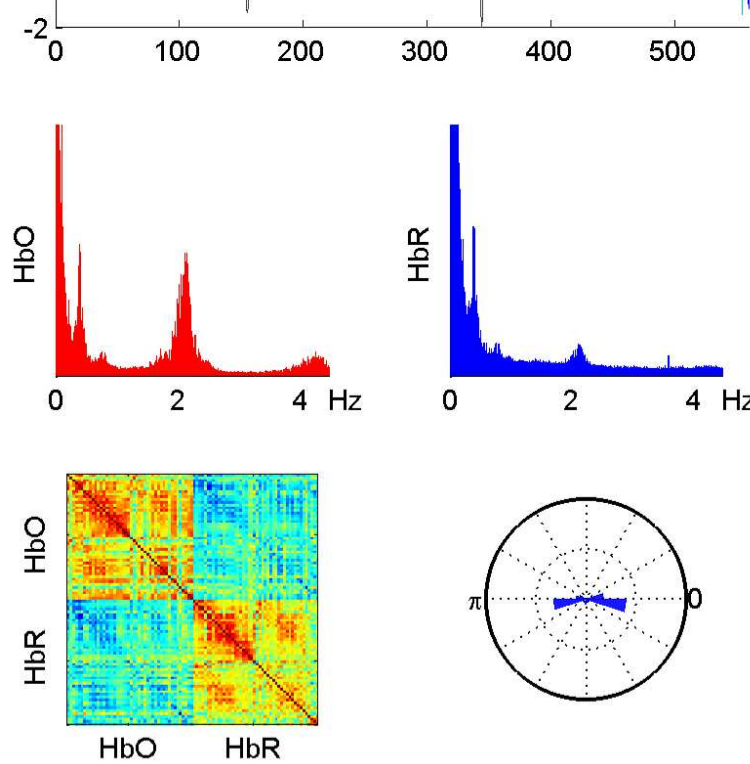

RS4_SL_4366

Filtered
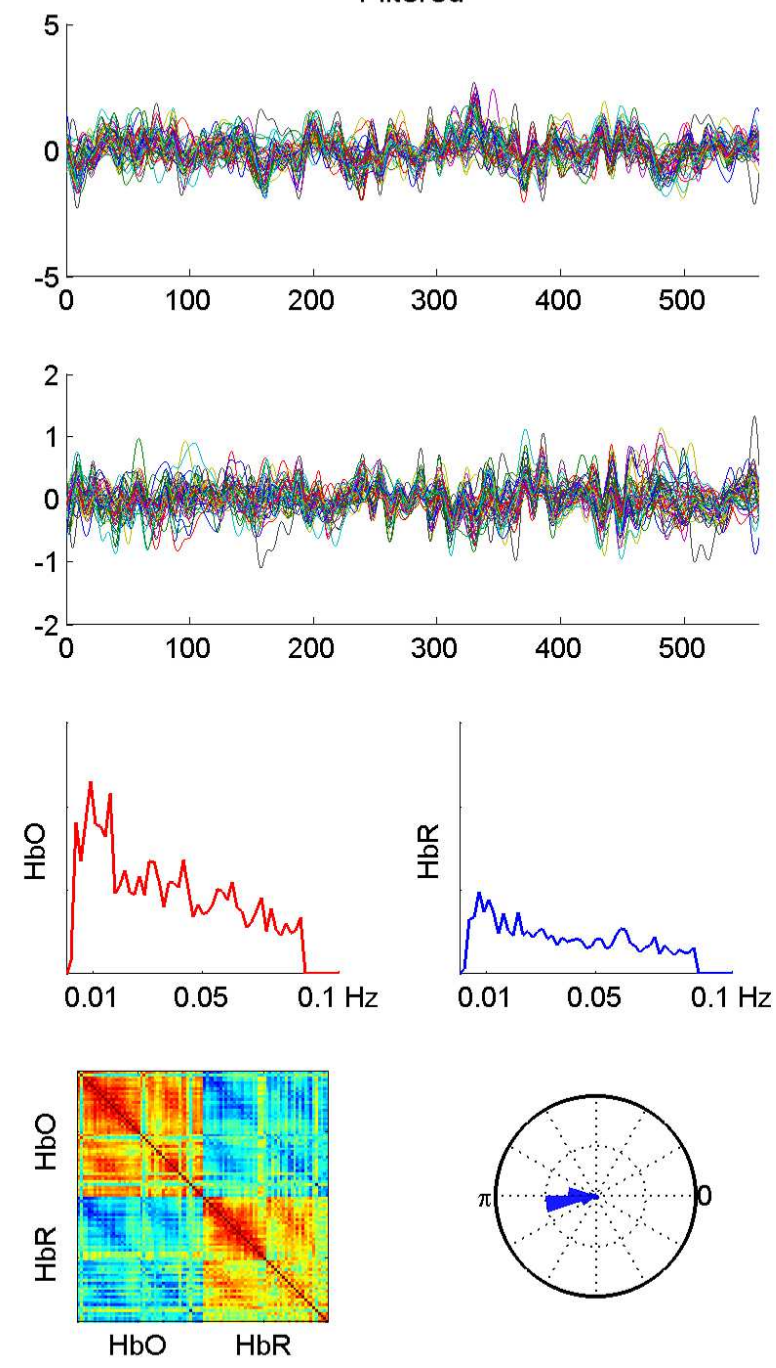
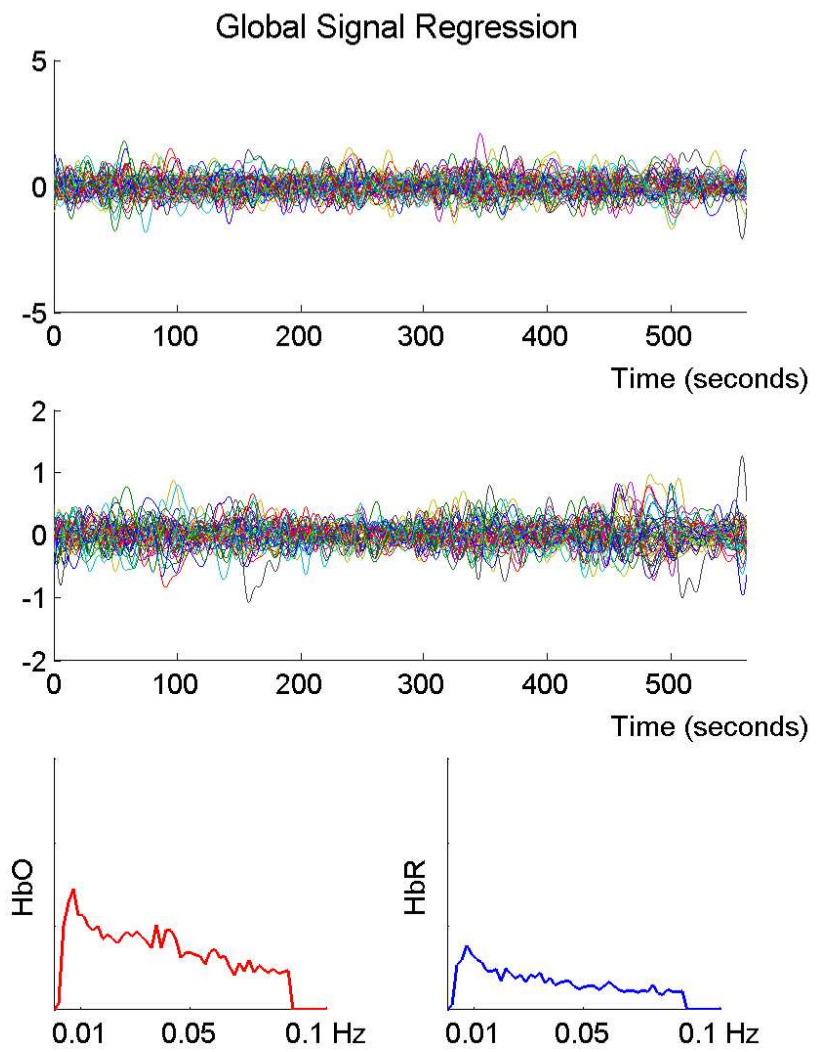
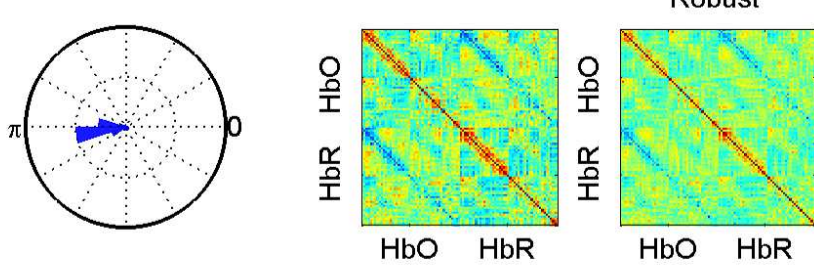

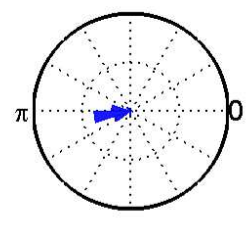


RS4 SL 4365 - 760 nm
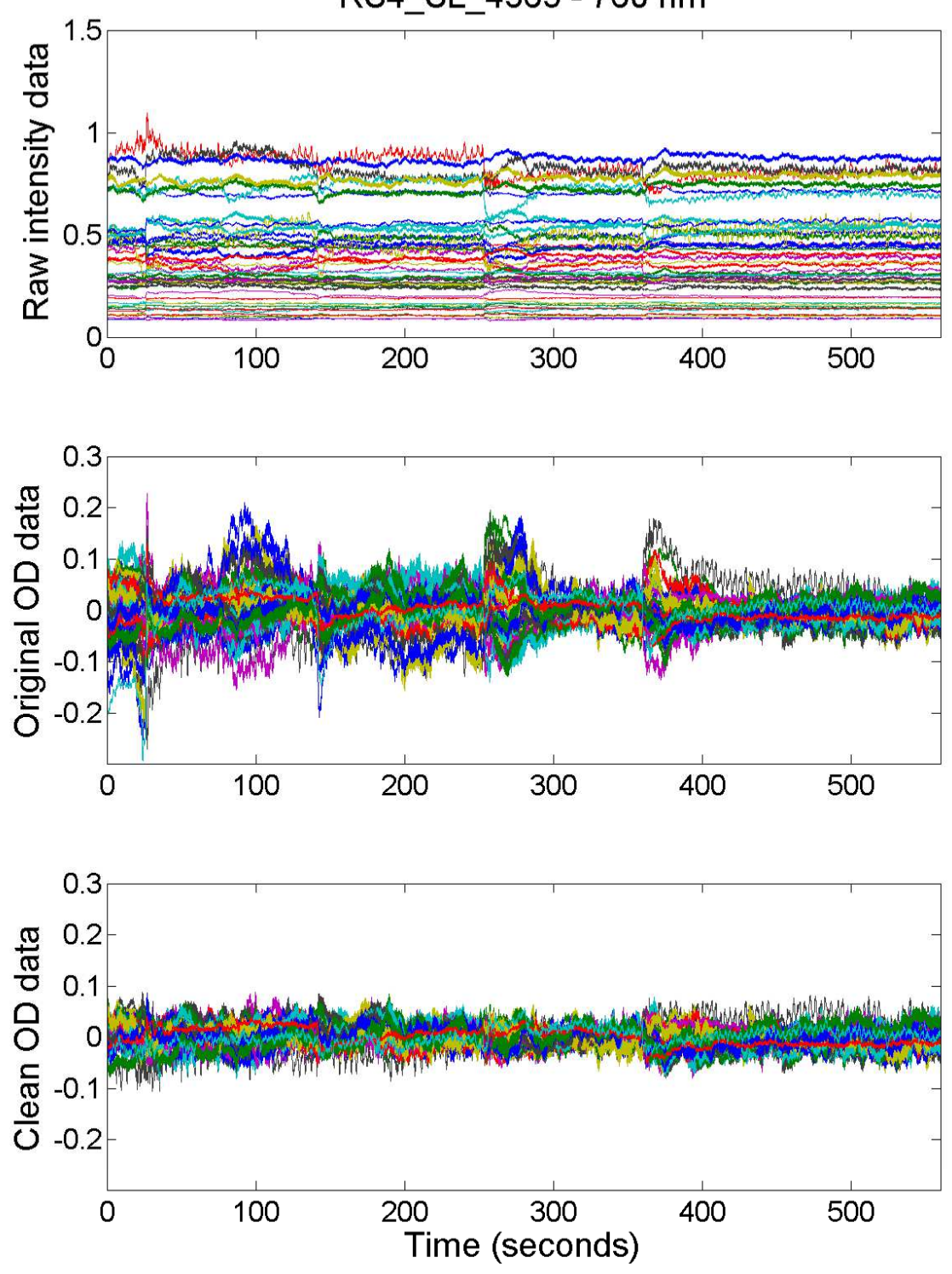

RS4 SL $4365-850 \mathrm{~nm}$
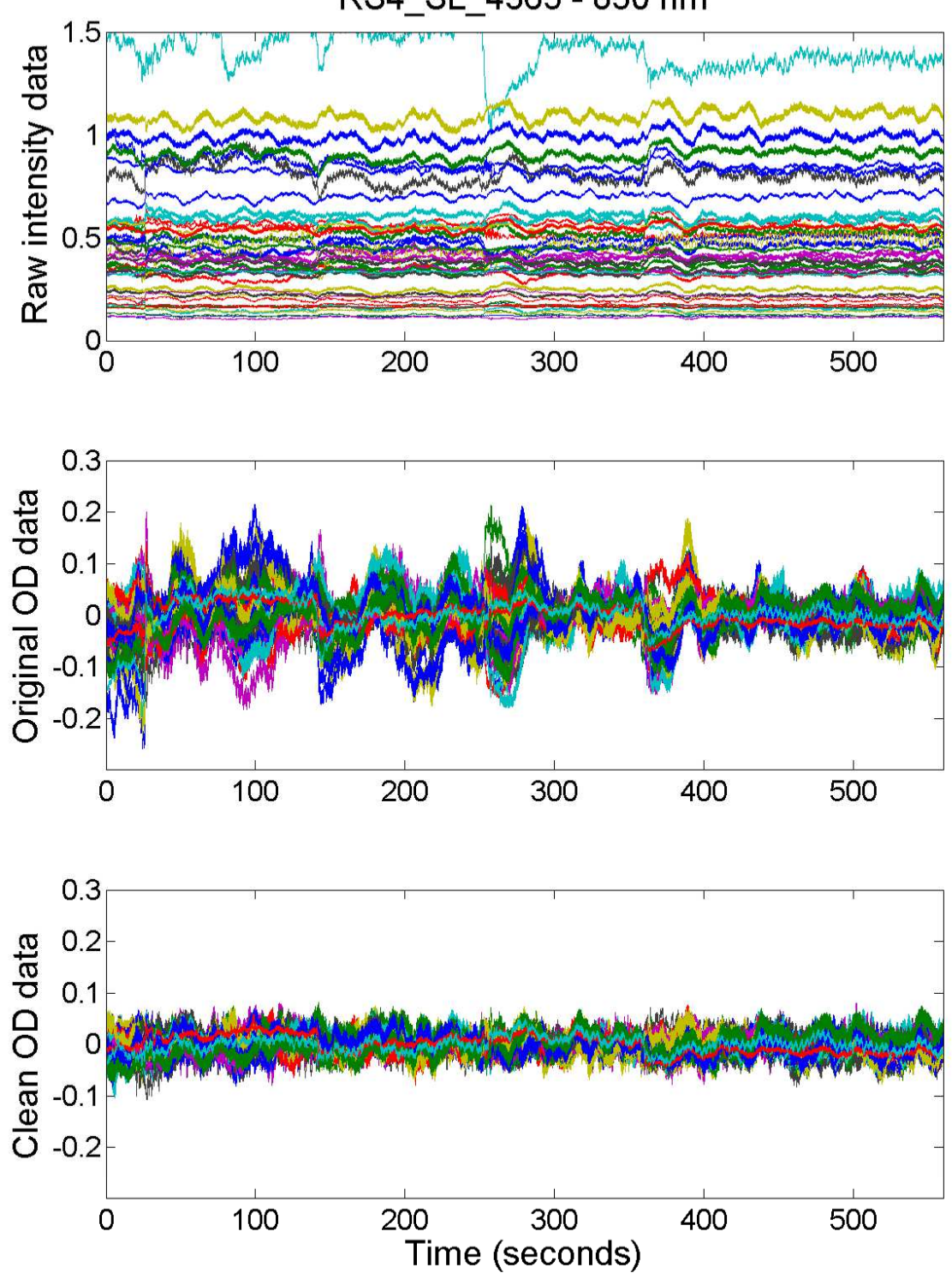
Raw
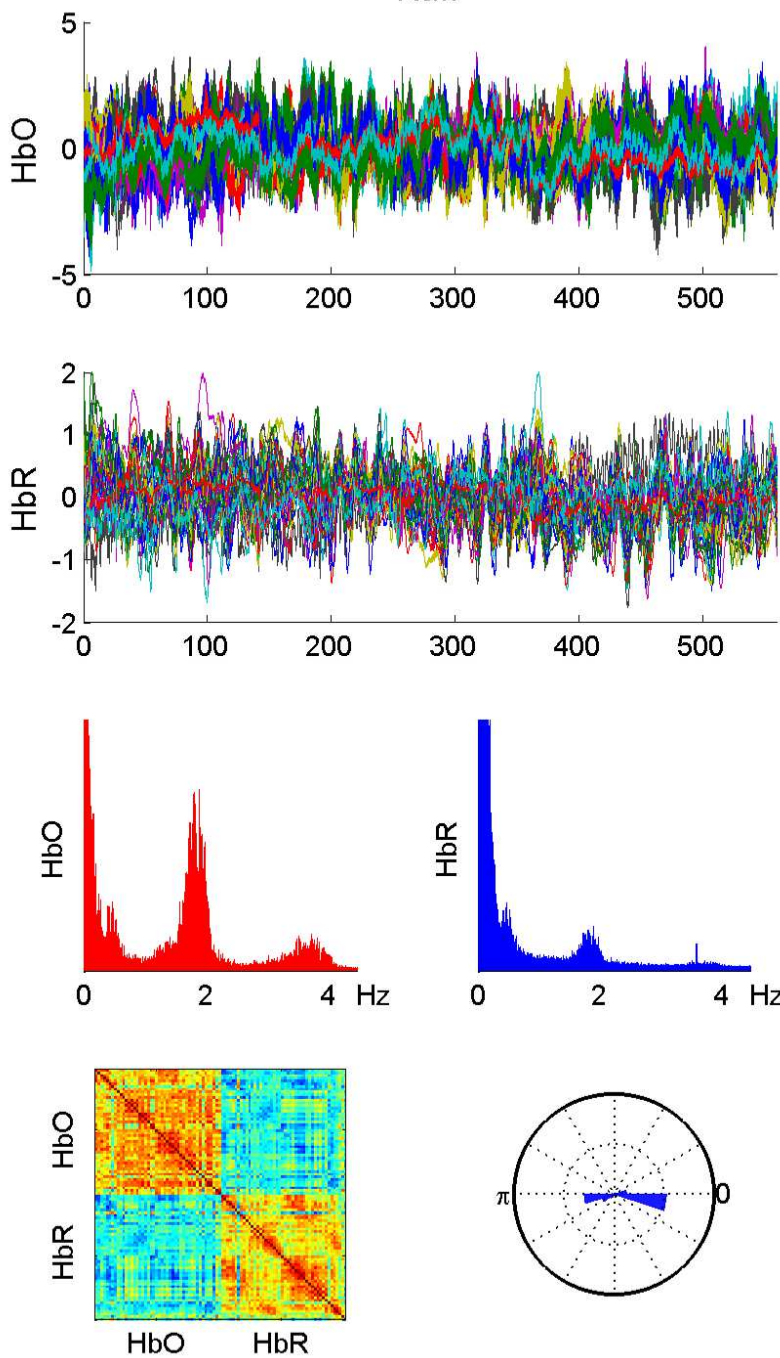

RS4 SL 4365

Filtered
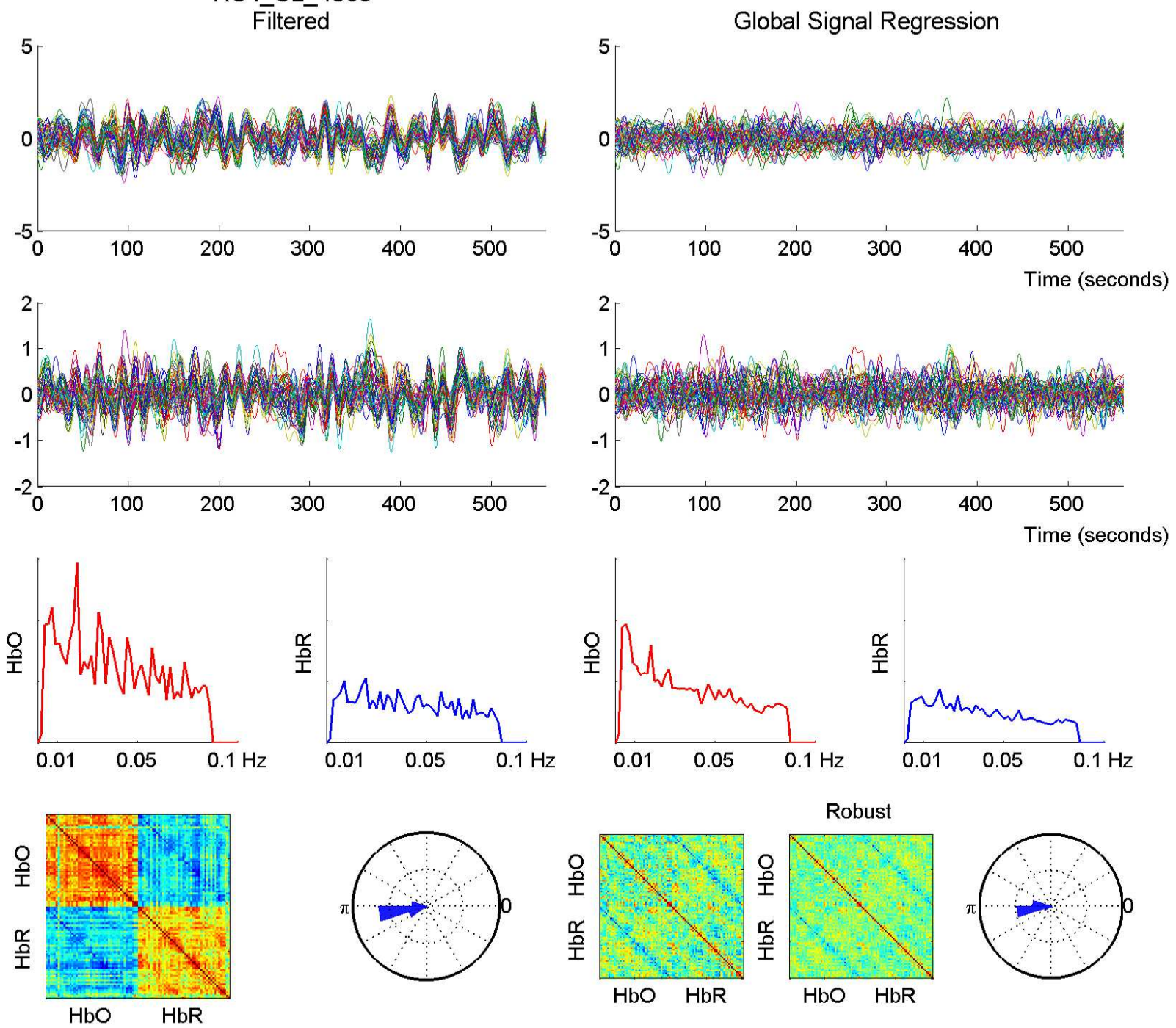
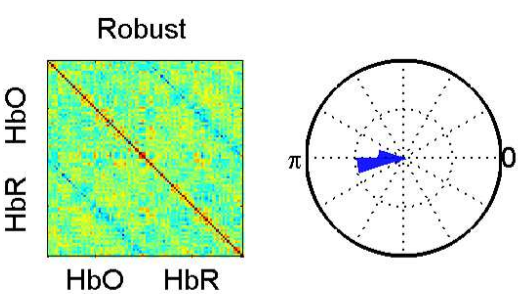

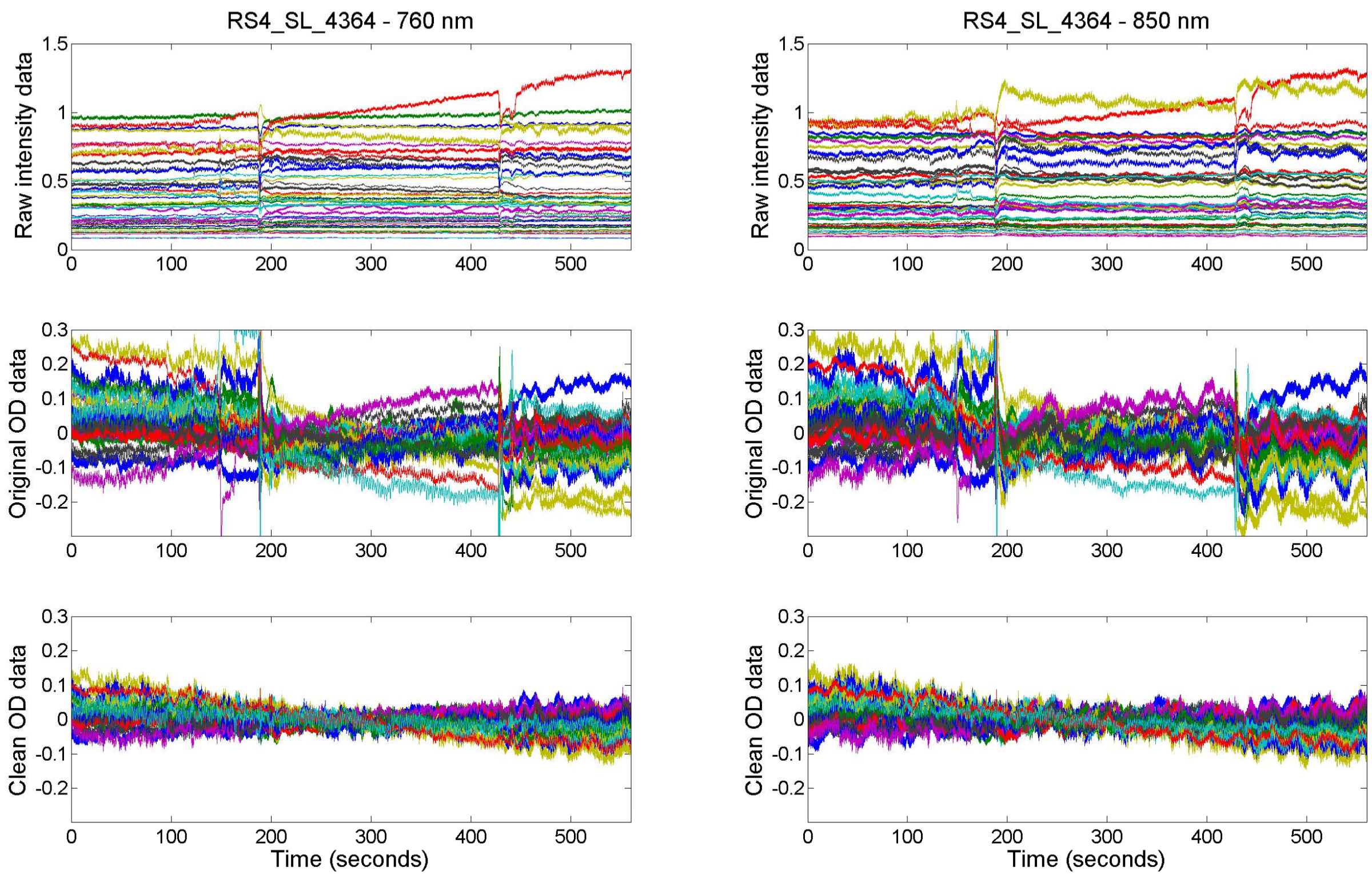
Raw

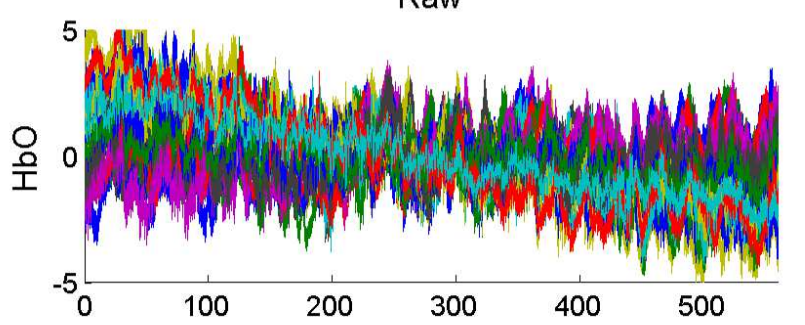

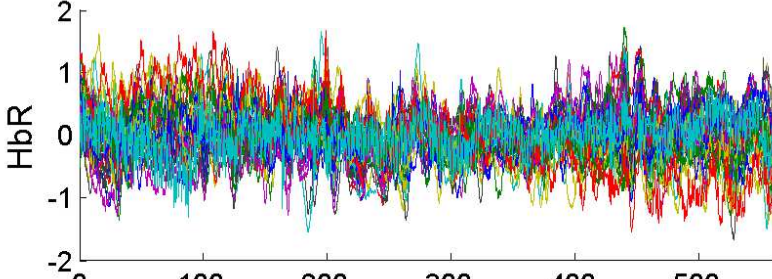
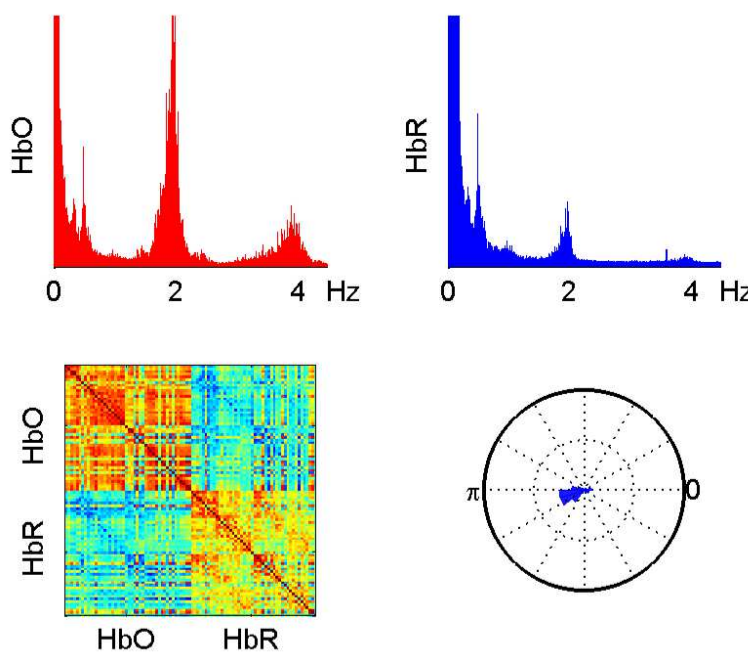

RS4 SL 4364

Filtered
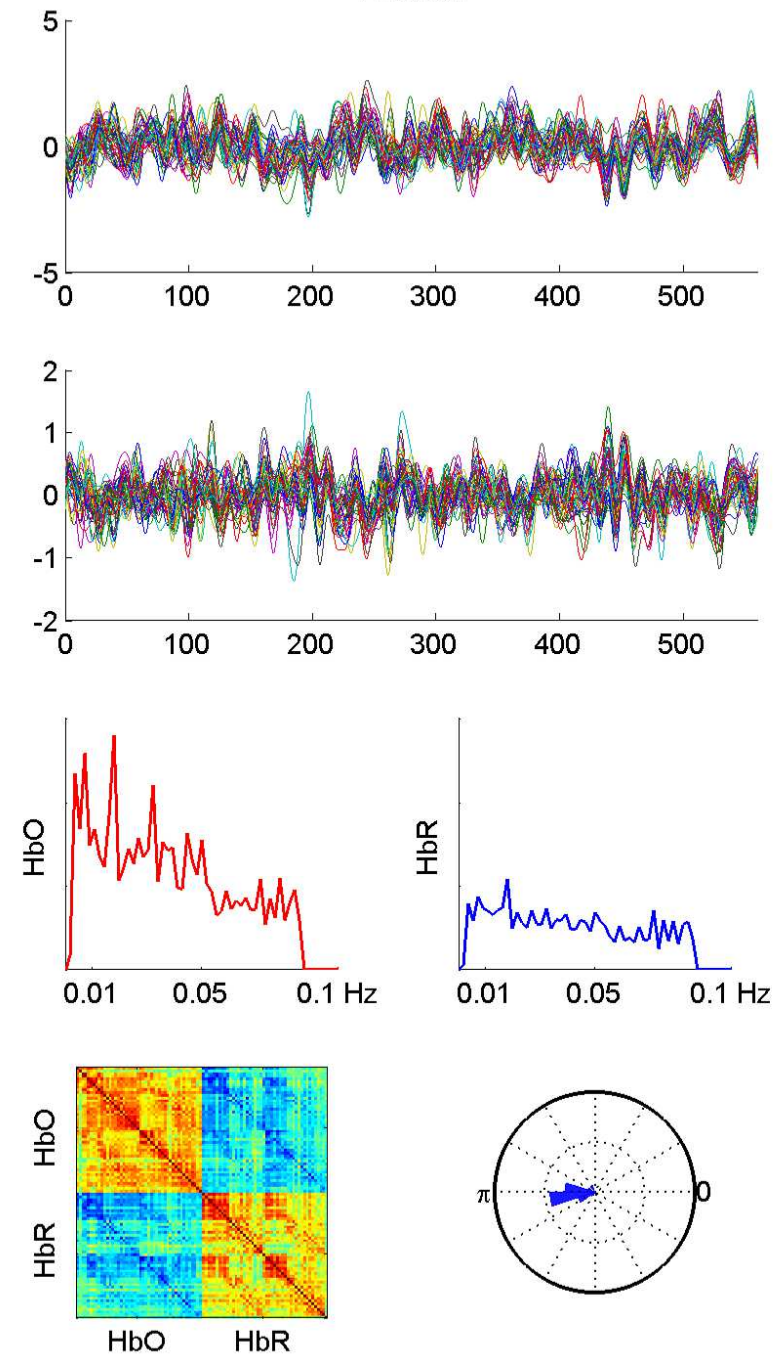

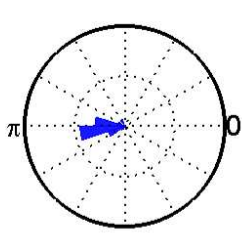

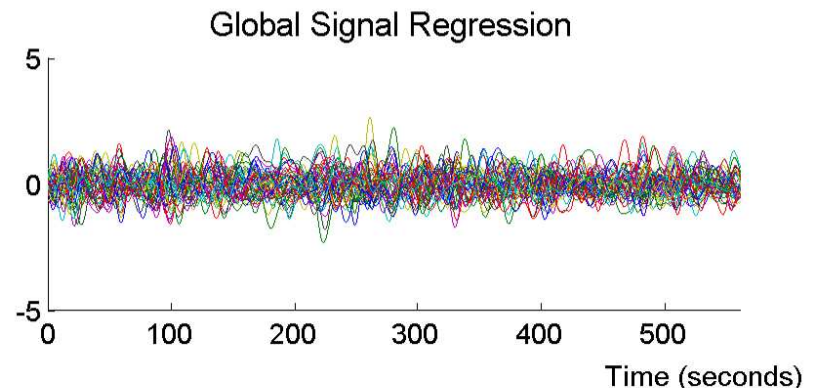
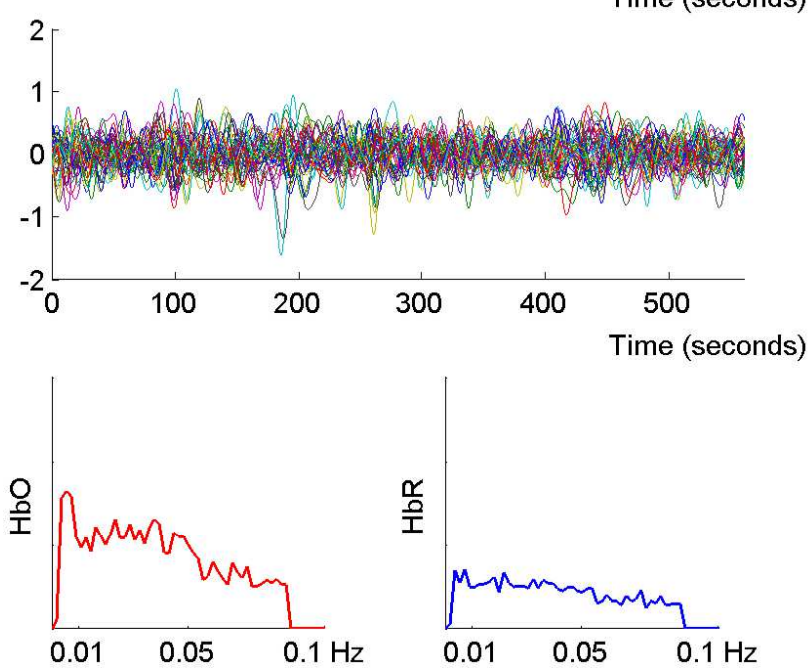
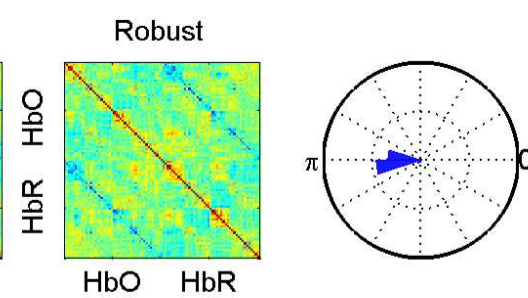

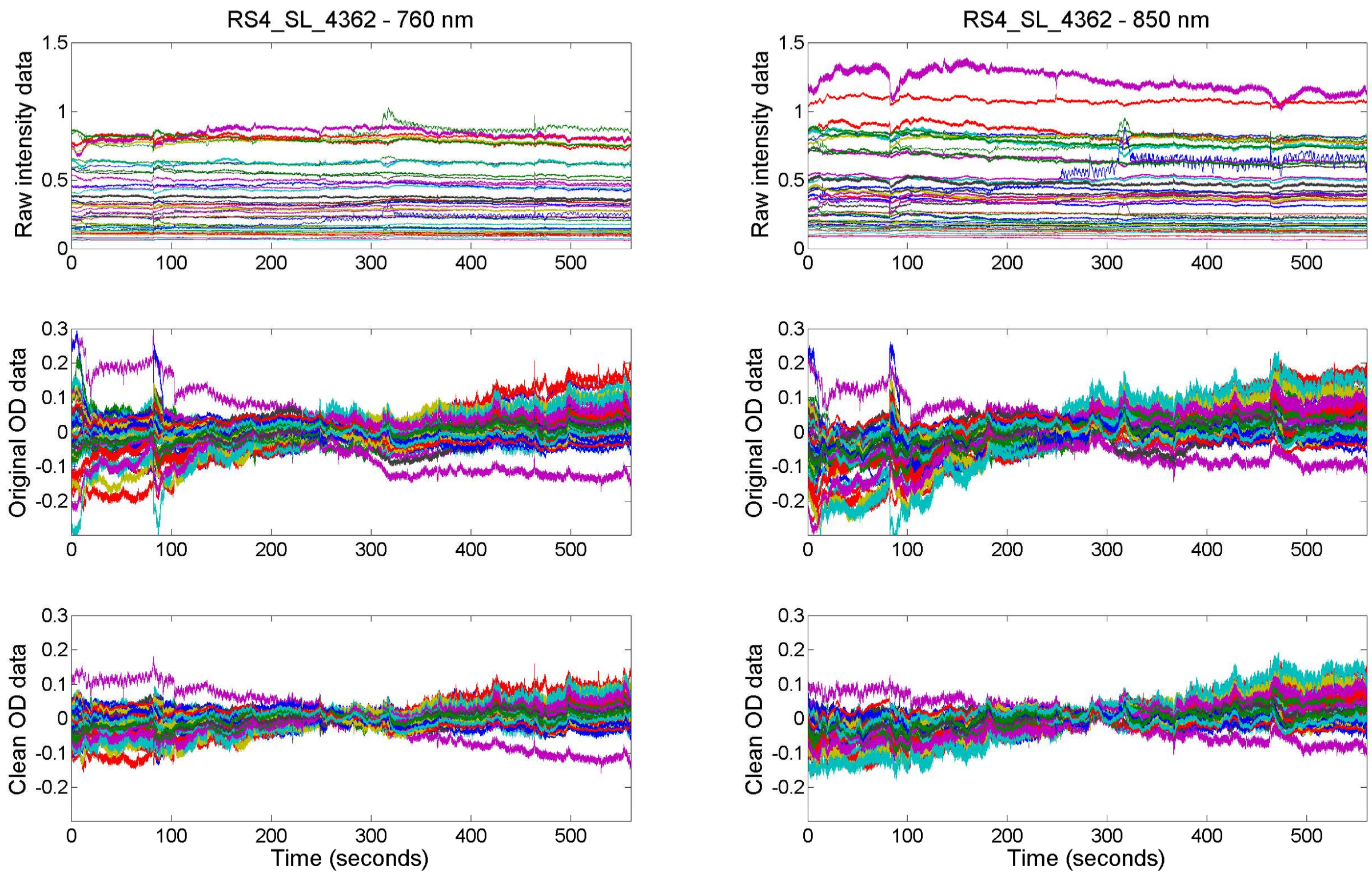

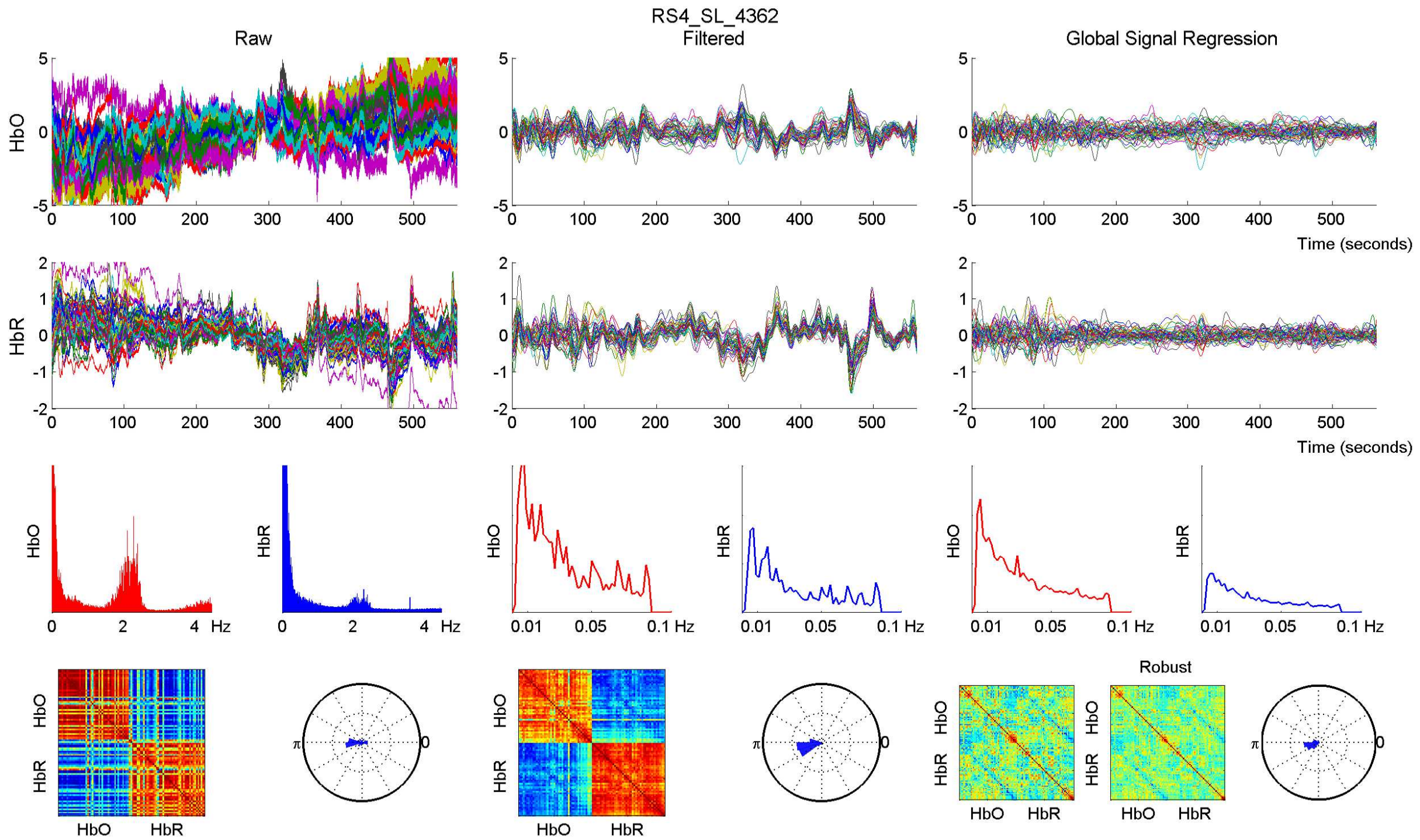

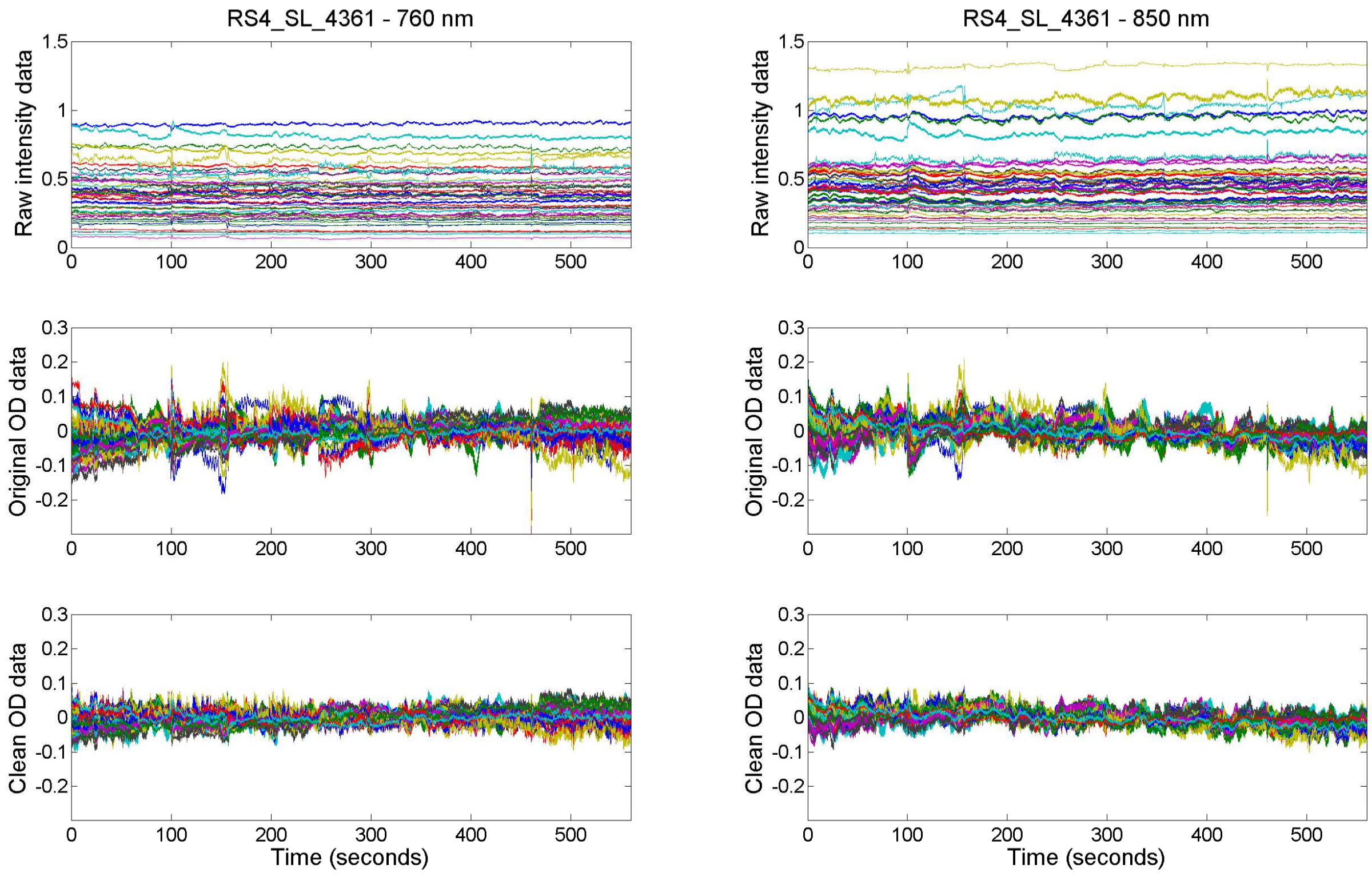
Raw
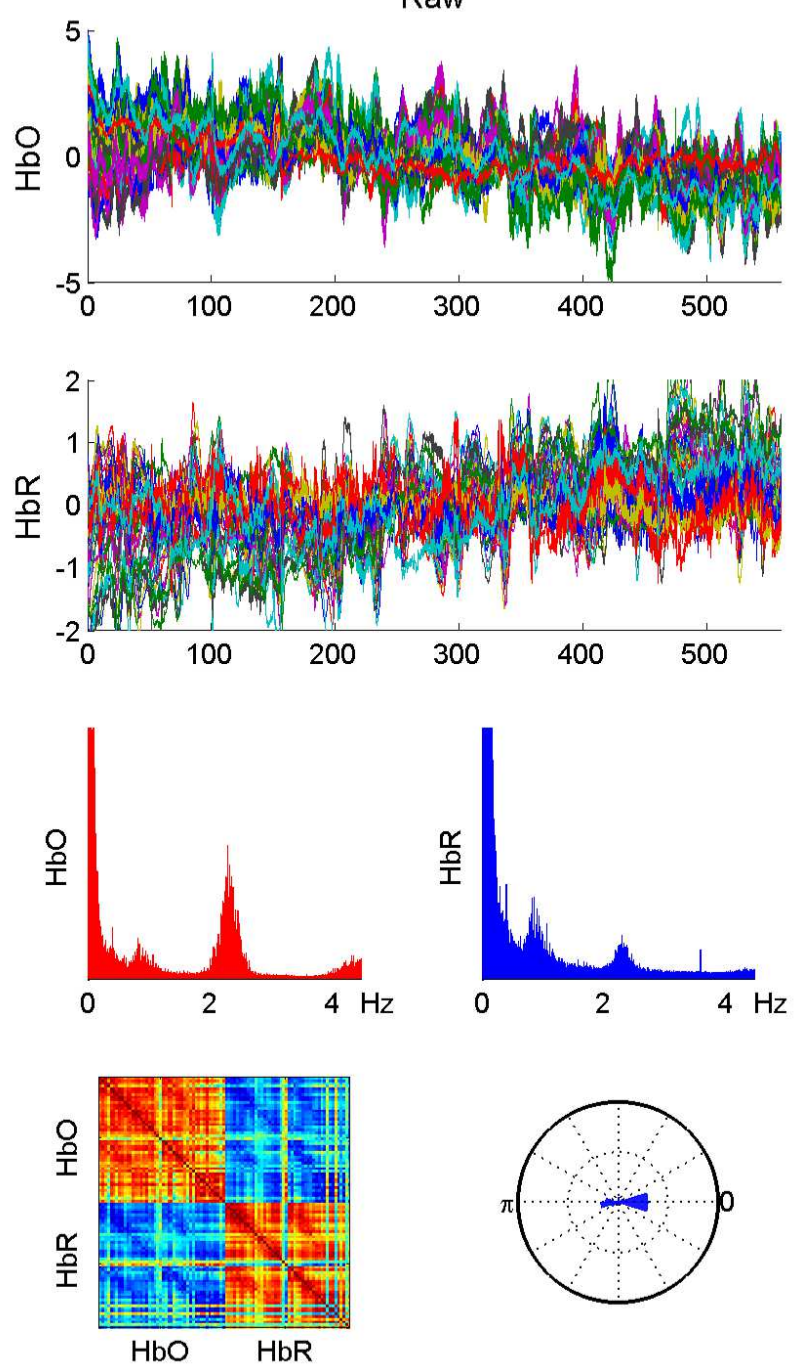

RS4 SL 4361

Filtered
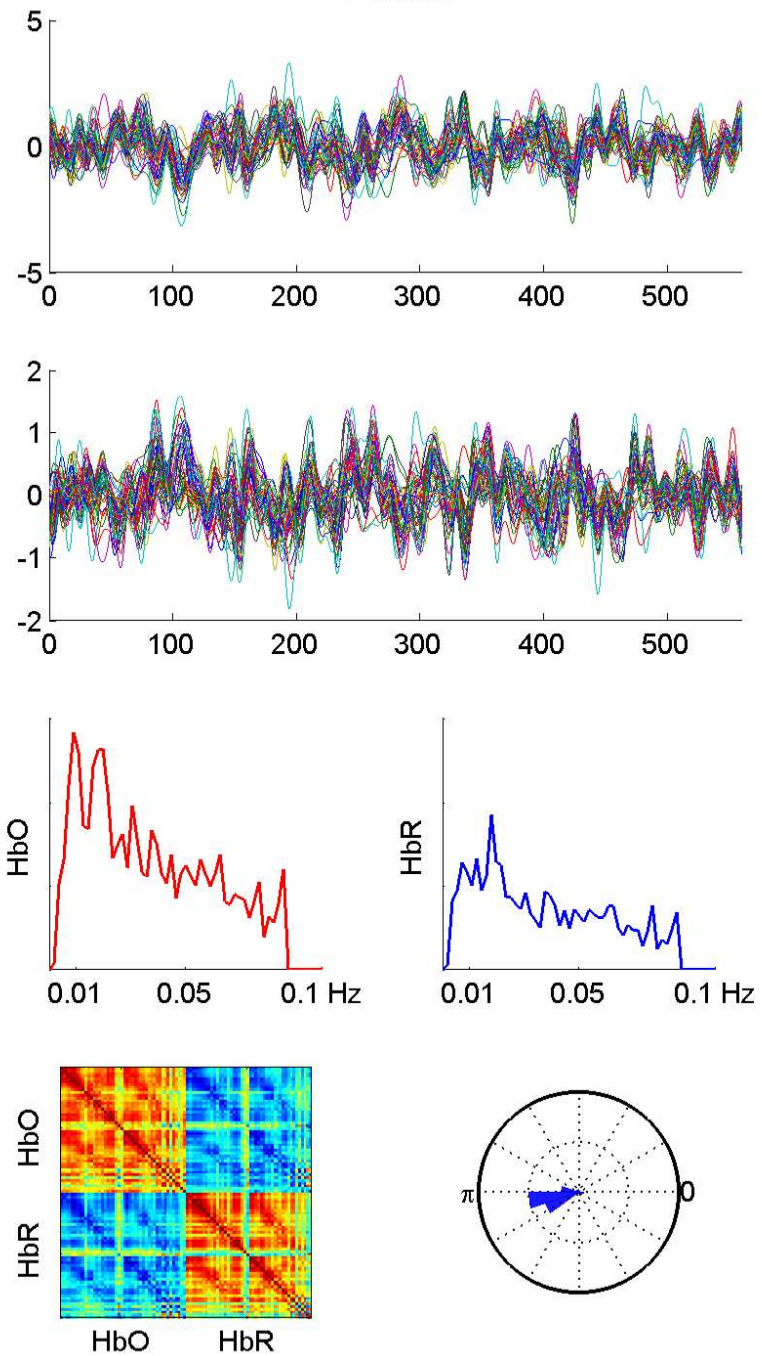
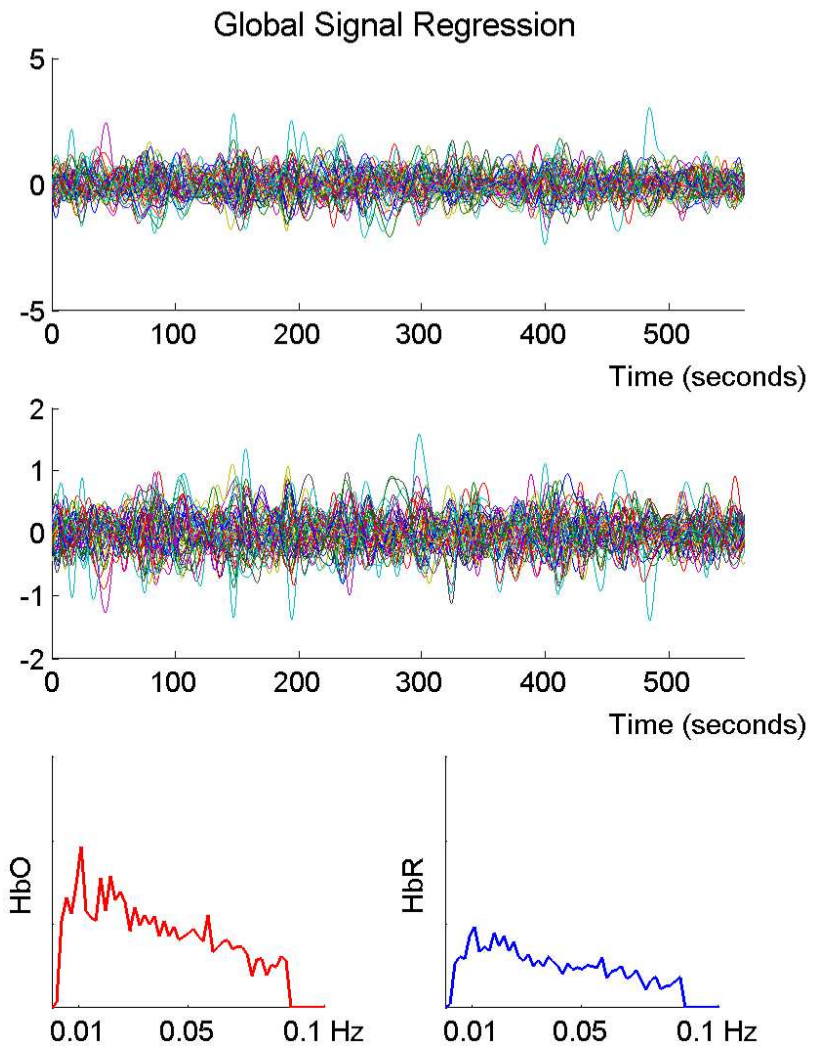
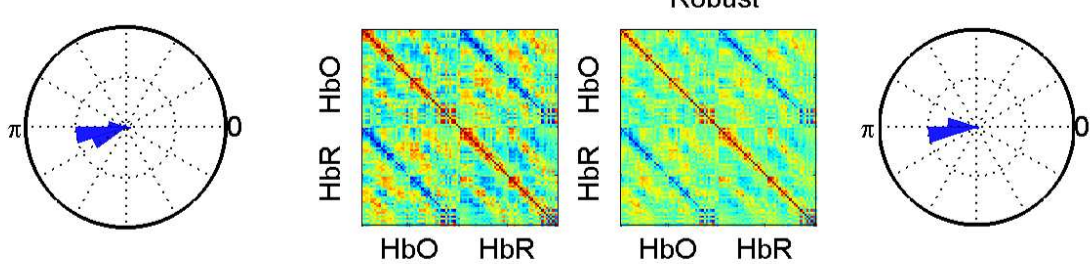

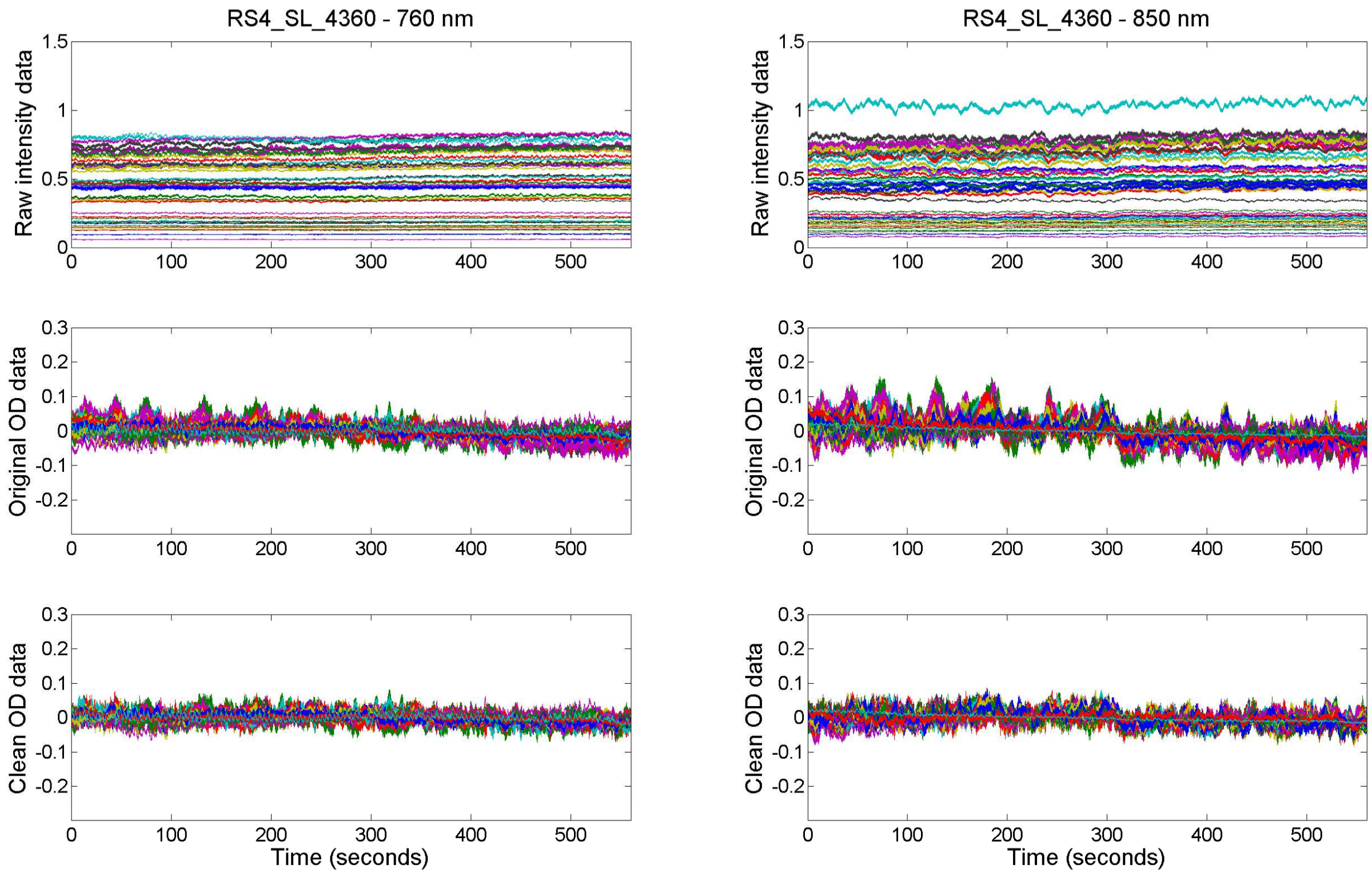
Raw

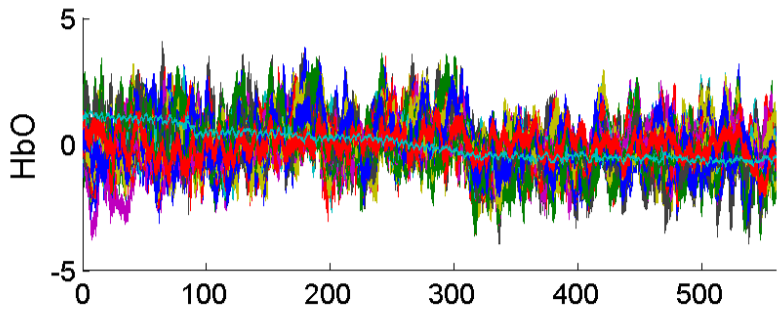

${ }_{-1}^{2}{ }_{-1}^{1}{ }^{2}$.
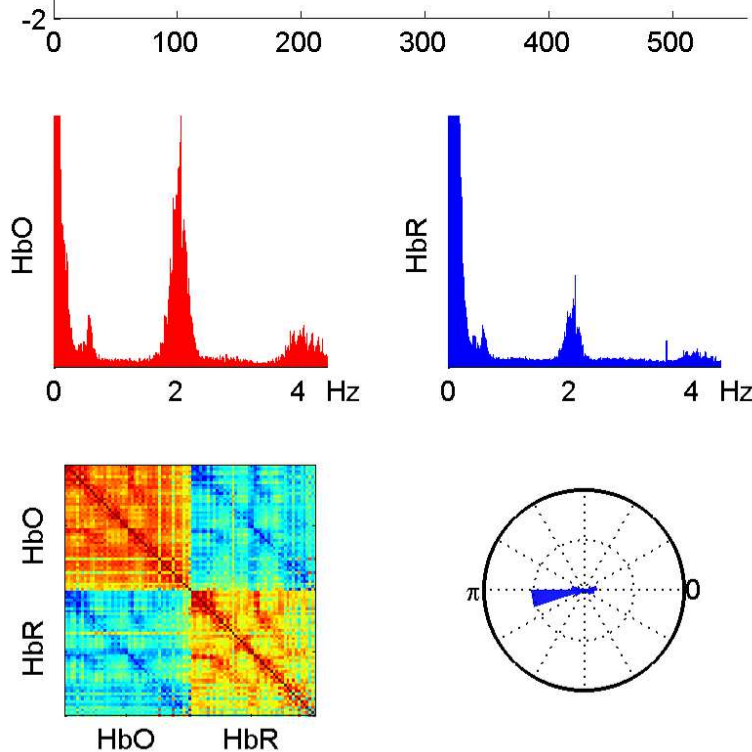

RS4_SL_4360

Filtered
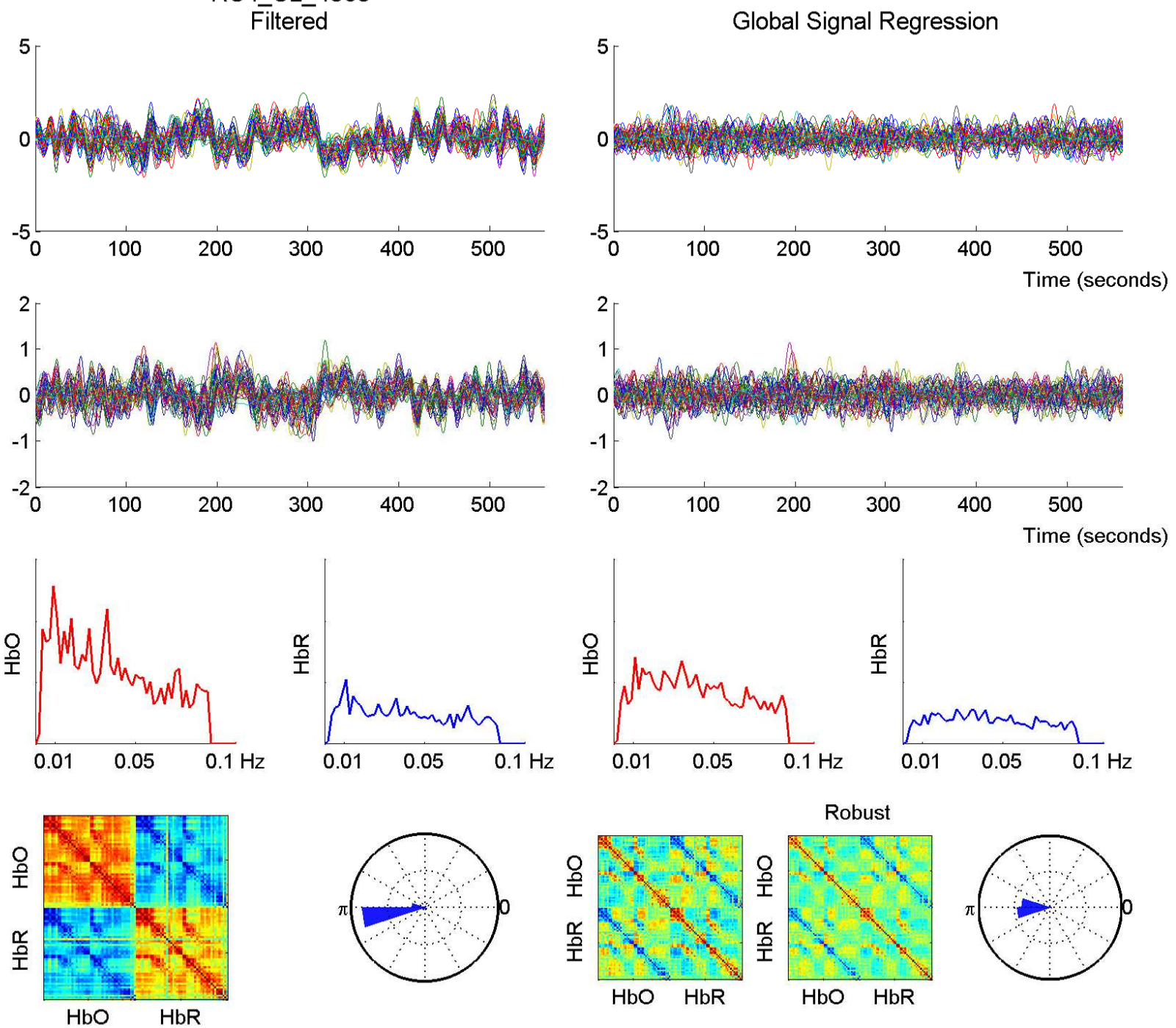
RS4 SL 4355 - 760 nm
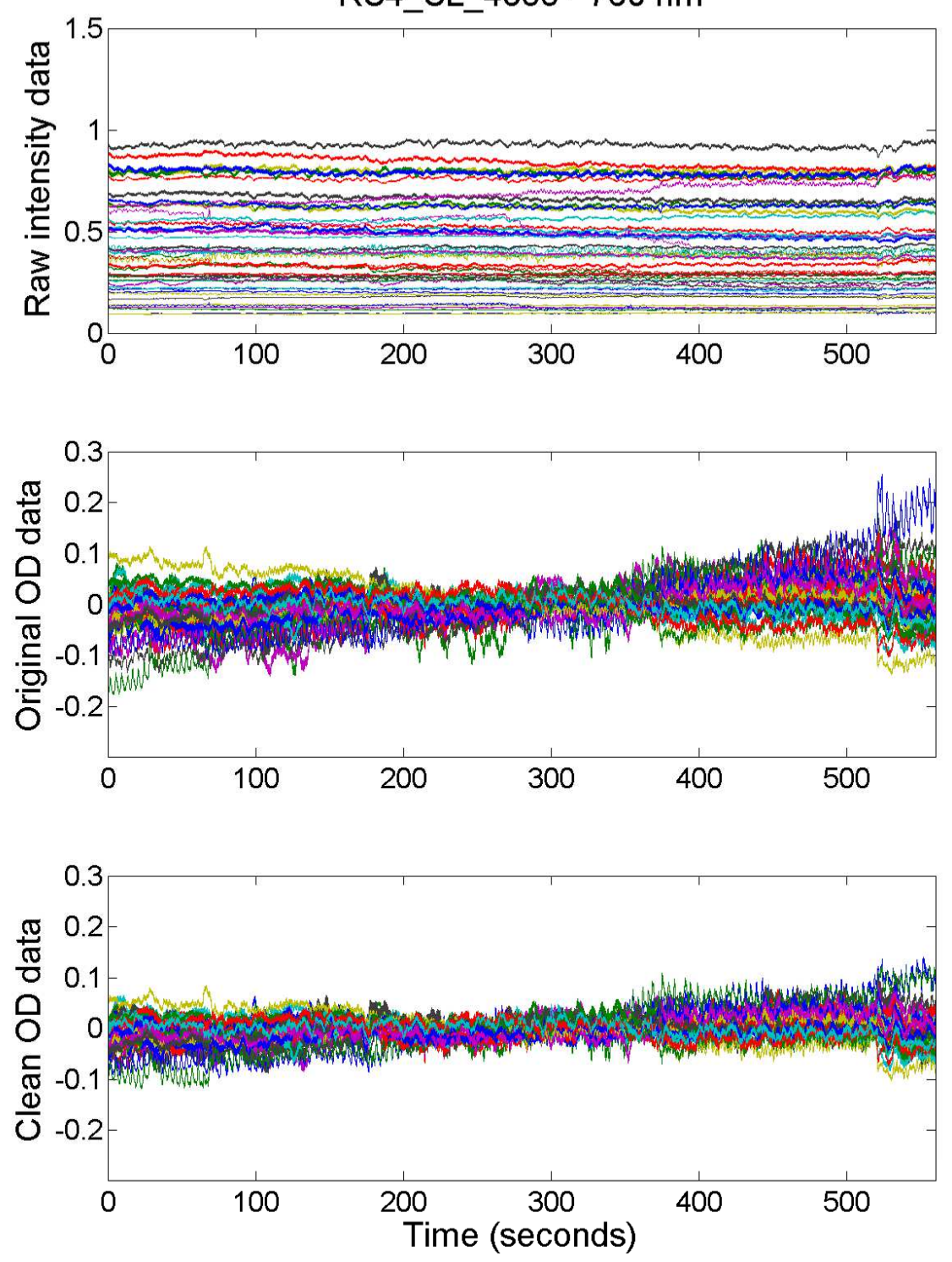

RS4 SL $4355-850 \mathrm{~nm}$
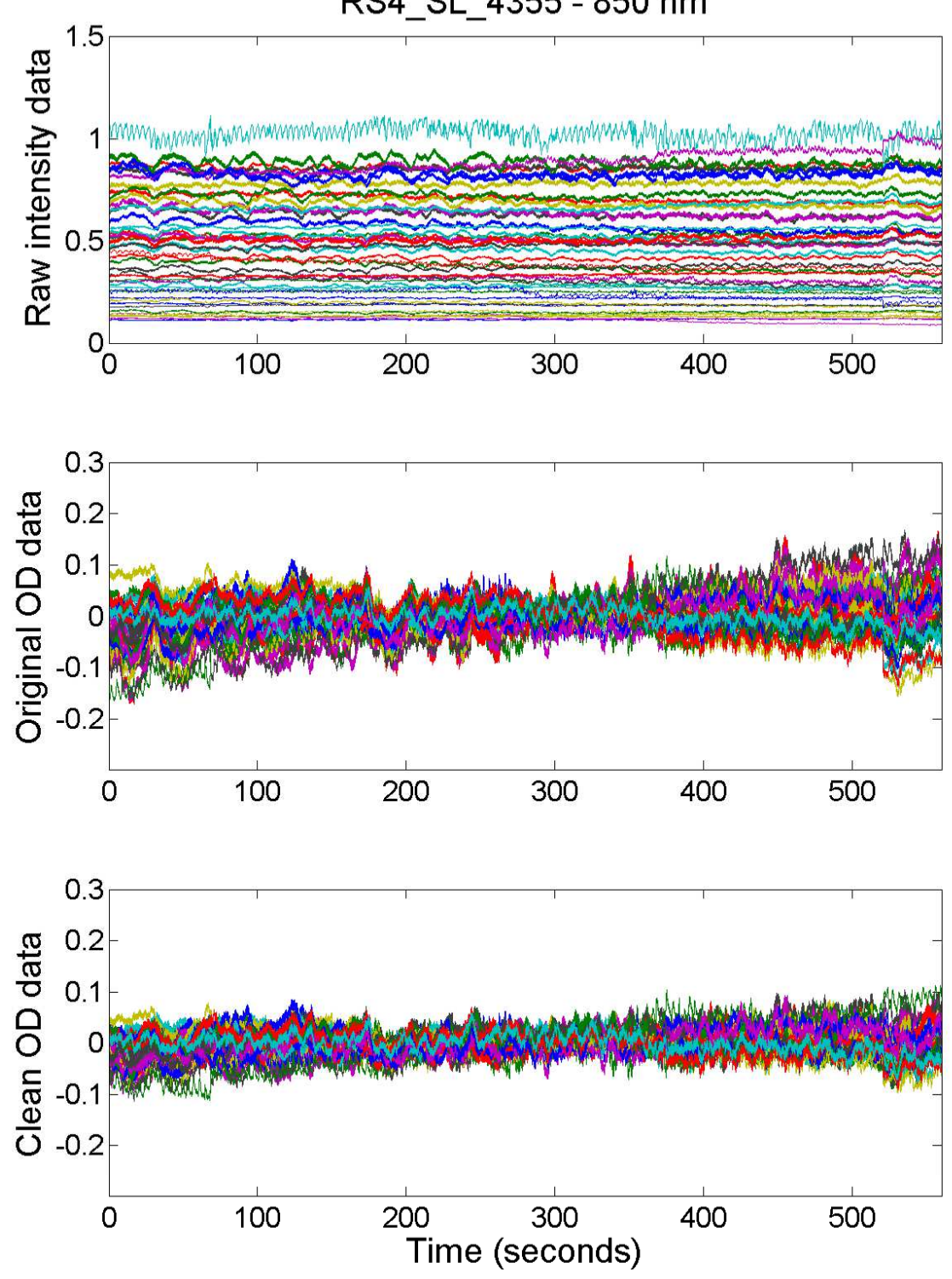
Raw
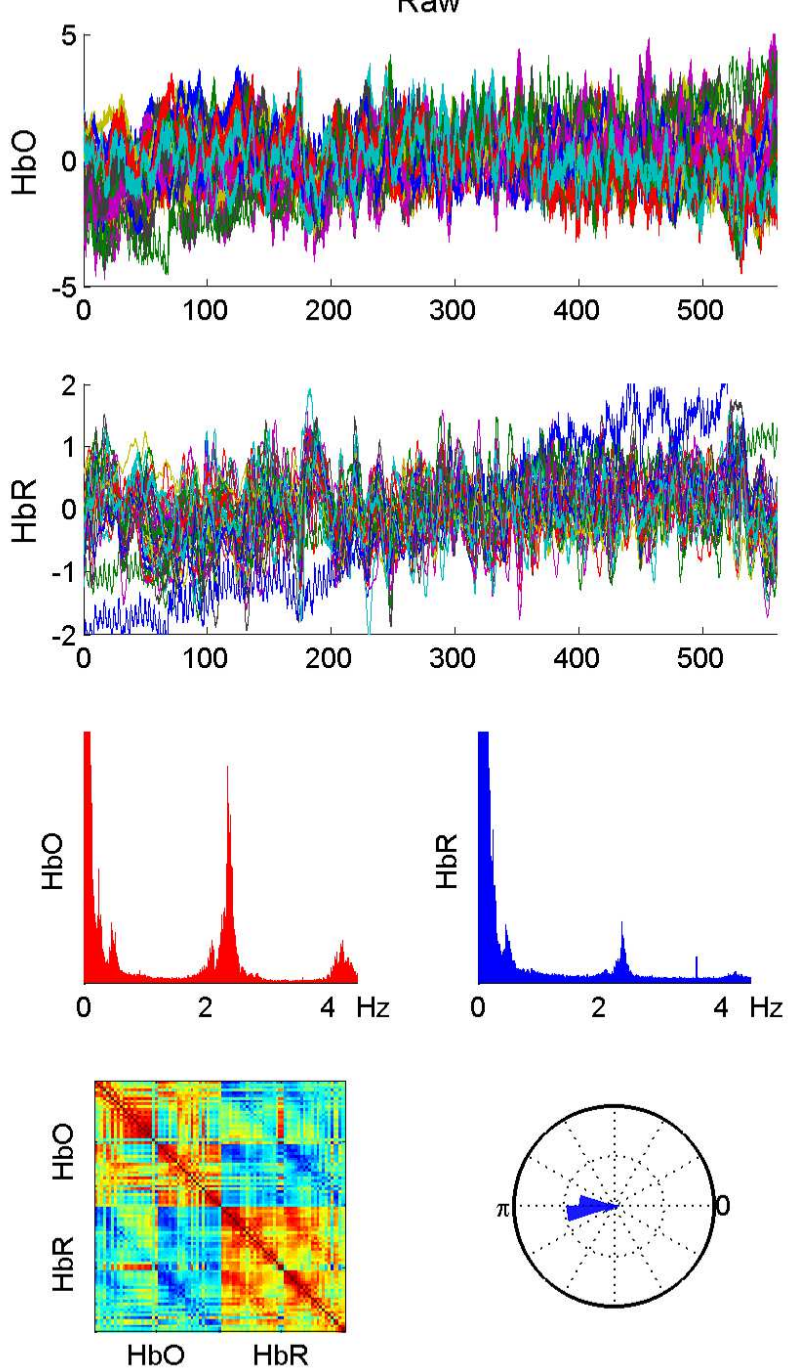

RS4 SL 4355

Filtered
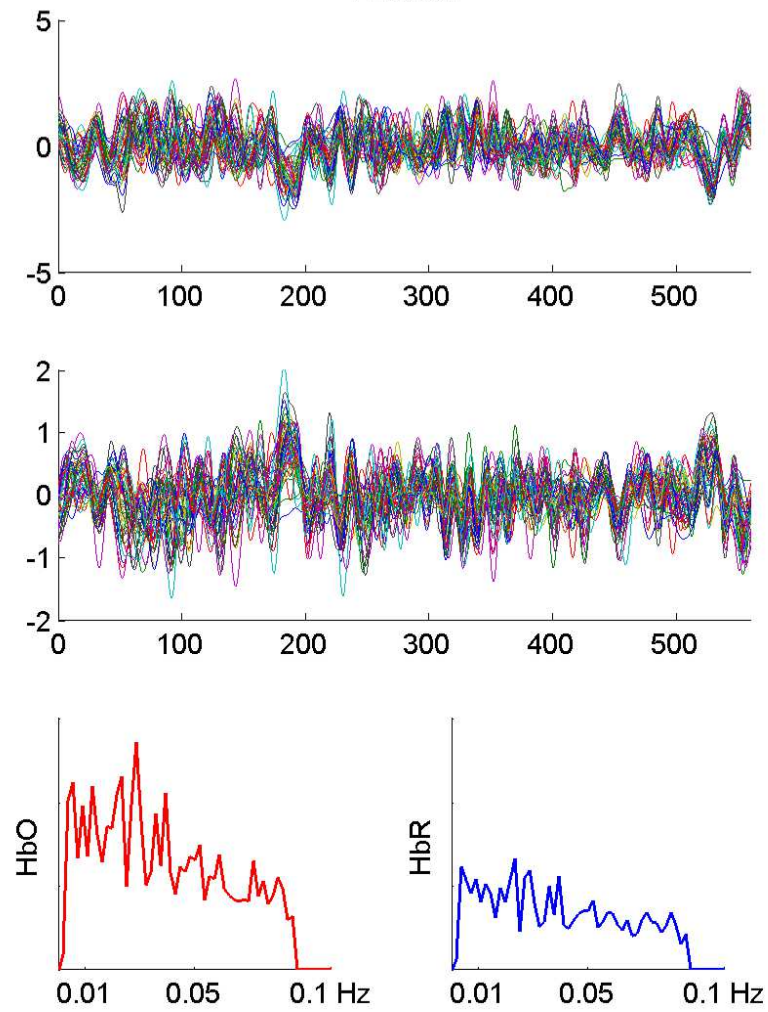
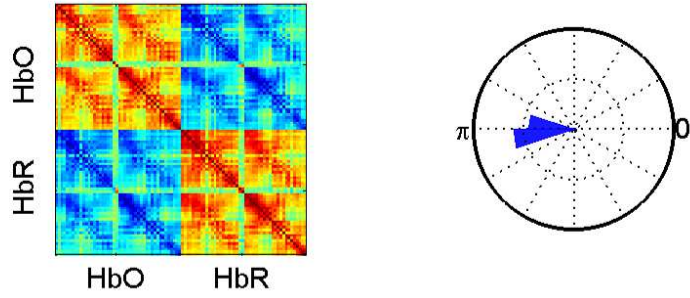

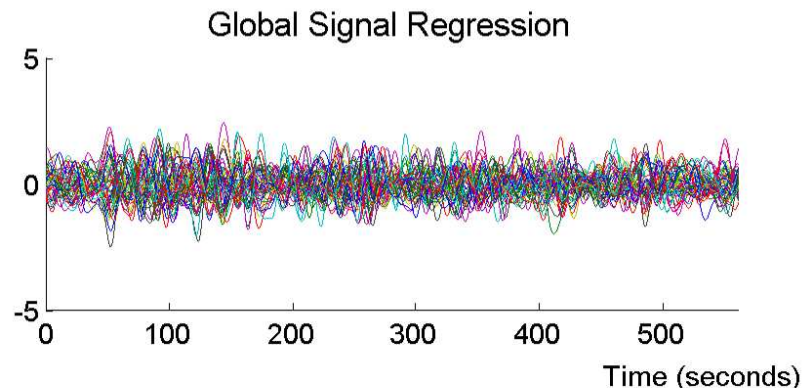

2
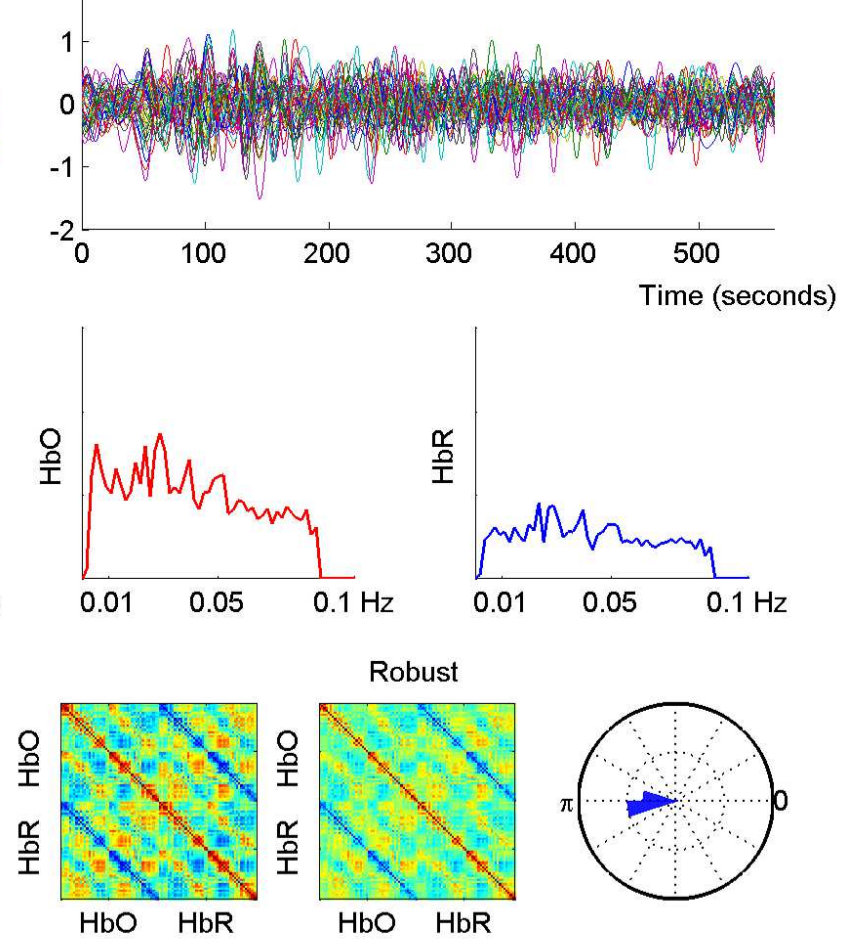

Robust
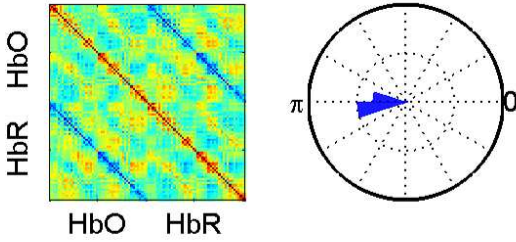

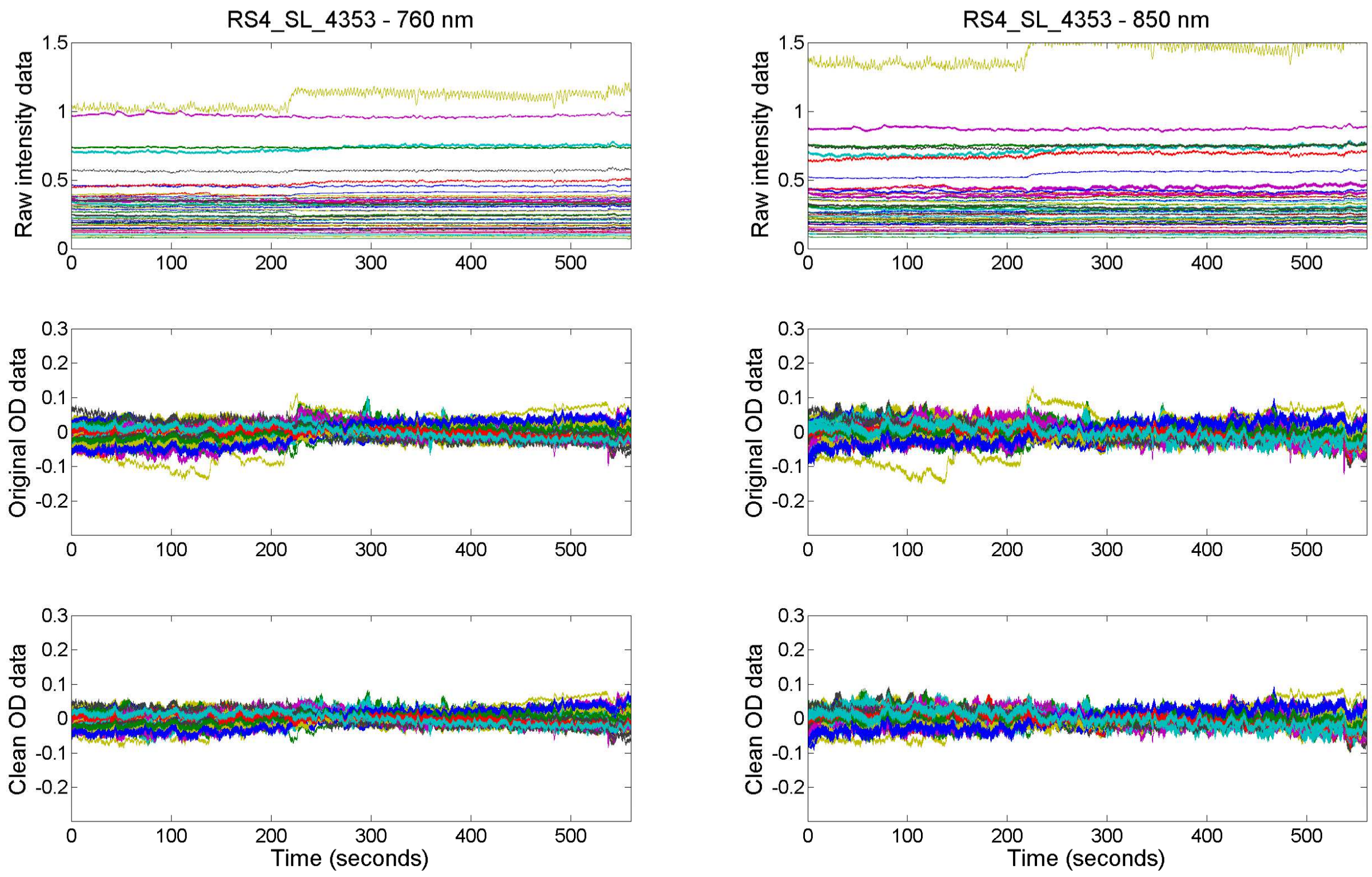
Raw
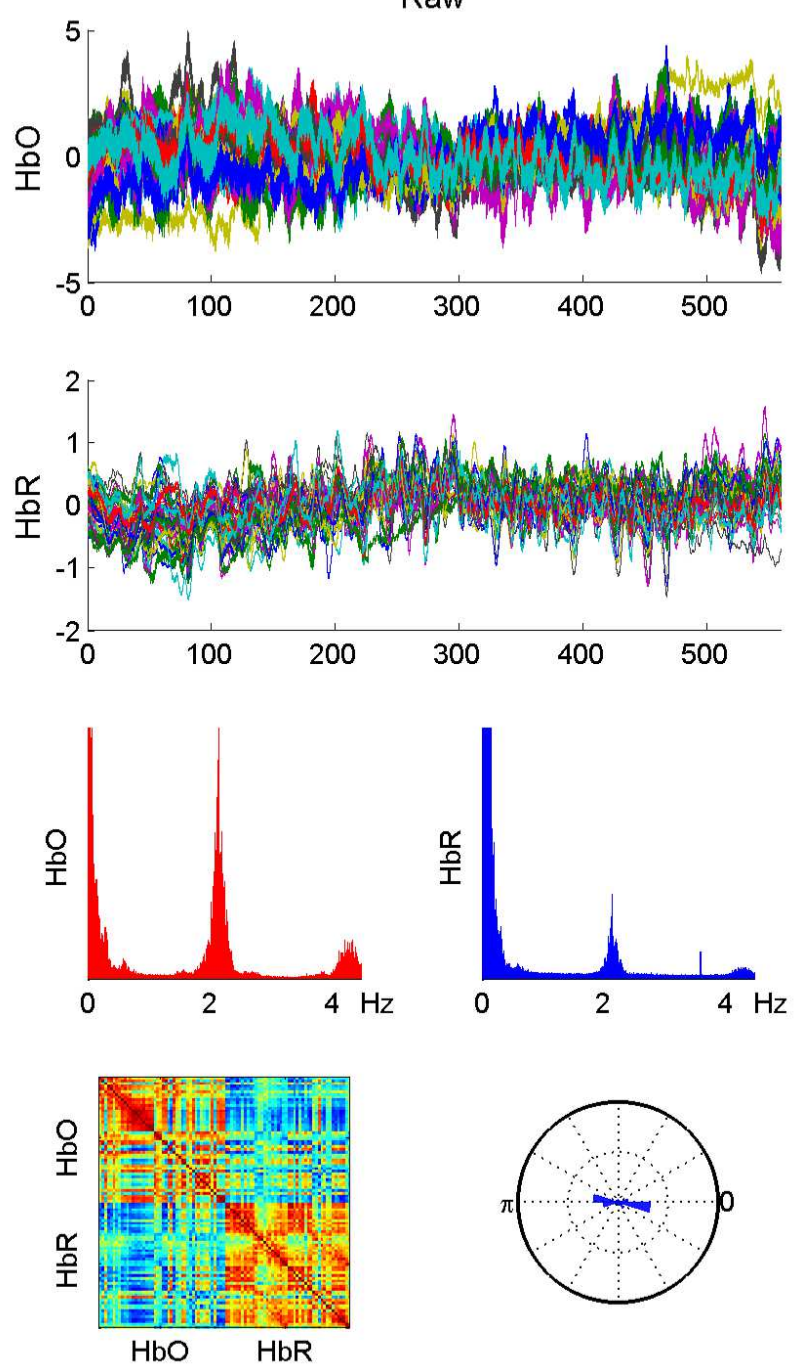

RS4_SL_4353

Filtered
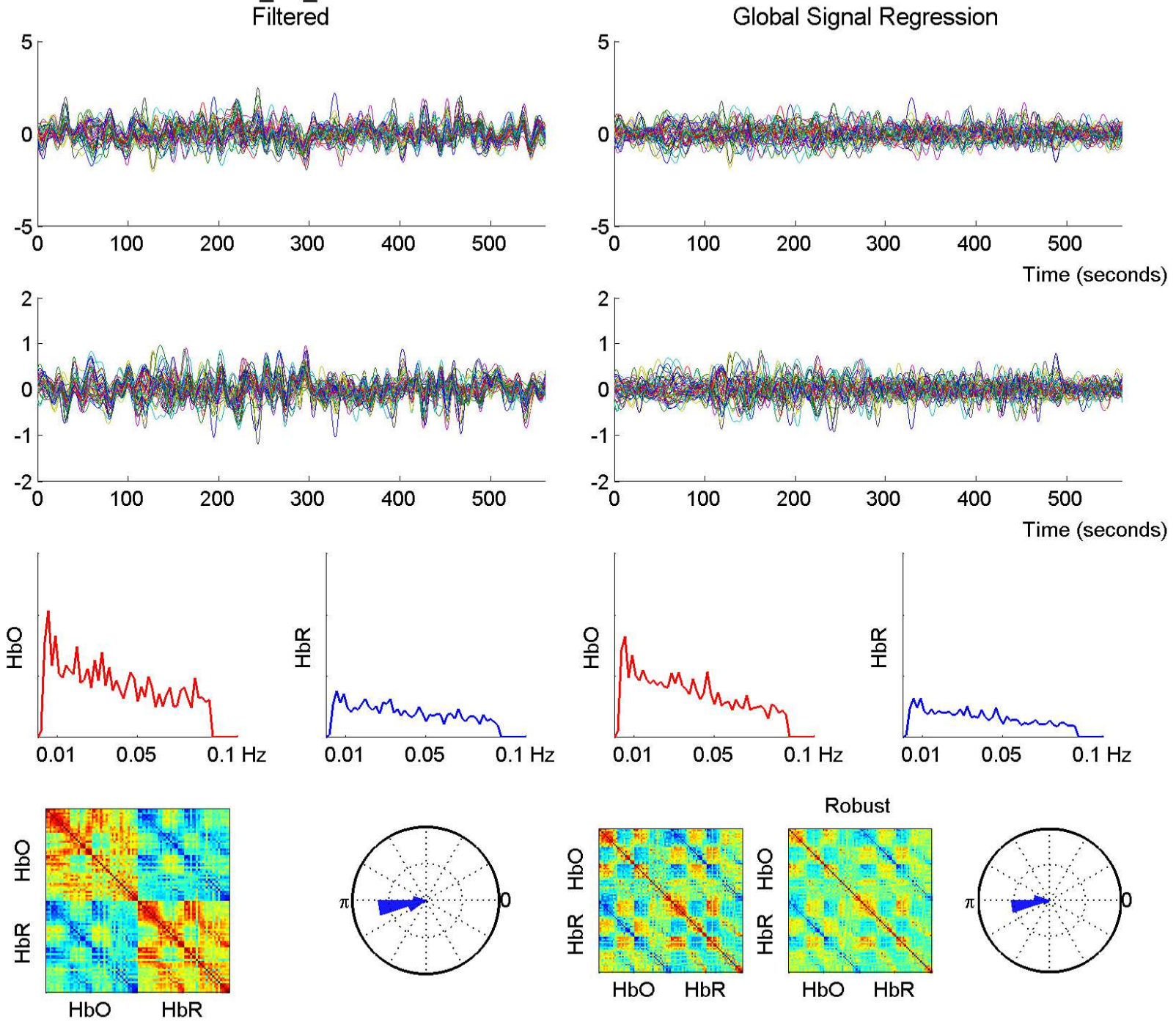
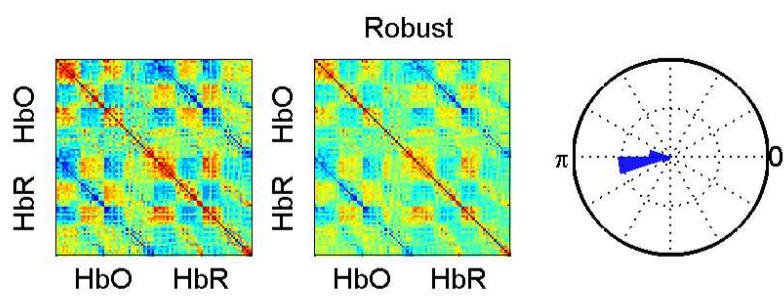
RS4 SL 4352 - 760 nm
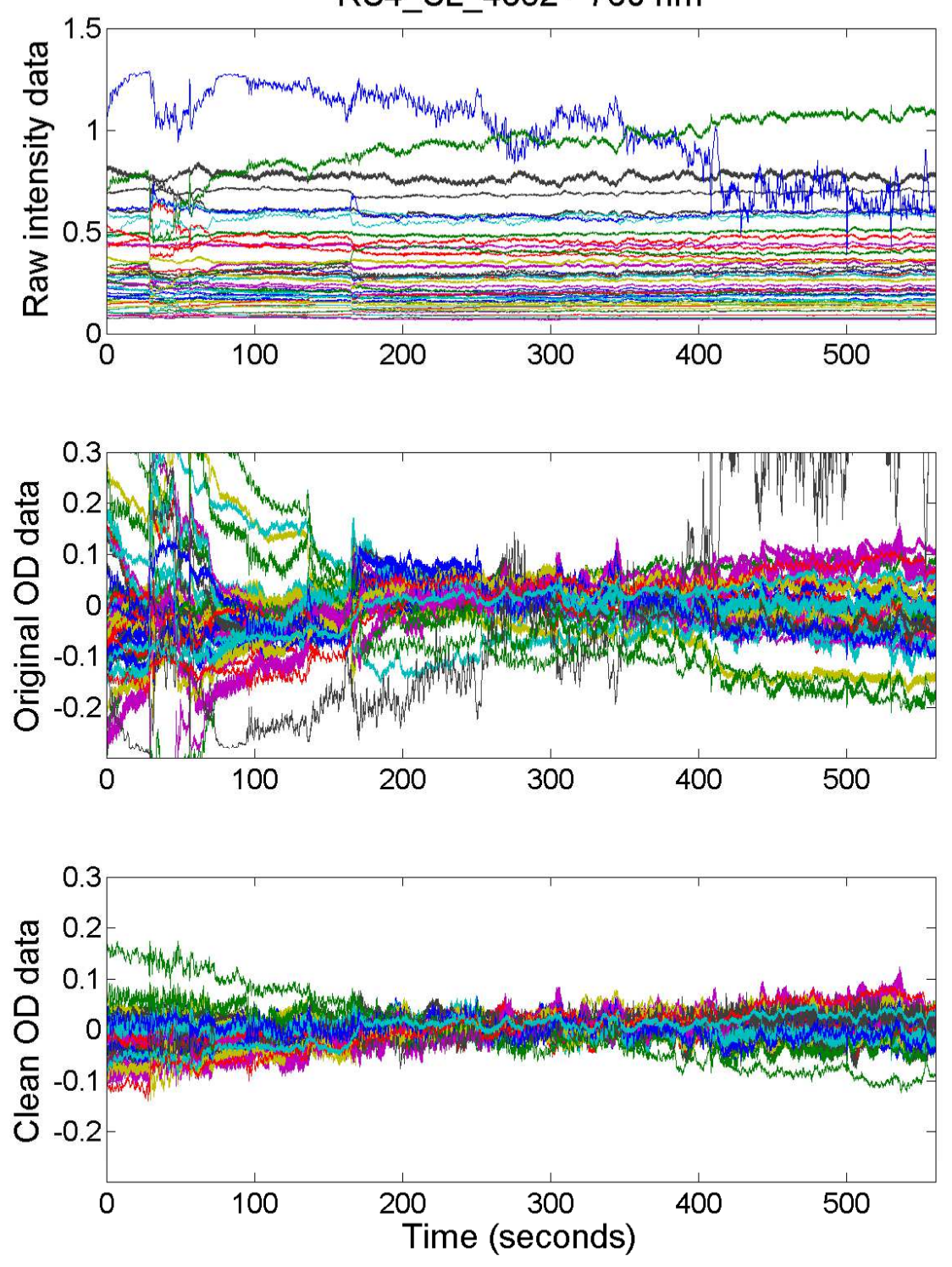
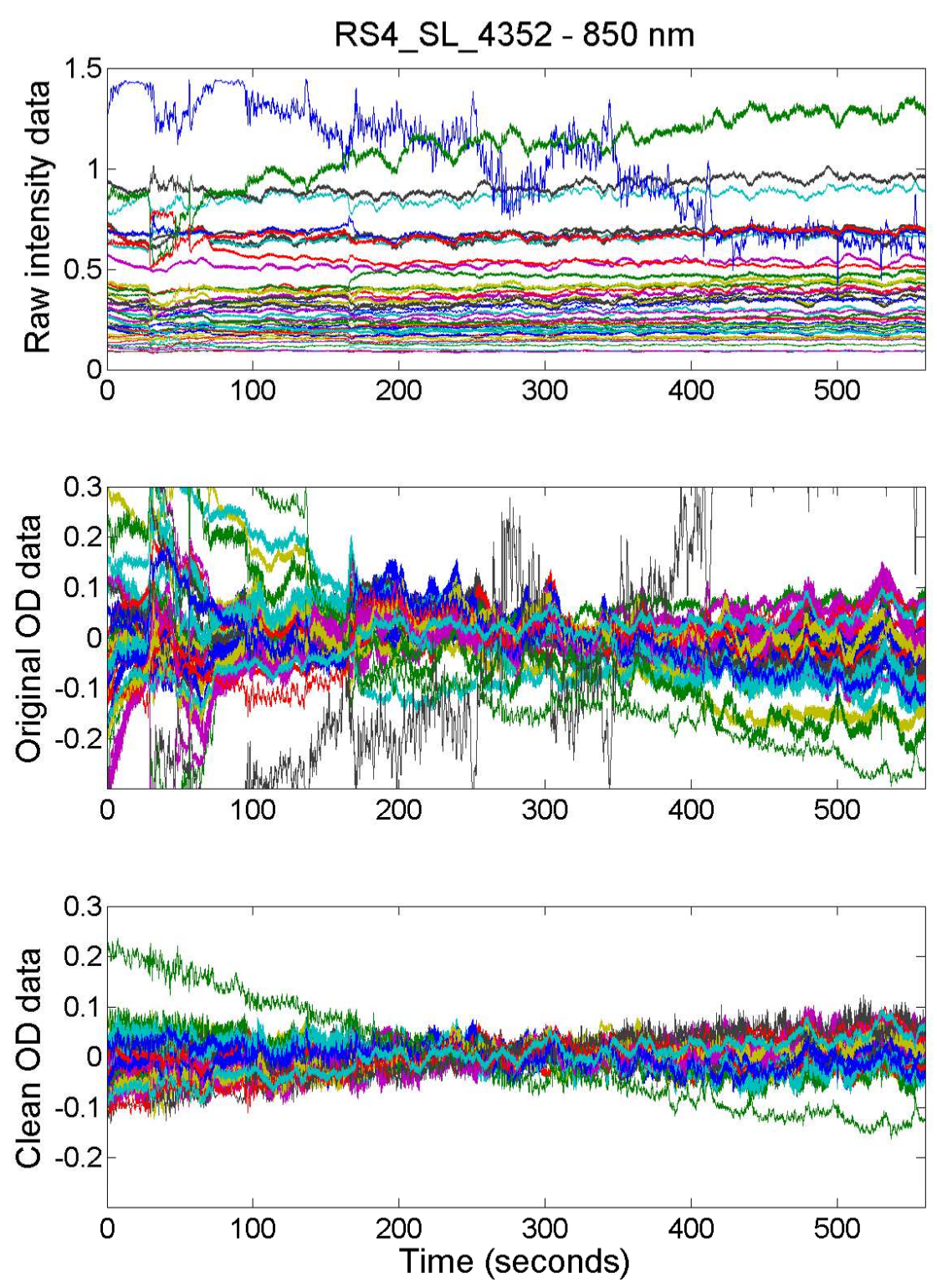
RS4_SL_4352
Raw

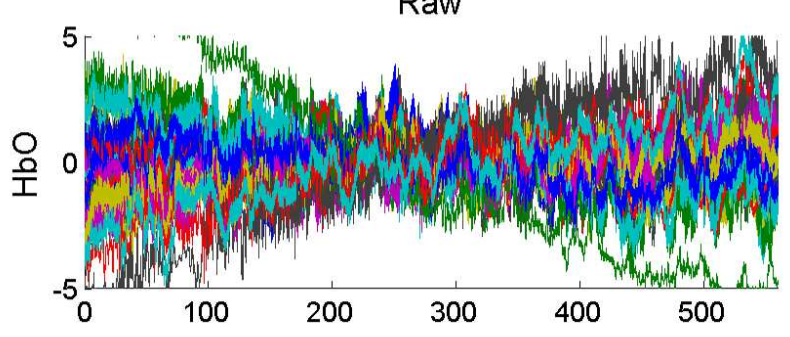

r.

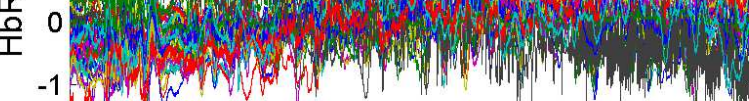

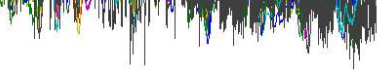
$\begin{array}{cccccc}-2 & 1 & 1 & 1 & 1 \\ 0 & 100 & 200 & 300 & 400 & 500\end{array}$
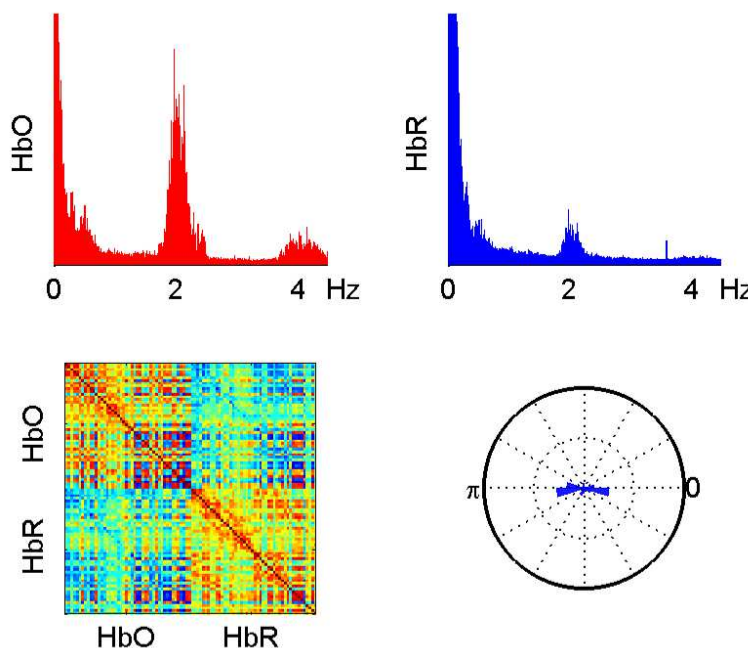

Filtered
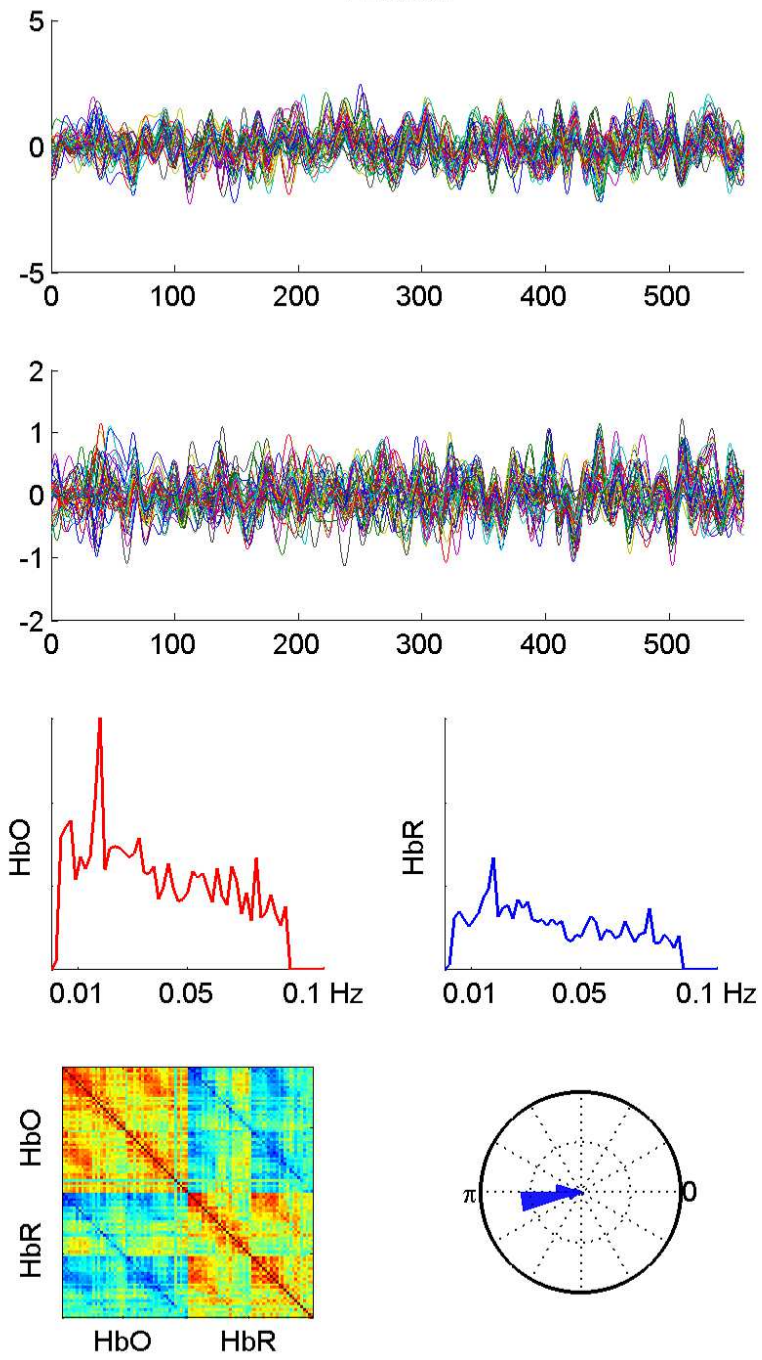

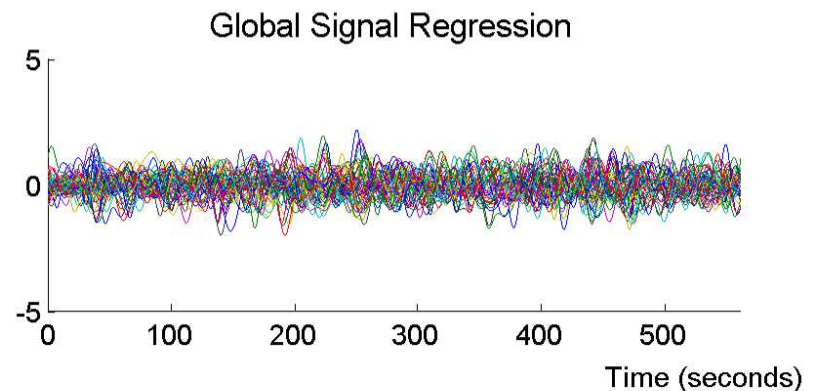

2
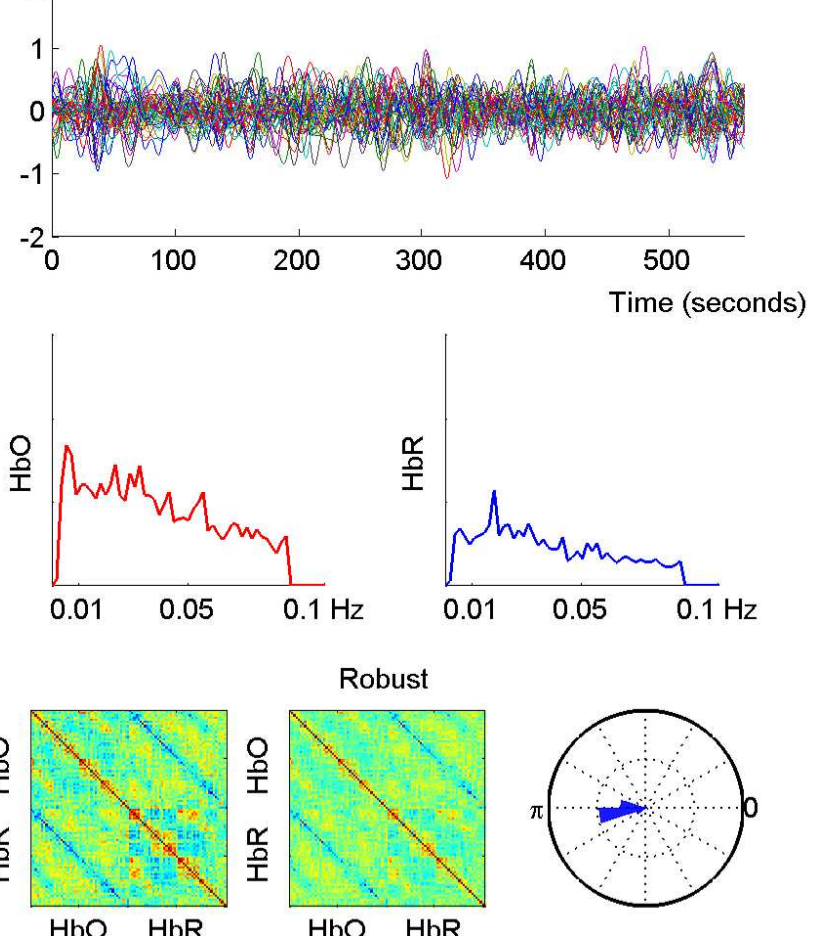

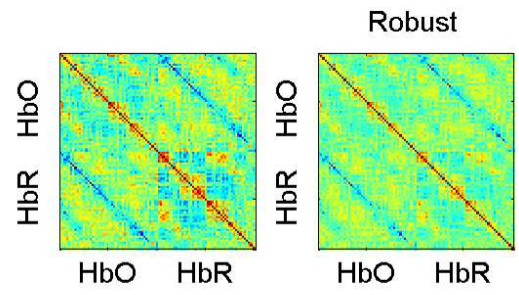



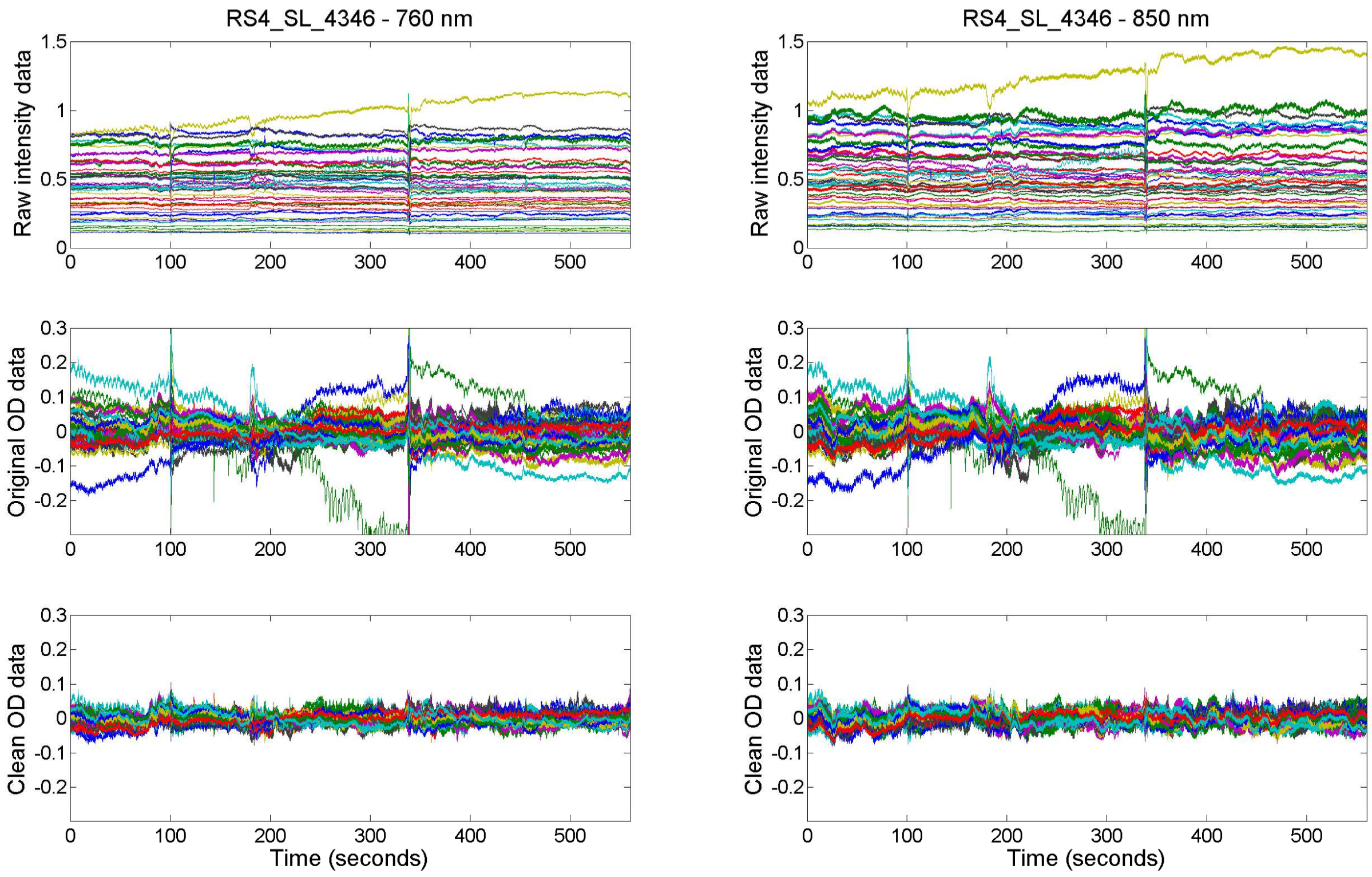
Raw
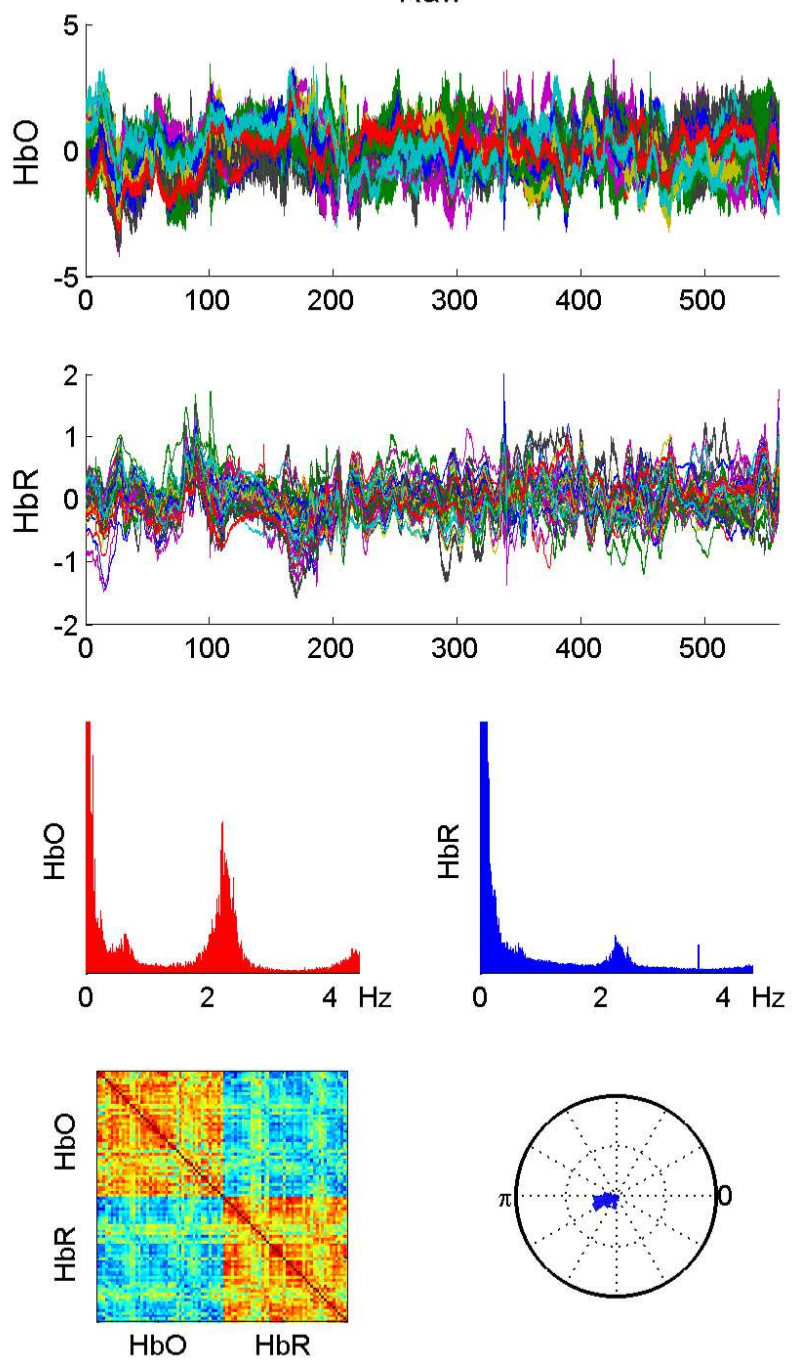

RS4_SL_4346

Filtered
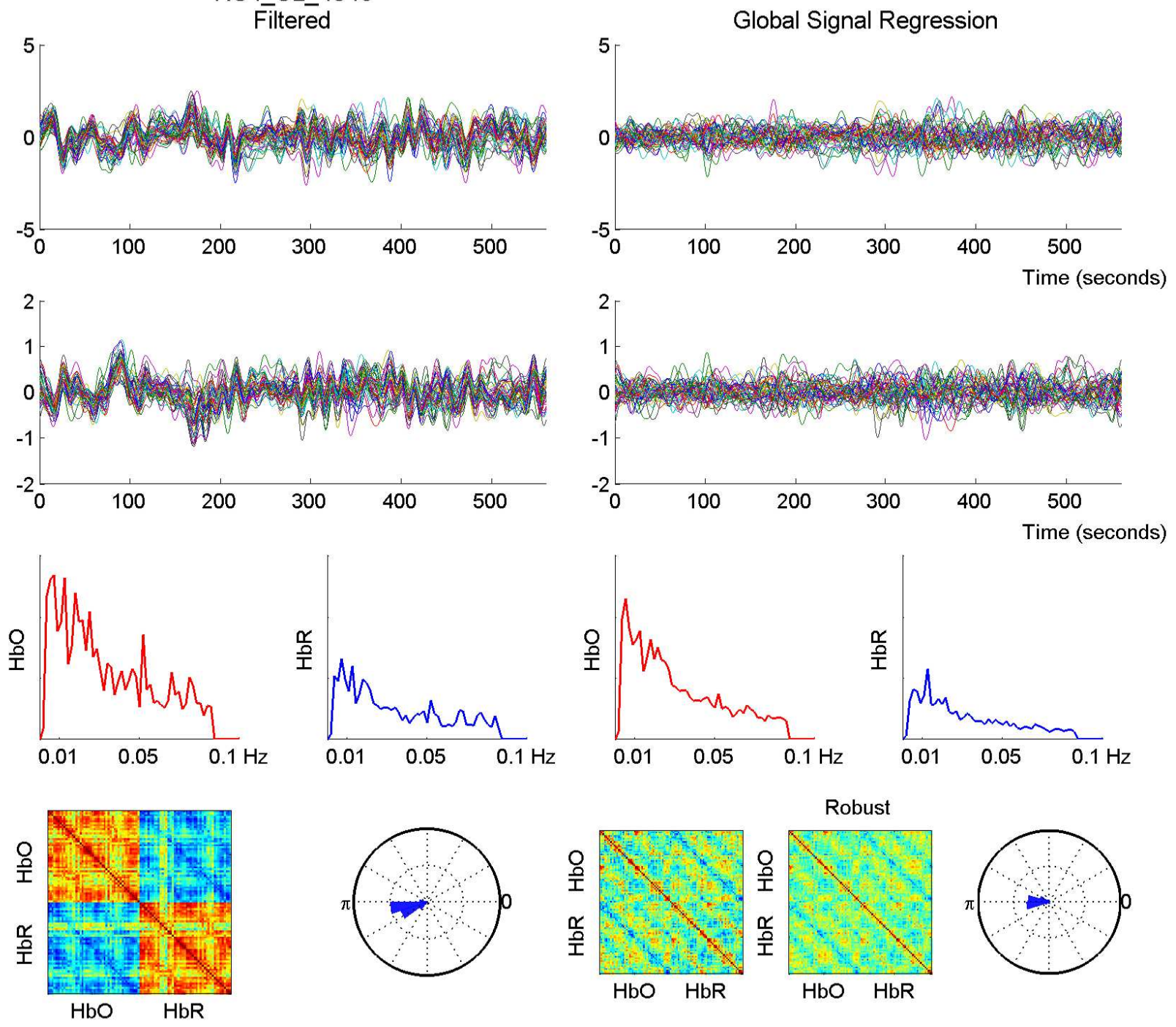
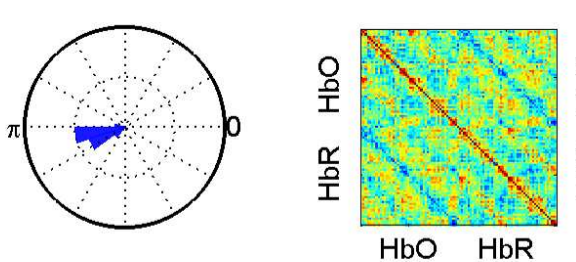

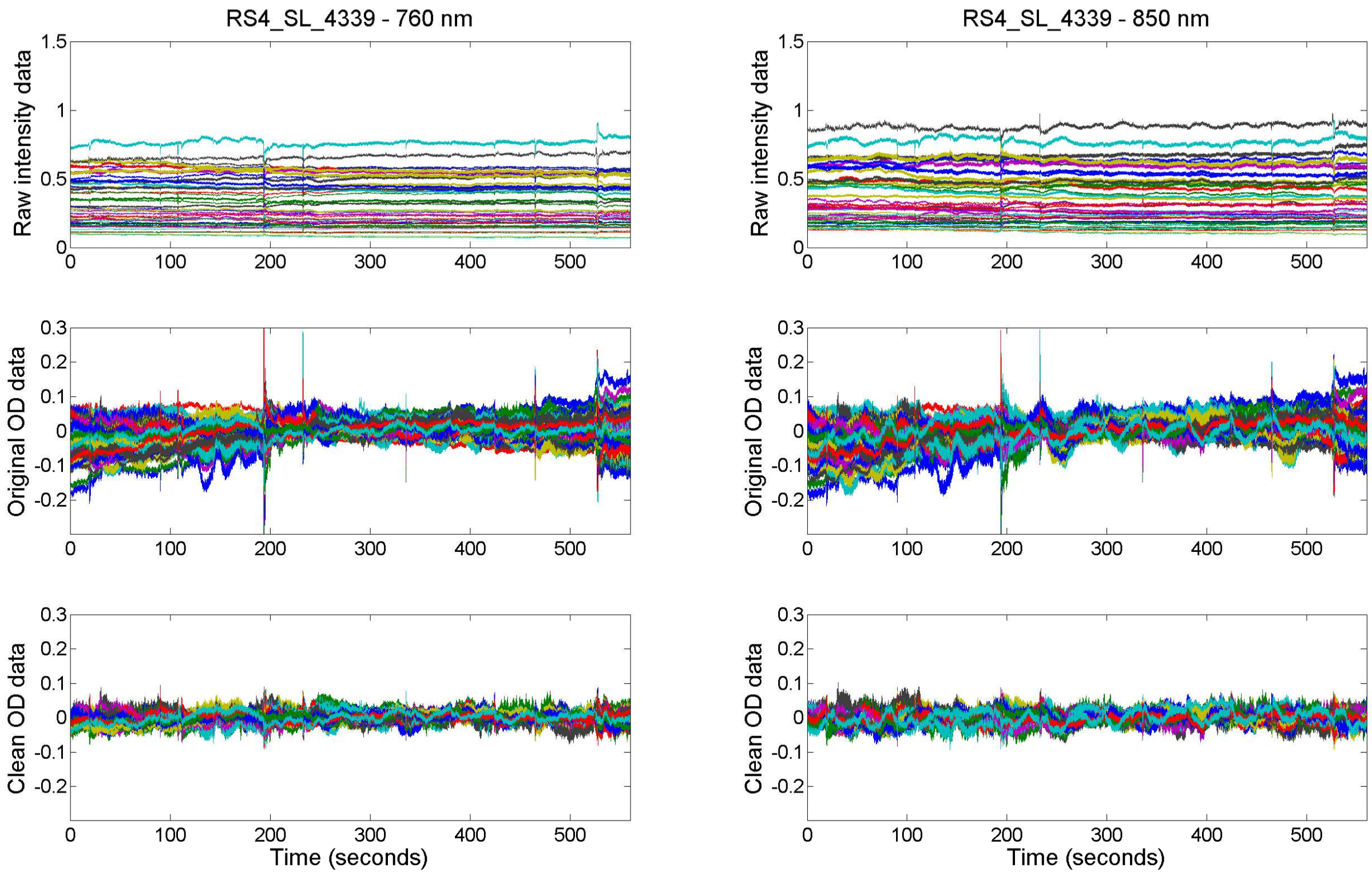

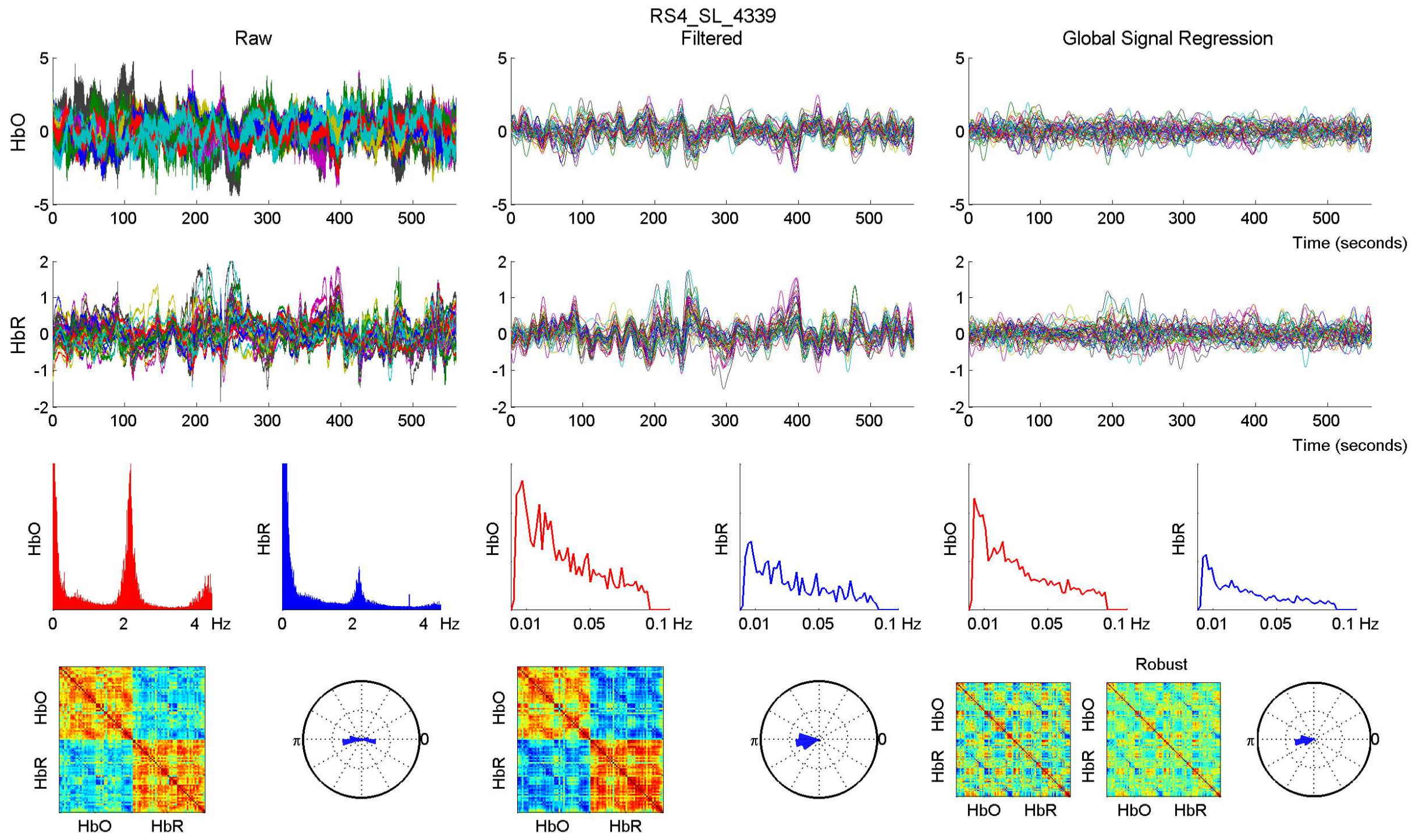

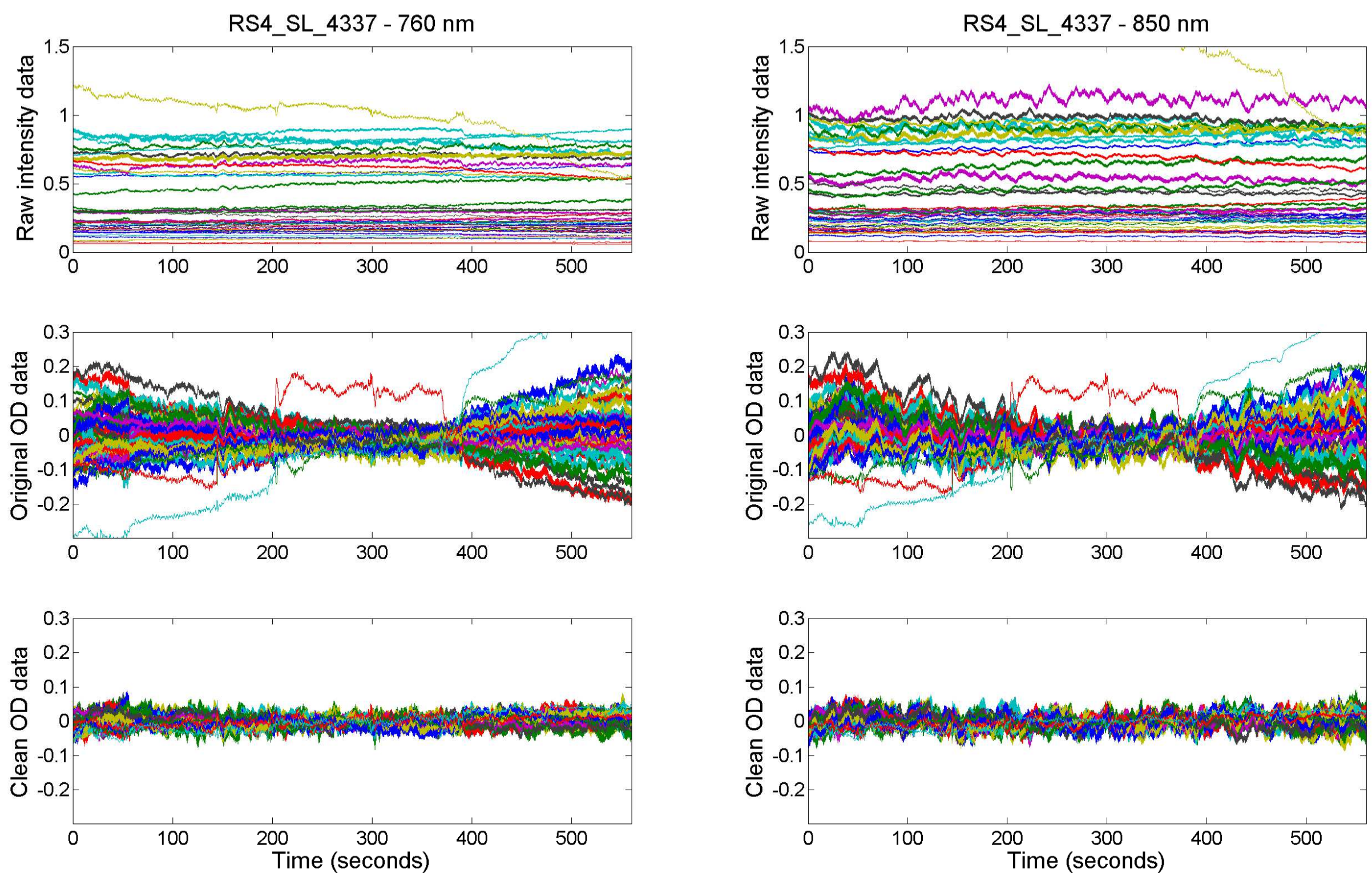
Raw

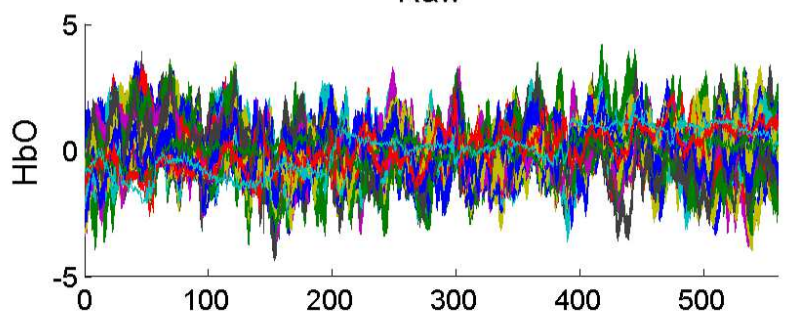

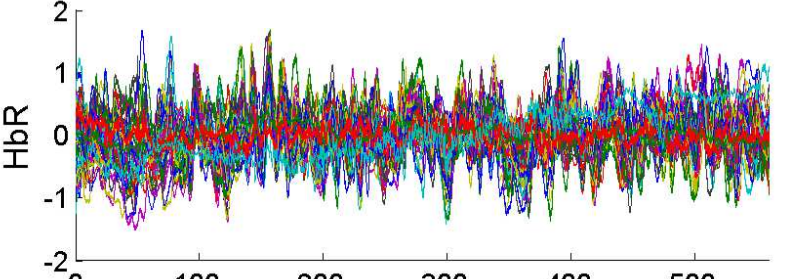
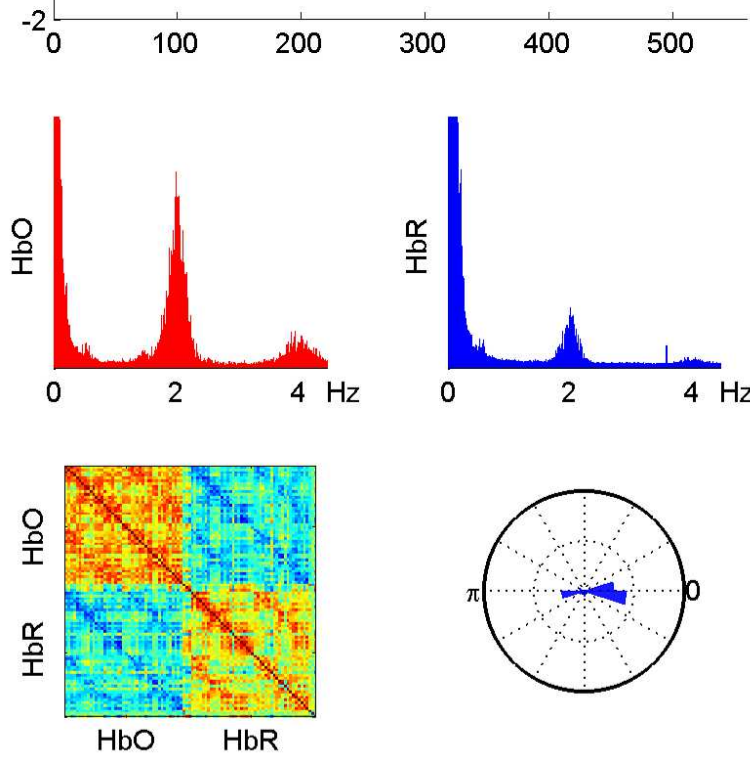
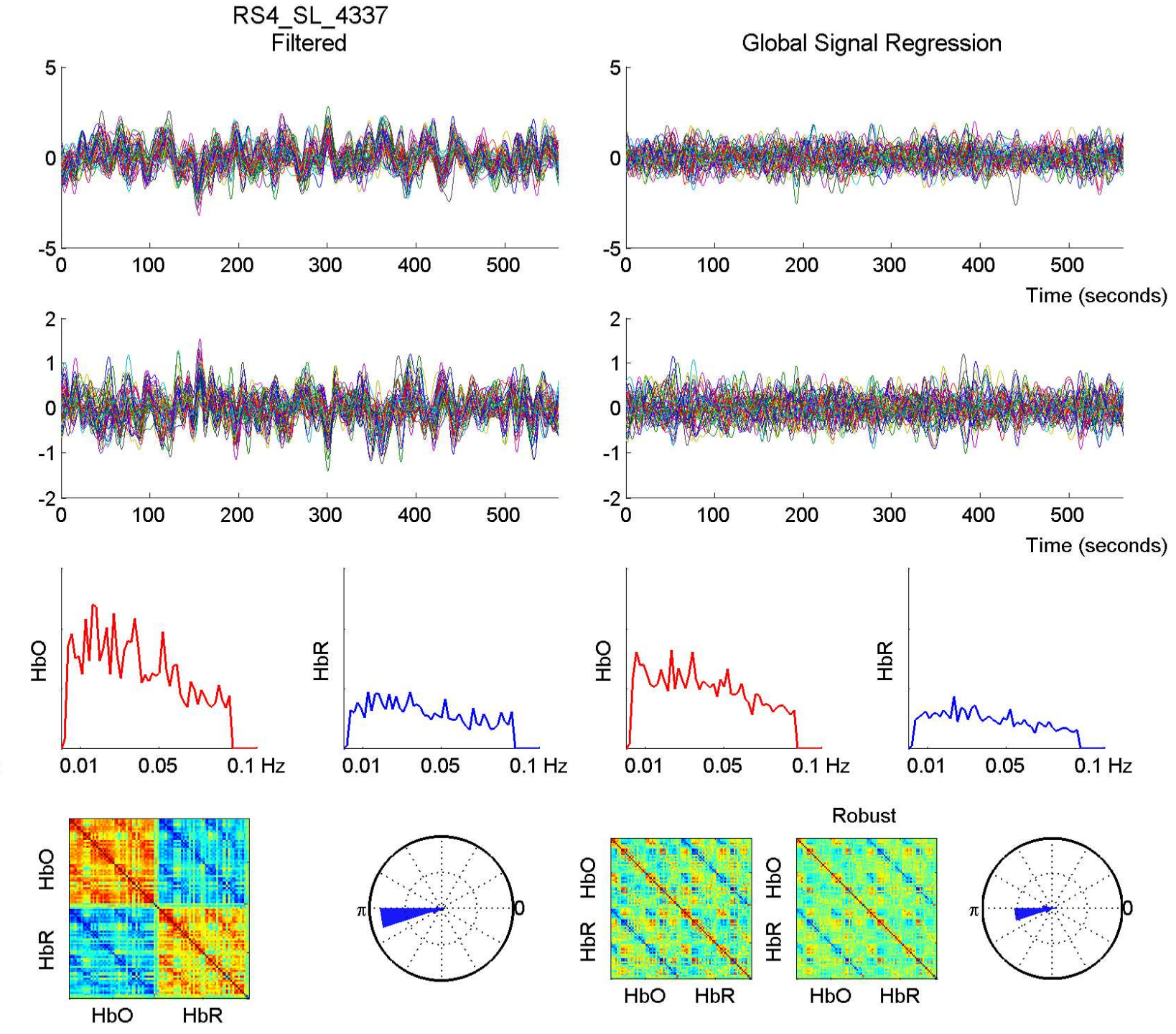
RS4 SL 4336 - 760 nm
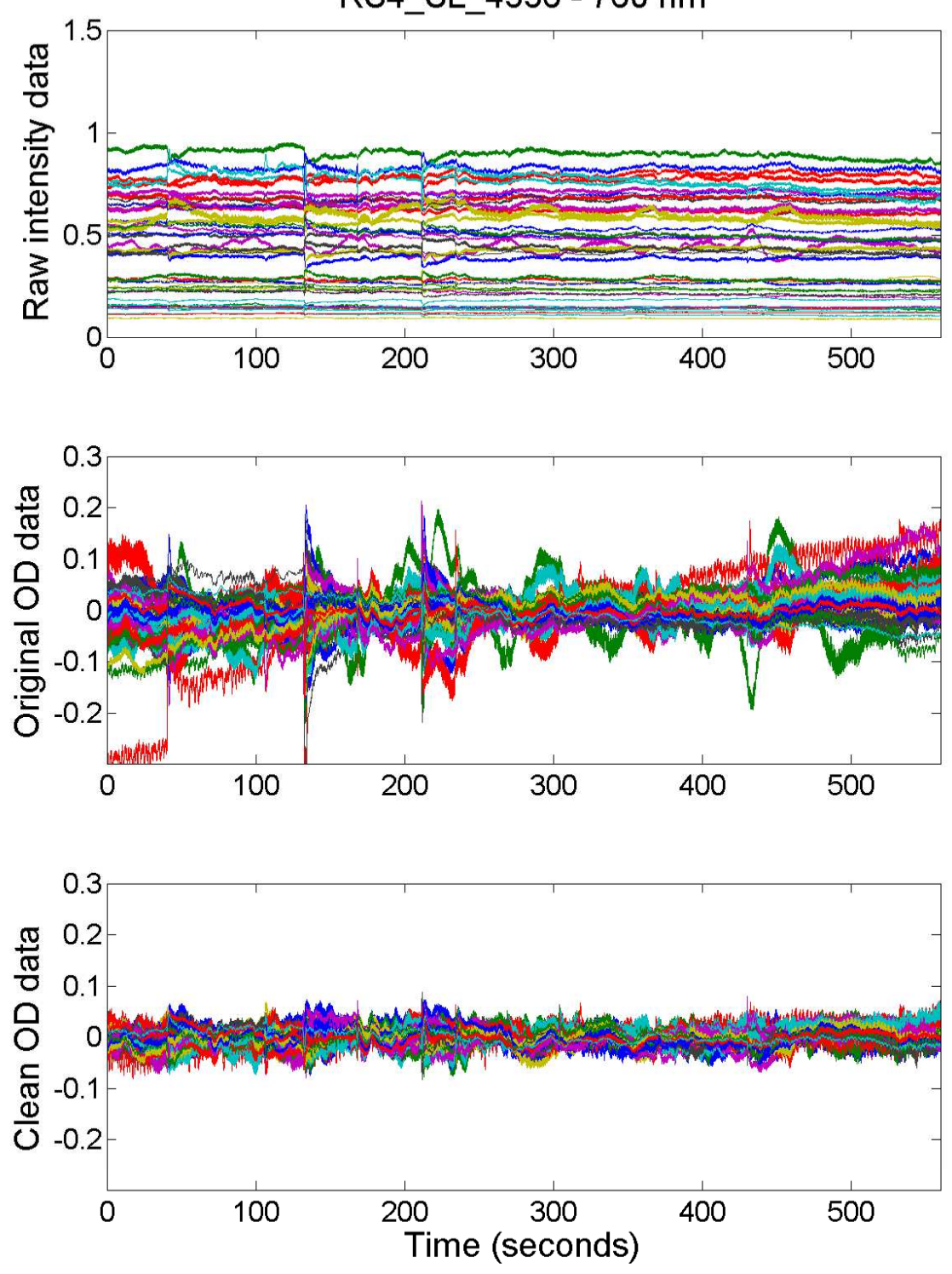

RS4 SL $4336-850 \mathrm{~nm}$
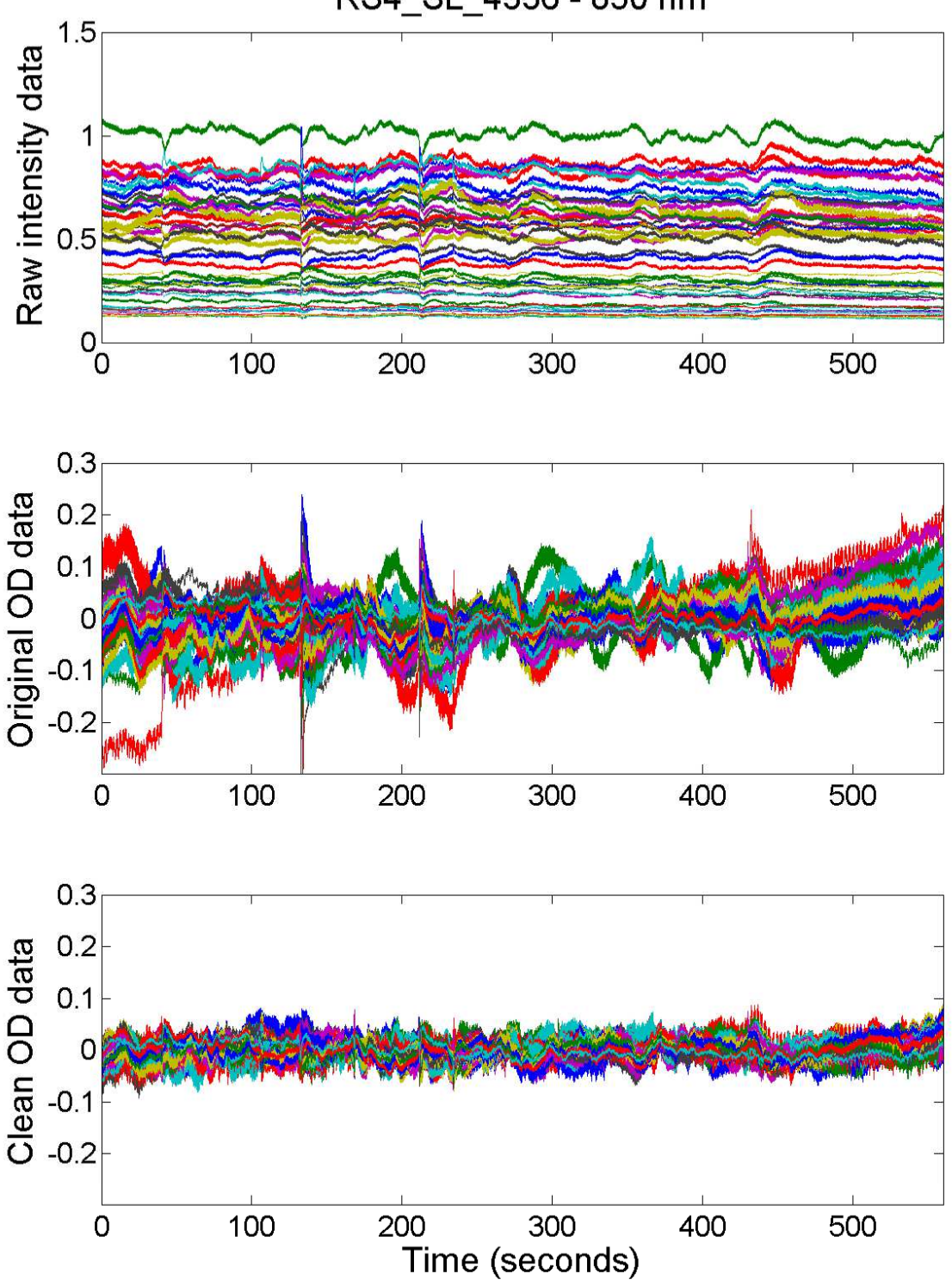
Raw
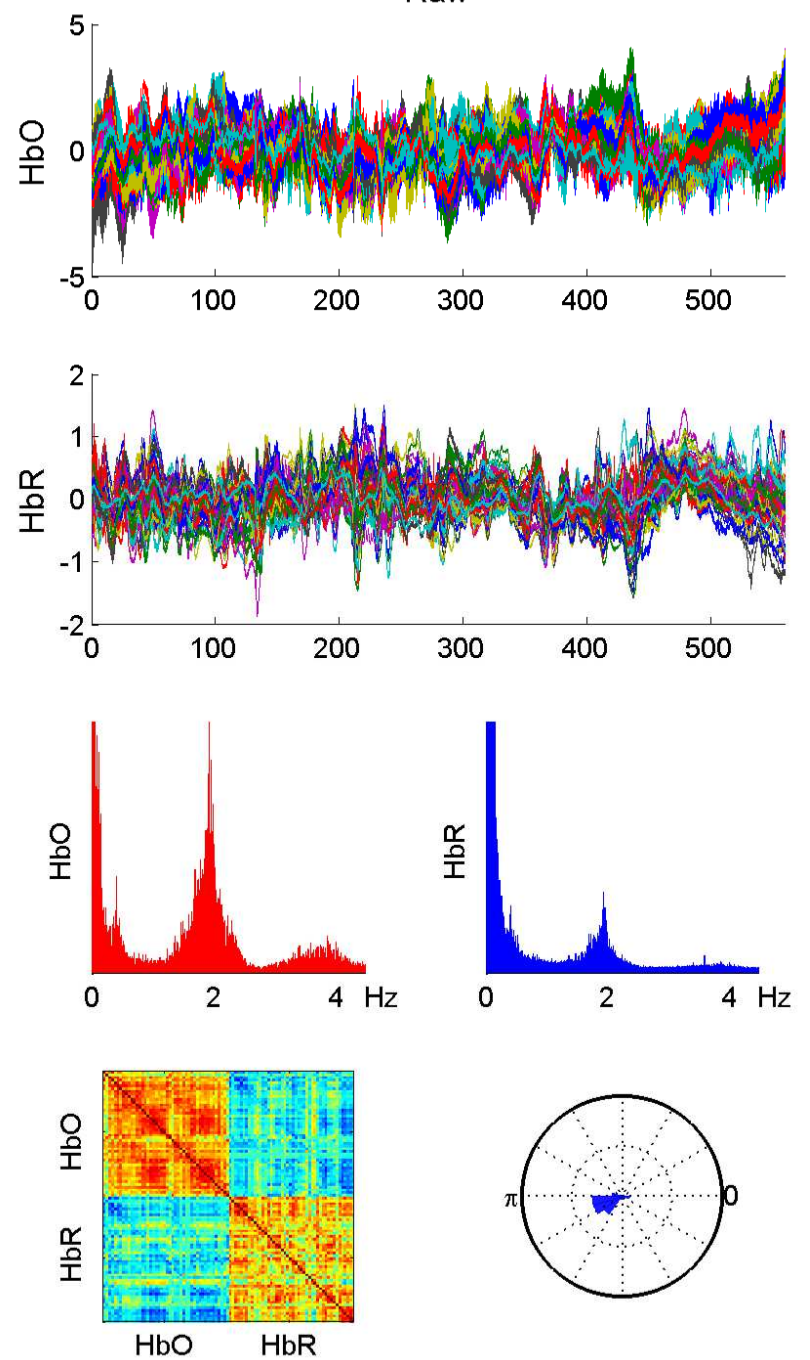

RS4_SL_4336

Filtered
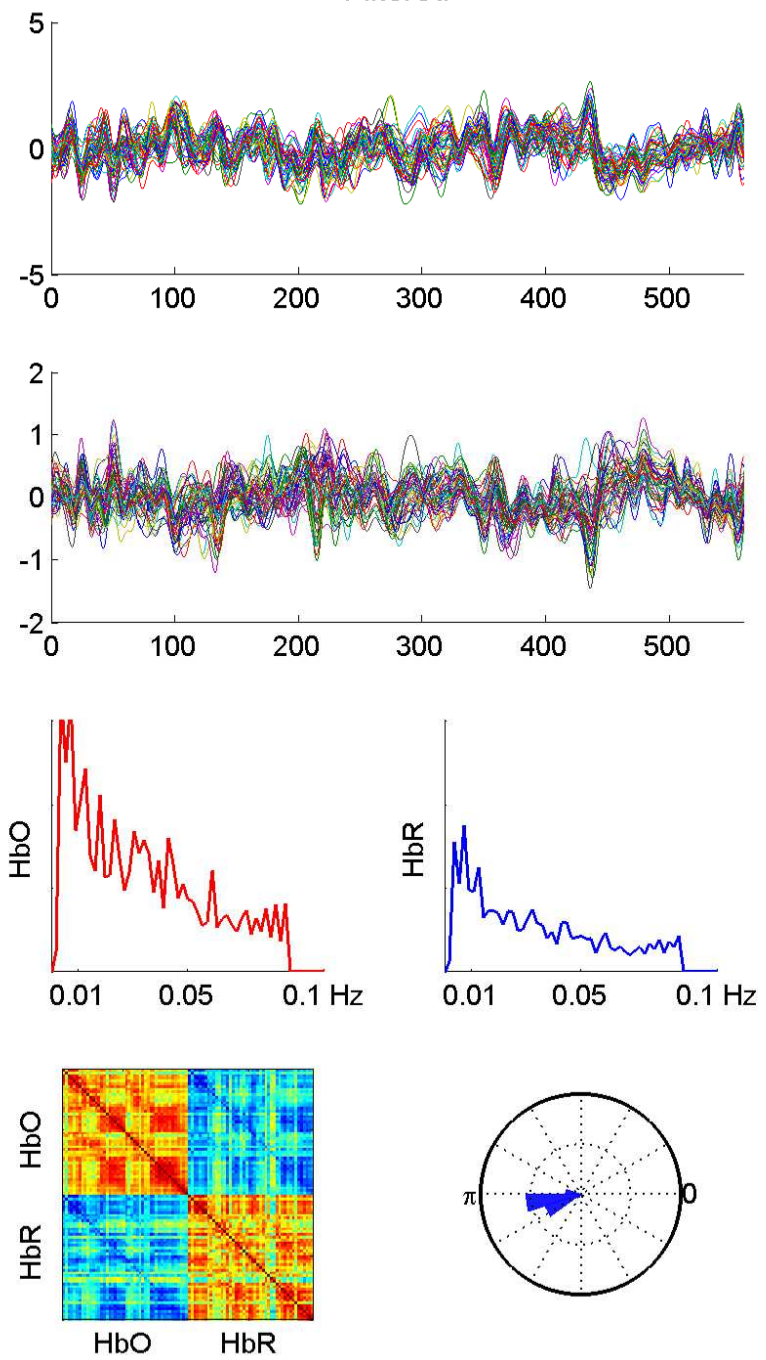

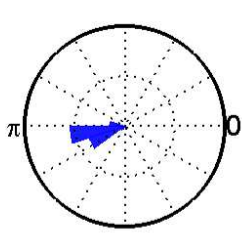

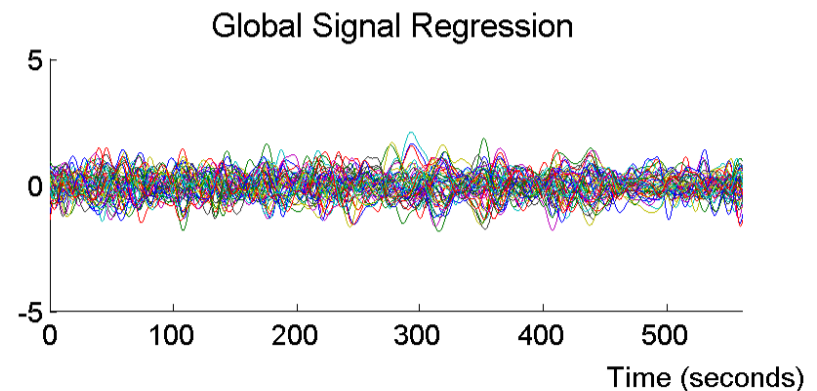

2
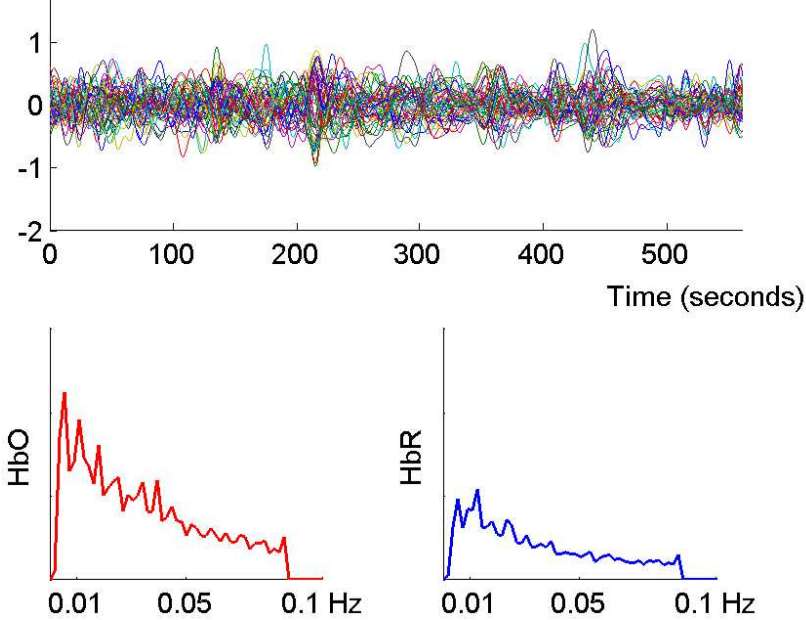
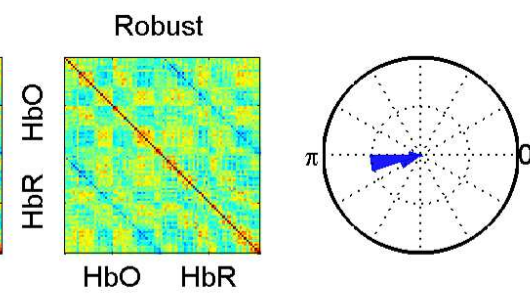
RS4 SL 4335 - 760 nm
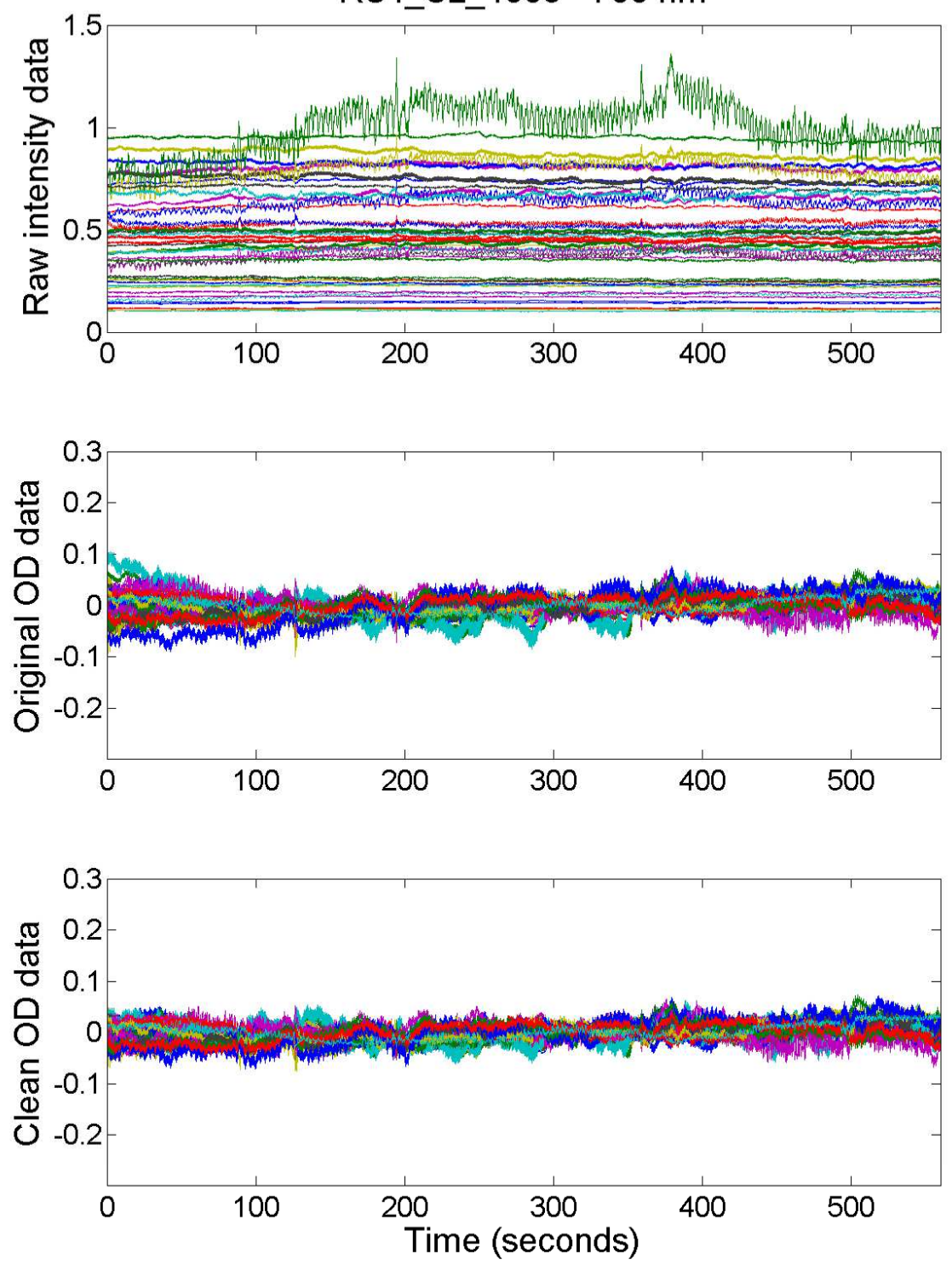

RS4 SL $4335-850 \mathrm{~nm}$
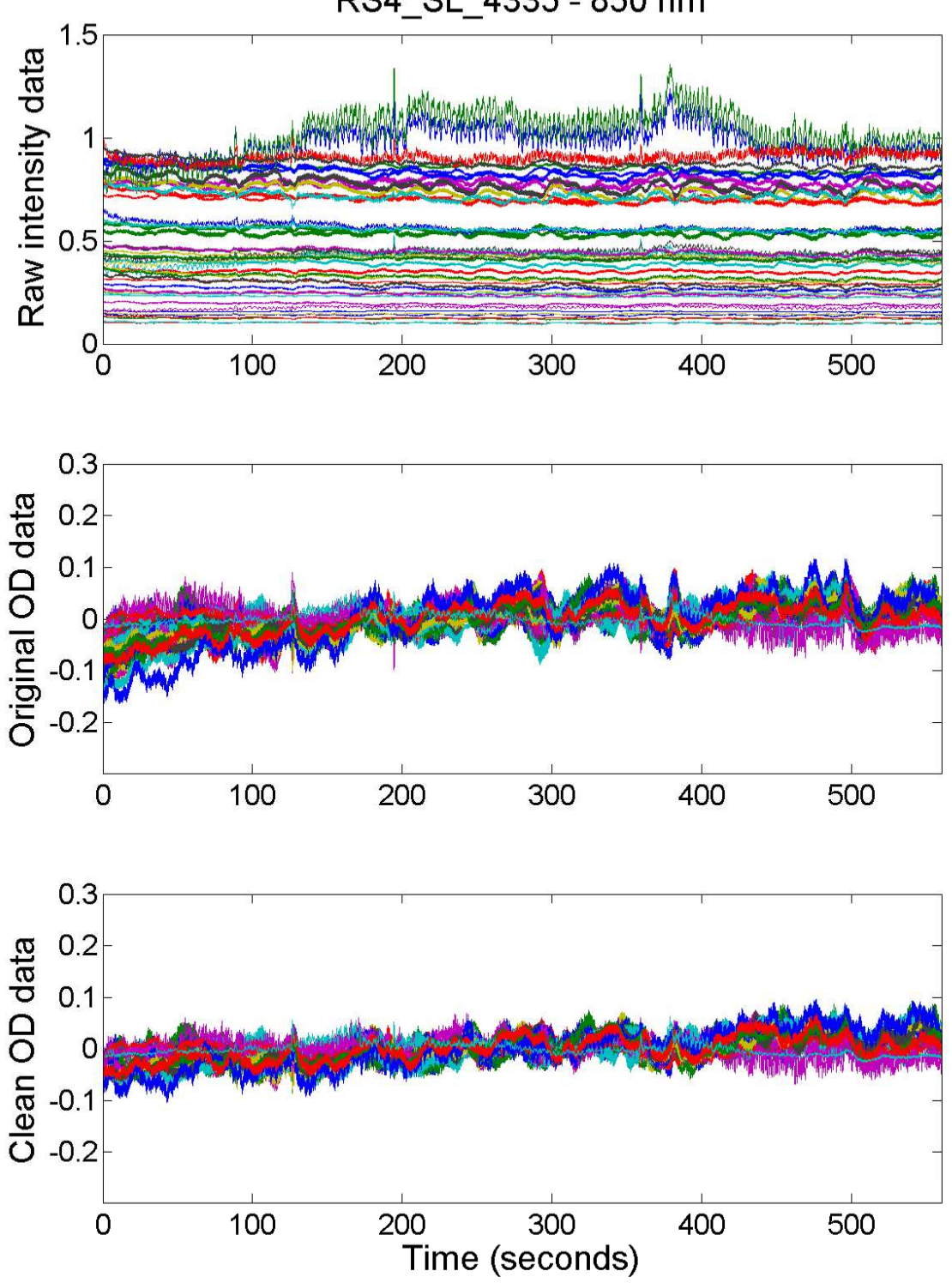

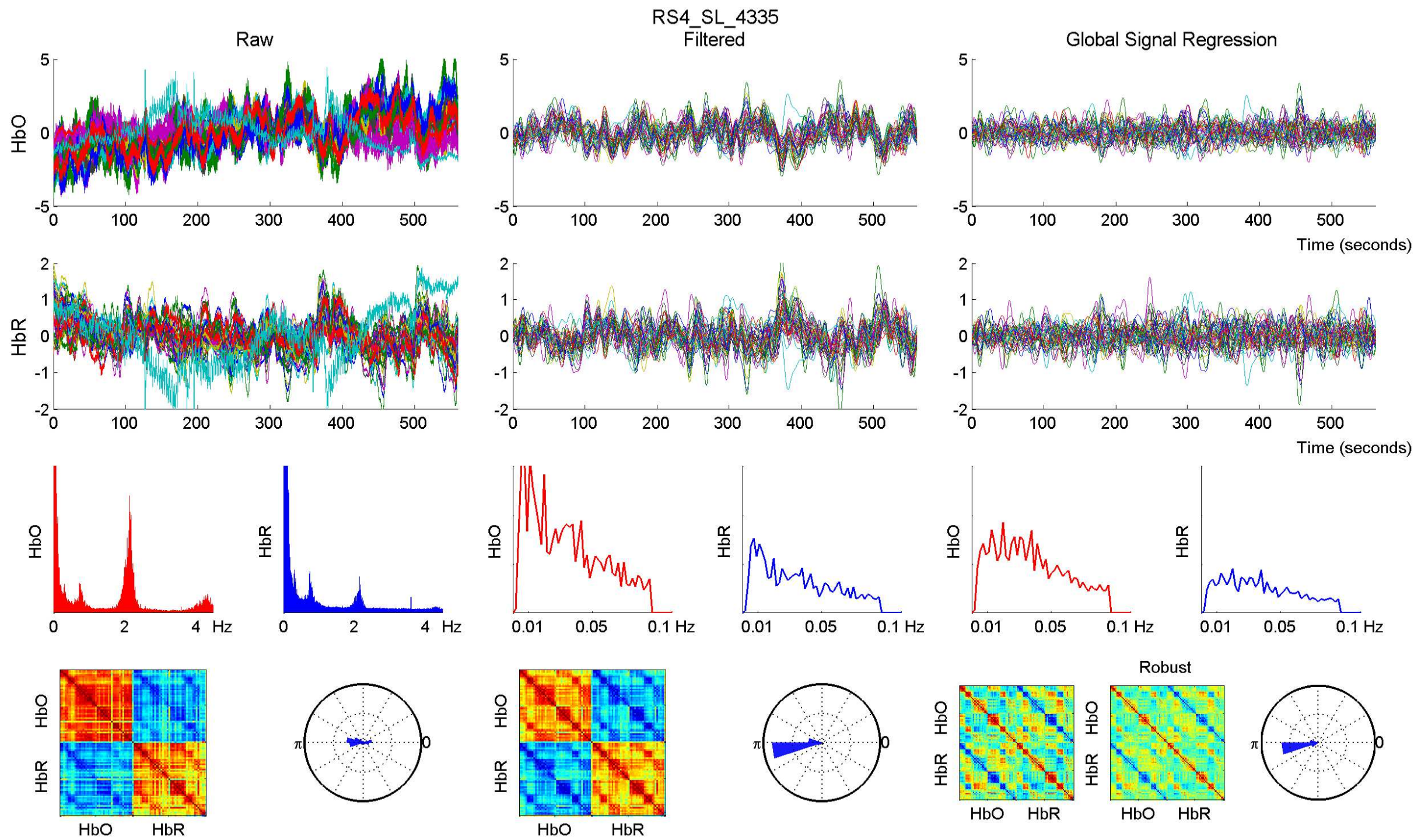

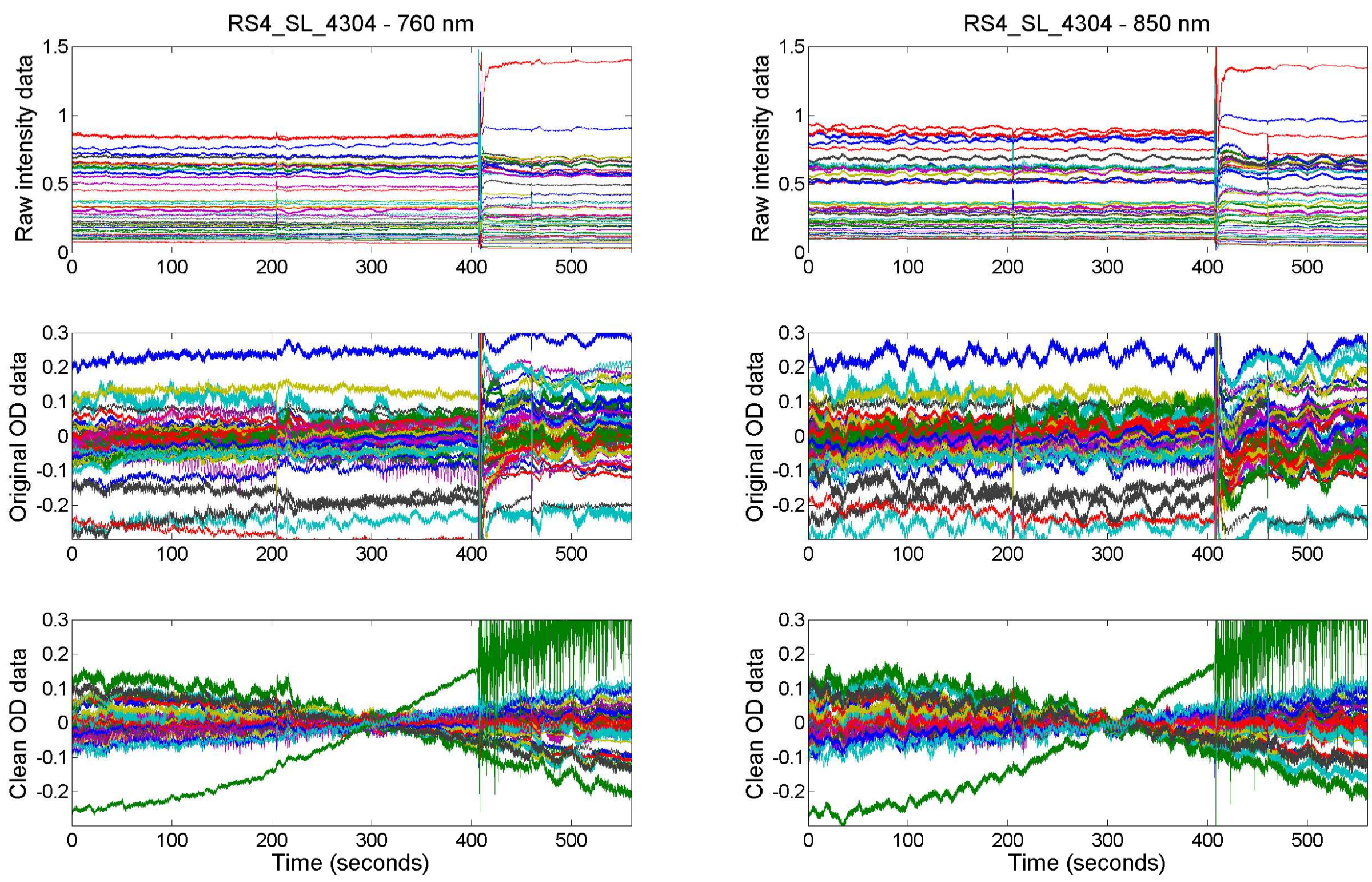
RS4_SL_4304

Raw
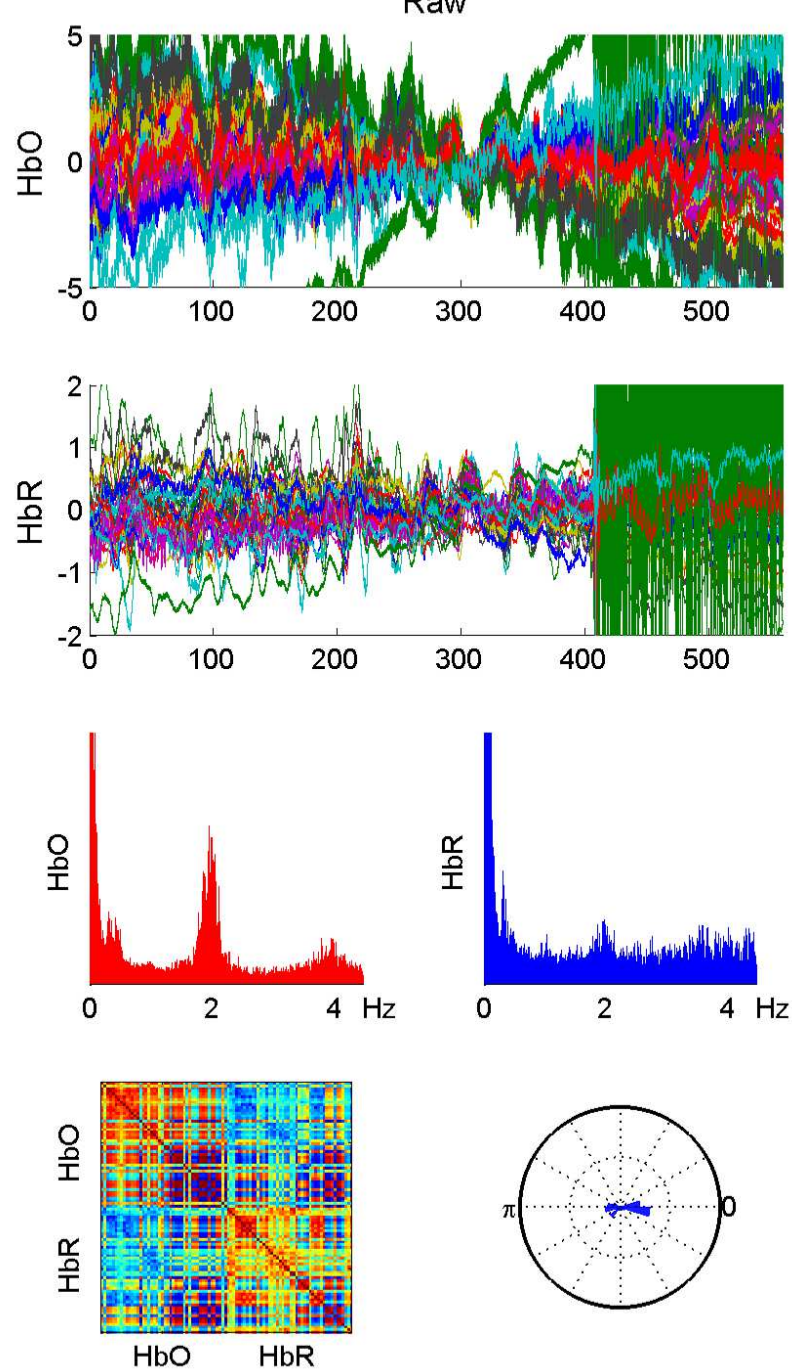
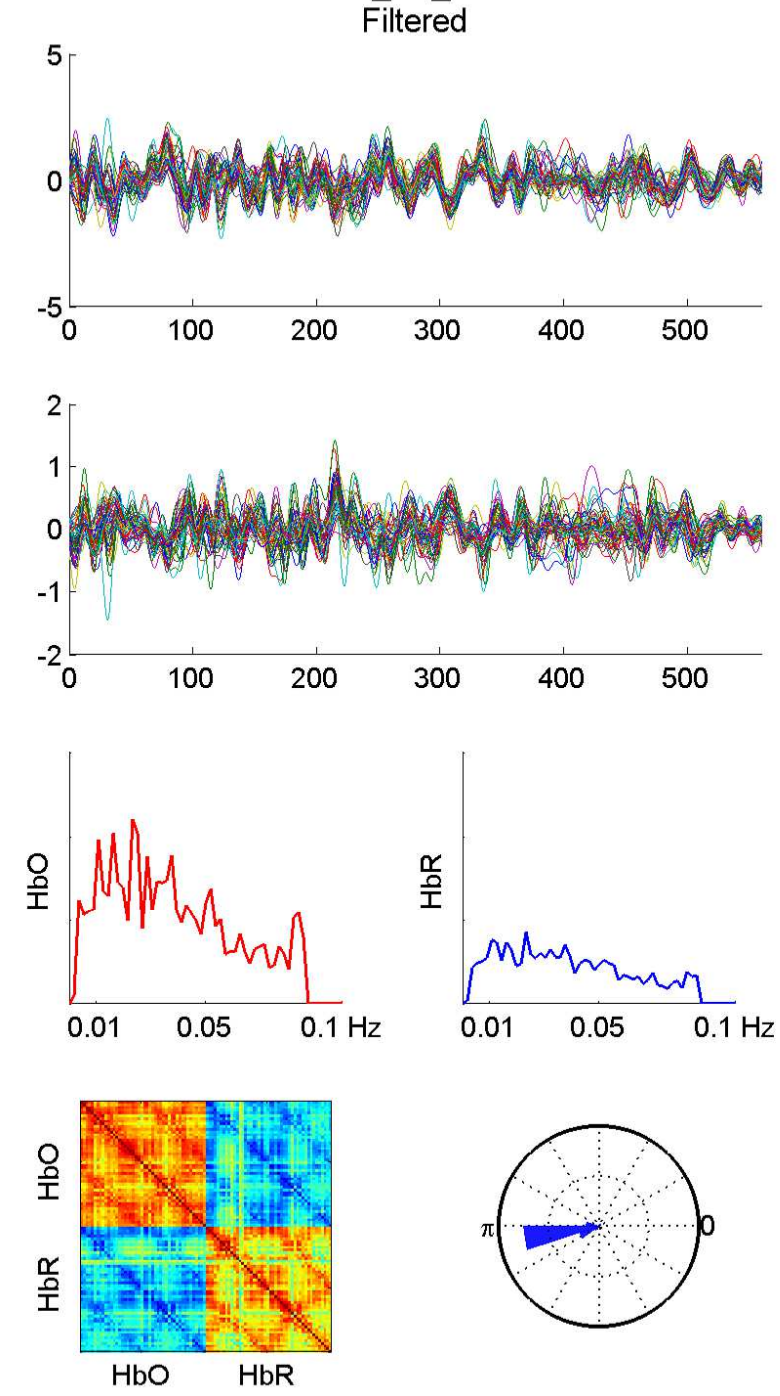
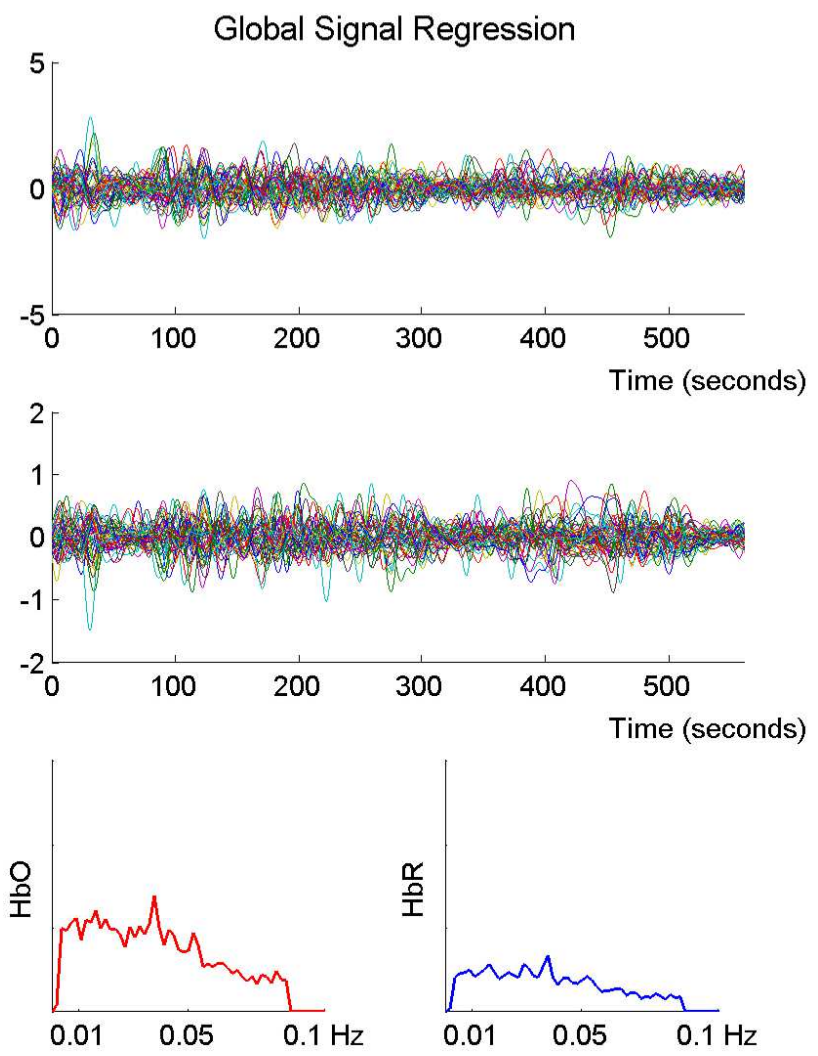
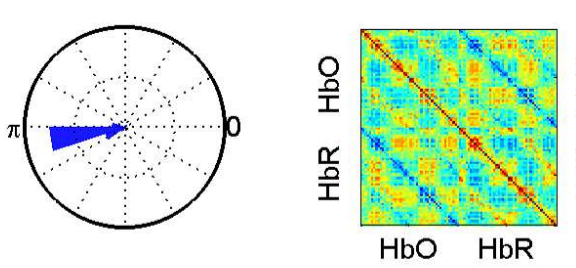
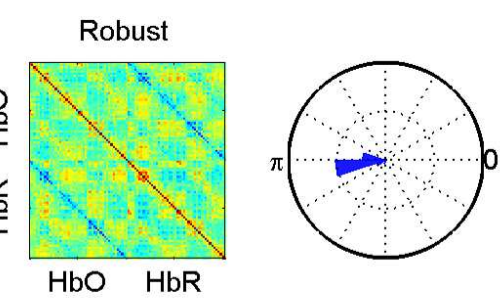
RS4 SL $4301-760 \mathrm{~nm}$
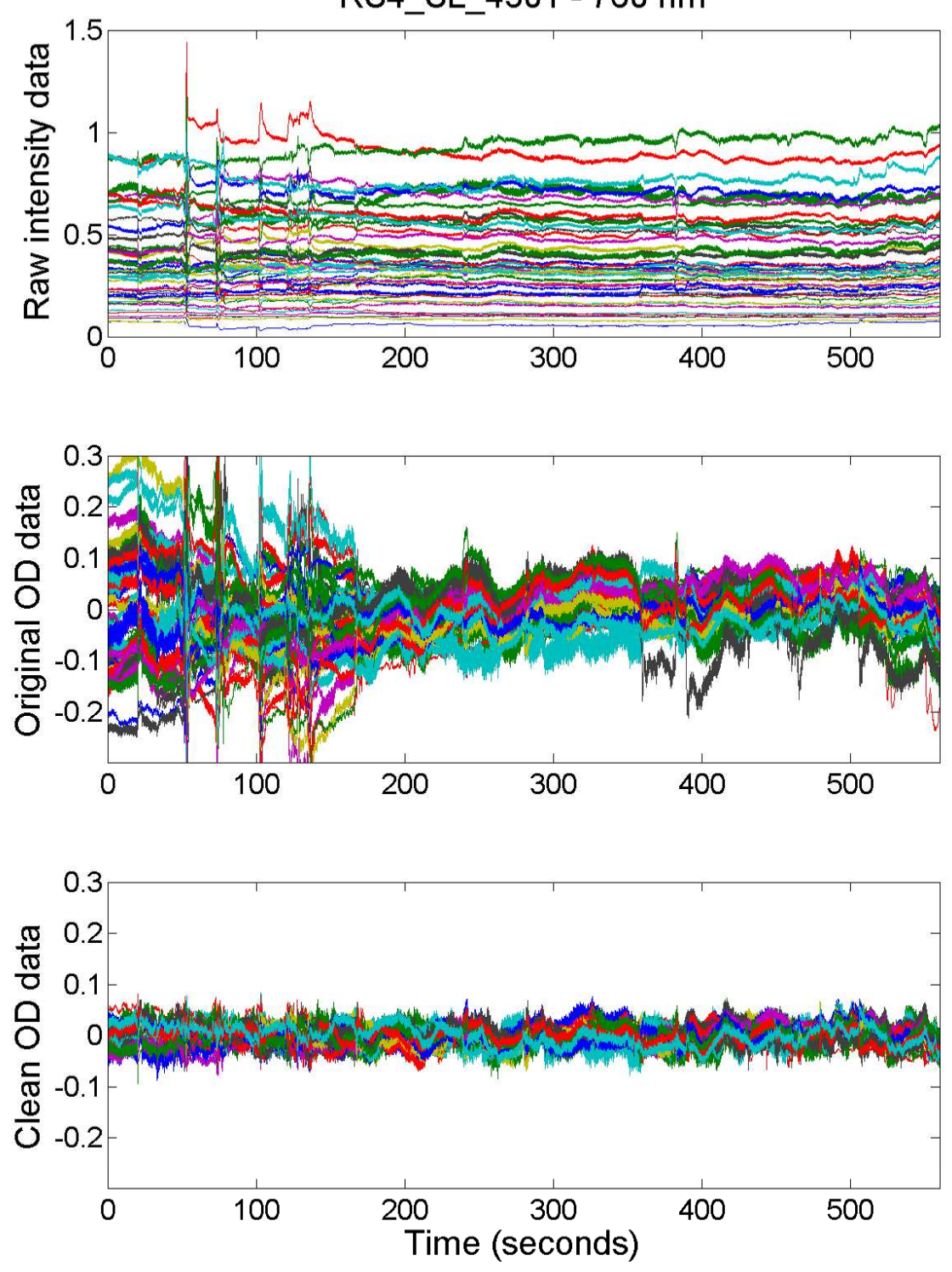

RS4 SL $4301-850 \mathrm{~nm}$
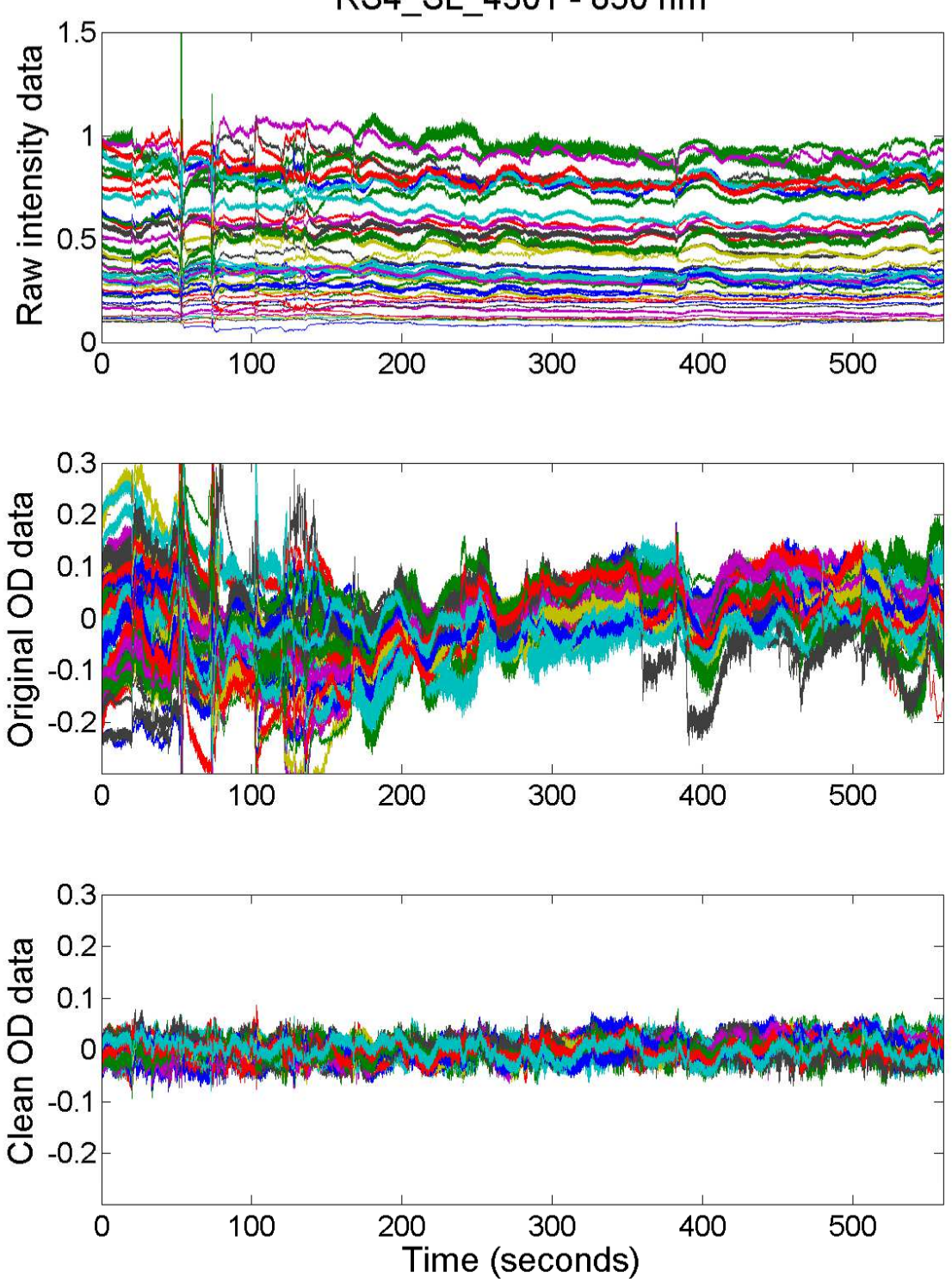
Raw

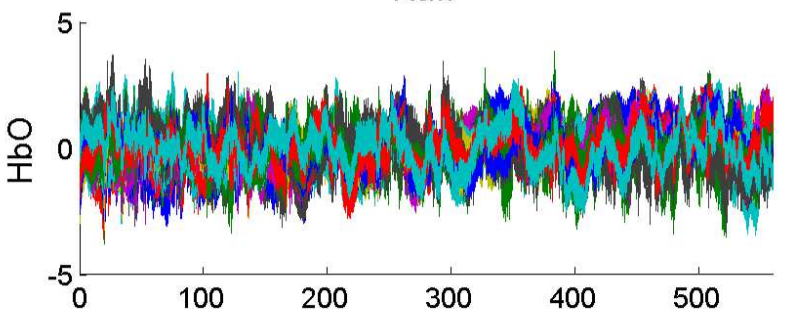

${ }_{0}^{2}{ }_{0}^{2}{ }_{0}^{2}$
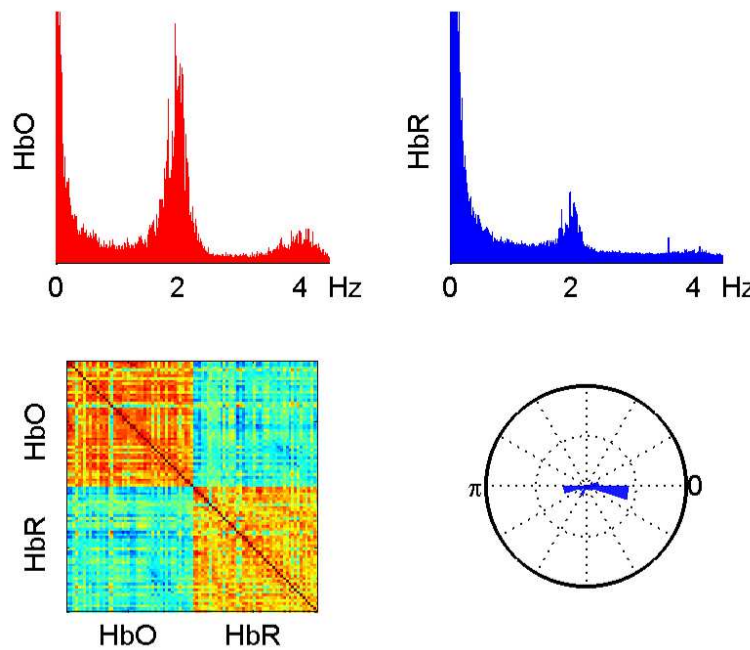

RS4 SL 4301

Filtered
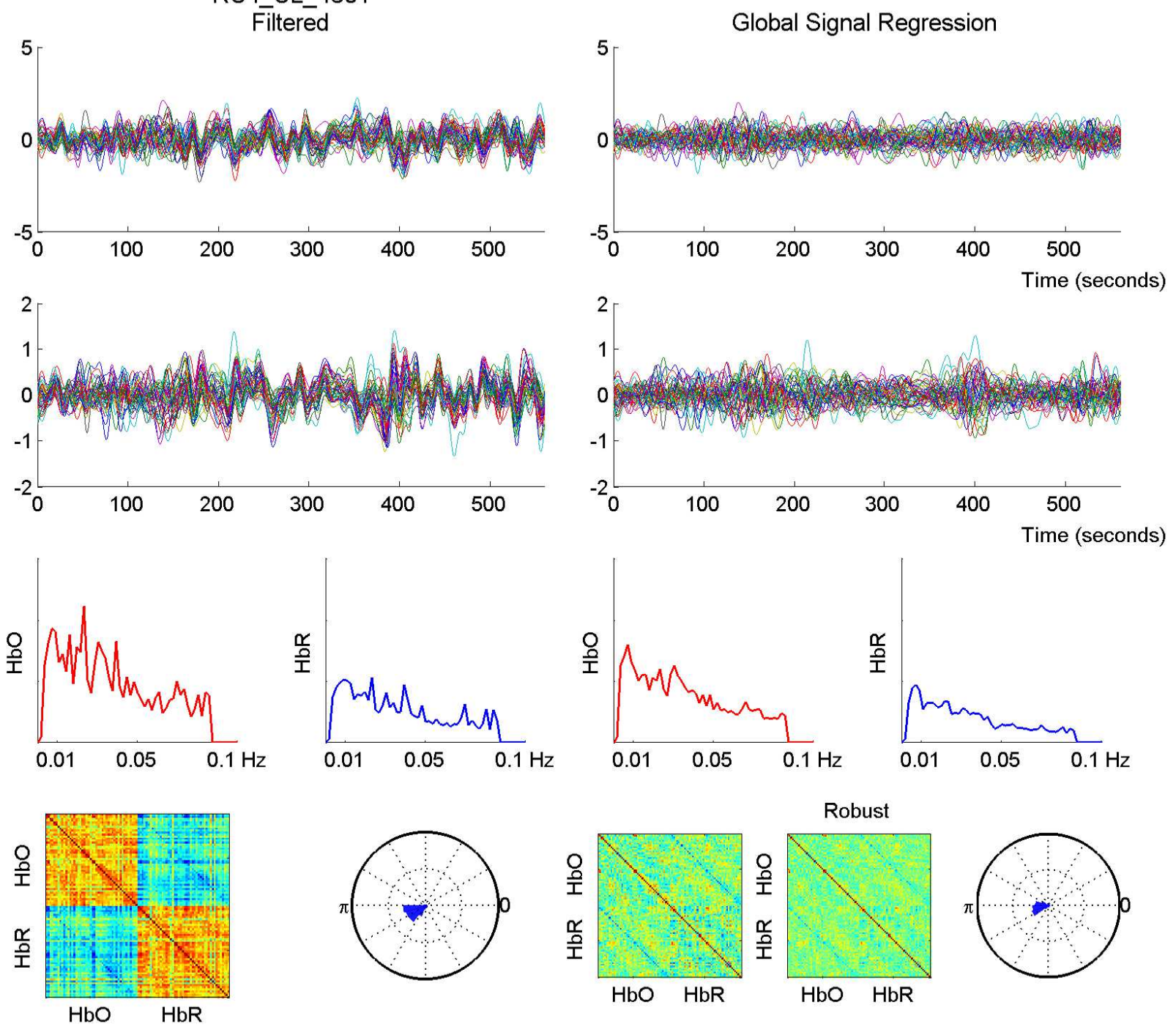
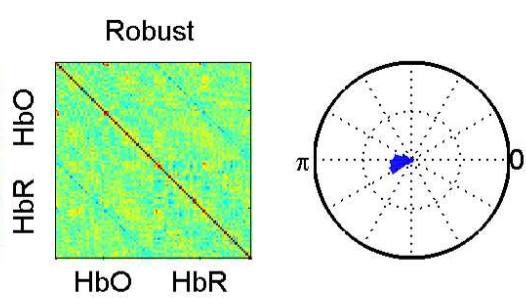

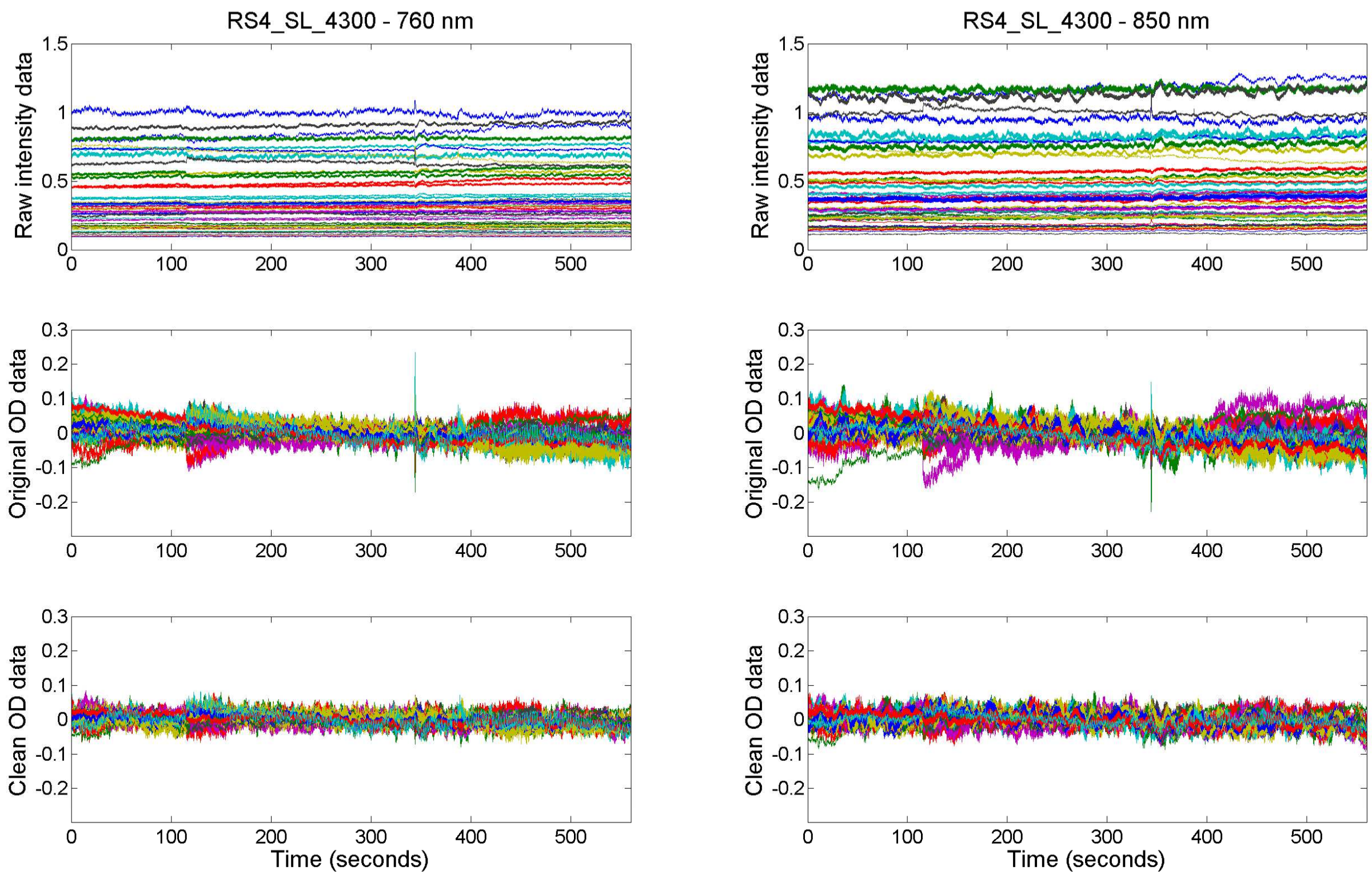
Raw

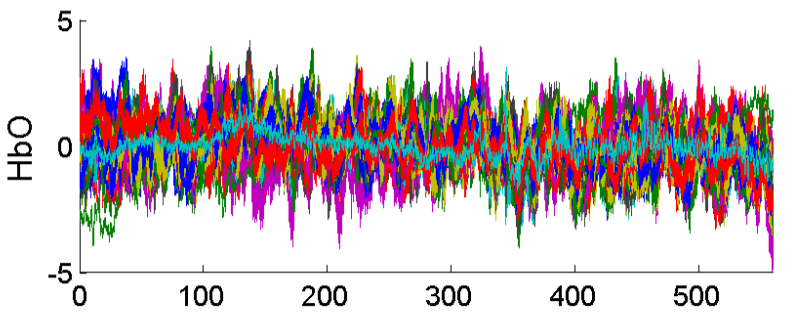

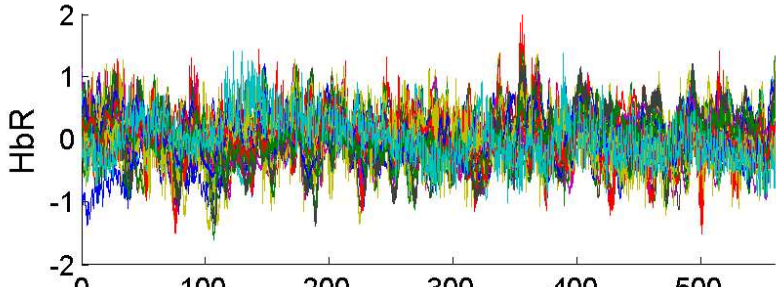
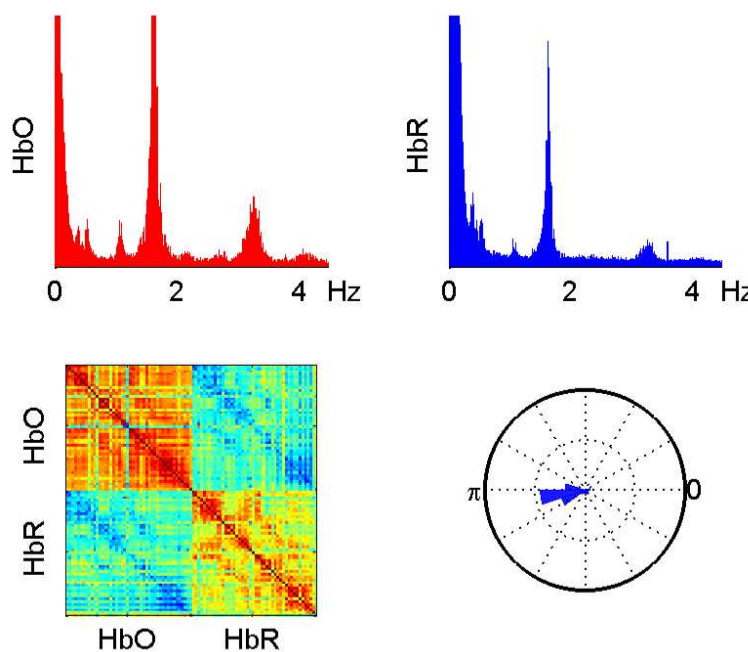

RS4 SL 4300

Filtered

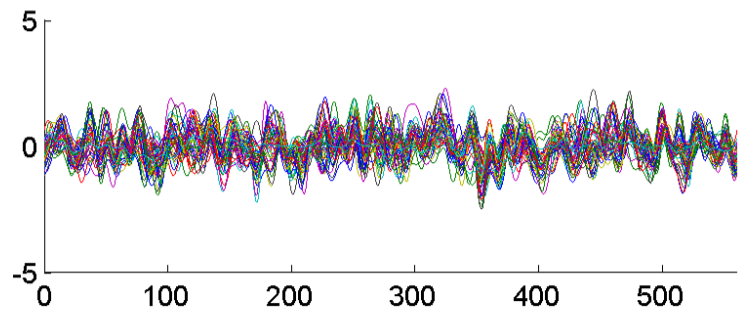

2

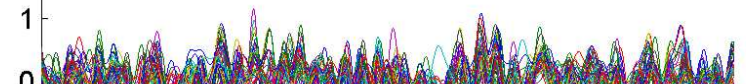

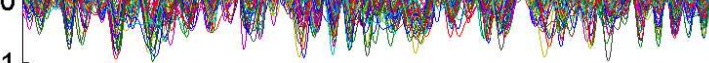
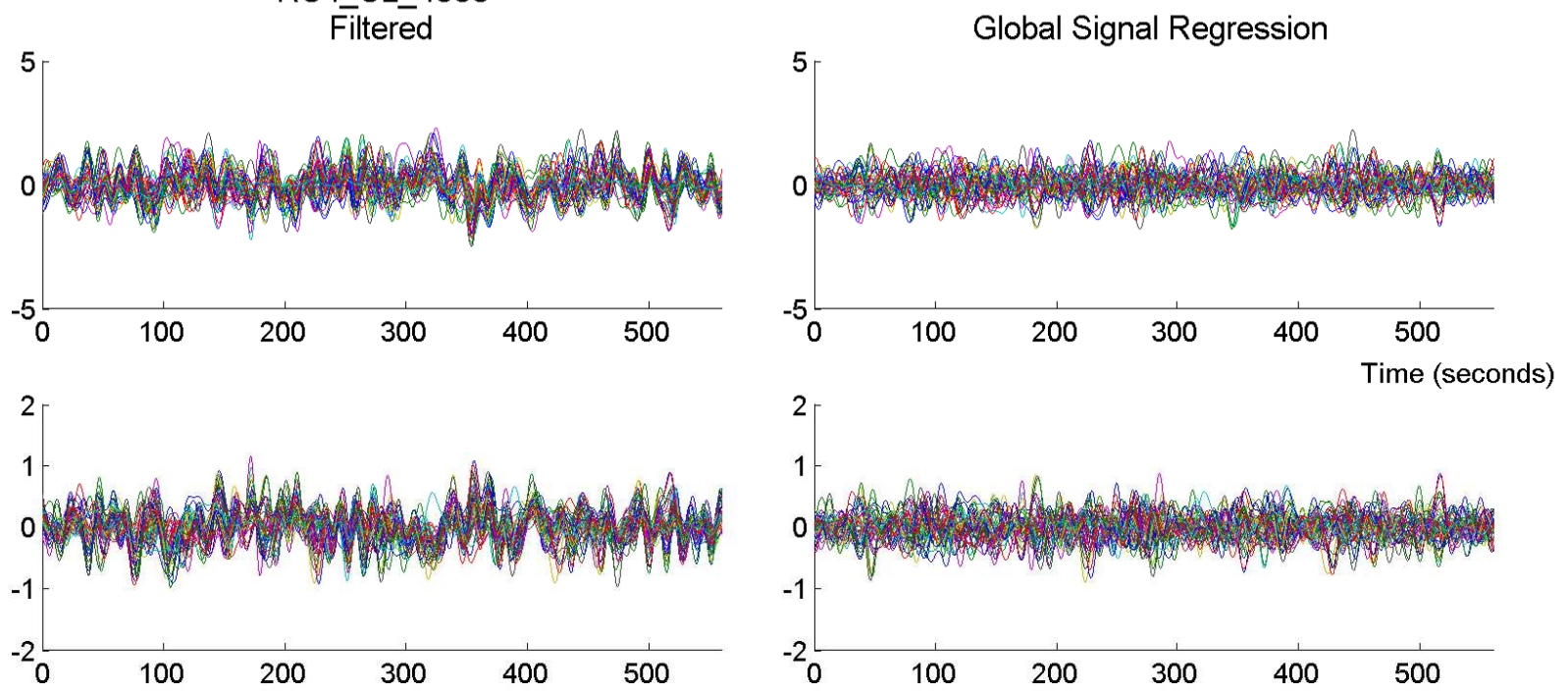

2
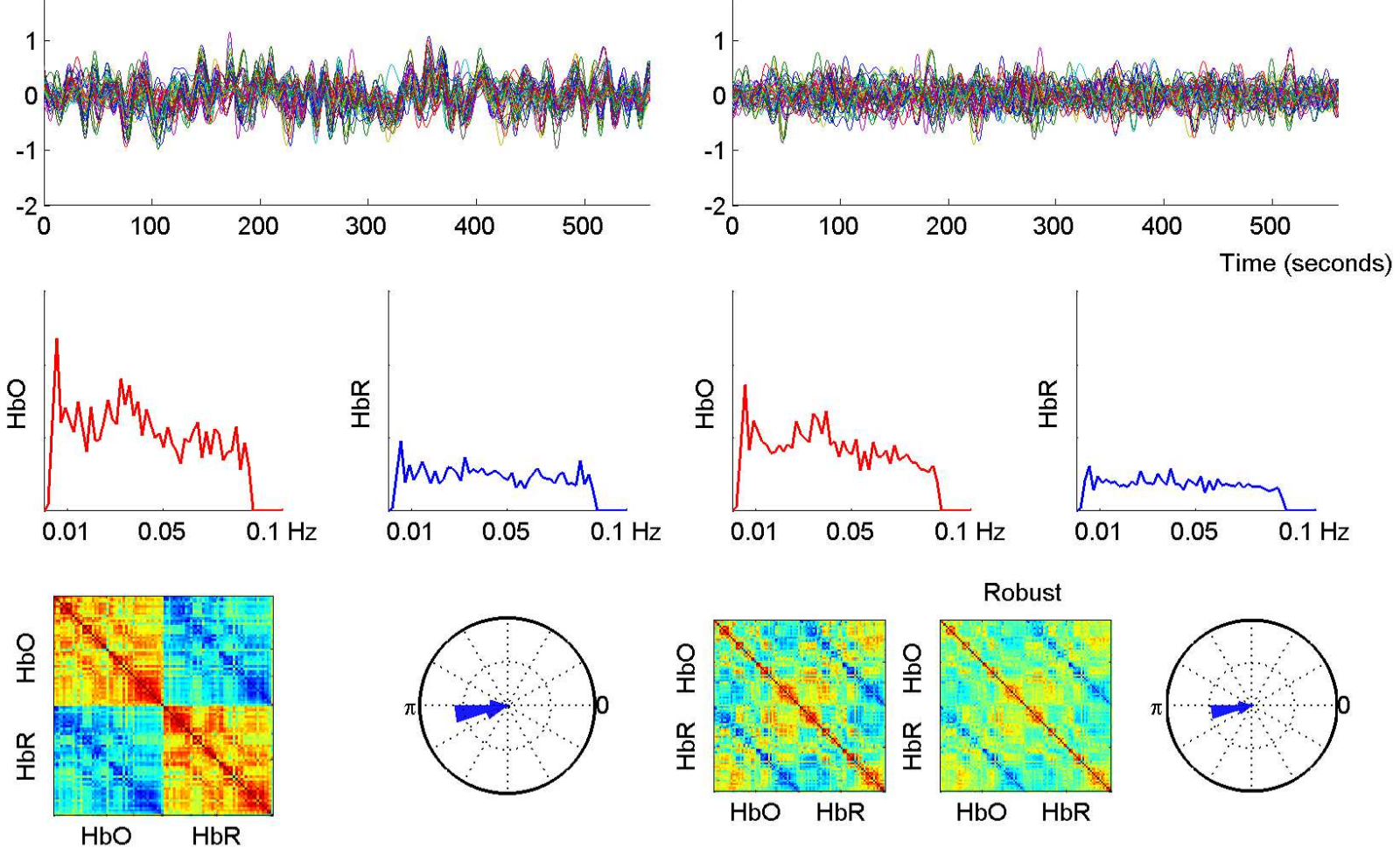
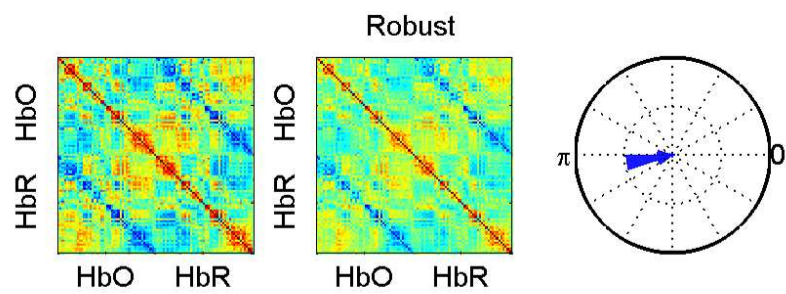
RS4 SL 4297 - 760 nm
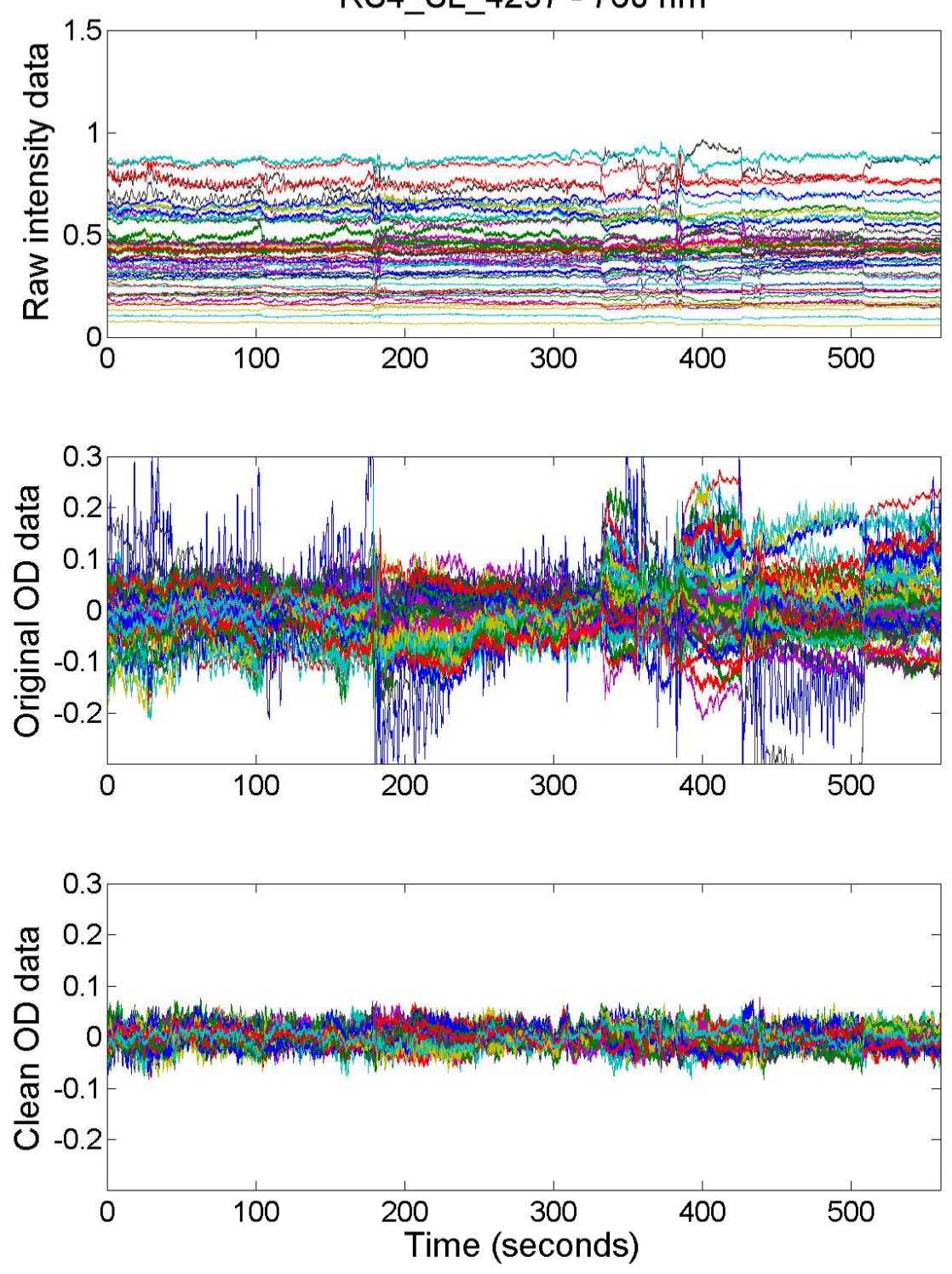

RS4 SL $4297-850 \mathrm{~nm}$
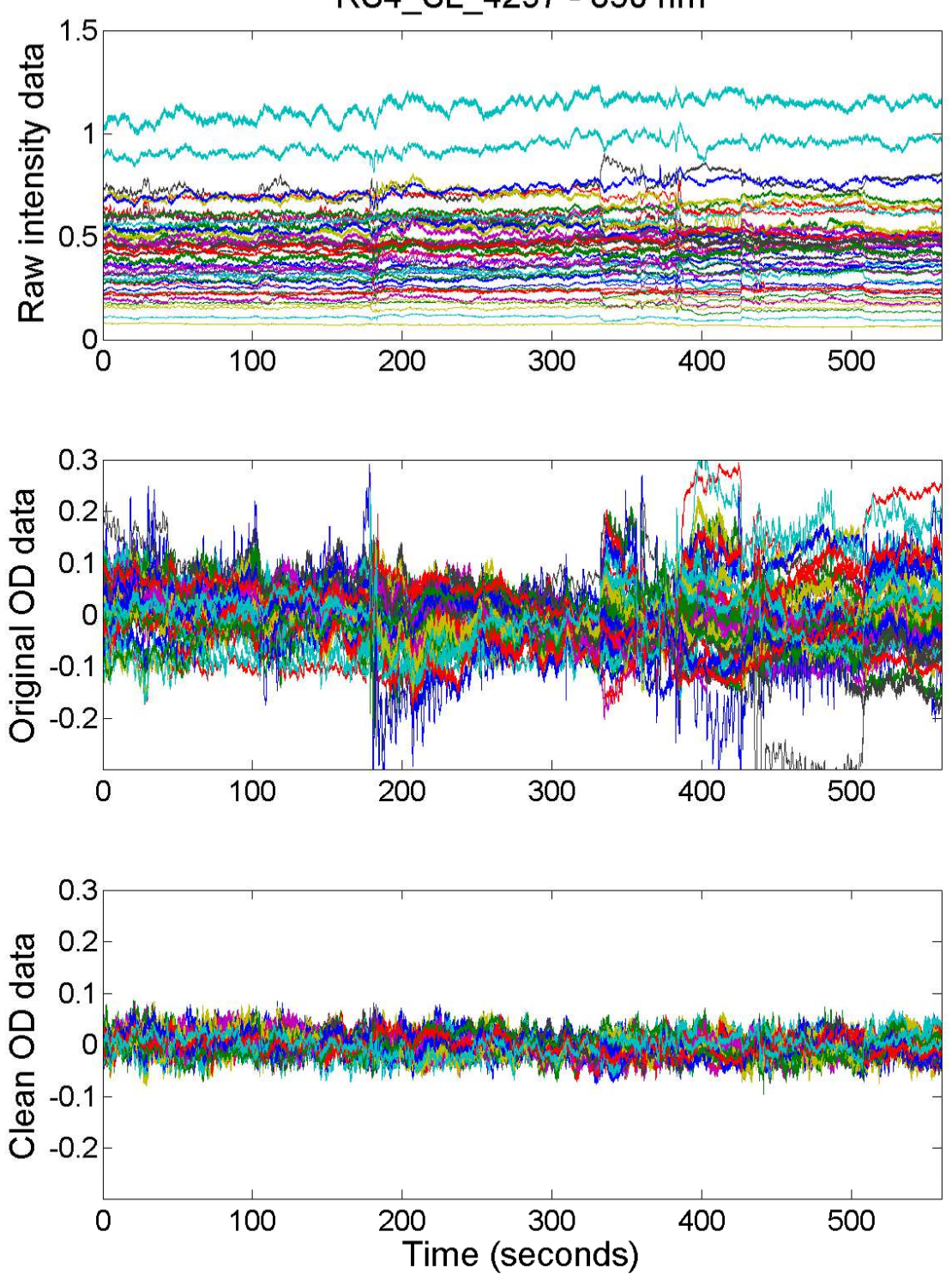
Raw
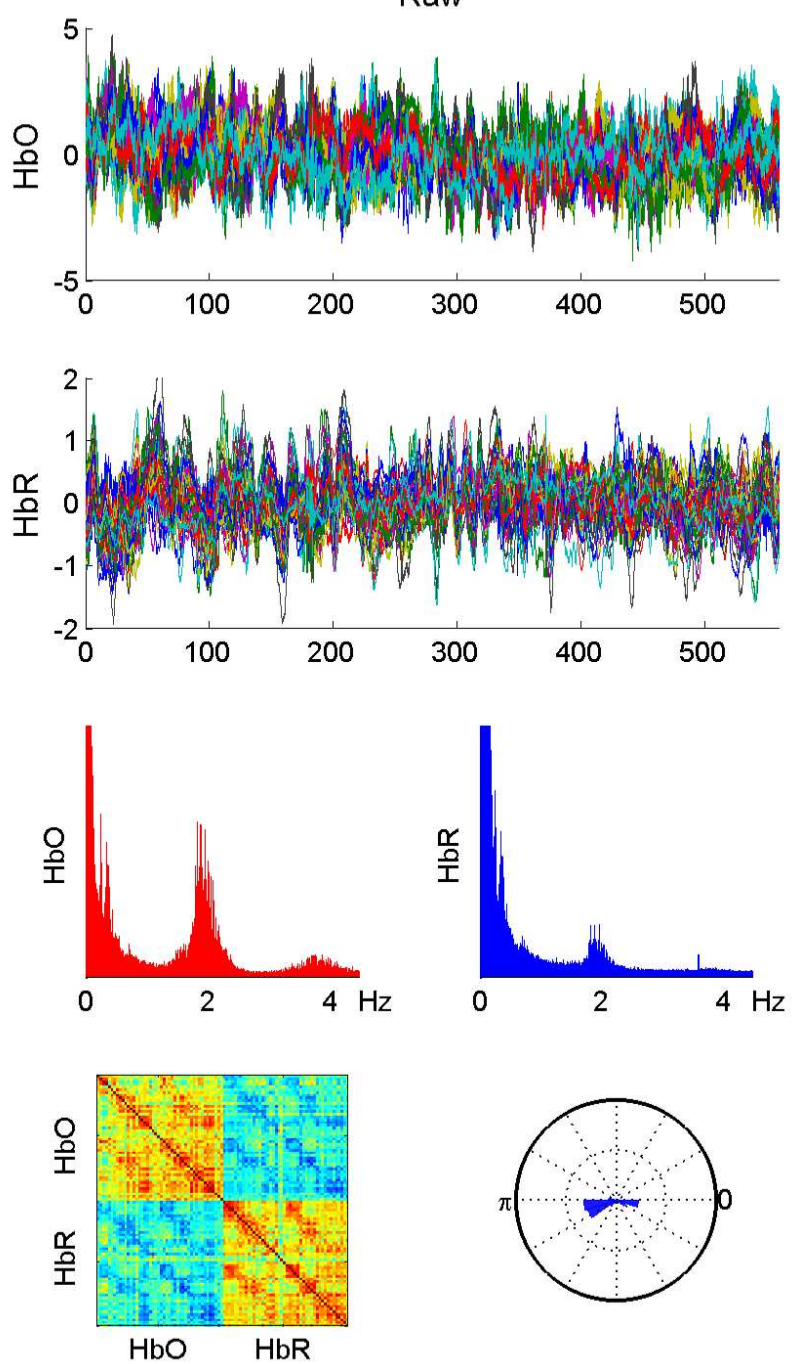

RS4 SL 4297

Filtered
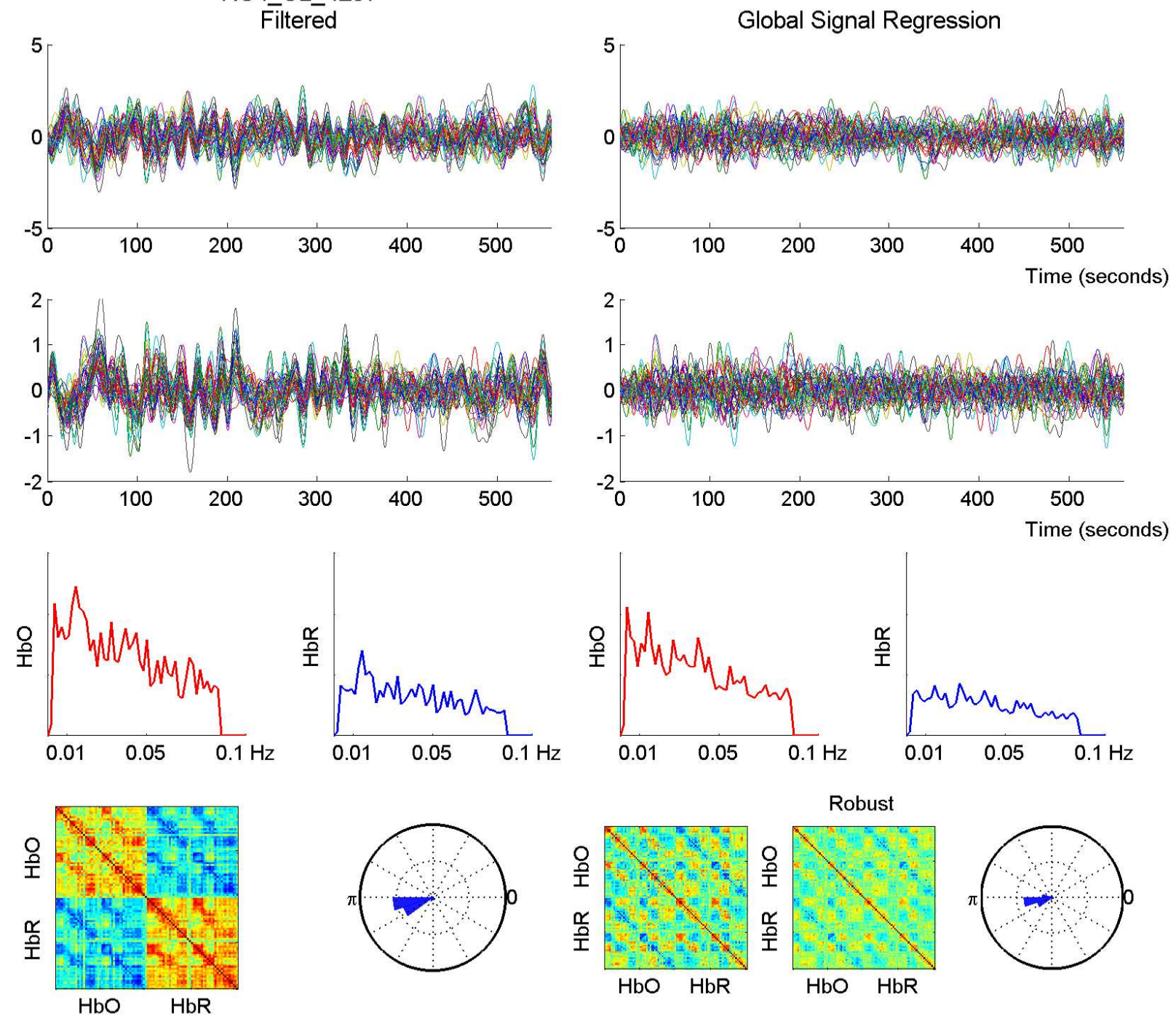
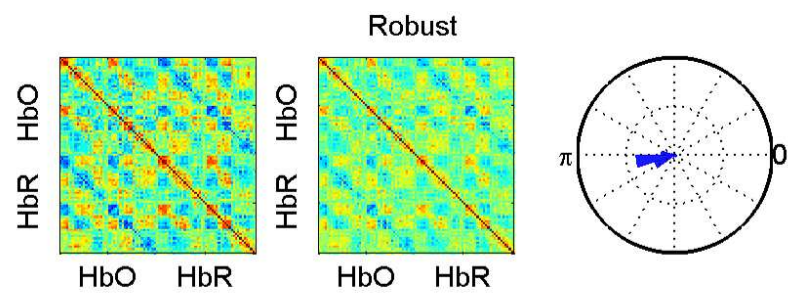
RS4 SL 4296 - 760 nm
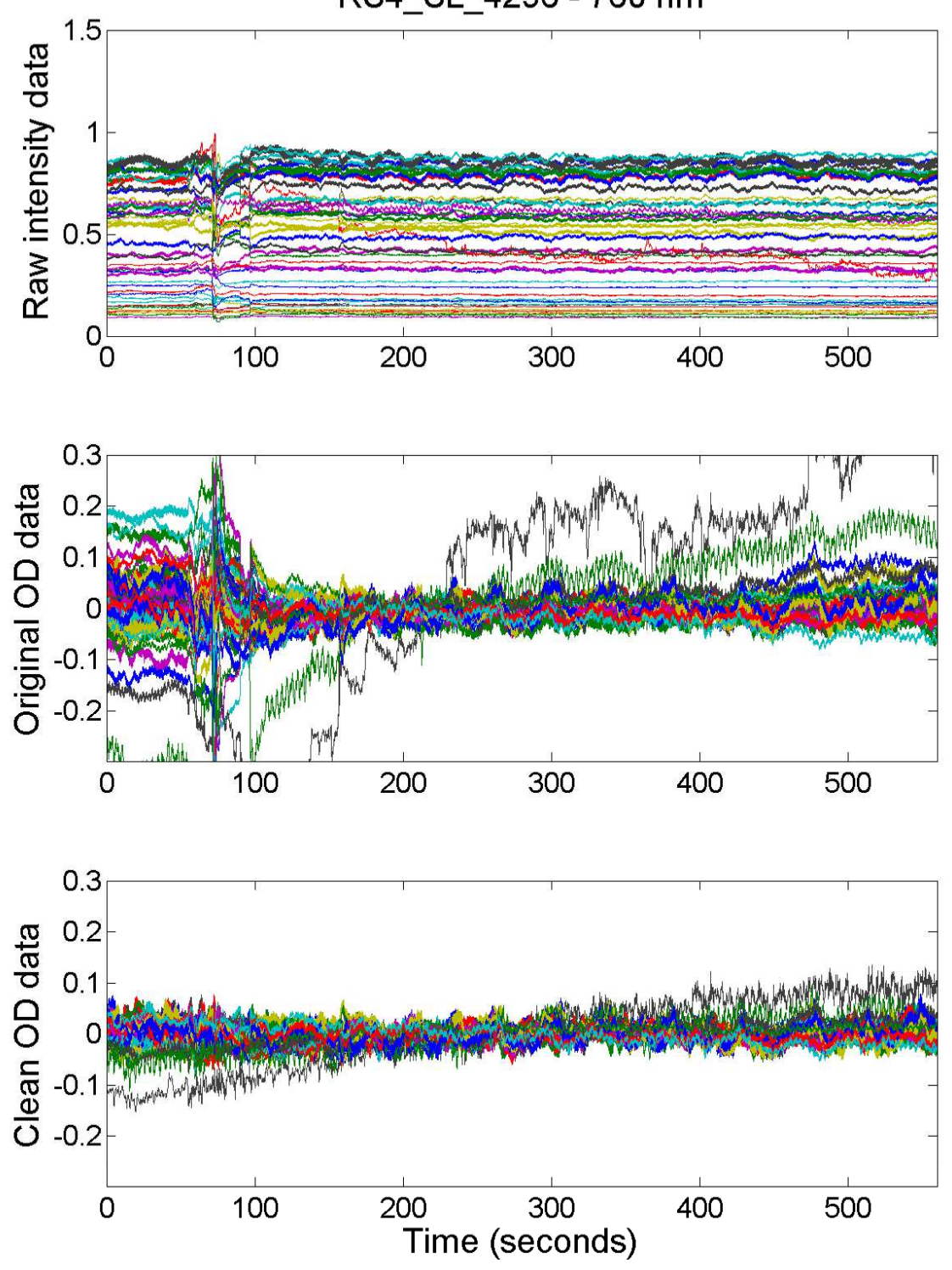

RS4 SL $4296-850 \mathrm{~nm}$
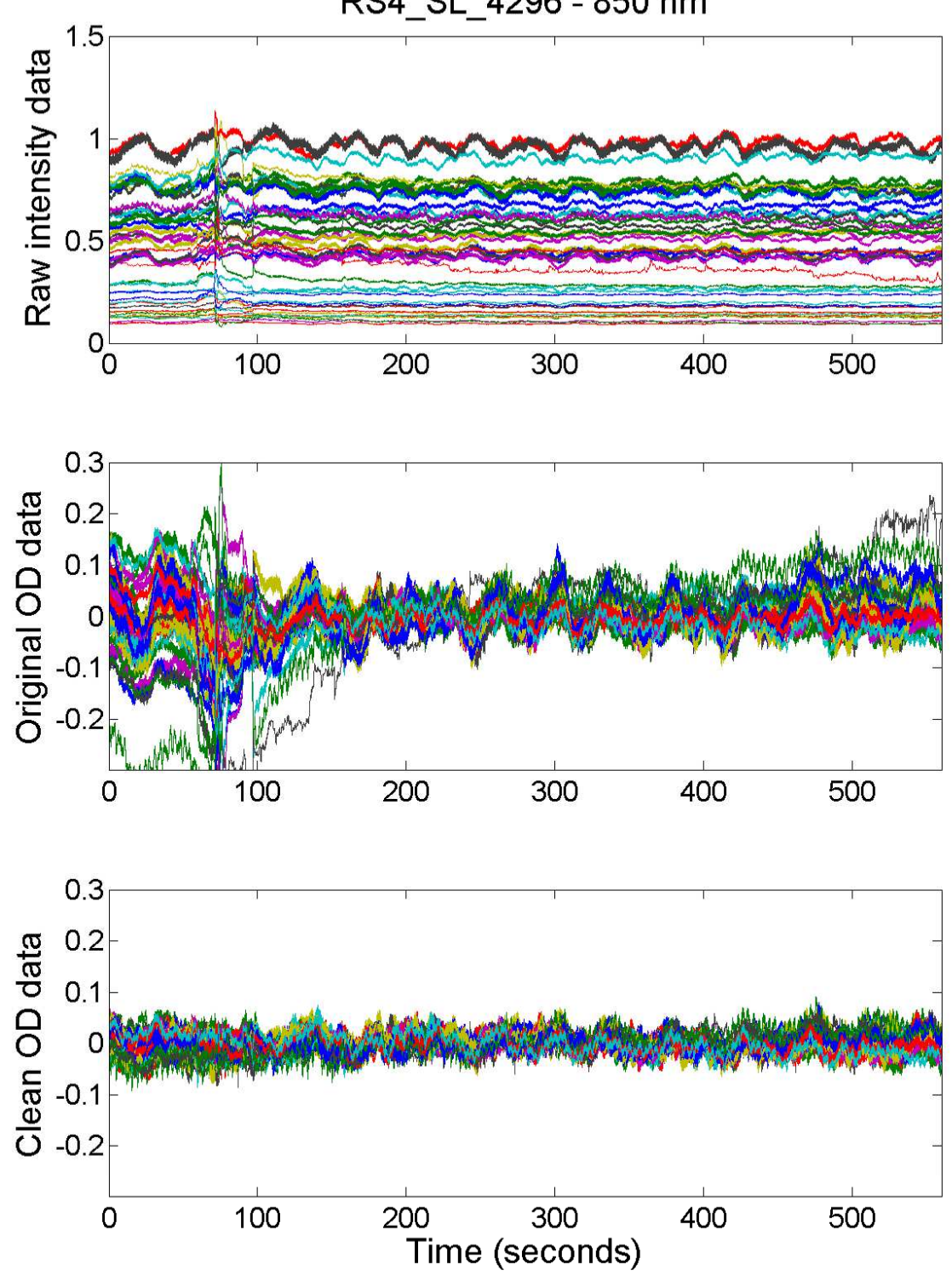
Raw
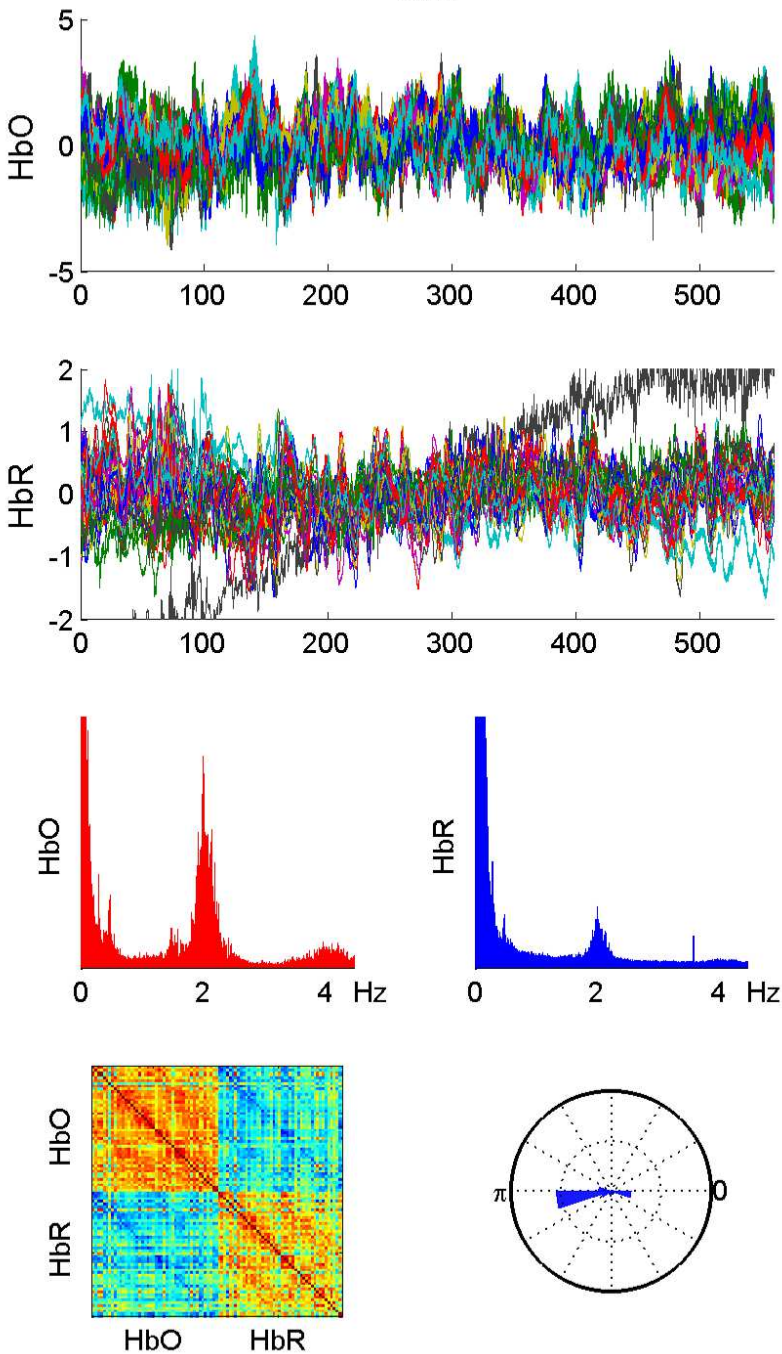

RS4_SL_4296

Filtered
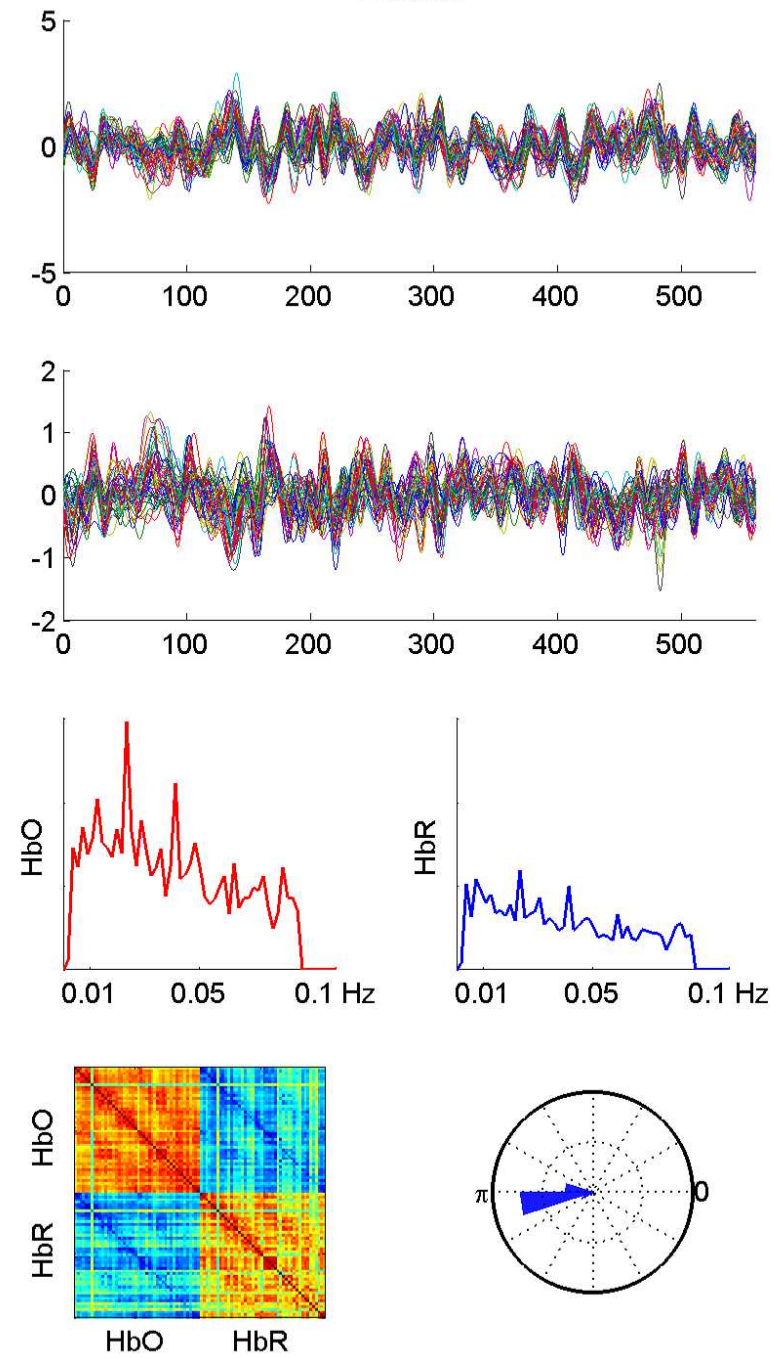

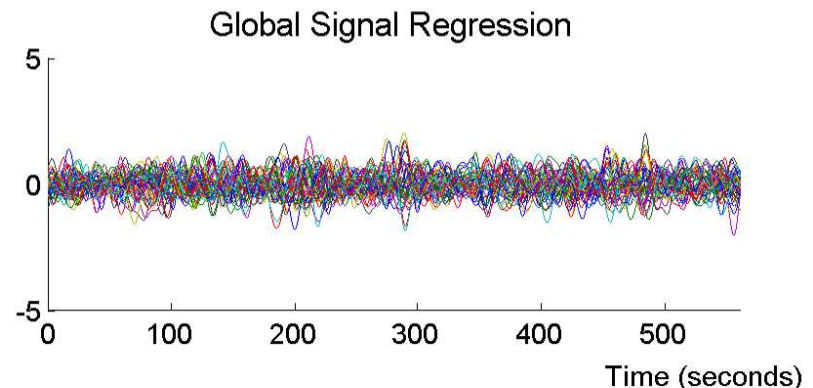

2
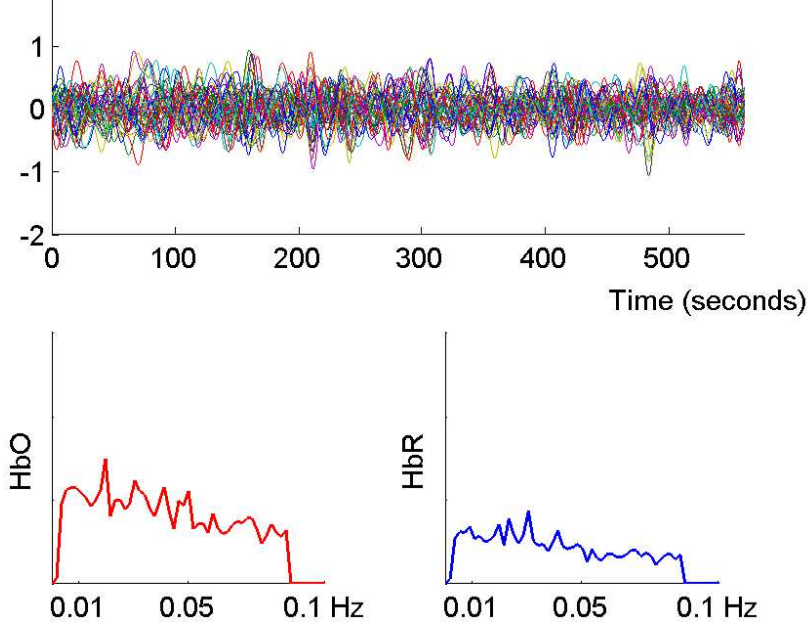
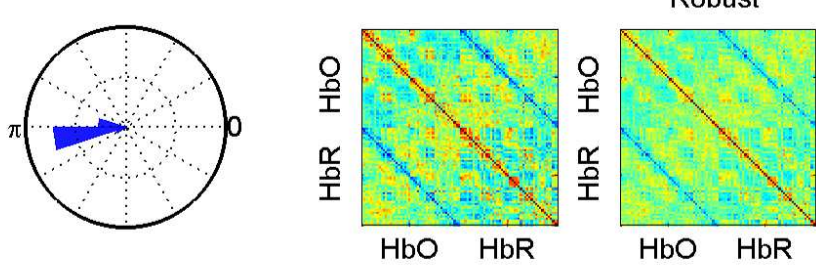

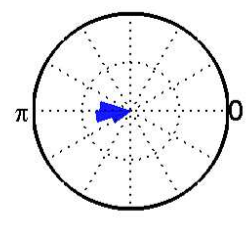


RS4 SL $4295-760$ nm
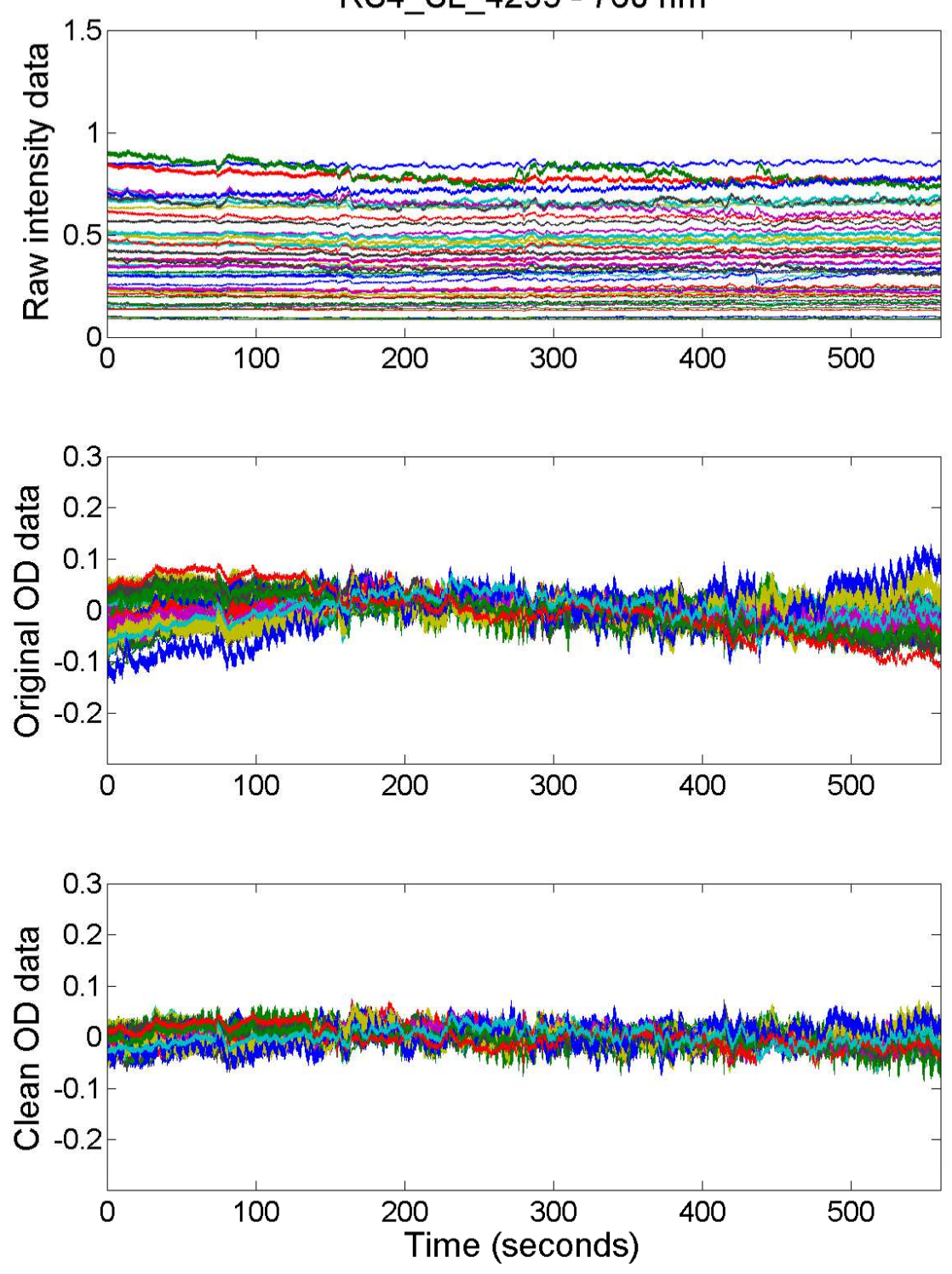

RS4 SL $4295-850 \mathrm{~nm}$
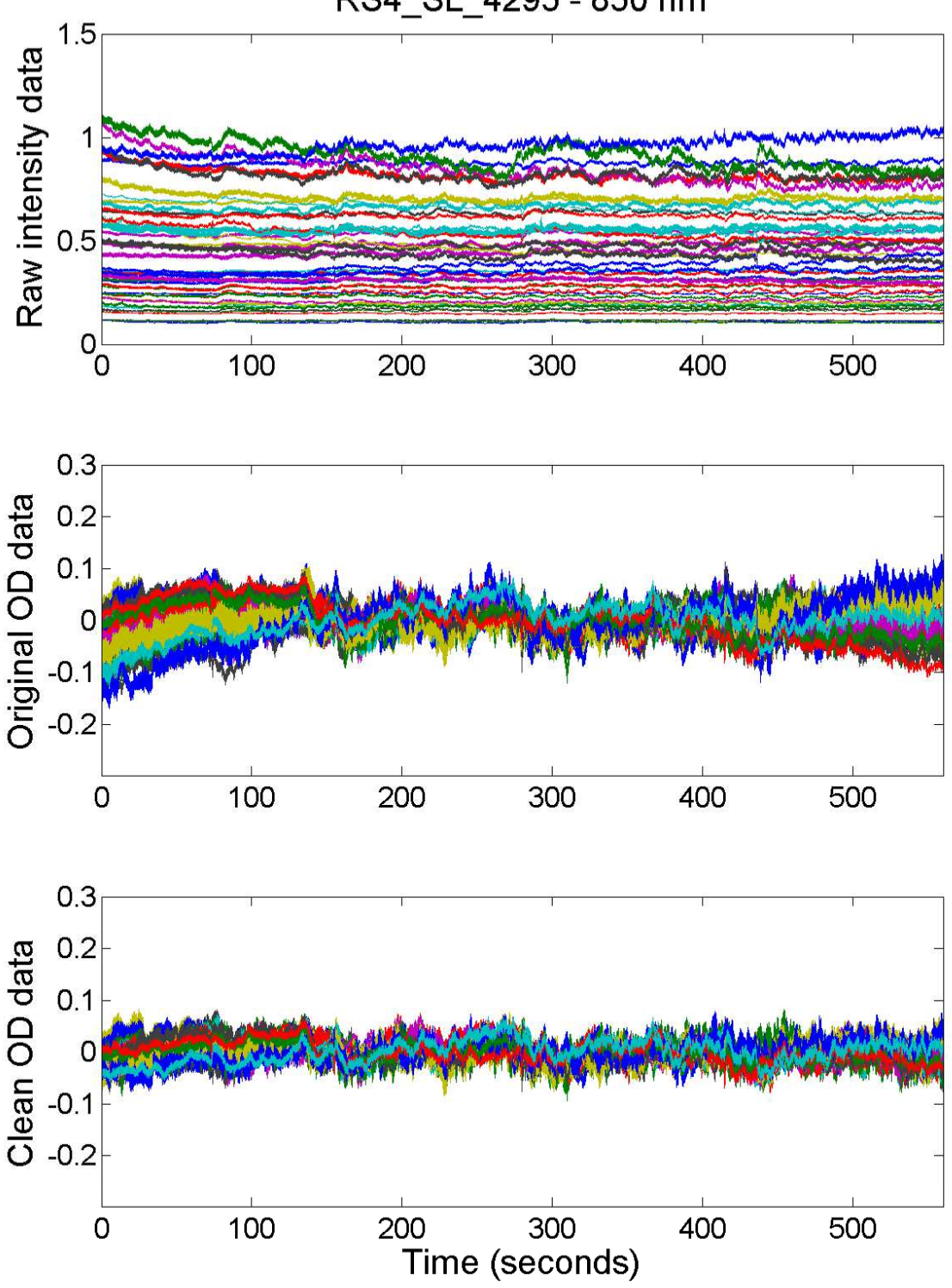
Raw

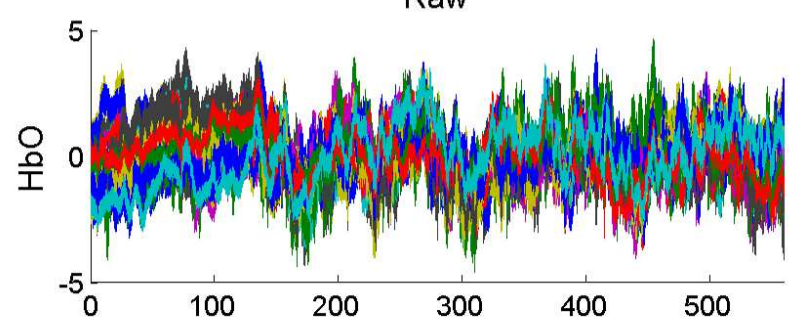

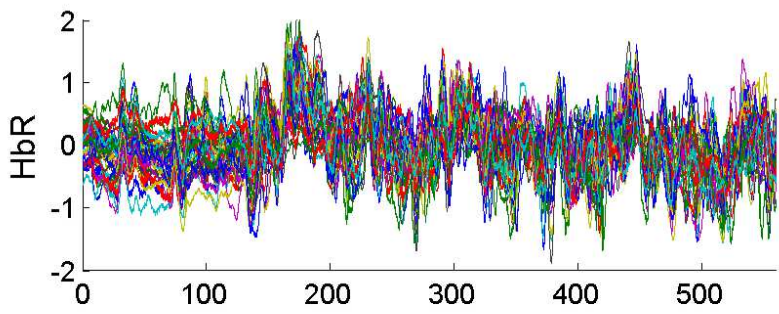
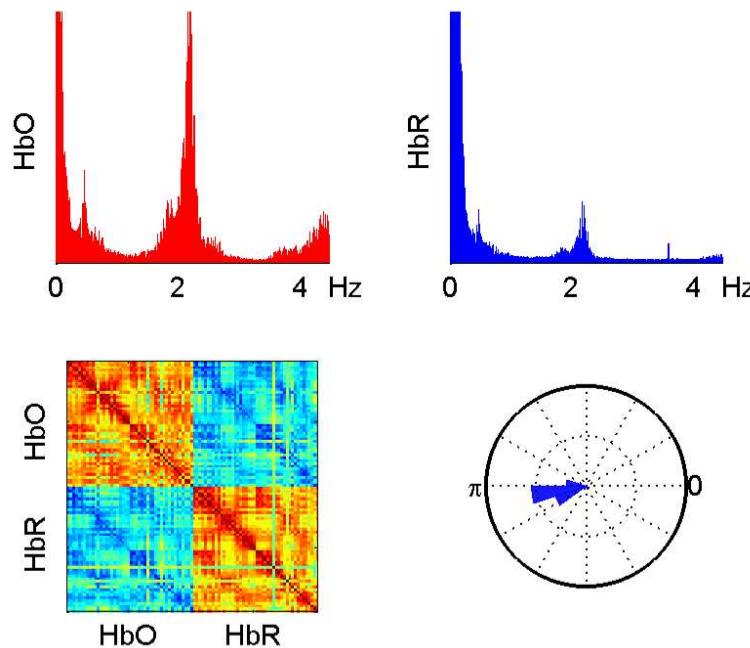

RS4_SL_4295

Filtered
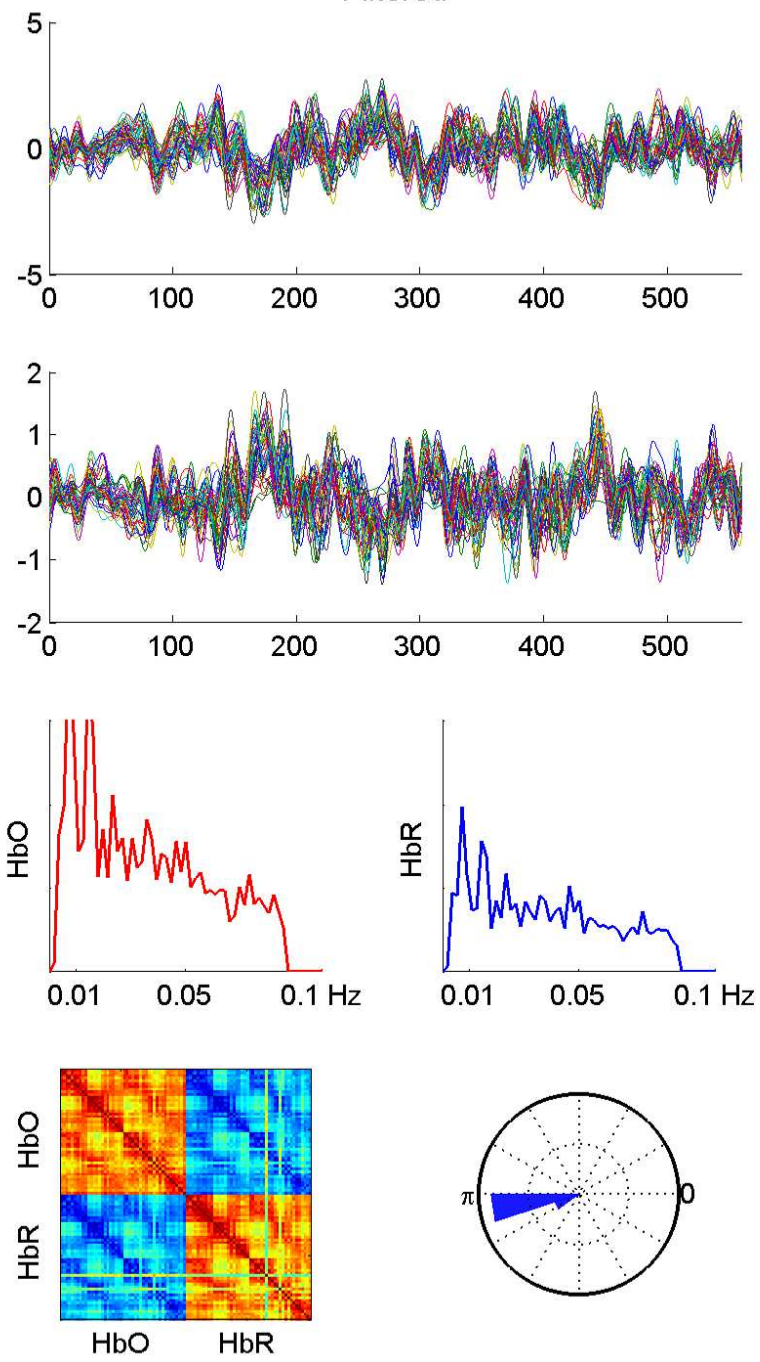

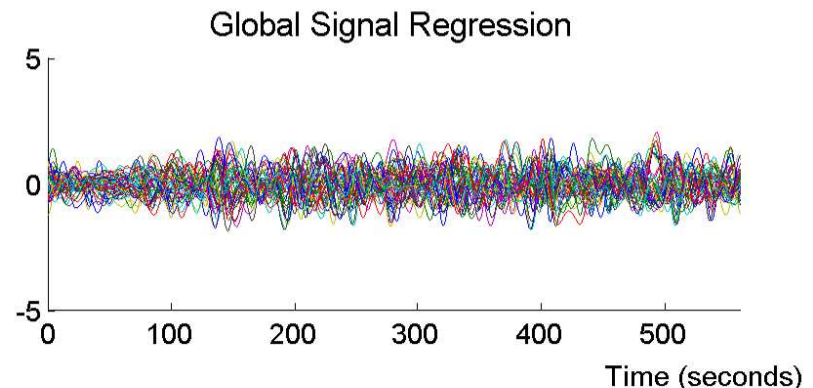

2
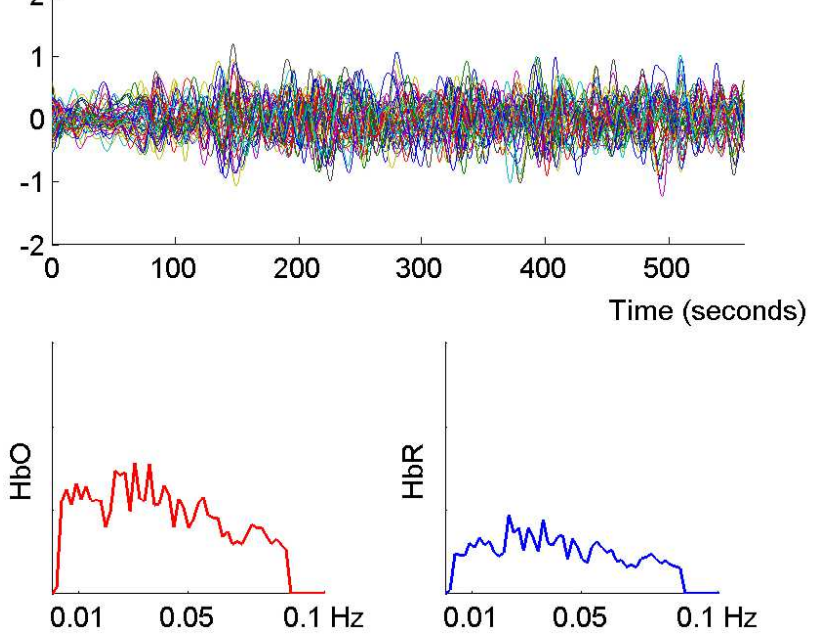
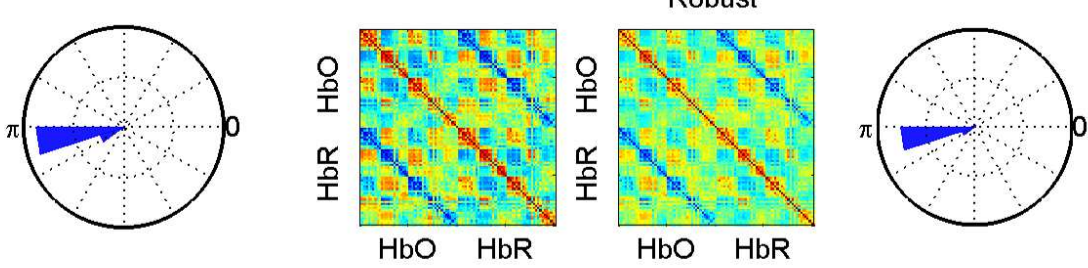

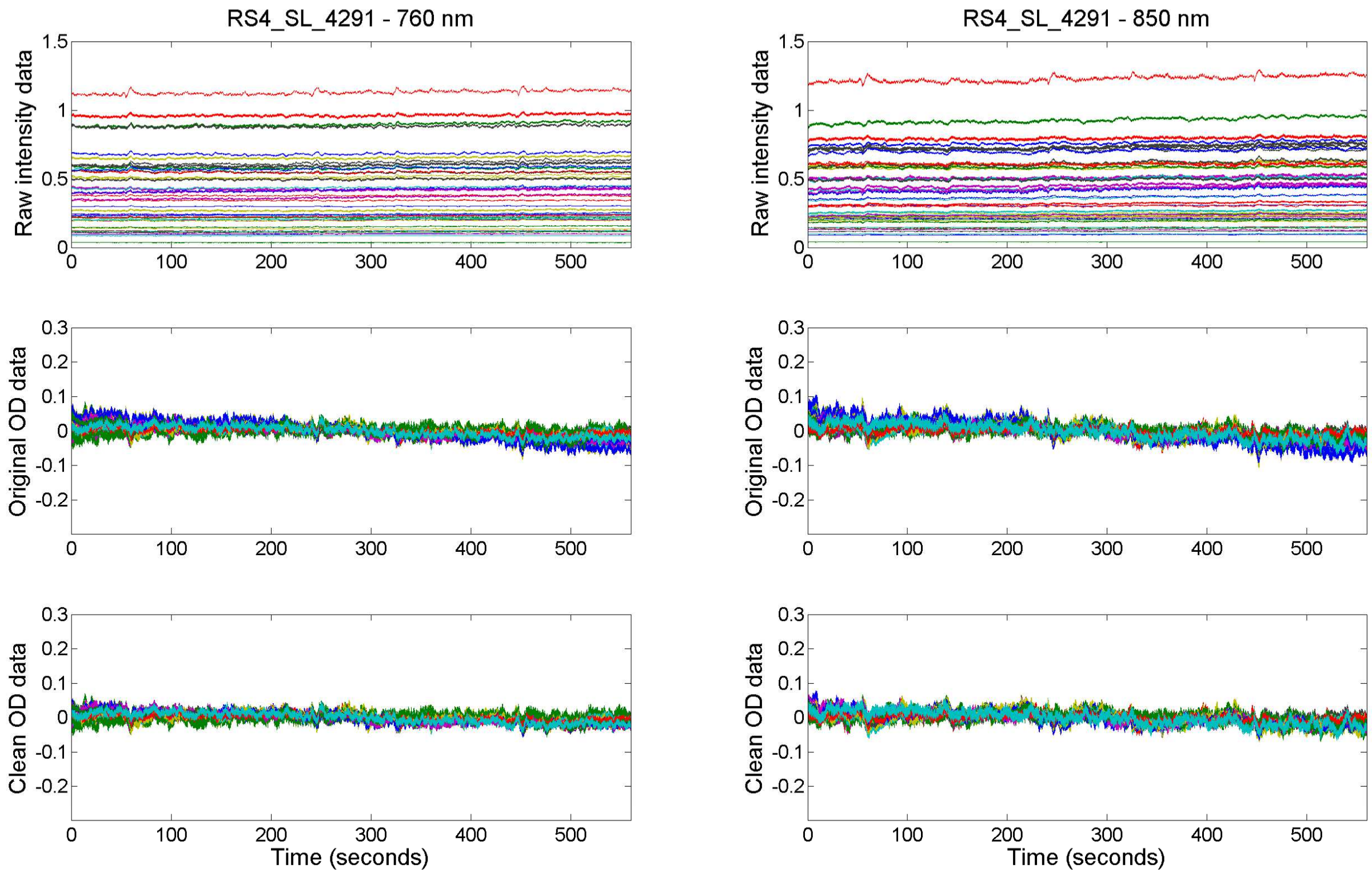
Raw

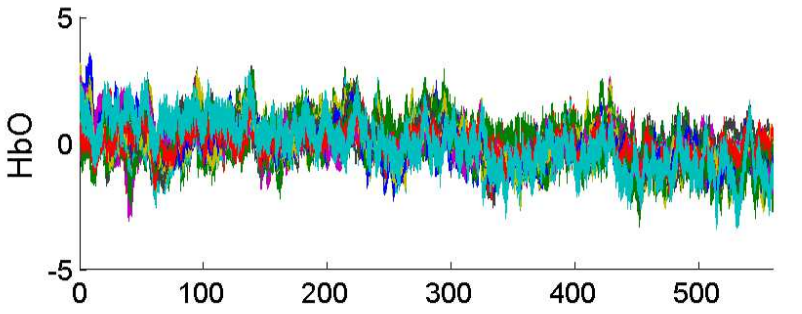

2

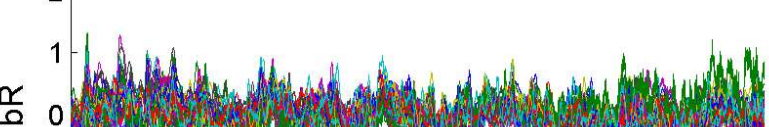
$\frac{0}{\mathrm{c}}{ }_{-1}^{0}$.
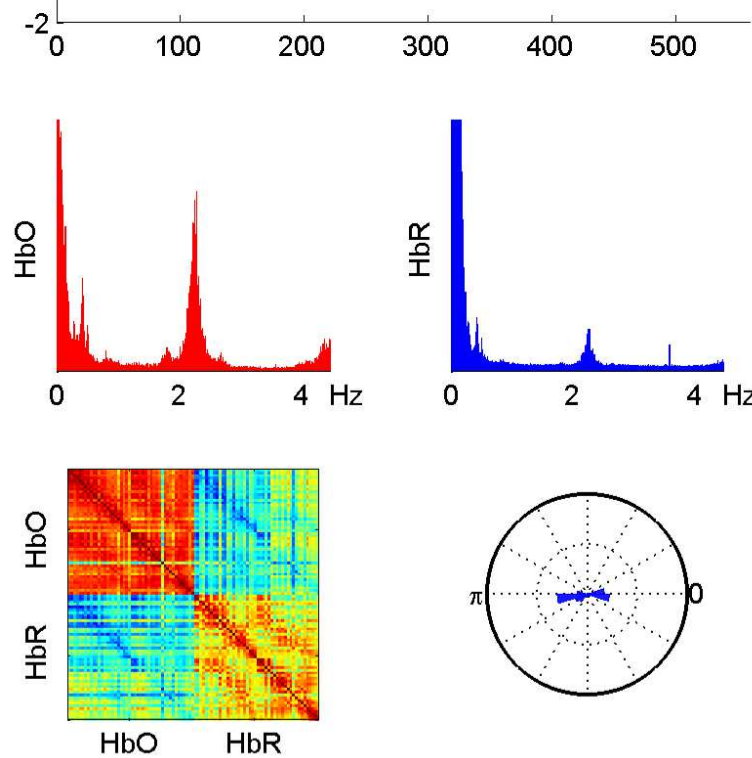

RS4 SL 4291

Filtered
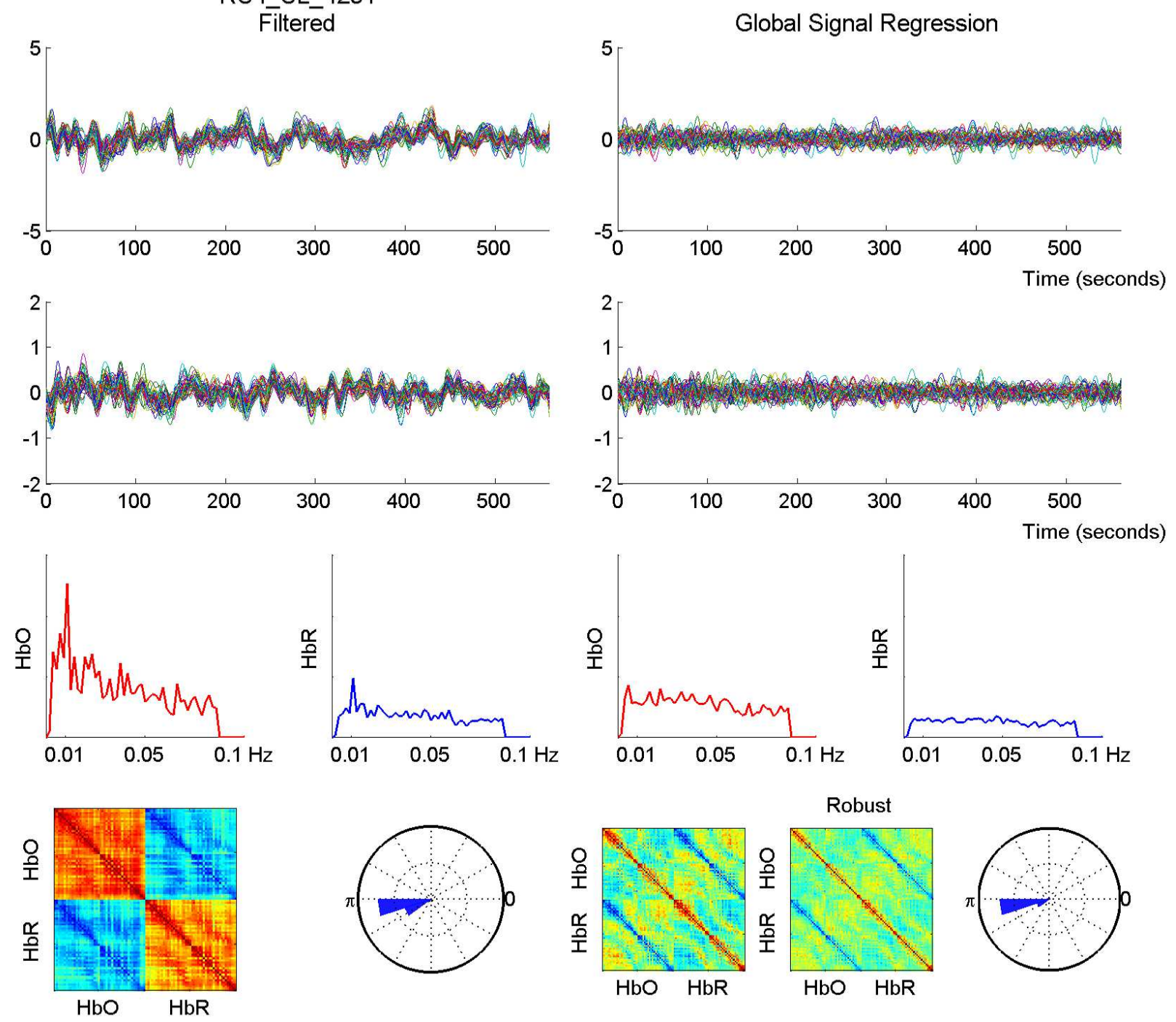

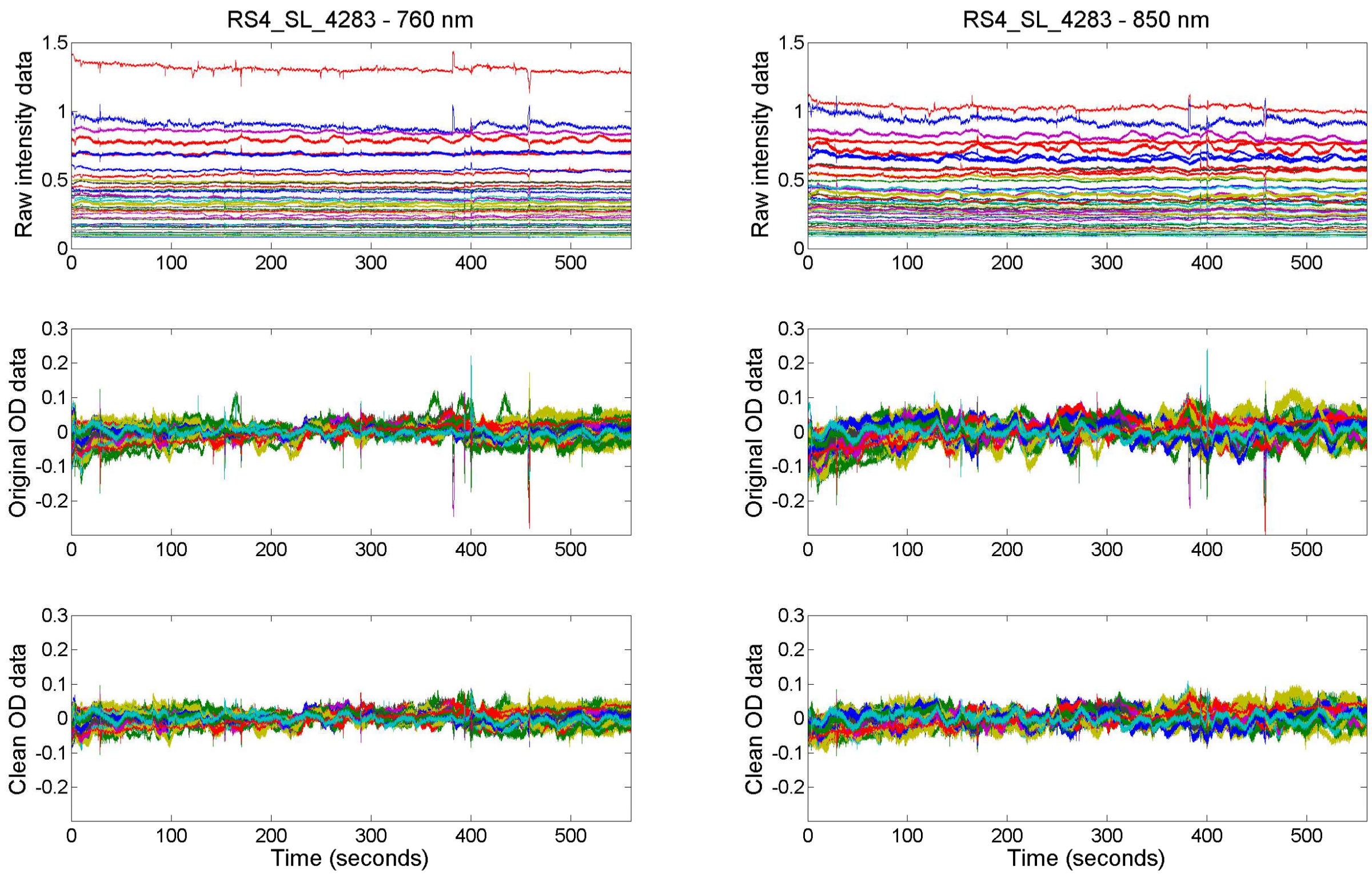
Raw
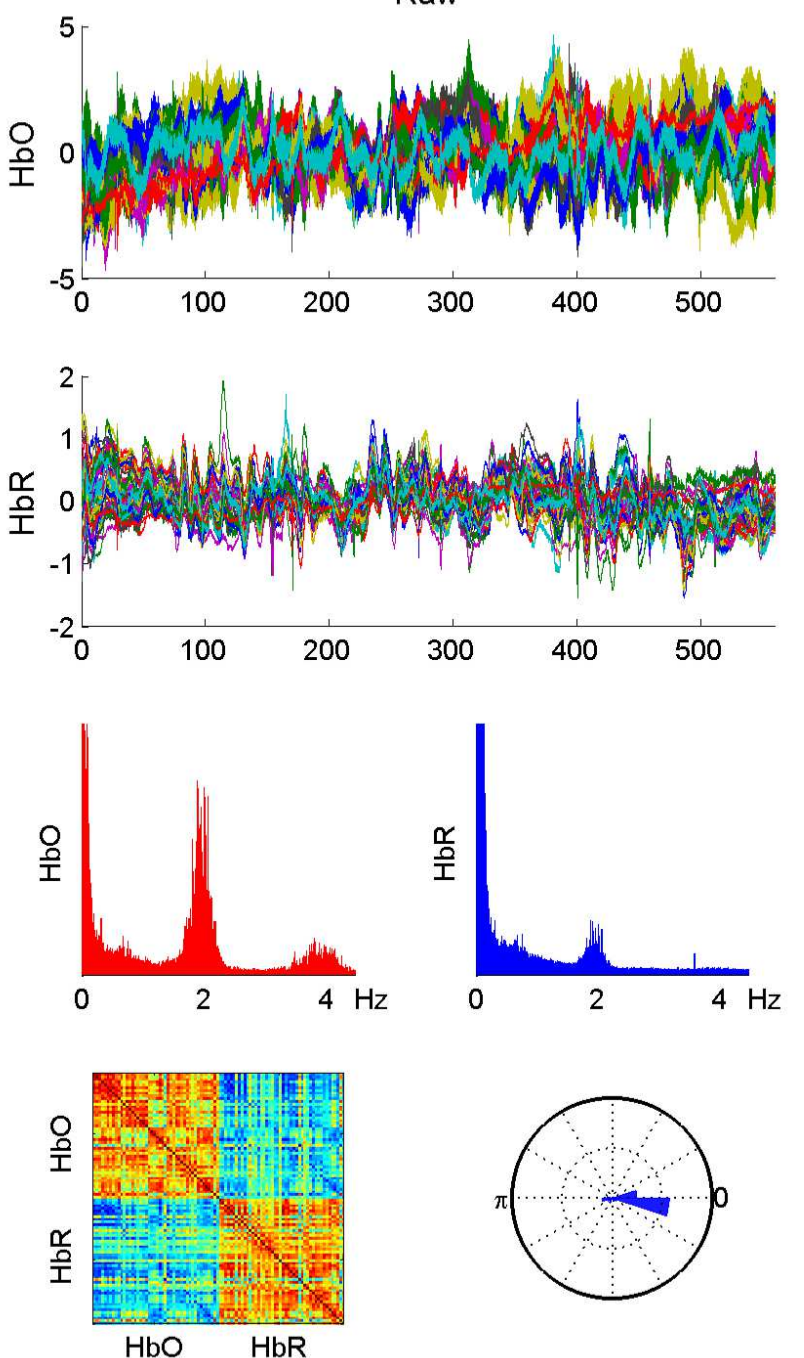

RS4 SL 4283

Filtered
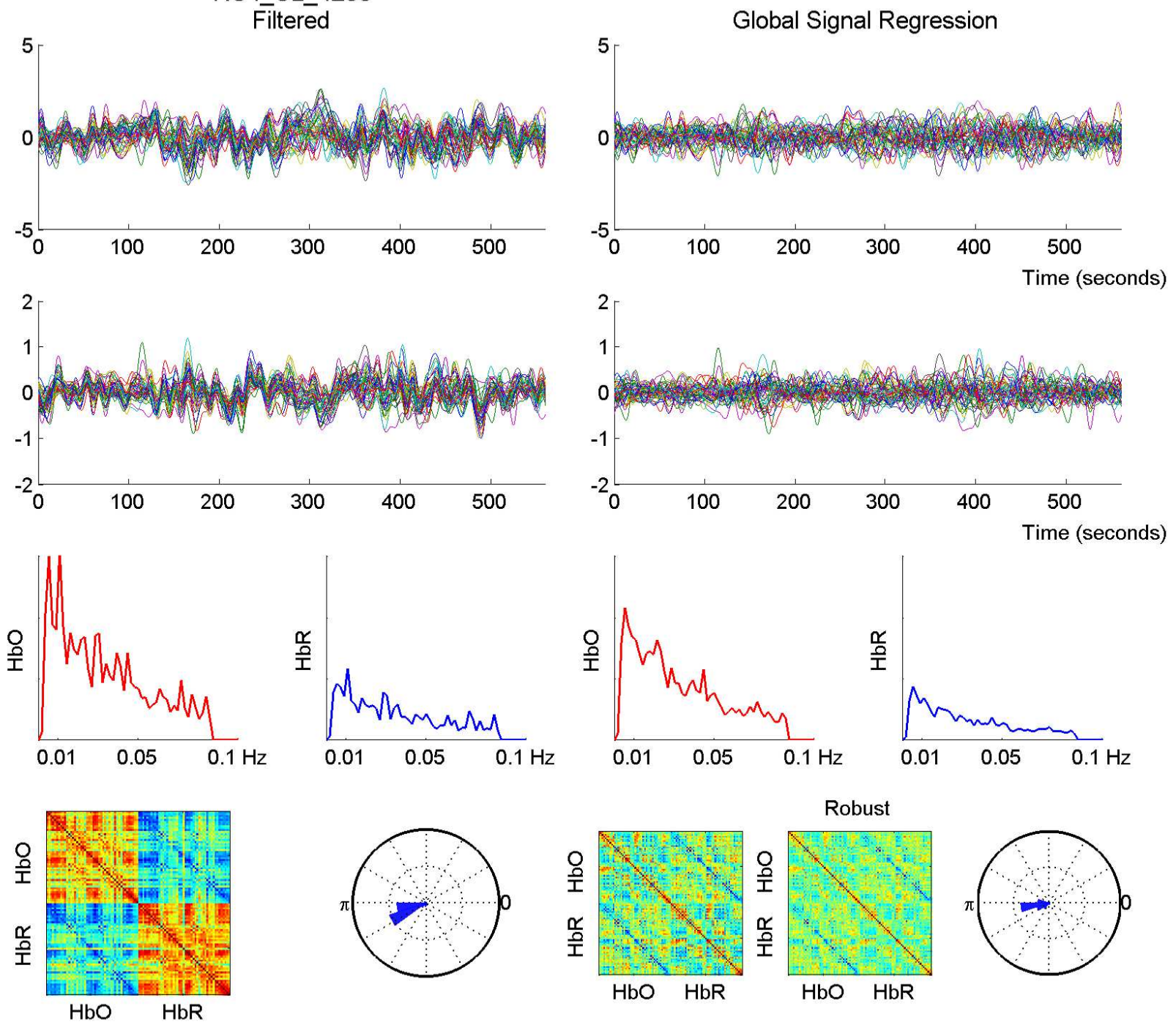

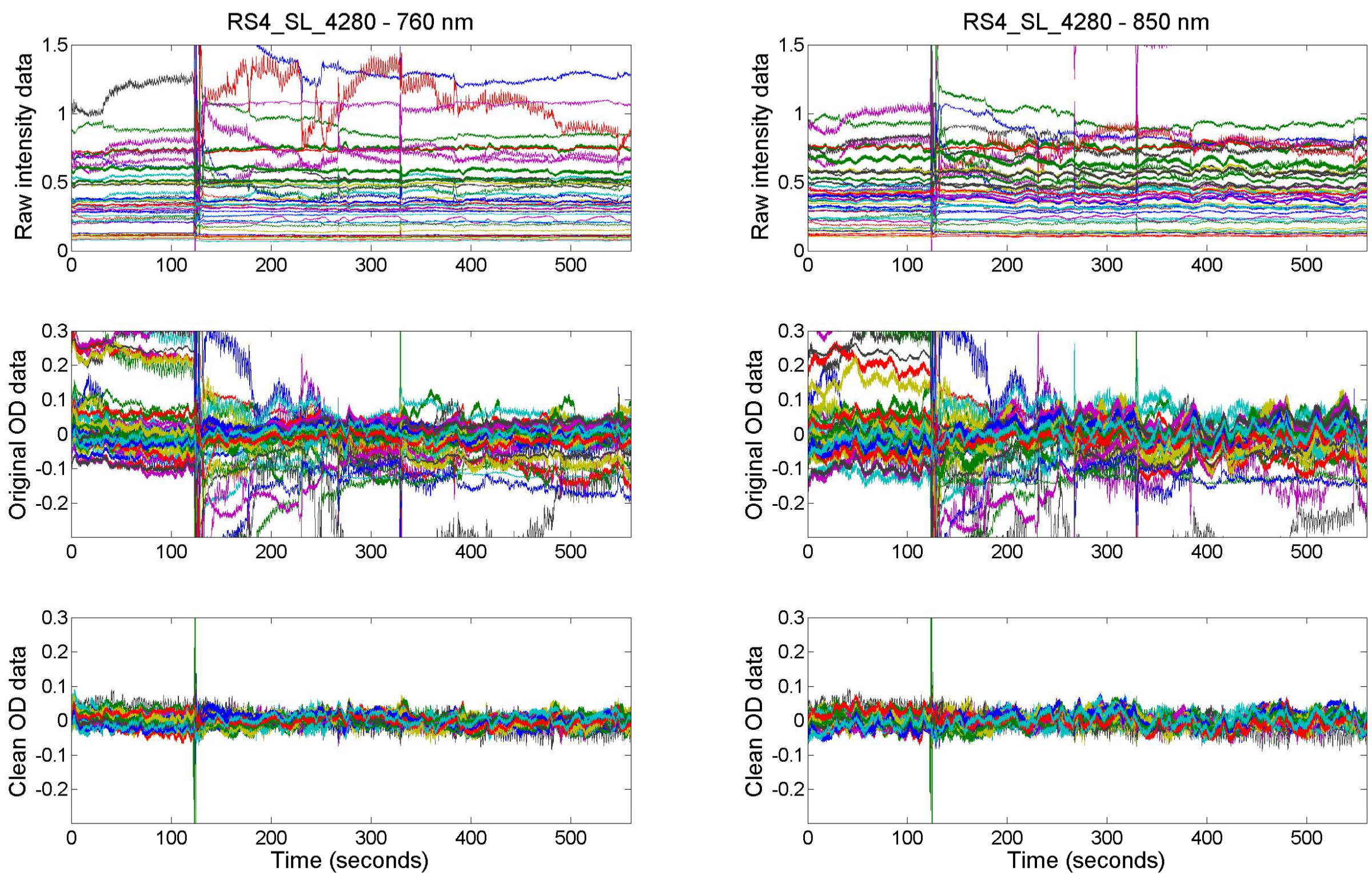

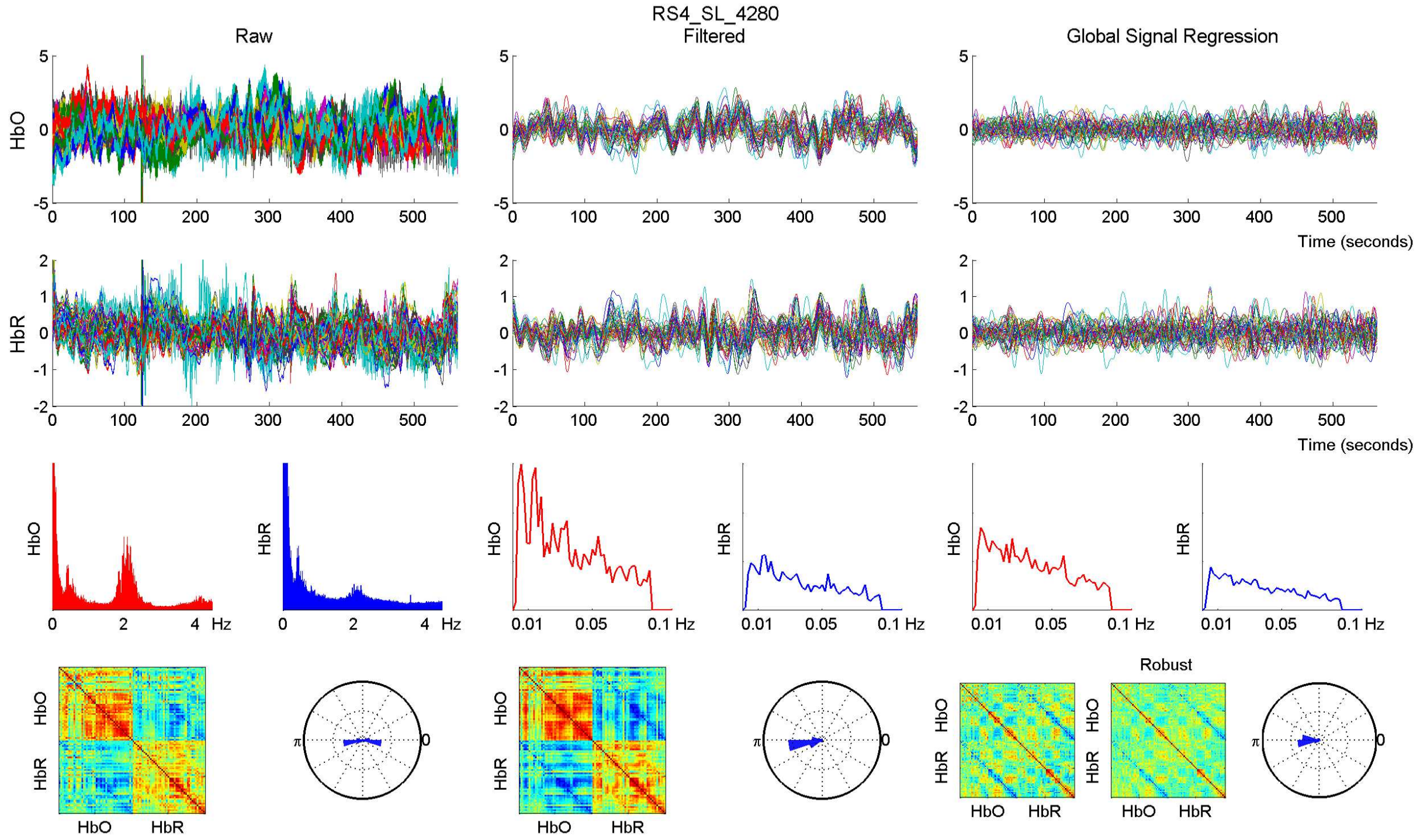

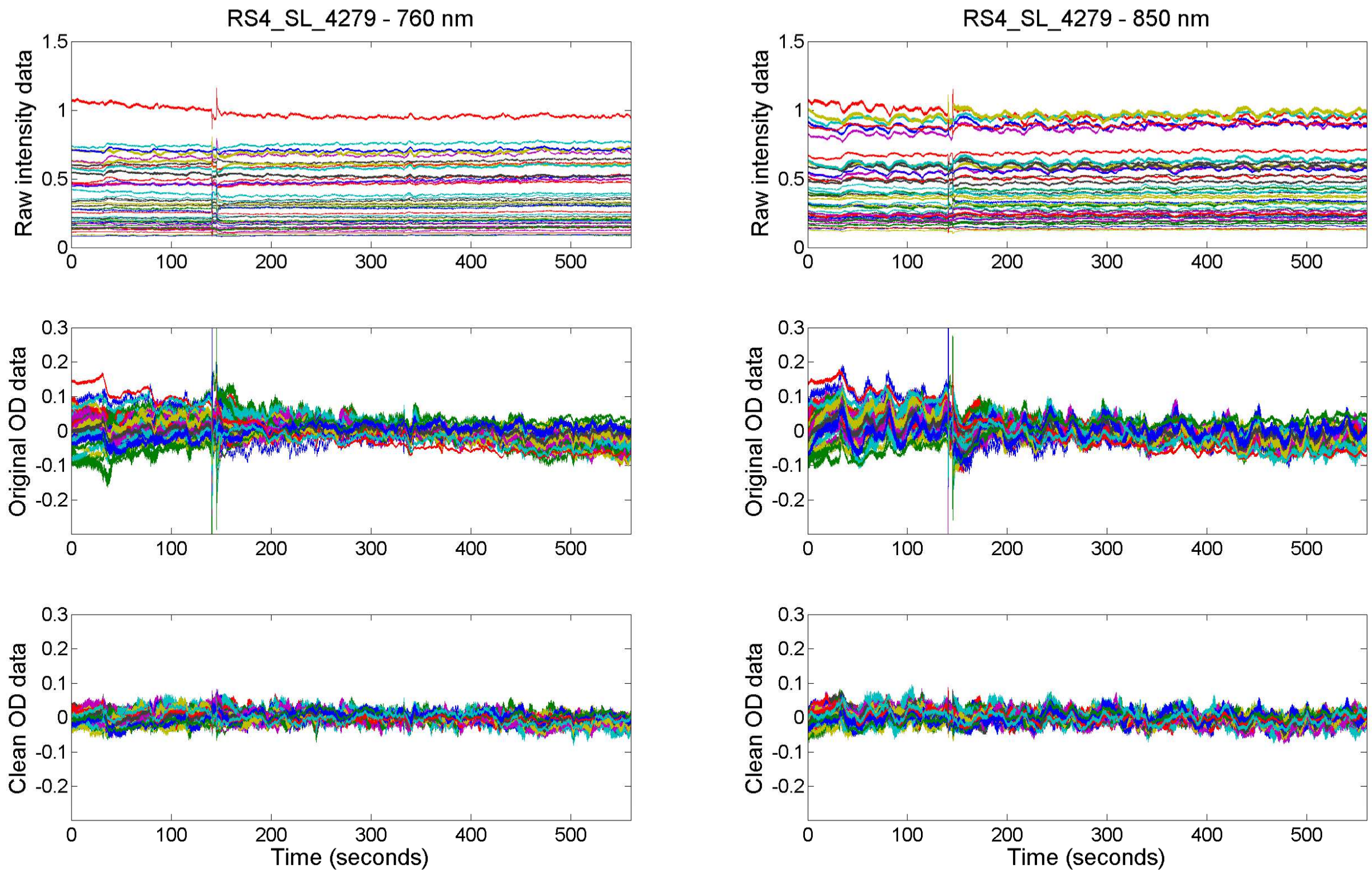
RS4_SL_4279

Raw

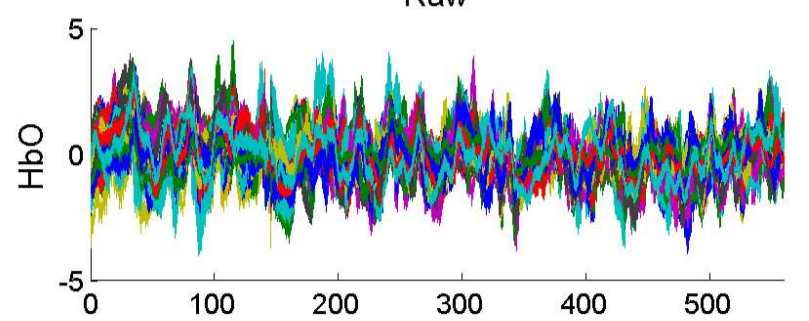

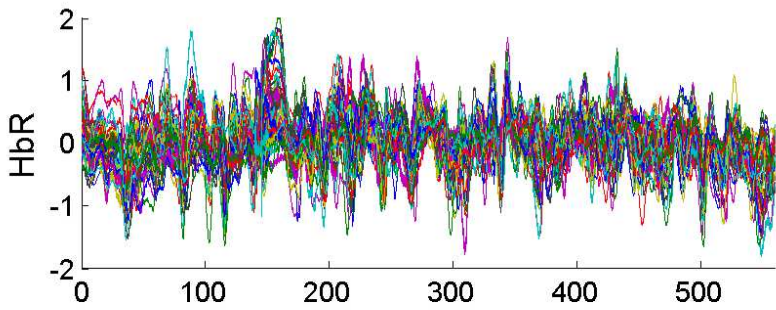
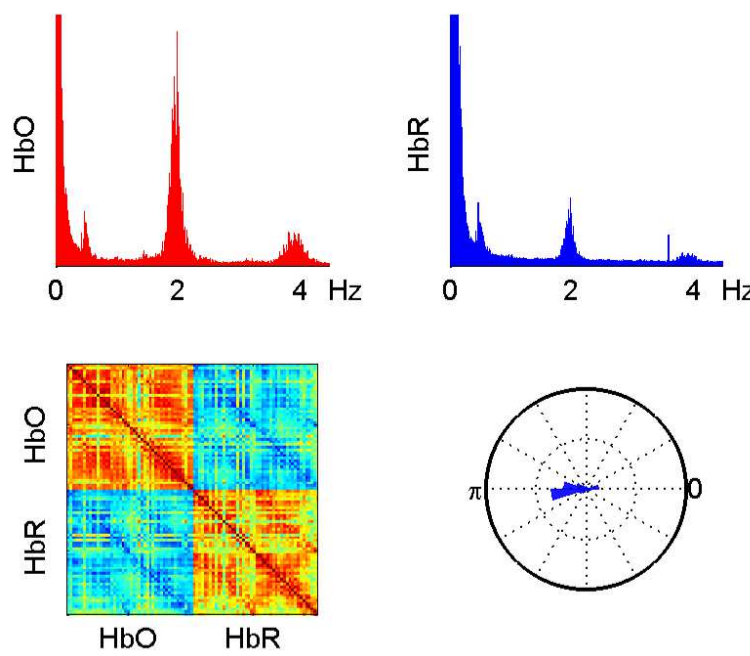

Filtered

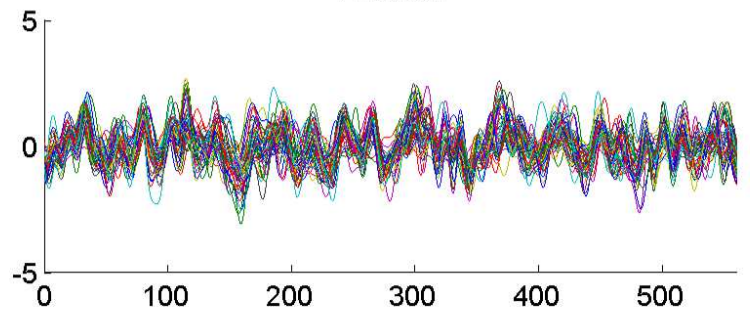

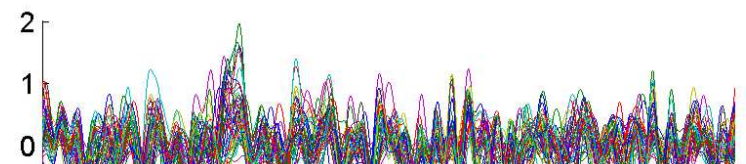

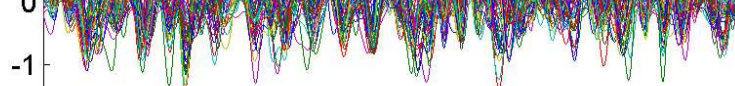
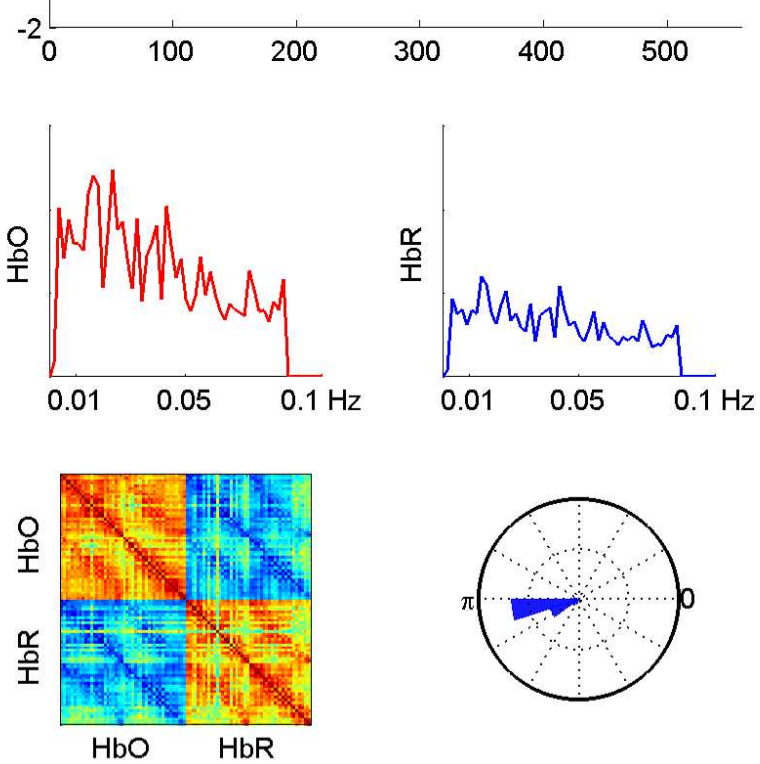

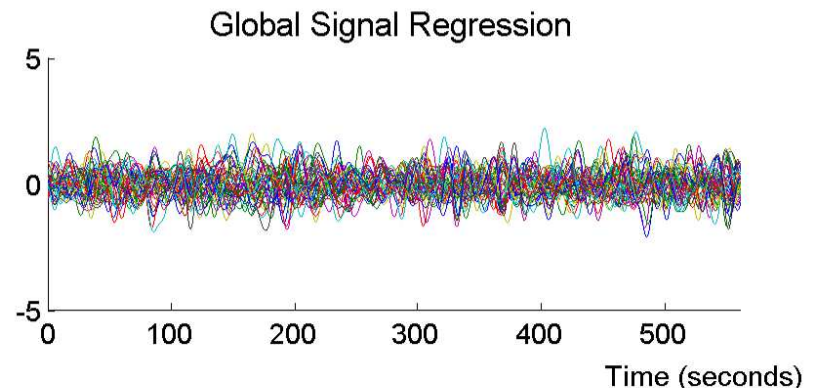

2
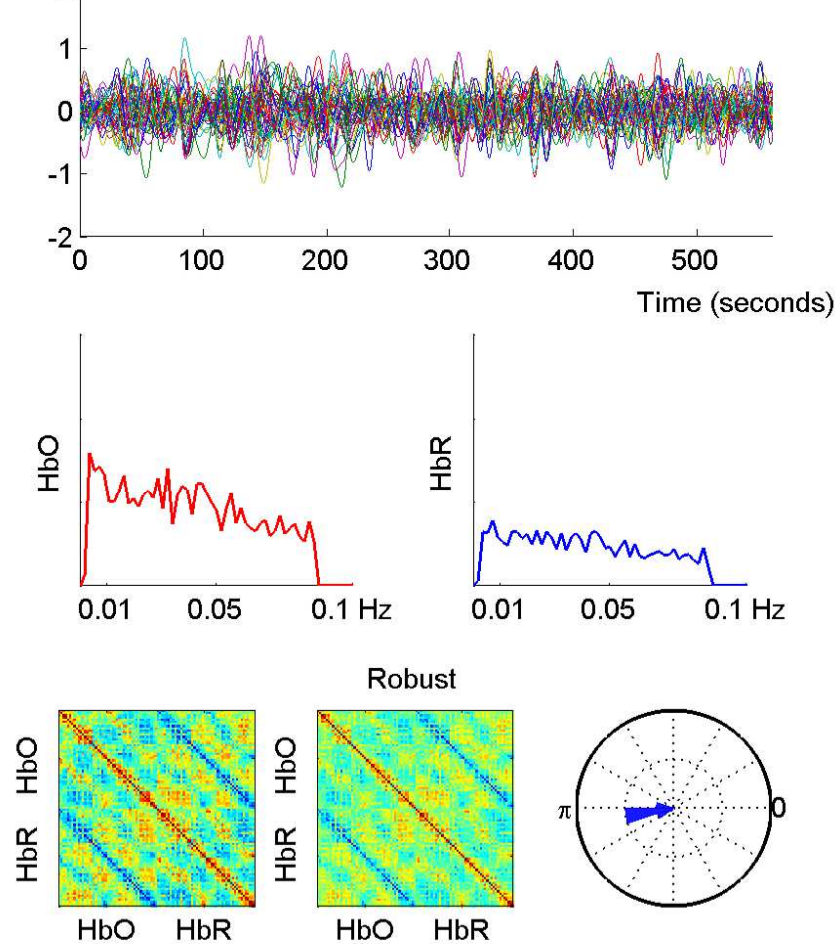

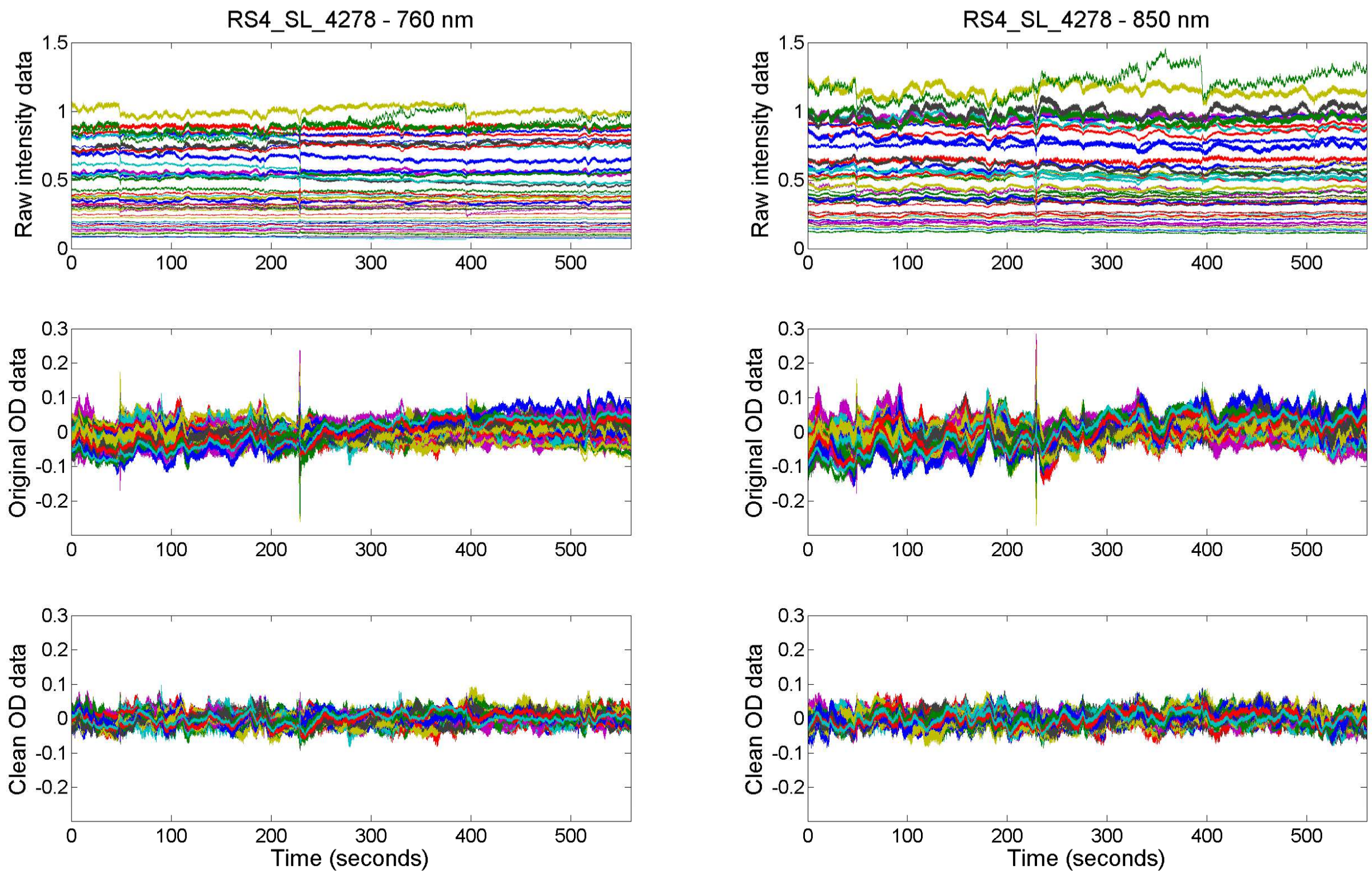
Raw

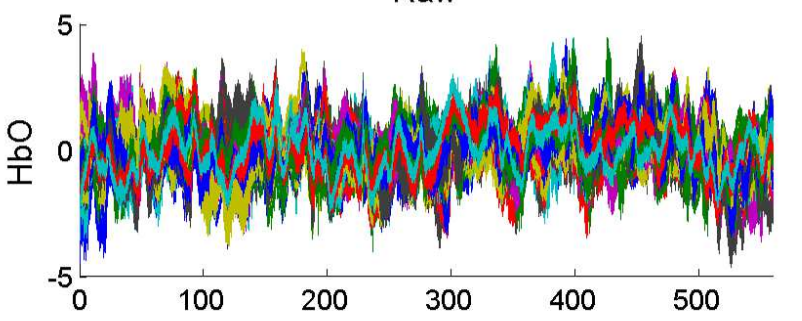

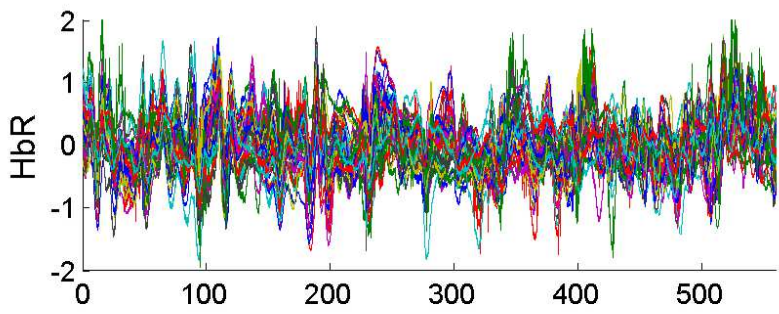
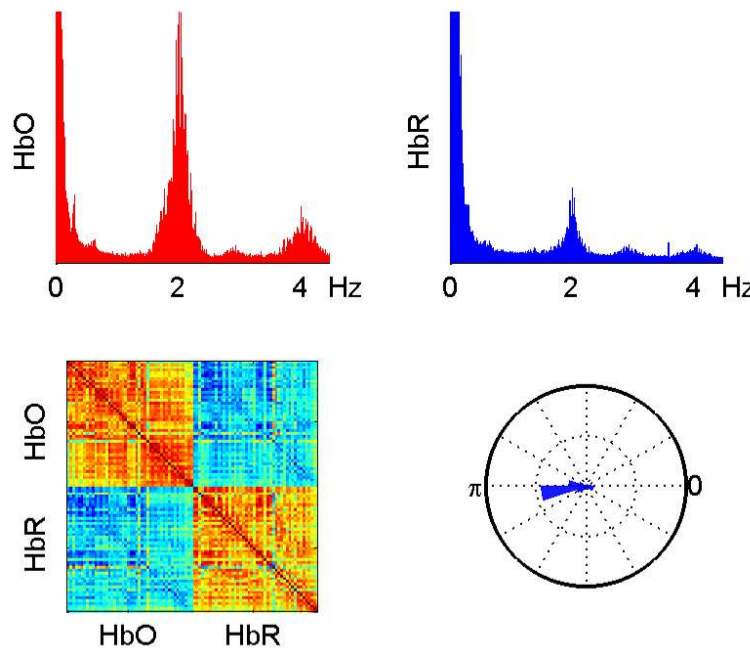

RS4_SL_4278

Filtered
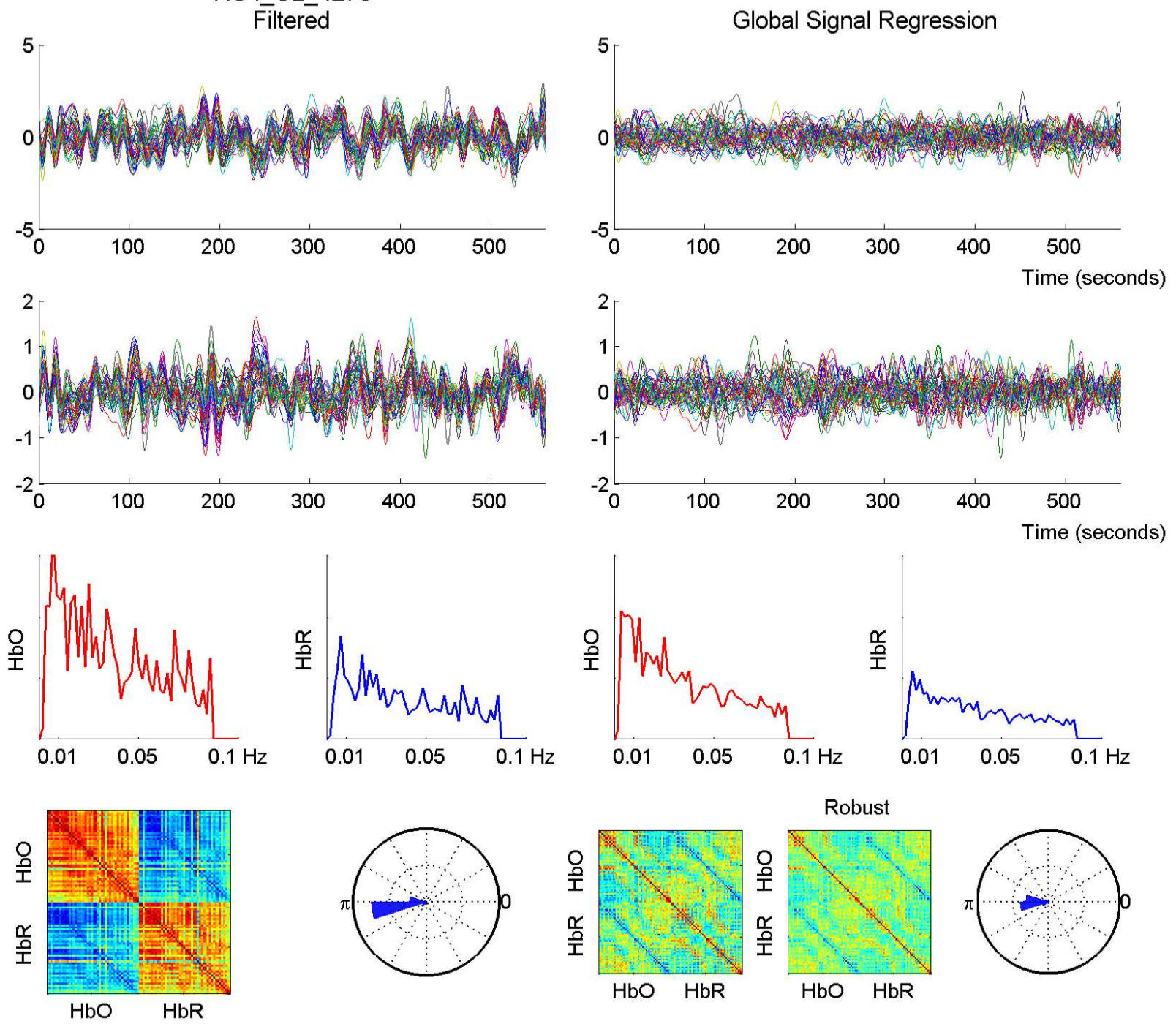
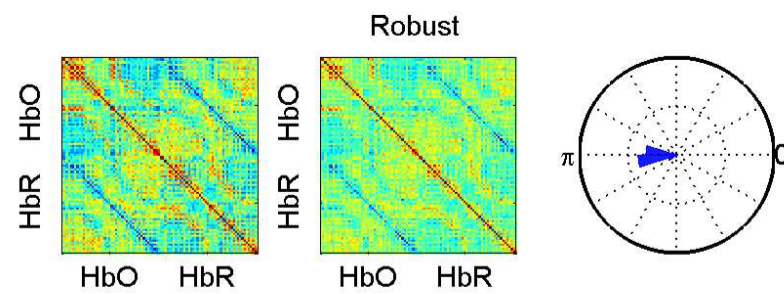
RS4 SL 4275 - 760 nm
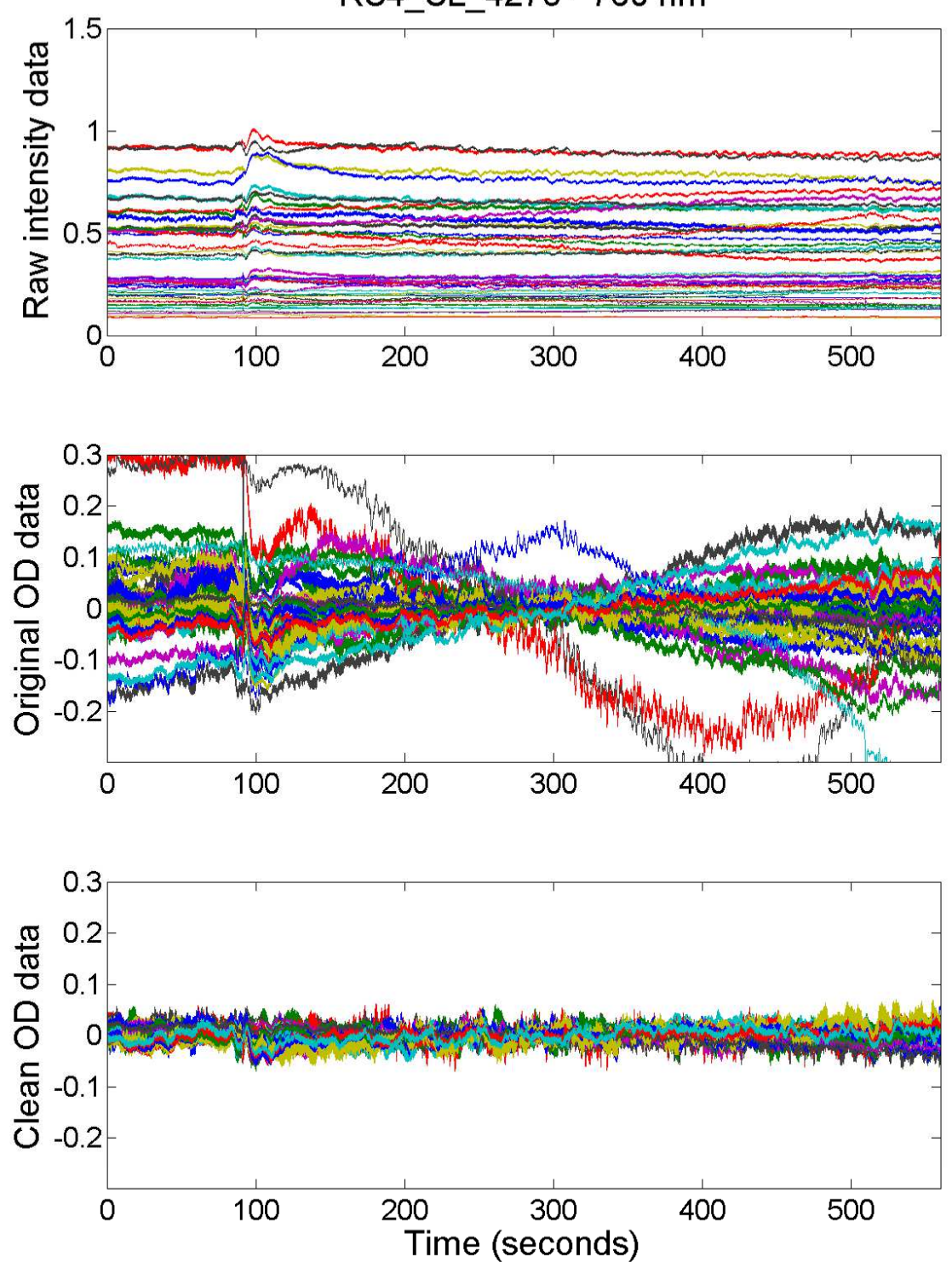

RS4 SL $4275-850 \mathrm{~nm}$
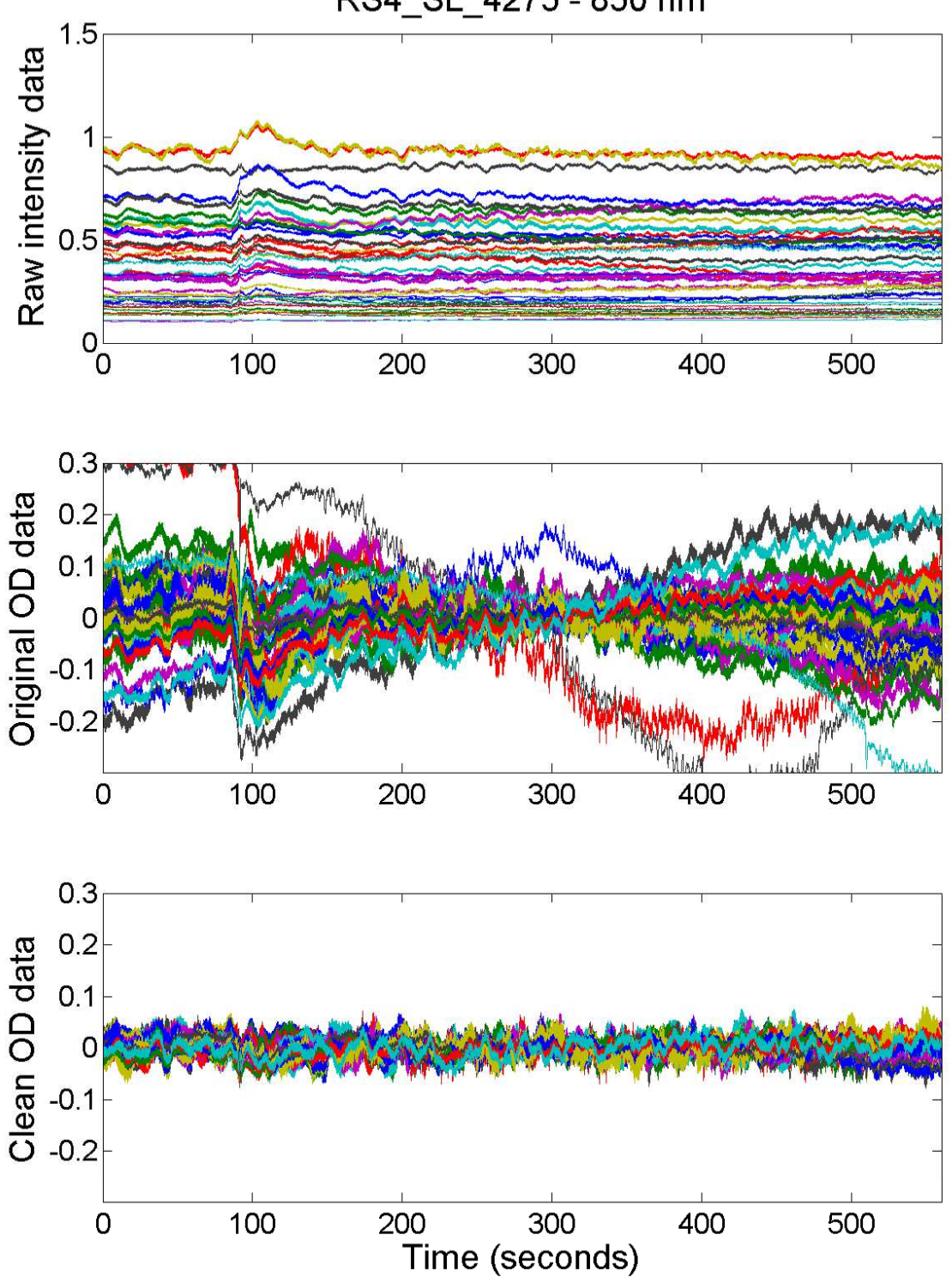
Raw
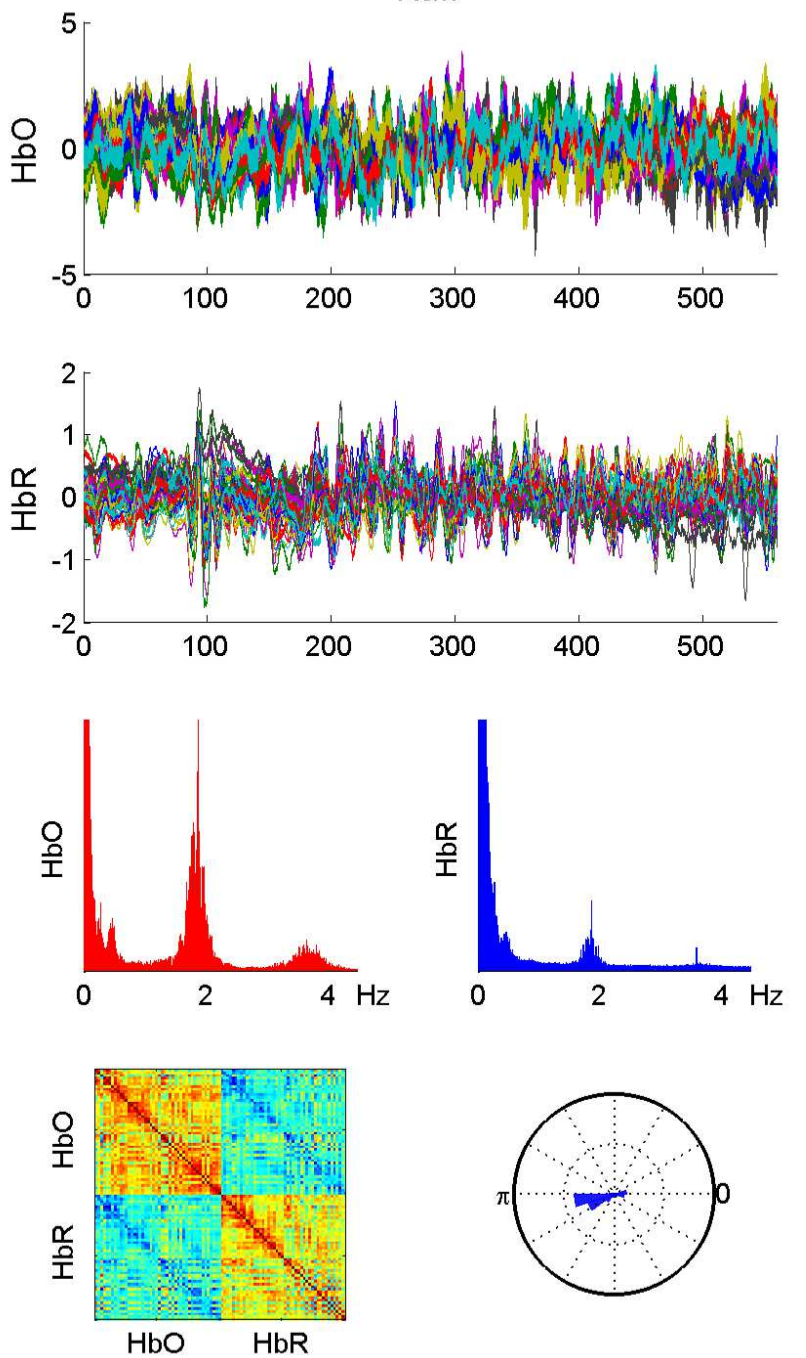

RS4 SL 4275

Filtered
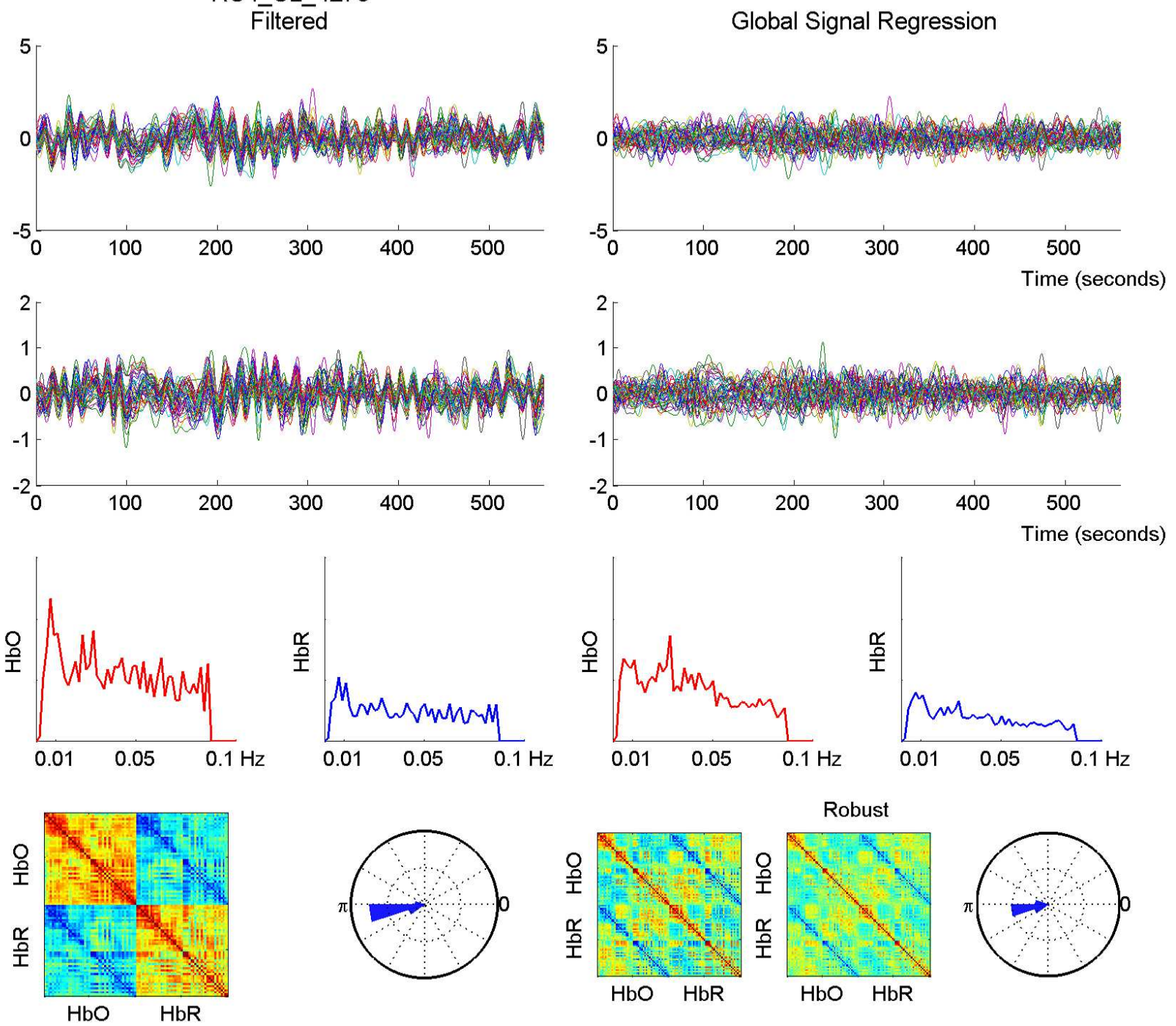
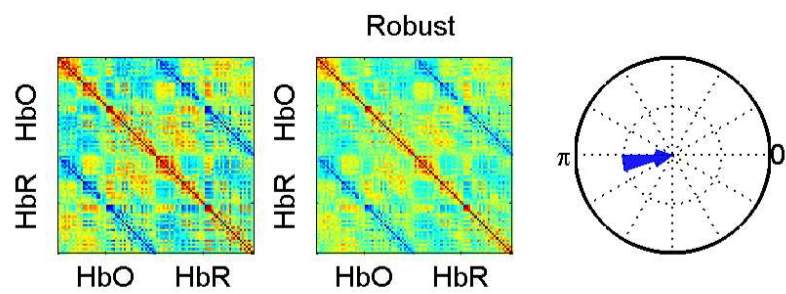

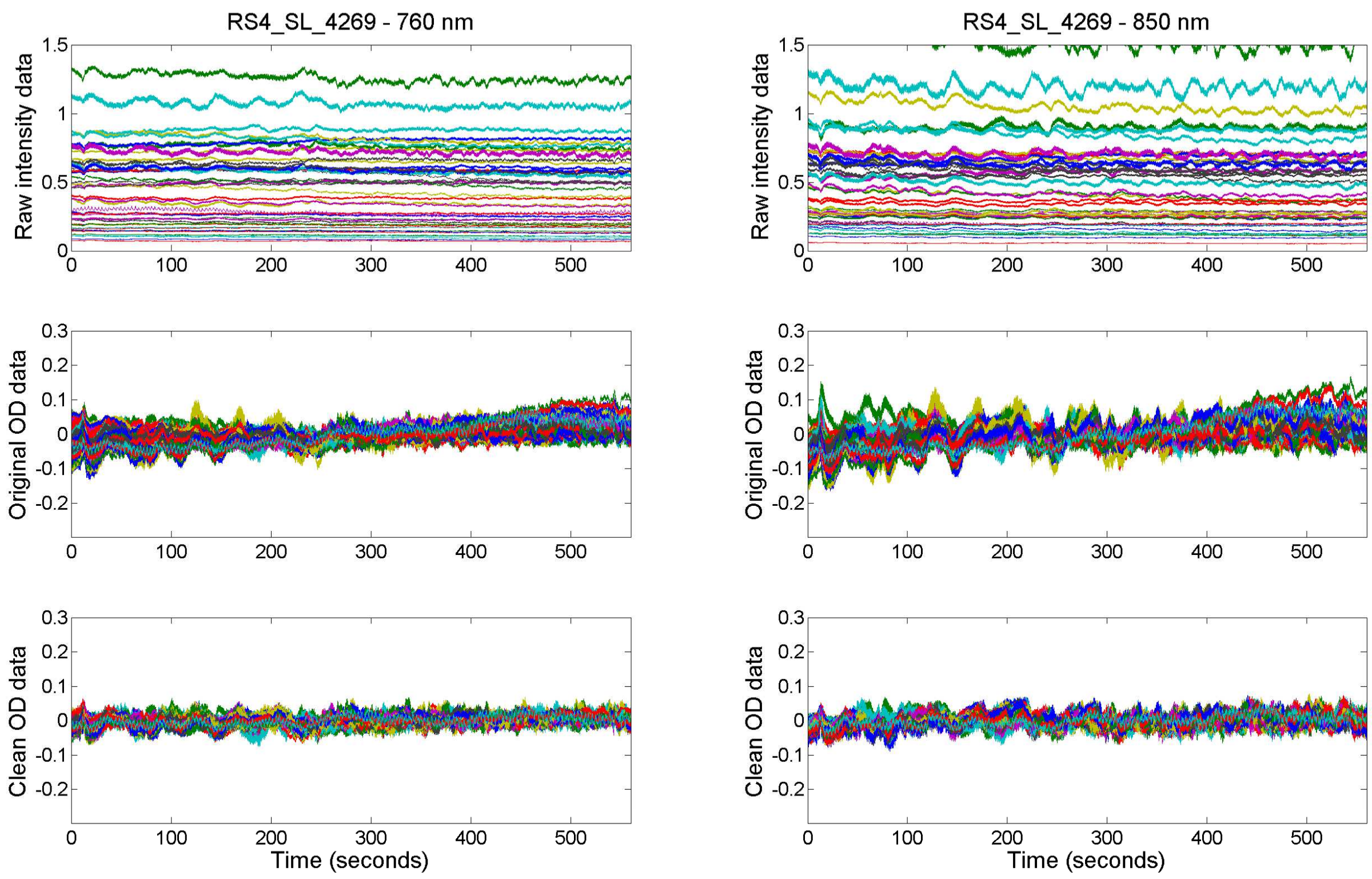
RS4_SL_4269

Raw
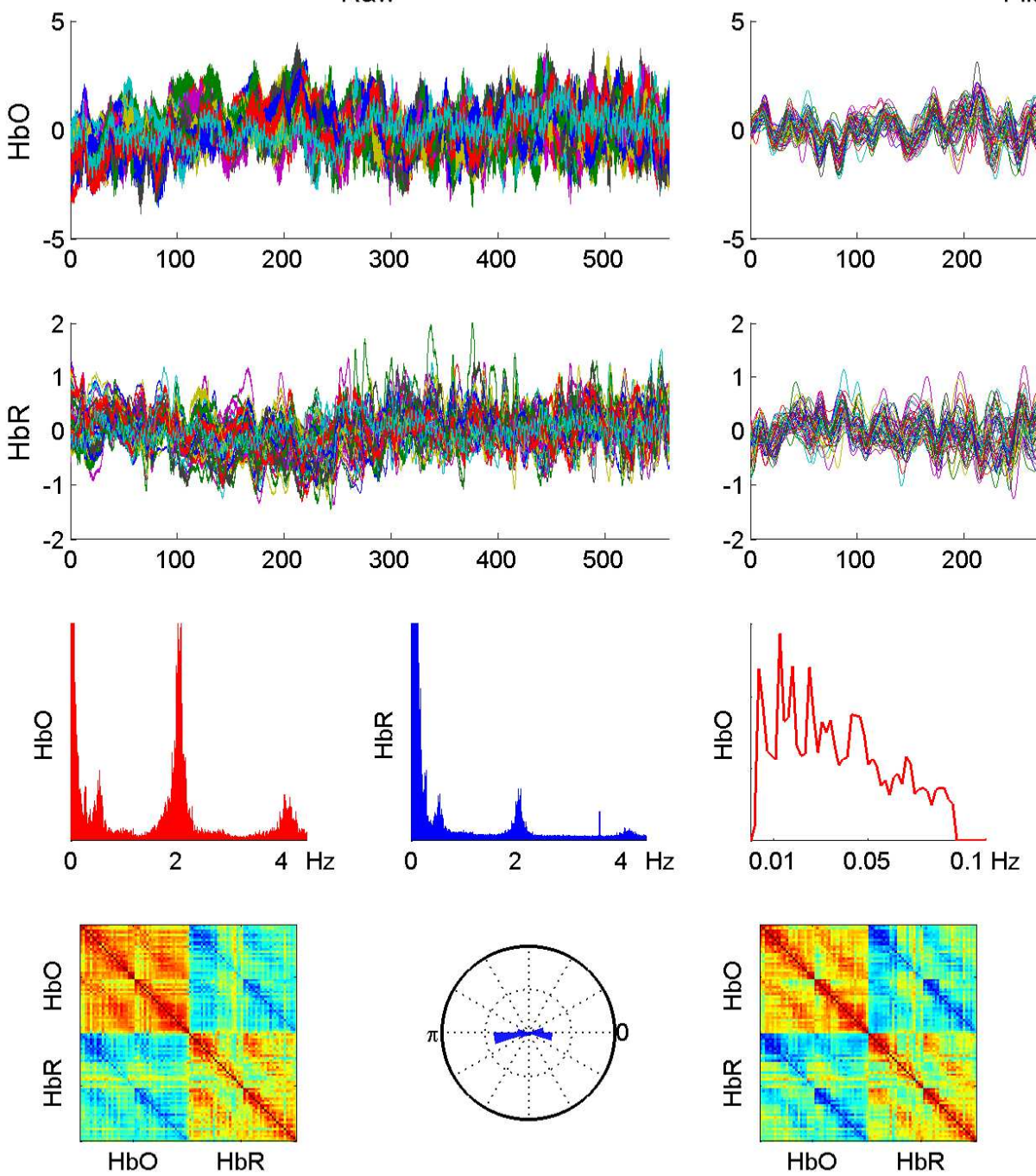
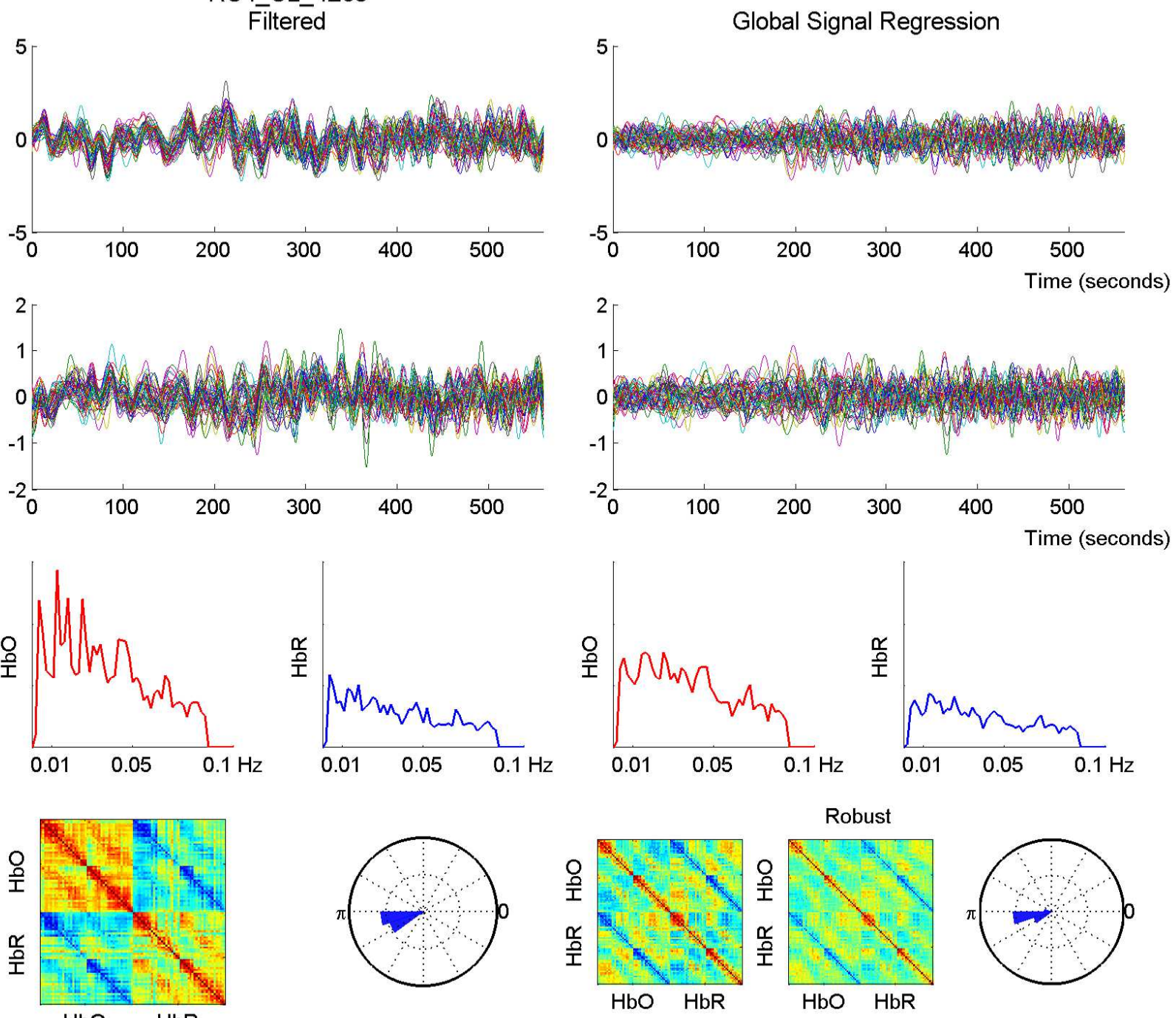

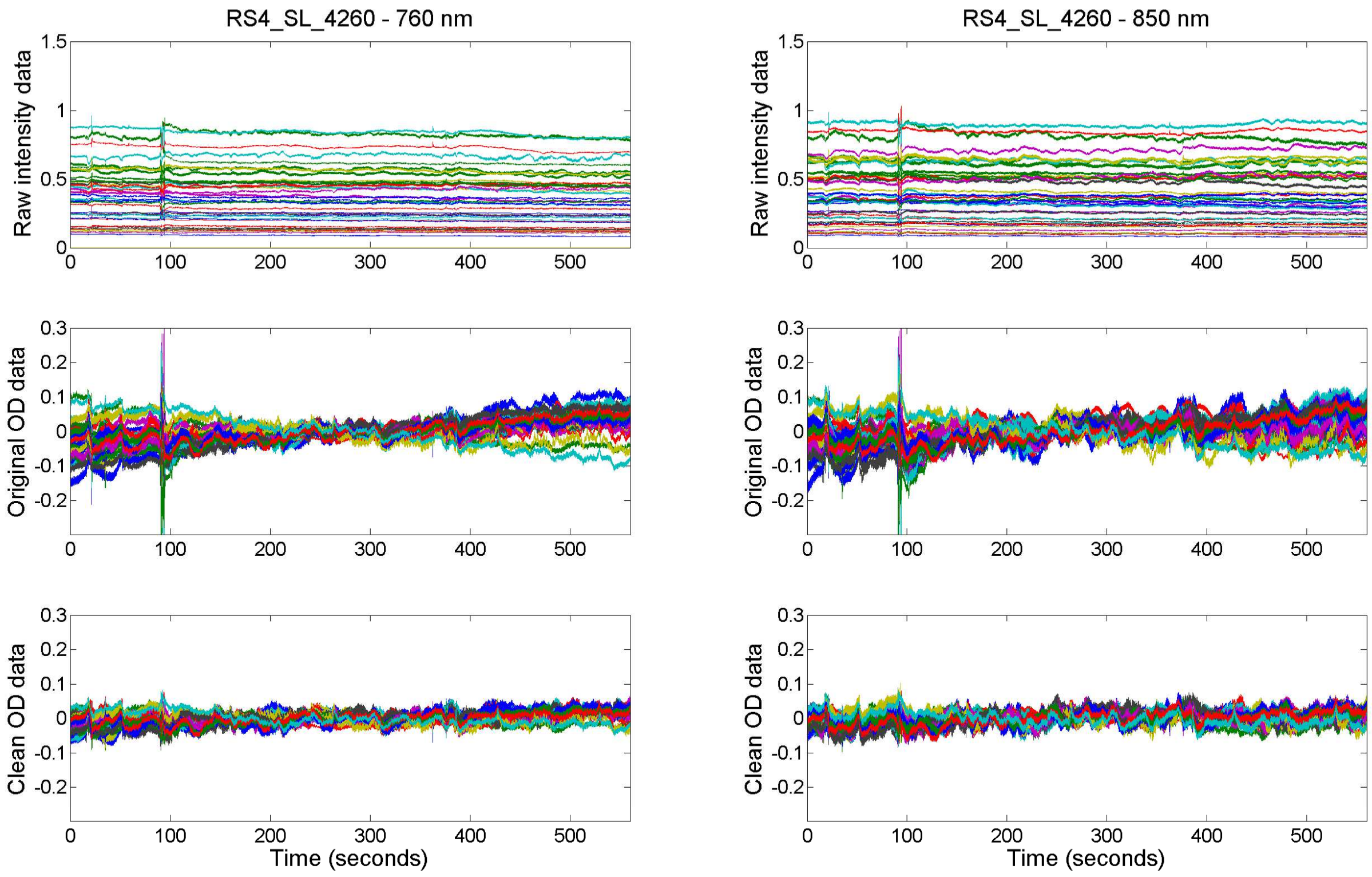
RS4_SL_4260

Raw

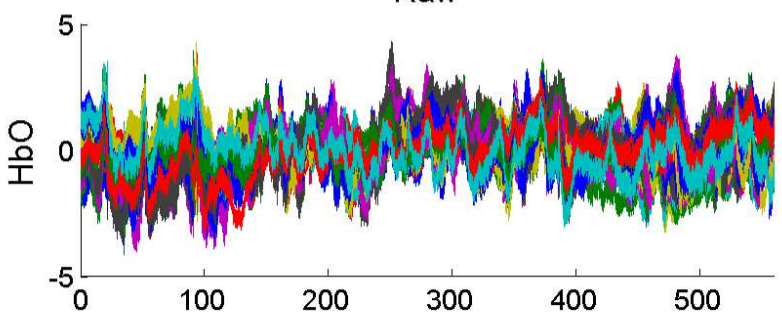

$$
\text { Filtered }
$$
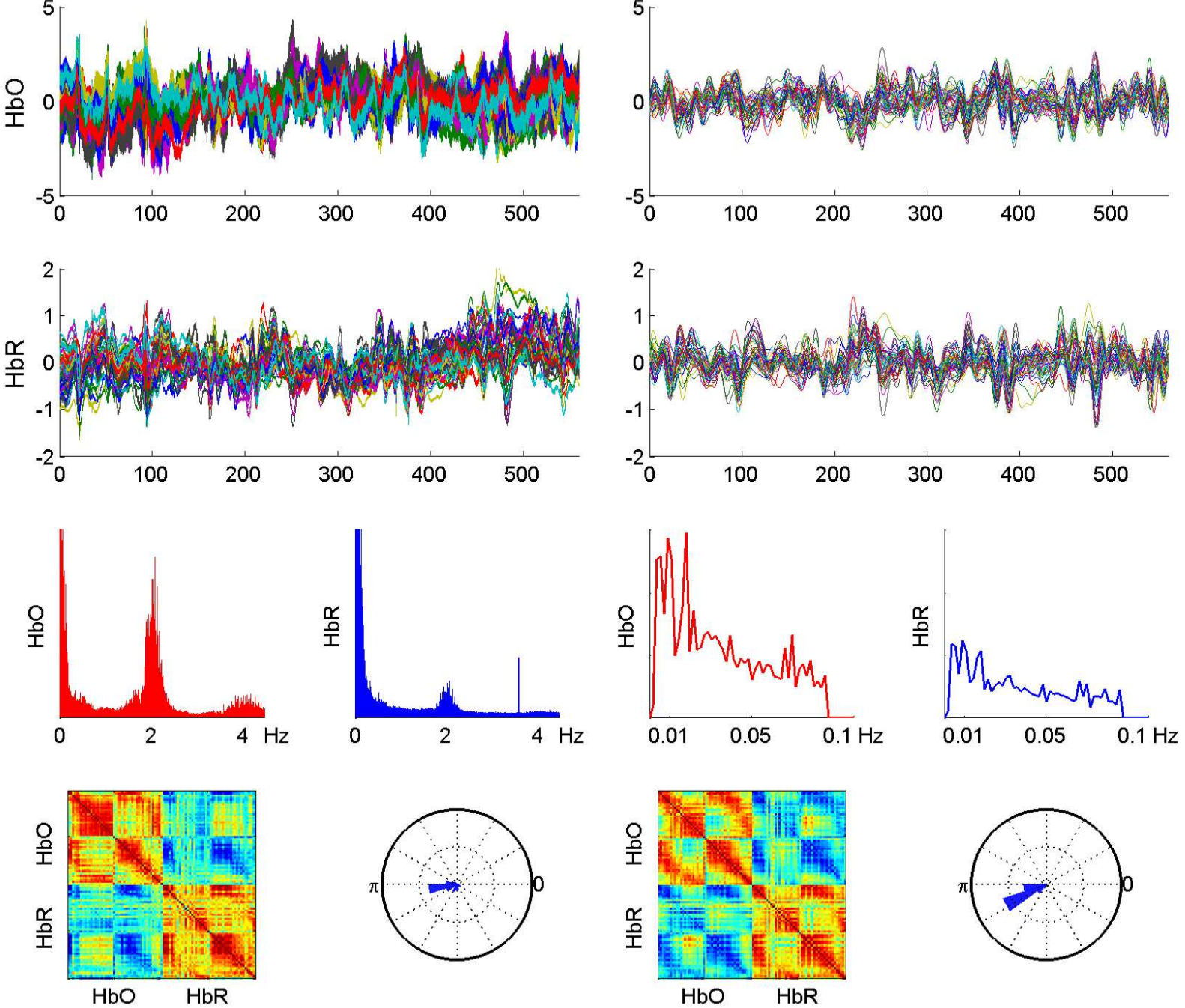
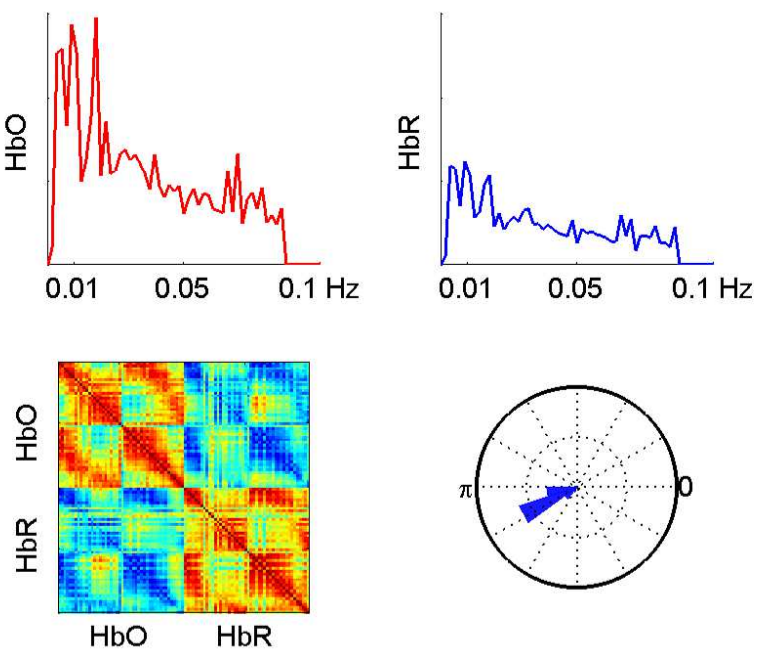

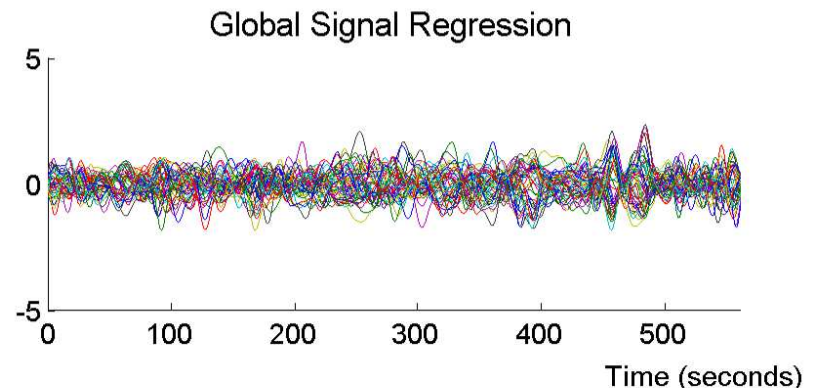

2
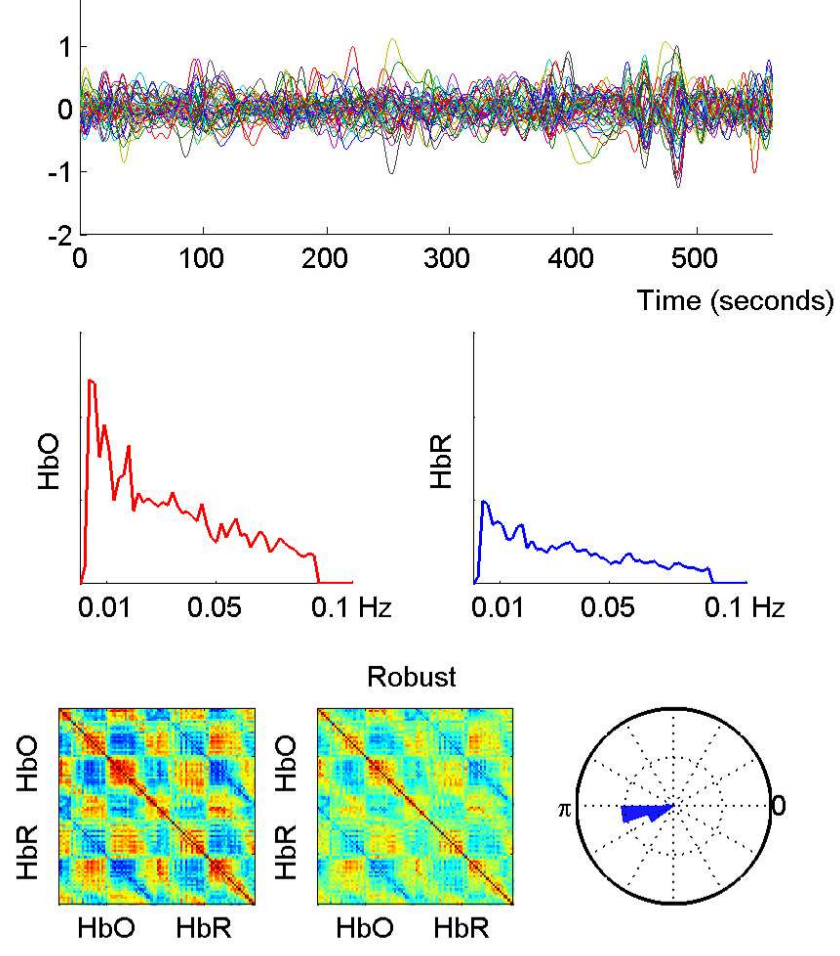
RS4 SL 4252 - 760 nm
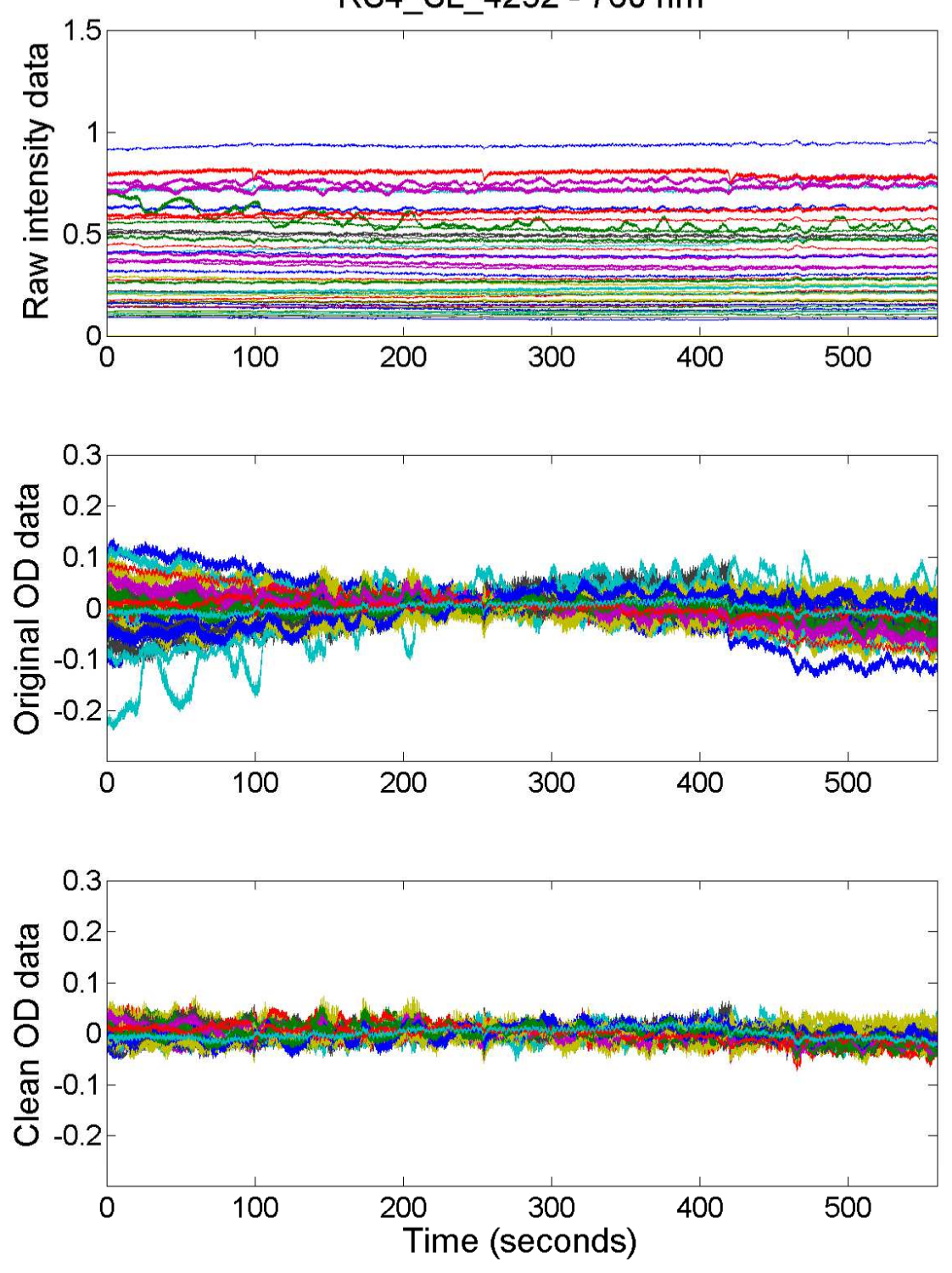

RS4 SL $4252-850 \mathrm{~nm}$
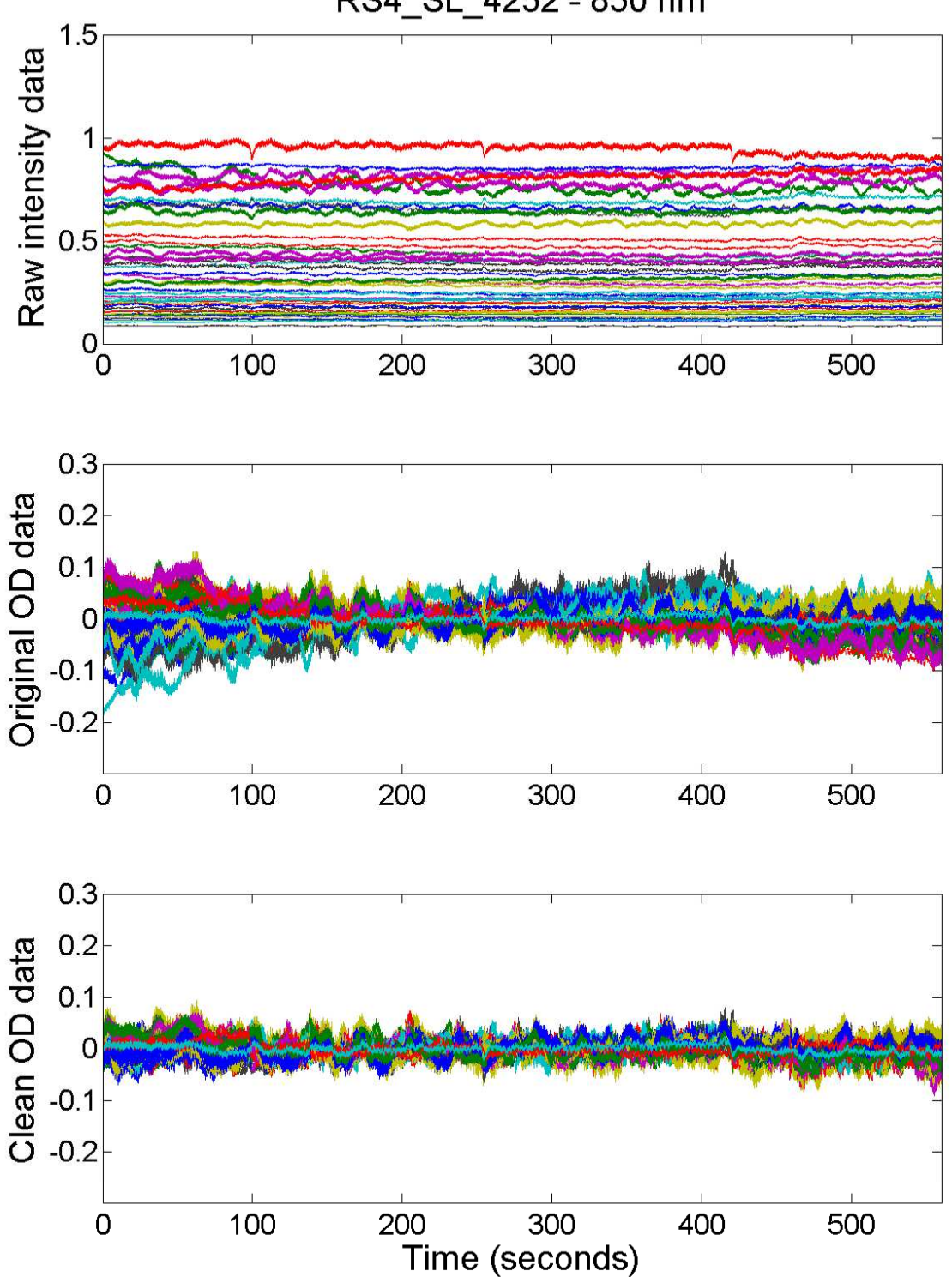
Raw
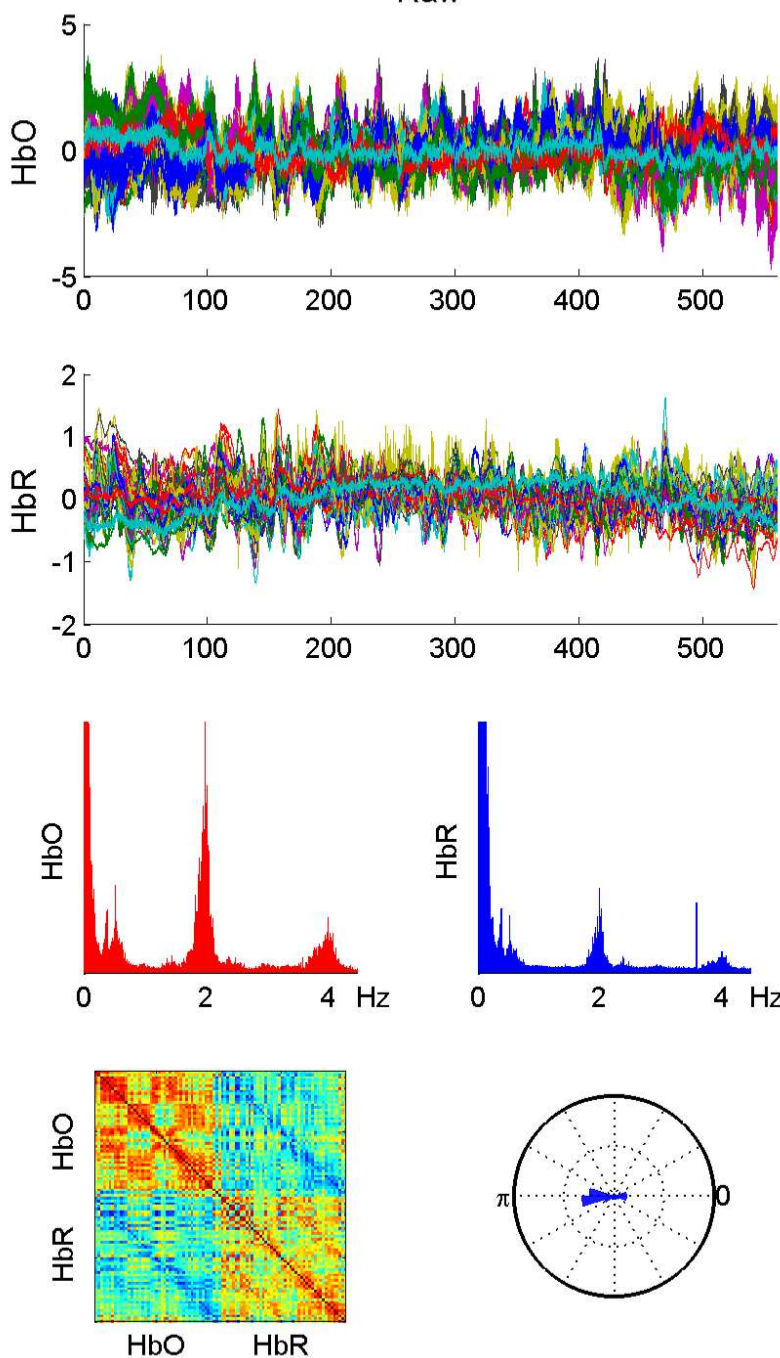

RS4_SL_4252

Filtered
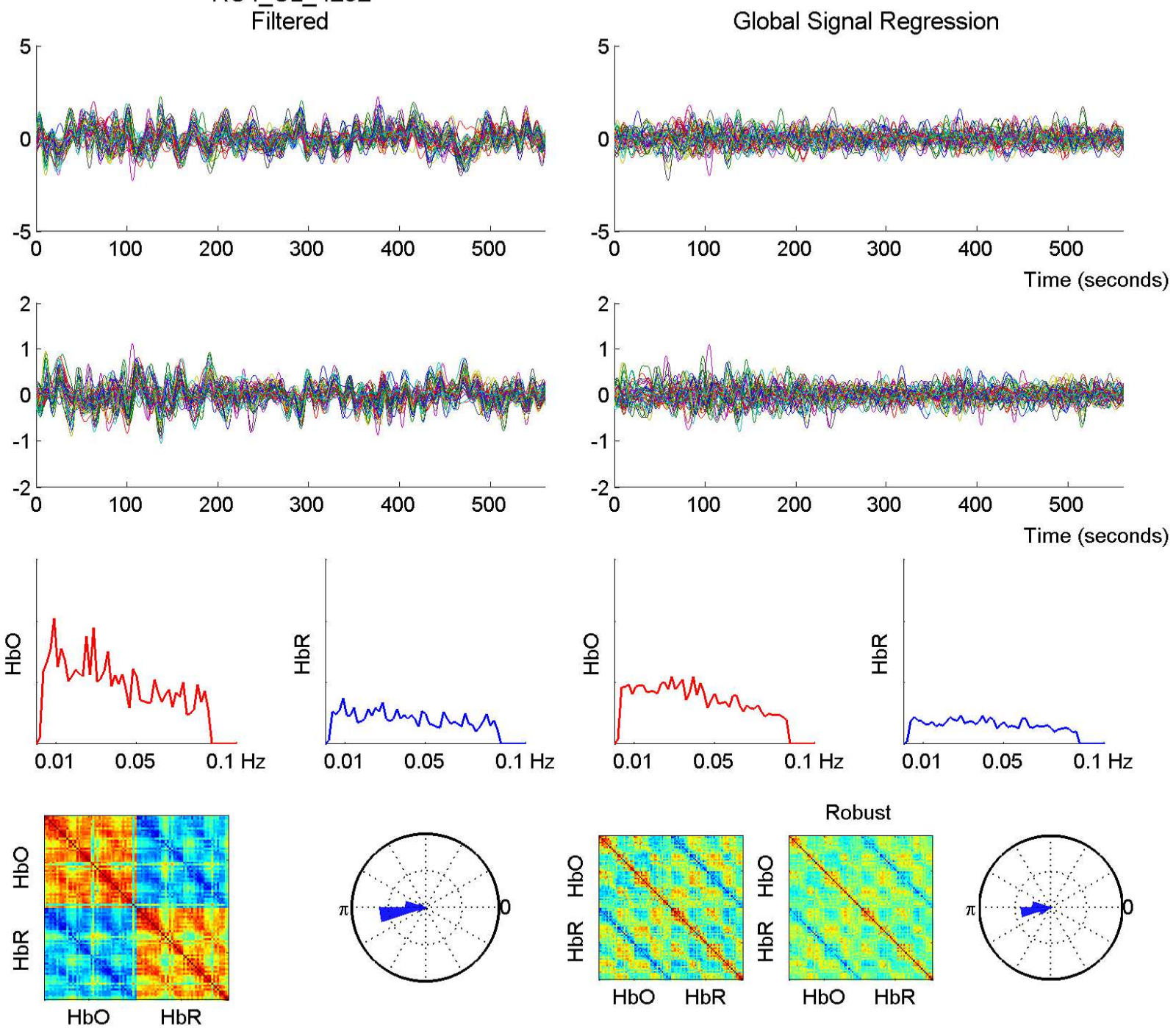
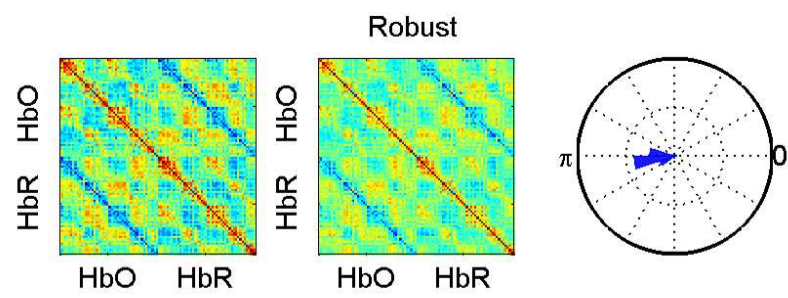

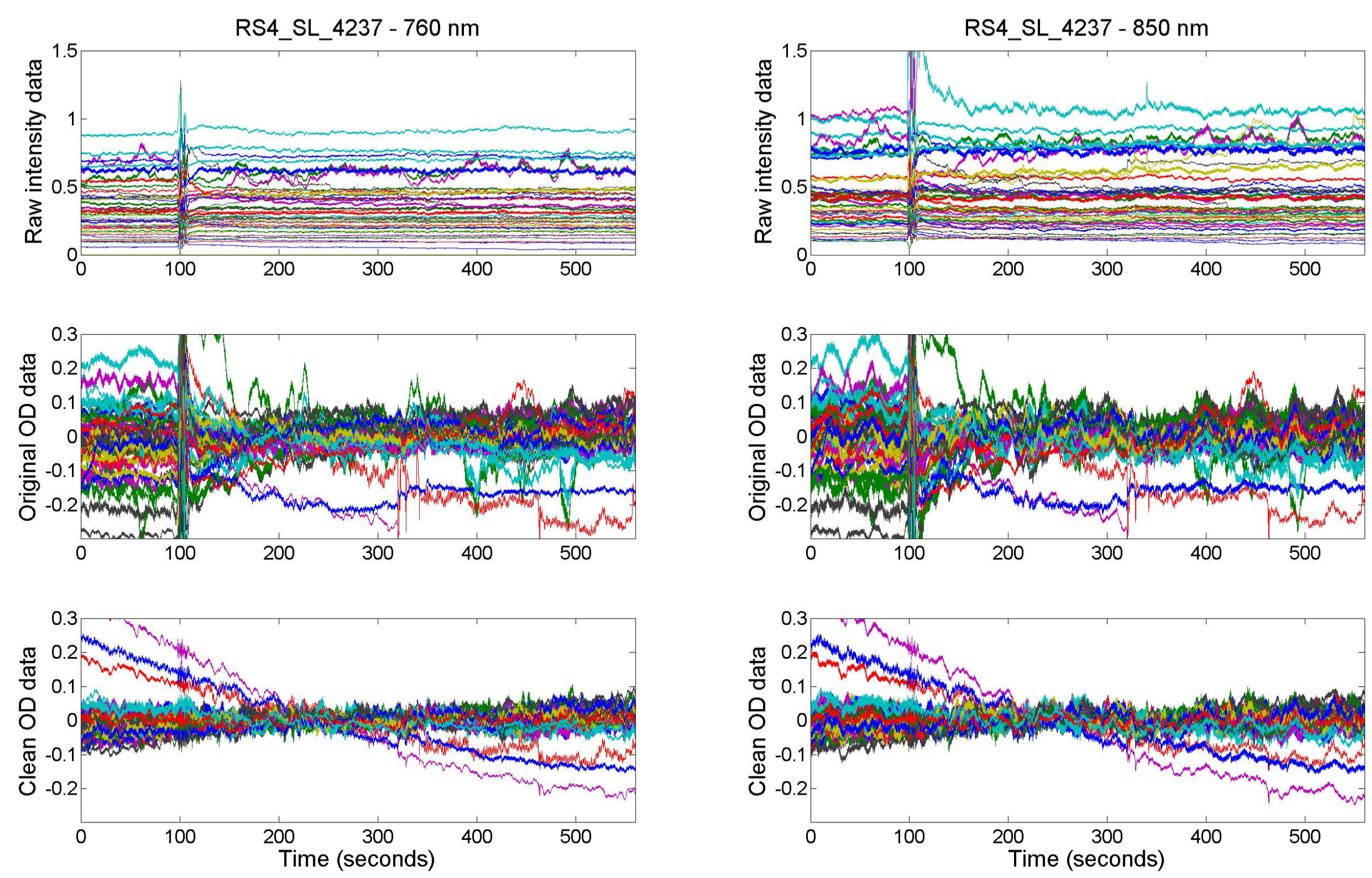
Raw

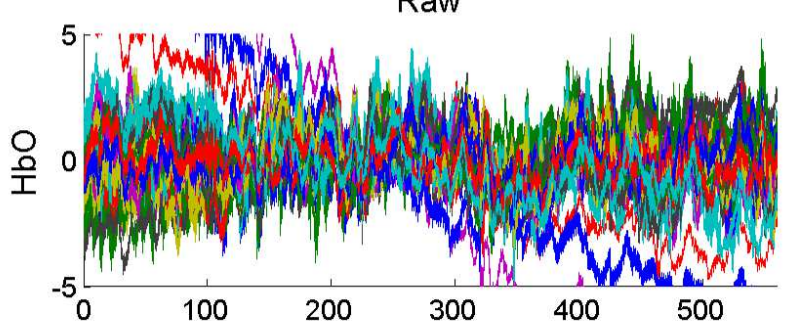

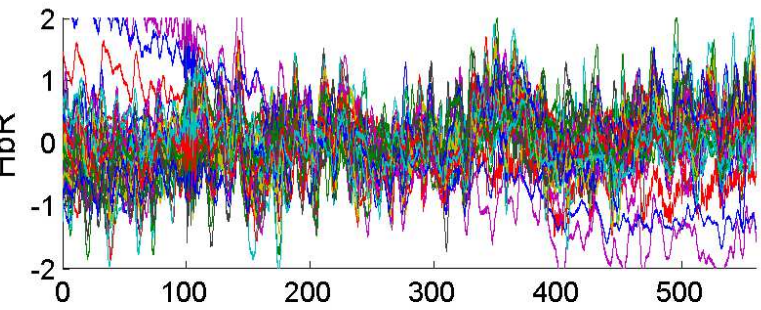
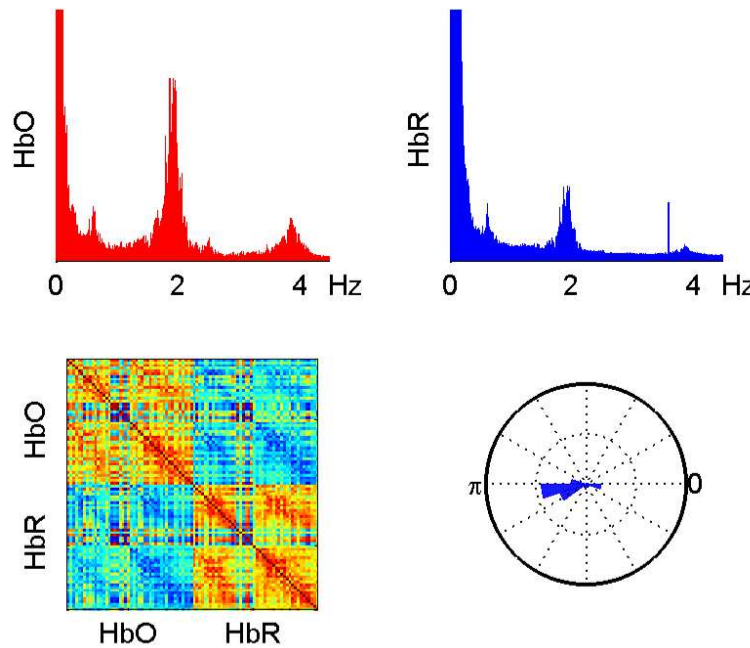

RS4_SL_4237

Filtered
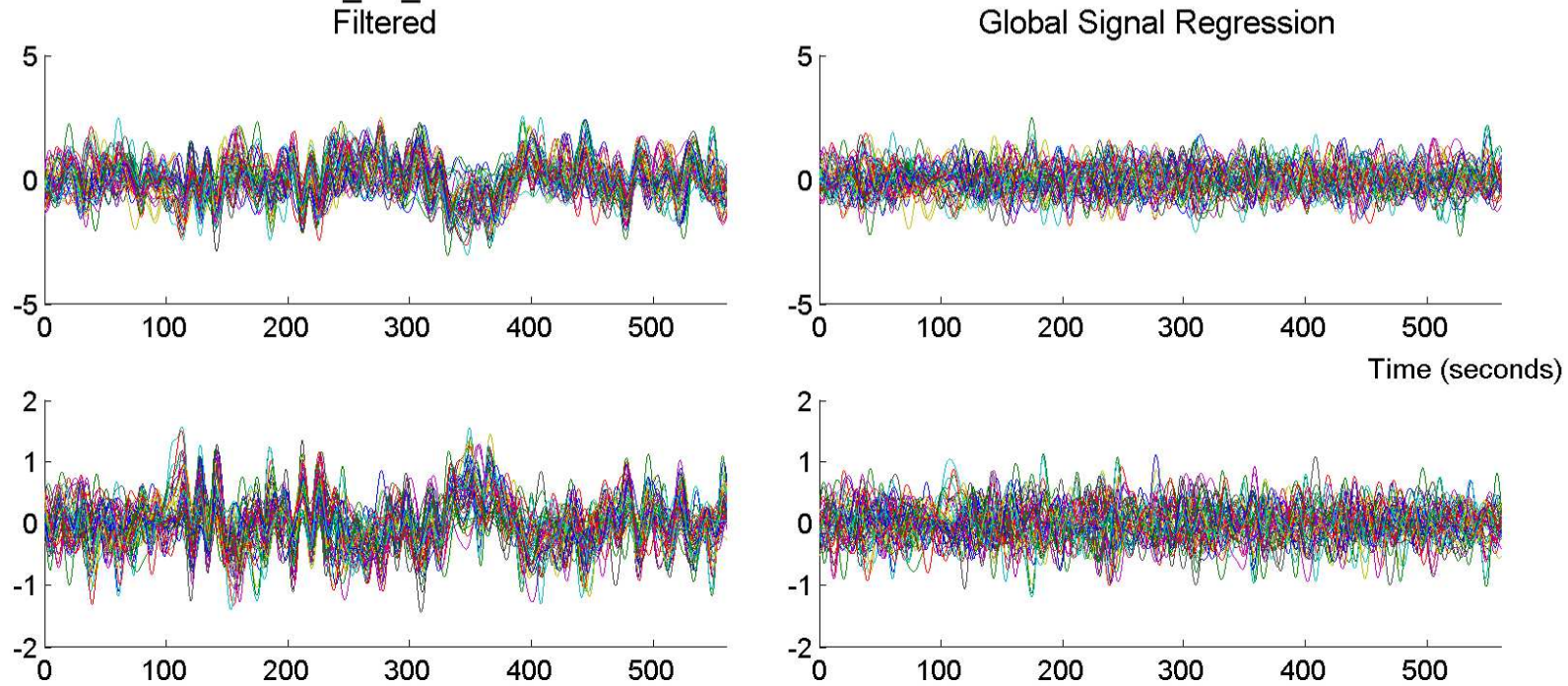

2
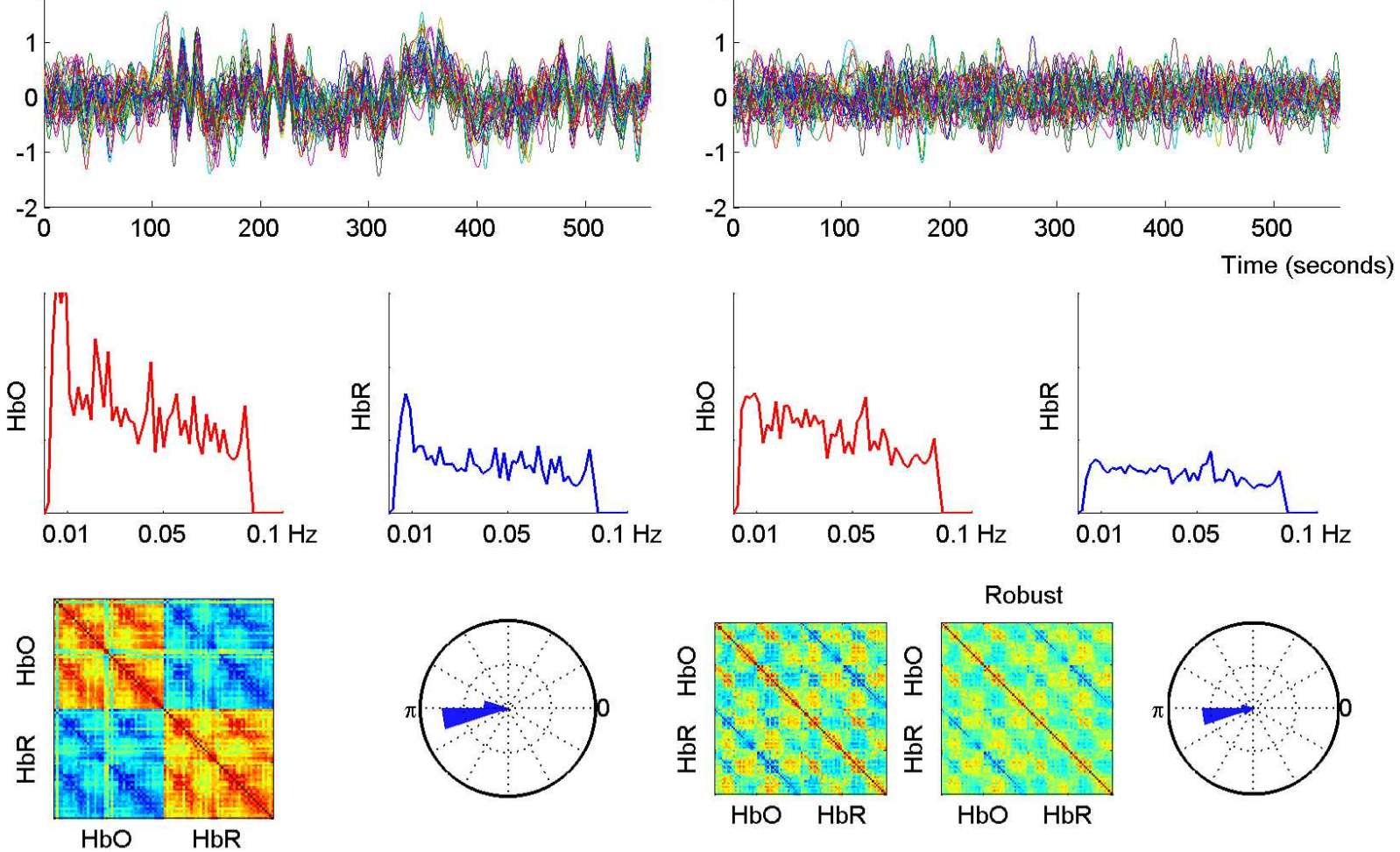
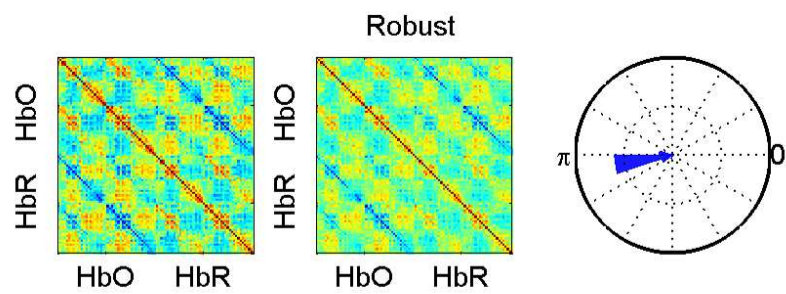

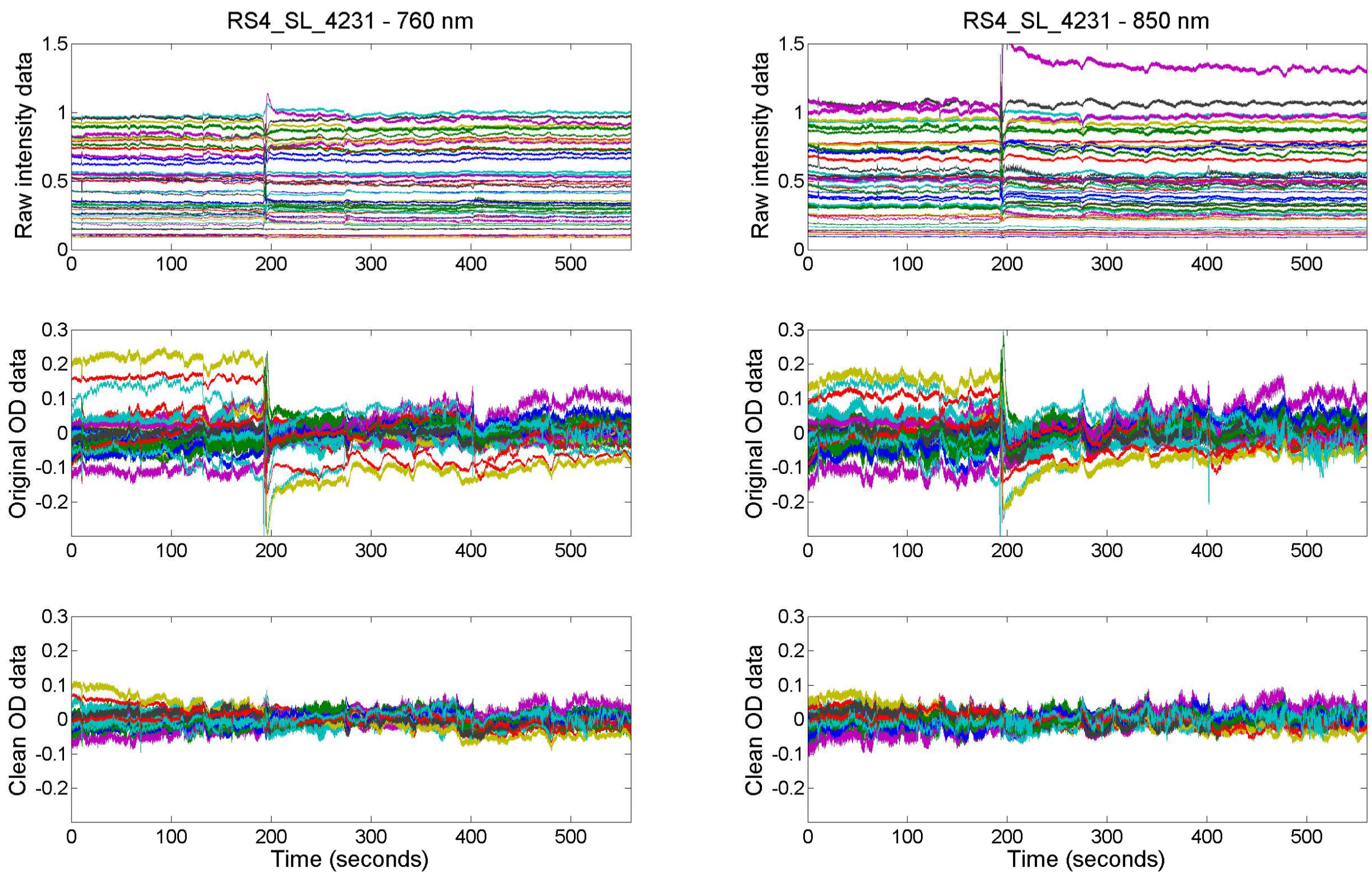
Raw
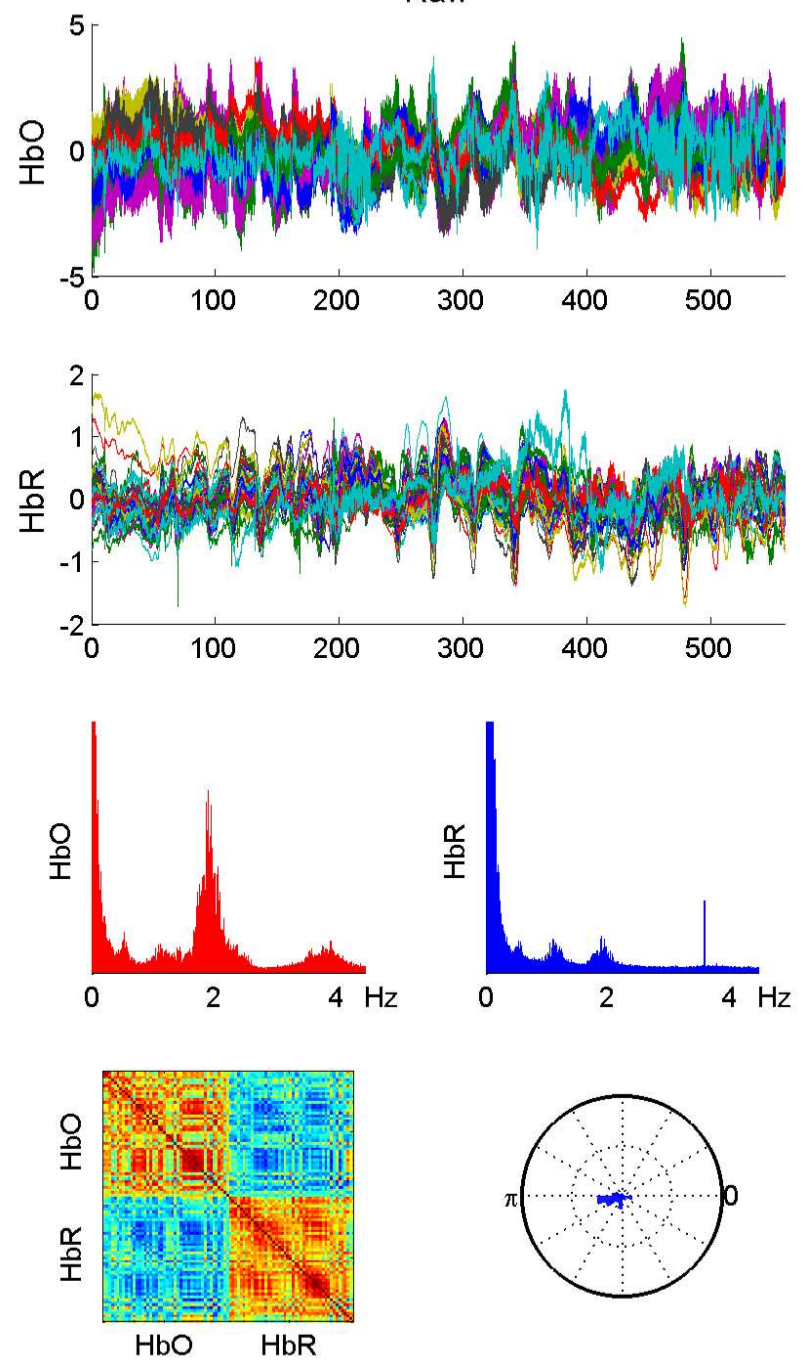

RS4_SL_4231

Filtered

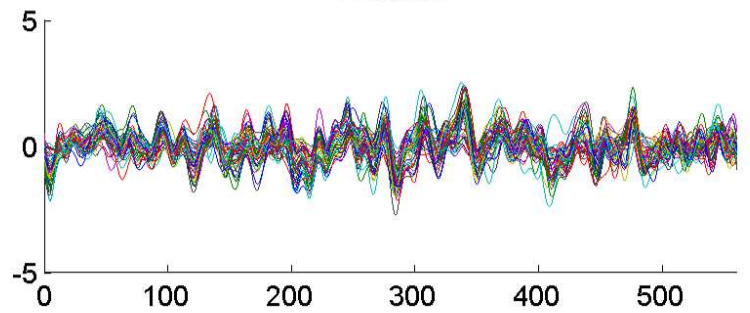

2
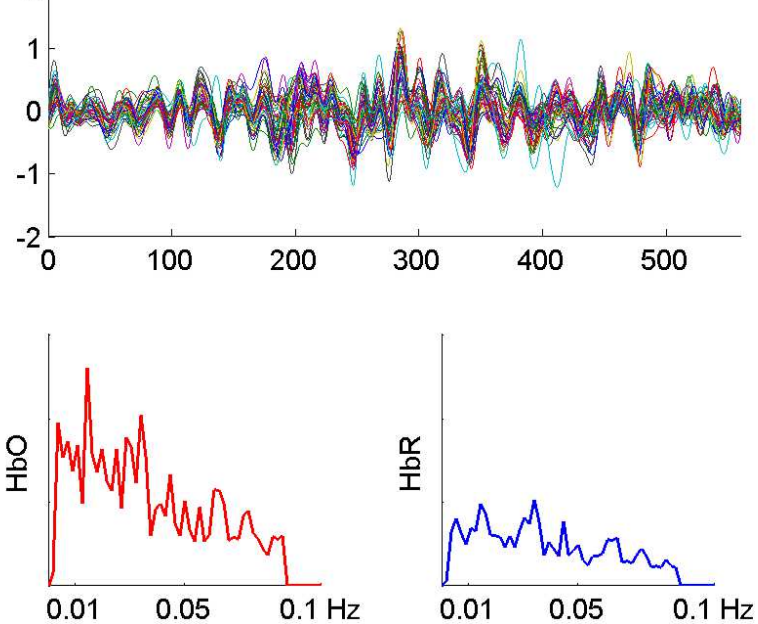

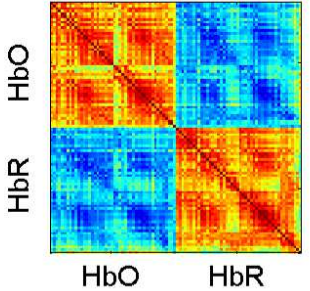

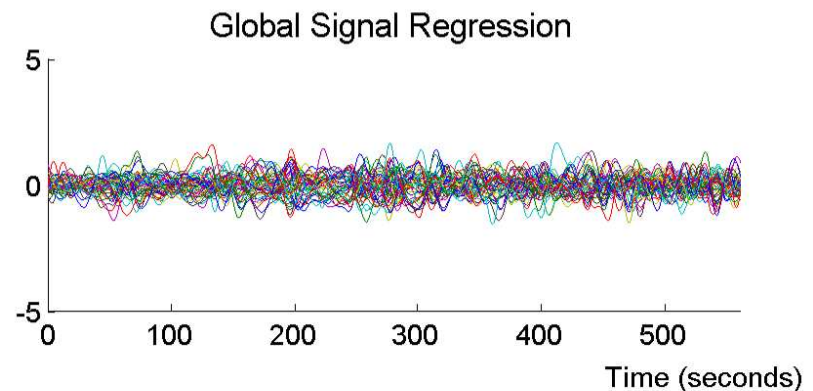

2
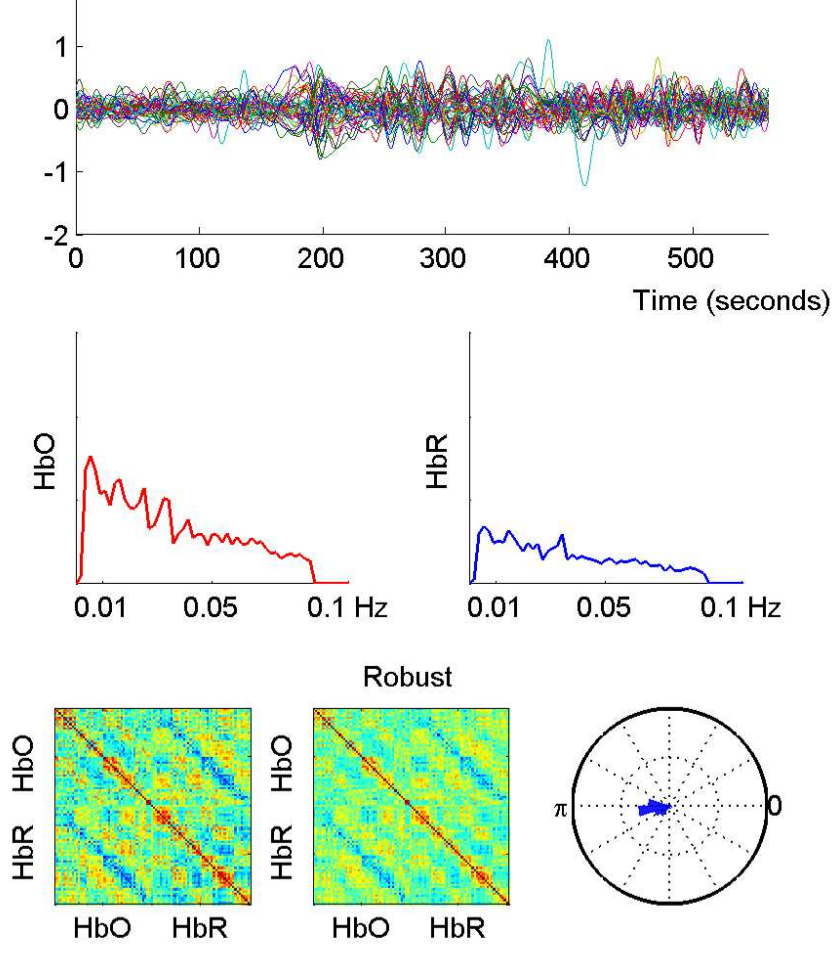

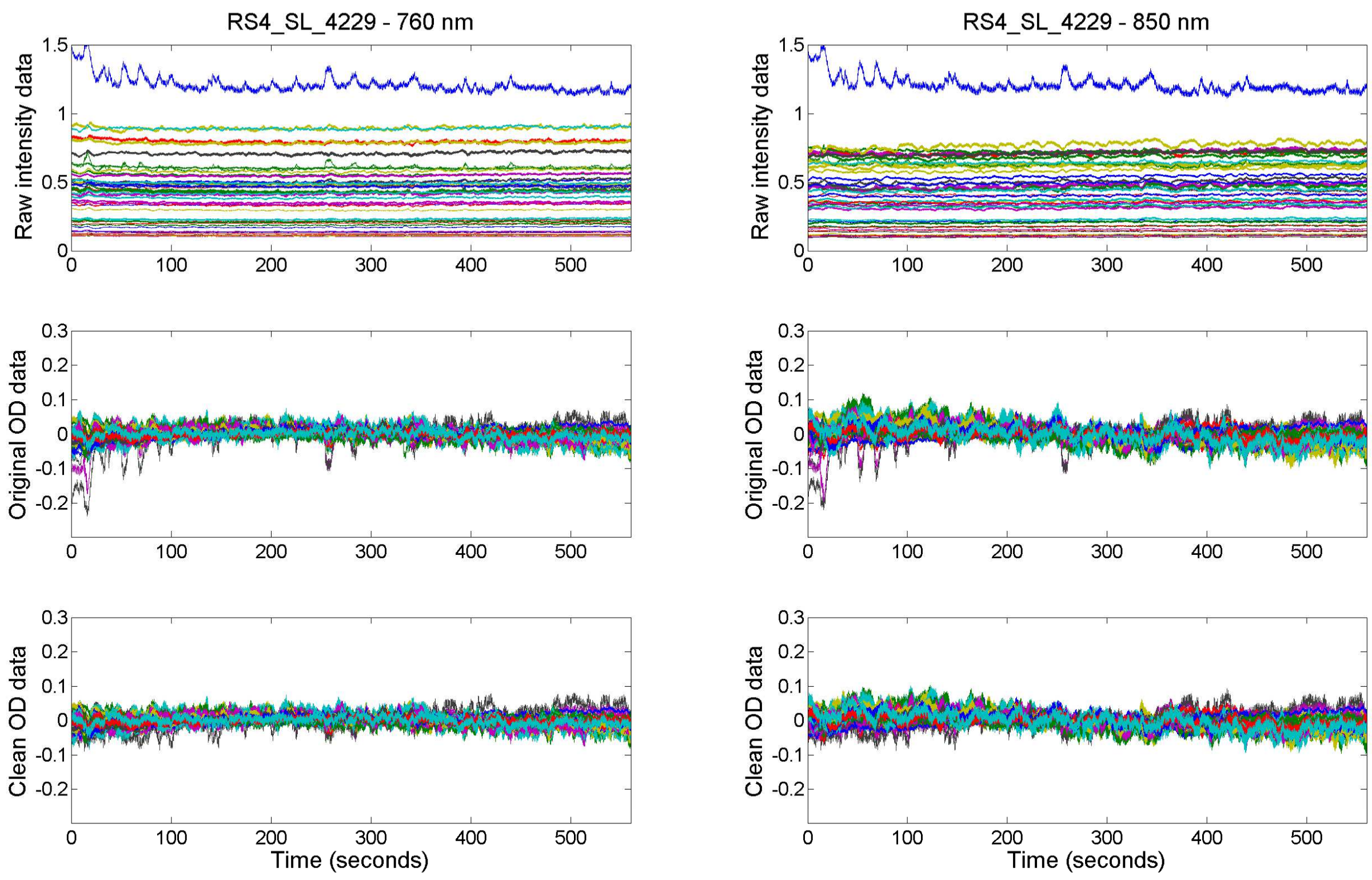
RS4_SL_4229

Raw
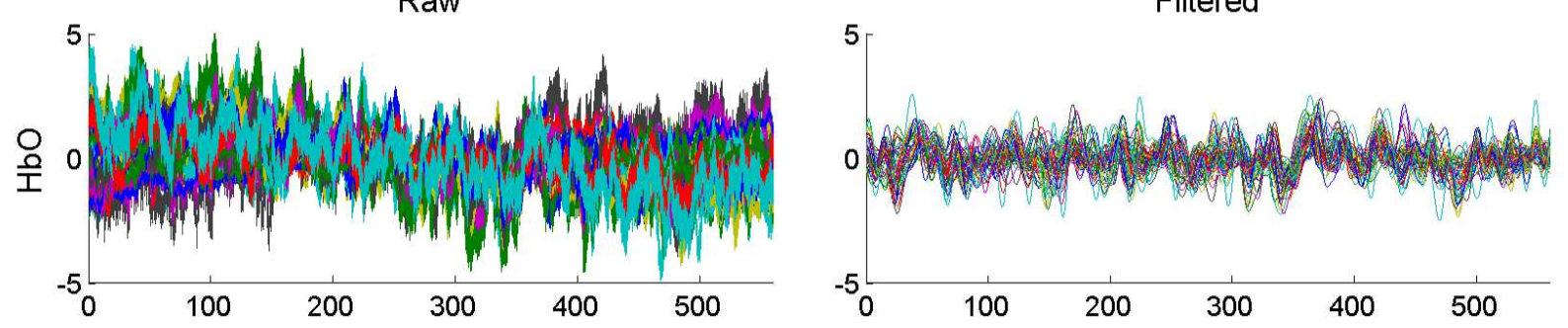

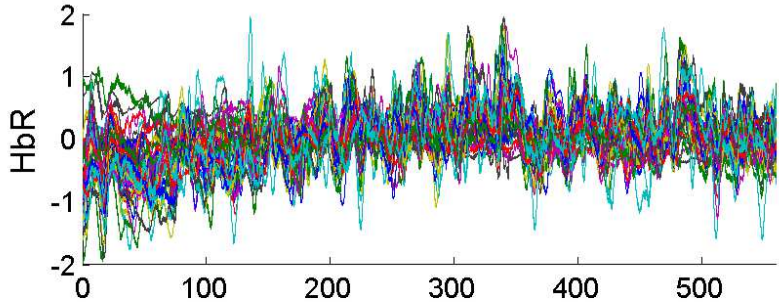
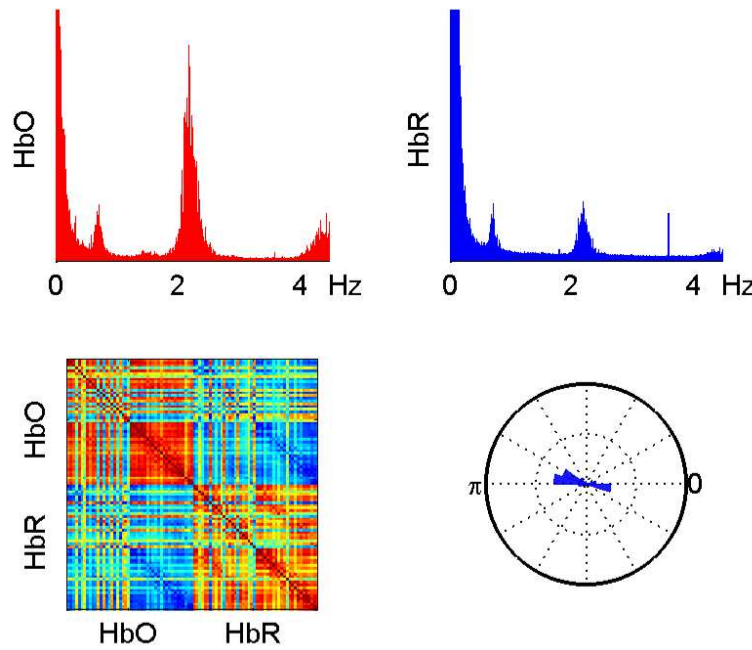

2

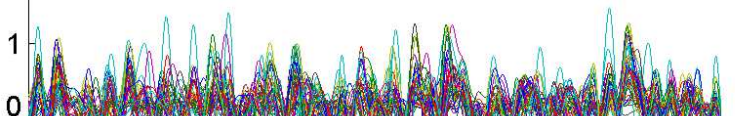

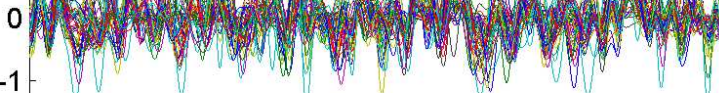
-1 -
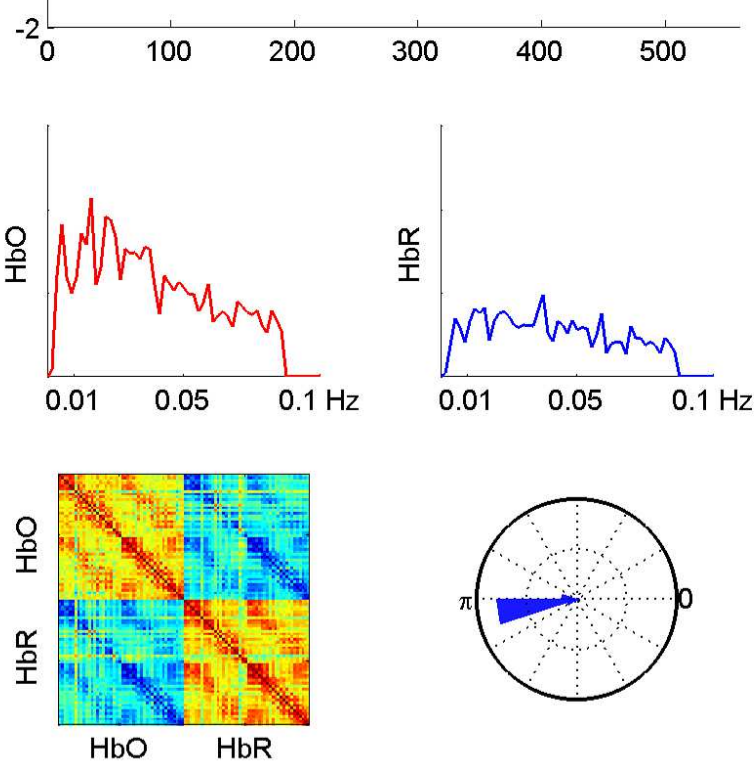

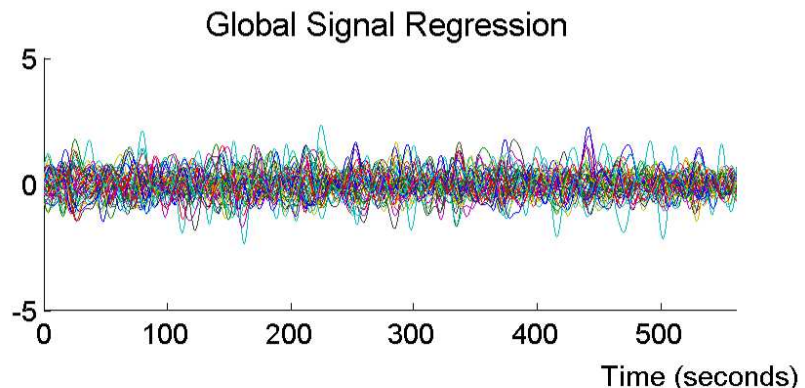

2
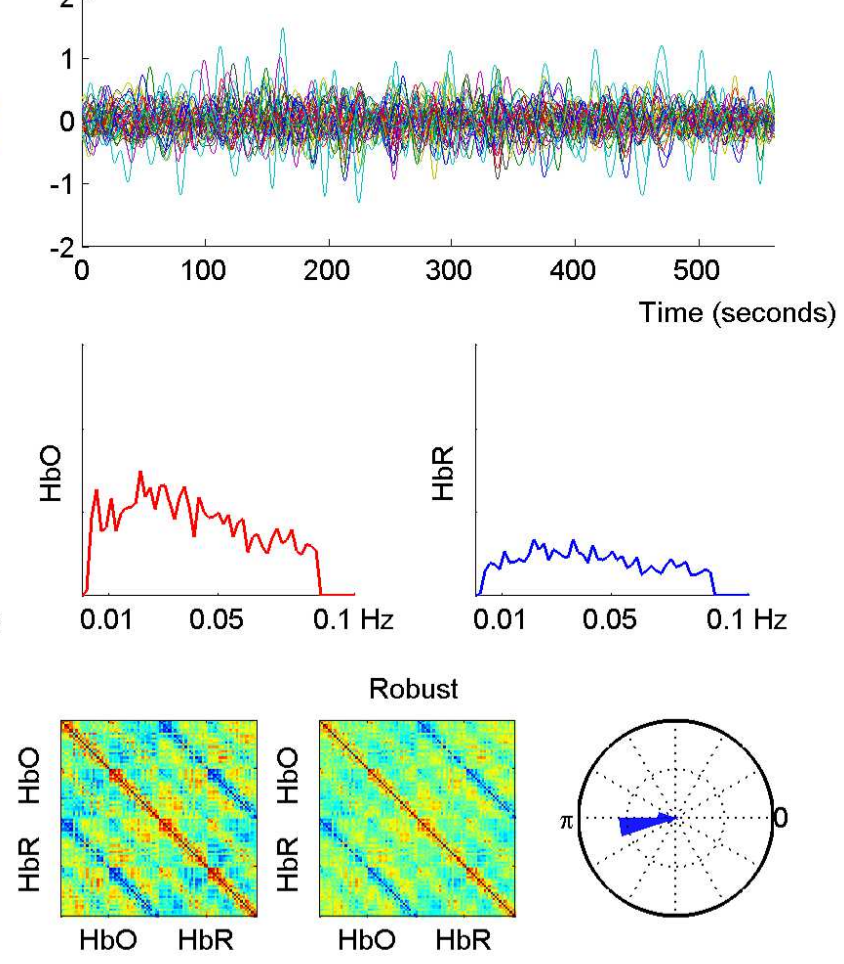

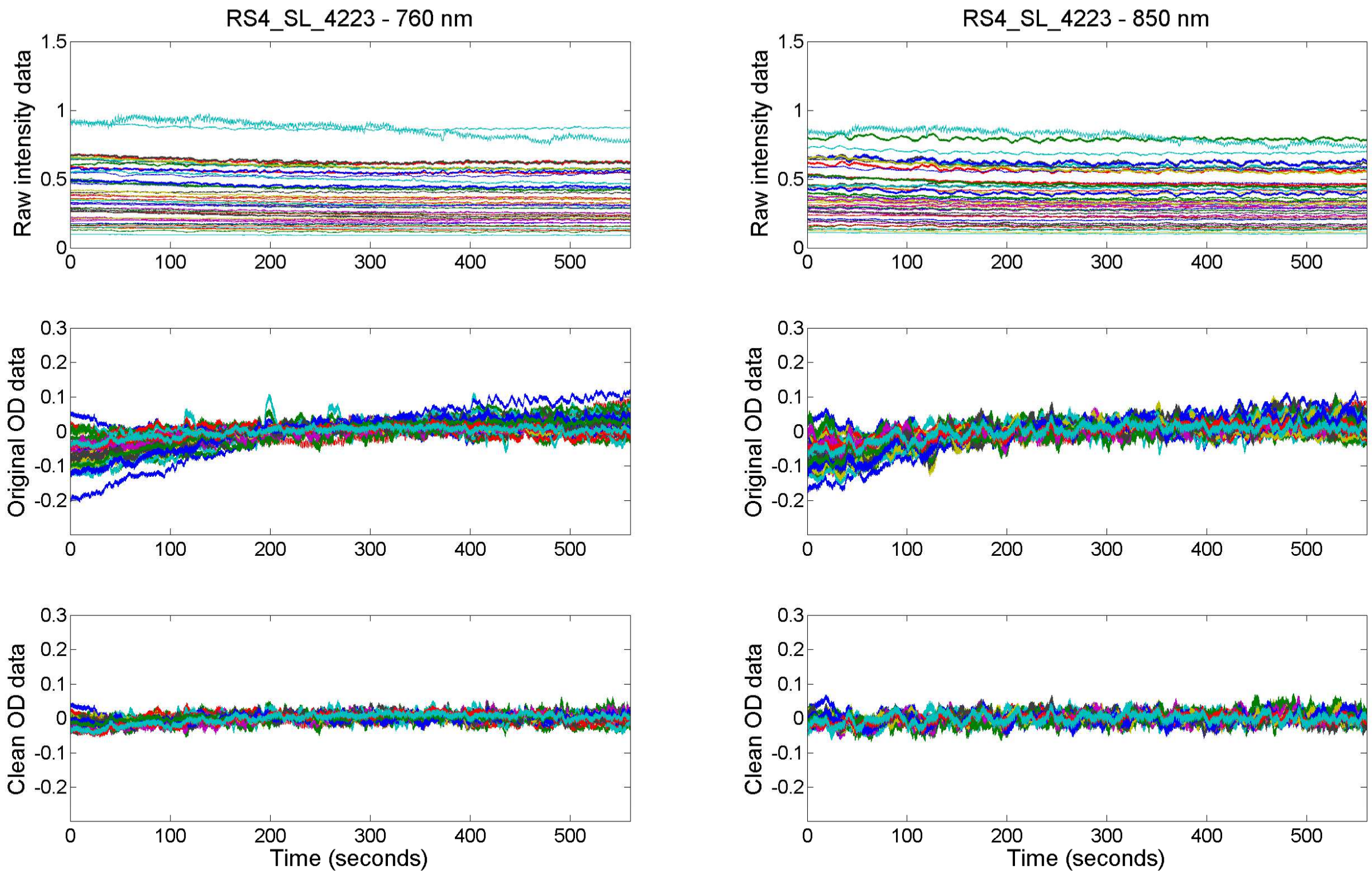
RS4_SL_4223

Raw

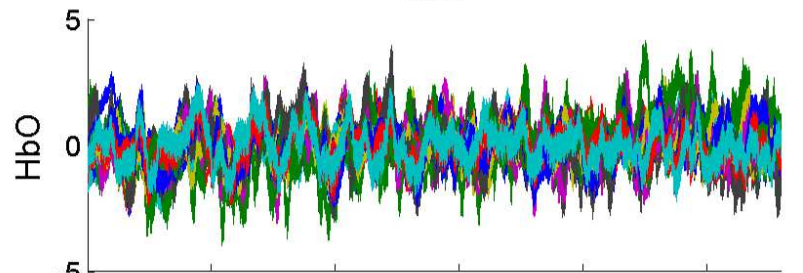

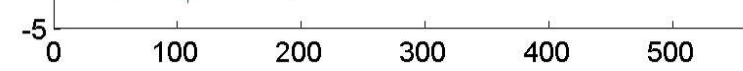

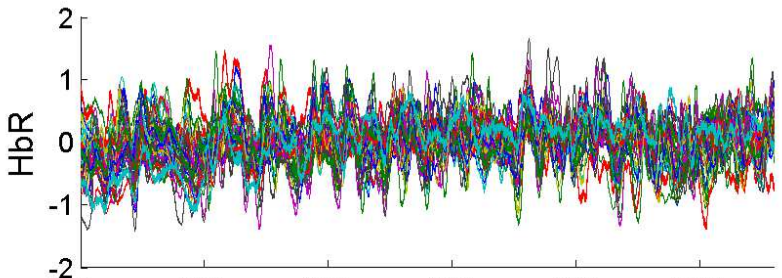
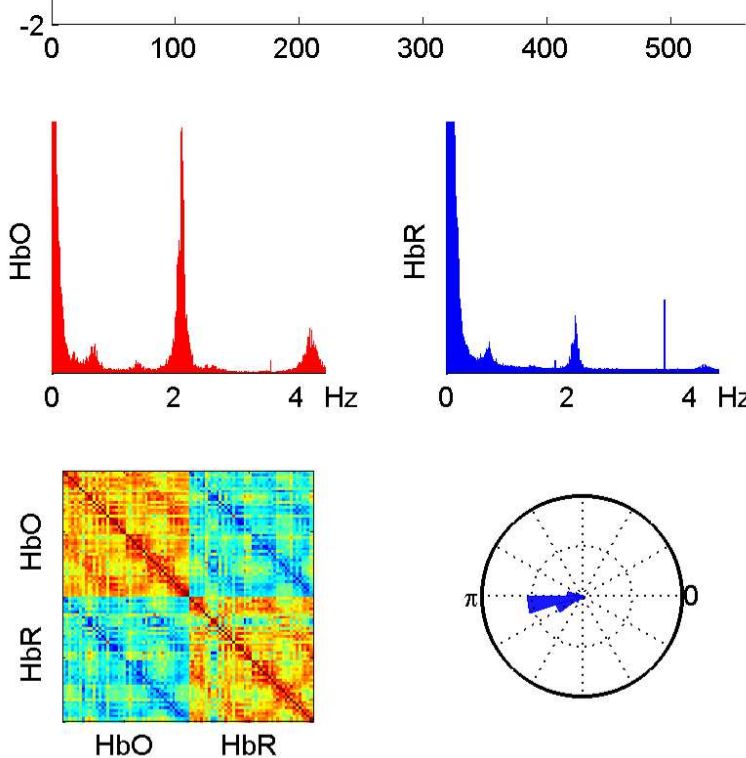

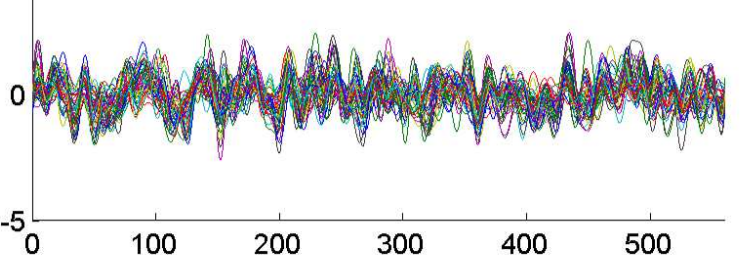

2

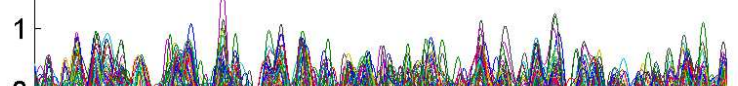

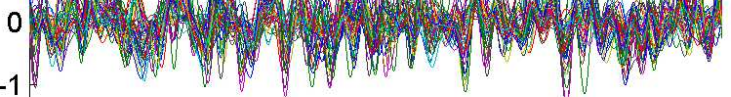
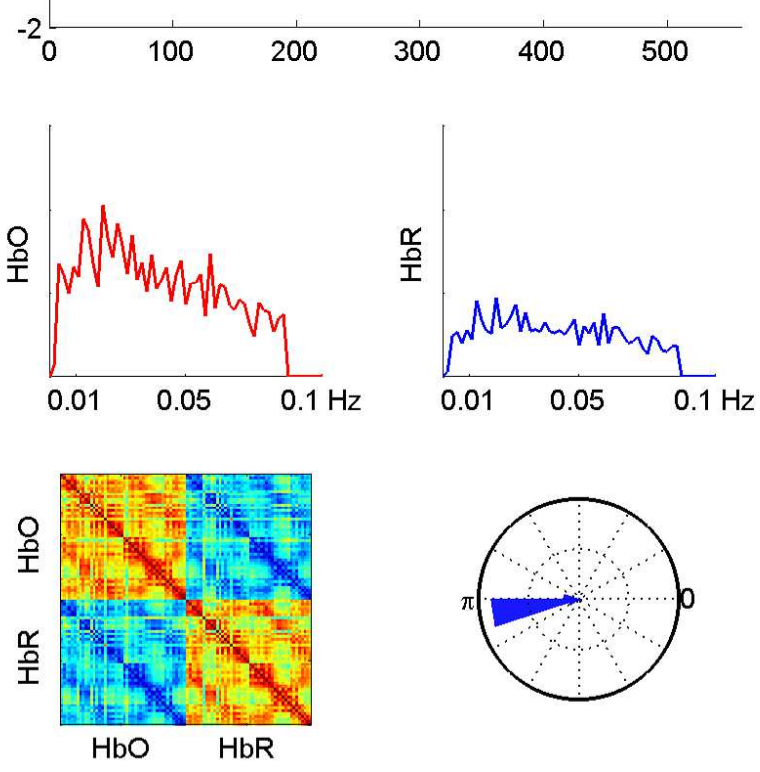

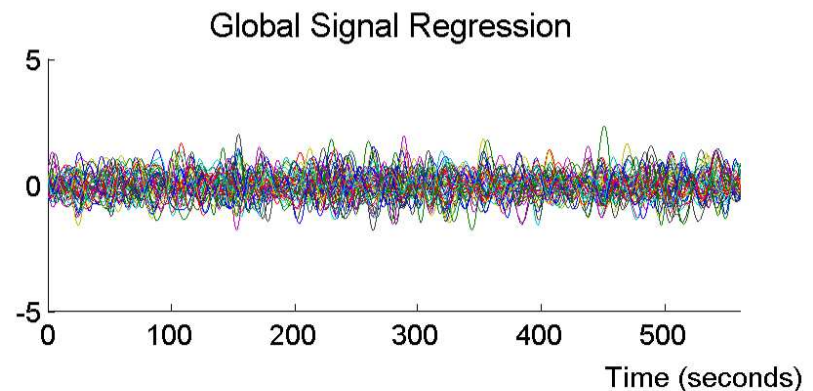

2
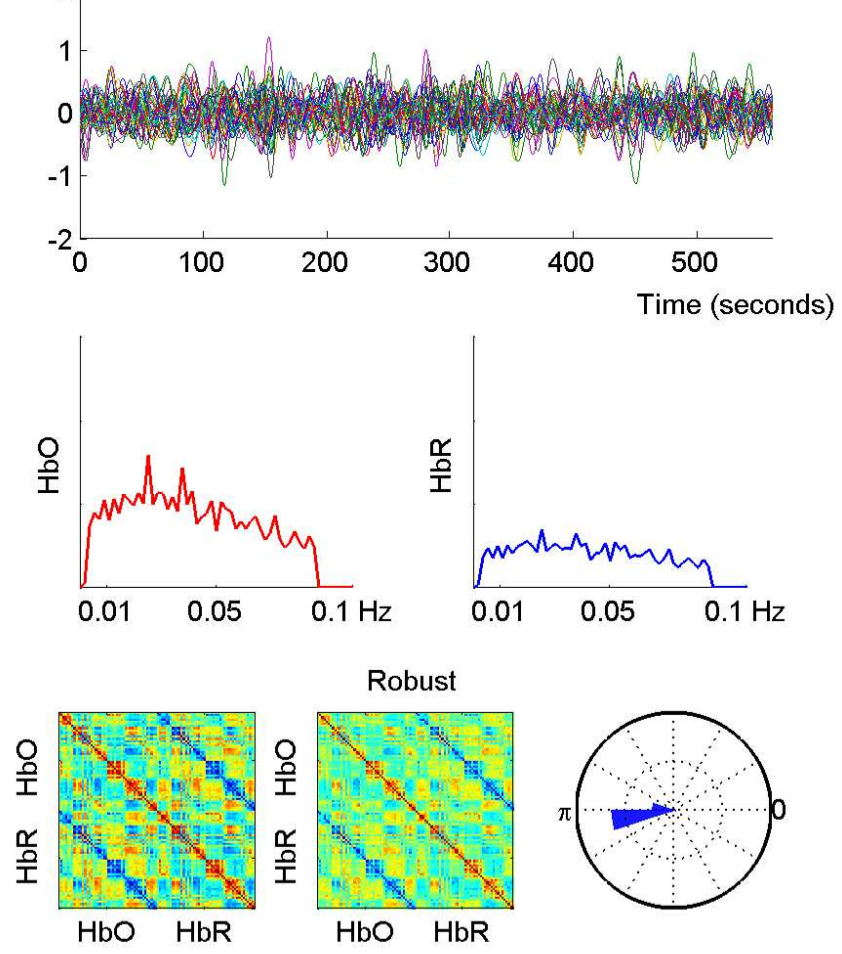


RS4_SL_4219
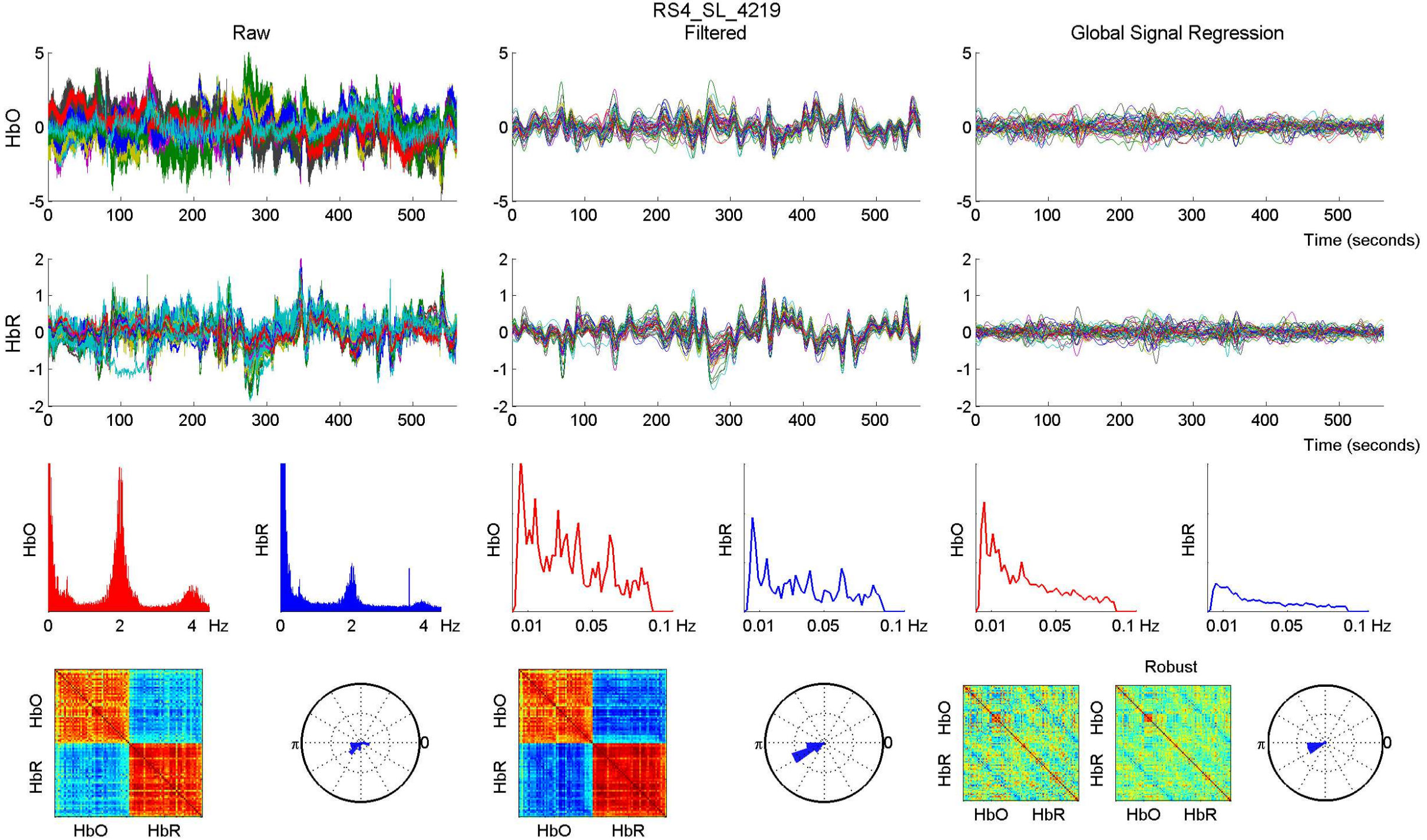

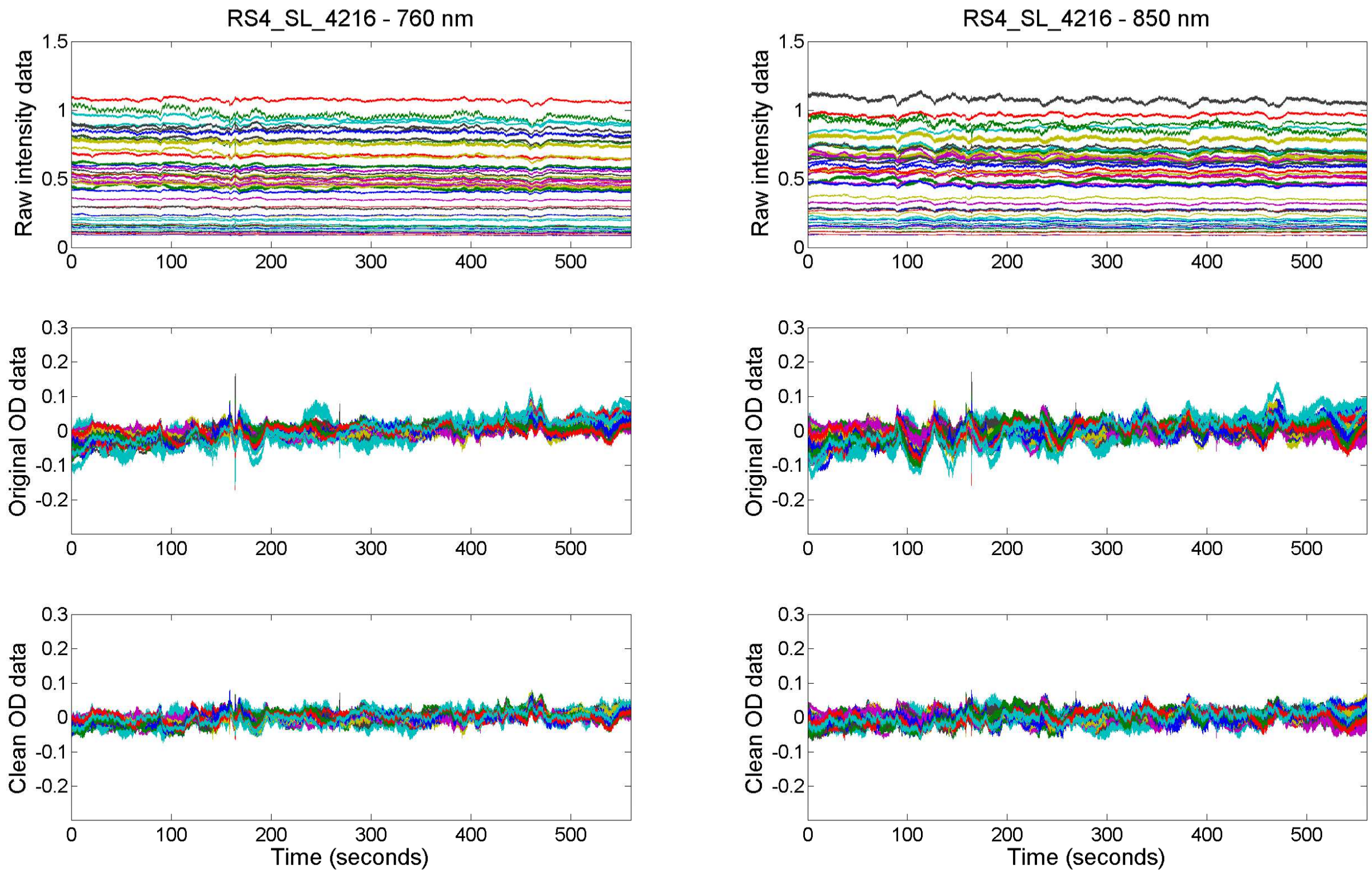
Raw

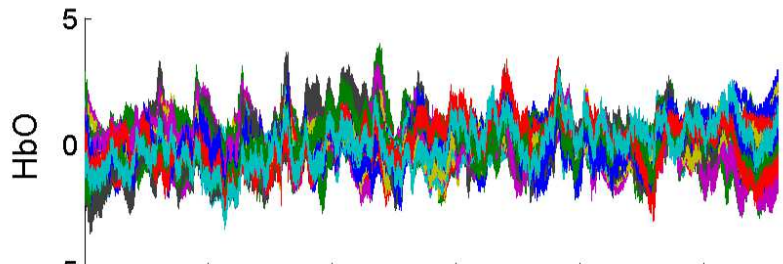
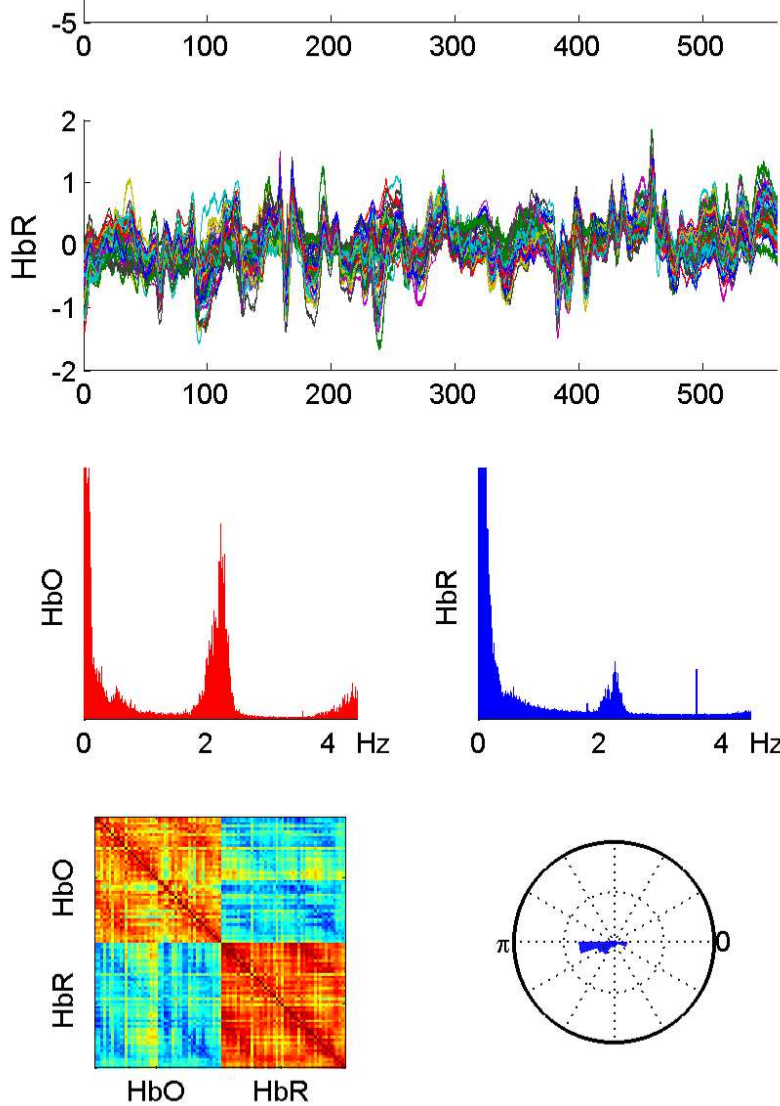

RS4_SL_4216

Filtered
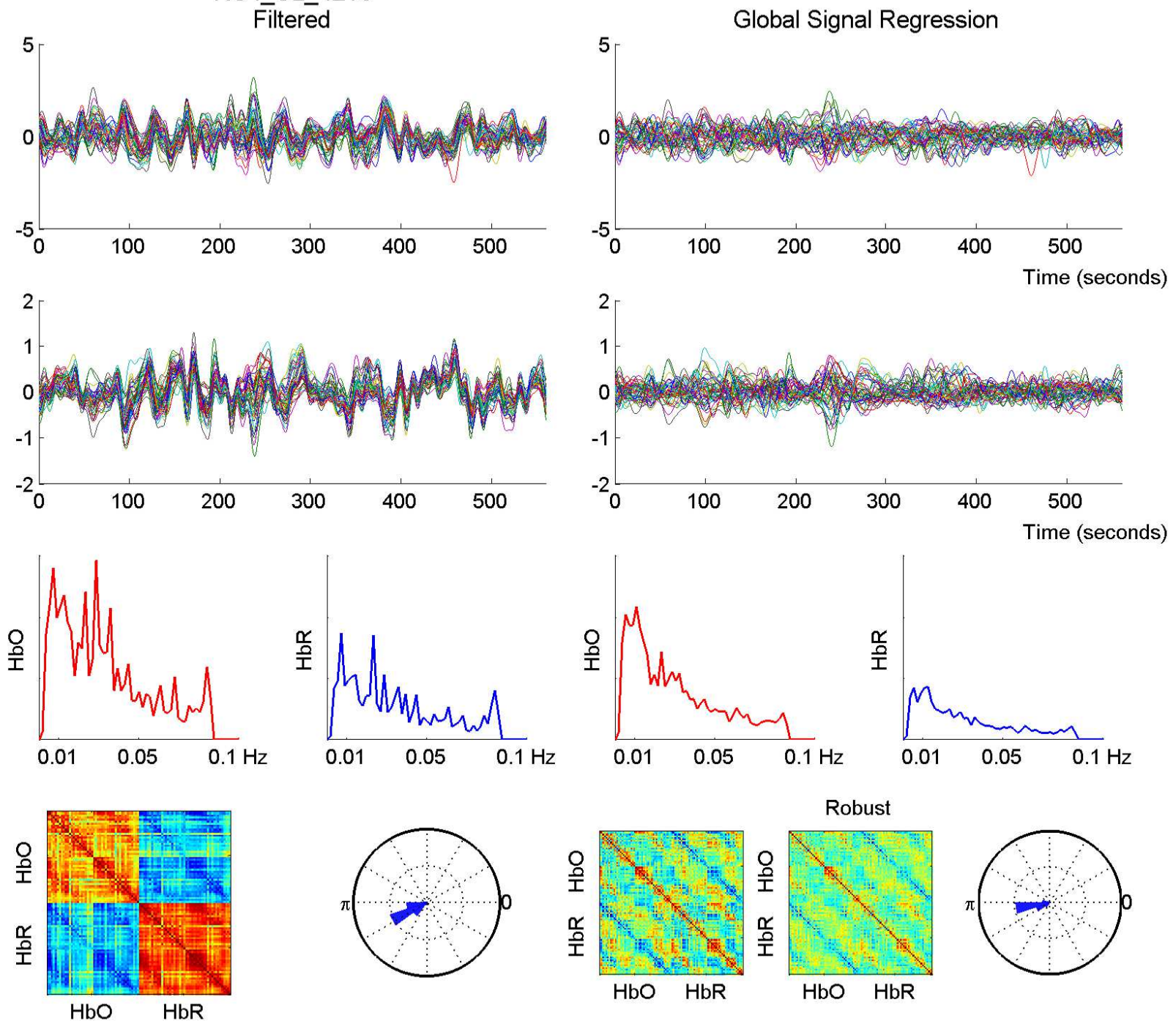

Robust
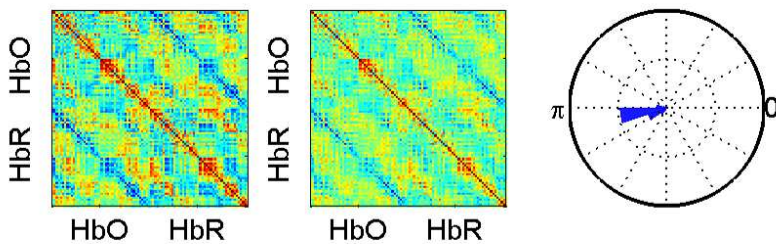

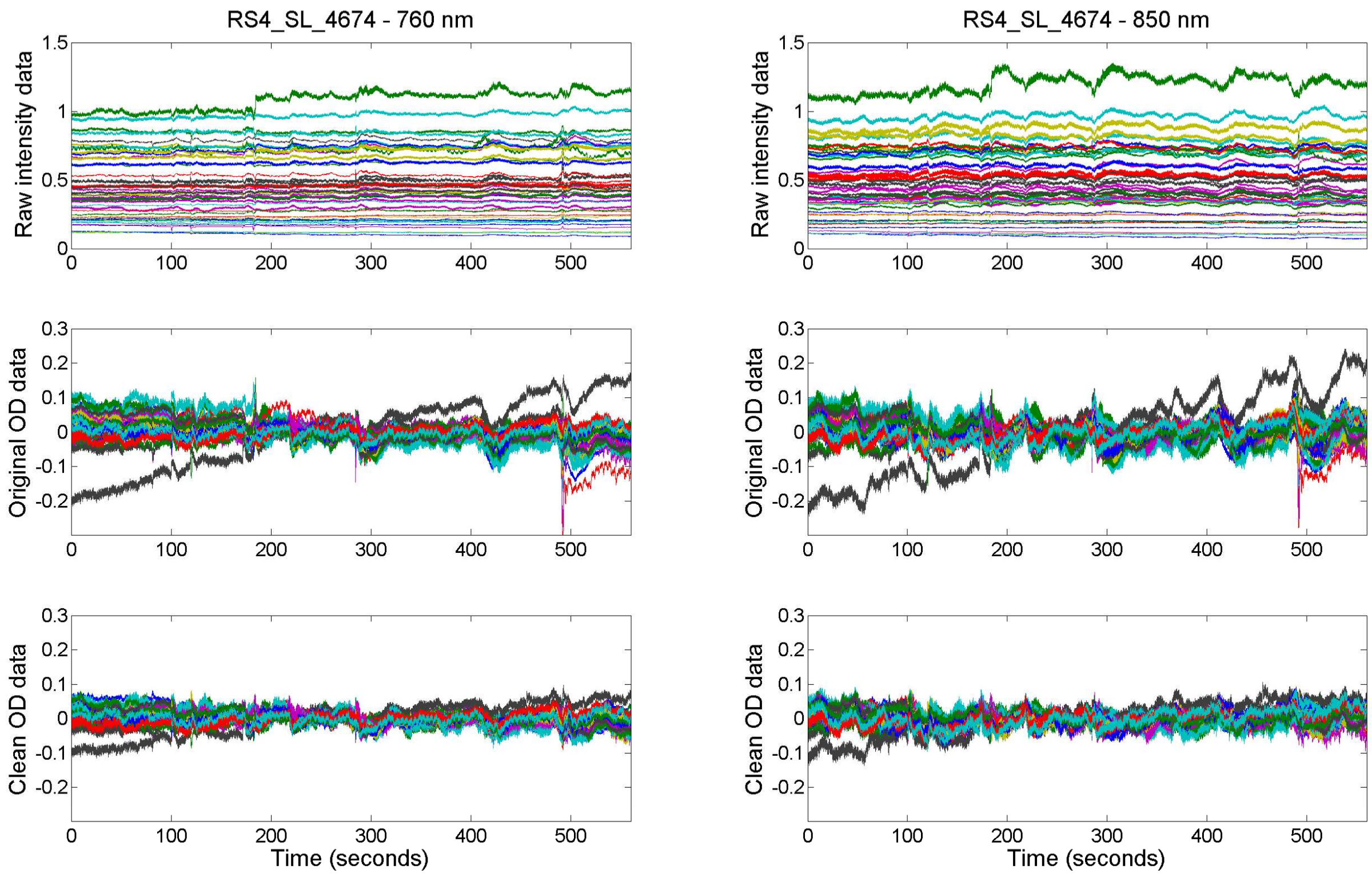
RS4_SL_4674
Raw
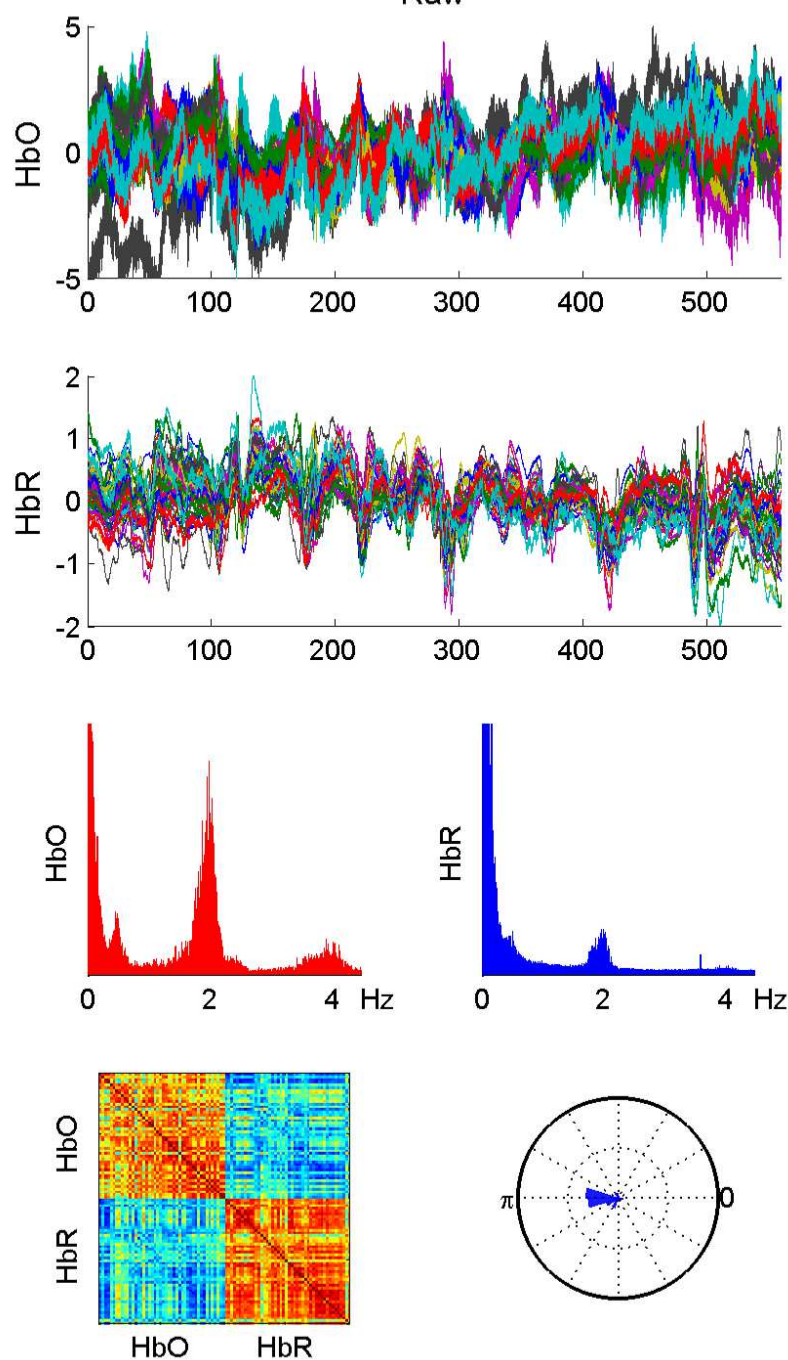
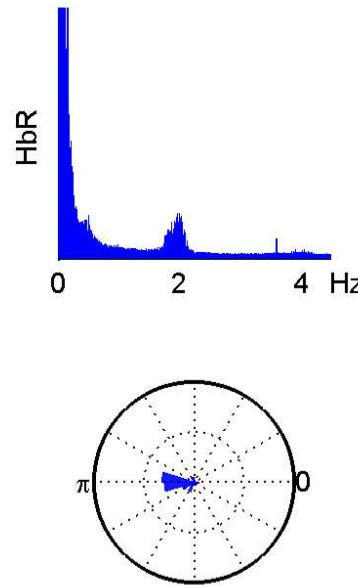

Filtered
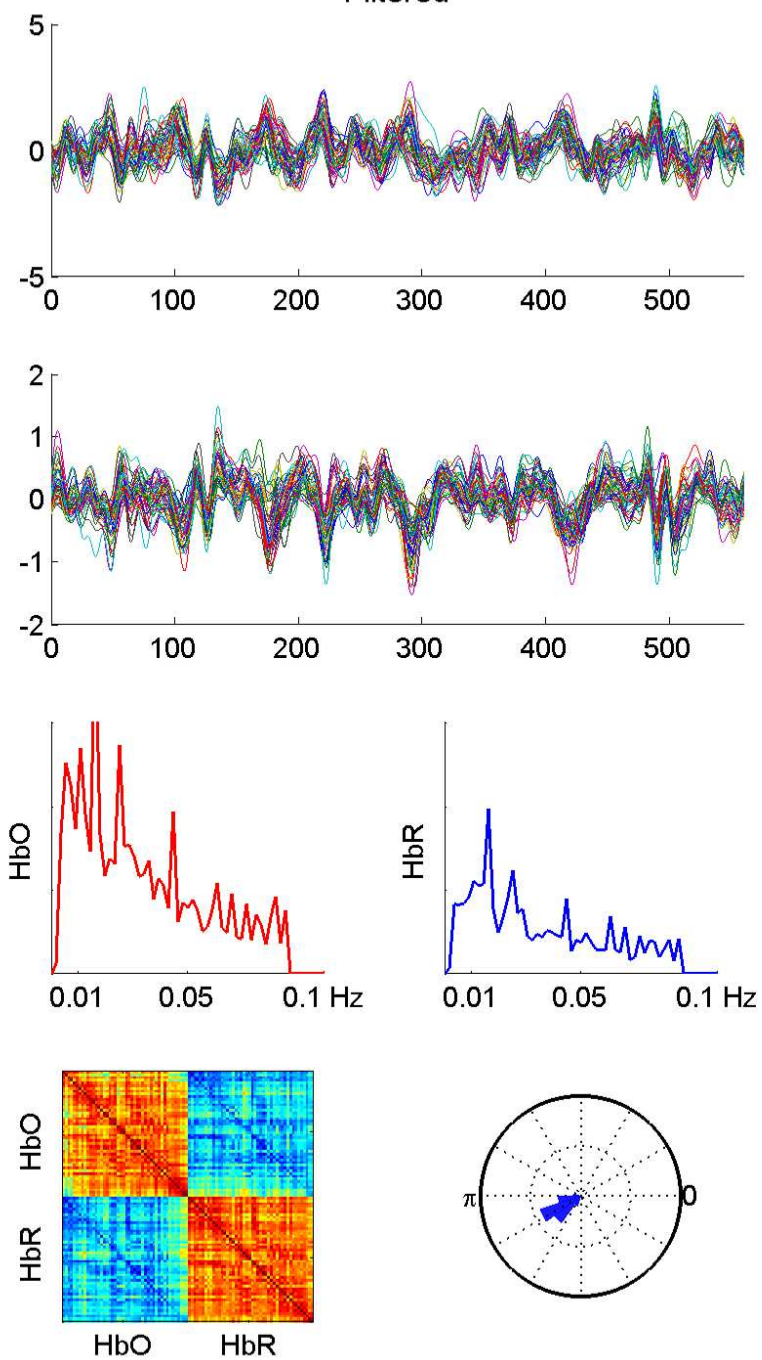

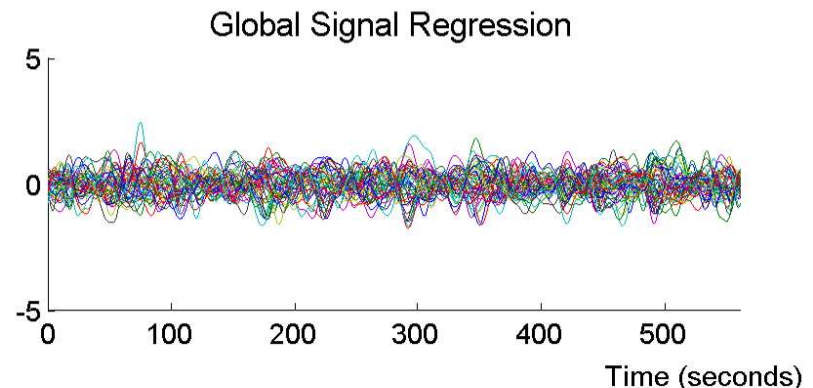

2
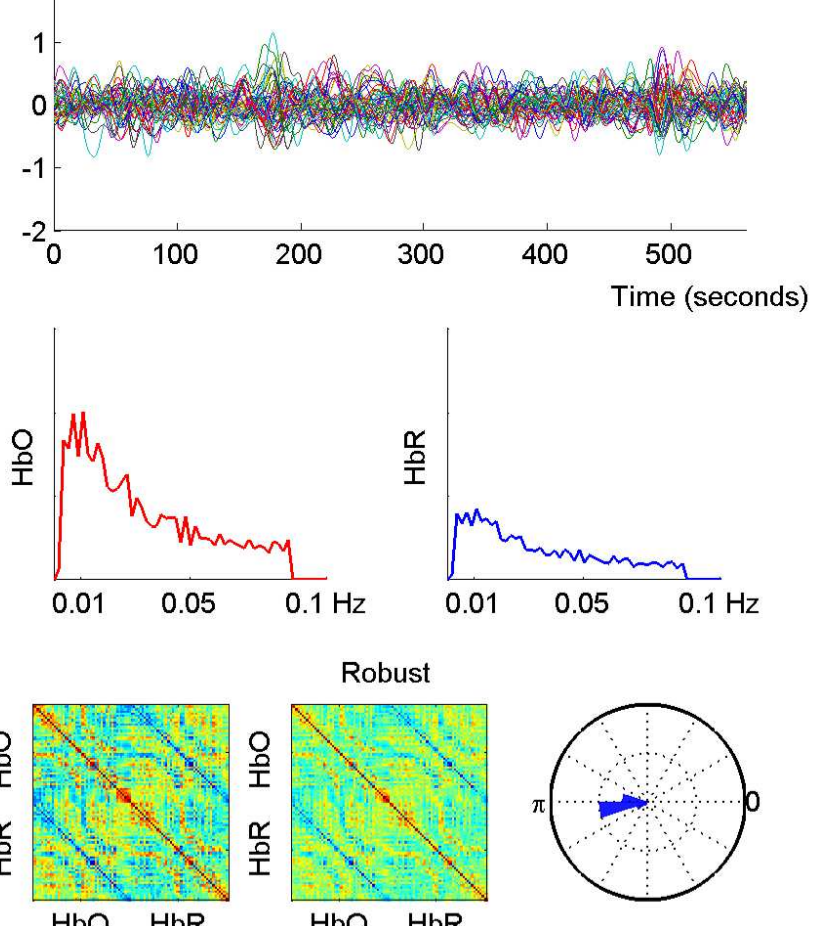

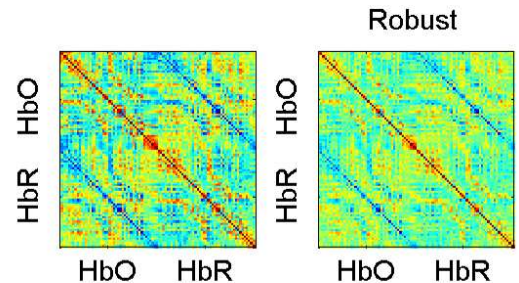


RS4 SL 4653 - 760 nm
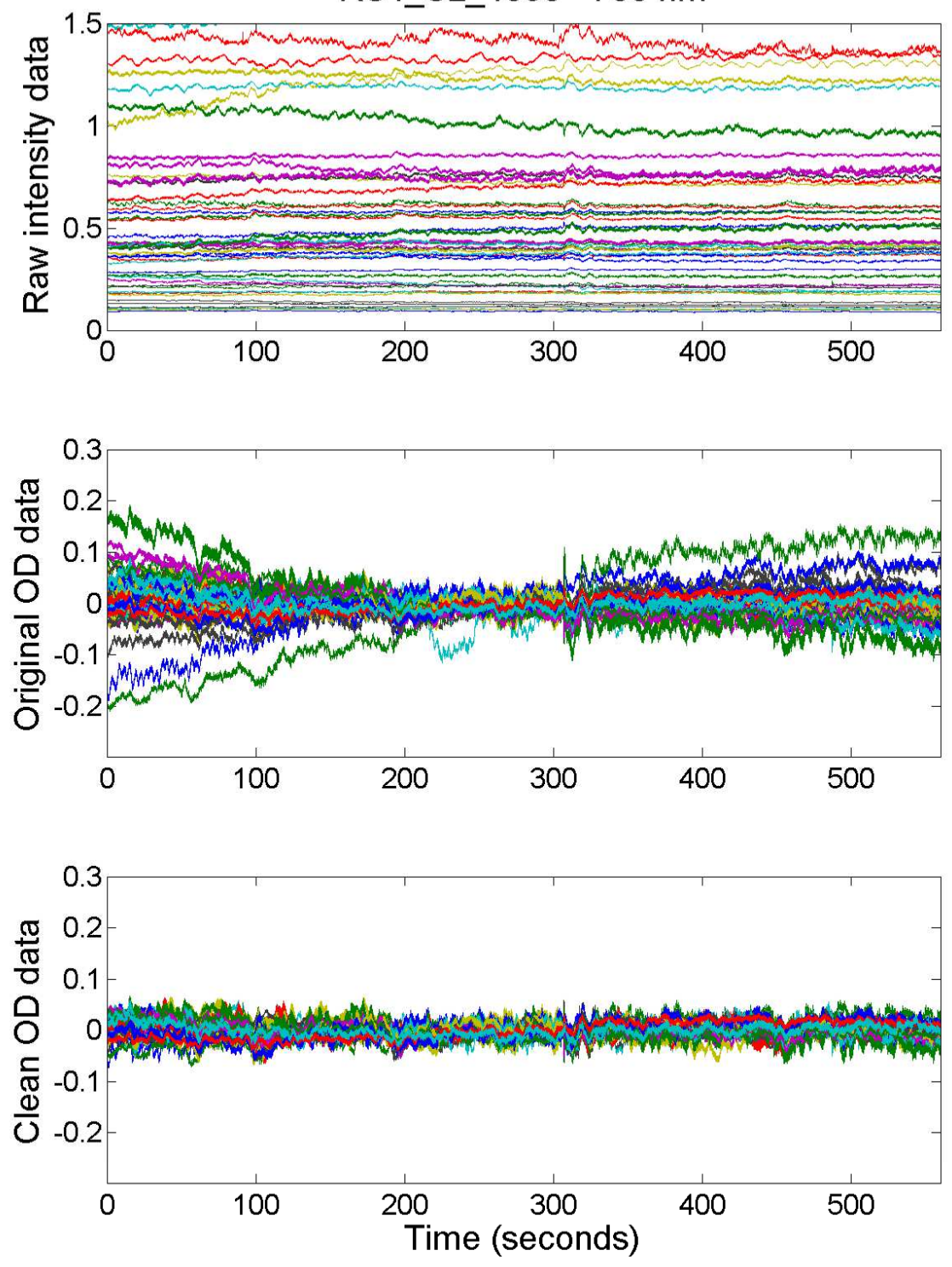

RS4 SL $4653-850 \mathrm{~nm}$
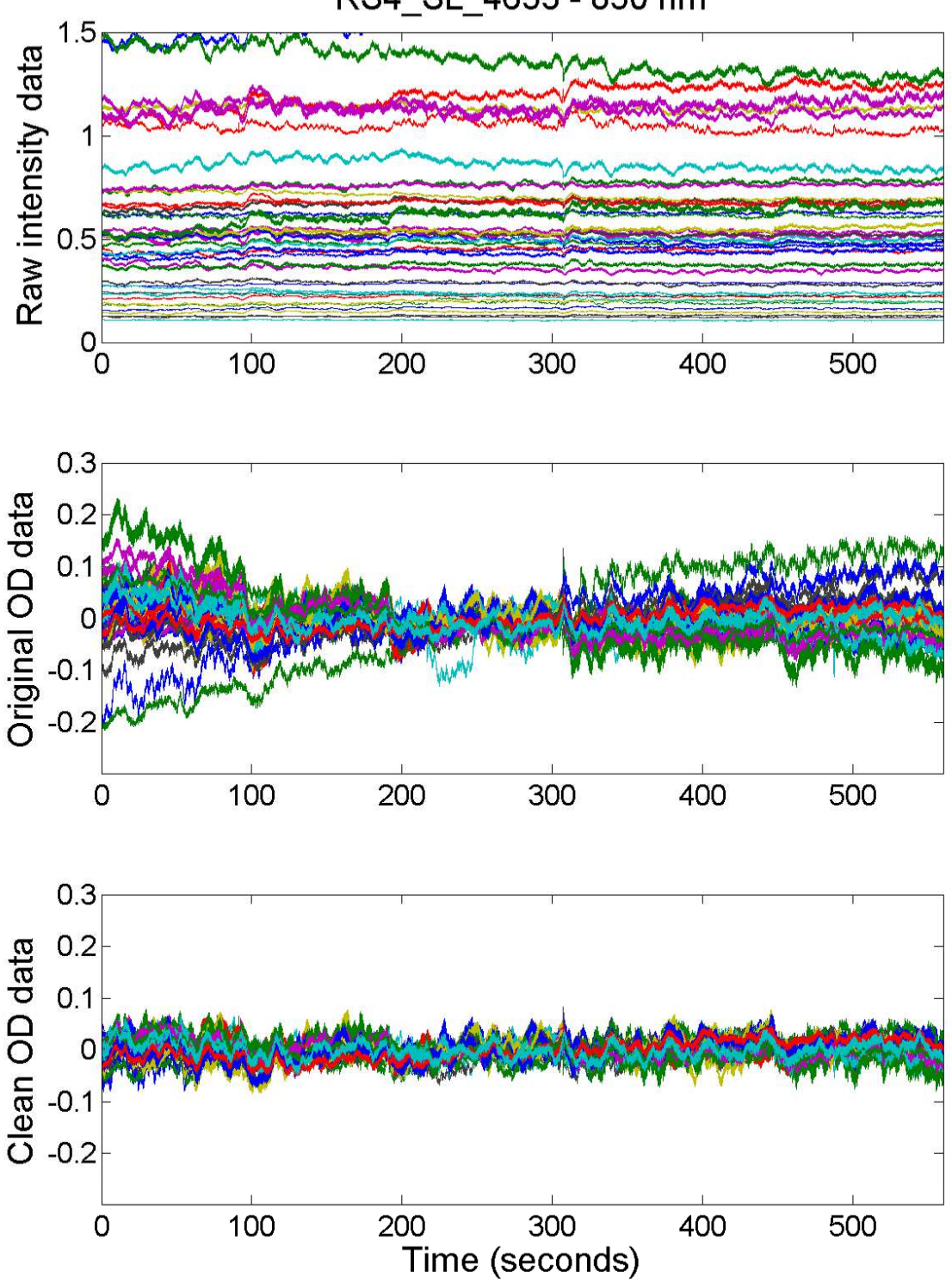
Raw

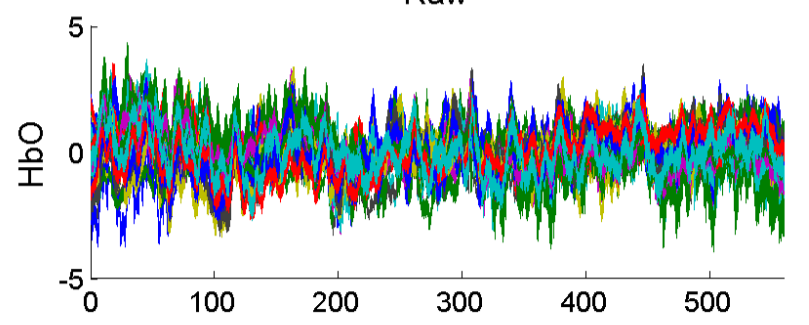

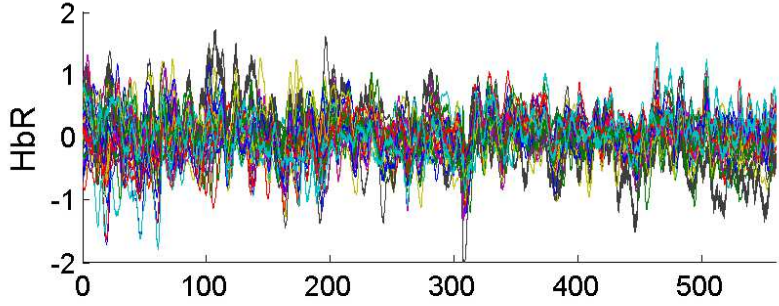
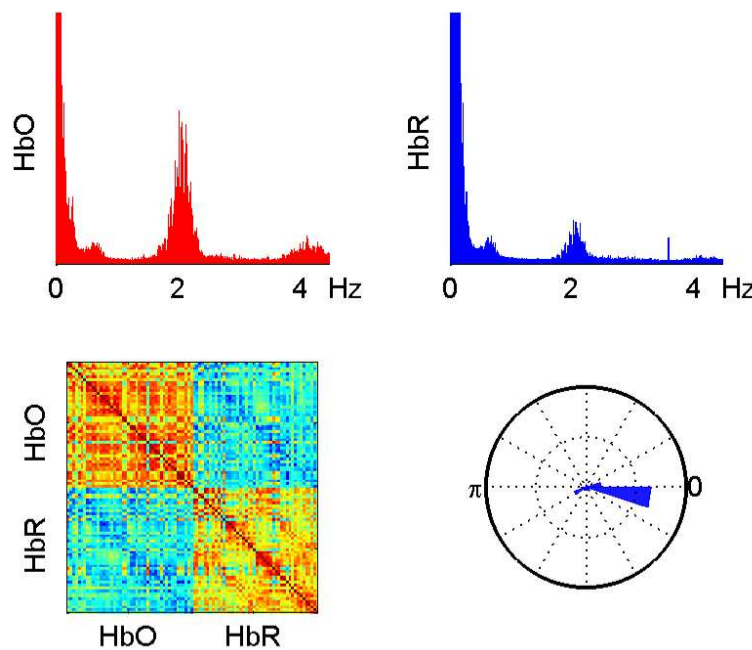

RS4 SL 4653

Filtered

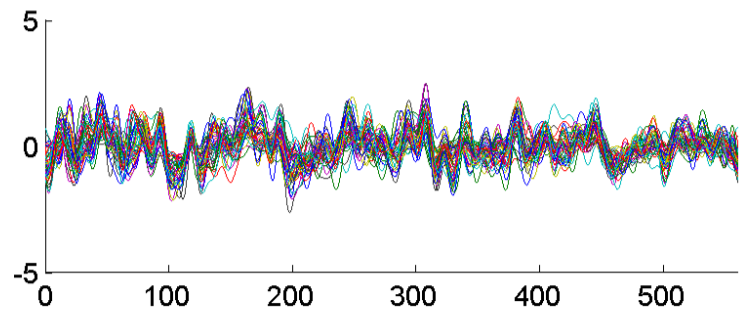

2

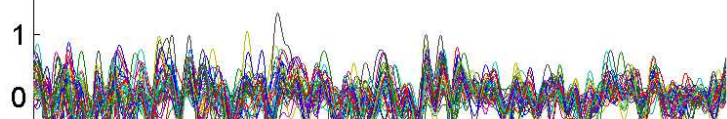

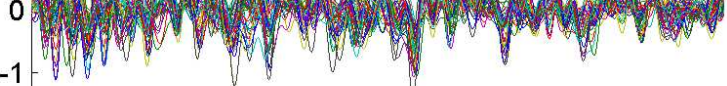
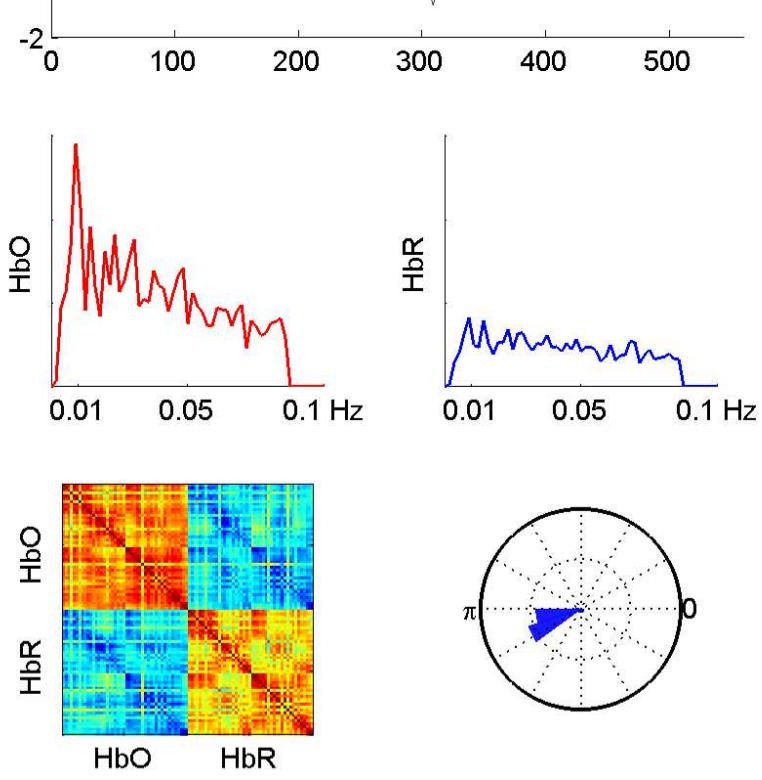

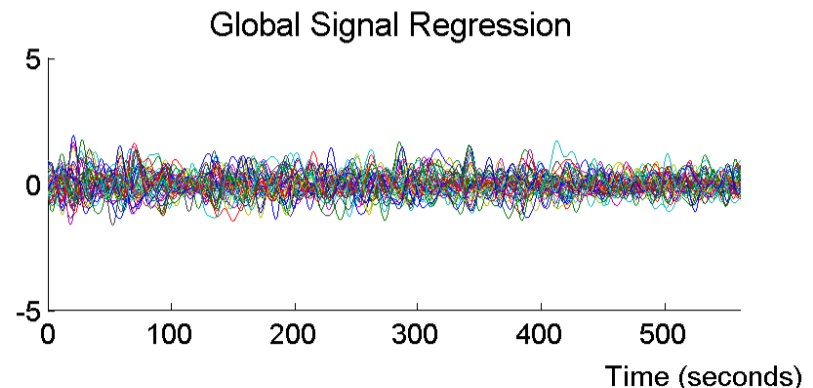

2
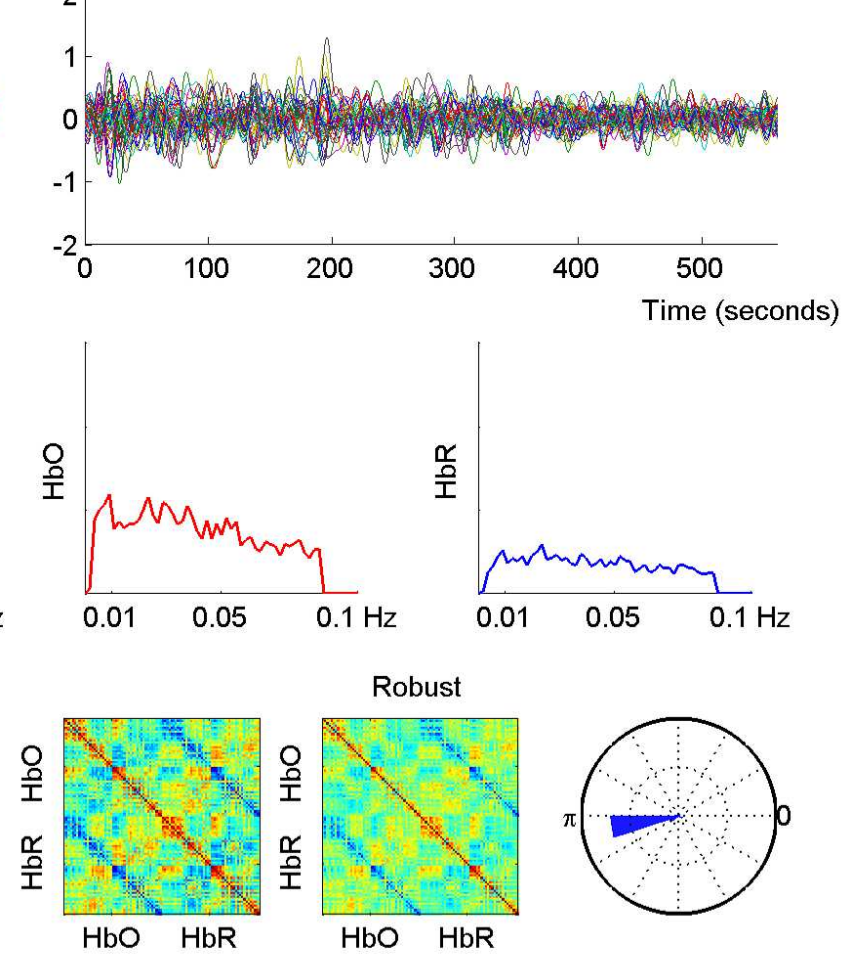

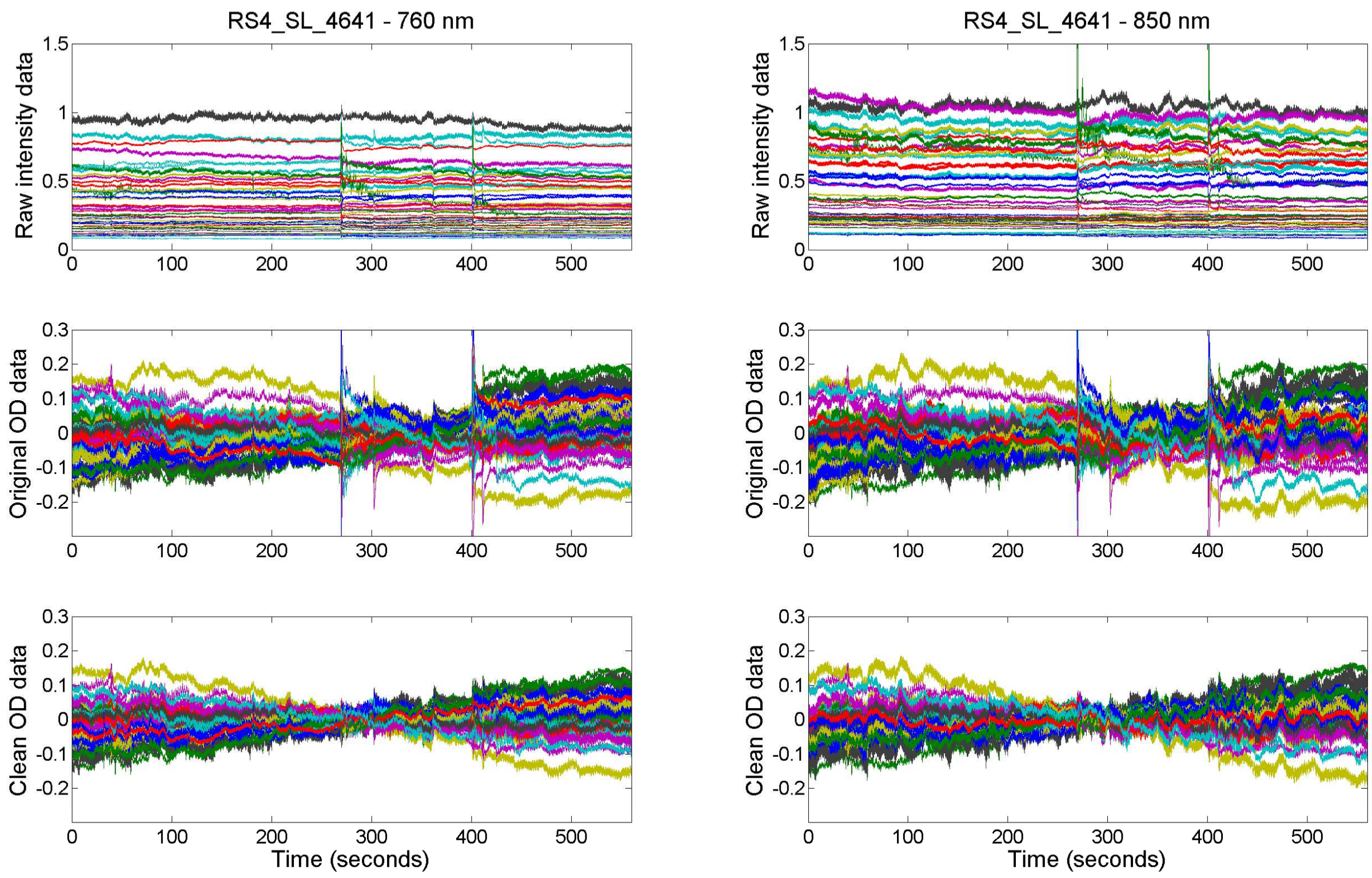

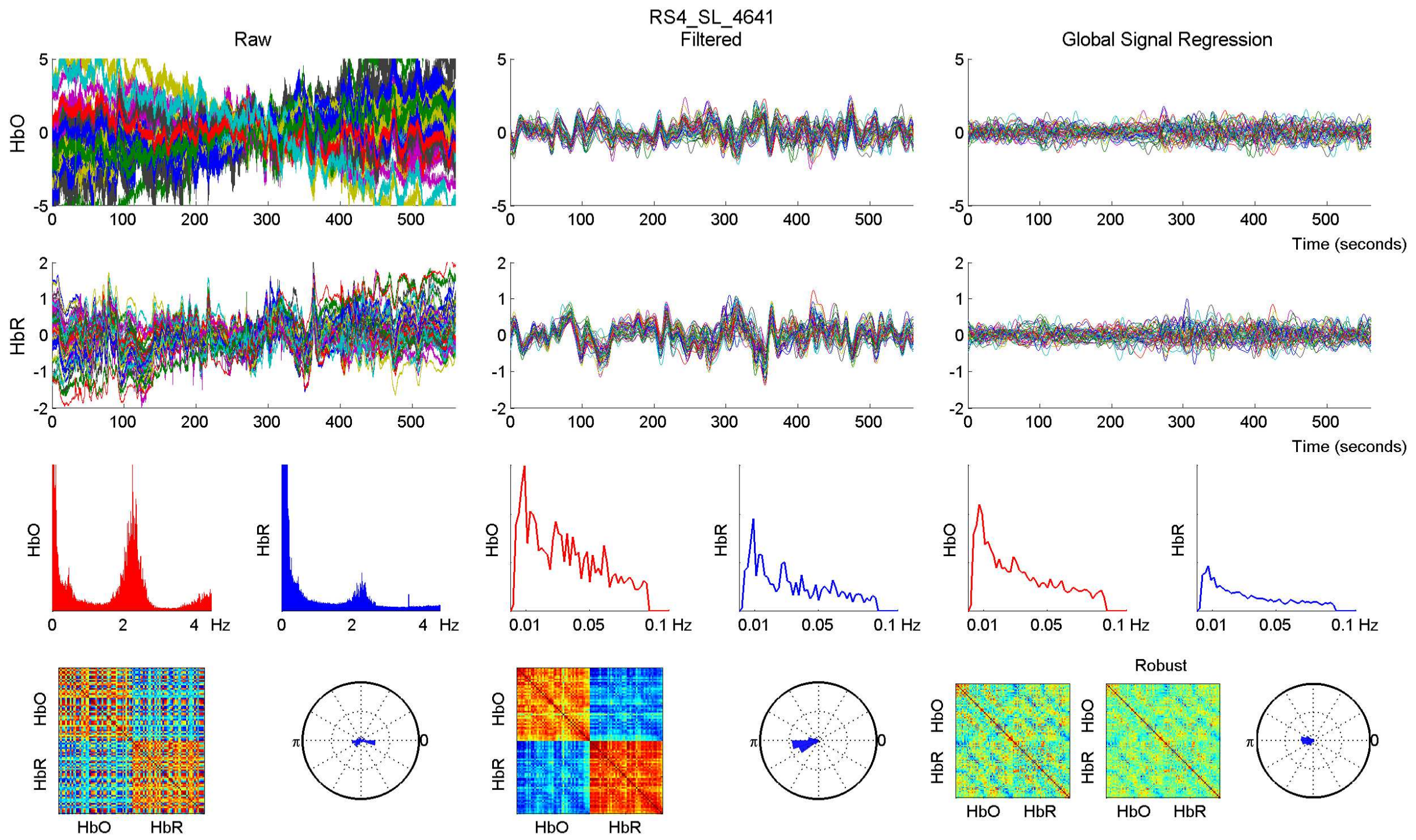
RS4 SL $4618-760$ nm
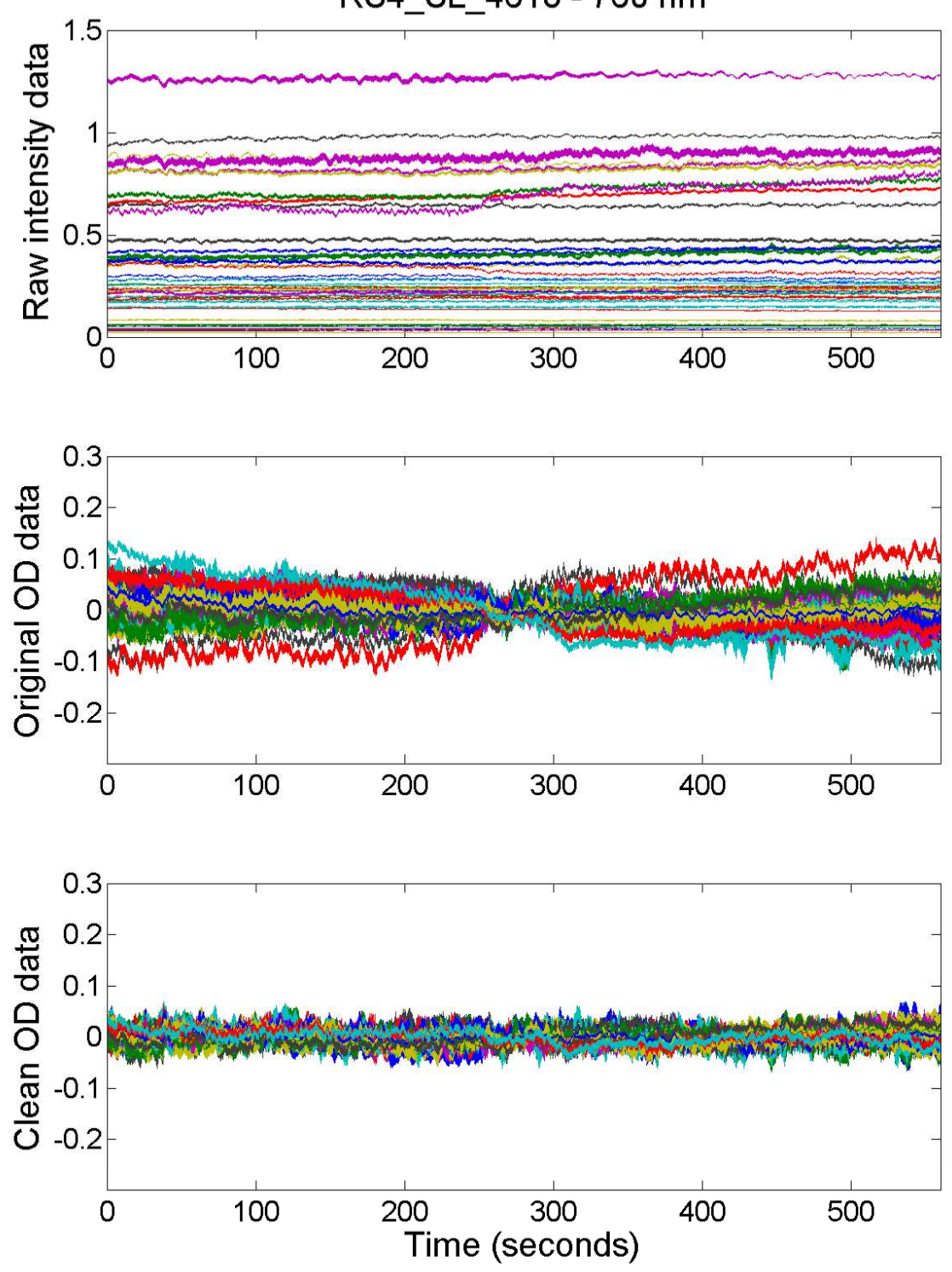

RS4 SL $4618-850 \mathrm{~nm}$
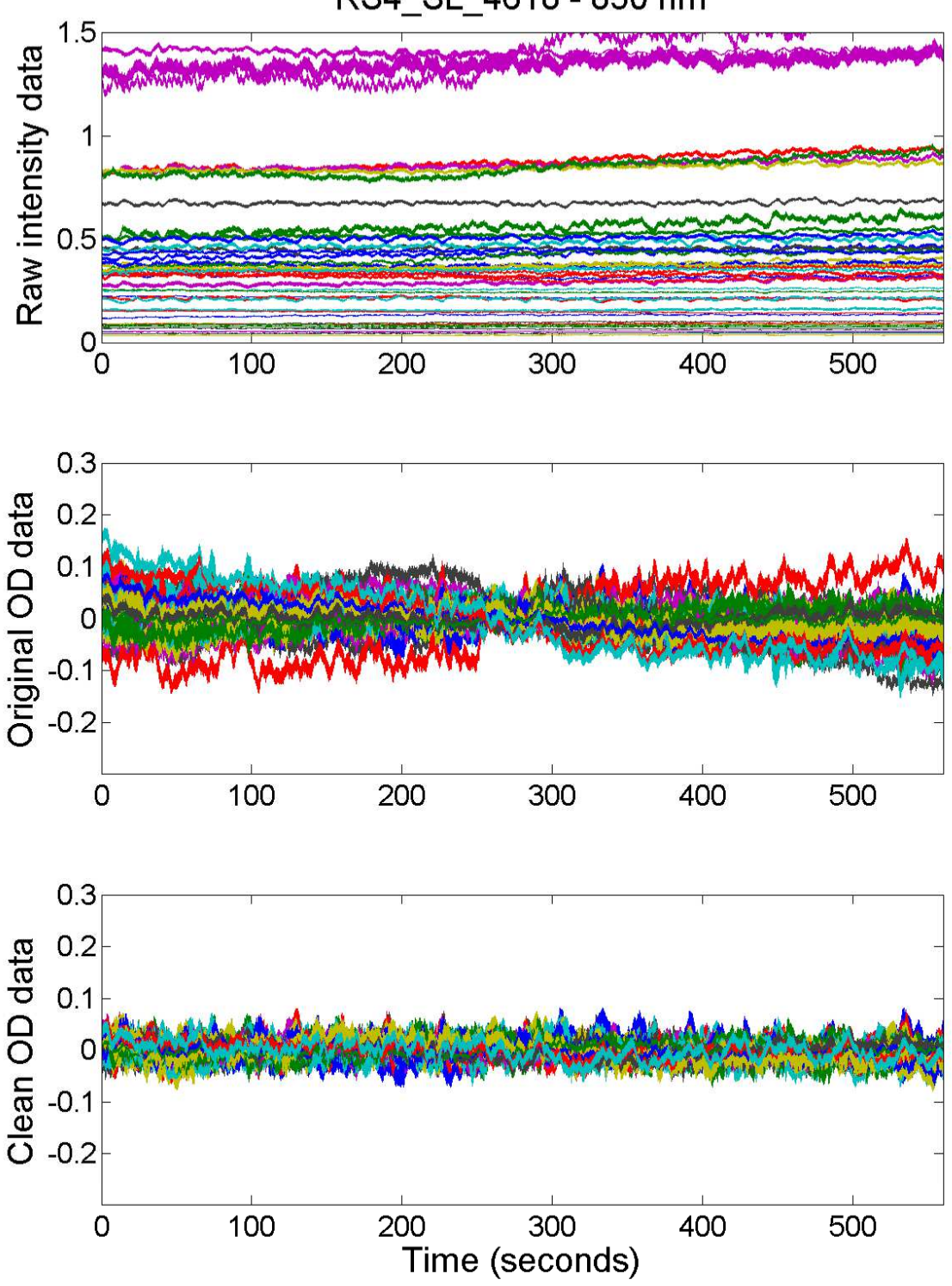
Raw

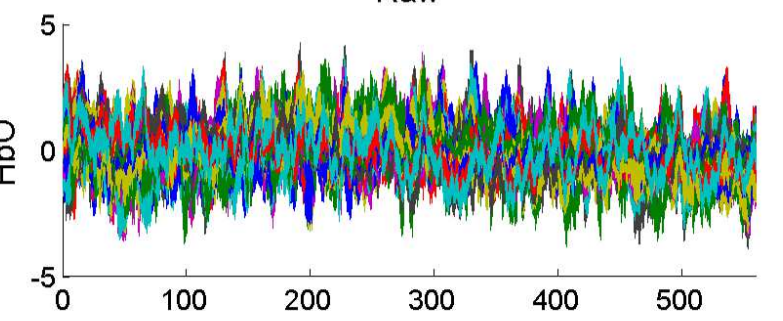

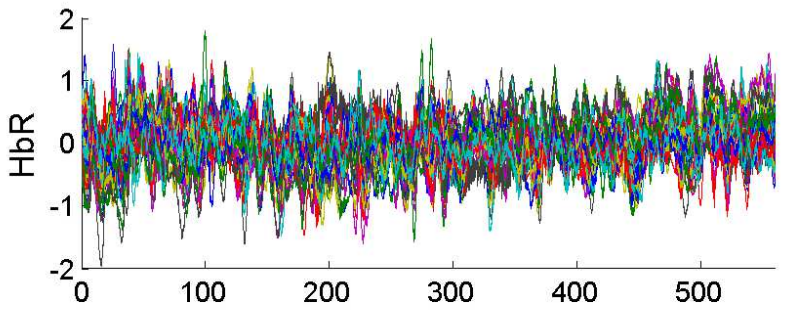
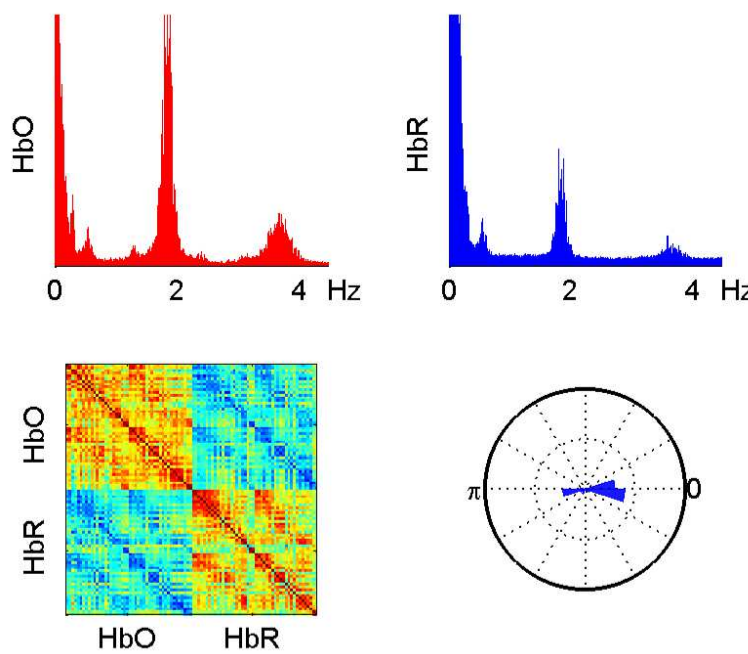

RS4 SL 4618

Filtered
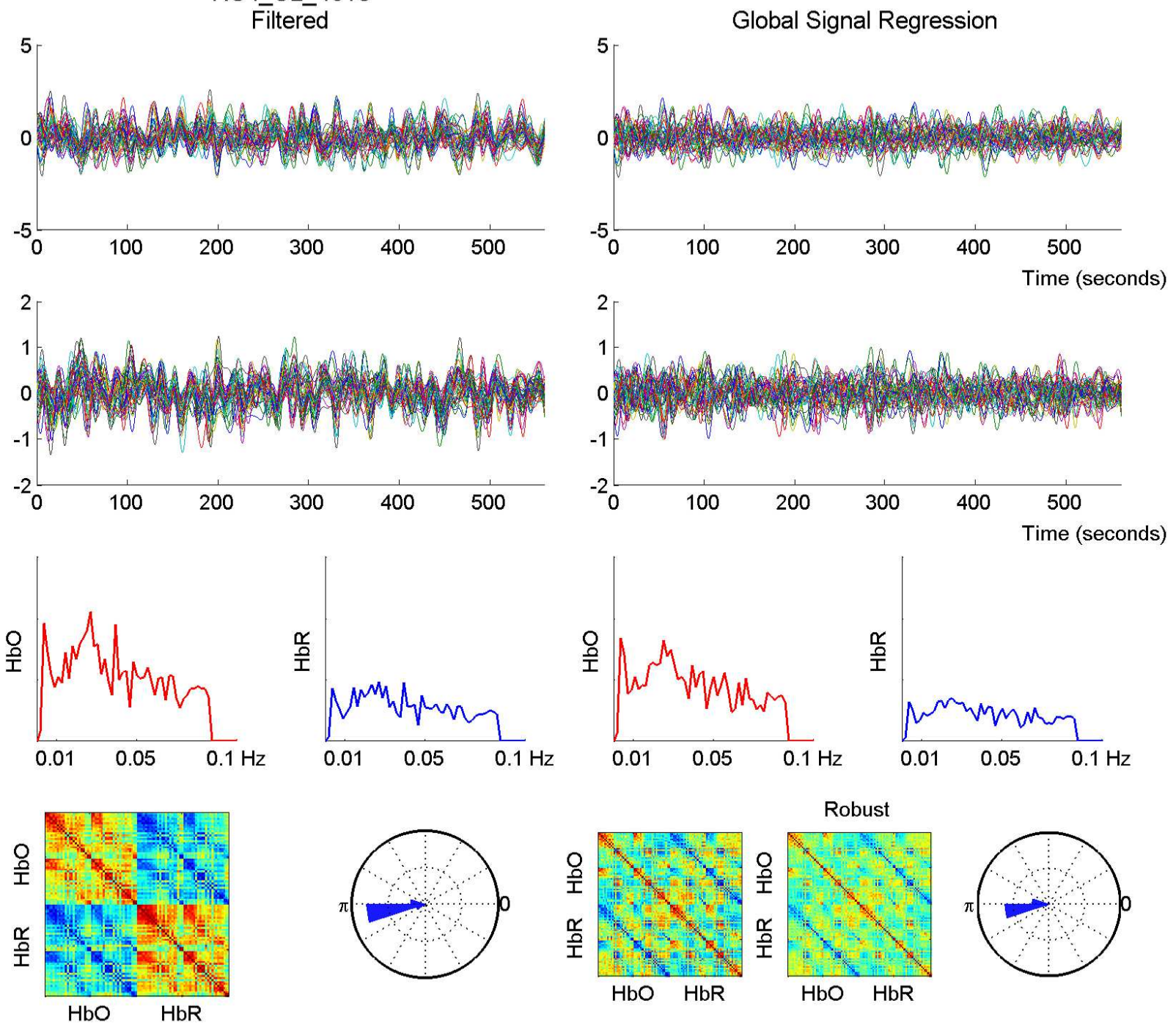

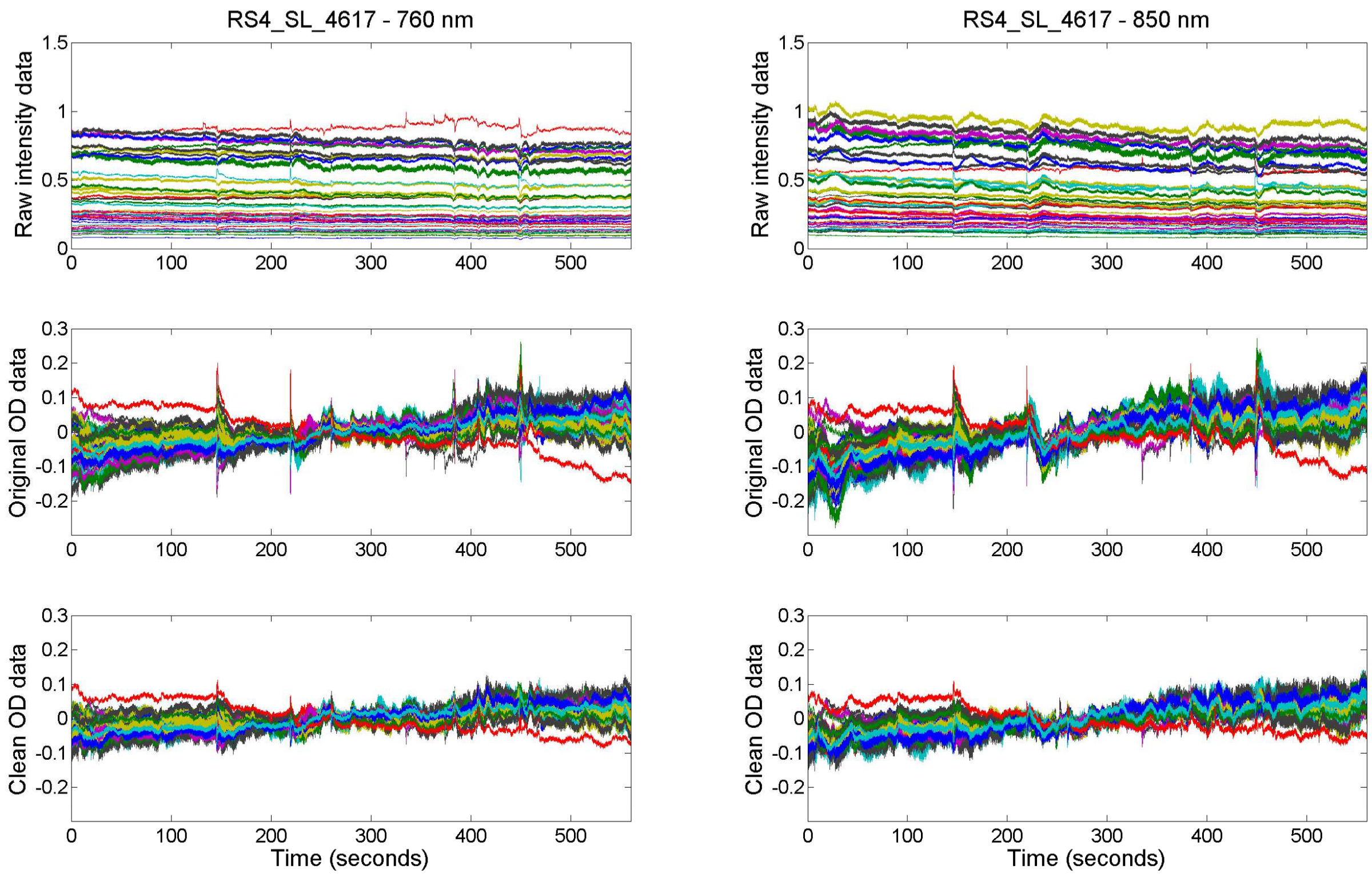
Raw
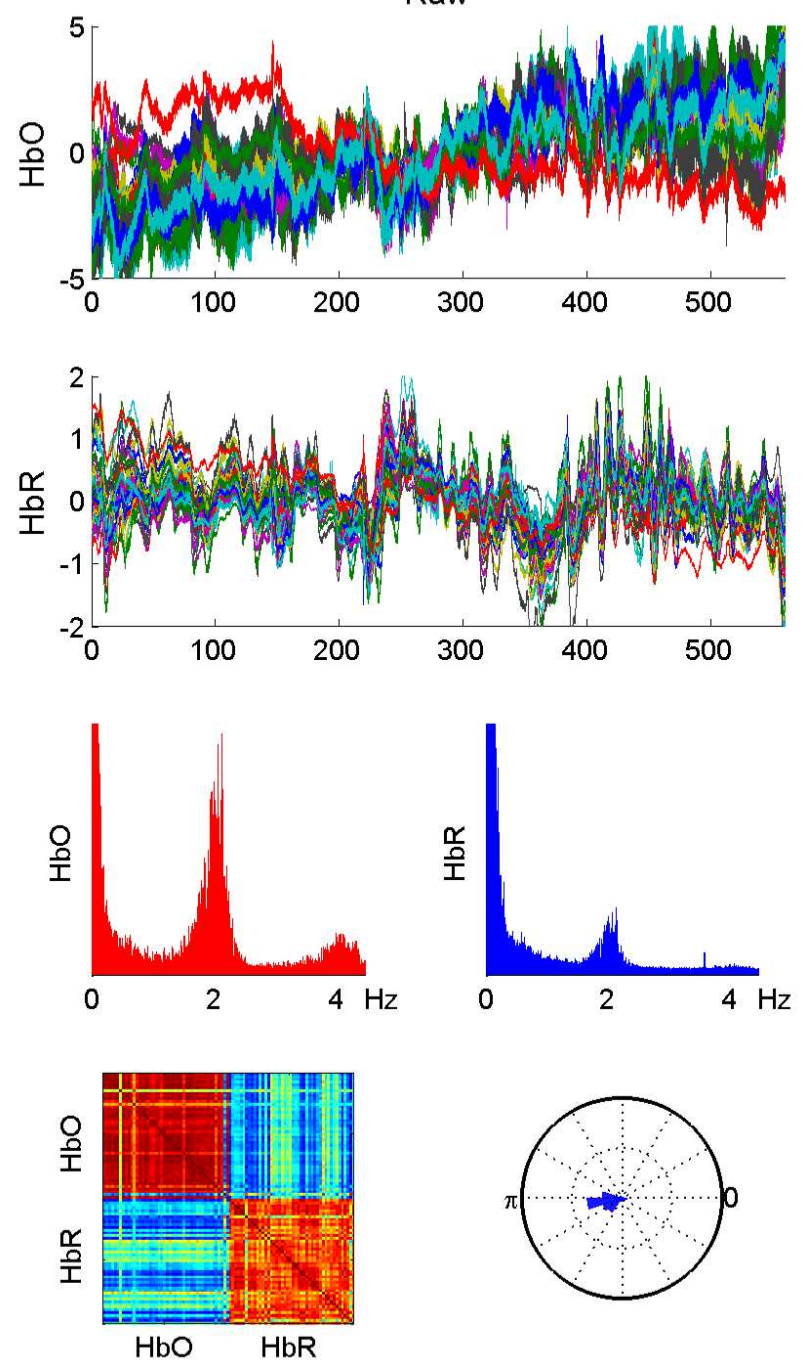

RS4_SL_4617

Filtered
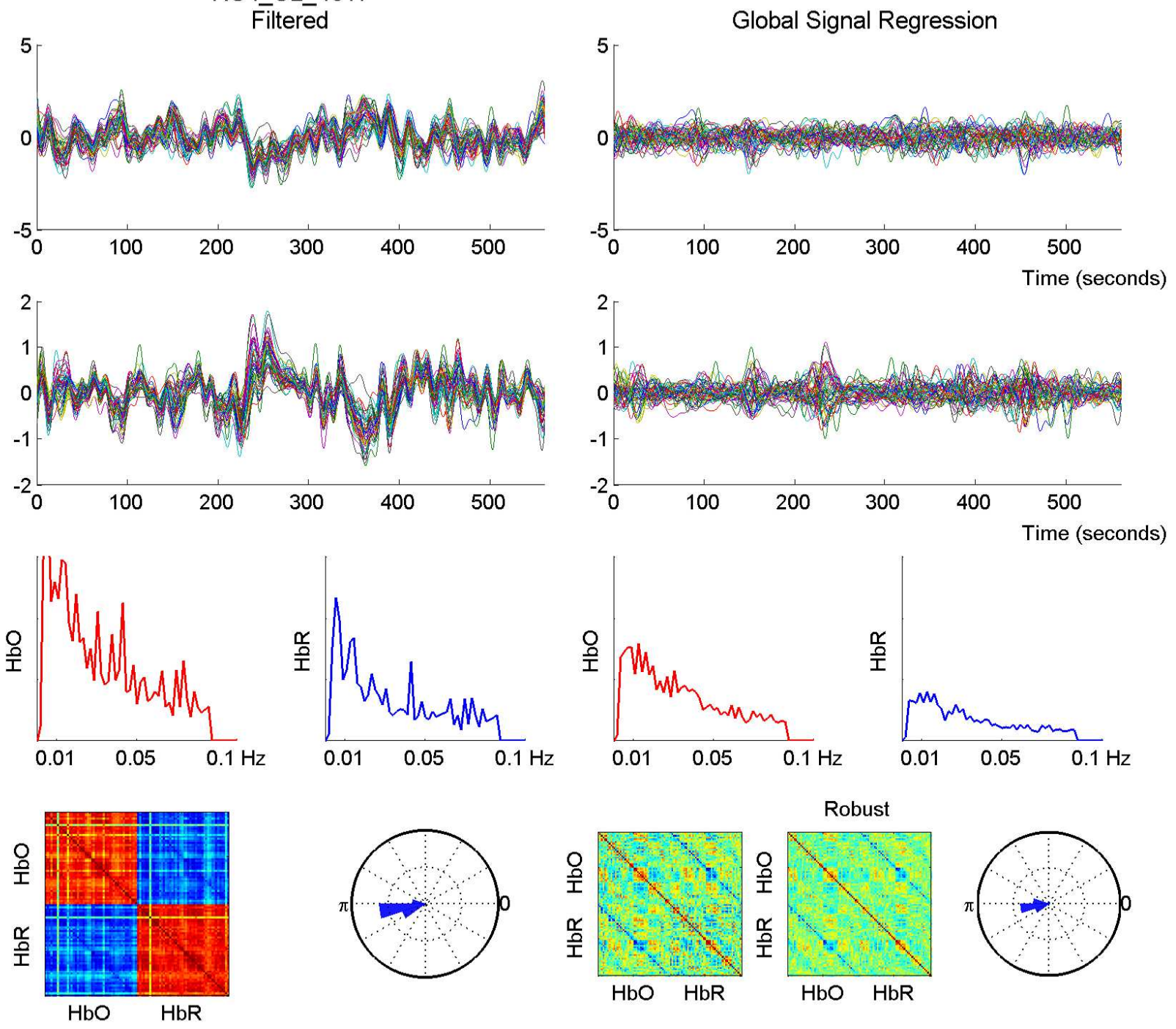

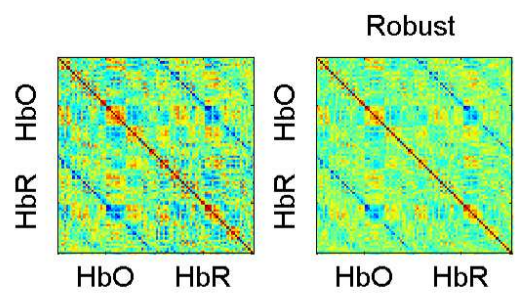

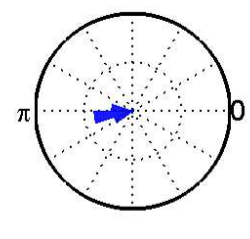


RS4 SL 4615 - 760 nm
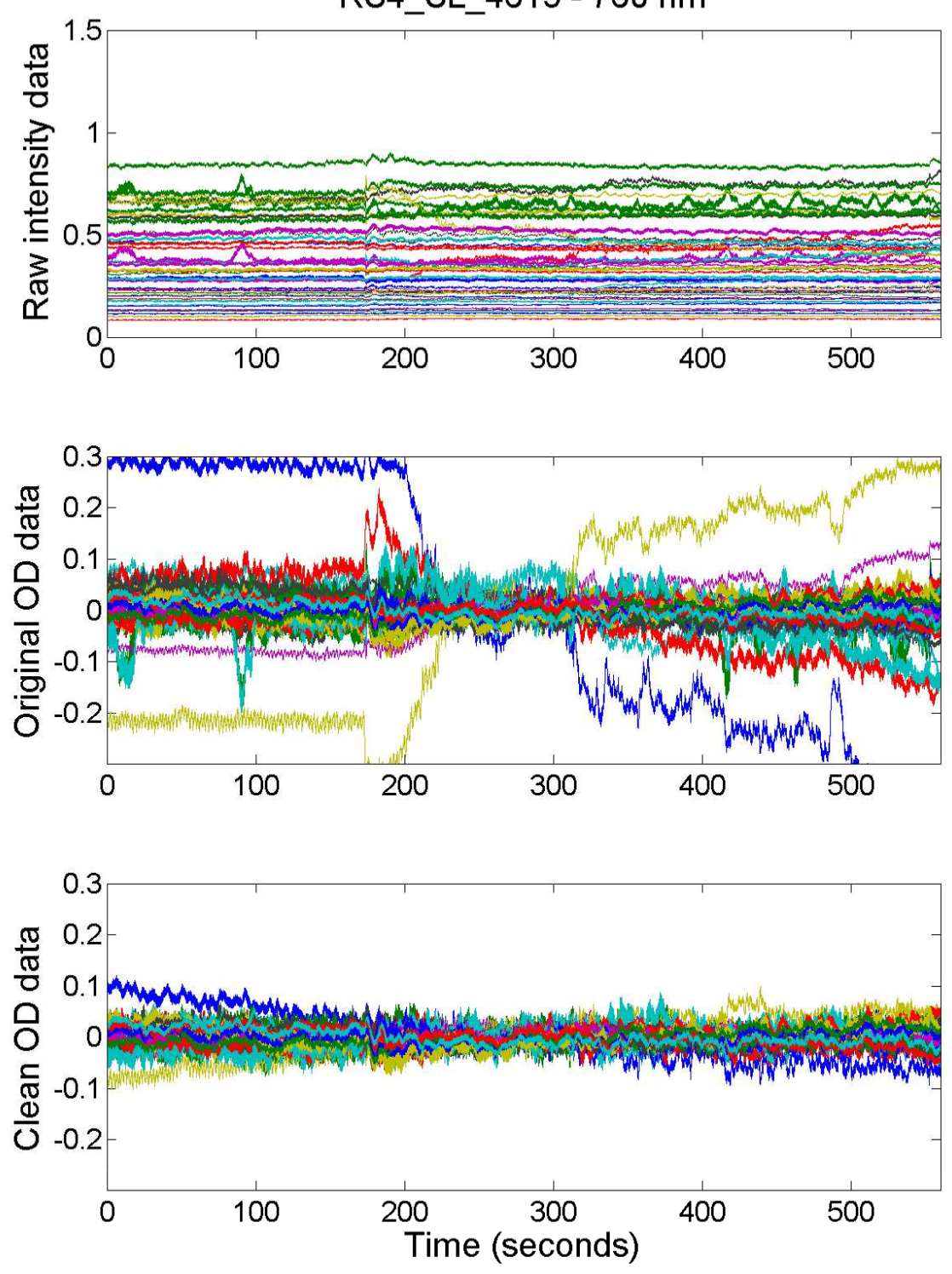

RS4 SL $4615-850 \mathrm{~nm}$
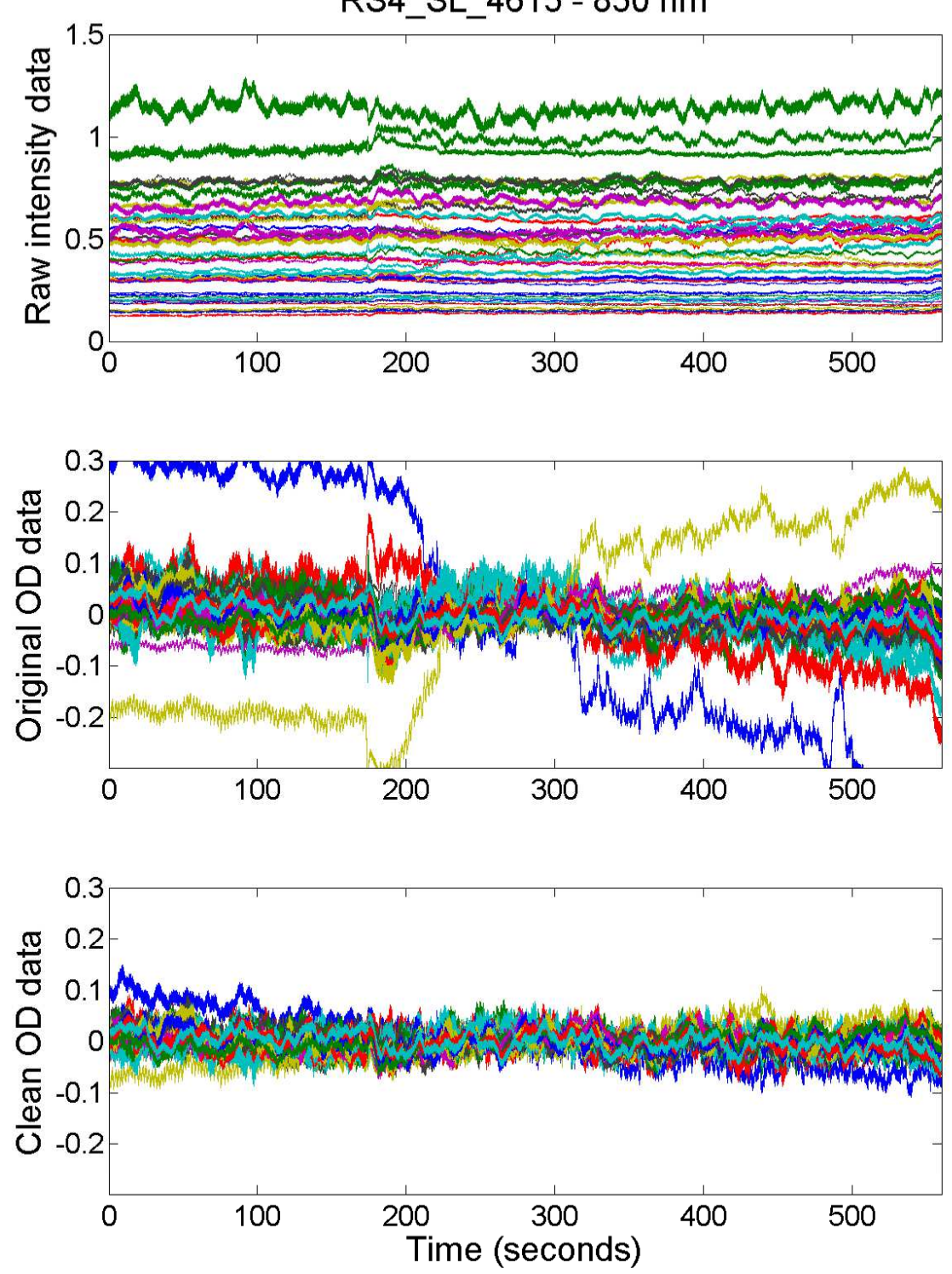
Raw

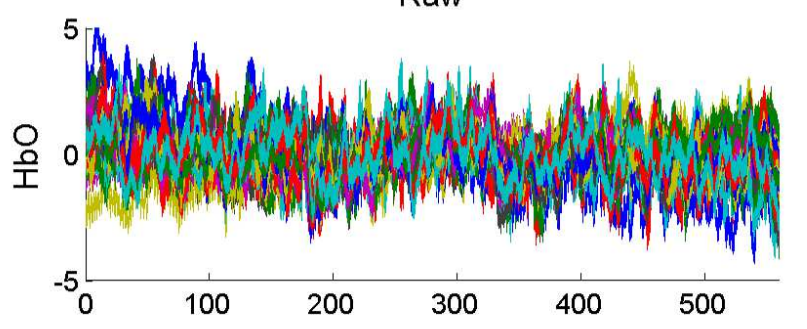

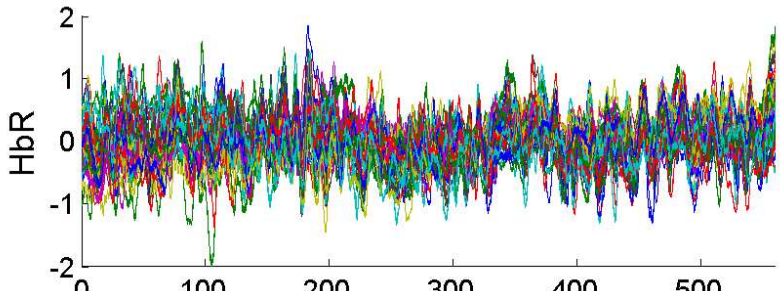
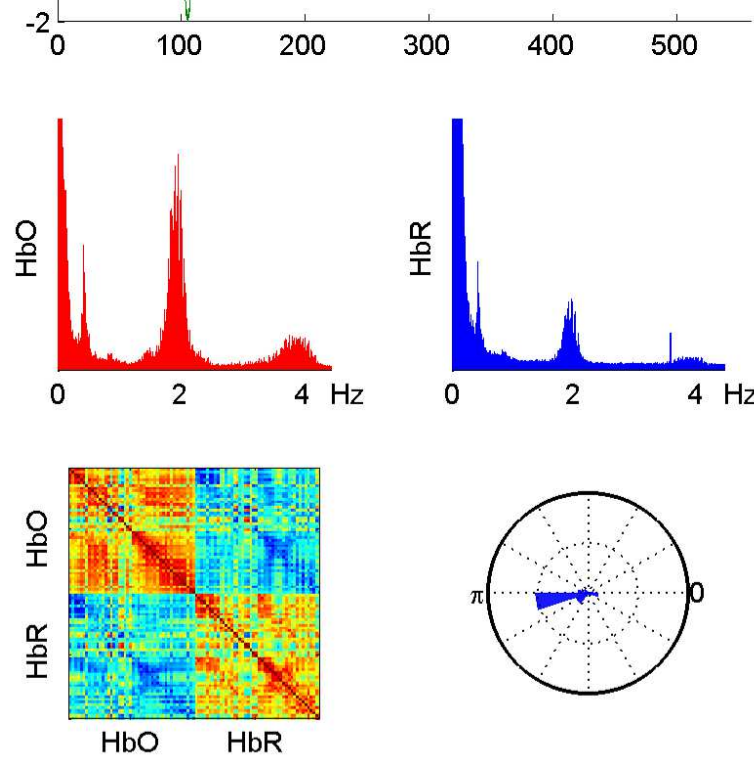
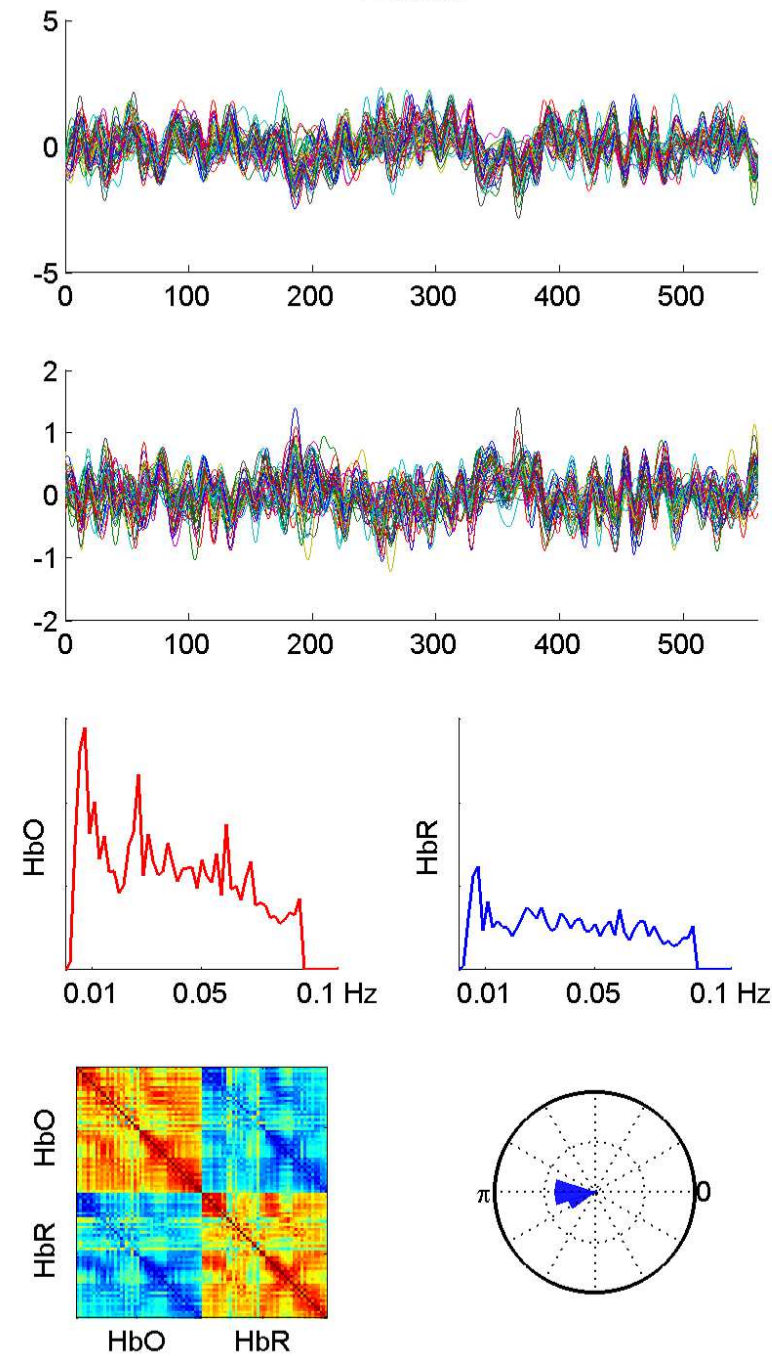

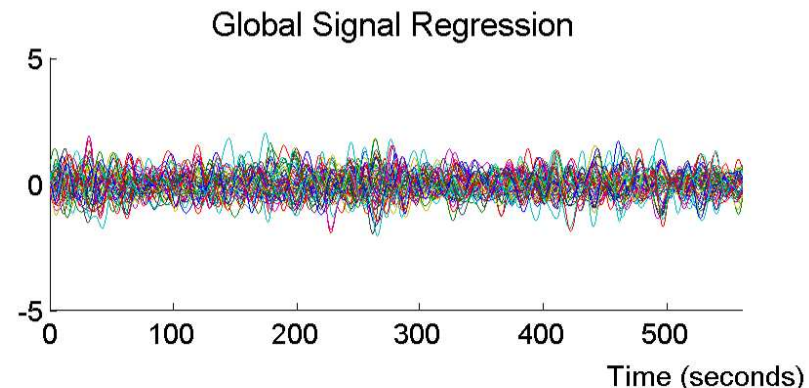

2
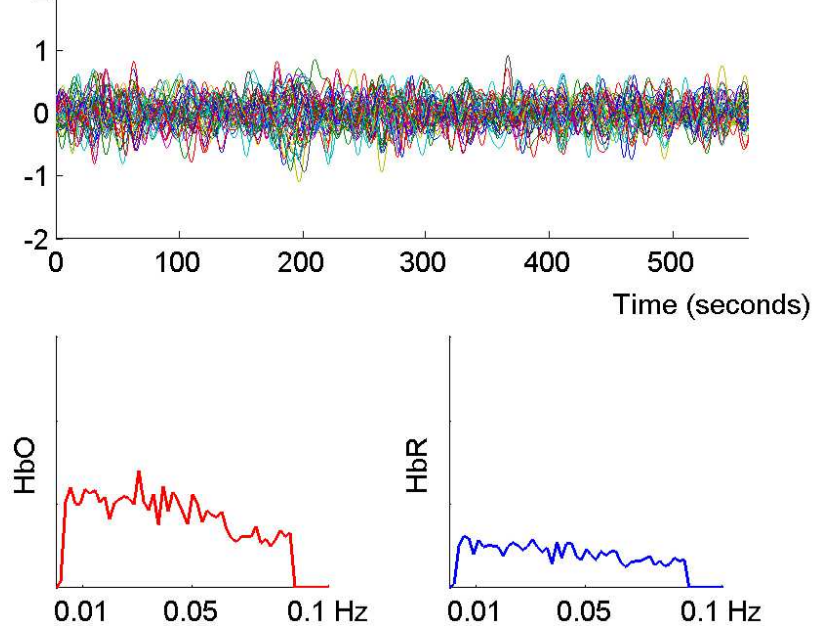
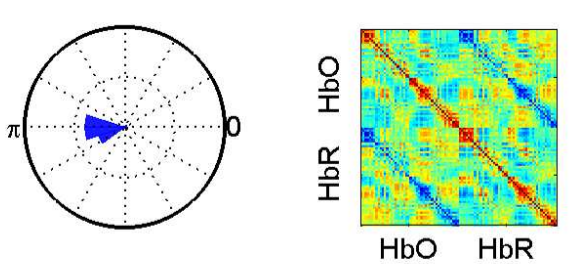
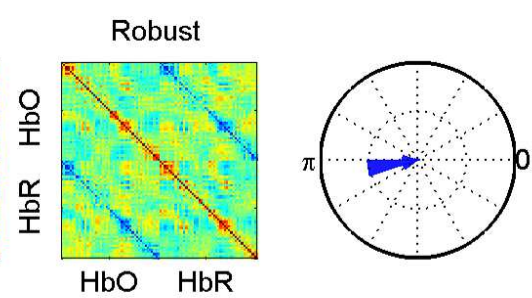

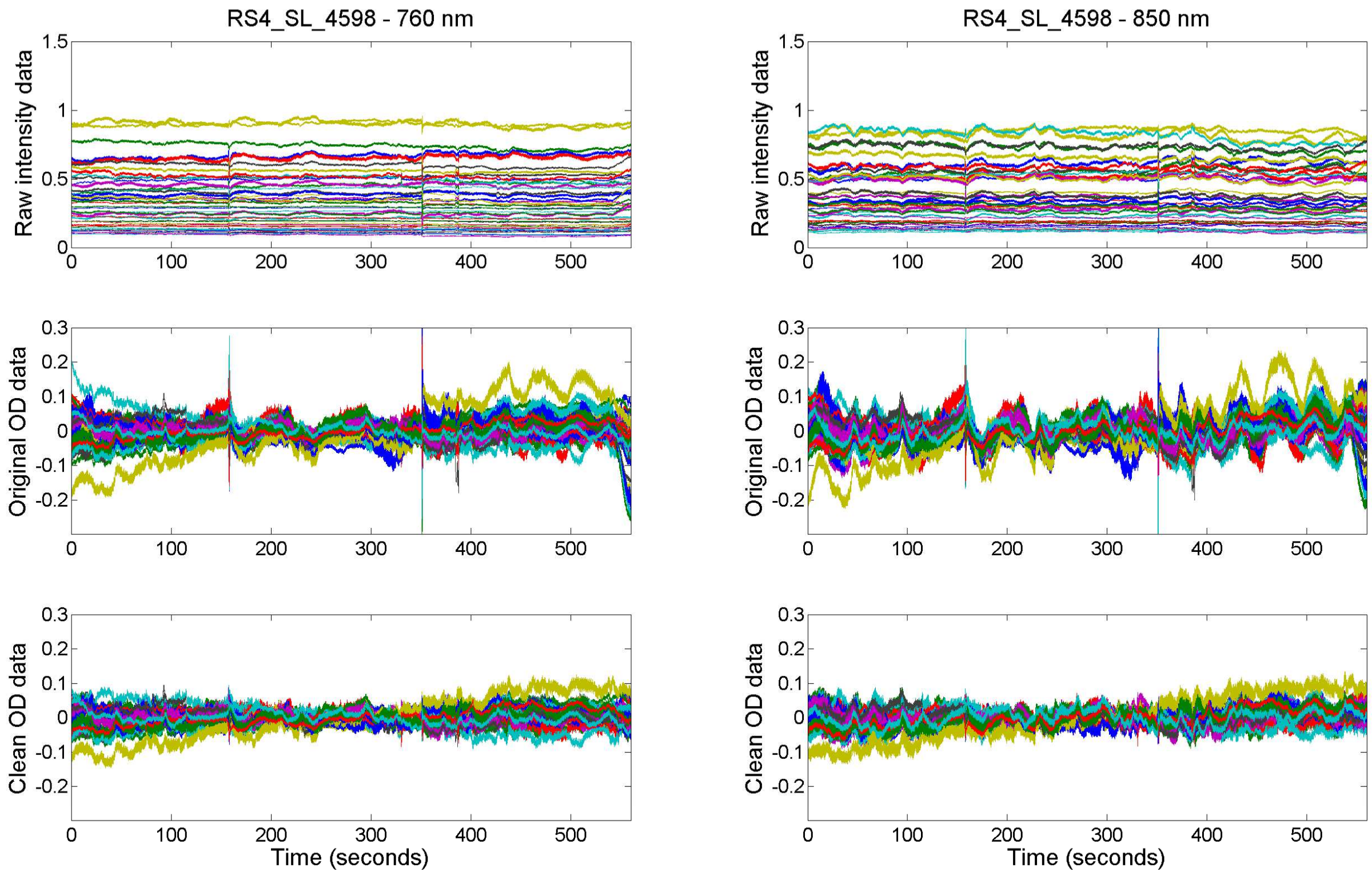
Raw
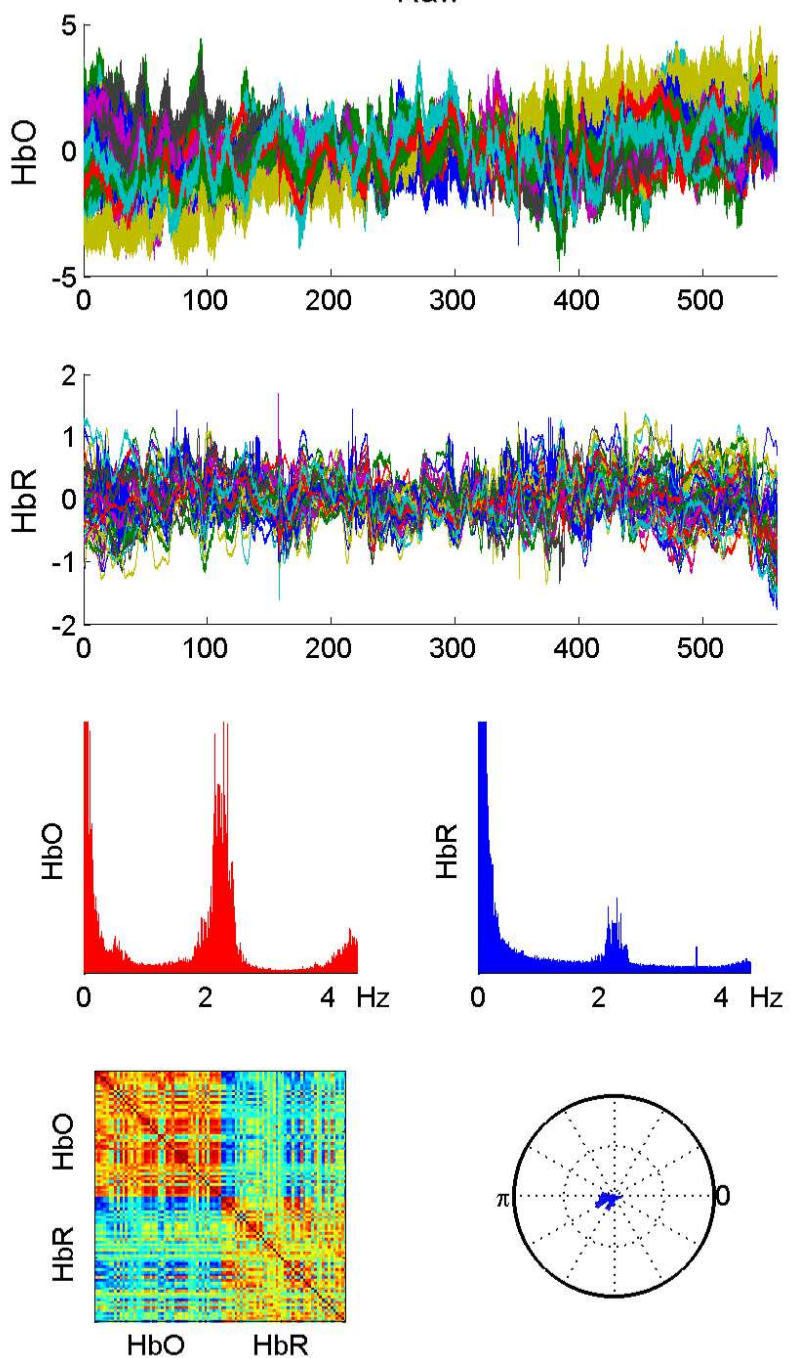

RS4 SL 4598

Filtered
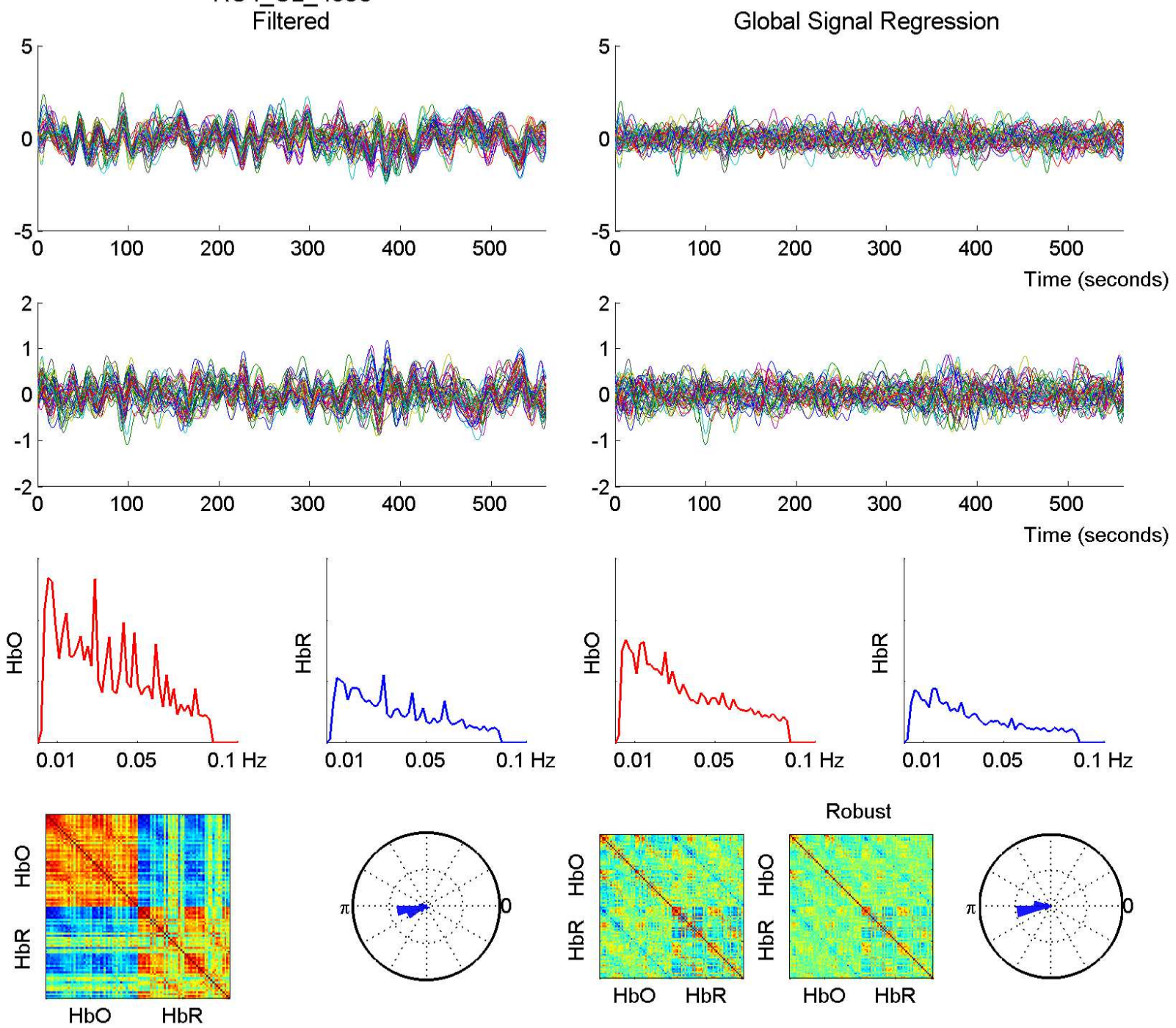
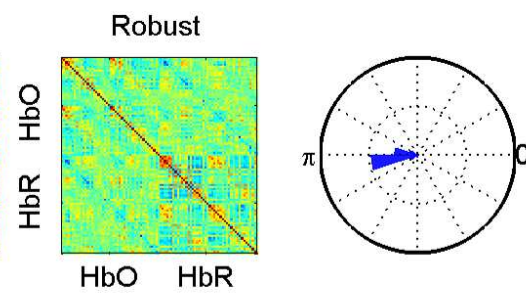

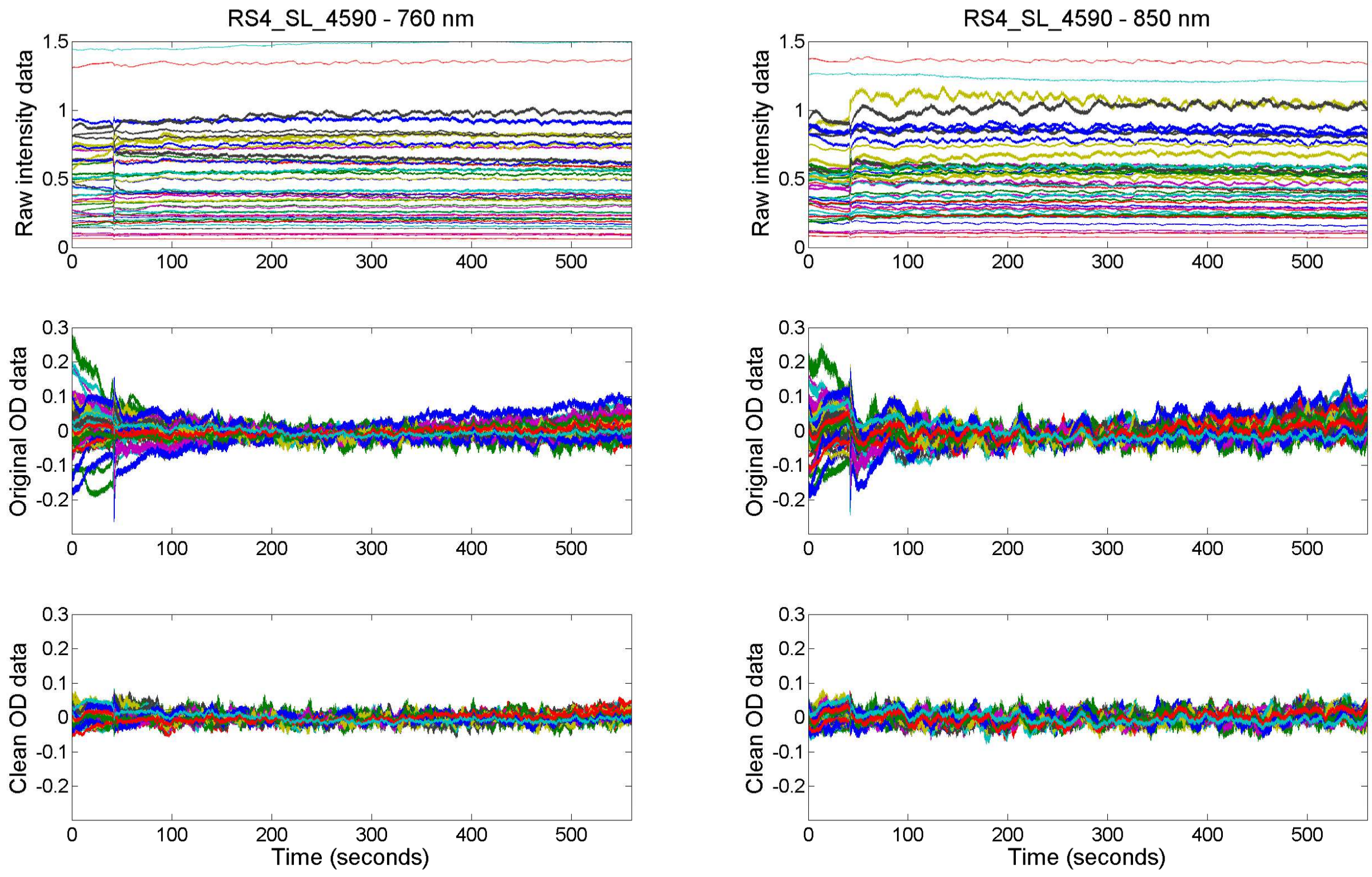
Raw

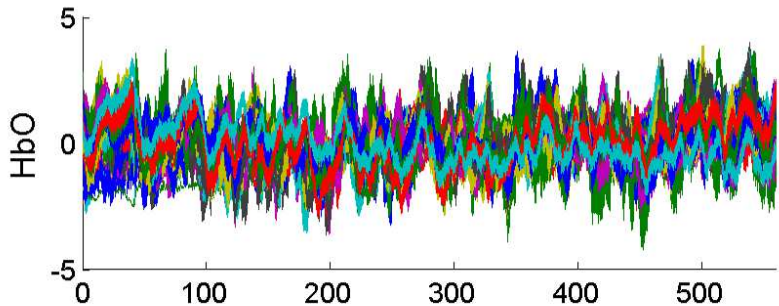

2

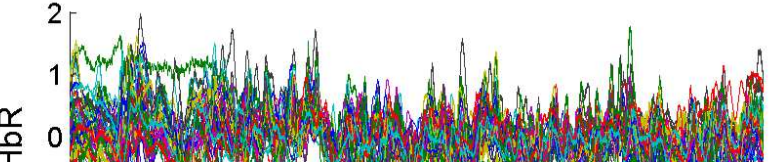

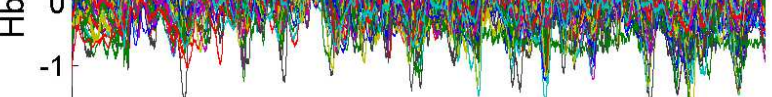
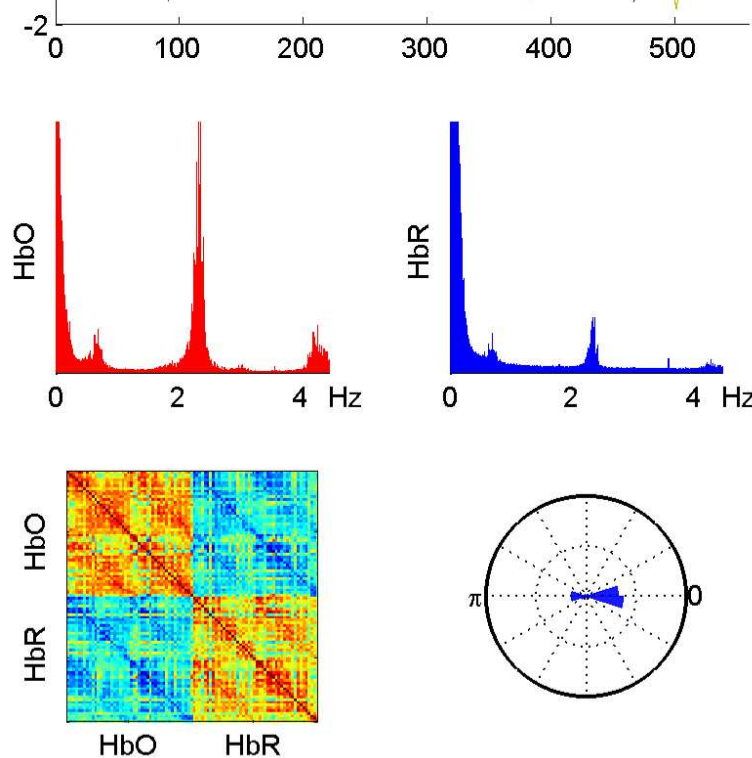

RS4 SL 4590

Filtered
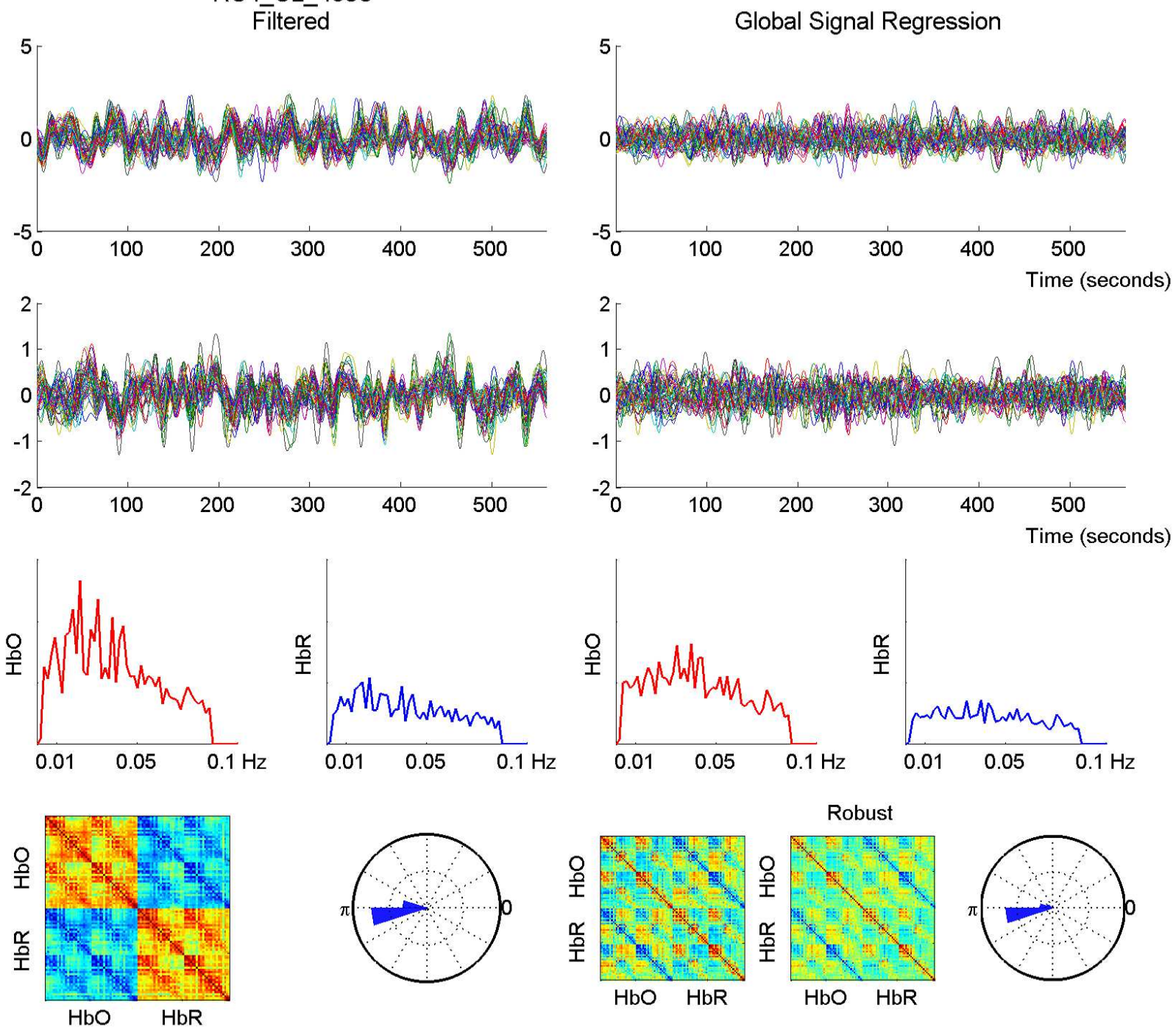

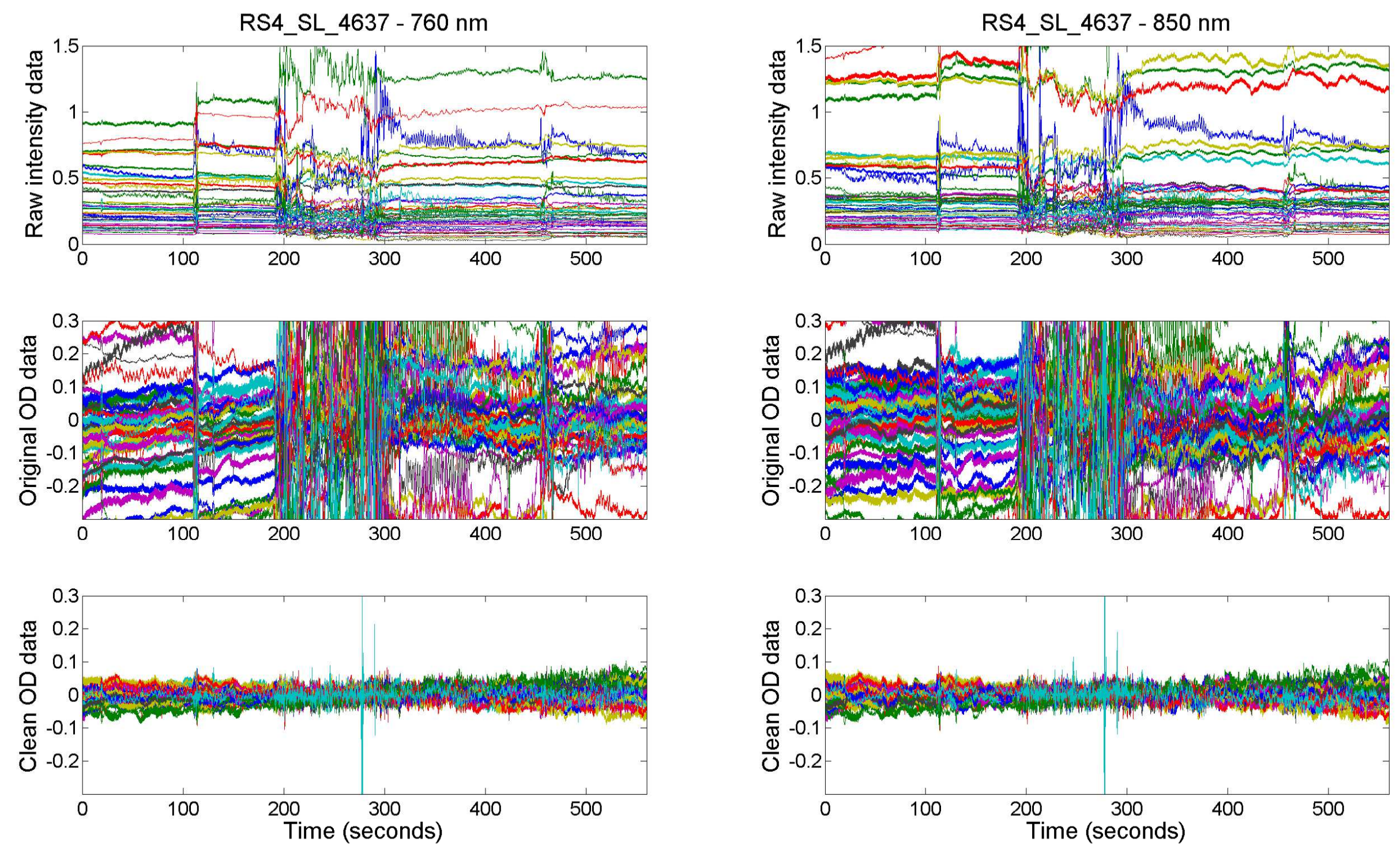

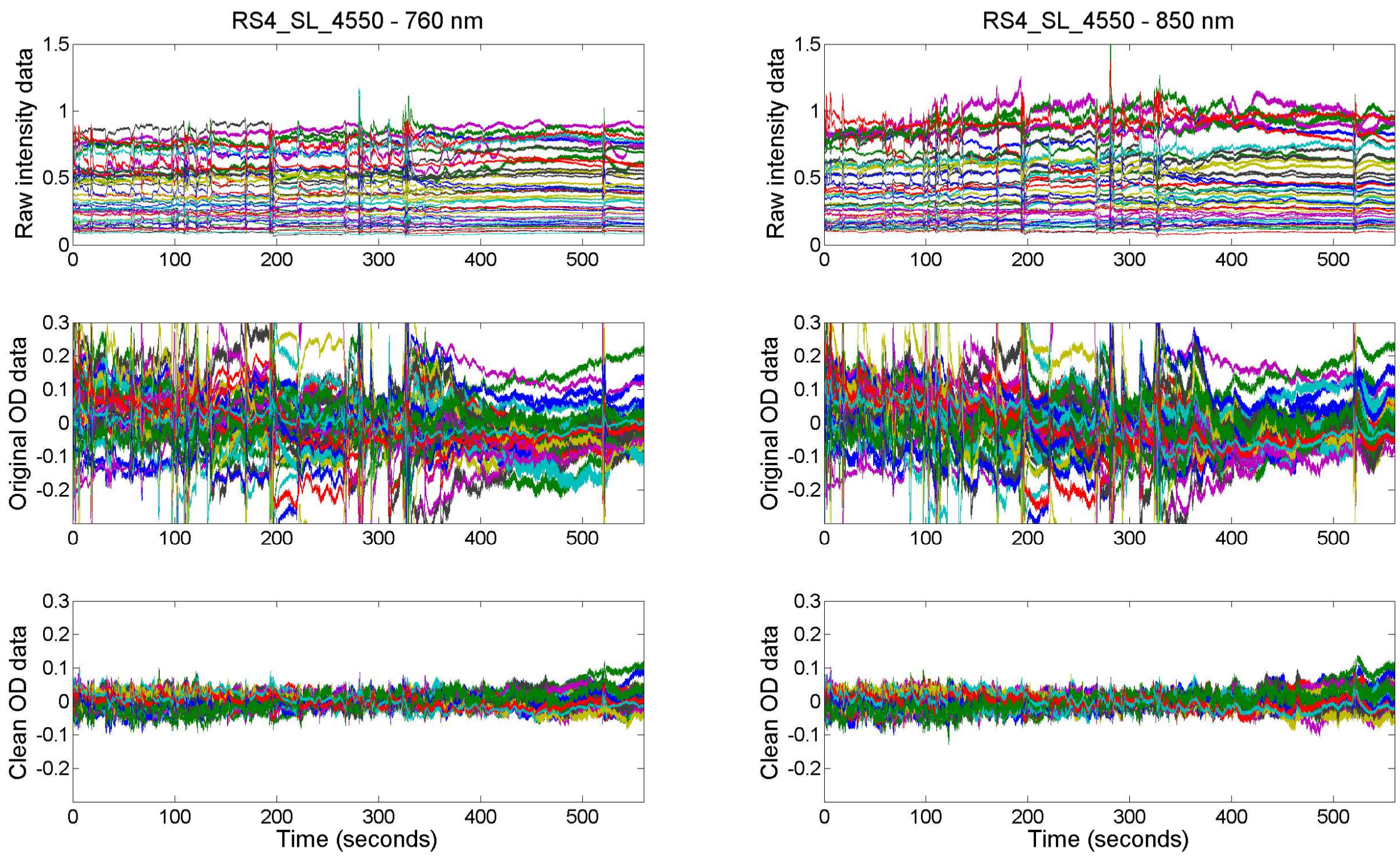

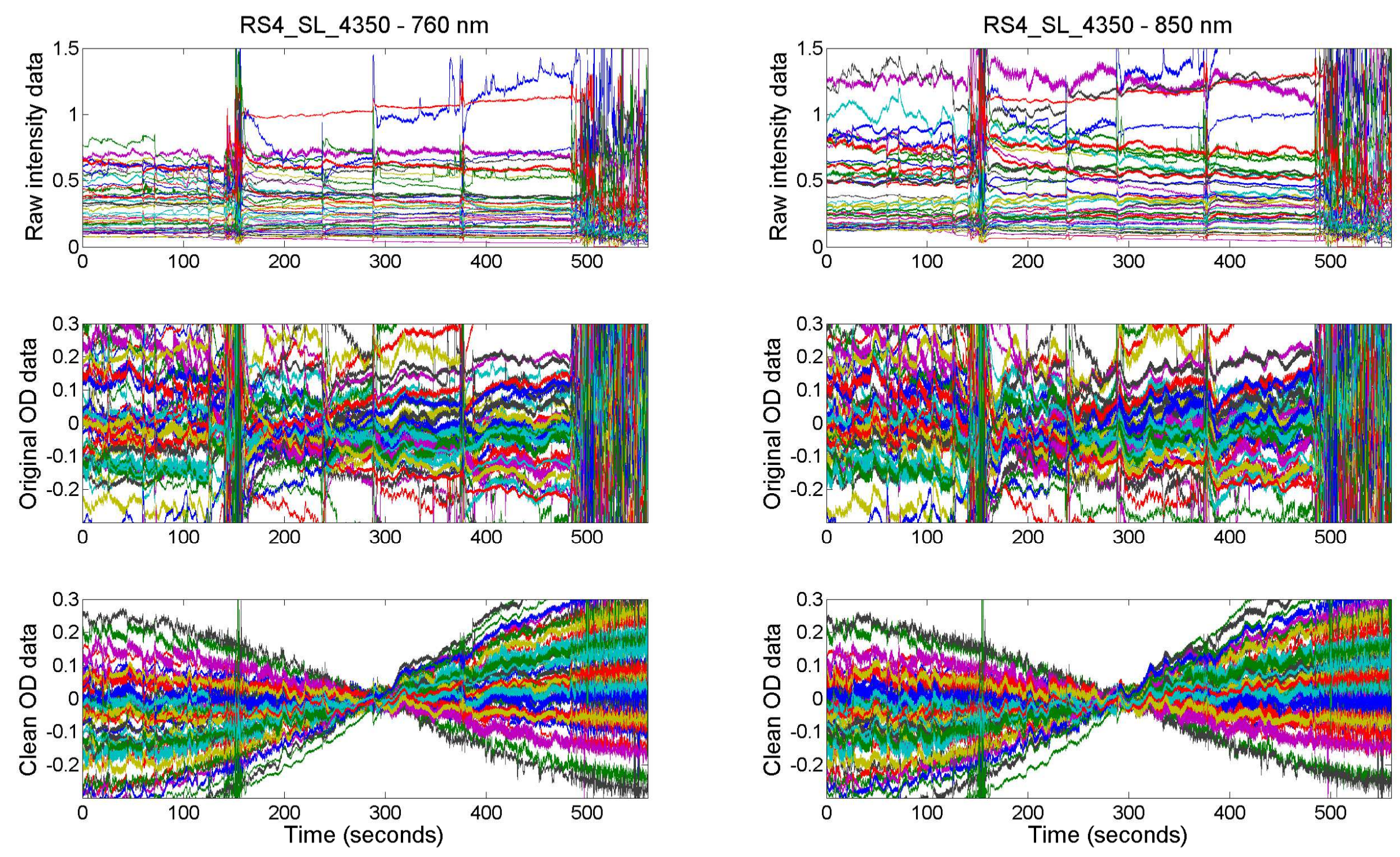

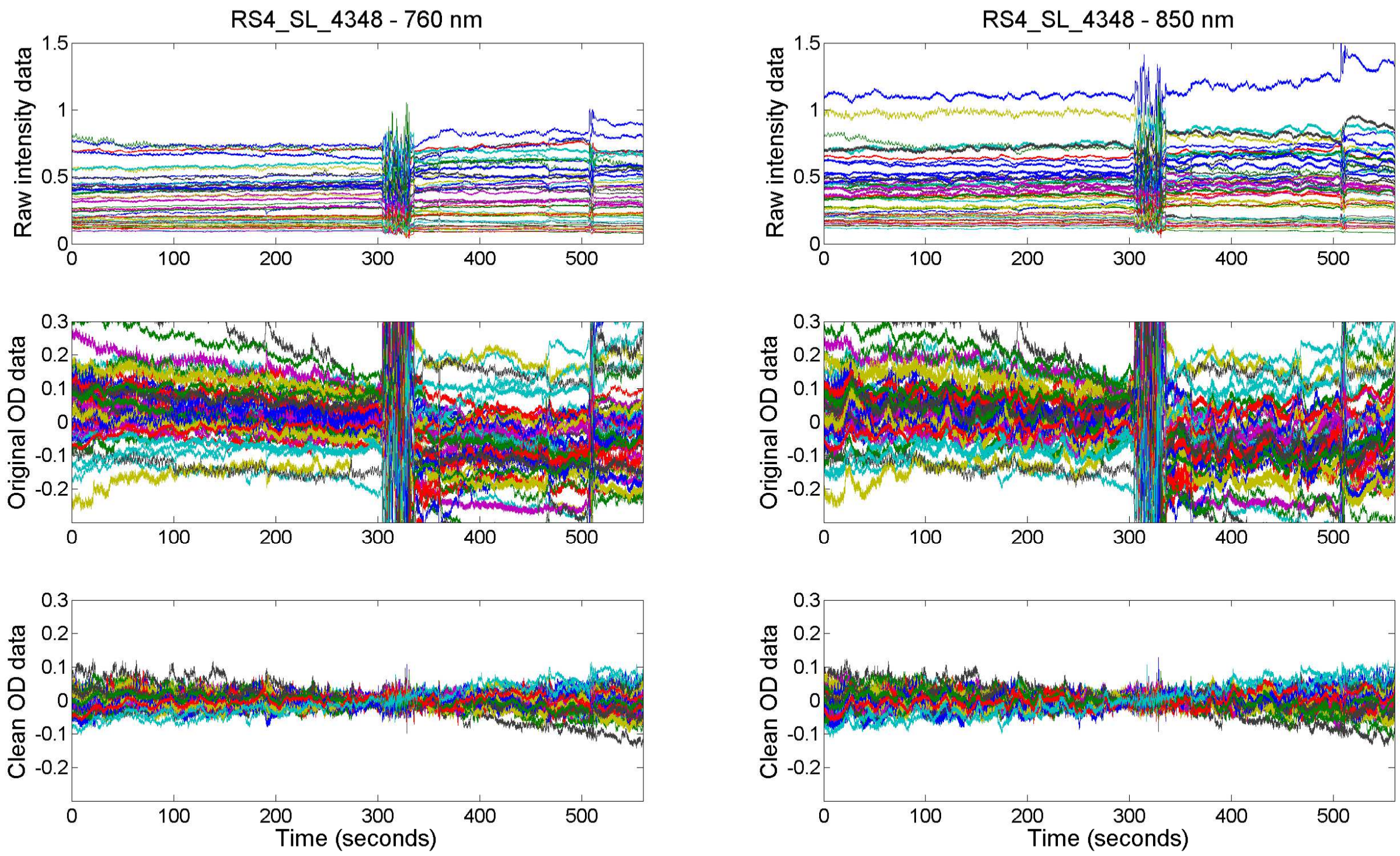

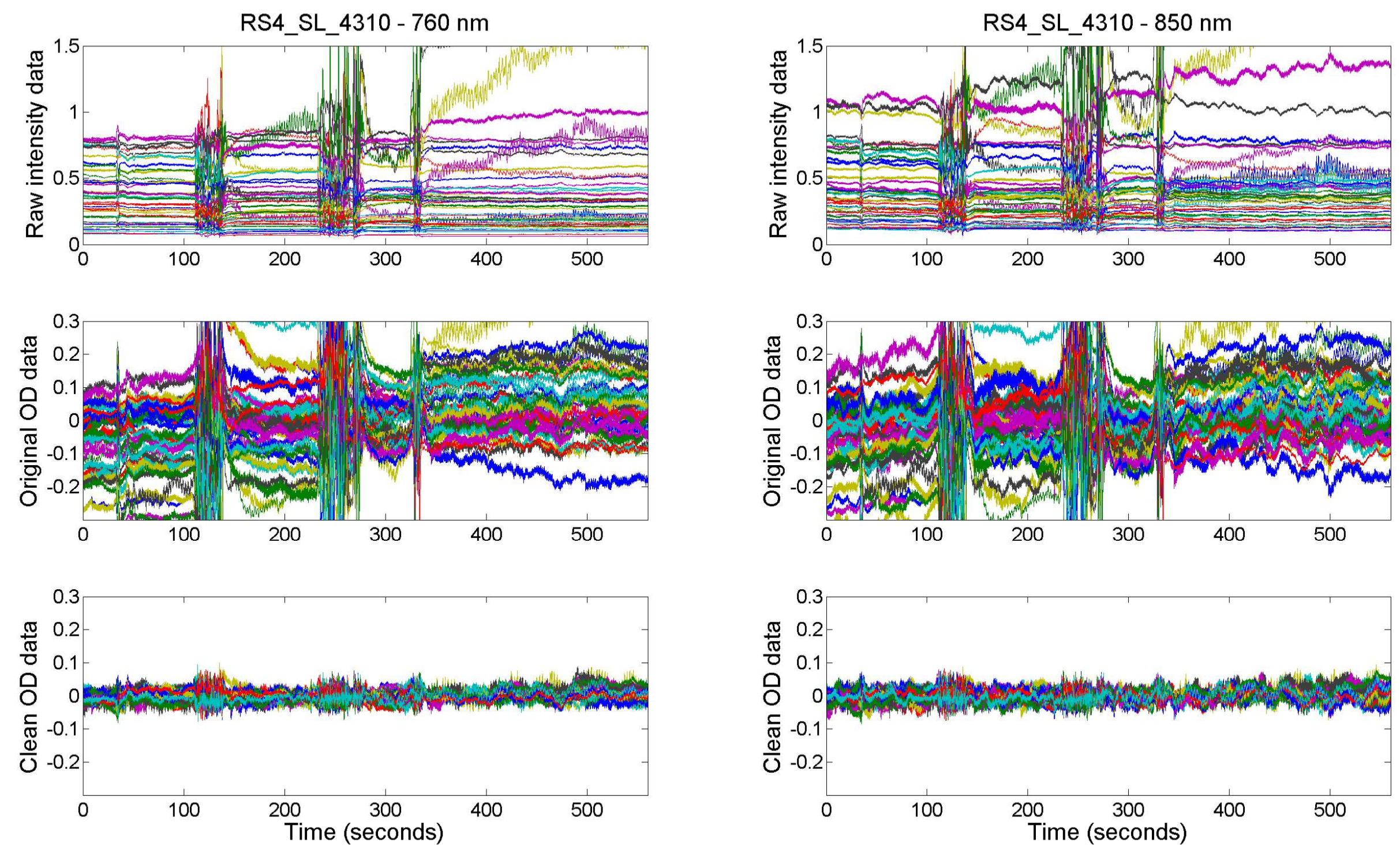